\title{
Verformungsbezogene mittragende Breite niedriger Verbundträger
}

\author{
Von der Fakultät Bau- und Umweltingenieurwissenschaften \\ der Universität Stuttgart zur Erlangung der Würde eines Doktors der \\ Ingenieurwissenschaften (Dr.-Ing.) genehmigte Abhandlung
}

vorgelegt durch

Andreas Rieg

aus Schwäbisch Gmünd

\begin{abstract}
Hauptberichter: Prof. Dr.-Ing. Ulrike Kuhlmann
Mitberichter: $\quad$ Prof. Dr.-Ing. Jörg Lange
\end{abstract}

Tag der mündlichen Prüfung: 26. Juni 2006

Institut für Konstruktion und Entwurf der Universität Stuttgart 
Mitteilungen des Instituts für Konstruktion und Entwurf; Nr. 2006-2

Andreas Rieg

Verformungsbezogene mittragende Breite niedriger Verbundträger

Herausgeber

Prof. Dr.-Ing. Ulrike Kuhlmann

Pfaffenwaldring 7

70569 Stuttgart

Telefon: (0711) 68566245

Telefax: (0711) 68566236

Redaktion Matthias Konrad

D 93

(c) Institut für Konstruktion und Entwurf; Stuttgart 2006

Alle Rechte vorbehalten

ISSN 1439-3751 


\section{Kurzdarstellung}

Aufgrund der vergleichsweise großen Schlankheit von Verbundträgern niedriger Bauhöhe wird bei diesen Trägern in sehr vielen Fällen die Einhaltung von Verformungsgrenzwerten zum querschnittsbestimmenden Aspekt. Das Verformungsverhalten dieser Träger wird dabei maßgeblich durch die große Biegesteifigkeit des Betongurts und dessen Rissbildung bestimmt. Einer der maßgebenden Einflüsse auf die rechnerischen Verformungen ist die mittragende Breite des Betongurts, dessen Wert durch die bestehenden Normregeln ohne Berücksichtigung des speziellen Trag- und Verformungsverhaltens niedriger Verbundträger abgeschätzt wird.

In der vorliegenden Arbeit wird daher das Verformungsverhalten und die mittragende Breite niedriger Verbundträger unter Berücksichtigung der Biegetragwirkung und der Rissbildung des Betongurts untersucht. Diese Untersuchungen zeigen unter anderem, dass für die Spannungs- und die Verformungsberechnung unterschiedliche Werte der mittragenden Breite anzusetzen sind. Anhand umfangreicher experimenteller, analytischer und numerischer Untersuchungen werden die Einflüsse auf die mittragende Breite analysiert. Auf Basis der Ergebnisse einer ausführlichen Parameterstudie für den Bereich des Hoch- und Geschossbaus wird ein Berechnungsansatz für die verformungsbezogene mittragende Breite entwickelt und ein Vorschlag zur quasi-elastischen Verformungsberechnung niedriger Verbundträger gemacht. Es zeigt sich, dass die verformungsbezogene mittragende Breite im Besonderen von der Biegetragwirkung des Betongurts, dessen Rissbildung, dem Verhältnis von Gurtbreite zu Trägerlänge, der Steifigkeitsverteilung im Verbundträger und der Belastungshöhe abhängt.

Mit dem entwickelten Berechungsansatz ist eine realistische Verformungsberechung und wirtschaftliche Dimensionierung niedriger Verbundträger möglich.

\section{Abstract}

Due to the high slenderness of composite girders with shallow height their deflection behaviour is of great importance. In many cases the cross sectional dimensions are determined by the limitation of the deflections. In contrary to high composite beams the deflection behaviour of these girders is influenced especially by the bending state and cracking behaviour of the concrete slab. Among others one main parameter for these is the effective width of the slab, whereas the rules for the calculation of the effective width in the corresponding codes do not take into account the special aspects of these girder types.

Therefore the presented thesis reports from the investigations of the deflection behaviour and the effective width of shallow composite girders taking into account the bending state and the cracking of the concrete slab. At first it is shown that for the calculation of stresses a different value of the effective width must be taken into account than for the calculation of deflections. On basis of extensive experimental, analytical and numerical investigations the influences on the effective width are analysed. A wide-ranging parametric study in the range of common multi-story buildings leads to the development of an approach for the calculation of the effective width due to deflections and a proposal for a quasi-elastic calculation of the deflections of shallow composite girders. At this it turns out that the effective width due to deflections is influenced above all by the bending state of the concrete slab, its cracking behaviour, its ratio width to length, the ratio of the individual stiffness components of the composite cross section and the load level.

On basis of the proposed approach for the calculation of the effective width the deflections of shallow composite girders can be determined realistically. This leads to a more economic design of these girder types than in the common practice. 



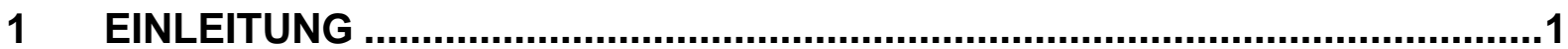

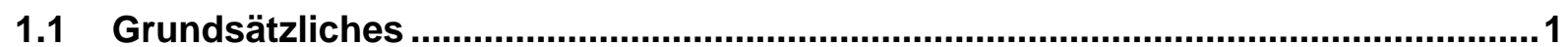

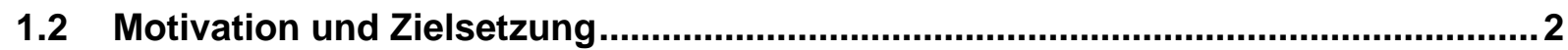

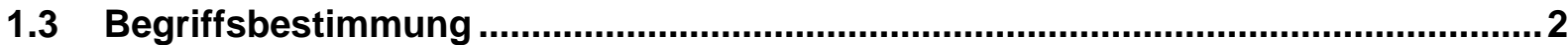

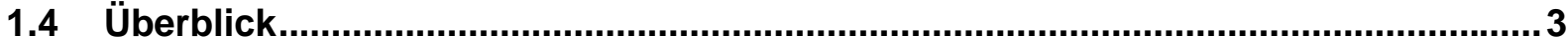

2 VERFORMUNGEN NIEDRIGER VERBUNDTRÄGER.................................5

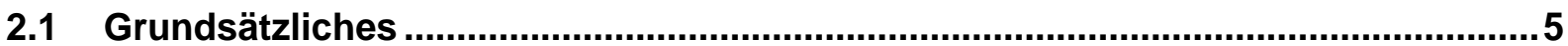

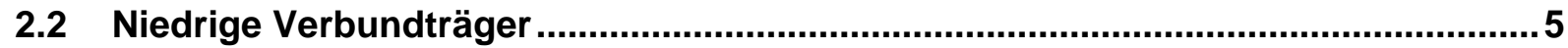

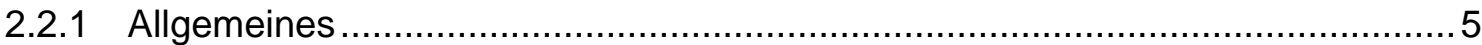

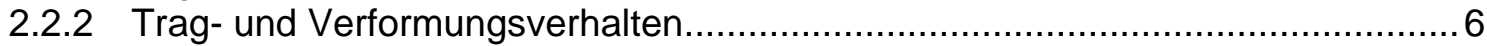

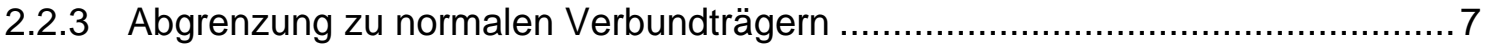

2.3 Verformungsberechnung niedriger Verbundträger ..................................................

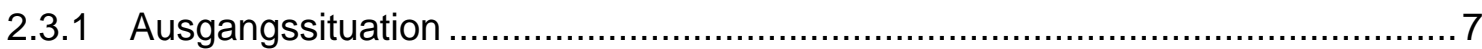

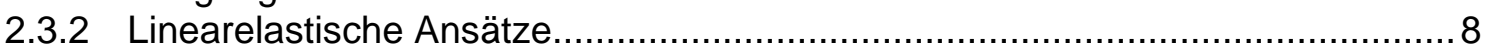

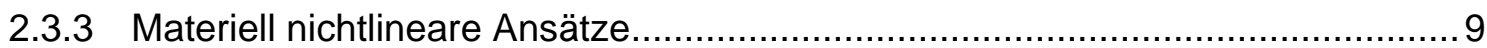

2.3.3.1 Grundsätzliches................................................................... 9

2.3.3.2 Momenten-Krümmungs-Beziehungen (M-к-Linien) .......................... 9

2.3.3.3 Berechnung mit M-N-K-Linien an den Teilquerschnitten ................... 11

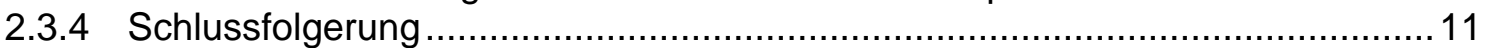

3 DIE MITTRAGENDE BETONGURTBREITE - STAND DER TECHNIK ..........13

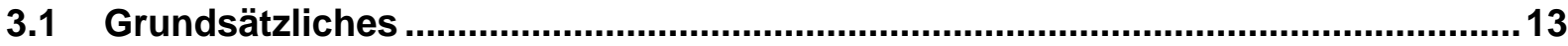

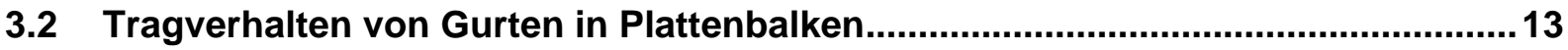

3.3 Definition der mittragenden Breite ....................................................................... 14

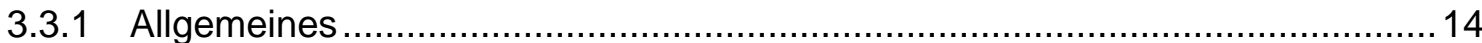

3.3.2 Mittragende Breite $b_{m}$ für die Spannungsberechnung ............................... 16

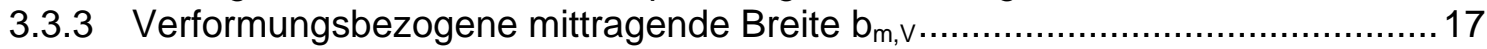

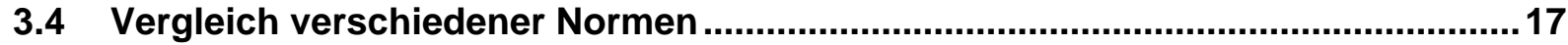

3.5 Stand der bisherigen wissenschaftlichen Untersuchungen ............................... 19

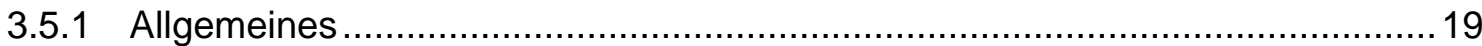

3.5.2 Untersuchungen zur Scheibentragwirkung des (Beton-) Gurts ......................... 21

3.5.3 Untersuchungen zur Biegetragwirkung des (Beton-) Gurts ............................24 
4 DIE MITTRAGENDE BREITE FÜR DEN LINEARELASTISCHEN FALL ........29

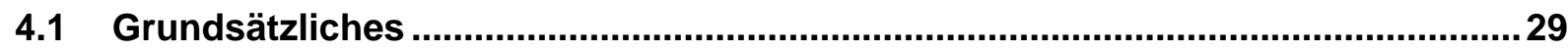

4.2 Reiner Scheiben- und Biegezustand des Betongurts ...........................................29

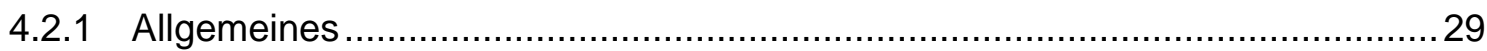

4.2.2 Einflüsse auf die mittragende Breite des Scheibenzustands............................30

4.2.3 Einflüsse auf die mittragende Breite des Biegezustands ................................ 31

4.3 Kombinierte Wirkung des Scheiben- und Biegezustands ...................................34

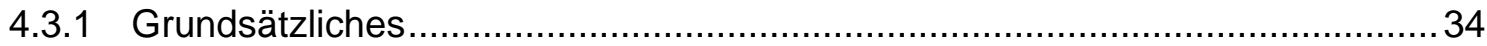

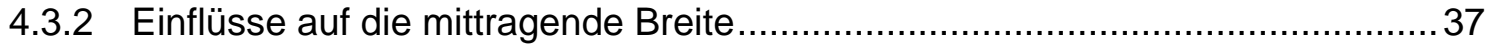

4.3.3 Verformungsbezogene mittragende Breite für elastisches

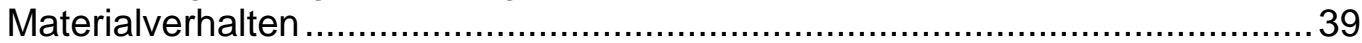

4.4 Schlussfolgerungen aus den linearelastischen Untersuchungen ........................42

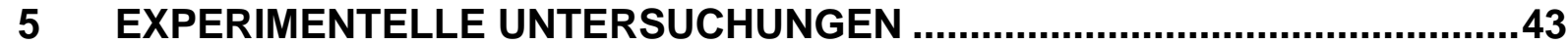

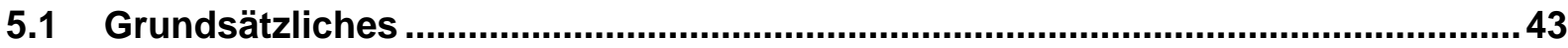

5.2 Grenzzustand der Gebrauchstauglichkeit .......................................................... 43

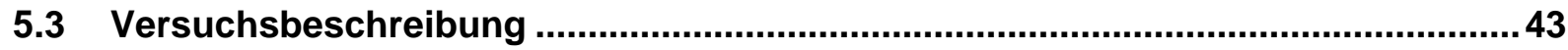

5.3.1 Gesichtspunkte zum Entwurf der Versuchsträger ................................... 43

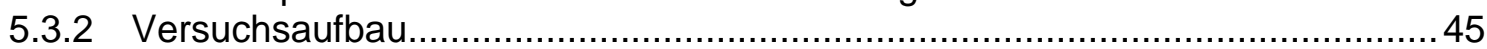

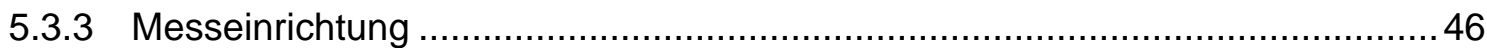

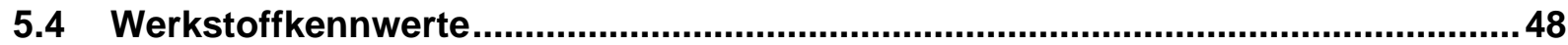

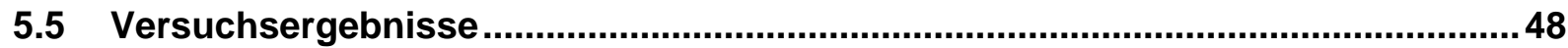

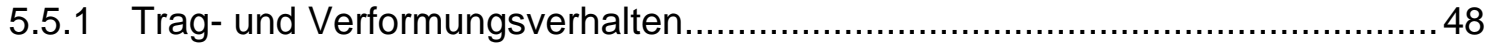

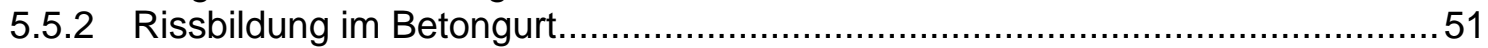

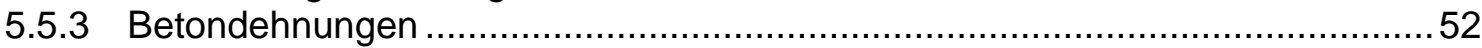

5.5.4 Mittragende Breite des Betongurts ...................................................... 54

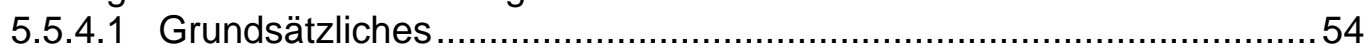

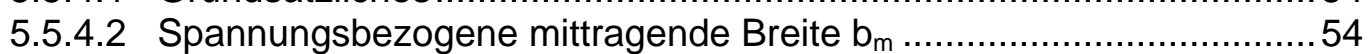

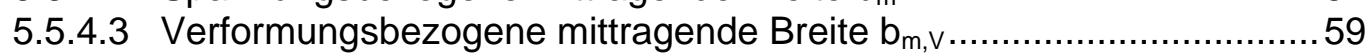

5.6 Zusammenfassung der wichtigsten Versuchsergebnisse .....................................64

6 ANALYTISCHES BERECHNUNGSMODELL ZUR VERFORMUNGSBERECHNUNG NIEDRIGER VERBUNDTRÄGER..............65

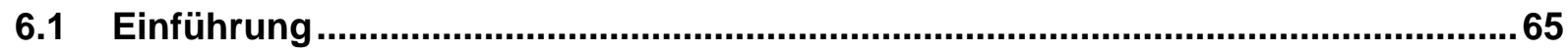

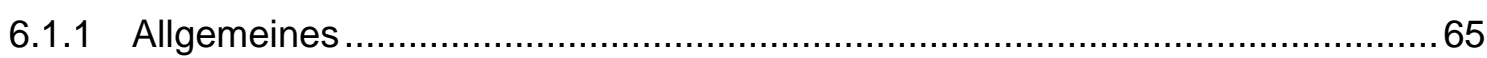

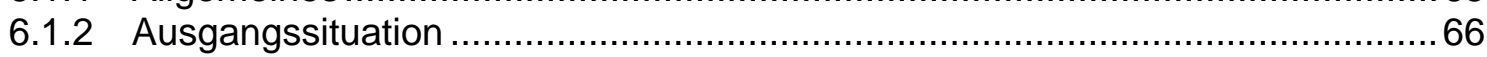

6.2 Beschreibung des analytischen Berechnungsmodells......................................67

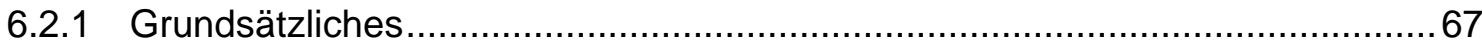




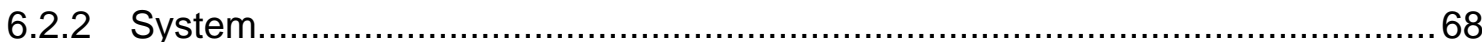

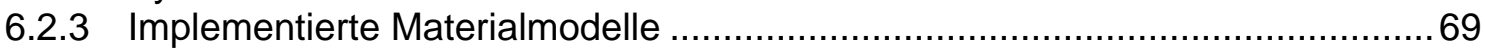

6.2.4 M-к-Linien und Breite des Betongurts ..................................................... 70

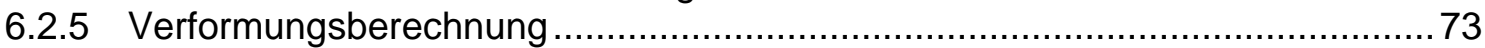

6.3 Voraussetzungen und Vereinfachungen ..............................................................

6.4 Verifikation des analytischen Berechnungsmodells ..............................................75

6.4 .1 Grundsätzliches................................................................................... 75

6.4.2 Vergleich mit den eigenen experimentellen Untersuchungen ........................76

6.4.3 Vergleich mit den experimentellen Untersuchungen von Amadio et al.

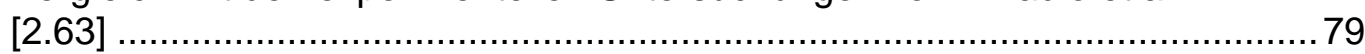

6.4.4 Vergleich mit den experimentellen Untersuchungen von Kuhlmann, Fries

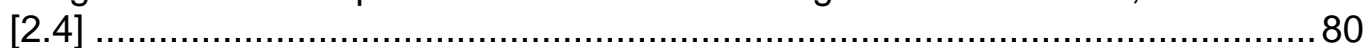

6.4.5 Fazit der Vergleiche mit Versuchsergebnissen ............................................. 81

7 HERLEITUNG EINES BERECHNUNGSANSATZES FÜR DIE VERFORMUNGSBEZOGENE MITTRAGENDE BREITE NIEDRIGER VERBUNDTRÄGER

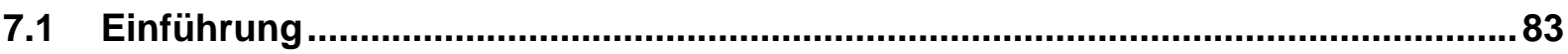

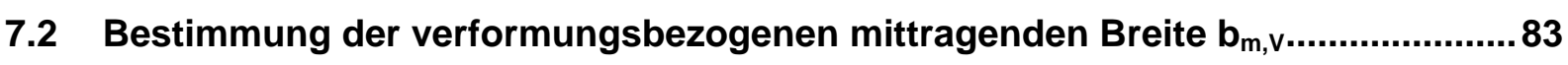

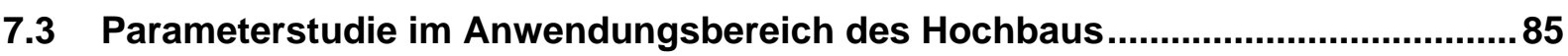

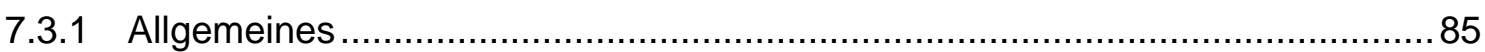

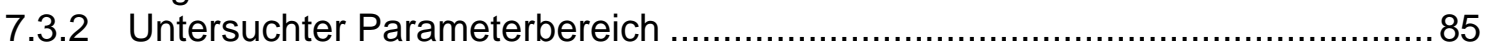

7.3.3 Einflüsse auf die verformungsbezogene mittragende Breite $b_{m, v} \ldots \ldots \ldots \ldots \ldots \ldots . . . \ldots 6$

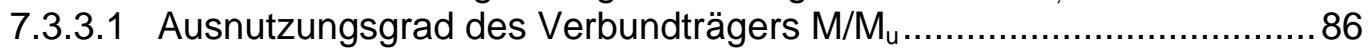

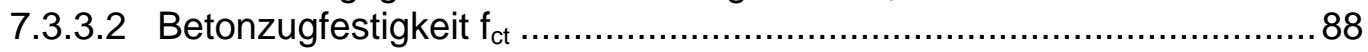

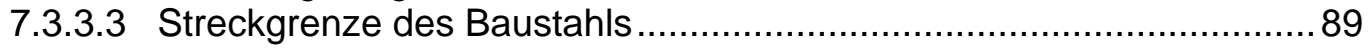

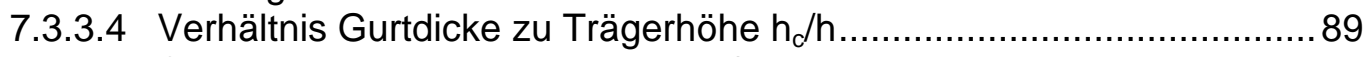

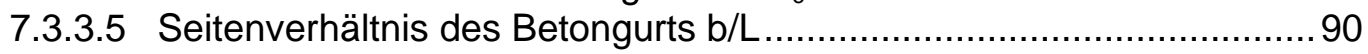

7.3.3.6 Querschnitt des Baustahlträgers .................................................. 91

7.3.3.7 Druckfestigkeit des Betongurts ................................................. 92

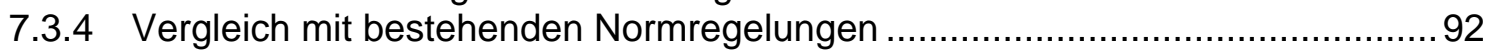

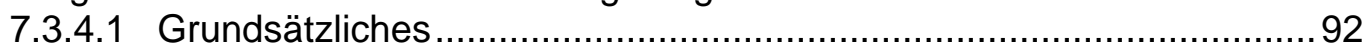

7.3.4.2 Vergleich mit EN 1994-1-1 und DIN 18800-5 ................................ 92

7.3.4.3 Vergleich mit DIN 1045-1 .......................................................... 94

7.3.4.4 Schlussfolgerung aus dem Vergleich mit den bestehenden

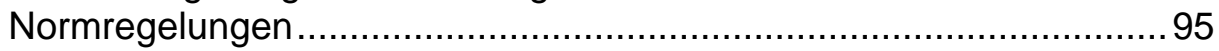

7.4 Berechnungsansatz für die verformungsbezogene mittragende Breite

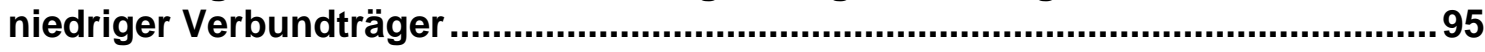

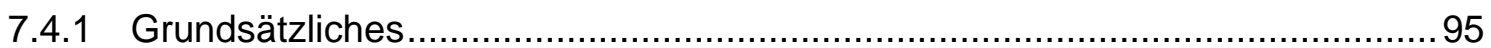

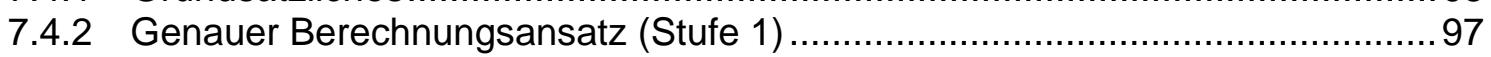

7.4.3 Vereinfachter Berechnungsansatz (Stufe 2)........................................... 101

7.4.4 Schlussfolgerung aus dem entwickelten Berechnungsansatz...................... 102

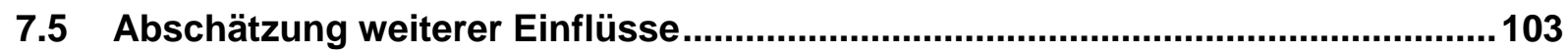

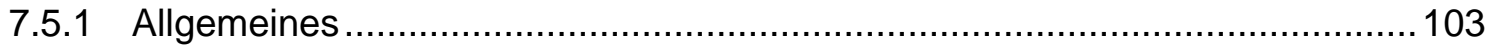

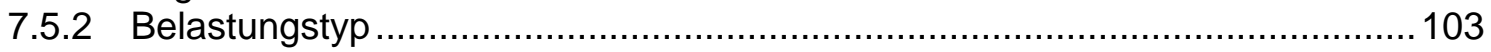




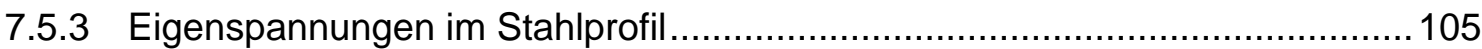

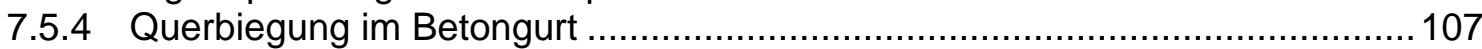

8 VORSCHLAG ZUR QUASI-ELASTISCHEN

VERFORMUNGSBERECHNUNG NIEDRIGER VERBUNDTRÄGER............111

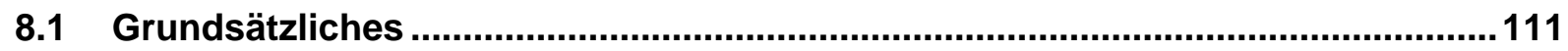

8.2 Beschreibung des quasi-elastischen Berechnungsvorschlags...........................112

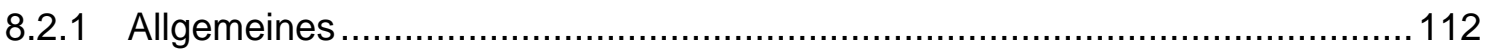

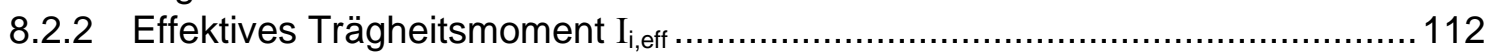

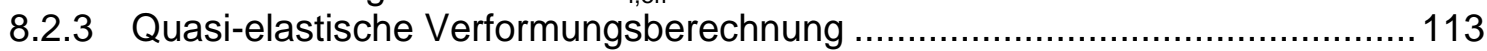

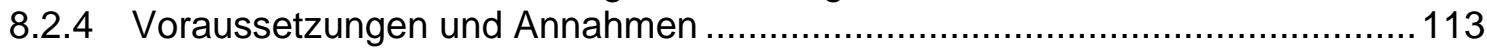

8.3 Abminderungsfaktor $\alpha_{c}$ zur quasi-elastischen Verformungsberechnung

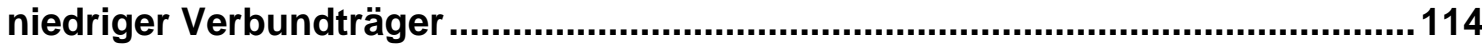

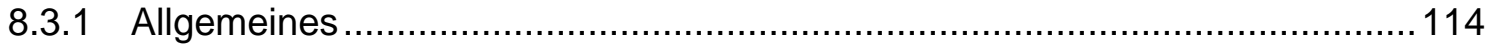

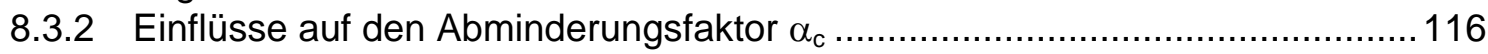

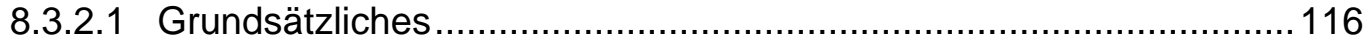

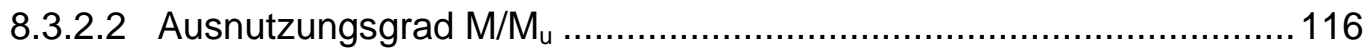

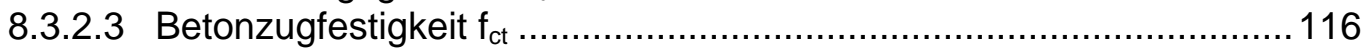

8.3.2.4 Streckgrenze $f_{y}$ des Stahlträgers ........................................... 117

8.3.2.5 Dicke des Betongurts $\mathrm{h}_{\mathrm{c}}$ und Trägerhöhe.................................. 117

8.3.2.6 Seitenverhältnis des Betongurts b/L ......................................... 118

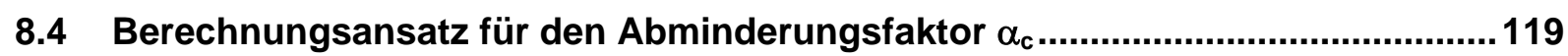

8.4.1 Herleitung des Berechnungsansatzes........................................................... 119

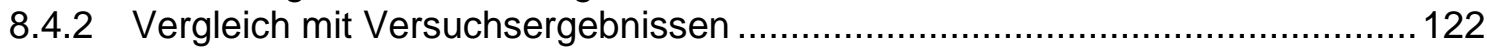

8.4.3 Vergleich mit dem analytischen Berechnungsmodell .................................. 125

8.4.4 Vergleich mit bestehenden Regelungen..................................................... 126

8.4.4.1 EN 1994-1-1 und DIN 18800-5 .............................................. 126

8.4.4.2 DIN 1045-1 ..................................................................... 127

8.4.5 Schlussfolgerungen aus dem quasi-elastischen Berechnungsansatz............ 128

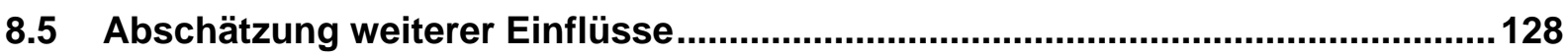

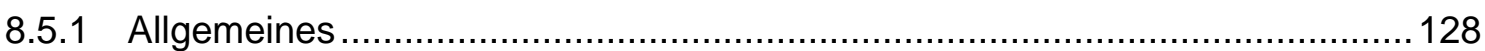

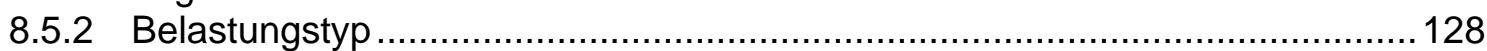

8.5.3 Eigenspannungen im Stahlprofil...................................................... 130

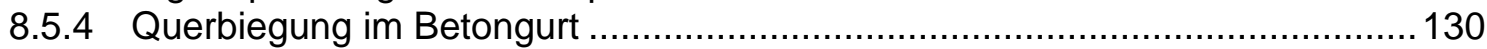

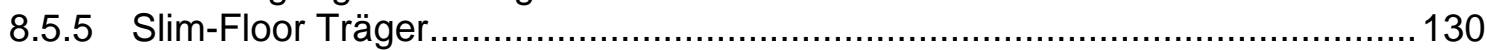

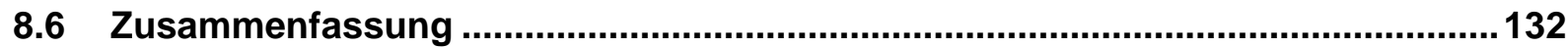

9 EINFLUSS VON KRIECHEN UND SCHWINDEN DES BETONS ..................133

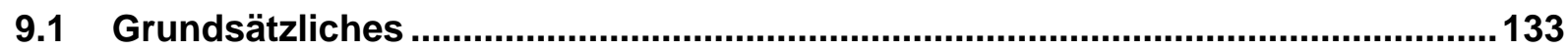

9.2 Berücksichtigung von Kriechen und Schwinden beim analytischen

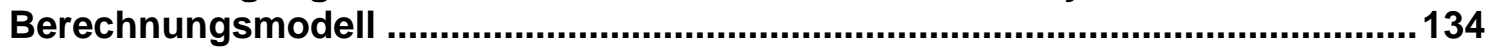

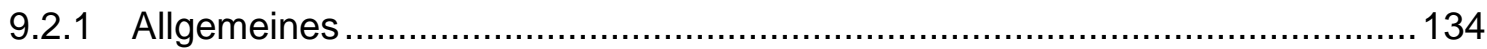


9.2.2 Materialmodelle für den Beton unter Berücksichtigung von Kriechen und Schwinden

9.2.3 Erweiterung des analytischen Berechnungsmodells für Kriechen und Schwinden des Betons....

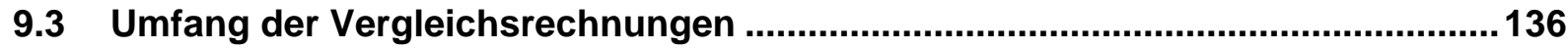

9.4 Verformungsbezogene mittragende Breite .................................................... 136

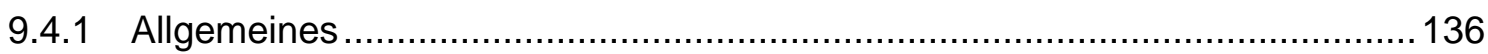

9.4.2 Vergleich des Berechnungsansatzes für Kriechen .................................... 137

9.4.3 Vergleich des Berechnungsansatzes für Schwinden ............................... 138

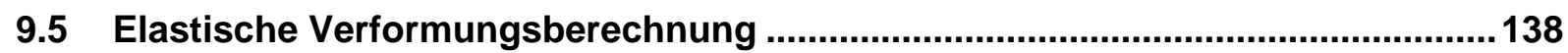

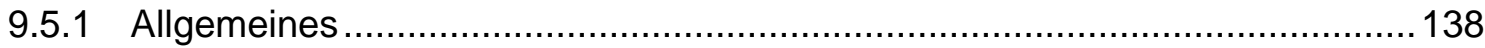

9.5.2 Vergleich des quasi-elastischen Berechnungsansatzes für Kriechen .............. 141

9.5.3 Vergleich des quasi-elastischen Berechnungsansatzes für Schwinden........... 141

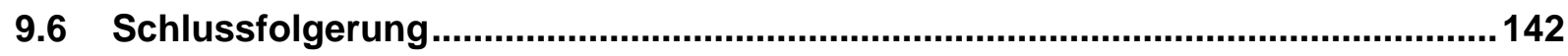

10 ZUSAMMENFASSUNG UND AUSBLICK.............................................143

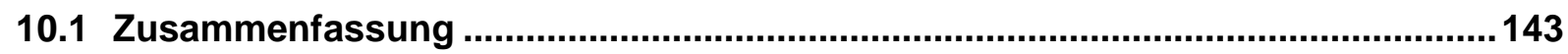

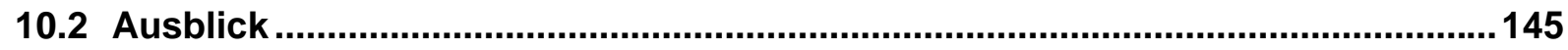

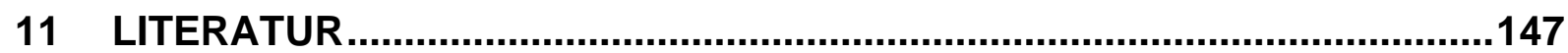

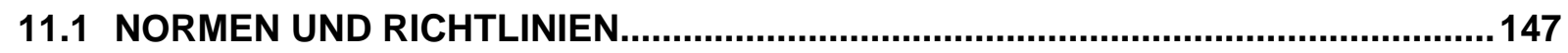

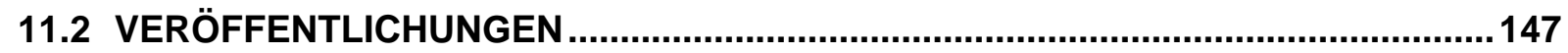

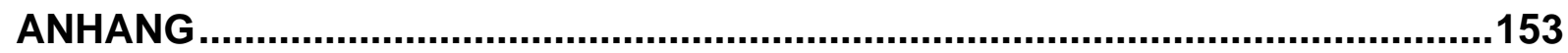

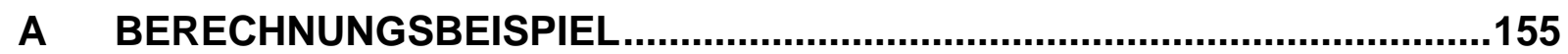

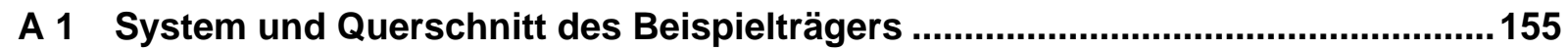

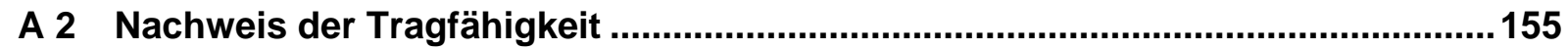

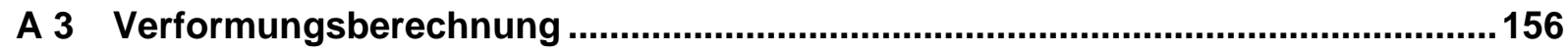

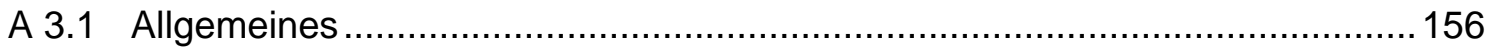

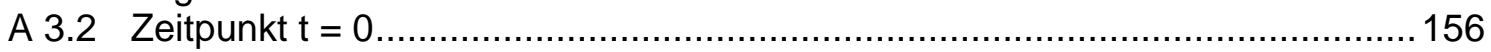

A 3.3 Zeitpunkt $t=\infty$ (nach Abschluss von Kriechen und Schwinden) ....................... 158

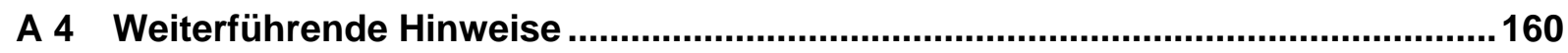

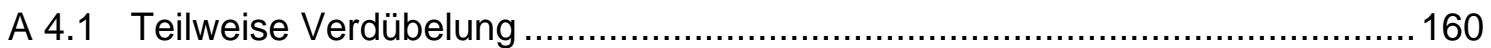

A 4.2 Eigenspannungen im Stahlträger ........................................................ 160 



\section{Einleitung}

\subsection{Grundsätzliches}

Wirtschaftliche Vorteile als auch architektonische Bedürfnisse führen in letzter Zeit vermehrt zum Einsatz von Verbundträgern mit niedriger Bauhöhe und Slim-Floor Trägern, siehe Abbildung 1-1. Sie stellen eine wirtschaftliche Alternative zu herkömmlichen Stahlbetonträgern und -decken dar. Im Vergleich zu herkömmlichen Stahlbetonkonstruktionen zeichnen sich Verbundträger niedriger Bauhöhe und Slim-Floor Träger durch eine hohe werksseitige Vorfertigung, einfache Verbindungstechnik und Montage, verkürzte Bauzeit sowie eine hohe Tragfähigkeit aus [2.1]-[2.25].

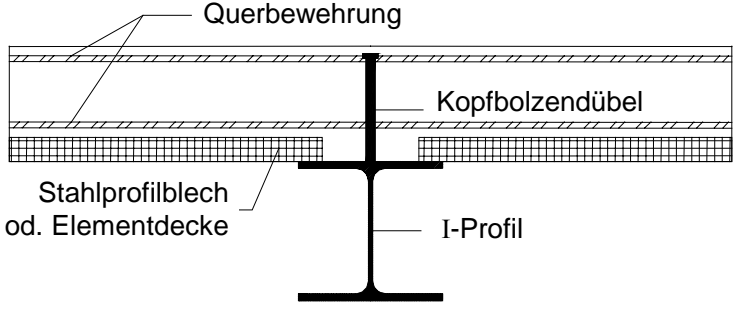

(a) Verbundträger niedriger Bauhöhe

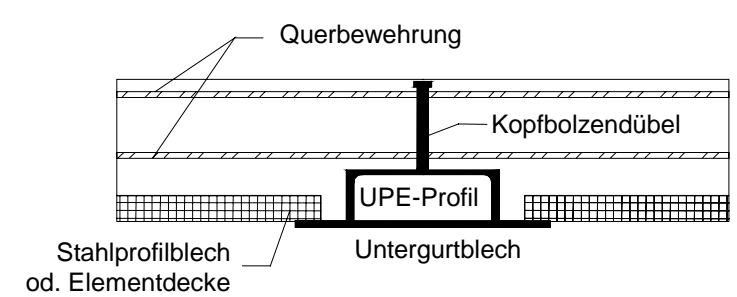

(b) Slim-Floor Träger

Abbildung 1-1: Verbundträger niedriger Bauhöhe und Slim-Floor Träger

Die vergleichsweise große Tragfähigkeit führt dazu, dass Verbundträger niedriger Bauhöhe mit einer großen Schlankheit ausgeführt werden. Da die Steifigkeit im Vergleich zur Tragfähigkeit überproportional stark mit der Bauhöhe abnimmt, sind bei Verbundträgern niedriger Bauhöhe die Verformungen für die Nutzung und die Dimensionierung dieser Träger besonders wichtig [2.21]. Im Gegensatz zu hohen Verbundträgern wird bei diesen Trägern in sehr vielen Fällen die Einhaltung von Verformungsgrenzwerten unter Gebrauchslasten zum bemessungsrelevanten Aspekt. Verbundträger niedriger Bauhöhe zeigen dabei ein anderes Trag- und Verformungsverhalten als normale Verbundträger. Infolgedessen sind die bekannten Verfahren zur Dimensionierung von Verbundträgern, die für hohe Verbundträger abgeleitet wurden, für niedrige Verbundträger besonders hinsichtlich einer wirklichkeitsnahen Verformungsberechnung unzutreffend [2.6], [2.7]. Im Allgemeinen werden mit diesen Verfahren zu große Verformungswerte ermittelt. Zur Einhaltung der gängigen Verformungsgrenzwerte im Grenzzustand der Gebrauchstauglichkeit sind deshalb in vielen Fällen größere Querschnitte erforderlich, als sich aus dem Nachweis der Tragfähigkeit im Grenzzustand der Tragfähigkeit ergeben. Die hohe Wirtschaftlichkeit dieser Trägerformen kann dann nicht ausgenutzt werden. Darüber hinaus führt die rechnerische Überschätzung der Verformungen z. B. aus falschen Überhöhungsvorgaben zu Problemen in der Ausführung und Nutzung [2.1], [2.7], [2.21].

Das Verformungsverhalten der betrachteten niedrigen Verbundträger wird dabei maßgeblich durch die vergleichsweise große Biegesteifigkeit des Betongurts und dessen Rissbildung, die bereits unter vergleichsweise geringen Gebrauchslasten auch im Bereich positiver Momente einsetzt, bestimmt. Die bestehenden Regeln zur Verformungsberechnung von Verbundträgern in den einschlägigen Normen tragen diesem Zustand jedoch nicht bzw. nur eingeschränkt Rechnung [2.1], [2.4], [2.7]. In [2.4] wurde für Slim-Floor Träger ein Beitrag zur Verformungsberechnung durch Untersuchung des Einflusses einer nachgiebigen Verdübelung und der Rissbildung des Betongurts geleistet. Ein weiterer Einfluss auf die rechnerischen Verformungen ist die angenommene mittragende Breite des Betongurts, deren Wert durch die bestehenden Normenregeln pauschal und ohne Berücksichtigung des speziellen Trag- und Verformungsverhaltens niedriger Verbundträger abgeschätzt wird. Auf die rechne- 
rische Tragfähigkeit von Verbundträgern hat diese zwar keinen großen Einfluss, für die rechnerische Steifigkeit stellt diese jedoch einen wesentlichen Einflussfaktor dar.

\subsection{Motivation und Zielsetzung}

Die im Rahmen dieser Arbeit unternommenen Untersuchungen zur mittragenden Breite niedriger Verbundträger motivieren sich aus mehreren Aspekten: Zum einen zeigt ein Vergleich der verschiedenen Regeln zur mittragenden Breite in den diversen nationalen und internationalen Normen des Stahl-, Massiv- und Verbundbaus sehr große Unterschiede, deren Ursachen sich zunächst nicht erschließen. Zum anderen zeigten erste Voruntersuchungen, dass die bestehenden Regeln für die Berechnung hoher Verbundträger entwickelt wurden und daher dem speziellen Tragverhalten niedriger Verbundträger nicht Rechnung tragen [2.26]. Darüber hinaus ist der Einfluss der mittragenden Breite auf die rechnerische Steifigkeit sehr viel größer als auf die Tragfähigkeit. Wegen der Verformungsempfindlichkeit dieser Träger hat die mittragende Breite daher einen großen Einfluss auf deren Dimensionierung. Erste Untersuchungen in [2.2], [2.4] legen nahe, dass die tatsächlich vorhandene Steifigkeit in der Regel sehr viel größer ist, als sich dies mit den Regeln zur mittragenden Breite in den gültigen Normen ergibt.

Die vorliegende Arbeit hat sich daher zum Ziel gesetzt, die mittragende Betongurtbreite niedriger Verbundträger unter Berücksichtigung des spezifischen Trag- und Verformungsverhaltens dieser Trägerformen zu untersuchen und einen realistischen Berechnungsansatz für die verformungsbezogene mittragende Breite zu entwickeln, auf dessen Basis eine wirklichkeitsnahe Ermittlung der Steifigkeiten und Verformungen dieser Träger möglich ist. Eine heute noch in vielen Fällen übliche - Überdimensionierung dieser Träger auf Grund unzutreffender rechnerischer Verformungen soll damit vermieden werden.

Die Untersuchungen beschränken sich dabei im Wesentlichen auf das Verformungsverhalten von Verbundträgern herkömmlicher Bauart mit niedriger Bauhöhe in Einfeldträgersystemen unter Gebrauchslasten für den Bereich des normalen Hoch- und Geschossbaus. Die Betrachtung der mittragende Breite zur Ermittlung der Querschnittstragfähigkeit im Bruchzustand (Traglast) oder die Untersuchung des negativen Momentenbereichs von durchlaufenden Trägersystemen hätten den Rahmen der vorliegenden Arbeit überschritten.

\subsection{Begriffsbestimmung}

Der Begriff der mittragenden Breite dient der Vereinfachung. Er wurde eingeführt, um das eigentlich dreidimensionale Tragverhalten von Plattenbalken rechnerisch auf ein eindimensionales Problem zu reduzieren. Er wird darüber hinaus in verschiedenen Bereichen auch für unterschiedliche Problemstellungen verwendet - z. B. neben der Beschreibung der Schubweichheit breiter Gurte, beim Ausbeulen gedrückter Querschnittsteile oder bei Platten.

Diese Arbeit widmet sich im Wesentlichen der Auswirkung der Schubweichheit breiter Betongurte in Verbundträgern und speziell deren Einfluss auf die Trägerverformung. Die Schubweichheit des Gurtes führt in einer Querschnittsbetrachtung zu einer ungleichmäßigen Querverteilung der Längsdehnungen und -spannungen des Gurtes. Im Rahmen dieser Arbeit wird der gängigen Praxis folgend dies mit mittragender Breite $\boldsymbol{b}_{\boldsymbol{m}}$ bezeichnet. Zur Verdeutlichung wird teilweise stattdessen auch der Begriff „spannungsbezogene mittragende Breite $\boldsymbol{b}_{\boldsymbol{m}}$ " verwendet. Dabei kann diese in die Werte des reinen Scheibenzustands, des reinen Biegezustands und der kombinierten Wirkung dieser Zustände unterschieden werden. Wenn in dieser Arbeit auf den Zusatz "Scheibenzustand“ bzw. „Biegezustand“ verzichtet wird, handelt es sich im Allgemeinen um die kombinierte Wirkung. Zur Unterscheidung werden folgende Formelzeichen vereinbart: 
- $b_{m, s} \quad$ (spannungsbezogene) mittragende Breite des Scheibenzustands

- $b_{m, B} \quad$ (spannungsbezogene) mittragende Breite des Biegezustands

- $\mathrm{b}_{\mathrm{m}} \quad$ (spannungsbezogene) mittragende Breite aus der kombinierten Wirkung des Scheiben- und Biegezustands

Während die (spannungsbezogene) mittragende Breite in Trägerlängsrichtung im Allgemeinen veränderlich ist, ist jedoch für eine Verformungsberechnung ein konstanter Wert von großem Vorteil. Hierfür wird die verformungsbezogene mittragende Breite $\boldsymbol{b}_{m, v}$ eingeführt. Der Begriff bringt dabei zum Ausdruck, dass sich die so definierte Breite auf die Verformung des Trägers bezieht. Hierfür wird als Formelzeichen verwendet:

- $b_{m, \mathrm{v}} \quad$ verformungsbezogene mittragende Breite

Die Definition dieser Größen ist in Abschnitt 3.3 ausführlich dargestellt.

\section{4 Überblick}

Die Untersuchungen zur mittragenden Breite, der verformungsbezogenen mittragenden Breite und des Verformungsverhaltens niedriger Verbundträger in der vorliegenden Arbeit unterteilen sich in experimentelle, analytische und numerische Betrachtungen. Die Arbeit ist dabei wie folgt strukturiert:

In Kapitel 2 wird zunächst der Begriff „Verbundträger niedriger Bauhöhe“ definiert, das besondere Trag- und Verformungsverhalten dieser Träger diskutiert und eine Abgrenzung zu hohen Verbundträgern gezogen. Daran anschließend werden in Kapitel 3 die Definition der mittragenden Breite und die bisherigen Untersuchungen zu diesem Thema zur Einordnung der eigenen Untersuchungen aufgearbeitet und kurz zusammengefasst.

Als Grundlage für die weiteren Untersuchungen werden in Kapitel 4 die Zusammenhänge bei elastischem Materialverhalten aufgezeigt. Die im Rahmen dieser Arbeit und eines AiFForschungsvorhabens [2.27] durchgeführten experimentellen Untersuchungen werden in Kapitel 5 beschrieben und ausgewertet. Das zur Untersuchung des Trag- und Verformungsverhaltens niedriger Verbundträger und der verformungsbezogenen mittragenden Breite entwickelte analytische Berechnungsmodell wird in Kapitel 6 beschrieben und verifiziert. Nach Diskussion der wesentlichen Einflussparameter in Kapitel 7 wird aufbauend auf einer umfangreichen Parameterstudie ein handhabbarer Berechnungsansatz für die verformungsbezogene mittragende Breite zur wirklichkeitsnahen Verformungsberechnung dieser Träger entwickelt. Zur vereinfachten quasi-elastischen Verformungsberechnung niedriger Verbundträger wird in Kapitel 8 ein Vorschlag zur Bestimmung einer effektiven Trägersteifigkeit vorgestellt, der eine realistische Verformungsberechung dieser Träger ermöglicht. Vergleichsrechnungen in Kapitel 9 zeigen, dass die entwickelten Berechnungsansätze auch bei Berücksichtigung von Kriechen und Schwinden des Betons in guter Näherung verwendet werden können.

Abschließend werden in Kapitel 10 die wesentlichen Erkenntnisse der vorliegenden Arbeit zusammengefasst und Ansatzpunkte für weitere notwendige Untersuchungen zur wirklichkeitsnahen Verformungsberechung von Verbundträgern angesprochen. 



\section{Verformungen niedriger Verbundträger}

\subsection{Grundsätzliches}

Im Folgenden soll eine kurze Erklärung des Begriffs „niedrige Verbundträger“ die Einordnung der in dieser Arbeit vorgenommenen Untersuchungen ermöglichen. Daran anschließend wird ein Überblick über die gebräuchlichen Verfahren der Verformungsberechnung für diese niedrigen Verbundträger gegeben.

\section{$2.2 \quad$ Niedrige Verbundträger}

\subsubsection{Allgemeines}

Auf Grund der vergleichsweise dicken Plattendicken, geringen Stützweiten und geringen Lasten reichen im Geschossbau in vielen Fällen relativ kleine Stahlträger im Verbund mit den Decken als Unterzüge aus. Auch auf Grund vereinfachter Installationsmöglichkeiten und nicht zuletzt architektonischer Belange wird dabei zunehmend versucht, die Gesamthöhe des Deckensystems zu minimieren. Einen Extremfall dieser niedrigen Verbundträger stellen die so genannten Slim-Floor Träger dar, bei denen der Stahlträger in die Decke integriert ist und somit eine ebene Deckenuntersicht bzw. eine Verbundflachdecke entsteht. Abbildung 11 zeigt typische Beispiele solcher niedriger Verbundträger und Slim-Floor Träger.

Niedrige Verbundträger und Slim-Floor Träger haben im Vergleich zu herkömmlichen Stahlbetonkonstruktionen zahlreiche Vorteile durch die Kombination der Stahl- und Stahlbetonbauweise. Durch die Möglichkeit zur flexiblen Grundrissgestaltung und freien architektonischen Gestaltung (geringe Bauhöhe, ebene Deckenuntersicht, etc.) können gerade Verbundflachdecken sehr effizient eingesetzt werden. Eine ausführliche Diskussion der Vor- und Nachteile dieser Trägerformen ist in [2.2], [2.4], [2.5], [2.11]-[2.25] gegeben. An dieser Stelle seien nur folgende Vorteile genannt:

- hohe Montagefreundlichkeit und Bauzeitverkürzung durch den Einsatz von vorgefertigten Bauelementen wie Stahlträgern, Betonfertigteilplatten, Stahlprofilblechen, etc.

- geringe Bauhöhe und damit geringe Geschosshöhe, das heißt Reduktion der Fassadenflächen und unter Umständen Ermöglichung zusätzlicher Geschosse

- geringes Eigengewicht der Deckenkonstruktion und Ersparnisse bei der Ausführung von Stützen, Wänden und Fundamenten

- geringere Finanzierungskosten durch frühere Mieteinnahmen und verkürzte Bauzeit

Ein Wirtschaftlichkeitsvergleich [2.28] unterschiedlicher Systeme der Verbundflachdecke mit einer konventionellen Stahlbeton-Flachdecke zeigt, dass zwar die Herstellungskosten der Verbundflachdecke nahezu identisch mit der Stahlbeton-Flachdecke sind, jedoch besitzen die möglichen Varianten der Verbundflachdecke eine Zeitersparnis auf der Baustelle von bis zu $20 \%$. Diese immer wichtiger werdende Bauzeitverkürzung macht das Verbundflachdeckensystem in einer Gesamtbetrachtung gegenüber der herkömmlichen Stahlbetonbauweise wirtschaftlicher. Untersuchungen in [2.14] zeigen, dass die Herstellungskosten von Verbundflachdecken zwar höher sind als bei vergleichbaren Unterzugsdecken, diese jedoch durch Vorteile bei der Installation und frühere Mieteinnahmen mehr als ausgeglichen werden. Ähnliche Werte sind auch für Verbundträger niedriger Bauhöhe zu erreichen [2.4], [2.27].

Die möglichen Spannweiten von Verbundträgern niedriger Bauhöhe betragen in Abhängigkeit von der Stützweite der quer verlaufenden Decke, der Belastungshöhe und der verwen- 
deten Beton- und Stahlgüte für übliche Fälle des Hochbaus $8 \div 15 \mathrm{~m}$. Slim-Floor Träger erreichen Spannweiten von bis zu $10 \mathrm{~m}$, bei einer Beschränkung der Deckenstärke auf $32 \mathrm{~cm}$, siehe Abbildung 2-2 [2.1], [2.5] [2.27]. Mit den möglichen Spannweiten von Verbundträgern niedriger Bauhöhe und Slim-Floor Trägern ist somit ein weiter Anwendungsbereich des normalen Geschossbaus abgedeckt.

\subsubsection{Trag- und Verformungsverhalten}

Bei normal hohen Verbundträgern wird im Gebrauchszustand das äußere Moment hauptsächlich durch das innere Kräftepaar aus der Verbundwirkung (Druckkraft $\mathrm{N}_{\mathrm{c}}$ im Beton und Zugkraft $\mathrm{N}_{\mathrm{a}}$ im Baustahl) und ein Biegemoment $\mathrm{M}_{\mathrm{a}}$ im Baustahl abgetragen, siehe Abbildung 2-1 (a). Das Biegemoment $M_{c}$ und die Biegesteifigkeit $I_{c}$ des Betongurts sind hierbei vernachlässigbar. Der Betongurt ist - zumindest im Gebrauchszustand - in Bereichen positiver Momente vollständig überdrückt und frei von Rissen (Zustand I).
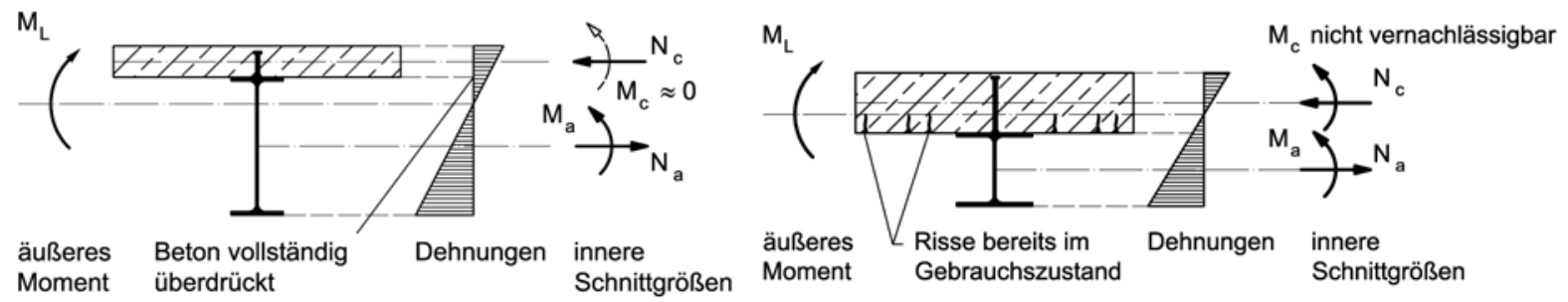

(a) normal hohe Verbundträger

(b) Verbundträger niedriger Bauhöhe

Abbildung 2-1: Tragverhalten von Verbundträgern [2.6]

Tabelle 2-1: $\quad$ Vergleich der Teilschnittgrößen von hohen Verbundträgern und Slim-Floor Trägern [2.6]

\begin{tabular}{|c|l|c|c|c|}
\hline \multicolumn{2}{|c|}{ Teilschnittgrößen } & $\mathbf{M}_{\mathbf{c}}$ & $\mathbf{M}_{\mathbf{a}}$ & $\mathbf{M}_{\mathbf{s t}}=\mathbf{N}_{\mathbf{a}} \cdot \mathbf{a}_{\mathbf{s t}}$ \\
\hline \multirow{2}{*}{$\mathrm{GZT}^{1)}$} & hohe Verbundträger & $5 \%$ & $5 \%$ & $90 \%$ \\
\cline { 2 - 5 } & Slim-Floor Träger & $30 \%$ & $5 \%$ & $65 \%$ \\
\hline \multirow{2}{*}{$\mathrm{GZG}^{2)}$} & hohe Verbundträger & $5 \%$ & $30 \%$ & $65 \%$ \\
\cline { 2 - 5 } & Slim-Floor Träger & $45 \%$ & $10 \%$ & $45 \%$ \\
\hline \multirow{2}{*}{ Zustand I $^{3)}$} & hohe Verbundträger & $5 \%$ & $30 \%$ & $65 \%$ \\
\cline { 2 - 5 } & Slim-Floor Träger & $60 \%$ & $5 \%$ & $35 \%$ \\
\hline
\end{tabular}

${ }^{1)}$ Grenzzustand der Tragfähigkeit (Lastniveau $\mathrm{q}_{\mathrm{Rd}}$ )

2) Grenzzustand der Gebrauchstauglichkeit (Lastniveau $\mathrm{q}_{\mathrm{GzG}} \approx 0,75 \cdot \mathrm{q}_{\mathrm{Rd}}$ )

${ }^{3)}$ im Zustand I, das heißt ohne Risse im Beton

Im Gegensatz dazu ist bei Verbundträgern niedriger Bauhöhe und Slim-Floor Trägern die Biegesteifigkeit $\mathrm{I}_{\mathrm{c}}$ des Betongurts so groß, dass sie nicht vernachlässigt werden kann und der Betongurt so große Biegemomente $M_{c}$ erhält, dass er bereits im Gebrauchszustand Risse bekommt, siehe Abbildung 2-1 (b). Dies zeigt sich auch bei einem Vergleich der Teilschnittgrößen dieser Trägerformen in Tabelle 2-1. Das Tragverhalten von niedrigen Verbundträgern liegt somit zwischen hohen Verbundträgern und gewöhnlichen Stahlbetonträgern. Dabei hat bei niedrigen Verbundträgern im Gegensatz zu hohen Verbundträgern die untere Längsbewehrung des Betongurts aufgrund der geometrischen Verhältnisse einen nennenswerten Einfluss auf das Trag- und Verformungsverhalten [2.6], [2.27]. 


\subsubsection{Abgrenzung zu normalen Verbundträgern}

Verbundträger niedriger Bauhöhe liegen bzgl. ihres Trag- und Verformungsverhaltens zwischen normal hohen Verbundträgern und Stahlbetonträgern. Vergleichbar liegt das Tragund Verformungsverhalten von Slim-Floor Trägern zwischen dem von normalen Verbundträgern und Stahlbetonflachdecken [2.4]. Es gibt jedoch keine „scharfe Grenze“ zwischen diesen Querschnittsformen. Der Übergang ist fließend.

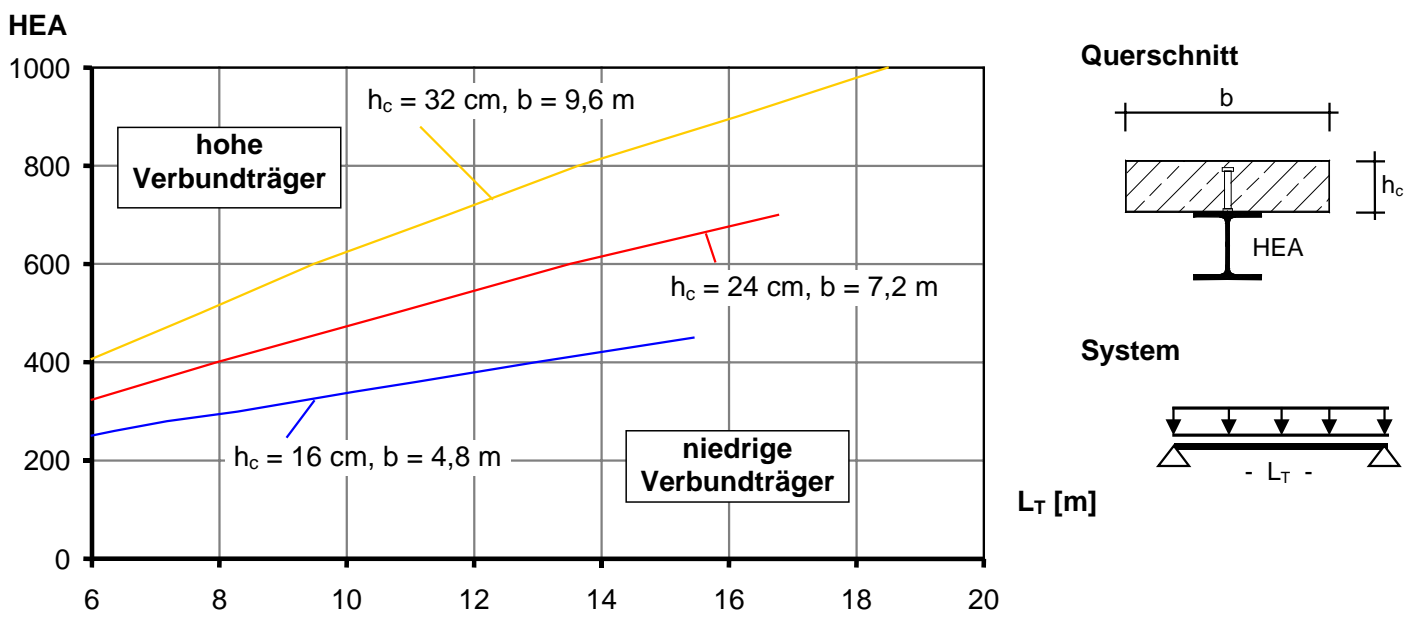

Abbildung 2-2: Abgrenzung zwischen niedrigen und hohen Verbundträgern [2.6]

In [2.6] wird zur Abgrenzung zwischen hohen und niedrigen Verbundträgern eine Beurteilung über die Rissbildung vorgeschlagen. Verbundträger niedriger Bauhöhe liegen dann vor, wenn der Betongurt bereits unter Gebrauchslasten im Bereich positiver Momente Risse an der Unterseite aufweist. In Abbildung 2-2 ist dieses Abgrenzungskriterium für hochbautypische Randbedingungen (Nutzlast $p=3,5 \mathrm{kN} / \mathrm{m}^{2}$, Beton C 30/37, Baustahl S 355) dargestellt. Unterhalb der Linien sind niedrige Verbundträger angeordnet, oberhalb hohe Verbundträger. Verbundträger niedriger Bauhöhe erreichen somit Spannweiten bis zu ca. $15 \mathrm{~m}$ und das Anwendungsgebiet dieser Träger im normalen Hoch- und Geschossbau ist entsprechend groß [2.6], [2.27].

\subsection{Verformungsberechnung niedriger Verbundträger}

\subsubsection{Ausgangssituation}

Eine exakte Verformungsberechnung von Verbundträgern ist auf Grund der vielen nichtlinearen Einflüsse wie z. B. der Rissbildung, Kriechen und Schwinden des Beton, etc. grundsätzlich sehr aufwändig. Aus diesem Grund werden bei der Berechnung in der Planungspraxis im Allgemeinen Näherungslösungen verwendet. In der Regel kommt hierbei das Gesamtquerschnittsverfahren [2.29]-[2.31], das auf elastischen Ansätzen basiert, zum Einsatz. Bei niedrigen Verbundträgern stellt dies aufgrund der ausgeprägten Rissbildung bereits unter Gebrauchslasten eine in vielen Fällen nicht akzeptable Näherung dar, die meist zu einer Unterschätzung der Verformungen führt. Dass dies in der Praxis dennoch selten zu Schäden führt, liegt an weiteren Unzulänglichkeiten der vorgenommenen Berechnung, die dem entgegenwirken, und daher häufig zu einer Überschätzung der Verformungen führen kann. Zu diesen Fehlern zählen unter anderem:

- Unterschätzung der mittragenden Breite des Betongurts 
- Überschätzung der Einflüsse aus Kriechen und Schwinden des Betons

- Vernachlässigung des günstigen Einflusses einer vorhandenen, unplanmäßigen Durchlaufwirkung

- Überschätzung des Einflusses einer nachgiebigen bzw. teilweisen Verdübelung

Im Sinne einer sauberen ingenieurmäßigen Lösung sollte die Verformungsberechnung jedoch dem speziellen Verformungsverhalten von Verbundträgern und den entsprechenden Einflüssen ausreichend genau Rechnung tragen und nicht auf der Hoffnung beruhen, dass sich zwei Fehler gegenseitig aufheben. Die oben erwähnten Fehler bei der Verformungsberechnung lassen sich kurz folgendermaßen zusammenfassen:

Im Allgemeinen wird bei der Verformungsberechnung für den Betongurt dieselbe mittragende Breite angesetzt wie beim Nachweis der Tragfähigkeit. Die so ermittelte mittragende Breite ist dabei in vielen Fällen sehr viel kleiner als die tatsächliche mittragende Breite, wie die Untersuchungen in dieser Arbeit zeigen. Dies führt zu einer zu kleinen rechnerischen Steifigkeit der Verbundträger und zu großen rechnerischen Verformungswerten.

Im Konzept des Gesamtquerschnittsverfahrens wird der Betongurt über die gesamte Höhe als wirksam für Kriechen und Schwinden betrachtet. Tatsächlich ist dieser Einfluss jedoch nur in den nicht gerissenen bzw. überdrückten Bereichen des Betongurts vorhanden [2.6], [2.32]. Dies führt im Allgemeinen zu einer rechnerischen Überschätzung der Kriech- und Schwindverformungen niedriger Verbundträger. Darüber hinaus sind das tatsächliche Kriechund Schwindverhalten aufgrund einer guten Nachbehandlung des Betons vor Ort geringer als in der Berechnung angenommen.

Zur Vereinfachung der Bemessung von Verbundträgern und ihrer Anschlüsse werden diese in der Planungspraxis in sehr vielen Fällen trotz durchlaufender Betongurte als Einfeldträger ohne Durchlaufwirkung berechnet. Die tatsächlich vorhandene Durchlaufwirkung führt im Vergleich zur Berechnung als Einfeldträger jedoch zu deutlich kleineren Verformungen.

Eine nachgiebige bzw. teilweise Verdübelung führt auf Grund des unvollständigen Zusammenwirkens der Teilquerschnitte Beton und Stahl zu einer Vergrößerung der Verformungen im Vergleich zu einem entsprechenden Verbundträger mit vollständiger und starrer Verdübelung. Da die Tragfähigkeit und Steifigkeit der Verbundmittel im Bauwerk im Allgemeinen höher ist als in der Berechnung angenommen und die verwendeten Näherungslösungen diesen Einfluss häufig überschätzen, wird die Verformung der Verbundträger mit teilweiser Verdübelung vielfach deutlich überschätzt.

Im Folgenden werden die verschiedenen gebräuchlichen Ansätze zur Verformungsberechnung von Verbundträgern sowie ihre Anwendungsgrenzen und ihre Anwendbarkeit auf niedrige Verbundträger kurz vorgestellt. Die diskutierten Ansätze haben gemein, dass sie als Eingangsgröße unter anderem die mittragende Breite des Betongurts verwenden. Dabei wird die mittragende Breite in der Regel als in Trägerlängsrichtung konstanter Wert evtl. mit einer Abstufung im Stützbereich durchlaufender Träger angesetzt. Der tatsächliche Verlauf der mittragende Breite wird im Allgemeinen nicht berücksichtigt, vgl. Abschnitt 3.3.

\subsubsection{Linearelastische Ansätze}

Die Berechnung von Verbundträgern in der Planungspraxis erfolgt in der Regel mit dem Gesamtquerschnittsverfahren [2.29]-[2.31] seltener mit dem Verfahren nach Sattler [2.34]. Beide Verfahren beruhen auf modifizierten elastischen Ansätzen. Der entscheidende Vorteil vor allem des Gesamtquerschnittsverfahrens ist die vergleichsweise einfache Berechnung, die Anwendbarkeit des Superpositionsprinzips sowie die Möglichkeit zur einfachen Berück- 
sichtigung von Kriechen und Schwinden des Betons. Für die verschiedenen Lastfälle wie ständige Last (Index $L=B$ ), kurzzeitig wirkende Last (Index $L=0$ ), Schwinden (Index $L=S$ ) etc. wird jeweils ein ideelles Trägheitsmoment $\mathrm{I}_{\mathrm{i}, \mathrm{L}}$ des Verbundquerschnitts bestimmt, mit dessen Hilfe Verformungen auf Basis der „normalen“ Stabstatik berechnet werden. Für eine genaue Beschreibung dieser Verfahren wird auf die Literatur verwiesen.

Für hohe Verbundträger mit vergleichsweise dünnem Betongurt liefern diese Verfahren im Allgemeinen wirklichkeitsnahe Verformungswerte.

Bei Verbundträgern niedriger Bauhöhe und Slim-Floor Trägern führen diese Verfahren jedoch auf Grund der frühen Rissbildung im Beton bereits unter Gebrauchslasten zu einer zu großen rechnerischen Steifigkeit und stellen daher eine Näherung dar, die in vielen Fällen nicht ausreichend ist. Eine realistische Verformungsberechnung dieser Träger ist daher mit diesen elastischen Ansätzen in der Regel nicht möglich.

\subsubsection{Materiell nichtlineare Ansätze}

\subsubsection{Grundsätzliches}

Für eine realistische Bestimmung des Last-Verformungs-Verhaltens von Verbundträgern mit ausgeprägter Rissbildung des Betongurts, im Besonderen von niedrigen Verbundträgern und Slim-Floor Trägern, muss das nichtlineare Materialverhalten von Beton und Stahl berücksichtigt werden. Im Gebrauchslastzustand muss hierbei vor allem die Rissbildung im Beton berücksichtigt werden. Ein Plastizieren des Stahls tritt unter Gebrauchslasten in der Regel noch nicht auf. Bei Slim-Floor Trägern kann es jedoch bereits unter Gebrauchslasten auch zum Fließen der Stützbewehrung kommen.

Eine Möglichkeit zur Berechnung von Verformungen bei Verbundträgern mit ausgeprägter Rissbildung im Beton stellen Momenten-Krümmungs-Beziehungen des Gesamtquerschnitts, auch M-א-Linien genannt, dar [2.7], [2.32], [2.37] [2.73]-[2.75]. Die Berücksichtigung einer nachgiebigen Verdübelung und eines Schlupfs in der Verbundfuge ist hierbei jedoch nicht

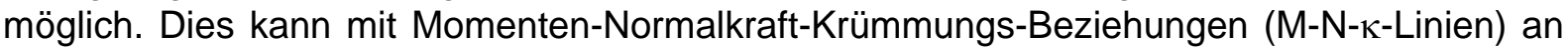
den Teilquerschnitten Betongurt und Baustahl erfolgen [2.4], [2.36].

\subsubsection{Momenten-Krümmungs-Beziehungen ( $M-\kappa-L i n i e n)$}

Momenten-Krümmungs-Beziehungen stellen den Zusammenhang des äußeren Moments des Gesamtquerschnitts und dessen Krümmung her. Die Berechnung erfolgt iterativ. Zunächst wird die Krümmung $\kappa$ des Querschnitts vorgegeben. Durch Variation des Dehnungszustands, aus dem der Spannungszustand des Verbundquerschnitts mit Kenntnis der Materialgesetze berechnet werden kann, wird das innere Kräftegleichgewicht im Verbundquerschnitt hergestellt. Bei der Berechnung der M-к-Linien können dabei unterschiedliche Materialgesetze berücksichtigt werden: Zum Beispiel kann beim Beton die Mitwirkung auf Zug berücksichtigt oder vernachlässigt werden. Weitere Informationen können [2.32] und [2.37] entnommen werden. Abbildung 2-3 zeigt beispielhaft die $M-\kappa-L i n i e$ eines niedrigen Verbundträgers.

Eine Vereinfachung zur kontinuierlichen Berechnung der M-к-Linie stellt die Linearisierung zwischen den maßgebenden Punkten dar. Diese sind:

- Beginn der Rissbildung im Beton ( $\left.\mathrm{M}_{\text {Riss }} / \kappa_{\text {Riss }}\right)$

- Beginn des Plastizierens des Baustahls $\left(\mathrm{M}_{\mathrm{el}} / \kappa_{\mathrm{el}}\right)$

- Baustahl ist vollständig plastiziert $\left(\mathrm{M}_{\mathrm{pl}} / \kappa_{\mathrm{pl}}\right)$ 
- Betonversagen auf Druck bzw. Zugversagen des Baustahls $\left(M_{u} / \kappa_{u}\right)$

- Evtl. kann auch das Fließen der Längsbewehrung als weiterer charakteristischer Punkt berücksichtigt werden.

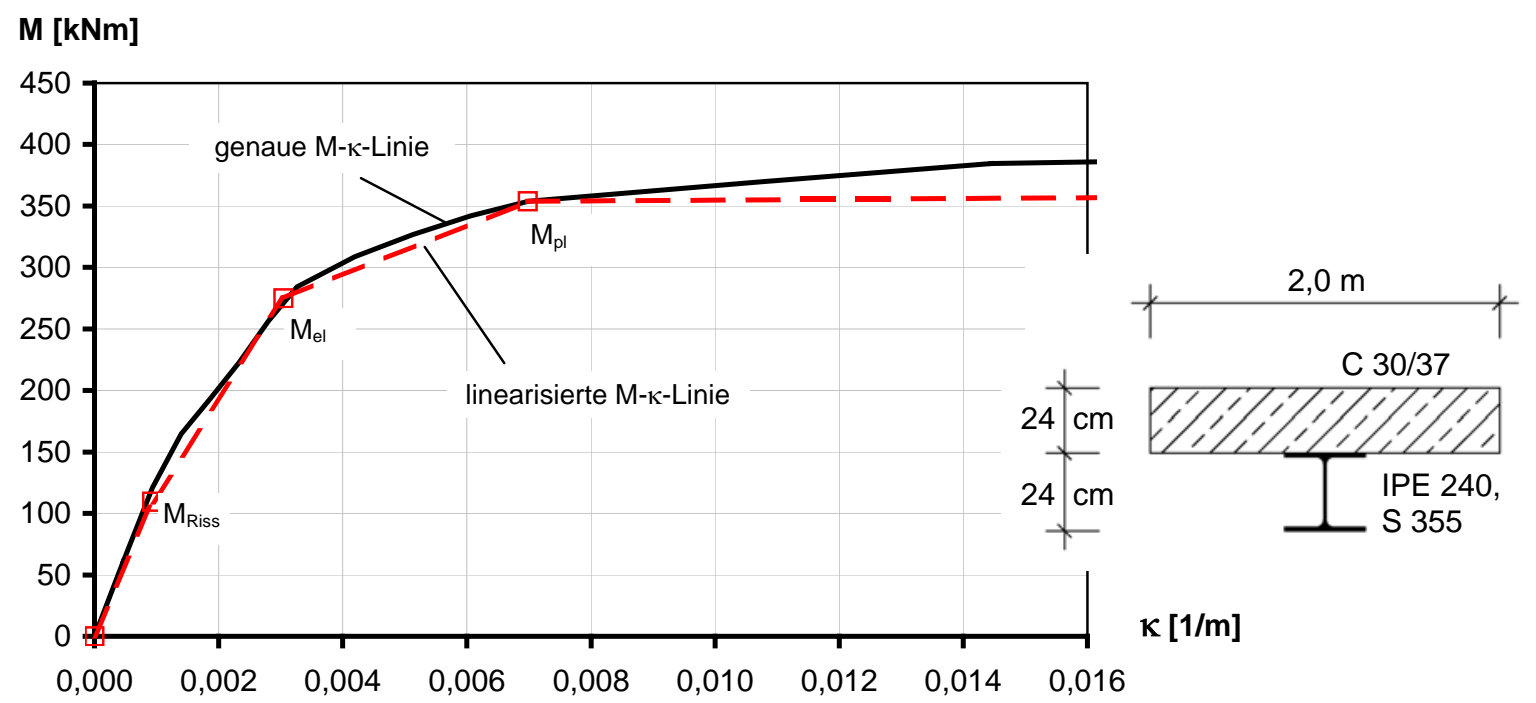

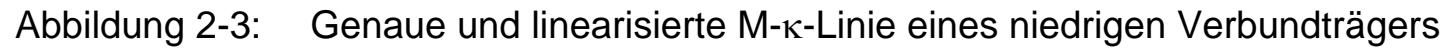

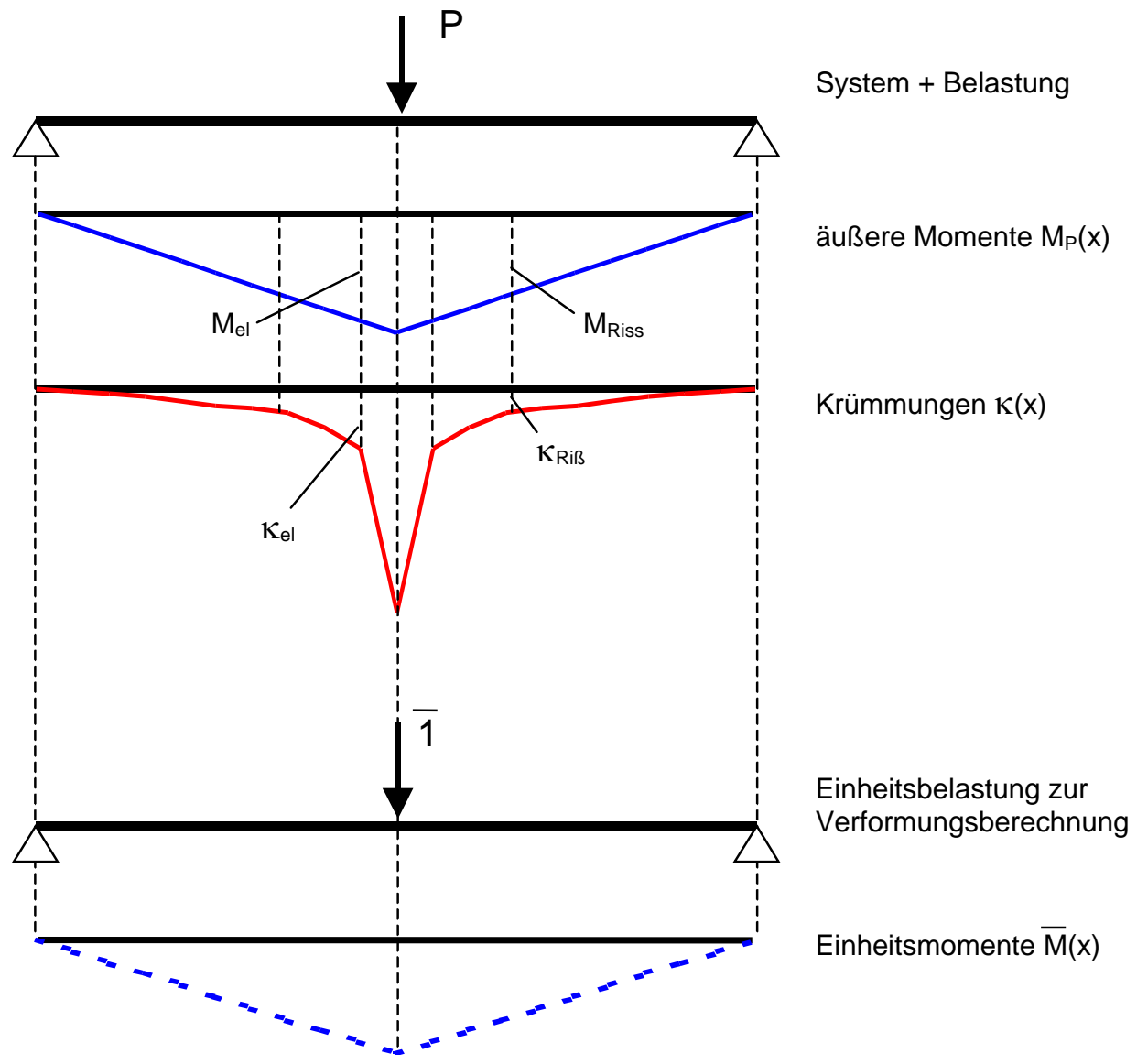

Abbildung 2-4: Vorgehen bei der Verformungsberechnung von Verbundträgern auf Basis von M-к-Linien [2.32], [2.37] 
Mit Kenntnis der M-к-Linie und des äußeren Momentenverlaufs kann der Krümmungsverlauf entlang der Trägerachse bestimmt und durch dessen Integration die Verformung des Verbundträgers berechnet werden, siehe Abbildung 2-4.

Die Gleichungen zur Berechnung von M-к-Linien und das Vorgehen bei der Verformungsberechnung von Verbundträgern sind ausführlich in [2.32] beschrieben.

\subsubsection{Berechnung mit M-N-א-Linien an den Teilquerschnitten}

Für Verbundträger, die einen ausgeprägten Schlupf zwischen Beton und Baustahl aufweisen, kann das in [2.4], [2.36] vorgestellte Verfahren zur Berechnung von Verformungen unter Berücksichtigung des nichtlinearen Materialverhaltens angewendet werden. Hierbei wird die Berechnung an den Teilquerschnitten Stahlbetongurt und Baustahlträger anstatt am Gesamtquerschnitt vorgenommen. Die Berechnung ist vergleichsweise aufwändig und wird nur in Sonderfällen empfohlen.

Für eine genaue Beschreibung des Vorgehens bei der Berechnung sei an dieser Stelle auf [2.4], [2.36] verwiesen.

\subsubsection{Schlussfolgerung}

Aufgrund der Rissbildung im Betongurt von niedrigen Verbundträgern bereits im Gebrauchslastzustand ist eine realistische Berechnung der Trägerdurchbiegung nur unter Berücksichtigung des nichtlinearen Materialverhaltens möglich. Dies kann z. B. mit M-к-Linien oder M-N$\kappa$-Linien erfolgen. Für eine wirklichkeitsnahe Verformungsberechnung muss darüber hinaus gegebenenfalls die Querkraftverformung berücksichtigt werden. 



\section{Die mittragende Betongurtbreite - Stand der Technik}

\subsection{Grundsätzliches}

Nach der grundsätzlichen Beschreibung des Trag- und Verformungsverhaltens niedriger Verbundträger in Kapitel 2 werden im Folgenden speziell das Tragverhalten breiter Gurte und die Hintergründe für die Einführung der mittragenden Breite kurz aufgearbeitet. Daran anschließend werden die bestehenden Normregelungen und die bekannten Veröffentlichungen zur mittragenden Breite kurz dargestellt. Dabei zeigt sich, dass diese Regelungen nur die spannungsbezogene mittragende Breite jedoch nicht die verformungsbezogene beschreiben und dem speziellen Trag- und Verformungsverhalten niedriger Verbundträger nicht Rechnung tragen. Für eine wirklichkeitsnahe Verformungsberechnung niedriger Verbundträger sind daher die in dieser Arbeit vorgestellten Untersuchungen zur verformungsbezogenen mittragenden Breite niedriger Verbundträger notwendig.

\subsection{Tragverhalten von Gurten in Plattenbalken}

Plattenbalken im weiteren Sinn sind Tragwerke, die aus einem oder mehreren schmalen Trägern (Stege) und einem oder mehreren Gurten, die kraftschlüssig an diese Träger angeschlossen sind, bestehen. Bei Verbundträgern im Geschossbau bestehen diese im Allgemeinen aus Stahlträgern mit aufliegender Betonplatte, die über Verbundmittel schubfest miteinander verbunden sind. Abbildung 3-1 zeigt eine Auswahl an möglichen Plattenbalkenquerschnitten. Die Untersuchungen in dieser Arbeit beschränken sich auf Verbundträger mit nur einer Gurtplatte an der Oberseite des Stahlträgers.
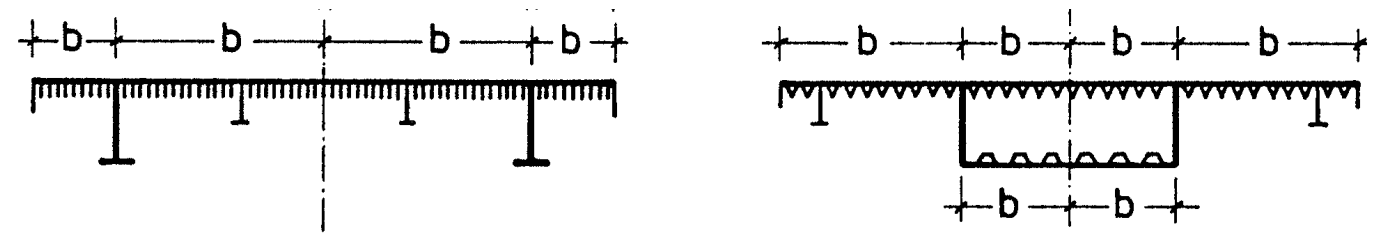

(a) Stahlquerschnitte
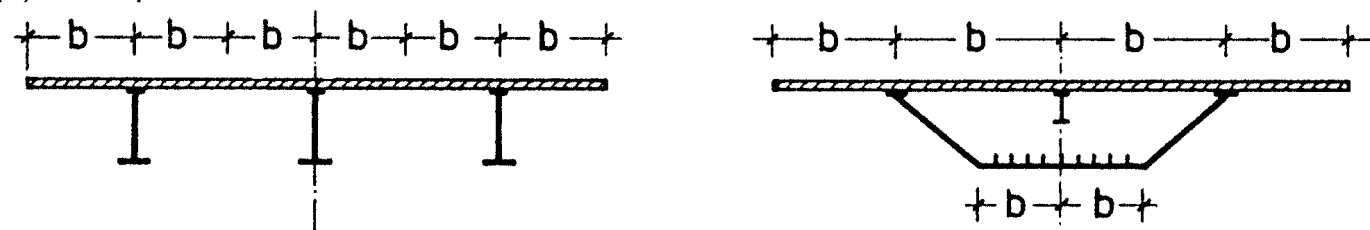

(b) Verbundquerschnitte
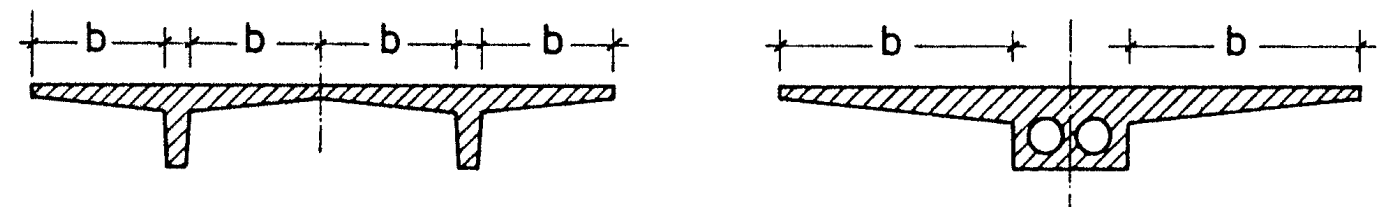

(c) Betonquerschnitte

Abbildung 3-1: Mögliche Plattenbalkenquerschnitte [2.38]

Die Gurte der Plattenbalken erfüllen im Allgemeinen zwei Aufgaben im Tragwerk: Zum einen wirken sie als Platten, die die zwischen den Trägern angreifenden Lasten in Querrichtung über Biegung zu den Trägern abtragen. Die Träger wirken hierbei als Auflager für die Platte. Zum anderen wirken die Gurte als Druck- bzw. Zuggurt im Plattenbalken in Trägerlängsrichtung mit. Auf Grund der Schubweichheit entziehen sich die vom Träger weiter entfernten Bereiche des Gurtes jedoch dieser Lastabtragung in Längsrichtung. Der Gurt wirkt im Plattenbalken somit nicht vollständig mit. Aufgrund des einachsigen Lastabtrags der Plattenbal- 
ken und zur Vereinfachung der Rechnung ist es jedoch wünschenswert, den Plattenbalken in Längsrichtung mit der normalen technischen Biegelehre zu berechnen. Dies führt auf den Begriff der mittragenden Breite [2.38].

Das Tragverhalten des Gurtes in Trägerlängsrichtung lässt sich dabei grundsätzlich in einen Scheibenzustand und einen Biegezustand unterscheiden, siehe Abbildung 3-2 [2.27], [2.35]. Der Scheibenzustand resultiert aus der Dehnsteifigkeit des Gurtes und der schubfesten Verbindung mit dem Stahlträger. Die Gurtnormalkraft ist Teil des inneren Kräftepaars im Verbundquerschnitt. Beim Scheibenzustand wird der Gurt als biegeschlaffe Scheibe betrachtet. Der Biegezustand resultiert aus der jedoch immer vorhandenen Biegesteifigkeit des Gurtes und der Kopplung der vertikalen Verformungen des Gurtes und des Stahlträgers. Hieraus ergibt sich ein Biegemoment im Gurtquerschnitt.

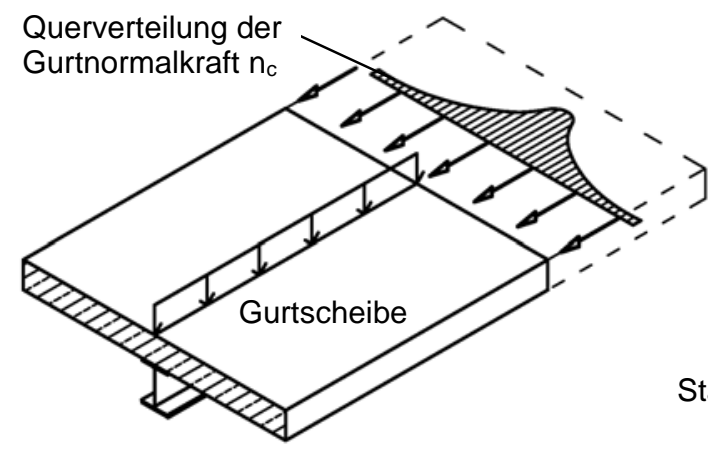

(a) Scheibenzustand

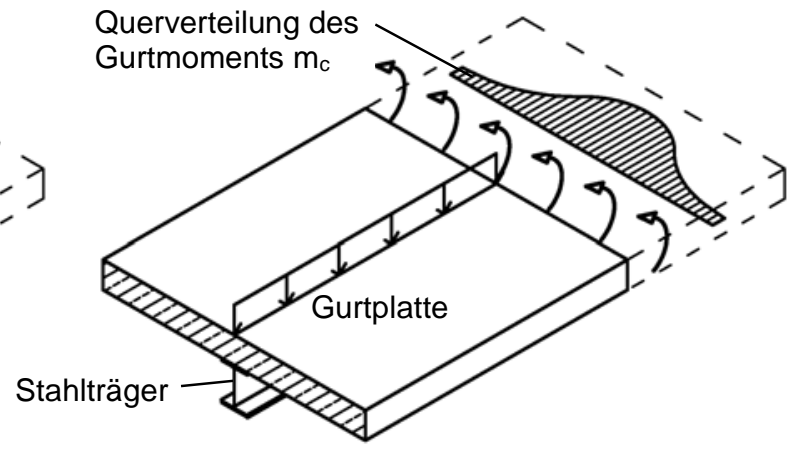

(b) Biegezustand

Abbildung 3-2: $\quad$ Scheiben- und Biegezustand des Gurtes von Verbundträgern [2.27], [2.35]

Die alleinige Betrachtung des Scheibenzustands unter Vernachlässigung der Biegesteifigkeit des Gurtes ist beispielsweise für reine Stahlträger oder hohe Verbundträger mit dünnen Betongurten eine ausreichend gute Näherung des tatsächlichen Tragverhaltens. Bei niedrigen Verbundträgern ist die Biegesteifigkeit des Betongurts jedoch so groß, dass diese für eine realistische Bestimmung des Trag- und Verformungsverhaltens nicht vernachlässigt werden kann [2.26], [2.27], [2.35].

\subsection{Definition der mittragenden Breite}

\subsubsection{Allgemeines}

Aufgrund der Schubweichheit der Gurtscheibe ist die Spannungsverteilung in Querrichtung des Gurtes nicht konstant, sondern nimmt vom Steg des Plattenbalkens nach außen ab, siehe Abbildung 3-3. Somit wirkt nicht die gesamte Gurtscheibe im Plattenbalken mit. Plattenbalken zeigen daher ein ausgeprägtes dreidimensionales Tragverhalten und lassen sich als eindimensionales Tragelement nur näherungsweise erfassen.

Um dennoch die technische Biegelehre verwenden zu können, wurde die mittragende Breite eingeführt. Die mittragende Breite ist hierbei eine fiktive Größe, mit deren Hilfe der Plattenbalken auf einen voll wirksamen Querschnitt reduziert wird und als eindimensionaler Träger berechnet werden kann.

Die so definierte mittragende Breite bezieht sich nach dieser Definition somit auf die Querverteilung der Gurtlängsspannungen an einer bestimmten Stelle des Plattenbalkens und wird folglich in dieser Arbeit als "(spannungsbezogene) mittragende Breite“ bezeichnet. Wie bekannt ist und die weiteren Ausführungen in dieser Arbeit zeigen, verändert sich die Größe 
der spannungsbezogenen mittragenden Breite in Trägerlängsrichtung und stellt somit keinen Querschnittswert dar, siehe Abbildung 3-5. In der Literatur liegen hierzu eine Vielzahl von Untersuchungen und Veröffentlichungen vor, z. B. [2.38]-[2.63].

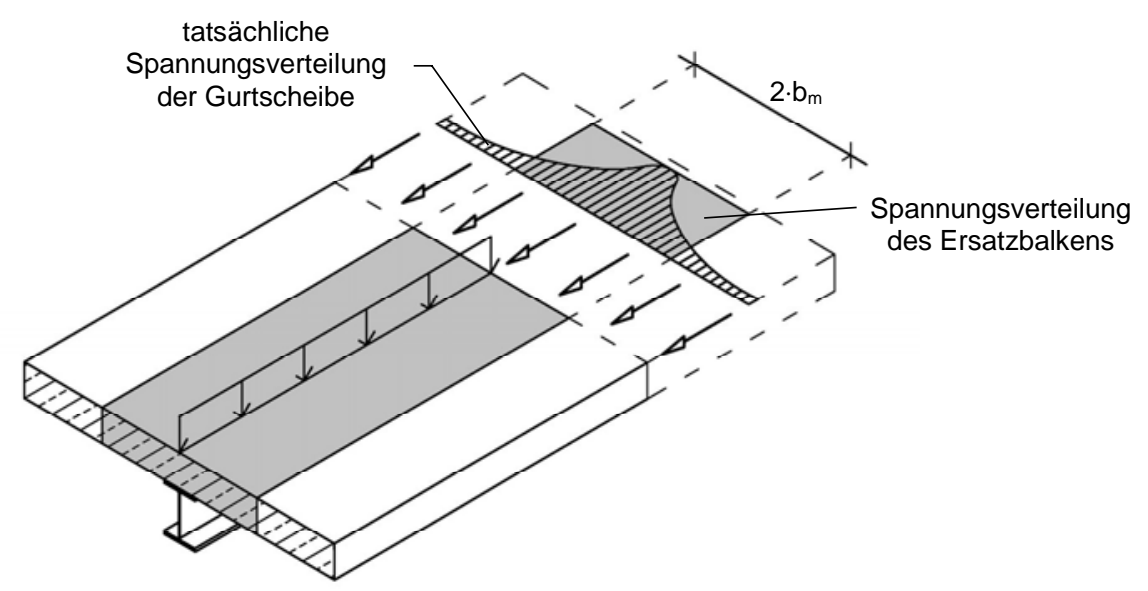

Abbildung 3-3: Definition der mittragenden Breite $b_{m}$ für die Spannungsberechnung

Für die Verformungsberechnung eines Plattenbalkens mit Hilfe der technischen Biegelehre muss strenggenommen der Verlauf der mittragenden Breite in Trägerlängsrichtung und deren Einfluss auf die Biegesteifigkeit des Trägers berücksichtigt werden. Da dies für eine praktische Berechnung viel zu aufwändig ist, wird im Allgemeinen ein konstanter Wert evtl. mit einer Abstufung im Stützbereich angesetzt. Hierfür wird in dieser Arbeit der Begriff der „verformungsbezogenen mittragenden Breite" eingeführt, siehe Abschnitt 3.3.3.

Zur Unterscheidung werden in dieser Arbeit der Index "m" als Hinweis auf den Spannungsbezug und der Index "m,V" für den Verformungsbezug verwendet.

$M_{u} / M_{u}\left(b_{m}=3 m\right)$

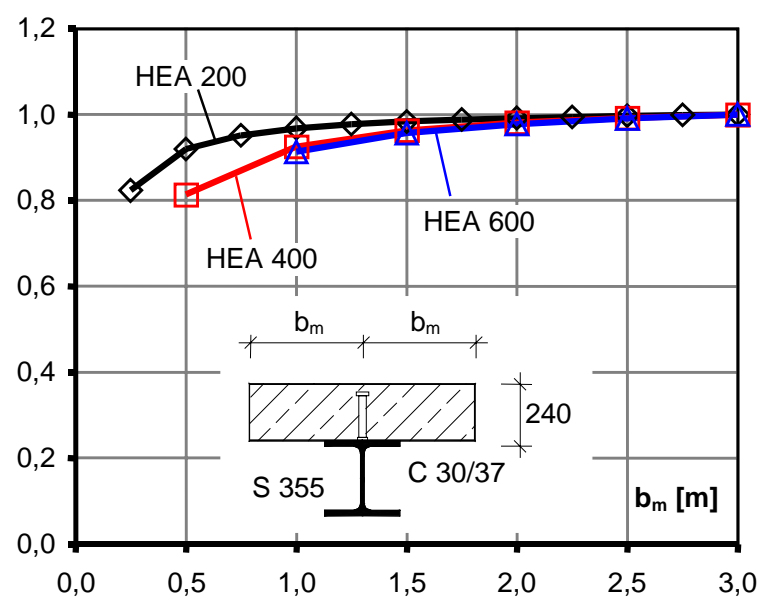

(a) Tragfähigkeit der Verbundquerschnitte

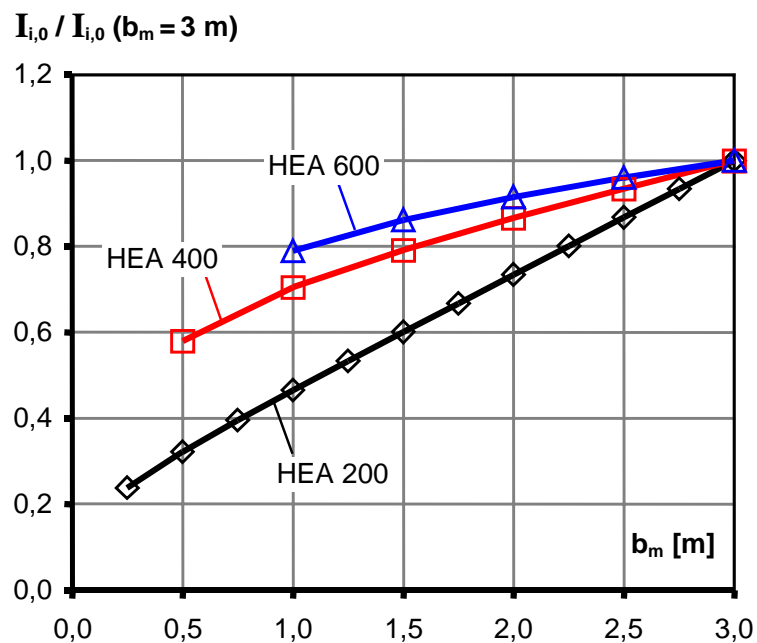

(b) Steifigkeit der Verbundquerschnitte

Abbildung 3-4: Einfluss der mittragenden Breite $b_{m}$ auf die Tragfähigkeit und Steifigkeit üblicher Verbundquerschnitte

Wie anhand der Berechnung der Tragfähigkeit gängiger Verbundquerschnitte zu erkennen ist, hat die Größe der mittragenden Breite im Rahmen baupraktischer Randbedingungen nur einen sehr geringen Einfluss auf die Querschnittstragfähigkeit, siehe Abbildung 3-4. Dies gilt insbesondere für Bereiche, in denen der Betongurt unter Druck steht. Dies liegt unter ande- 
rem daran, dass sich durch eine Variation der mittragenden Breite zwar die Druckzonenhöhe im Beton, aber nur unwesentlich der Hebelarm im Verbundquerschnitt verändert und daher die Tragfähigkeit nahezu unverändert bleibt. Auf die rechnerische Steifigkeit der Verbundquerschnitte hat die mittragenden Breite im Gegensatz dazu jedoch einen sehr großen Einfluss - insbesondere bei niedrigen Verbundträgern und Slim-Floor Trägern, siehe Abbildung 3-4. Grund hierfür ist, dass vor allem bei niedrigen Verbundträgern die Biegesteifigkeit $\mathrm{I}_{\mathrm{c}}$ des Betongurts einen maßgebenden Einfluss auf die elastisch berechnete Gesamtsteifigkeit $\mathrm{I}_{i, 0}$ des Verbundquerschnitts hat und sich $\mathrm{I}_{\mathrm{c}}$ proportional zur mittragenden Breite verhält. Da bei hohen Verbundträgern der Einfluss der Biegesteifigkeit des Betongurts und der mittragenden Breite auf die Gesamtsteifigkeit des Verbundträgers vergleichsweise klein ist und deren Querschnittsdimensionierung im Allgemeinen durch den Nachweis der Tragfähigkeit bestimmt wird, ist bei diesen Trägern die genaue Kenntnis der mittragenden Breite von keiner allzu großen Bedeutung. Bei niedrigen Verbundträgern und Slim-Floor Trägern ist jedoch im Gegensatz dazu der Einfluss der Biegesteifigkeit des Betongurts und der mittragenden Breite auf die Gesamtsteifigkeit sehr groß. Da die Dimensionierung dieser Träger in vielen Fällen durch deren Verformung bestimmt wird, ist hier die Kenntnis der tatsächlichen mittragenden Breite von großer wirtschaftlicher Bedeutung. Hierin liegt die Motivation dieser Arbeit.

Diese Arbeit widmet sich daher der Untersuchung der verformungsbezogenen mittragenden Breite $b_{m, v}$ speziell niedriger Verbundträger.

\subsubsection{Mittragende Breite $b_{m}$ für die Spannungsberechnung}

Die Definition der mittragenden Breite $b_{m}$ über die Querverteilung der Gurtlängsspannungen kann als klassische Definition betrachtet werden. In dieser Arbeit wird der Index " $\mathrm{m}$ " als Hinweis auf den Spannungsbezug der mittragenden Breite verwendet.
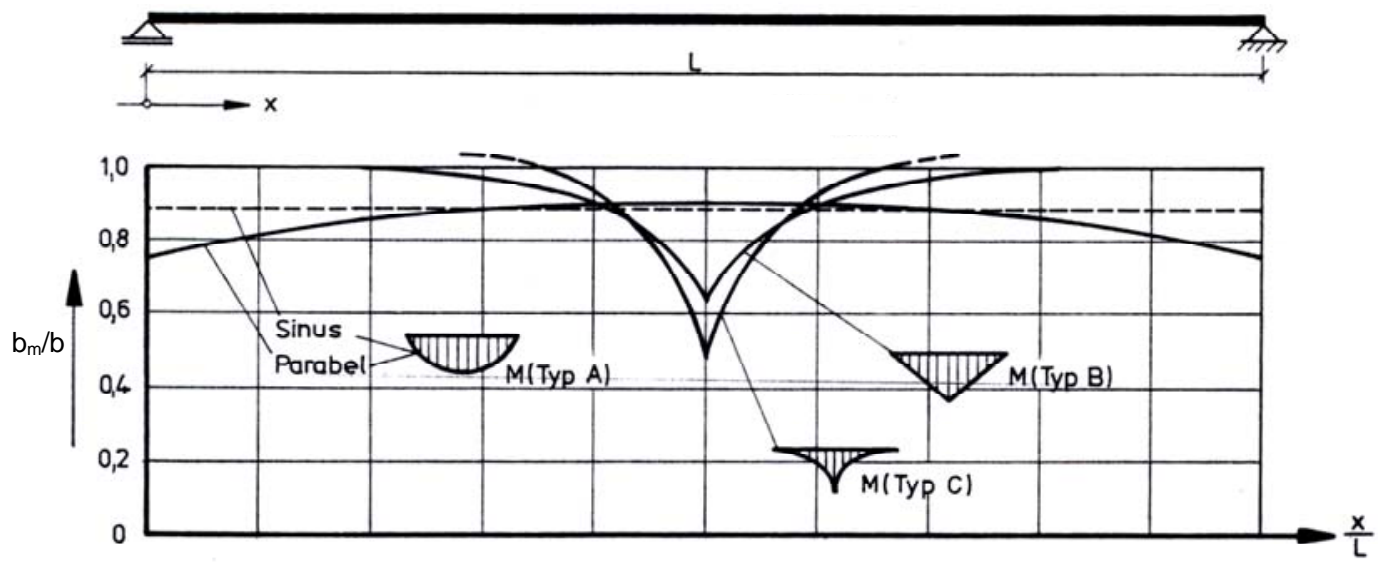

Abbildung 3-5: Verlauf der spannungsbezogenen mittragenden Breite in Trägerlängsrichtung bei elastischem Materialverhalten [2.38]

Die mittragende Breite $b_{m}$ ist durch die Forderung definiert, dass bei Berechnungen nach der technischen Biegelehre an einem eindimensionalen Ersatzbalken, dessen Breite des Betonquerschnitts gleich $b_{m}$ ist, die gleiche Spannung an der Verbindungslinie zwischen Steg und Gurtscheibe ermittelt wird wie mit einer genauen Berechnung, die den nichtlinearen Spannungszustand des Gurtes berücksichtigt [2.56]. Bei elastischem Materialverhalten ist dies gleichbedeutend mit folgender Definition: Die mittragende Breite ist definiert als jene Breite, die unter der Annahme einer konstanten Spannungsverteilung im Gurt die gleiche Spannungsresultierende liefert wie die tatsächliche Spannungsverteilung über die gesamte Gurtbreite. Dabei ist der über dem Steg auftretende Größtwert der Spannung über die Breite der 
mittragenden Breite konstant wirksam, siehe Abbildung 3-3 bzw. Gleichung (3-1). Die mittragende Breite bezieht sich hierbei auf die Berechnung der maximalen Gurtspannung im Hinblick auf den Nachweis der Tragfähigkeit des Plattenbalkens und ändert sich in Trägerlängsrichtung, siehe Abbildung 3-5.

$$
\mathrm{b}_{\mathrm{m}}=\frac{1}{\sigma_{\mathrm{x}, \max }} \cdot \int \sigma_{\mathrm{x}} \mathrm{dy}
$$

Bei der überwiegenden Zahl der bekannten Veröffentlichungen werden die Gurte als biegeschlaffe Scheiben betrachtet und die Biegetragwirkung vernachlässigt. Bei den hier betrachteten Verbundträgern niedriger Bauhöhe sind die Biegetragwirkung und die Biegesteifigkeit des Betongurts jedoch so groß, dass diese nicht vernachlässigt werden können [2.26], [2.27], [2.35].

\subsubsection{Verformungsbezogene mittragende Breite $b_{m, v}$}

Wie oben bereits erwähnt, ist die (spannungsbezogene) mittragende Breite in Trägerlängsrichtung und damit auch die Steifigkeit des Verbundquerschnitts veränderlich. Dies gilt im Allgemeinen auch für Träger mit prismatischem, in Trägerlängsrichtung konstantem Querschnitt. Für eine exakte Verformungsberechnung muss daher der Verlauf der Querschnittssteifigkeit und auch der (spannungsbezogenen) mittragenden Breite in Trägerlängsrichtung berücksichtigt werden. Für eine praktische Berechnung ist solch ein Vorgehen jedoch viel zu aufwändig. In vielen Fällen wird daher vereinfachend der Wert im maßgebenden Querschnitt konstant über die gesamte Trägerlänge eventuell mit einer Abstufung im Stützbereich angesetzt. Für hohe Verbundträger, bei denen der Beitrag des Betongurts und vor allem dessen Biegesteifigkeit $\mathrm{I}_{\mathrm{c}}$ zur Gesamtsteifigkeit nur gering ist, stellt dies im Allgemeinen eine ausreichende Näherung dar. Dies gilt nicht zuletzt deshalb, weil die Querschnittssteifigkeit im höchstbelasteten Bereich den größten Einfluss auf die Trägerdurchbiegung hat. Bei niedrigen Verbundträgern ist diese Vereinfachung jedoch unter anderem aus folgenden Gründen nicht akzeptabel:

- Der Beitrag des Betongurts und insbesondere dessen Biegesteifigkeit $\mathrm{I}_{\mathrm{c}}$ zur Gesamtsteifigkeit des Verbundträgers ist sehr groß.

- Die Rissbildung im Betongurt führt zu einem sehr ungleichmäßigen Verlauf der mittragenden Breite in Trägerlängsrichtung mit einem Minimum an der höchstbelasteten Trägerstelle. Der globale Ansatz der mittragenden Breite an der höchstbelasteten Stelle führt daher zu einer deutlichen Unterschätzung der Trägersteifigkeit und einer Überschätzung der Durchbiegung.

Aus diesem Grund wird in dieser Arbeit die verformungsbezogene mittragende Breite $b_{m, v}$ eingeführt und folgendermaßen definiert: Die Durchbiegungsberechnung am Ersatzbalken unter Annahme der in Trägerlängsrichtung konstanten Breite, die gleich der verformungsbezogenen mittragenden Breite $b_{m, v}$ ist, ergibt die gleiche Durchbiegung wie beim realen Plattenbalken.

Zur Unterscheidung wird für den Verformungsbezug der mittragenden Breite der Index „m, $\mathrm{V}^{“}$ verwendet.

\subsection{Vergleich verschiedener Normen}

In den nationalen und europäischen Normen des Stahlbeton- und Verbundbaus im Hochund Brückenbau ist die mittragende Breite von Betongurten unterschiedlich geregelt, wie die 
folgende Zusammenfassung für den Feldbereich von Plattenbalken mit parabelförmigem Momentenverlauf zeigt, siehe Abbildung 3-7 und Abbildung 3-8.

Eine Unterscheidung in die mittragende Breite $b_{m}$ für die Spannungsberechnung und die mittragende Breite $b_{m, v}$ für die Verformungsberechnung wird dabei mit Ausnahme von DIN 1075 [1.6] nicht vorgenommen.

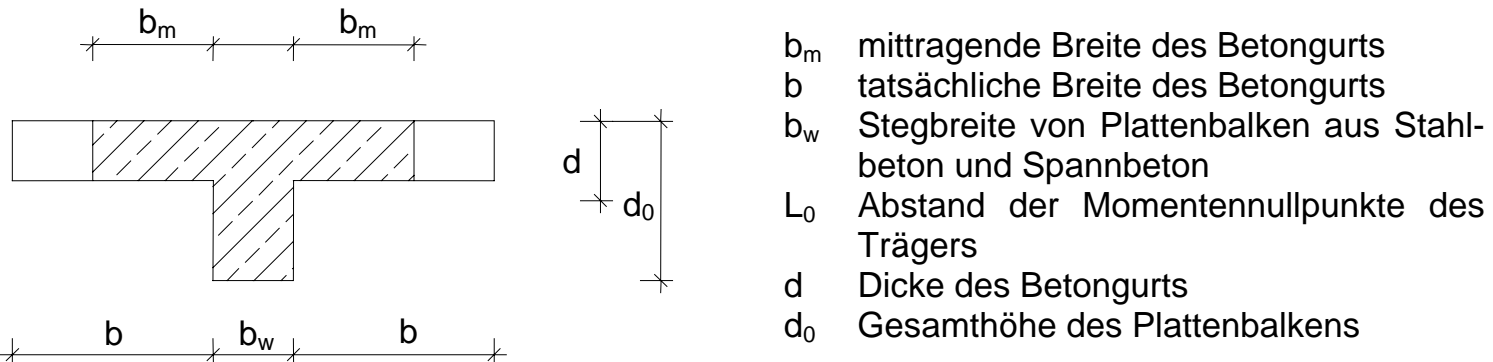

Abbildung 3-6: Bezeichnungen zur mittragenden Breite

Der Vergleich der Regel zur mittragenden Breite in EN 1994-1-1 [1.1] mit den unterschiedlichen nationalen Regelungen zeigt eine große Bandbreite an „möglichen“ Werten für die mittragende Breite, siehe Abbildung 3-7. Dabei liefert EN 1994-1-1 sehr viel kleiner Werte für die mittragende Breite als vor allem gegenüber den Regeln für Stahlbetonplattenbalken in Heft 240 [2.58] und DIN 1045-1 [1.8]. In Heft 240 ist hierbei eine starke Abhängigkeit von der Dicke des Betongurts, ausgedrückt durch das Verhältnis $\mathrm{d} / \mathrm{d}_{0}$, erkennbar. Je dicker der Betongurt ( $d / d_{0}$ wird größer) desto größer ist die mittragende Breite. Dies liegt unter anderem daran, dass bei den Regeln in Heft 240 der Einfluss der Biegetragwirkung und Biegesteifigkeit des Betongurts auf die mittragende Breite berücksichtigt ist. Im Vergleich zu EN 1994-11 ergibt sich für $\mathrm{d} / \mathrm{d}_{0}=0,3$ eine um $50 \div 160 \%$ größere mittragende Breite. Ein ähnlicher Einfluss der Dicke des Betongurts auf die mittragende Breite ist auch bei den betrachteten Verbundträgern niedriger Bauhöhe zu erwarten.

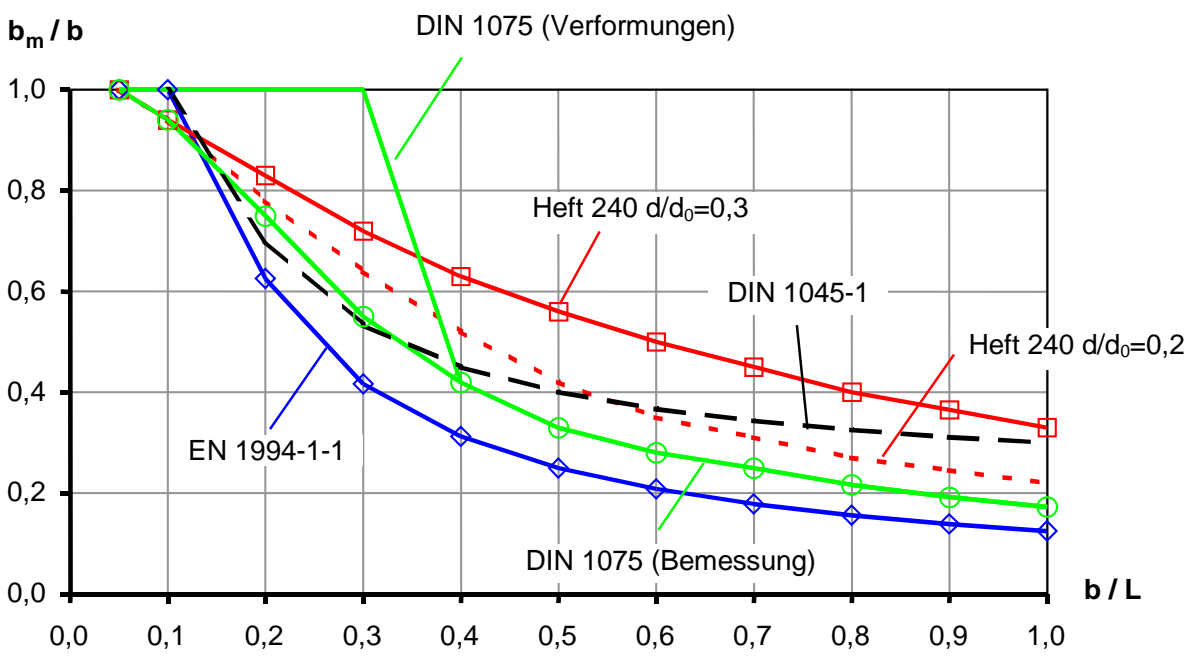

Abbildung 3-7: Vergleich der mittragenden Betongurtbreite nationaler Normen für den Feldbereich von Plattenbalken mit parabelförmigem Momentenverlauf

Der Vergleich der unterschiedlichen europäischen Normen in Abbildung 3-8 zeigt ebenfalls eine große Bandbreite an „möglichen“ Werten für die mittragende Breite. EN 1994-1-1 [1.1], prEN 1994-2 [1.2] und ENV 1992-1-1 [1.3] ergeben für die mittragende Breite die kleinsten Werte. BS 5400-5 [1.9] unterscheidet für die mittragende Breite in verschiedene Systeme und Belastungsformen. Gerade für eine gleichmäßige Belastung ergeben sich vergleichs- 
weise große Werte, die eine gute Übereinstimmung mit den Werten in Heft 240 zeigen. Dies liegt vermutlich an der Berücksichtigung der Biegetragwirkung des Betongurts. Die im Bereich sehr kleiner Verhältnisse $b / L$ relativ kleinen Werte der mittragenden Breite für eine mittige Einzellast von BS 5400-5 sind für die dort betrachteten Verbundbrücken weniger interessant, da hier die Hauptbelastung aus dem Eigengewicht der Träger kommt und somit gleichmäßig verteilt ist. Ob die in ENV 1992-2 [1.4] zur Verformungs-, Schnittgrößen- und Schwingungsberechnung angegebene Regel $b_{m}=b$ realistische Werte liefert, ist zumindest für große Verhältnisse b/L (also kurze Träger mit sehr breiten Gurten) zweifelhaft. Dies würde unterstellen, dass der Betongurt überhaupt keiner Schubverformung unterliegt.

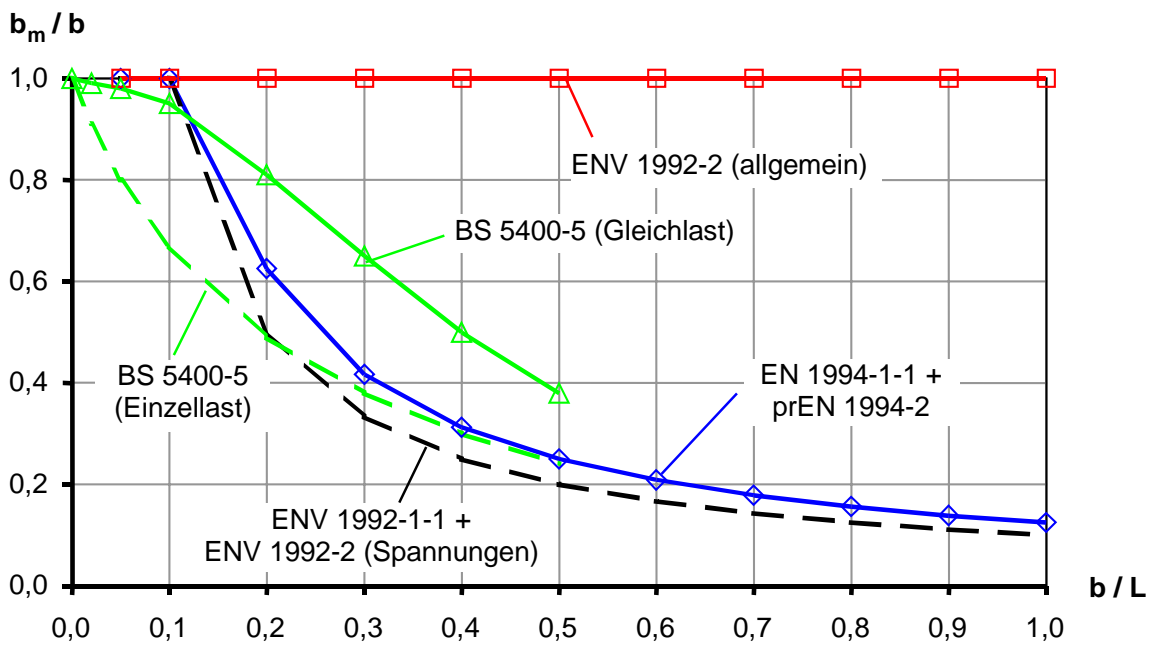

Abbildung 3-8: Vergleich der mittragenden Betongurtbreite europäischer Normen für den Feldbereich von Plattenbalken mit parabelförmigem Momentenverlauf

Zwar haben diese unterschiedlichen Werte der mittragenden Breite $b_{m}$ für die Berechnung der Tragfähigkeit im Grenzzustand der Tragfähigkeit keine große Auswirkung, für die rechnerische Steifigkeit im Grenzzustand der Gebrauchstauglichkeit sind sie jedoch von großer Bedeutung, siehe Abbildung 3-4.

Die dargestellten zur Zeit gültigen Normregelungen zur mittragenden Breite können, vor allem weil sie die Rissbildung im Betongurt und die große Biegesteifigkeit des Betongurts bei Verbundträgern niedriger Bauhöhe und Slim-Floor Trägern nicht berücksichtigen, keine realistische Aussage über die mittragende Breite der betrachteten Verbundträger machen.

\subsection{Stand der bisherigen wissenschaftlichen Untersuchungen}

\subsubsection{Allgemeines}

Wie in Abschnitt 3.2 beschrieben, setzt sich das Tragverhalten von Betongurten in Plattenbalken grundsätzlich aus einem Scheiben- und einem Biegezustand zusammen. Für elastisches Materialverhalten können diese beiden Zustände nach dem Prinzip der Superposition voneinander getrennt werden, siehe unter anderem [2.27], [2.35]. Für beide Zustände kann dann getrennt eine entsprechende mittragende Breite bestimmt werden: $b_{m, s}$ für den Scheibenzustand und $b_{m, B}$ für den Biegezustand. Die mittragende Breite des reinen Biegezustands ist dabei deutlich größer als die des reinen Scheibenzustands, siehe Abbildung 3-9 bzw. Kapitel 4.

Der überwiegende Teil der bekannten Untersuchungen zur mittragenden Breite berücksichtigt den reinen Scheibenzustand unter Vernachlässigung der Biegesteifigkeit und Biegetrag- 
wirkung des Gurtes. Dabei werden in erster Linie Träger untersucht, die ein sehr kleines Verhältnis Gurtdicke zu Trägerhöhe aufweisen, z. B. reine Stahlträger, hohe Verbundträger oder hohe Stahlbeton-/Spannbetonträger mit dünnem Betongurt. In diesen Fällen ist die Biegetragwirkung des Betongurts und dessen Biegesteifigkeit in der Tat so gering, dass diese im Vergleich zur Scheibentragwirkung vernachlässigt werden können. Für niedrige Verbundträger trifft dies jedoch nicht zu. Die Vernachlässigung der Biegetragwirkung des Betongurts stellt hier eine unzureichende Näherung des tatsächlichen Tragverhaltens dar [2.27], [2.35].

$\mathbf{b}_{\mathrm{m}} / \mathbf{b}$

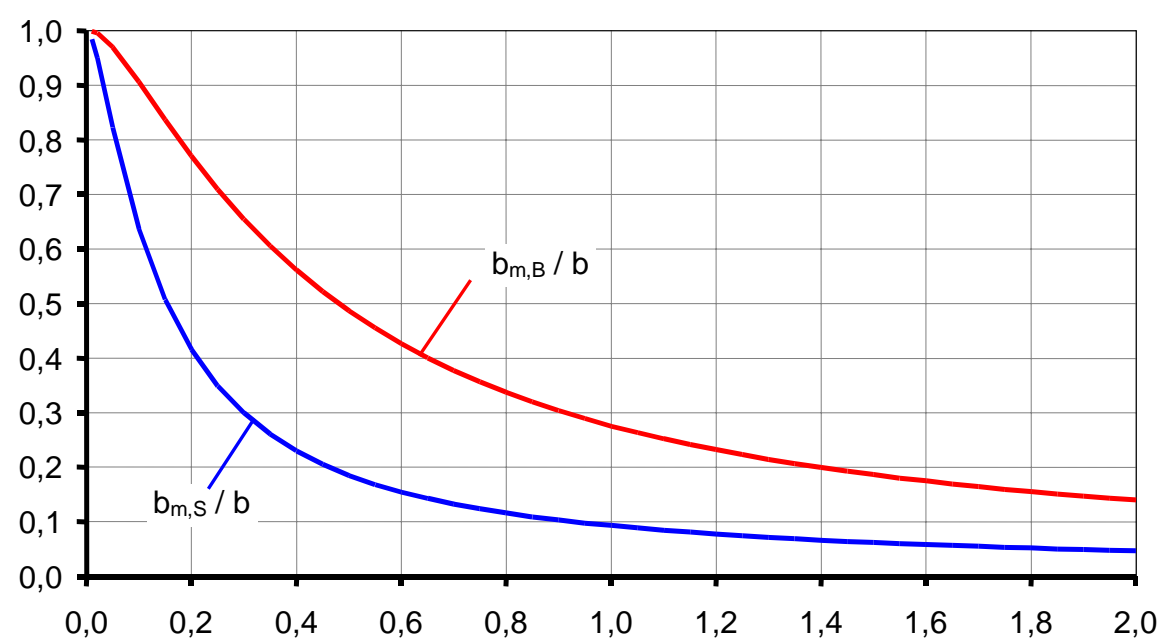

\section{Querschnitt:}

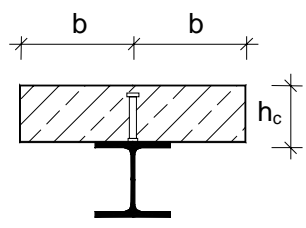

System:

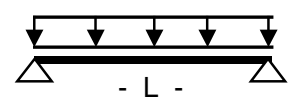

b / L

Abbildung 3-9: Mittragende Breite $b_{m, B}$ und $b_{m, s}$ des einstegigen Plattenbalkens in Feldmitte unter Gleichlast [2.26], [2.27], [2.35]

Forschungsarbeiten, die den Einfluss der Biegetragwirkung des Gurtes auf die mittragende Breite berücksichtigen, beschränken sich im Wesentlichen auf elastisches Materialverhalten. Der Einfluss der Rissbildung im Betongurt auf die mittragende Breite für die Biegetragwirkung wird hierbei jedoch nicht berücksichtigt. Doch gerade dieser Einfluss ist für das Tragund Verformungsverhalten niedriger Verbundträger und Slim-Floor Träger von entscheidender Bedeutung [2.27], [2.35]. Die bekannten Arbeiten zur mittragenden Breite unter Berücksichtigung der Biegetragwirkung des Betongurts zeigen jedoch qualitativ, dass mit zunehmender Biegetragwirkung und Biegesteifigkeit des Gurtes die mittragende Breite zunimmt, ein möglicher Einfluss der Rissbildung des Betons hierauf bleibt jedoch ungeklärt.

Grundsätzlich liegt es nahe, auf Grund der geometrischen und statischen Übereinstimmungen niedrige Verbundträger hinsichtlich der mittragenden Breite des Betongurts mit Stahlbeton-Plattenbalken zu vergleichen. In beiden Systemen wirkt der Betongurt zum einen als quergespannte Decke und zum anderen als breiter Flansch des Trägerquerschnitts. Dennoch gibt es entscheidende Unterschiede zwischen beiden Querschnitten:

- Da bei Verbundträgern niedriger Bauhöhe und Slim-Floor Trägern der Betongurt im Vergleich zur Gesamthöhe des Trägers sehr viel dicker ist $\left(d / d_{0}>0,3\right)$ als bei Stahlbeton-Plattenbalken trägt dieser hier stärker auf Biegung ab und erfährt eine stärkere Rissbildung als der Betongurt von Stahlbeton-Plattenbalken.

- Im Vergleich zu Stahlbetonträgern ergeben sich bei Verbundträgern im Allgemeinen größere Druckkräfte im Betongurt. Dies liegt zum einen an der größeren Normalkraft der Stahlträger von Verbundträgern im Vergleich zur Bewehrung von Stahlbetonträgern und zum anderen an der gedrungeneren Ausführung von Verbundträgern. 
Im Folgenden wird eine kurze Zusammenfassung der Ergebnisse der wesentlichen Arbeiten zur mittragenden Breite von Plattenbalken des Stahl-, Verbund- und Stahlbetonbaus gegeben und die in der vorliegenden Arbeit vorgenommenen Untersuchungen in diesen Rahmen eingeordnet. Dabei werden die bekannten Veröffentlichungen nach folgender Systematik geordnet:

- Untersuchungen zur reinen Scheibentragwirkung des (Beton-) Gurts unter Vernachlässigung der Biegetragwirkung

- Untersuchungen zur kombinierten Scheiben- und Biegetragwirkung des (Beton-) Gurts

\subsubsection{Untersuchungen zur Scheibentragwirkung des (Beton-) Gurts}

Diese Untersuchungen vernachlässigen im Allgemeinen die Biegetragwirkung des Betongurts und gehen von der Annahme aus, dass der Betongurt aus der Plattenbalkenwirkung nur durch eine Normalkraft $\mathrm{N}_{\mathrm{c}}$ aus dem inneren Kräftepaar beansprucht wird $\left(\mathrm{I}_{\mathrm{c}, 0} \approx 0\right.$ und $\left.M_{c} \approx 0\right)$. Veröffentlichungen, die zu dieser Gruppe zählen, liegen in großem Umfang vor.

Die Veröffentlichungen zu diesem Thema gehen bis zum Beginn des 20. Jahrhunderts zurück und sind mit den Namen v. Karman [2.39], Metzer [2.40] und Chawalla [2.41] verbunden. Ausgehend von der Elastizitätstheorie und der Scheibendifferentialgleichung wird die mittragende Breite für verschiedene Randbedingungen mit Hilfe von Reihenansätzen bestimmt. Die Belastung wird hierbei in Stegebene aufgebracht, das heißt die Beanspruchung des Gesamtsystems Plattenbalkendecke wird im Sinne des Superpositionsprinzip in eine Plattenaufgabe des Gurtes mit der Abtragung der Lasten auf die Hauptträger und die Weiterleitung über die Plattenbalkenwirkung zu den Auflagern unterteilt. Im elastischen Fall beeinflusst die evtl. vorhandene Querbiegung im Gurt daher die mittragende Breite nicht. Die Untersuchungen gehen von prismatischen Hauptträgern mit in Trägerlängsrichtung gleichbleibendem Querschnitt und isotropen Gurtquerschnitten aus. Dabei zeigt sich, dass die mittragende Breite unter anderem von folgenden Parametern beeinflusst wird:

- Seitenverhältnis b/L des Gurtes

- Statisches System des Trägers (Einfeld-, Durchlauf-, Kragträger)

- Belastung (Gleichlast, Einzellasten, etc.)

- Querschnittsausbildung (einseitiger oder zweiseitiger Gurt, Orthotropie des Gurtes, etc.)

Erste umfangreiche Zahlen- und Tabellenwerke für die mittragende Breite von Stahlbetonplattenbalken wurden von Koepcke und Denecke [2.47] veröffentlicht. In den Tafeln werden die mittragende Breite und Beiwerte zur Berechnung der Scheibenspannungen in der Kontaktlinie zwischen Steg und Gurt sowie am Rand des Gurtes für Einfeld- und Durchlaufträger unter Gleichlast, Einzellasten und für Vorspannkräfte angegeben. Diese Untersuchungen auf Basis der Elastizitätstheorie beschränken sich dabei auf Querschnitte mit dünnen Gurten, elastisches Materialverhalten und prismatische Querschnitte.

Von Schmidt, Peil und Born [2.38], [2.49] wird für orthotrope Gurte in Plattenbalken die Problematik durch Einführung des Begriffs des mitwirkenden Gurtquerschnitts erweitert. Die sehr umfangreichen Tabellen auf Basis der Elastizitätstheorie berücksichtigen dabei eine Vielzahl von unterschiedlichen Randbedingungen wie Belastungstyp (Einzellast, Gleichlast, Vorspannung, symmetrisch, antimetrisch, etc.), Querschnittsform des Hauptträgers, Flächenverhältnis von Gurt zu Hauptträger, Orthotropiefaktor des Gurtes, Ausbildung von Längsversteifungen, etc. Die Untersuchungen basieren auf einer elastischen Lösung der Scheibendifferen- 
tialgleichung ohne Berücksichtigung einer Rissbildung von Betongurten, Plastizierung von Stahlquerschnitten oder der Biegesteifigkeit des Betongurts.

In [2.56] wird von Sedlacek und Bild ein weiterer Ansatz zur Bestimmung der mittragenden Breite auf Basis der Elastizitätstheorie gegeben. Es zeigt sich, dass für die Ermittlung der mittragenden Breite von zweistegigen Querschnitten nur die symmetrischen Lastanteile (Biegeverformung) zu berücksichtigen sind, da der Einfluss der antimetrischen Lasten (Torsion) nur gering ist. Als maßgebende Parameter werden das Seitenverhältnis b/L der Gurtscheibe, die Lagerungsbedingungen und der Belastungstyp identifiziert. Für parabelförmige Momentenverteilungen ergeben sich im Bereich mittlerer Seitenverhältnisse b/L deutlich größere mittragende Breiten als nach EN 1994-1-1, siehe Abbildung 3-10. Im Vergleich zu DIN 1045-1 ergeben sich für größere Seitenverhältnisse b/L deutlich kleinere Werte.
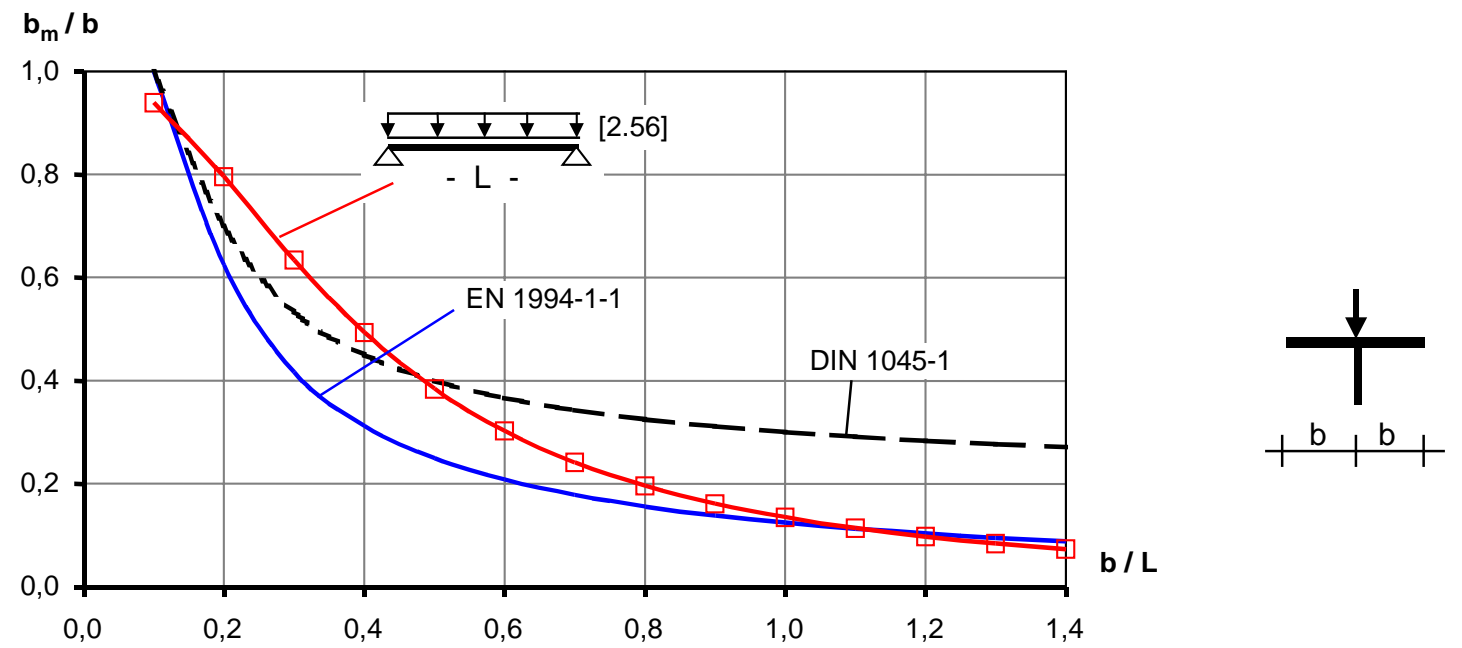

Abbildung 3-10: Vergleich der mittragenden Breite nach Sedlacek und Bild [2.56] mit EN 1994-1-1 und DIN 1045-1
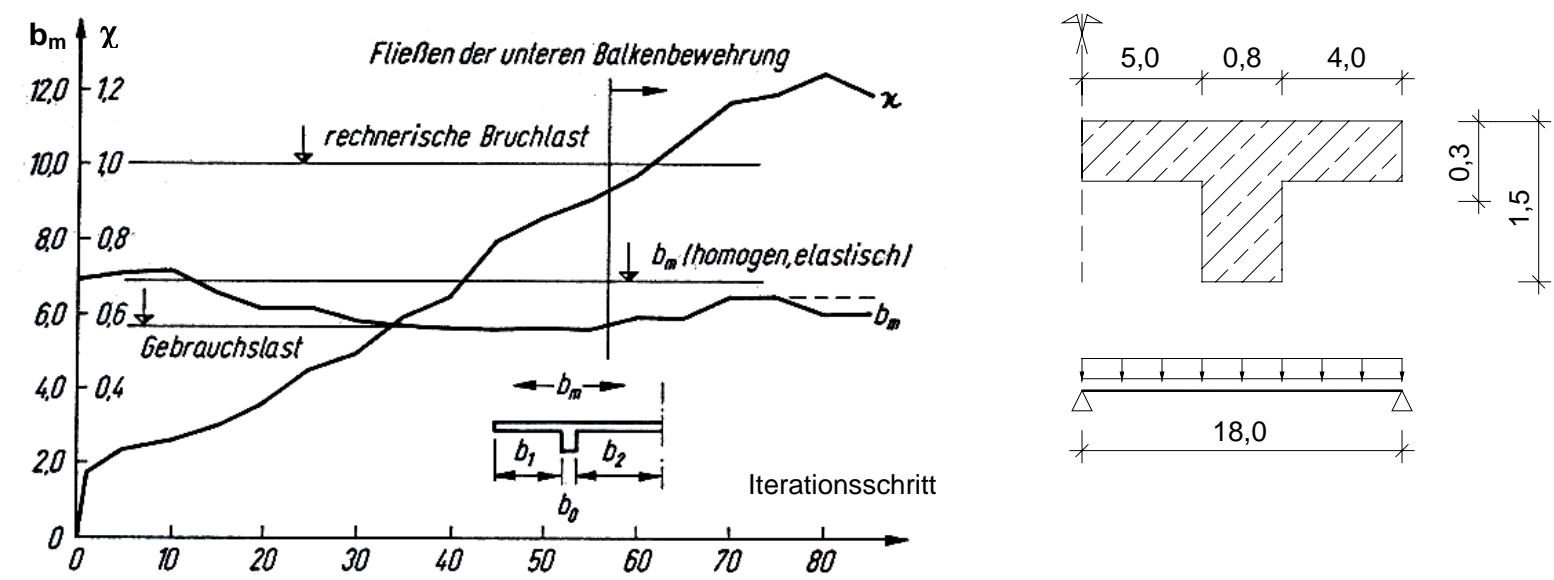

(a) Mittragende Breite $b_{m}$ und Ausnutzungsgrad $\chi=M / M_{u}$

(b) System und Querschnitt [m]

Abbildung 3-11: Abhängigkeit der mittragenden Breite von der Belastung eines StahlbetonPlattenbalkens nach Grünberg [2.52]

Von Albrecht wird der Einfluss der Plastizierung von Stahlgurten auf die mittragende Breite untersucht und eine Zunahme der mittragenden Breite mit zunehmender Belastung und Plastizierung festgestellt [2.48]. 
Untersuchungen von Stegbauer [2.51] und Grünberg [2.52] an Stahlbeton-Plattenbalken mit Hilfe der Finite Elemente Methode berücksichtigen zwar die Rissbildung, beschränken sich jedoch auf dünne Betongurte mit $d / d_{0}<0,2$. An ausgewählten Beispielen stellt Grünberg einen Einfluss der Rissbildung des Betongurts bei Stahlbeton-Plattenbalken auf die mittragende Breite fest, deren Größe von der Querschnittsform abhängt, siehe Abbildung 3-11. Für typische Plattenbalken des Hochbaus stellt er eine geringfügige Steigerung der mittragenden Breite im Vergleich zum Zustand I fest. Für andere Randbedingungen ermittelt er jedoch auch kleinere mittragende Breiten. Die ermittelten Werte für die mittragende Breite der untersuchten Systeme sind jedoch nennenswert größer als die entsprechenden Werte nach EN 1994-1-1 und ENV 1992-1-1 und zeigen eine gute Übereinstimmung mit den Werten nach DIN 1045-1.

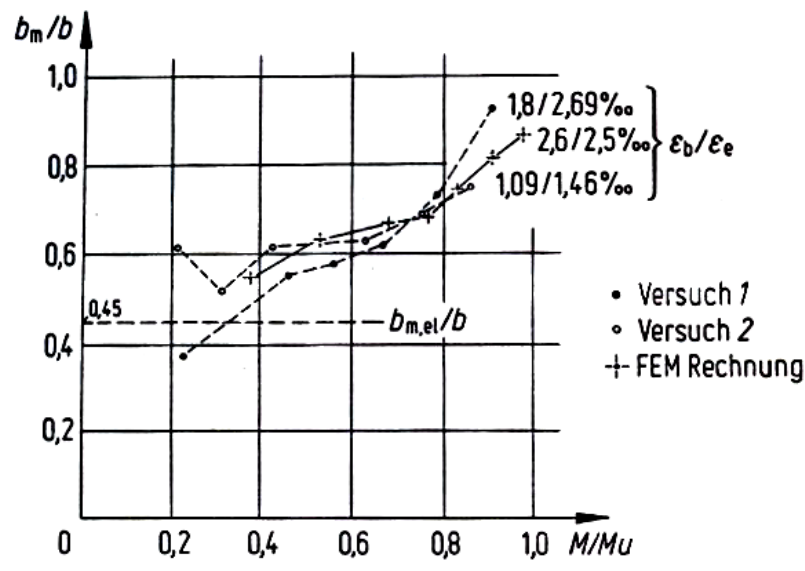

(a) Mittragende Breite $b_{m}$

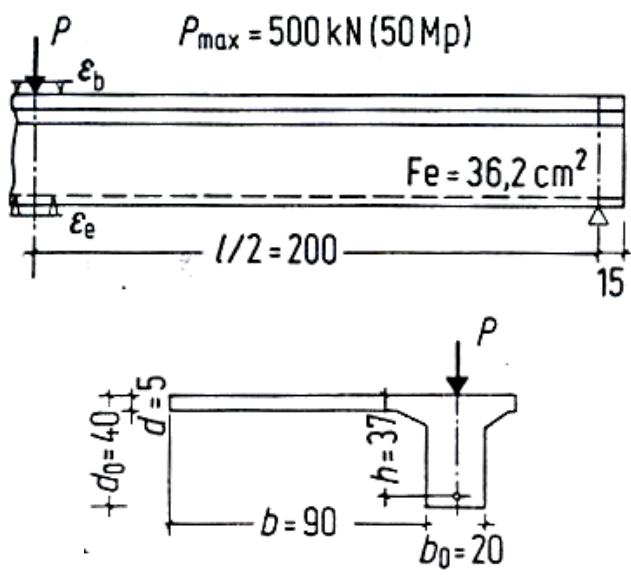

(b) System und Querschnitt [cm]

Abbildung 3-12: Abhängigkeit der mittragenden Breite von der Belastung eines StahlbetonPlattenbalkens nach Stegbauer [2.51]
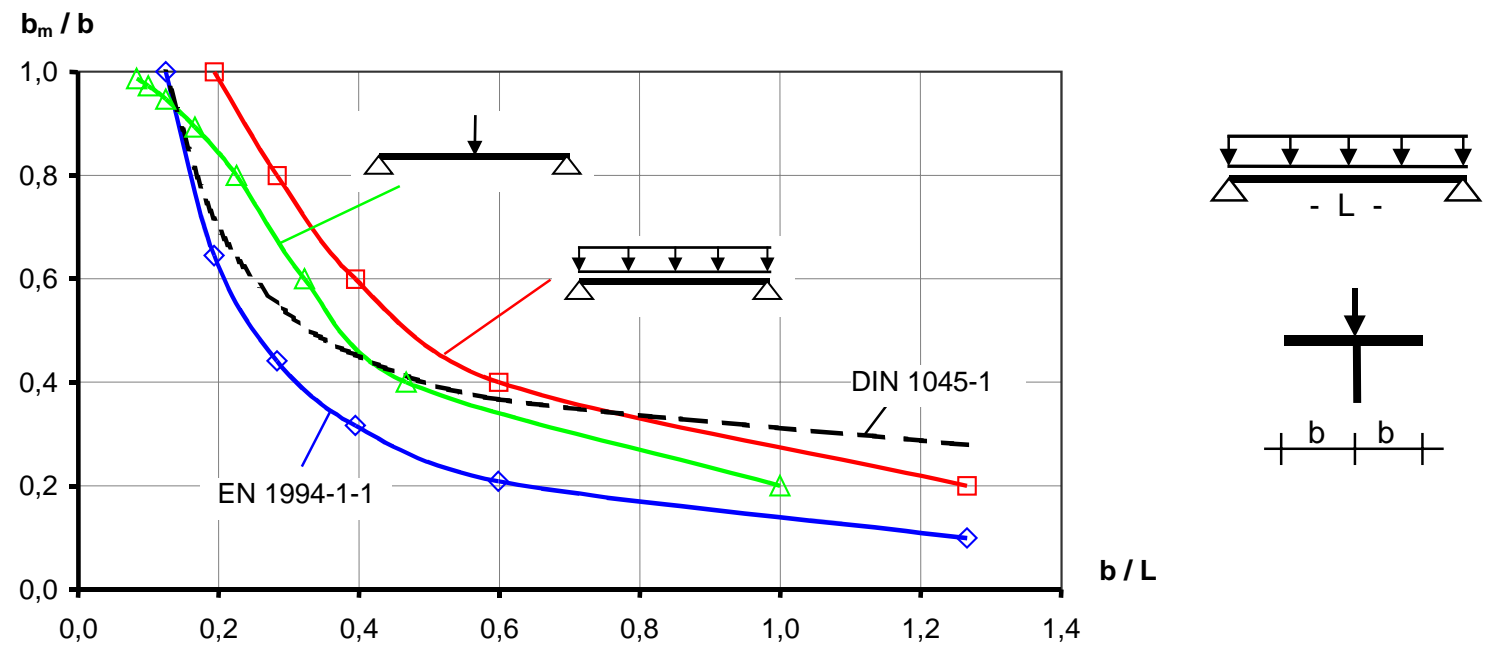

Abbildung 3-13: Vergleich der mittragenden Breite nach Stegbauer [2.51] mit EN 1994-1-1 und DIN 1045-1

Stegbauer stellt bei seinen Untersuchungen eine Steigerung der mittragenden Breite mit zunehmender Belastung fest, siehe Abbildung 3-12 [2.51]. Wenn eine ausreichend dimensionierte Querbewehrung vorhanden ist, beeinflusst die Rissbildung in Steg und Platte der untersuchten Systeme die mittragende Breite im Grenzzustand der Tragfähigkeit nur wenig. 
Der Einfluss der vorhandenen Querbiegung im Gurt auf die mittragende Breite ist gering. Auf Basis einer Parameterstudie werden Diagramme zur Ermittlung der mittragenden Breite im Grenzzustand der Tragfähigkeit unter Vernachlässigung der Biegetragwirkung des Gurtes angegeben. Die so ermittelten Werte für die mittragende Breite sind deutlich größer als die entsprechenden Werte nach EN 1994-1-1 und für kleine und mittlere Seitenverhältnisse b/L auch im Vergleich zu den Werten nach DIN 1045-1, siehe Abbildung 3-13. Diese Werte liegen auf Grund der Vernachlässigung der Biegetragwirkung des Betongurts auf der sicheren Seite. Auf Grund einer fehlenden systematischen Analyse aller Einflussfaktoren und der Begrenzung der Untersuchungen auf dünne Gurte, können diese Erkenntnisse jedoch nicht auf Verbundträger niedriger Bauhöhe übertragen werden. Sie zeigen jedoch die Notwendigkeit, die Rissbildung des Betongurts bei der Ermittlung der mittragenden Breite zu berücksichtigen.

Von Holtkamp wird die mittragende Breite von durchlaufenden Verbundträgern im Stützbereich unter Berücksichtigung der Rissbildung untersucht [2.57]. Er stellt beispielhaft an der Berechnung verschiedener Verbundträger eine starke Abhängigkeit der mittragenden Breite von der Rissbildung und der Belastungshöhe fest. Darüber hinaus stellt er fest, dass die mittragende Breite im Sinne der klassischen Definition mit Bezug auf die Spannung in Stegebene des Plattenbalkens größer werden kann als die tatsächliche geometrische Breite des Gurts. Dies liegt daran, dass die Betonzugspannungen im Zustand II durch die Mitwirkung des Betons zwischen den Rissen mit zunehmender Dehnung abnehmen und die Dehnung des Gurtes vom Steg zum Rand kleiner wird. Daher liegt die maximale Betonzugspannung im Zustand II nicht über dem Steg, sondern die Betonspannungen nehmen vom Steg zum Rand hin zu. Somit kann es nach der klassischen Definition zu größeren Werten für die mittragende Breite als die geometrische Gurtbreite kommen. Eine quantitative Aufbereitung der Ergebnisse in Form eines Berechnungsvorschlags der mittragenden Breite für die untersuchten Stützbereiche von Verbundträgern wird jedoch nicht gemacht.

Weyer und Uhlendahl [2.60], [2.61] untersuchen an typischen dreifeldrigen Verbunddeckbrücken den Einfluss der Rissbildung des Betongurts im Stützbereich auf die Rissesicherung. Die verwendeten Materialgesetze berücksichtigen hierbei das Mitwirken des Betons zwischen den Rissen und behandeln den Stahl linearelastisch. Die Untersuchungen zeigen, dass zwar wie zu erwarten mit der Rissbildung die Zugkraft im Betongurt abnimmt, die Querverteilung der Längsspannungen im Gurt jedoch ausgeglichener werden und damit die mittragende Breite im Zustand II gegenüber dem elastischen Fall um ca. 20 $\div 50 \%$ größer wird.

Die Untersuchungen zur Scheibentragwirkung des Betongurts zeigen, dass die mittragende Breite wesentlich vom Seitenverhältnis des Gurtes sowie der Lagerungs- und Belastungsbedingungen bestimmt wird. Die Rissbildung im Beton zeigt einen großen Einfluss auf die mittragende Breite und führt eher zu einer Vergrößerung der mittragenden Breite. Eine systematische Auswertung dieses Einflusses insbesondere bei Verbundträgern niedriger Bauhöhe liegt jedoch nicht vor.

\subsubsection{Untersuchungen zur Biegetragwirkung des (Beton-) Gurts}

Erste Untersuchungen über die mittragende Breite von breiten Gurten für die Biegetragwirkung ( $\mathrm{I}_{\mathrm{c}, 0}$ und $\mathrm{M}_{\mathrm{c}}$ werden nicht vernachlässigt) wurden von Marguerre [2.42] verfasst. Basierend auf der Elastizitätstheorie wird durch Anwendung des Superpositionsprinzips für einen Plattenbalken mit U-Querschnitt und sinusförmiger Belastung der Hauptstege der Scheibenzustand des Gurtes vom Biegezustand getrennt und die Spannungsverteilungen der beiden Zustände durch Lösung der entsprechenden Differentialgleichungen bestimmt. Aus den Spannungsverteilungen werden so dann die mittragende Breite für die Biege- und Scheibentragwirkung ermittelt, siehe Abbildung 3-14. Die mittragende Breite $b_{m, B}$ der Biegetragwirkung ist dabei deutlich größer als für die Scheibentragwirkung $b_{m, s}$. Die Einzelwerte $b_{m, s}$ und $b_{m, B}$ 
sind für das untersuchte System im Wesentlichen nur vom Seitenverhältnis b/L des Gurts abhängig. Mit Kenntnis der mittragenden Breite $b_{m, s}$ und $b_{m, B}$ kann die effektive Biegesteifigkeit des Plattenbalkens mit Gleichung (3-2) bestimmt werden. Mit Gleichung (3-3) kann daraus ein mittlerer Wert für die mittragende Breite $b_{m}$ bestimmt werden. Dieser ist neben dem Seitenverhältnis b/L des Gurtes wesentlich von der Steifigkeitsverteilung im Querschnitt abhängig. Mit zunehmendem Verhältnis der Gurtdicke zu Trägerhöhe $h_{c} / h$ nimmt $b_{m} z u$.

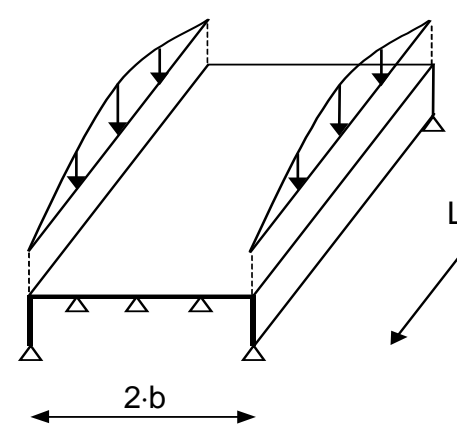

(a) System

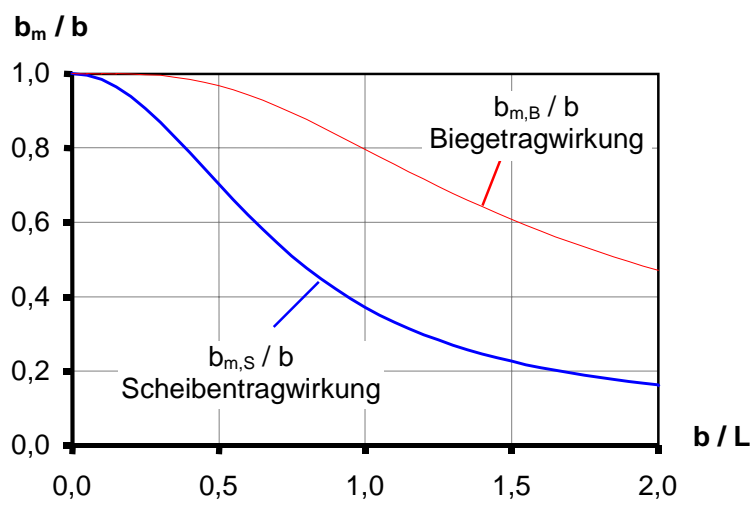

(b) mittragende Breite $b_{m}$

Abbildung 3-14: Mittragende Gutbreite für die Scheiben- und Biegetragwirkung nach Marguerre [2.42]

$$
\begin{aligned}
& I_{i, 0}=I_{a}+I_{c, 0}+S_{i, 0} \cdot a_{S t} \\
& I_{i, 0}=I_{a}+\frac{b_{m, B} \cdot h_{c}^{3}}{12 \cdot n_{0}}+\frac{\frac{b_{m, S} \cdot h_{c}}{n_{0}} \cdot A_{a}}{\frac{b_{m, S} \cdot h_{c}}{n_{0}}+A_{a}} \cdot a_{S t}^{2}
\end{aligned}
$$

mit

$\mathrm{I}_{\mathrm{i}, 0}$

$\mathrm{I}_{\mathrm{a}}$

$\mathrm{I}_{\mathrm{c}, 0}=\mathrm{I}_{\mathrm{c}} / \mathrm{n}_{0}$

$\mathrm{S}_{\mathrm{i}, 0}$

$A_{a}$

$\mathrm{n}_{0}=\mathrm{E}_{\mathrm{a}} / \mathrm{E}_{\mathrm{cm}}$

$b_{m, s}, b_{m, B}$

$\mathrm{h}_{\mathrm{c}}$

$a_{S t}$
Trägheitsmoment des Plattenbalkenquerschnitts

Trägheitsmoment des Stegquerschnitts

Trägheitsmoment des Gurtquerschnitts statisches Moment des Plattenbalkenquerschnitts Querschnittsfläche des Stegquerschnitts Reduktionszahl $\left(\mathrm{E}_{\mathrm{a}}=\mathrm{E}-\mathrm{Modul}\right.$ des Steges, $\mathrm{E}_{\mathrm{cm}}=\mathrm{E}-\mathrm{Modul}$ des Gurtes)

mittragende Breite des Gurtes für den reinen Scheibenbzw. Biegezustand

Dicke des Gurtes

Schwerpunktabstand zwischen Gurt- und Stegquerschnitt

$I_{i, 0}=I_{a}+\frac{b_{m} \cdot h_{c}^{3}}{12 \cdot n_{0}}+\frac{\frac{b_{m} \cdot h_{c}}{n_{0}} \cdot A_{a}}{\frac{b_{m} \cdot h_{c}}{n_{0}}+A_{a}} \cdot a_{S t}^{2}$

mit

$b_{m}$

mittlerer Wert der mittragenden Breite 


\begin{tabular}{|c|c|c|c|c|c|c|c|c|c|c|c|c|c|c|c|c|c|c|c|c|c|}
\hline $\mathrm{d} / d_{0}$ & $l / b_{0}$ & $l_{j} b=0$ & $\ldots 1$ & 2 & 3 & 4 & 6 & 8 & 10 & 12 & 14 & 16 & 18 & 0 & 1 & 2 & 3 & 4 & 6 & 8 & 10 \\
\hline 0,10 & $\begin{array}{r}\leqq 10 \\
50 \\
100 \\
150 \\
\geq 200\end{array}$ & $\begin{array}{l}0 \\
0 \\
0 \\
0 \\
0\end{array}$ & $\begin{array}{l}0,18 \\
0,19 \\
0,21 \\
0,23 \\
0,27\end{array}$ & $\begin{array}{l}0,36 \\
0,37 \\
0,40 \\
0,43 \\
0,47\end{array}$ & $\begin{array}{l}0,53 \\
0,54 \\
0,56 \\
0,59 \\
0,62\end{array}$ & $\begin{array}{l}0,65 \\
0,66 \\
0,67 \\
0,69 \\
0,71\end{array}$ & $\begin{array}{l}0,78 \\
0,79 \\
0,80 \\
0,81 \\
0,81\end{array}$ & $\begin{array}{l}0,88 \\
0,37 \\
0,87 \\
0,88 \\
0,88\end{array}$ & $\begin{array}{l}0,92 \\
0,92 \\
0,92 \\
0,992 \\
0,93\end{array}$ & $\begin{array}{l}0,95 \\
0,95 \\
0,96 \\
0,96 \\
0,96\end{array}$ & $\begin{array}{l}0,98 \\
0,98 \\
0,98 \\
0,98 \\
0,98\end{array}$ & $\begin{array}{l}0,99 \\
0,99 \\
0,99 \\
0,99 \\
0,99\end{array}$ & $\begin{array}{l}1,00 \\
1,00 \\
1,00 \\
1,00 \\
1,00\end{array}$ & $\begin{array}{l}0 \\
0 \\
0 \\
0 \\
0 \\
0\end{array}$ & $\begin{array}{l}0,18 \\
0,19 \\
0,21 \\
0,24 \\
0,27\end{array}$ & $\begin{array}{l}0,38 \\
0,39 \\
0,42 \\
0,45 \\
0,48 \\
0,48\end{array}$ & $\begin{array}{l}0,57 \\
0,58 \\
0,60 \\
0,62 \\
0,64\end{array}$ & $\begin{array}{l}0,71 \\
0,72 \\
0,73 \\
0,75 \\
0,77\end{array}$ & $\begin{array}{l}0,89 \\
0,89 \\
0,89 \\
0,90 \\
0,90\end{array}$ & $\begin{array}{l}0,96 \\
0,96 \\
0,96 \\
0,96 \\
0,96\end{array}$ & $\begin{array}{l}1,00 \\
1,00 \\
1,00 \\
1,00 \\
1,00\end{array}$ \\
\hline 0,15 & $\begin{array}{r}\leqq \\
50 \\
50 \\
100 \\
150 \\
\geqq 200\end{array}$ & $\begin{array}{l}0 \\
0 \\
0 \\
0 \\
0\end{array}$ & $\begin{array}{l}0,19 \\
0,222 \\
0,30 \\
0,36 \\
0,40\end{array}$ & $\begin{array}{l}0,37 \\
0,42 \\
0,51 \\
0,60 \\
0,65\end{array}$ & $\begin{array}{l}0,53 \\
0,58 \\
0,660 \\
0,73 \\
0,79\end{array}$ & $\begin{array}{l}0,66 \\
0,699 \\
0,74 \\
0,80 \\
0,85\end{array}$ & $\begin{array}{l}0,79 \\
0,818 \\
0,83 \\
0,86 \\
0,89\end{array}$ & $\begin{array}{l}0,87 \\
0,88 \\
0,89 \\
0,91 \\
0,92\end{array}$ & $\begin{array}{l}0,92 \\
0,92 \\
0,93 \\
0,94 \\
0,95\end{array}$ & $\begin{array}{l}0,95 \\
0,96 \\
0,96 \\
0,96 \\
0,97\end{array}$ & $\begin{array}{l}0,98 \\
0,998 \\
0,98 \\
0,98 \\
0,98\end{array}$ & $\begin{array}{l}0,99 \\
0,99 \\
0,99 \\
0,99 \\
0,99\end{array}$ & $\begin{array}{l}1,00 \\
1,00 \\
1,00 \\
1,00 \\
1,00\end{array}$ & $\begin{array}{l}0 \\
0 \\
0 \\
0 \\
0 \\
0\end{array}$ & $\begin{array}{l}0,19 \\
0,233 \\
0,31 \\
0,37 \\
0,41\end{array}$ & $\begin{array}{l}0,39 \\
0,4.1 \\
0,53 \\
0,61 \\
0,666\end{array}$ & $\begin{array}{l}0,58 \\
0,62 \\
0,68 \\
0,74 \\
0,80\end{array}$ & $\begin{array}{l}0,72 \\
0,74 \\
0,78 \\
0,83 \\
0,88\end{array}$ & $\begin{array}{l}0,89 \\
0,90 \\
0,91 \\
0,92 \\
0,93\end{array}$ & $\begin{array}{l}0,97 \\
0,97 \\
0,97 \\
0,97 \\
0,98\end{array}$ & $\begin{array}{l}1,00 \\
1,00 \\
1,00 \\
1,00 \\
1,00\end{array}$ \\
\hline 0,20 & $\begin{array}{r}10 \\
50 \\
100 \\
150 \\
\geqq 200\end{array}$ & $\begin{array}{l}0 \\
0 \\
0 \\
0 \\
0\end{array}$ & $\begin{array}{l}0,21 \\
0,30 \\
0,40 \\
0,44 \\
0,45\end{array}$ & $\begin{array}{l}0,40 \\
0,52 \\
0,65 \\
0,70 \\
0,73\end{array}$ & $\begin{array}{l}0,57 \\
0,69 \\
0,79 \\
0,85 \\
0,89\end{array}$ & $\begin{array}{l}0,68 \\
0,78 \\
0,86 \\
0,91 \\
0,93\end{array}$ & $\begin{array}{l}0,81 \\
0,86, \\
0,89 \\
0,94 \\
0,95\end{array}$ & $\begin{array}{l}0,87 \\
0,90 \\
0,92 \\
0,95 \\
0,96\end{array}$ & $\begin{array}{l}0,92 \\
0,94 \\
0,95 \\
0,966 \\
0,97\end{array}$ & $\begin{array}{l}0,96 \\
0,96 \\
0,97 \\
0,97 \\
0,98\end{array}$ & $\begin{array}{l}0,98 \\
0,998 \\
0,98 \\
0,988 \\
0,98\end{array}$ & $\begin{array}{l}0,99 \\
0,99 \\
0,99 \\
0,99 \\
1,90\end{array}$ & $\begin{array}{l}1,00 \\
1,000 \\
1,00 \\
1,00 \\
1,00\end{array}$ & $\begin{array}{l}0 \\
0 \\
0 \\
0 \\
0\end{array}$ & $\begin{array}{l}0,21 \\
0,30 \\
0,41 \\
0,44 \\
0,45\end{array}$ & $\begin{array}{l}0,42 \\
0,54 \\
0,66 \\
0,71 \\
0,74\end{array}$ & $\begin{array}{l}0,61 \\
0,71 \\
0,80 \\
0,86 \\
0,89\end{array}$ & $\begin{array}{l}0,74 \\
0,82 \\
0,87 \\
0,91 \\
0,93\end{array}$ & $\begin{array}{l}0,90 \\
0,92 \\
0,94 \\
0,96 \\
0,97\end{array}$ & $\begin{array}{l}0,97 \\
0,97 \\
0,93 \\
0,98 \\
0,99\end{array}$ & $\begin{array}{l}1,00 \\
1,00 \\
1,00 \\
1,00 \\
1,00\end{array}$ \\
\hline 0,30 & $\begin{array}{r}\cong 10 \\
50 \\
100 \\
150 \\
\geqq 200\end{array}$ & $\begin{array}{l}0 \\
0 \\
0 \\
0 \\
0 \\
\end{array}$ & $\begin{array}{l}0,28 \\
0,422 \\
0.45 \\
0.46 \\
0.46\end{array}$ & $\begin{array}{l}0,48 \\
0,65 \\
0,73 \\
0,75 \\
0,77\end{array}$ & $\begin{array}{l}0,63 \\
0,83 \\
0,90 \\
0,91 \\
0,92\end{array}$ & $\begin{array}{l}0,72 \\
0,87 \\
0,92 \\
0,93 \\
0.94 \\
0.94\end{array}$ & $\begin{array}{l}0,81 \\
0,990 \\
0,94 \\
0,95 \\
0,95\end{array}$ & $\begin{array}{l}0,87 \\
0,92 \\
0,95 \\
0,966 \\
0,97\end{array}$ & $\begin{array}{l}0,92 \\
0,994 \\
0,96 \\
0,97 \\
0,99\end{array}$ & $\begin{array}{l}0,96 \\
0.96 \\
0.97 \\
0.98 \\
0,99\end{array}$ & $\begin{array}{l}0,98 \\
0,98 \\
0.98 \\
0.99 \\
0,99 \\
\end{array}$ & $\begin{array}{l}0,99 \\
0,999 \\
0,999 \\
1,00 \\
1,00\end{array}$ & $\begin{array}{l}1,00 \\
1,000 \\
1,00 \\
1,00 \\
1,00\end{array}$ & $\begin{array}{l}0 \\
0 \\
0 \\
0 \\
0 \\
\end{array}$ & $\begin{array}{l}0,28 \\
0,42 \\
0,45 \\
0,46 \\
0,47 \\
0,47\end{array}$ & $\begin{array}{l}0,50 \\
0,69 \\
0,74 \\
0,76 \\
0,77 \\
\end{array}$ & $\begin{array}{l}0,65 \\
0.83 \\
0,90 \\
0,92 \\
0,92 \\
\end{array}$ & $\begin{array}{l}0,77 \\
0,83 \\
0,94 \\
0,95 \\
0,96\end{array}$ & $\begin{array}{l}0,91 \\
0,93 \\
0,95 \\
0,97 \\
0,99 \\
0.93\end{array}$ & $\begin{array}{l}0,97 \\
0,97 \\
0,98 \\
0,999 \\
0,99 \\
\end{array}$ & $\begin{array}{l}1,00 \\
1,00 \\
1,00 \\
1,00 \\
1,00\end{array}$ \\
\hline & & & & & & & & & & & $b_{m} / b$ & & & & & & & & & & \\
\hline
\end{tabular}

Abbildung 3-15: Mittragende Breite nach Brendel [2.44]
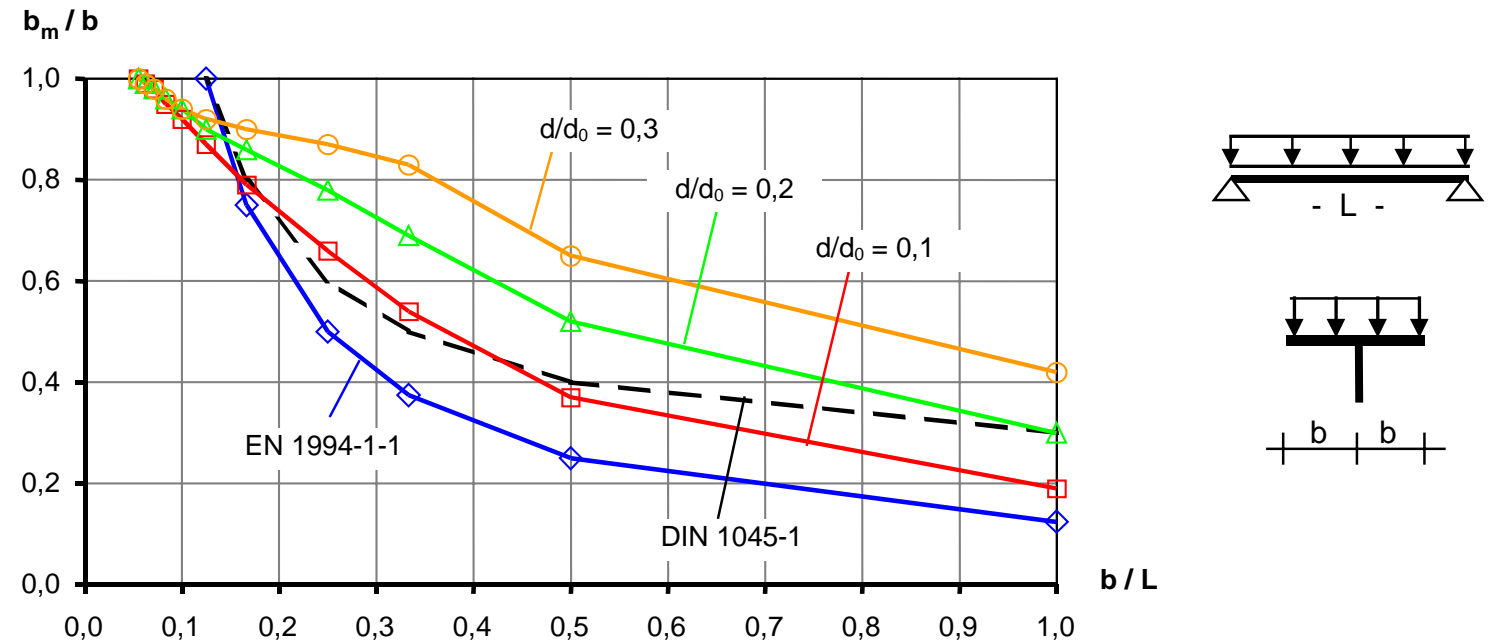

Abbildung 3-16: Vergleich der mittragenden Breite nach Brendel [2.44] mit EN 1994-1-1 und DIN 1045-1

Aufbauend auf den Untersuchungen von Marguerre werden von Brendel in [2.44] weitere Systeme untersucht und die mittragende Breite $b_{m}$ für die kombinierte Scheiben- und Biegetragwirkung des Gurtes in Form von Tabellen in Abhängigkeit verschiedener Querschnittsparameter aufbereitet, siehe Abbildung 3-15. Es zeigt sich eine starke Abhängigkeit der mittragenden Breite von der Biegesteifigkeit des Betongurts. Mit zunehmender Dicke des Betongurts d im Vergleich zur Trägerhöhe $d_{0}$ wird die mittragende Breite größer. Im Vergleich zu EN 1994-1-1 und DIN 1045-1 ergeben sich deutlich größere Werte für die mittragende Breite, siehe Abbildung 3-16. In Versuchen an Stahlbeton-Plattenbalken wird von ihm eine Zunahme der mittragenden Breite mit der Belastungshöhe erkannt. Von Rose [2.46], Severn [2.45], Schröder und Stritzke [2.50] werden für weitere Systeme, Randbedingungen und Belastungen ebenfalls auf Basis der Elastizitätstheorie die mittragende Breite unter Berücksichtigung der Biegetragwirkung des Betongurts ermittelt. Diese führen auf ähnliche Ergebnisse wie [2.44]. 
Von Amadio und Fragiacomo [2.62] wird mit Hilfe der Finite Elemente Methode an ausgewählten Beispielen der Einfluss einer nachgiebigen Verdübelung und des nichtlinearen Materialverhaltens auf die mittragende Breite von Verbundträgern untersucht. Die Untersuchungen zeigen, dass eine nachgiebige Verdübelung zu einer Reduktion der mittragenden Breite im Vergleich zu einer starren Verdübelung führen kann. In Feldmitte und im Stützbereich ermitteln sie eine gute Übereinstimmung mit den Werten nach EN 1994-1-1. Die Untersuchungen zeigen, dass mit zunehmender Belastung die mittragende Breite teilweise sehr stark anwächst. Eine Verallgemeinerung dieser Ergebnisse ist jedoch nicht möglich, da sich die untersuchten Träger auf Systeme mit einem Seitenverhältnis $b / L<0,2$ und mit dünnen Betongurten $(\mathrm{d}<10 \mathrm{~cm})$ beschränken und die Biegetragwirkung des Betongurts zwar berücksichtigt wird, jedoch bei den untersuchten Querschnitten sehr klein ist. In [2.63] wird über Versuche an Verbundträgern berichtet. Dabei wird eine starke Abhängigkeit der mittragenden Breite von der Belastungshöhe beobachtet. Ein Versuch unter positiver Momentenbelastung mit einem Seitenverhältnis $b / L=0,21$ zeigt eine deutlich größere mittragende Breite als nach EN 1994-1-1, siehe Abbildung 3-17. Mit zunehmender Belastung nimmt die mittragende Breite zu, bis mit Erreichen des Bruchzustands nahezu die gesamte Breite des Betongurts mitwirkt.

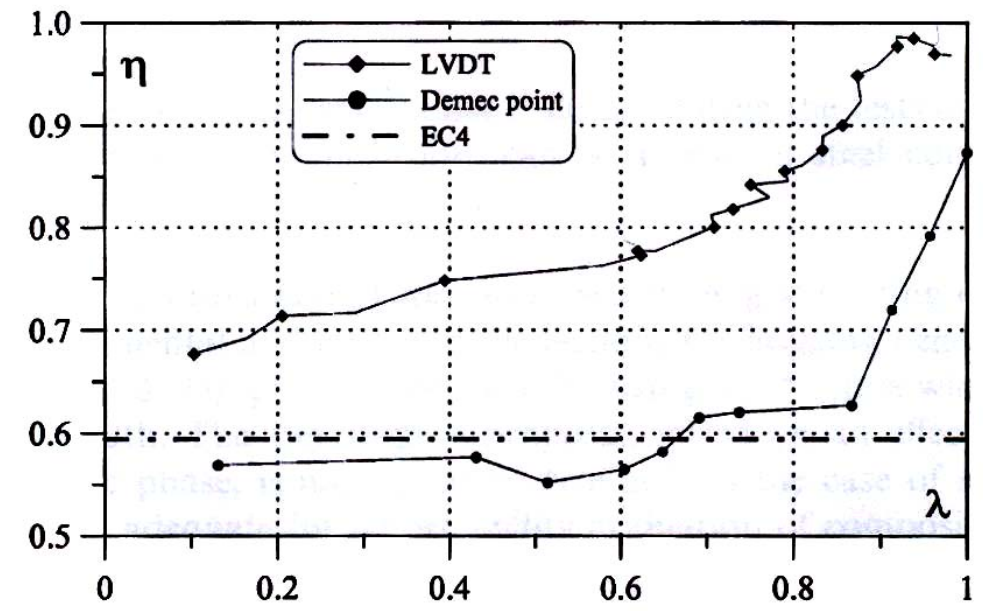

(a) Mittragende Breite $b_{m}=\eta \cdot b$ in Abhängigkeit des Ausnutzungsgrades $\lambda=M / M_{u}$
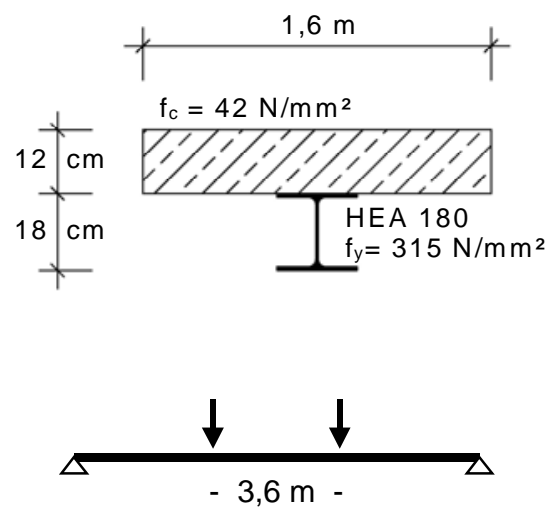

(b) System und Querschnitt

Abbildung 3-17: Abhängigkeit der mittragenden Breite von der Belastung eines Verbundträgers nach Amadio et al. [2.63]

Die Untersuchungen zur Biegetragwirkung des Betongurts zeigen, dass die mittragende Breite des reinen Biegezustands deutlich größer ist als für den reinen Scheibenzustand. In der kombinierten Wirkung des Scheiben- und Biegezustands ist die mittragende Breite daher von der Steifigkeitsverteilung zwischen Gurt- und Stegquerschnitt abhängig und nimmt unter anderem mit zunehmender Dicke des Gurtes und abnehmender Gesamthöhe des Trägers zu. Eine systematische Untersuchung des Einflusses der Rissbildung im Beton insbesondere bei Trägern mit großer Biegesteifigkeit des Gurtes liegt jedoch nicht vor.

\subsection{Schlussfolgerung aus den bisherigen Untersuchungen}

Zur mittragenden Breite von Plattenbalkenquerschnitten mit breiten Gurten liegt eine Vielzahl von Untersuchungen vor. Als wesentliche Einflüsse auf die mittragende Breite werden hierbei folgende Parameter identifiziert:

- Seitenverhältnis b/L des Gurtes 
- Lagerungsbedingungen und Belastungsform des Plattenbalkens

- betrachtete Trägerstelle

- Querschnitt des Trägers, vor allem Verhältnis der Biegesteifigkeit des Gurtes zur Gesamtsteifigkeit des Trägers

- Rissbildung im Betongurt

- Fließen der Bewehrung bzw. des Stahlträgers

- im Bereich nichtlinearen Materialverhaltens die Belastungshöhe

Die vorliegenden Untersuchungen beschränken sich dabei im Wesentlichen auf die Elastizitätstheorie, die reine Scheibentragwirkung, die Berechnung der Traglast oder lassen eine umfangreiche Parameterstudie im Bereich niedriger Verbundträger vermissen. Untersuchungen zur kombinierten Biege- und Scheibentragwirkung des Betongurts unter Berücksichtigung der Rissbildung des Betongurts in Feldbereichen von Verbundträgern insbesondere im Hinblick auf das Verformungsverhalten liegen nicht vor. Gerade diese Einflüsse sind jedoch bei Verbundträgern niedriger Bauhöhe entscheidend. Die vorliegenden Arbeiten zeigen jedoch, dass die Biegetragwirkung des Betongurts, die Rissbildung im Beton und damit die Belastungshöhe einen großen Einfluss auf die mittragende Breite haben und für eine wirklichkeitsnahe Ermittlung der mittragenden Breite berücksichtigt werden müssen. Die Untersuchungen dieser Einflüsse ist daher Gegenstand der vorliegenden Arbeit. 


\section{Die mittragende Breite für den linearelastischen Fall}

\subsection{Grundsätzliches}

Wie in Kapitel 2 dargestellt weisen niedrige Verbundträger bereits unter Gebrauchslasten ein ausgeprägtes nichtlineares Tragverhalten auf, das besonders durch die Rissbildung im Beton bestimmt wird. Zum besseren Verständnis wird die mittragende Breite jedoch zunächst für linearelastisches Materialverhalten betrachtet, um in einem ersten Schritt vor allem den Einfluss der Biegetragwirkung des Betongurts näher zu klären. Zunächst werden die mittragende Breite des reinen Scheiben- und des reinen Biegezustands, daran anschließend deren kombinierte Wirkung diskutiert. Die Berechnungen orientieren sich dabei zum großen Teil an den Untersuchungen von Rose [2.46]. Eine ausführliche Darstellung der Berechnungen ist in [2.27] enthalten.

Die folgenden Darstellungen zur mittragenden Breite bei linearelastischem Materialverhalten beruhen auf der bekannten Literatur, sind aber für das Verständnis der nachfolgenden nichtlinearen Untersuchungen nach Auffassung des Verfassers sehr hilfreich. Die Ergebnisse der elastischen Untersuchungen bilden dabei die Basis für die nichtlinearen Untersuchungen in den weiteren Kapiteln.

Elastizitätstheorie bedeutet dabei, dass Dehnungen so klein sind, dass

- der Beton auf Zug keine Risse bekommt (Zustand I),

- der Beton auf Druck sich näherungsweise linear verhält,

- der Beton keinen Langzeiteinflüssen unterliegt und

- der Stahl nicht plastiziert.

Im Folgenden werden die linearelastischen Werte der mittragenden Breite des reinen Scheibenzustands und des reinen Biegezustands für die wichtigsten Belastungsfälle im Hochbau angegeben und anschließend die kombinierte Wirkung aus Scheiben- und Biegezustand diskutiert.

\subsection{Reiner Scheiben- und Biegezustand des Betongurts}

\subsubsection{Allgemeines}

Unter Annahme, dass die Elastizitätstheorie gültig ist, kann durch Anwendung des Superpositionsprinzips der Scheibenzustand des Gurtes vom Biegezustand getrennt werden. Für beide Zustände können der Spannungs- und Dehnungszustand des Gurtes unter Beachtung der Randbedingungen aus der Differentialgleichung der Scheibe bzw. Platte bestimmt werden. Für Verbundträger im Hochbau ist dabei die Annahme eines isotropen und elastischen Materialverhaltens ausreichend genau, solange der Betongurt im Zustand I ist.

Der Scheibenzustand resultiert aus der Dehnsteifigkeit des Gurtes und der schubfesten Verdübelung mit dem Steg des Plattenbalkens. Der Gurt trägt dabei die Druckkomponente des inneren Kräftepaars des Steg- und Gurtquerschnitts ab. Im Biegezustand wirkt der Betongurt eines Plattenbalkens als Platte. Er resultiert aus der immer vorhandenen Biegesteifigkeit des Gurtes und der Kopplung der vertikalen Verformungen von Gurt und Steg. Dabei trägt der Gurt entsprechend dem Verhältnis seiner Biegesteifigkeit zur Gesamtsteifigkeit des Trägers einen Anteil der äußeren Belastung über Biegung ab. 
Gleichung (4-1) stellt die Differentialgleichung der isotropen Scheibe dar, Gleichung (4-2) diejenige der isotropen Platte.

$$
\frac{\partial^{4} \Phi}{\partial y^{4}}+2 \cdot \frac{\partial^{4} \Phi}{\partial x^{2} \partial y^{2}}+\frac{\partial^{4} \Phi}{\partial x^{4}}=0
$$

mit $\quad \Phi$

$\Phi \quad$ Airysche Spannungsfunktion

x Koordinate der Gurtscheibe in Längsrichtung des Trägers

y Koordinate der Gurtscheibe in Querrichtung des Trägers

$$
\frac{\partial^{4} w}{\partial y^{4}}+2 \cdot \frac{\partial^{4} w}{\partial x^{2} \partial y^{2}}+\frac{\partial^{4} w}{\partial x^{4}}=0
$$

mit

$$
\begin{array}{ll}
\text { w } & \text { Durchbiegung der Gurtplatte } \\
\text { x } & \text { Koordinate der Gurtplatte in Längsrichtung des Trägers } \\
\text { y } & \text { Koordinate der Gurtplatte in Querrichtung des Trägers }
\end{array}
$$

Eine exakte Lösung der Differentialgleichungen ist im Allgemeinen nicht möglich. Die Lösungen können näherungsweise numerisch oder mittels Fourierreihenentwicklung erfolgen. Dies führt auf vergleichsweise komplizierte Formeln [2.27]. Im Folgenden werden die Ergebnisse dieser Berechnungen kurz zusammengestellt und die Einflüsse auf die mittragende Breite diskutiert.

\subsubsection{Einflüsse auf die mittragende Breite des Scheibenzustands}

Aus den Berechnungen der mittragenden Breite des reinen Scheibenzustands durch Lösung der Scheibendifferentialgleichung können folgende Schlussfolgerungen gezogen werden, die mit den Angaben in der zuvor beschriebenen Literatur übereinstimmen [2.27]:

- Der Haupteinflussparameter für die mittragende Breite $b_{m, s}$ ist das Seitenverhältnis der Gurtscheibe $b / L$. Für zunehmende Werte $b / L$ nimmt die bezogene mittragende Breite $b_{m, s} / b$ rasch $a b$, siehe $z$. B. Abbildung 4-1.

- Die Belastungsfunktion hat einen nennenswerten Einfluss auf die mittragende Breite. Die mittragende Breite $b_{m, s}$ für Gleichlast ist an der maßgebenden Trägerstelle deutlich größer als bei mittiger Einzellast, siehe z. B. Abbildung 4-1 und Abbildung 4-2.

- Die mittragende Breite $b_{m, s}$ im Rahmen der im normalen Hoch- und Geschossbau üblichen Querschnitte ist nur unwesentlich vom Querschnittstyp abhängig. Die mittragende Breite bei Querschnitten mit einer Behinderung der Querverschiebung an den Längsrändern ist geringfügig größer als ohne.

- Die mittragende Breite $b_{m, s}$ variiert in Trägerlängsrichtung, siehe Abbildung 4-2. Bei Belastung des Trägers mit einer Gleichlast ist die mittragende Breite in Feldmitte maximal und nimmt hin zu den Auflagern stetig ab. Bei einer Belastung des Trägers mit einer konzentrierten Einzellast kommt es auf Grund der Spannungsspitze an der Stelle der Einzellast zu einer starken Einschnürung der mittragenden Breite. Einen Sonderfall stellt die einfach harmonische Belastung dar, für die die mittragende Breite in Trägerlängsrichtung konstant ist.

- Die mittragende Breite $b_{m, s}$ des reinen Scheibenzustands ist unabhängig von der Höhe der äußeren Belastung des Plattenbalkens. Dies gilt jedoch nur, solange sich die Gurtscheibe linearelastisch verhält und im Zustand I ist. Bei nicht-linearelastischem Materialverhalten ist die mittragende Breite zusätzlich von der Belastungshöhe abhängig. 
- Die mittagende Breite $b_{m, s}$ des reinen Scheibenzustands ist unabhängig von der Dicke der Gurtscheibe $h_{c}$. In der kombinierten Wirkung mit dem immer vorhandenen Biegezustand des Betongurts ist die mittragende Breite jedoch von der Dicke des Betongurts bzw. der Steifigkeitsverteilung im Gesamtquerschnitt abhängig.

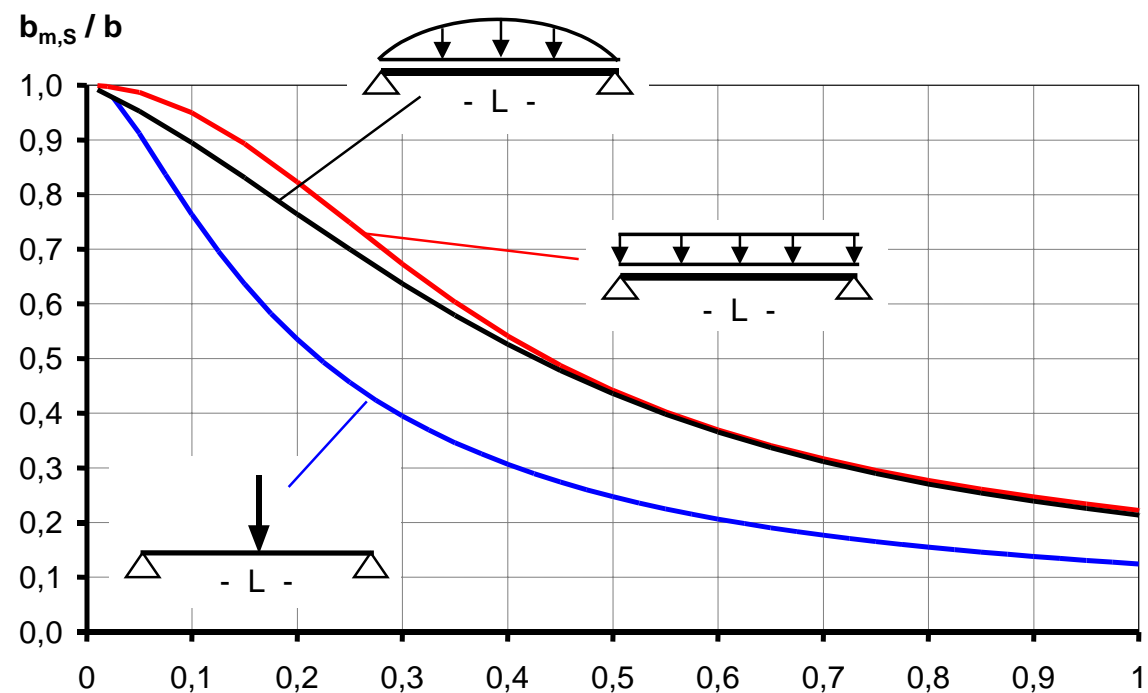

Querschnitt:

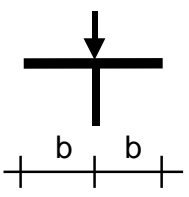

b / L

Abbildung 4-1: Mittragende Breite $b_{m, s}$ der Gurtscheibe des einstegigen Plattenbalkens in Feldmitte für verschiedene Belastungsfunktionen [2.27]

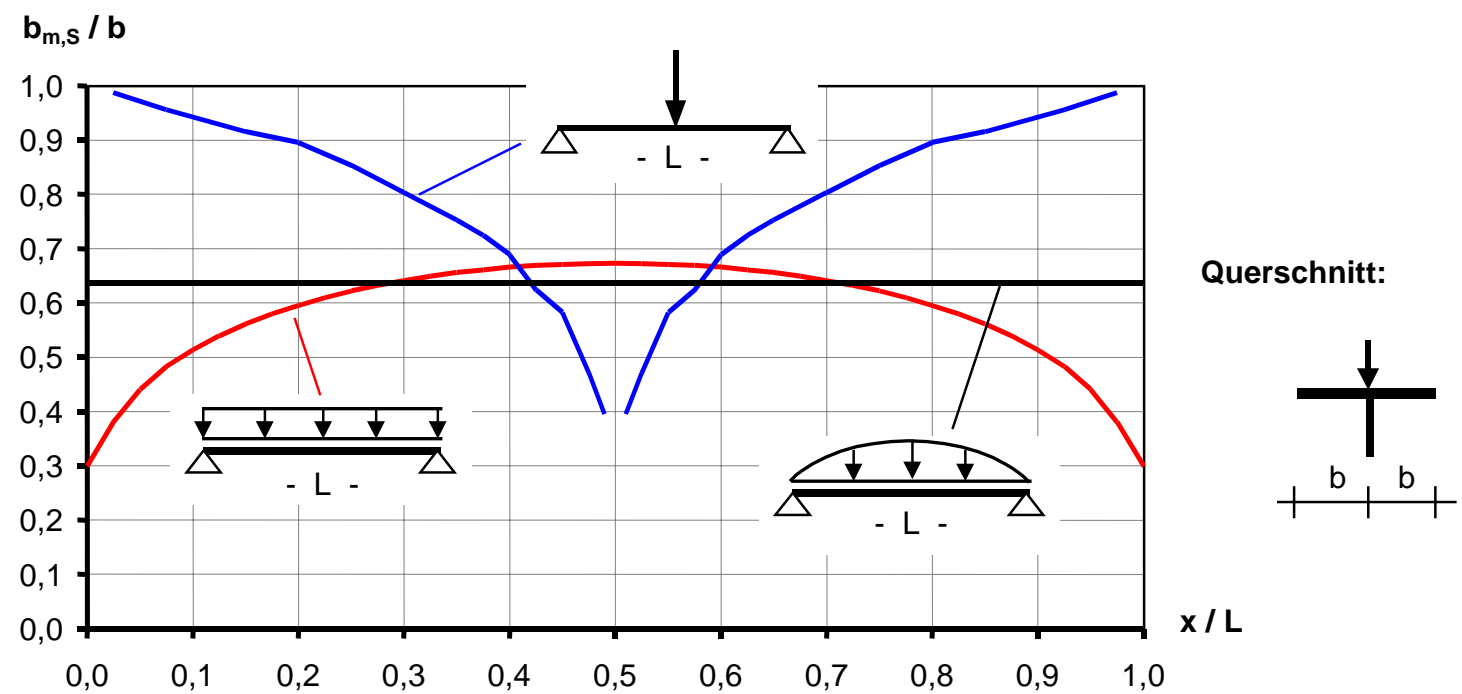

Abbildung 4-2: Verlauf der mittragenden Breite $b_{m, s}$ der Gurtscheibe des einstegigen Plattenbalkens in Trägerlängsrichtung (Seitenverhältnis $b / L=0,3$ ) [2.27]

\subsubsection{Einflüsse auf die mittragende Breite des Biegezustands}

Aus den Berechnungen der mittragenden Breite des reinen Biegezustands durch Lösung der Plattendifferentialgleichung können folgende Schlussfolgerungen gezogen werden, die mit den Angaben in der zuvor beschriebenen Literatur übereinstimmen [2.27]:

- Der Haupteinflussparameter für die mittragende Breite $b_{m, B}$ des Biegezustands ist das Seitenverhältnis des Gurts b/L. Für zunehmende Werte b/L nimmt die bezogene mittragende Breite $b_{m, B} / b$ rasch $a b$, siehe Abbildung 4-3. 
- Der Belastungstyp des Trägers hat einen nennenswerten Einfluss auf die mittragende Breite. Die mittragende Breite $b_{m, B}$ für Gleichlast ist an der maßgebenden Trägerstelle deutlich größer als bei mittiger Einzellast, siehe Abbildung 4-3 und Abbildung 4-4.

- Die mittragende Breite $b_{m, B}$ variiert in Trägerlängsrichtung, siehe Abbildung 4-4. Bei Belastung des Trägers mit einer Gleichlast ist die mittragende Breite in Feldmitte maximal und nimmt in Richtung zu den Auflagern stetig ab. Bei einer Belastung des Trägers mit einer konzentrierten Einzellast kommt es aufgrund der Spannungsspitze an der Stelle der Einzellast zu einer Einschnürung der mittragenden Breite. Ein Sonderfall stellt die einfach harmonische Belastung dar: Hier ist die mittragende Breite in Trägerlängsrichtung konstant.

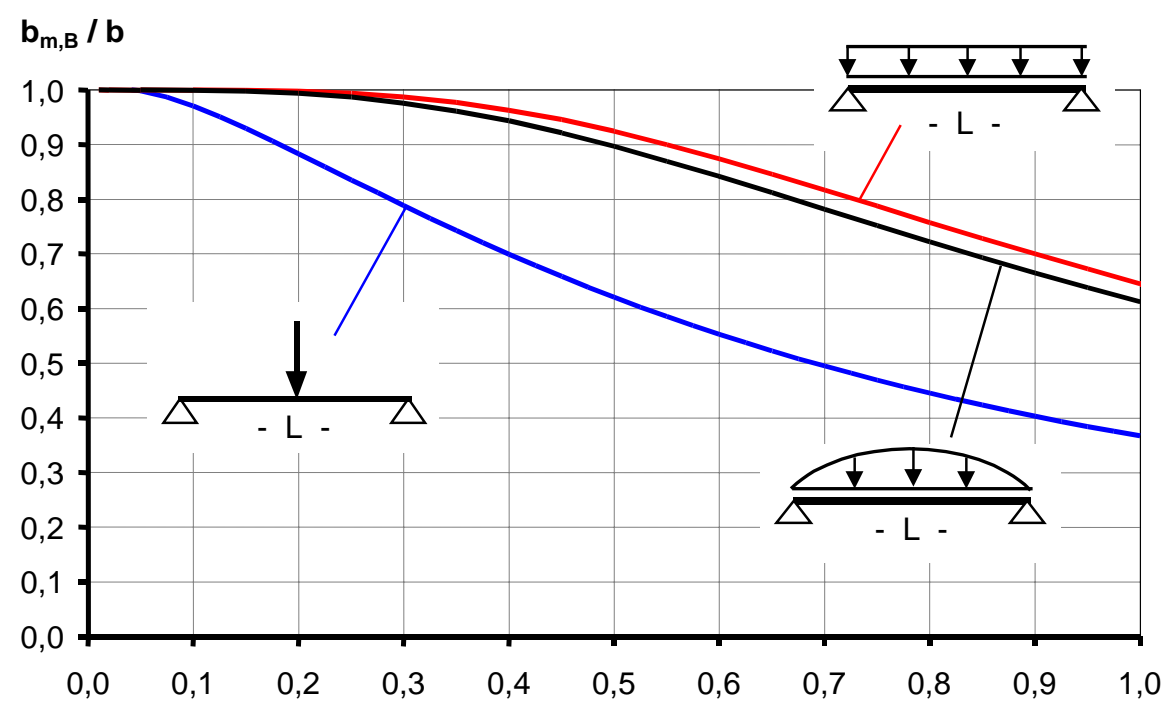

Querschnitt:

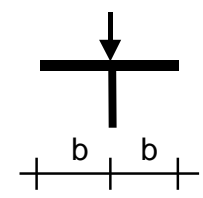

b / L

Abbildung 4-3: $\quad$ Mittragende Breite $b_{m, B}$ der Gurtplatte des einstegigen Plattenbalkens in Trägermitte [2.27]

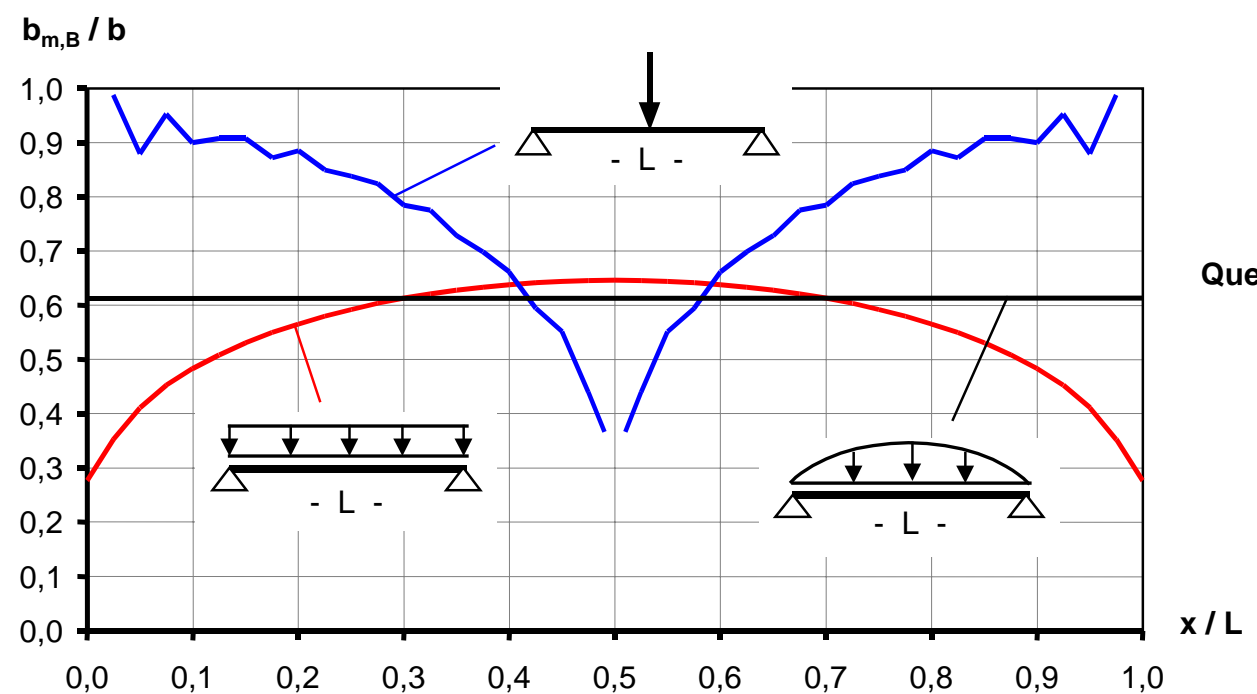

Abbildung 4-4: Verlauf der mittragenden Breite $b_{m, B}$ der Gurtplatte des einstegigen Plattenbalkens in Trägerlängsrichtung [2.27]

- Die mittragende Breite $b_{m, B}$ des reinen Biegezustands ist unabhängig von der Höhe der äußeren Belastung des Plattenbalkens. Dies gilt jedoch nur, solange sich die Gurtplatte linearelastisch verhält und im Zustand I ist. Bei nicht-linearelastischem Ma- 
terialverhalten ist die mittragende Breite zusätzlich von der Belastungshöhe abhängig.

- Die mittagende Breite $b_{m, B}$ des reinen Biegezustands ist unabhängig von der Dicke $h_{c}$ der Gurtplatte. In der kombinierten Wirkung mit dem immer vorhandenen Scheibenzustand des Betongurts ist die mittragende Breite jedoch von der Dicke des Betongurts bzw. der Steifigkeitsverteilung im Gesamtquerschnitt abhängig, siehe Abschnitt 4.3.

- Die mittragende Breite $b_{m, B}$ des reinen Biegezustands ist im Allgemeinen deutlich größer die des reinen Scheibenzustands $b_{m, s}$, siehe Abbildung 4-6.

- Der Querschnittstyp des Trägers (einstegig oder mehrstegig) hat auf die mittragende Breite $b_{m, B}$ nur einen geringfügigen Einfluss. Die mittragende Breite bei Behinderung der Querverdrehung an den Längsrändern des Betongurts (mehrstegiger Querschnitt) ist geringfügig größer als ohne, siehe Abbildung 4-5.

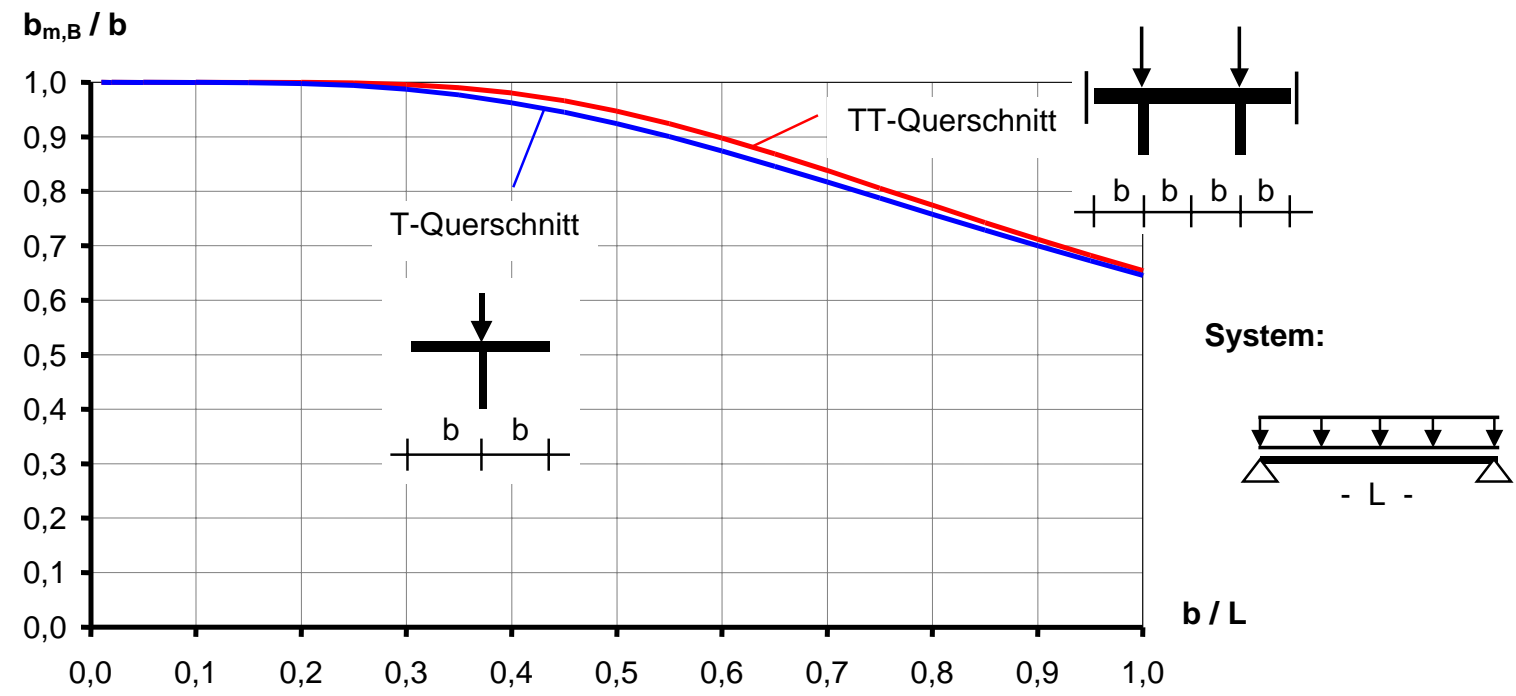

Abbildung 4-5: Mittragende Breite $b_{m, B}$ der Gurtplatte von Plattenbalken in Trägermitte unter Gleichlast in Abhängigkeit des Querschnittstyps [2.27]

$\mathbf{b}_{\mathrm{m}} / \mathbf{b}$

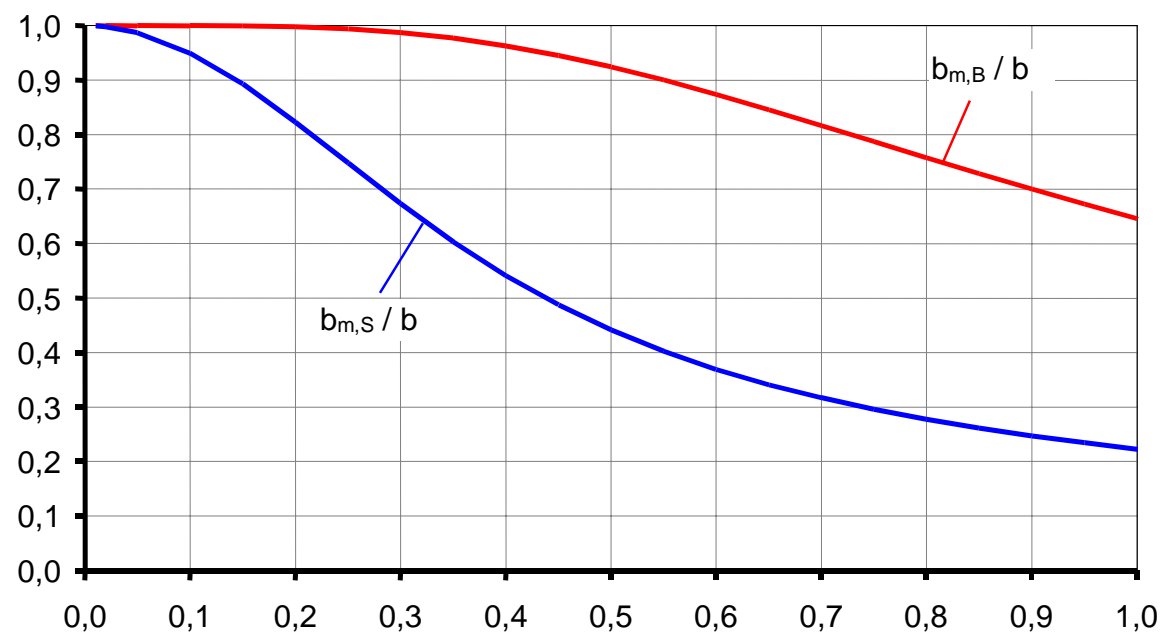

Querschnitt:

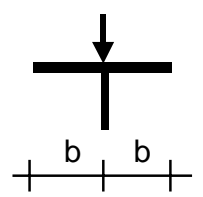

System:

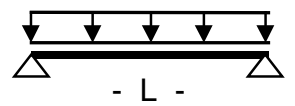

b / L

Abbildung 4-6: Mittragende Breite $b_{m, B}$ der Gurtplatte und $b_{m, s}$ der Gurtscheibe des einstegigen Plattenbalkens in Trägermitte unter Gleichlast [2.26], [2.27] 


\subsection{Kombinierte Wirkung des Scheiben- und Biegezustands}

\subsubsection{Grundsätzliches}

Das Tragverhalten von breiten Gurten in Plattenbalken und Verbundträgern kann wie bereits erwähnt in einen Scheibenzustand und einen Biegezustand unterteilt werden, siehe Abschnitt 4.1. Solange der Betongurt im Zustand I ist und sich Beton und Stahl linearelastisch verhalten, kann der Scheiben- vom Biegezustand durch Anwendung des Superpositionsprinzip getrennt werden. Mit Kenntnis der mittragenden Breite des Scheiben- und Biegezustands kann die Biegesteifigkeit $\mathrm{I}_{\mathrm{i}, 0}$ des Verbundquerschnitts im elastischen Fall nach Gleichung (4-3) berechnet werden. Die Eigenbiegesteifigkeit $I_{c}$ des Betongurts wird dabei mit der mittragenden Breite $b_{m, B}$ des Biegezustands und der Steiner-Anteil $S_{i, 0} \cdot a_{S t}$ der Teilquerschnitte mit der mittragenden Breite $b_{m, s}$ des Scheibenzustands berechnet.

$$
\begin{aligned}
& I_{i, 0}=I_{a}+I_{c, 0}+S_{i, 0} \cdot a_{S t} \\
& I_{i, 0}=I_{a}+\frac{b_{m, B} \cdot h_{c}^{3}}{12 \cdot n_{0}}+\frac{\frac{b_{m, S} \cdot h_{c}}{n_{0}} \cdot A_{a}}{\frac{b_{m, S} \cdot h_{c}}{n_{0}}+A_{a}} \cdot a_{S t}^{2}
\end{aligned}
$$

$\begin{array}{ll}\text { mit } & \text { effektives Trägheitsmoment des Verbundquerschnitts } \\ \mathrm{I}_{\mathrm{i}, 0} & \text { Trägheitsmoment des Stahlquerschnitts } \\ \mathrm{I}_{\mathrm{c}, 0} & \text { reduziertes Trägheitsmoment des Betonquerschnitts } \\ \mathrm{S}_{\mathrm{i}, 0} & \text { statisches Moment des Betonquerschnitts } \\ \mathrm{A}_{\mathrm{a}} & \text { Querschnittsfläche des Stahlquerschnitts } \\ \mathrm{n}_{0}=\mathrm{E}_{a} / \mathrm{E}_{\mathrm{cm}} & \text { Reduktionszahl ( } \mathrm{E}_{\mathrm{a}}=\mathrm{E}-\text { Modul von Stahl, } \mathrm{E}_{\mathrm{cm}}=\mathrm{E}-\text { Modul } \\ & \text { von Beton) } \\ \mathrm{b}_{\mathrm{m}, \mathrm{S}}, \mathrm{b}_{\mathrm{m}, \mathrm{B}} & \text { mittragende Breite des Betongurts für den reinen Schei- } \\ & \text { ben- bzW. Biegezustand } \\ \mathrm{h}_{\mathrm{c}} & \text { Dicke des Betongurts } \\ \mathrm{a}_{\mathrm{St}} & \text { Schwerpunktabstand zwischen Beton- und Stahlquer- } \\ & \text { schnitt }\end{array}$

Im Gegensatz dazu wird in der Ingenieurpraxis im Allgemeinen die Eigenbiegesteifigekeit $\mathrm{I}_{\mathrm{c}}$ des Betongurts mit der mittragenden Breite $b_{m, s}$ des Scheibenzustands berechnet. Im Vergleich zu dieser Praxis ergibt sich mit Gleichung (4-3) ein realistischerer Rechenwert für die Biegesteifigkeit des Verbundquerschnitts, der für niedrige Verbundträger deutlich größer ist als die heute übliche Praxis, siehe Abbildung 4-7.

In Abbildung 4-7 ist das Verhältnis der rechnerischen Trägheitsmomente des Verbundquerschnitts dargestellt, wenn die mittragende Breite $b_{m, B}$ des Biegezustands bei der Berechnung berücksichtigt (Wert $\mathrm{I}_{\mathrm{i}, 0}$ nach Gleichung (4-3)) bzW. vernachlässigt wird (Wert $\mathrm{I}_{\mathrm{i}, 0, \mathrm{~s}}$ berechnet mit $\left.I_{c}=b_{m, s} \cdot h_{c}{ }^{3} / 12\right)$. Es zeigt sich, dass die Vernachlässigung der mittragenden Breite $b_{m, B}$ des Biegezustands $\left(\rightarrow \mathrm{I}_{\mathrm{i}, 0, \mathrm{~S}}\right.$ ) bei hohen Verbundträgern mit dünnen Betongurten keine nennenswerte Abweichung ergibt. Die Werte $\mathrm{I}_{\mathrm{i}, 0, \mathrm{~S}}$ und $\mathrm{I}_{\mathrm{i}, 0}$ sind ungefähr gleich groß. Dies liegt 
daran, dass bei hohen Verbundträgern mit einem vergleichsweise dünnen Gurt der Anteil der Biegesteifigkeit $I_{c}$ des Betongurts an der Gesamtsteifigkeit $I_{i, 0}$ des Verbundträgers sehr klein ist. Somit stellt die Vernachlässigung des Biegezustands bei hohen Trägern eine ausreichend genaue Näherung dar. Bei den bekannten Verfahren zur Verbundträgerbemessung z. B. dem Gesamtquerschnittsverfahren [2.30] wird deshalb in guter Näherung zur Berechnung der Biegesteifigkeit $I_{c}$ des Betongurt anstatt der mittragenden Breite $b_{m, B}$ des Biegezustands der kleinere Wert $b_{m, s}$ verwendet.

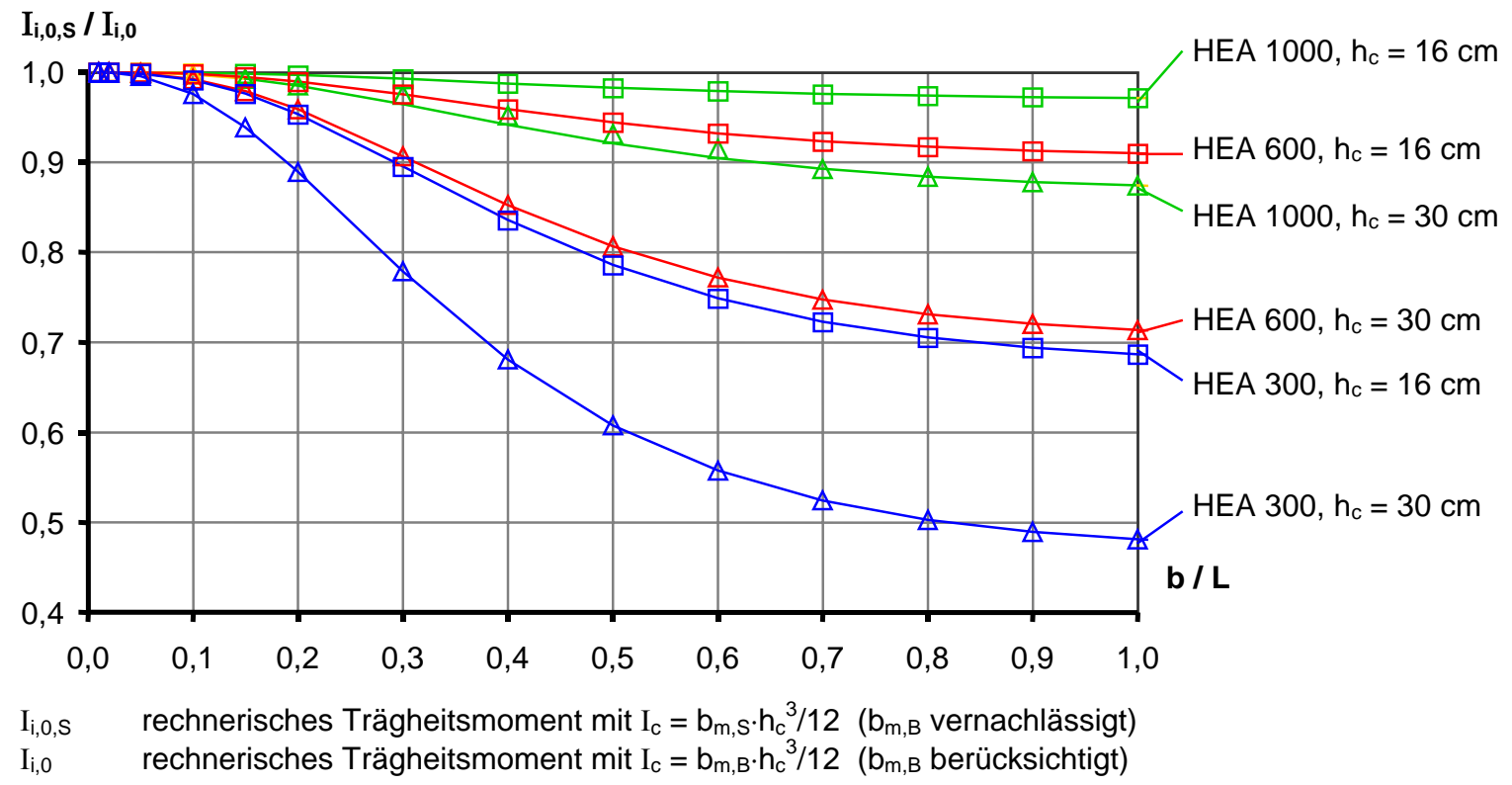

Abbildung 4-7: Einfluss der mittragenden Breite $b_{m, B}$ des Biegezustands auf die rechnerische Biegesteifigkeit von Verbundträgern

Bei Verbundträgern niedriger Bauhöhe ist der Anteil der Biegesteifigkeit $I_{c}$ des Betongurts an der Gesamtsteifigkeit des Verbundquerschnitts jedoch sehr groß, so dass der Fehler bei Verwendung von $b_{m, s}$ an Stelle von $b_{m, B}$ bei der Berechnung der Biegesteifigkeit $I_{c}$ sehr groß

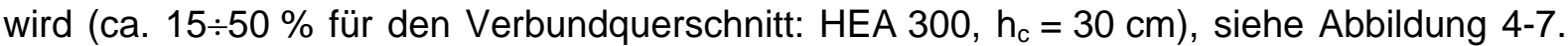
Ein weiterer Grund für den großen Einfluss des Biegezustands des Betongurts bei niedrigen Verbundträgern ist, dass niedrige Verbundträger im Vergleich zu hohen Trägern mit einer kleineren Stützweite und damit bei gleichem Trägerabstand einem größeren Seitenverhältnis b/L der Gurte ausgeführt werden. Bei größerem Seitenverhältnis b/L ist der relative Unterschied zwischen der mittragenden Breite des Scheiben- und Biegezustands größer als bei kleinem Seitenverhältnis. Die Vernachlässigung des Einflusses des Biegezustands auf die mittragende Breite führt daher bei niedrigen Verbundträgern zu einer nicht akzeptablen Abweichung. Der Unterschied, der durch Vernachlässigung der mittragenden Breite $b_{m, B}$ des Biegezustands entsteht, wird dabei größer, wenn

- die Dicke $h_{c}$ des Betongurts zunimmt,

- die Höhe $h_{a}$ des Stahlträgers kleiner wird,

- die Steifigkeit $\mathrm{I}_{\mathrm{a}}$ des Stahlquerschnitts kleiner wird und

- das Seitenverhältnis b/L größer wird, siehe Abbildung 4-7.

Eine realistische Berechnung der Biegesteifigkeit $\mathrm{I}_{\mathrm{i}, 0}$ von niedrigen Verbundträgern kann daher nur unter Berücksichtigung des Einflusses des Biegezustands des Betongurts auf die mittragende Breite erfolgen, da der Biegezustand des Betongurts einen wesentlichen Einfluss auf die mittragende Breite hat. 
Über die Biegesteifigkeit $\mathrm{I}_{\mathrm{i}, 0}$ des Verbundquerschnitts können die unterschiedlichen Werte der mittragenden Breite des reinen Scheiben- und Biegezustands in einen „mittleren“ Wert für die mittragende Breite $b_{m}$ durch Gleichsetzen der Gleichungen (4-3) und (4-4) umgerechnet werden. Der Wert der „mittleren“ mittragenden Breite $b_{m}$ liegt dabei zwischen den beiden Grenzkurven des reinen Scheiben- und reinen Biegezustands. Wie nahe er dabei an den jeweiligen Grenzkurven liegt, hängt vom Verhältnis der Steifigkeiten der Teilquerschnitte ab, siehe Abbildung 4-8. Für hohe Verbundträger mit dünnen Betongurten ist der Einfluss der Biegesteifigkeit $I_{c}$ des Betongurts auf die Gesamtsteifigkeit des Verbundquerschnitts klein. Die "mittlere" mittragende Breite $b_{m}$ liegt daher sehr nahe an der Grenzkurve des reinen Scheibenzustands. Bei Verbundträgern niedriger Bauhöhe ist der Anteil der Biegesteifigkeit $I_{c}$ des Betongurts jedoch sehr groß, so dass der Wert der „mittleren“ mittragenden Breite $b_{m}$ näher an der Grenzlinie des reinen Biegezustands liegt. Mit zunehmender Dicke des Betongurts $h_{c}$ und abnehmender Steifigkeit des Stahlquerschnitts wird die mittragende Breite größer und nähert sich zusehends der Grenzkurve des reinen Biegezustands an. Folgende Einflüsse auf die "mittlere“ mittragende Breite $b_{m}$ können festgestellt werden, siehe Abbildung 4-8:

- Mit zunehmender Dicke $h_{c}$ des Betongurts wird $b_{m}$ größer.

- Für niedrigere Stahlträger wird $b_{m}$ größer.

- Mit zunehmender Steifigkeit $I_{a}$ des Stahlquerschnitts wird $b_{m}$ kleiner.

- Mit zunehmendem Seitenverhältnis $b / L$ wird der Unterschied zwischen $b_{m, s}$ und $b_{m}$ größer.

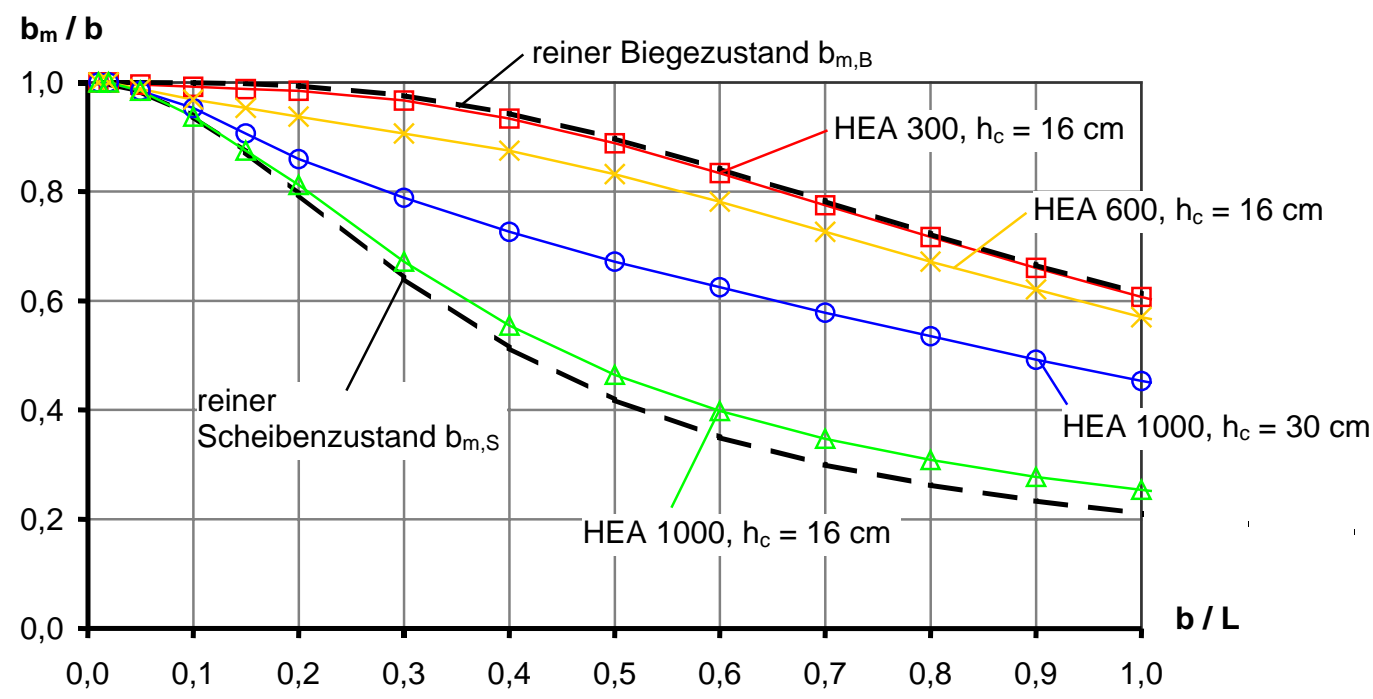

Abbildung 4-8: Einfluss des Biegezustands und der Dicke des Betongurts auf die „mittlere“ mittragende Breite $b_{m}$

Diese Überlegungen zeigen, dass für die wirklichkeitsnahe Berechnung der Steifigkeit von niedrigen Verbundträgern der Einfluss des Biegezustands des Betongurts auf die mittragende Breite berücksichtigt werden muss. Die vereinfachende Annahme, die (mittlere) mittragende Breite $b_{m}$ des Betongurts mit der des reinen Scheibenzustands $b_{m, s}$ gleichzusetzen und den Einfluss des Biegezustands auf die mittragende Breite zu vernachlässigen, führt bei diesen Trägern auf eine sehr große Unterschätzung der tatsächlichen Steifigkeit, die in vielen Fällen nicht akzeptabel ist. 


$$
\begin{aligned}
& I_{i, 0}=I_{a}+I_{c, 0}+S_{i, 0} \cdot a_{S t} \\
& I_{i, 0}=I_{a}+\frac{b_{m} \cdot h_{c}^{3}}{12 \cdot n_{0}}+\frac{\frac{b_{m} \cdot h_{c}}{n_{0}} \cdot A_{a}}{\frac{b_{m, s} \cdot h_{c}}{n_{0}}+A_{a}} \cdot a_{S t}^{2}
\end{aligned}
$$

mit

$$
\begin{array}{ll}
\mathrm{I}_{\mathrm{i}, 0} & \text { effektives Trägheitsmoment des Verbundquerschnitts } \\
\mathrm{b}_{\mathrm{m}} & \text { "mittlere“ mittragende Breite des Verbundquerschnitts }
\end{array}
$$

sonstige Bezeichnungen siehe Gleichung (4-3)

\subsubsection{Einflüsse auf die mittragende Breite}

Der maßgebende Einfluss auf die mittragende Breite ist das Seitenverhältnis $\mathbf{b} / \mathbf{L}$ des Betongurts [2.38]. Mit zunehmendem Verhältnis b/L nimmt die bezogene „mittlere“ mittragende Breite $b_{m} / b$ rasch $a b$. Bei gleichbleibender Stützweite $L$ und größer werdender Breite $b$ des Betongurts nimmt dabei zwar das Verhältnis $b_{m} / b$ ab, der absolute Wert der mittragenden Breite $b_{m}$ wird jedoch größer, siehe Abbildung 4-9. Die mittlere mittragende Breite $b_{m}$ nimmt bei gleichbleibendem Seitenverhältnis $b / L$ aber auch mit der Stützweite $L \quad z u$, siehe Abbildung 4-10. Grund hierfür ist: Bei konstantem Verhältnis b/L bleiben zwar die bezogenen Werte der mittragenden Breite für den Scheibenzustand $b_{m, s} / b$ und den Biegezustand $b_{m, B} / b$ gleich, aber da die tatsächliche Breite b zunimmt, werden auch die Einzelwerte $b_{m, s}$ und $b_{m, B}$ größer. Bei unverändertem Stahlprofil nimmt dadurch die Biegesteifigkeit des Betongurts und damit der Einfluss des Biegezustands auf die Gesamtsteifigkeit des Verbundquerschnitts sowie letztlich der Wert der mittleren mittragenden Breite $b_{m} z u$. Für größere Stützweiten $L$ nähert sich daher die mittragende Breite dem Grenzwert des reinen Biegezustands an, siehe Abbildung 4-10. Bei Querschnitten mit vergleichsweise geringer Biegesteifigkeit des Betongurts (kleine Plattendicke $h_{c}$, großer Stahlträger, etc.) ist dieser Effekt stärker ausgeprägt.

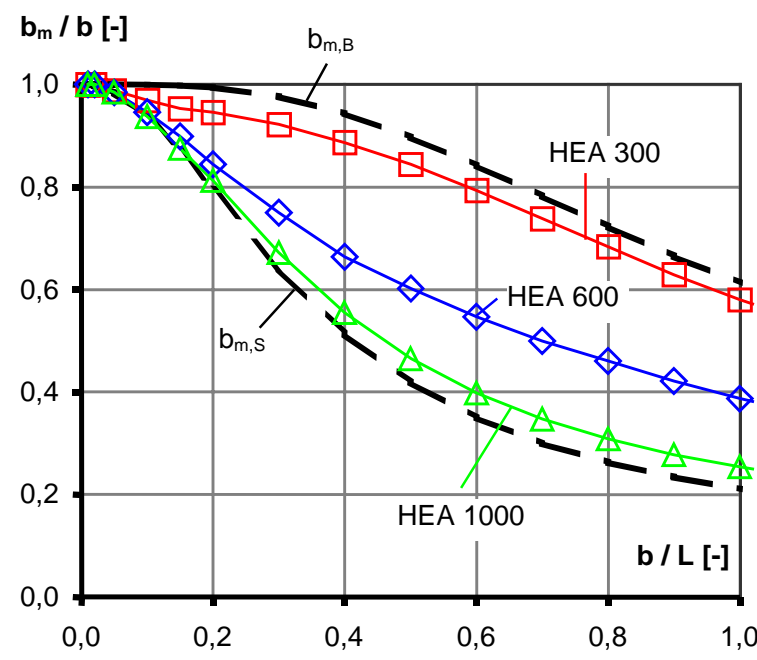

(a) bezogene mittragende Breite $b_{m} / b$

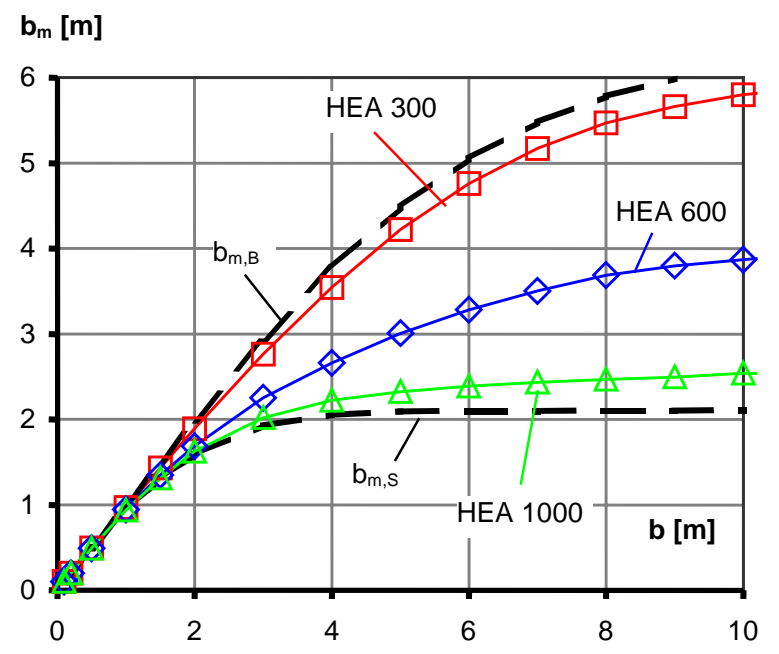

(b) absoluter Wert der mittragenden Breite $b_{m}$

System- und Querschnittswerte: $L=10 \mathrm{~m}, h_{c}=16 \mathrm{~cm}, E_{c}=3200 \mathrm{kN} / \mathrm{cm}^{2}$

Abbildung 4-9: $\quad$ Mittlere mittragende Breite $b_{m}$ in Abhängigkeit der Breite $b$ für verschiedene Stahlträgerhöhen bei gleichbleibender Stützweite und Plattendicke 
Die Untersuchungen der vorangegangenen Abschnitte zeigen, dass zwar die mittragende Breite für den reinen Scheibenzustand und den reinen Biegezustand jeweils für sich unabhängig von der Dicke des Betongurts sind, der mittlere Wert der mittragenden Breite $b_{m}$ ist jedoch deutlich von der Dicke $\boldsymbol{h}_{\boldsymbol{c}}$ des Betongurts abhängig, siehe Abbildung 4-8 und Abbildung 4-10. Dies liegt daran, dass die mittragende Breite des reinen Biegezustands deutlich größer ist als die des reinen Scheibenzustands. Da für größere Gurtdicken $h_{c}$ die Biegesteifigkeit $I_{c}$ des Betongurts stärker anwächst als die Dehnsteifigkeit, wird der Einfluss der mittragenden Breite des Biegezustands auf die mittlere mittragende Breite $b_{m}$ und damit auch der Wert $b_{m}$ größer. Grundsätzlich führen alle Einflüsse, die die Biegesteifigkeit des Betongurts im Verhältnis zur Dehnsteifigkeit vergrößern, auf eine größere mittragende Breite $b_{m}$. Dies bedeutet, dass die mittragende Breite größer wird mit kleiner werdender Höhe des Stahlprofils, kleiner werdendem Schwerpunktsabstand $a_{\text {st }}$ zwischen Beton und Stahl sowie geringerem Trägheitsmoment $\mathrm{I}_{\mathrm{a}}$ des Stahlprofils, etc.

Einen weiteren wichtigen Einfluss auf die mittragende Breite hat die Form der Belastung bzw. des Momentenverlaufs [2.38]. Dies gilt sowohl für die Größe der mittragenden Breite als auch deren Verlauf in Trägerlängsrichtung, siehe z. B. Abbildung 4-3 und Abbildung 4-4. Im maßgebenden Querschnitt ist so z. B. die mittragende Breite für eine Gleichlast deutlich größer als für eine Einzellast in Trägermitte.

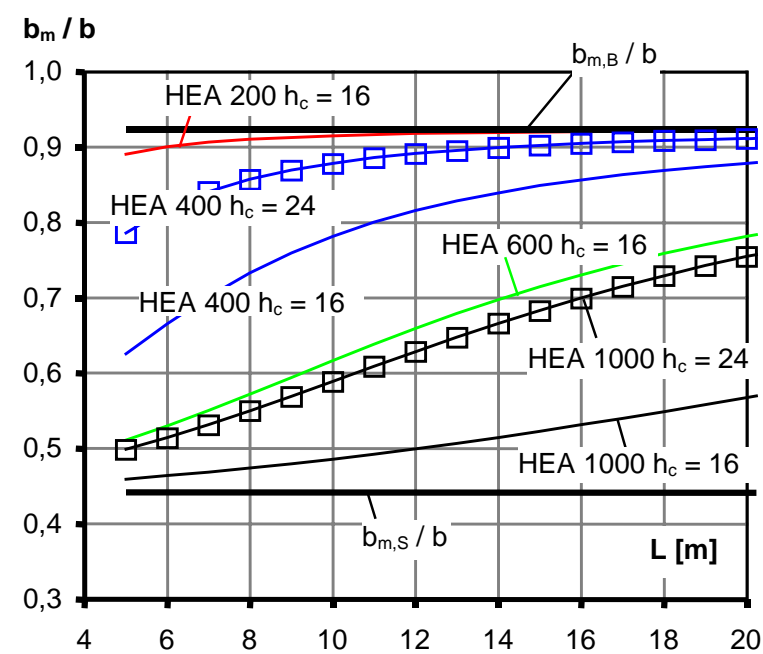

(a) bezogene mittragende Breite $b_{m} / b$

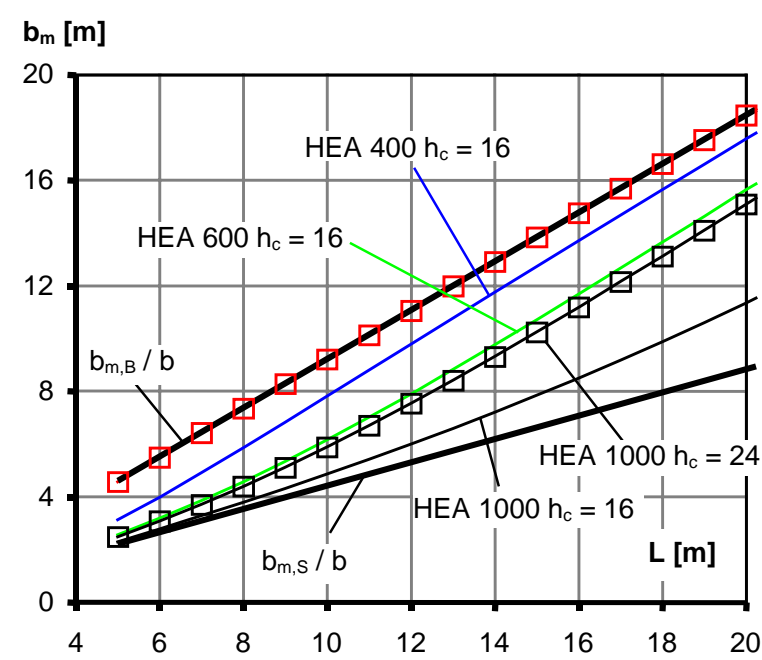

(b) absoluter Wert der mittragenden Breite $b_{m}$

Systemwerte: $\mathrm{b} / \mathrm{L}=0,5, E_{c}=3200 \mathrm{kN} / \mathrm{cm}^{2}, h_{c}[\mathrm{~cm}]$

Abbildung 4-10: Mittlere mittragende Breite $b_{m}$ in Abhängigkeit der Stützweite $L$ für verschieden Stahlträgerhöhen und Plattendicken $h_{c}$ bei gleichbleibendem Seitenverhältnis b/L

Die mittragende Breite des reine Scheiben- und Biegezustands ist von der Querdehnzahl $\mu$ des (Beton-)Gurts abhängig [2.27]. Für größere Werte der Querdehnzahl nimmt die mittragende Breite geringfügig $\mathrm{ab}$. Da die Querdehnzahl für Beton jedoch unabhängig von der Betongüte ist, hat dies bei baupraktischen Verbundträgern keinen Einfluss auf die mittragende Breite.

Wie den Gleichungen (4-3) und (4-4) zu entnehmen ist, ist das Trägheitsmoment $\mathrm{I}_{\mathrm{i}, 0}$ des Verbundquerschnitts vom Verhältnis der E-Moduli des Stahlträgers und des Betongurts abhängig. Abbildung 4-11 zeigt den Einfluss des E-Moduls des Betons auf die mittragende Breite $b_{m}$ des Verbundquerschnitts. Es zeigt sich, dass die mittragende Breite mit zunehmendem E-Modul des Betons größer wird. Dies liegt daran, dass bei größerem E-Modul des Betons die Reduktion der Betonquerschnittswerte $\left(I_{c} \rightarrow I_{c, 0}\right.$ und $\left.A_{c} \rightarrow A_{c, 0}\right)$ geringer wird und 
dadurch der Anteil der Biegesteifigkeit $\mathrm{I}_{\mathrm{c}, 0}$ des Betongurts an der Gesamtsteifigkeit des Verbundquerschnitts größer ist. Dies gilt somit auch für die mittragende Breite. Der Einfluss des E-Moduls des Betons ist dabei bei Querschnitten mit vergleichsweise kleinem Biegeanteil des Betongurts größer als bei solchen mit großem Biegeanteil des Betongurts. Für die beispielhaft untersuchten Querschnitte liegt der Einfluss des E-Moduls auf die mittlere mittragende Breite bei maximal $11 \%$ und im Rahmen baupraktischer Verhältnisse bei weniger als $4 \%$.

$\mathbf{b}_{\mathrm{m}} / \mathbf{b}$

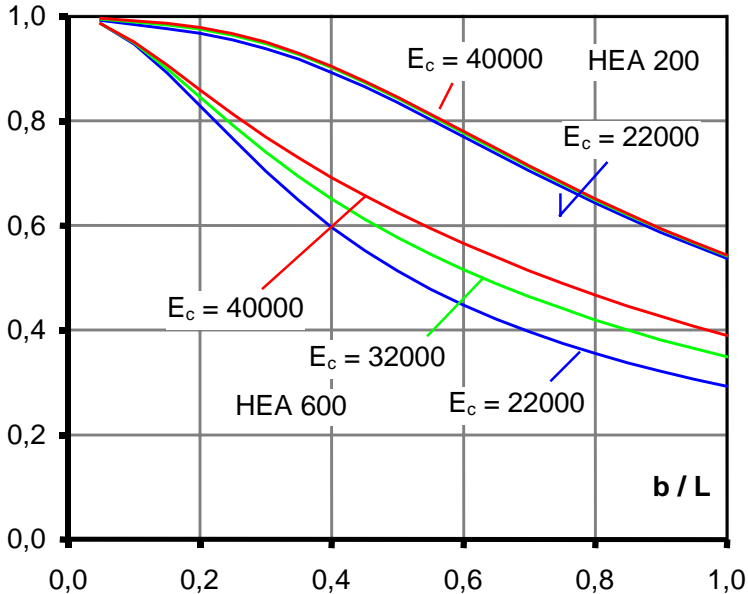

(a) $h_{c}=16 \mathrm{~cm}$ $b_{m} / b$

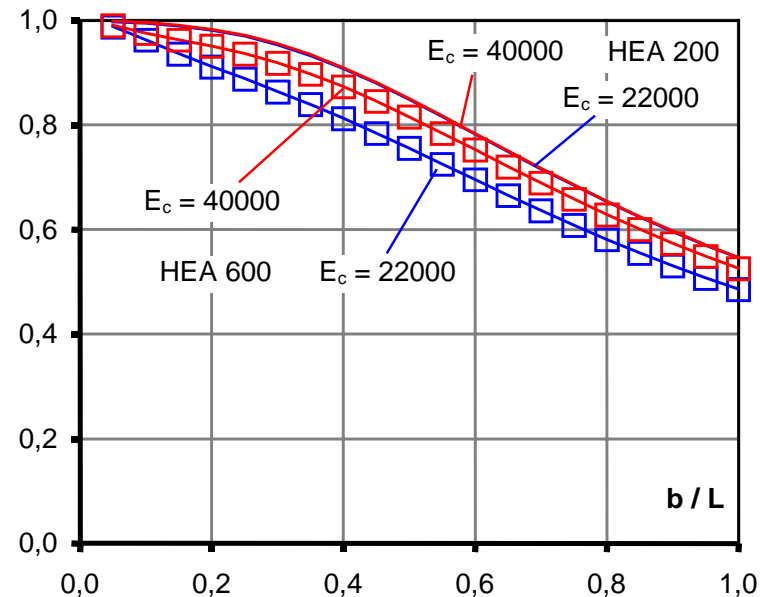

(b) $h_{c}=32 \mathrm{~cm}$

Abbildung 4-11: Einfluss des E-Moduls des Betons [N/mm²] auf die mittragende Breite $b_{m}$

Die Behinderung der Querdehnung bzw. Querverdrehung am Rand des Betongurts führt zu einer geringfügig größeren mittragende Breite als ohne, siehe z. B. Abbildung 4-5. Dieser Einfluss des Querschnittstyps auf die mittragende Breite ist jedoch in der Regel vernachlässigbar.

Prinzipiell hat die Orthotropie des Gurts einen Einfluss auf die mittragende Breite bzw. auf den mitwirkenden Gurtquerschnitt [2.38]. Bei den in dieser Arbeit betrachteten Trägern kann jedoch in ausreichender Genauigkeit von einem isotropen Verhalten des Betongurts ausgegangen werden.

Solange der Beton im Zustand I ist, hat die Bewehrung nur einen vernachlässigbaren Einfluss auf die Steifigkeit des Betongurts sowie des Verbundträgers und daher auch auf die mittragende Breite.

\subsubsection{Verformungsbezogene mittragende Breite für elastisches Materialverhalten}

Wie die voranbeschriebenen Untersuchungen zeigen, ist die spannungsbezogene mittragende Breite entlang der Trägerlängsrichtung im Allgemeinen nicht konstant, siehe $z$. B. Abbildung 4-4. Dadurch ist ebenso das Trägheitsmoments $\mathrm{I}_{i, 0}$ des Verbundträgers in Trägerlängsrichtung veränderlich. Für eine Verformungsberechnung bedeutet dies, dass strenggenommen nicht mit einer konstanten Steifigkeit und einer konstanten mittragenden Breite in Trägerlängsrichtung gerechnet werden kann. Um eine solche aufwändige Berechnung zu vermeiden, wurde die verformungsbezogene mittragende Breite $b_{m, v}$ eingeführt, siehe Abschnitt 3.3. Auf die verformungsbezogene mittragende Breite haben die oben beschriebenen Parameter einen vergleichbaren Einfluss wie auf die spannungsbezogene mittragende Breite. Darüber hinaus hat die Form der Belastung einen deutlichen Einfluss darauf, wie sehr die verformungsbezogene mittragende Breite $b_{m, v}$ von der spannungsbezogenen mittragenden Breite $b_{m}$ im maßgebenden Querschnitt abweicht, siehe Abbildung 4-12 und Abbildung 4-13. 
Für Träger unter Gleichlast ist der Unterschied zwischen der verformungsbezogenen mittragenden Breite $b_{m, v}$ und der spannungsbezogenen mittragenden Breite $b_{m}$ in Feldmitte vernachlässigbar klein. Im Beispiel in Abbildung 4-12 liegt die Abweichung zwischen beiden Werten für hochbautypische Querschnitte bei weniger als $5 \%$. Grund hierfür ist, dass für eine Gleichlast die mittragende Breite in Trägerlängsrichtung vergleichsweise ausgeglichen ist. In guter Näherung können daher bei elastischem Materialverhalten und Einfeldträgern unter Gleichlast Verformungen unter der Annahme berechnet werden, dass die spannungsbezogene mittragende Breite $b_{m}$ in Feldmitte über die gesamte Trägerlänge konstant ist.

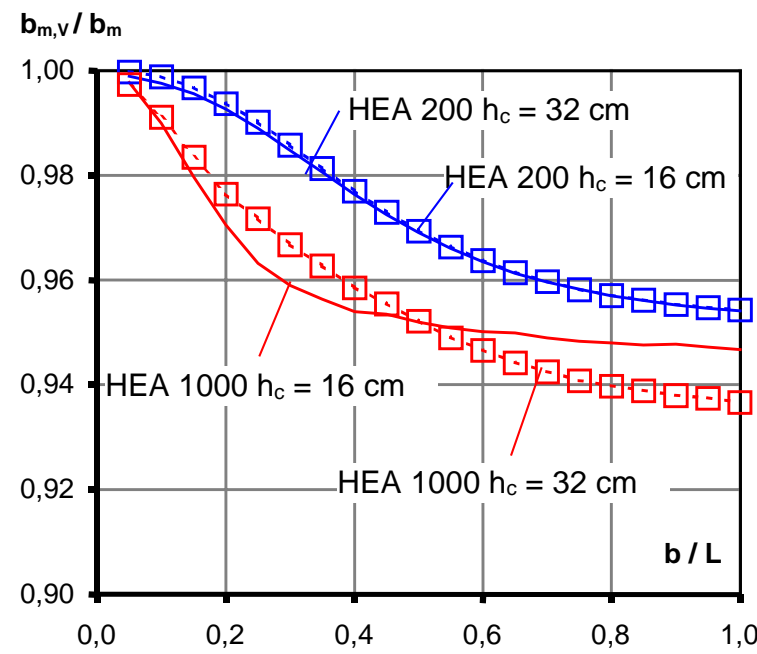

(a) Gleichlast

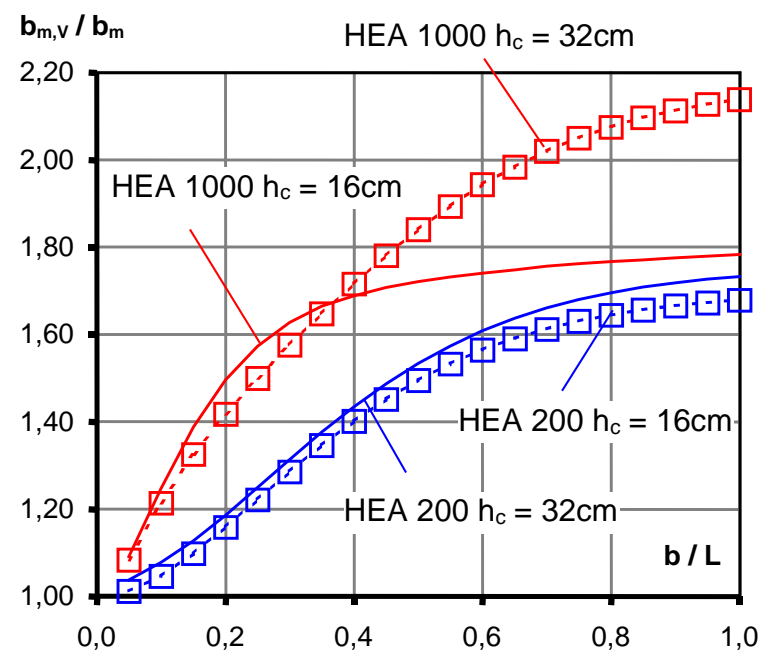

(b) mittige Einzellast

Abbildung 4-12: Vergleich der verformungsbezogenen mittragenden Breite $b_{m, v}$ und der spannungsbezogenen mittragenden Breite $b_{m}(x / L=0,5)$ für verschiedene Verbundquerschnitte

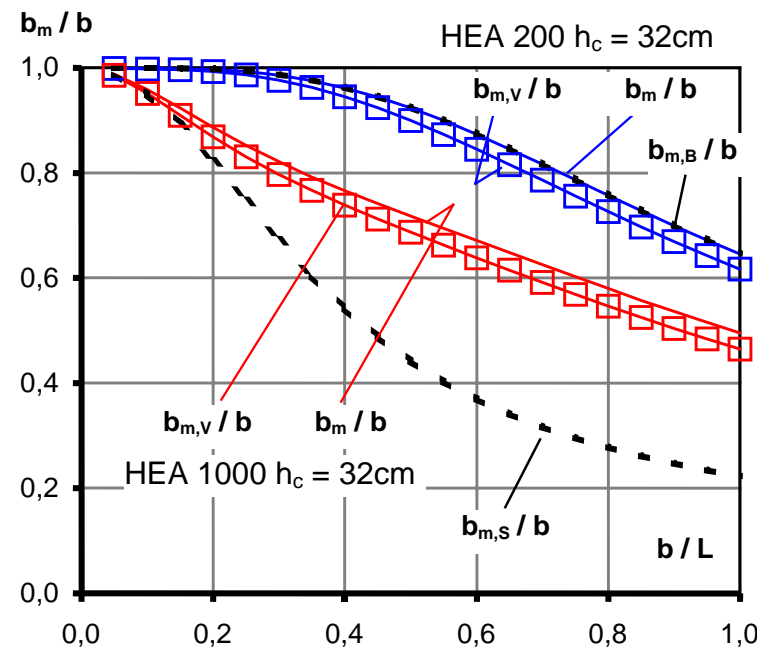

(a) Gleichlast

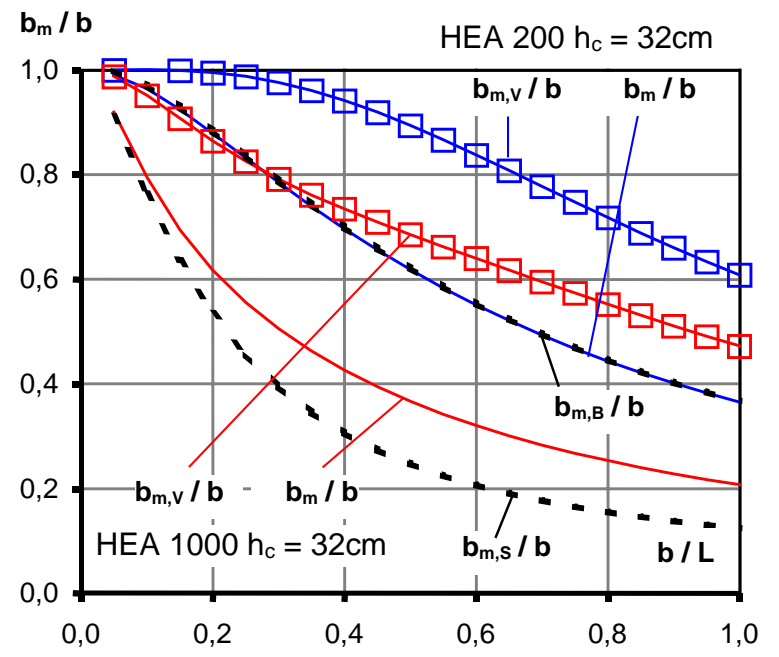

(b) mittige Einzellast

Abbildung 4-13: Vergleich der verformungsbezogenen mittragenden Breite $b_{m, v}$ und der spannungsbezogenen mittragenden Breite $b_{m}(x / L=0,5)$ für verschiedene Verbundquerschnitte

Für eine mittige Einzellast ist der Unterschied zwischen der verformungsbezogenen mittragenden Breite und der spannungsbezogenen mittragenden Breite in Feldmitte jedoch deutlich. Im Beispiel in Abbildung 4-12 beträgt der Unterschied zwischen den Werten $b_{m, v}$ und $b_{m}$ 
bis zu $100 \%$. Die verformungsbezogene mittragende Breite $b_{m, v}$ ist dabei deutlich größer als die spannungsbezogene mittragende Breite $b_{m}$ in Feldmitte, siehe Abbildung 4-13, da für die mittige Einzellast der Verlauf der mittragenden Breite in Trägerlängsrichtung stark variiert, siehe z. B. Abbildung 4-2 und Abbildung 4-4. Im Fall einer Einzellast in Trägermitte ist die vereinfachende Annahme $b_{m, v}=b_{m}$ daher im Allgemeinen unzureichend.

Wie Abbildung 4-14 zeigt, ist die verformungsbezogene mittragende Breite $b_{m, v}$ für Gleichlast und mittige Einzellast am Einfeldträger in der Regel ungefähr gleich groß. Der große Unterschied, wie er für die spannungsbezogene mittragende Breite $b_{m}$ in Feldmitte für diese Belastungsformen vorhanden ist, besteht bei der verformungsbezogenen mittragenden Breite nicht. Dies liegt daran, dass bei mittiger Einzellast die spannungsbezogene mittragende Breite in Feldmitte zwar sehr viel kleiner ist als bei Gleichlast, die spannungsbezogene mittragende Breite bei mittiger Einzellast mit zunehmendem Abstand von der Feldmitte jedoch sehr schnell größer wird und die entsprechenden Werte bei Gleichlast übersteigen, siehe Abbildung 4-2 und Abbildung 4-4. Da die verformungsbezogene mittragende Breite $b_{m, v}$ ein integraler Wert der spannungsbezogenen mittragenden Breite über die Trägerlänge ist, werden bei diesem die Unterschiede zwischen den Werten der spannungsbezogenen mittragenden Breite bei mittiger Einzellast und Gleichlast ausgeglichen.

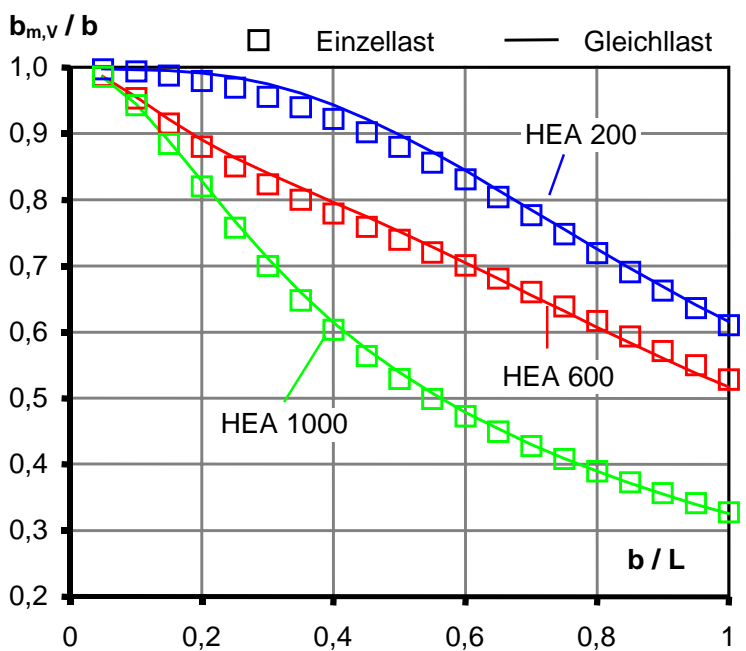

(a) $L=20 m$

Systemwerte: $h_{c}=16 \mathrm{~cm}, E_{c}=32000 \mathrm{~N} / \mathrm{mm}^{2}$

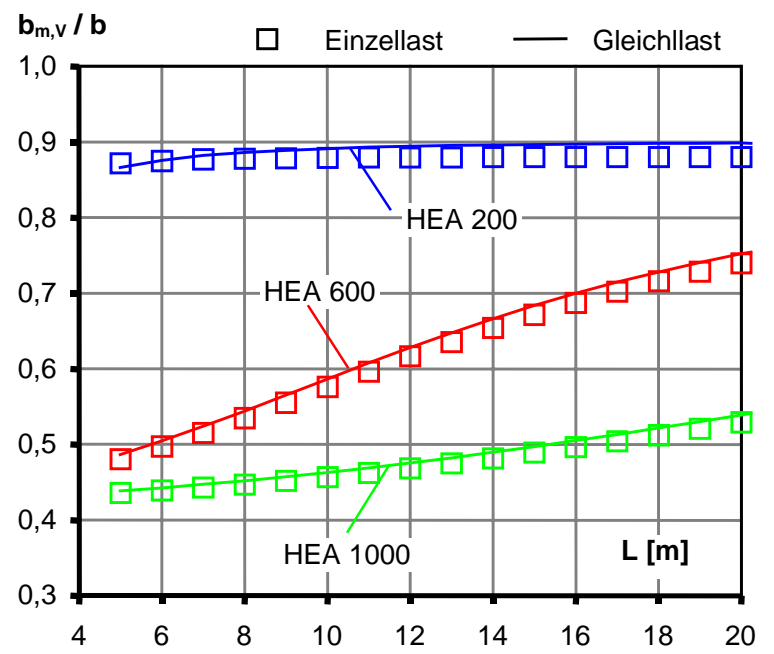

(b) $b / L=0,5$

Abbildung 4-14: Verformungsbezogene mittragende Breiten $b_{m, v}$ für Gleichlast und mittige Einzellast für verschiedene Verbundquerschnitte in Abhängigkeit des Seitenverhältnisses b/L

Generell gilt, dass die verformungsbezogene mittragende Breite $b_{m, v}$ im Wesentlichen denselben Einflüssen unterliegt wie die spannungsbezogene mittragende Breite $b_{m}$. Die maßgebenden Einflüsse sind somit:

- Mit zunehmendem Seitenverhältnis $b / L$ wird der bezogene Wert der verformungsbezogenen mittragenden Breite $b_{m, v} / b$ kleiner.

- Mit zunehmender Biegetragwirkung des Betongurts, zunehmender Plattendicke $\mathrm{h}_{\mathrm{c}}$, abnehmender Trägerhöhe, etc. wird die verformungsbezogene mittragende Breite $b_{m, v}$ größer. 


\subsection{Schlussfolgerungen aus den linearelastischen Untersuchungen}

Das Tragverhalten von Betongurten in Verbundträgern setzt sich aus einem Scheiben- und einem Biegezustand zusammen. Bei Annahme linearelastischen Materialverhaltens lassen sich diese beiden Zustände durch Anwendung des Superpositionsprinzips voneinander trennen. Die durchgeführten Untersuchungen zeigen, dass die mittragende Breite des reinen Biegezustands deutlich größer ist als die des reinen Scheibenzustands. Der Einfluss des Biegezustands nimmt dabei mit größer werdender Dicke des Betongurts und mit kleiner werdender Gesamthöhe des Verbundträgers bzw. Steifigkeit des Stahlquerschnitts zu.

Bei dem gängigen Vorgehen in der Ingenieurpraxis wird der Einfluss des Biegezustands des Betongurts auf die mittragende Breite vernachlässigt. Der Fehler, der dabei bei der Berechnung der Biegesteifigkeit des Verbundträgers gemacht wird, ist zwar bei hohen Verbundträgern mit im Verhältnis zur Stützweite kleinem Achsabstand vernachlässigbar, bei niedrigen Verbundträgern kann er jedoch $15 \div 50 \%$ betragen und sollte daher nicht vernachlässigt werden. Der Einfluss des Biegezustands auf die rechnerische Steifigkeit und die mittragende Breite des Verbundträgers nimmt dabei mit zunehmender Dicke des Betongurts und kleiner werdender Trägerhöhe zu.

Von den hier vorgestellten elastischen Untersuchungen zur mittragenden Breite von Verbundträgern lässt sich folgern, dass der Biegezustand des Betongurts einen wesentlichen Einfluss auf die mittragende Breite und die rechnerische Biegesteifigkeit niedriger Verbundträger hat. Der Einfluss nimmt mit zunehmender Dicke und Breite des Betongurts sowie kleiner werdender Gesamthöhe des Verbundträgers zu. Für einen realistischen Berechnungsansatz müssen diese Faktoren berücksichtigt werden.

Die in diesem Kapitel beschriebenen Untersuchungen beschränken sich auf Situationen, in denen sich der Verbundträger linearelastisch verhält - der Betongurt somit ungerissen im Zustand I ist. Da bei niedrigen Verbundträgern bereits im Gebrauchszustand Risse entstehen, muss dies für eine realistische Bestimmung der mittragenden Breite des Betongurts berücksichtigt werden. Dies wird in den Kapiteln 7 und 8 vorgenommen. Die in diesem Kapitel dargestellten linearelastischen Untersuchungen dienen als Grundlage für diese nichtlinearen Untersuchungen. 


\section{Experimentelle Untersuchungen}

\subsection{Grundsätzliches}

Im Rahmen eines AiF-Forschungsvorhabens [2.27] wurden sechs Versuche mit niedrigen Verbundträgern mit breiten Betongurten am Otto-Graf-Institut (MPA), Forschungs- und Materialprüfungsanstalt für das Bauwesen an der Universität Stuttgart durchgeführt. Ziel der Versuche war die experimentelle Untersuchung der mittragenden Breite und des Verformungsverhaltens niedriger Verbundträger sowie die Möglichkeit, die rechnerischen Untersuchungen mit diesen Versuchsergebnissen zu verifizieren. Für eine genaue Beschreibung der Versuche sei auf [2.27] verwiesen. Im Folgenden werden lediglich die wichtigsten Eckdaten und Ergebnisse dieser Versuche wiedergegeben.

\subsection{Grenzzustand der Gebrauchstauglichkeit}

In der Regel ist das Verformungsverhalten von Verbundträgern vor allem im Gebrauchszustand und nicht im Bruchzustand von Interesse. In der Planung von Bauwerken werden die Verformungen dabei in der Regel im Grenzzustand der Gebrauchstauglichkeit untersucht. Für die Auswertung der Versuche muss hierfür zunächst das Lastniveau, das dem Grenzzustand der Gebrauchstauglichkeit entspricht, bestimmt werden. Dies ist insbesondere deshalb von großer Bedeutung, da das Verformungsverhalten niedriger Verbundträger stark von der Belastungshöhe abhängig ist.

Die Bruchlasten der Versuchsträger waren unter Anderem wegen der Wiederfestigung des Stahls und der Zunahme der spannungsbezogenen mittragenden Breite im Bruchzustand größer als eine normale Bemessung ergibt. Für die Auswertung der Versuche werden daher das Lastniveau im Grenzzustand der Tragfähigkeit (GZT) bzw. das Lastniveau im Grenzzustand der Gebrauchstauglichkeit (GZG) auf Basis der gemessenen Werkstoff- und Querschnittswerte mittels folgender Annahmen berechnet, anstatt diese aus den experimentellen Bruchlasten zu bestimmen:

- Für die Querschnittstragfähigkeit von Verbundträgern ist der Teilsicherheitsbeiwert des Baustahls $\gamma_{\mathrm{M}, \mathrm{a}}=1,1$ maßgebend.

- Auf Seite der Einwirkungen kann von einem mittleren Teilsicherheitsbeiwert $\gamma_{F}=1,4$ ausgegangen werden.

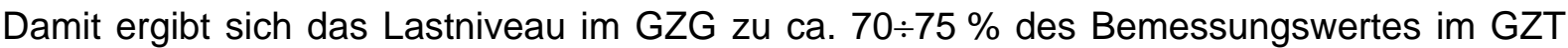
bzw. $60 \div 70 \%$ der charakteristischen Last im GZT. Das so ermittelte Lastniveau im GZG beträgt ca. $50 \%$ der Bruchlasten im Versuch.

\subsection{Versuchsbeschreibung}

\subsubsection{Gesichtspunkte zum Entwurf der Versuchsträger}

Die Versuche wurden unter Berücksichtigung folgender Randbedingungen und Überlegungen konzipiert:

- Verwendung praxisgerechter Werkstoffe, Querschnitte und Abmessungen im Maßstab 1:1 
- Verwendung von Verbundquerschnitten niedriger Bauhöhe, bei denen die elastische Nulllinie im Beton liegt. Das heißt, bereits im Gebrauchszustand entstehen Risse im Beton.

- Verwendung eines sehr breiten Betongurts, um eine klare Aussage über den Verlauf der Betonspannungen und -dehnungen in Querrichtung des Betongurts zu erhalten sowie einen deutlichen Einfluss der Schubverformung des Betongurts auf das Tragund Verformungsverhalten der Versuchsträger zu garantieren.

- Verwendung statisch bestimmter Systeme, um klare Aussagen durch die Kenntnis des Verlaufs der Gesamtschnittgrößen und Auflagerreaktionen allein aus den Lagerungs- und Belastungsbedingungen ohne Kenntnis der Steifigkeitsverteilung im Träger zu erhalten.

- Verwendung einer Zweipunktbelastung zur ausreichend genauen Approximation einer gleichmäßig verteilten Belastung in Trägerlängsrichtung, die den wichtigsten Lastfall in der Praxis darstellt.

- Anordnung der Lastpunkte in Stegebene des Stahlträgers, um eine Exzentrizität in Querrichtung, ein damit verbundenes Querbiegemoment des Betongurts zu vermeiden und eine bessere Übereinstimmung mit analytischen Untersuchungen zu erreichen.

- Die Versuchsträger wurden während dem Betonieren und der Zwischenlagerung in kurzen Abständen unterstützt, um einen Eigengewichtsverbund zu erzeugen, wie er bei Verbundträgern niedriger Bauhöhe im Allgemeinen in der Praxis ausgeführt wird.

- Zwischen Betonieren und Versuchsdurchführung wurden entsprechend der üblichen Ausschalfrist in der Praxis ca. 28 Tage geplant.

Ausgehend von einem Basisversuch wurde bei jedem weiteren Versuch nur ein einziger Parameter variiert, um dessen Auswirkung auf das Verformungsverhalten und die mittragende Breite zu bestimmen, siehe Tabelle 5-1. Damit war es möglich, mit diesem beschränkten Versuchsprogramm eine möglichst große Anzahl an Parametern zu untersuchen.

Die ersten analytischen Untersuchungen [2.26] zeigten, dass die mittragende Breite sehr stark von der Biegetragwirkung und dem Seitenverhältnis des Betongurts abhängt. Aus diesem Grund wurden folgende Parameter im Versuchsprogramm variiert:

- Plattendicke: Da die Plattendicke einen maßgebende Einfluss auf die Biegetragwirkung des Betongurts hat, sollte dessen Einfluss auf die mittragende Breite untersucht werden.

- Plattenbreite: Durch Variation der Plattenbreite kann der Einfluss des Seitenverhältnisses des Betongurts auf die mittragende Breite experimentell untersucht werden.

- Betongüte: Durch die Variation der Betongüte kann der Einfluss der Zugfestigkeit des Betons und damit dessen Rissverhaltens auf die mittragende Breite untersucht werden.

- Verdübelungsgrad: Durch Variation des Verdübelungsgrads sollte zum einen das Verhältnis zwischen Biege- und Scheibentragwirkung des Betongurts sowie dessen Rissbildung beeinflusst werden und deren Einflüsse auf die mittragende Breite untersucht werden. 


\subsubsection{Versuchsaufbau}

Die Versuchsträger wurden als Einfeldträger mit einer Zweipunktbelastung in Stegebene des Stahlträgers durchgeführt, siehe Abbildung 5-1. Tabelle 5-1 zeigt die Abmessungen und verwendeten Werkstoffe der Versuchsträger im Überblick.

Schnitt 1 - 1

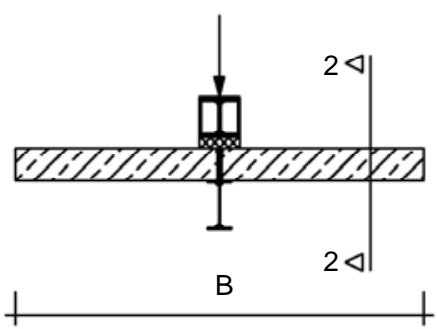

Schnitt 2 - $2 \quad$ Hydraulikzylinder

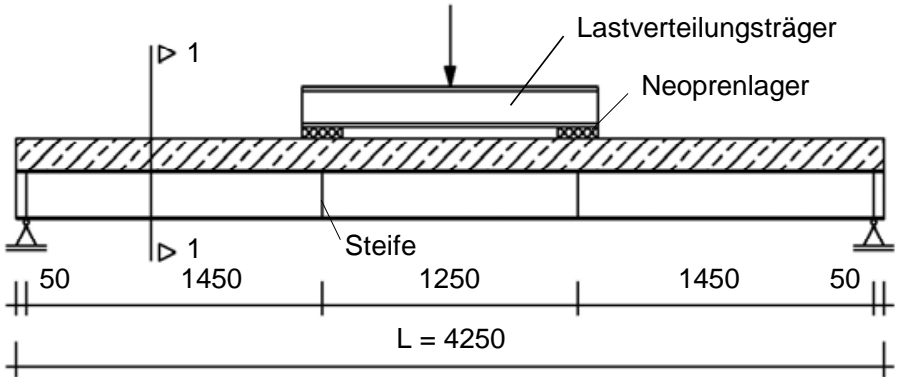

Abbildung 5-1: $\quad$ Prinzipieller Versuchsaufbau [2.27]

Tabelle 5-1: $\quad$ Abmessungen und Werkstoffe der Versuchsträger [2.27]

\begin{tabular}{|c|c|c|c|c|c|c|c|c|}
\hline $\begin{array}{c}\text { Versuchs- } \\
\text { Nr. }\end{array}$ & $\begin{array}{c}\text { Stahl- } \\
\text { querschnitt }\end{array}$ & $\begin{array}{c}\text { Stahl- } \\
\text { güte }\end{array}$ & $\begin{array}{c}\text { Beton- } \\
\text { güte }\end{array}$ & $\begin{array}{c}\text { Bewehrungs- } \\
\text { güte }\end{array}$ & $\mathbf{L}[\mathbf{m}]$ & $\mathbf{B}[\mathbf{m}]$ & $\mathbf{h}_{\mathbf{c}}[\mathbf{c m}]$ & $\eta^{\mathbf{1})}[-]$ \\
\hline VT 1 & IPE 240 & S 235 & C 20/25 & S 500 & 4,25 & 4,00 & 16 & 1,0 \\
\hline VT 2 & IPE 240 & S 235 & C 30/37 & S 500 & 4,25 & 4,00 & 16 & 1,0 \\
\hline VT 3 & IPE 240 & S 235 & C 20/25 & S 500 & 4,25 & 4,00 & 16 & $\mathbf{0 , 4}$ \\
\hline VT 4 & IPE 240 & S 235 & C 20/25 & S 500 & 4,25 & $\mathbf{5 , 4 0}$ & 16 & 1,0 \\
\hline VT 5 & IPE 240 & S 235 & C 20/25 & S 500 & 4,25 & 4,00 & $\mathbf{2 6}$ & 1,0 \\
\hline VT 6 & IPE 240 & S 235 & C 30/37 & S 500 & 4,25 & 4,00 & $\mathbf{2 6}$ & 1,0 \\
\hline
\end{tabular}

1) planmäßiger Verdübelungsgrad

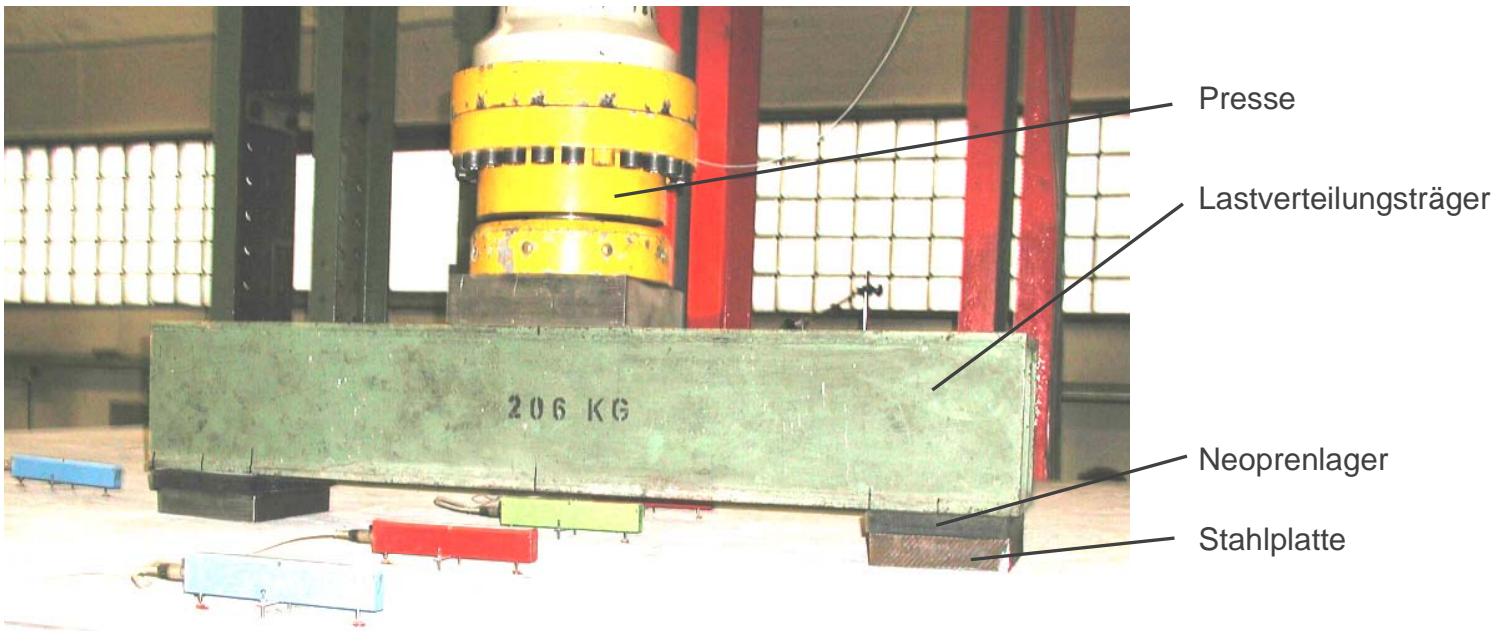

Abbildung 5-2: $\quad$ Lasteinleitungskonstruktion [2.27]

Die Belastung wurde mit einem servohydraulisch gesteuerten Prüfzylinder und einem Lastverteilungsträger auf die beiden Lastpunkte weggesteuert aufgebracht. Zwischen Lastverteilungsträger und Betongurt wurden bewehrte Neoprenlager mit ca. $3 \mathrm{~cm}$ Dicke und evtl. noch zusätzlich Stahlplatten zum Höhenausgleich angeordnet, siehe Abbildung 5-2. 
Die Träger wurden im Versuchsstand an beiden Auflagern verschieblich auf den Stahlträgern aufgelagert. Zur Kippsicherung wurde der Betongurt an beiden Trägerenden in vertikaler Richtung seitlich geführt, siehe Abbildung 5-3. Als horizontales Auflager in Trägerlängsrichtung diente der Hydraulikzylinder.

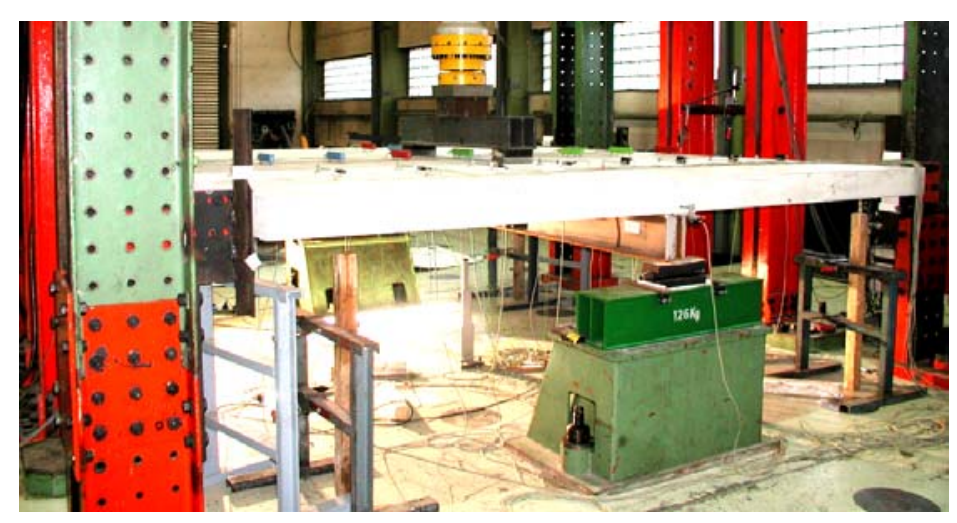

(a) Kippstabilisierung

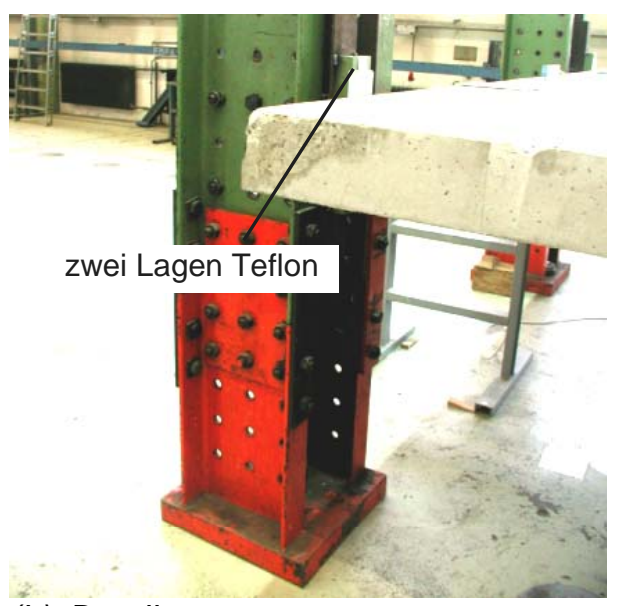

(b) Detail

Abbildung 5-3: Seitliche Führung zur Kippstabilisierung der Versuchsträger [2.27]

\subsubsection{Messeinrichtung}

Im Wesentlichen wurden folgende Messungen vorgenommen:

(a) Globale Messungen

W-5E Messung der Durchbiegung des Trägers in Feldmitte Achse E, gemessen an der Unterkante des Stahlträgers mittels eines 300 mm-Wegaufnehmers

S1 Messung des Endschlupfs zwischen Beton und Stahl an beiden Trägerenden mittels $25 \mathrm{~mm}$-Wegaufnehmern

(b) Messungen am Stahlträger mittels Dehnmessstreifen

DMS Messung der Dehnung des Stahlträgers am Ober-, Untergurt und Steg in Trägerlängsrichtung

DMS-R Messung des Dehnungszustands an den Auflagern des Stahlträgers am Steg mittels Dehnmessrosetten zur Bestimmung der Querkraft im Stahlträger

(c) Messungen an der Oberseite des Betongurts, siehe Abbildung 5-4

Bo-5 Messung der Längsdehnung des Betons in Feldmitte (Achse 5) an der Betonoberseite verteilt über die Breite des Betongurts mittels Setzdehnungsmesser. Messbasis $200 \mathrm{~mm}$

Bo-3 Messung der Längsdehnung des Betons im Viertelspunkt (Achse 3) an der Betonoberseite, verteilt über die Breite des Betongurts mittels $25 \mathrm{~mm}$-Wegaufnehmer. Messbasis $200 \mathrm{~mm}$ 
(d) Messungen an der Unterseite des Betongurts, siehe Abbildung 5-4

Bu-5 Messung der Längsdehnung des Betons in Feldmitte (Achse 5) an der Betonunterseite, verteilt über die Breite des Betongurts mittels $5 \mathrm{~mm}$-Wegaufnehmer. Messbasis $200 \mathrm{~mm}$

Bu-3 Messung der Längsdehnung des Betons in Feldmitte (Achse 3) an der Betonunterseite, verteilt über die Breite des Betongurts mittels $25 \mathrm{~mm}$-Wegaufnehmer. Messbasis $200 \mathrm{~mm}$

\section{Untersicht des Betongurts}

Schnitt 1 - 1

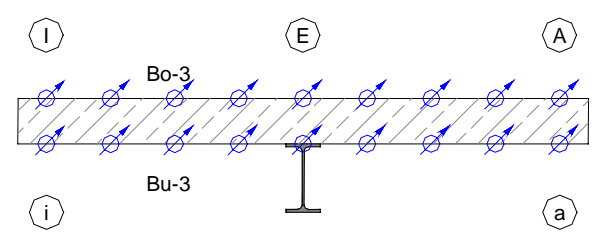

\section{Schnitt 2 - 2}
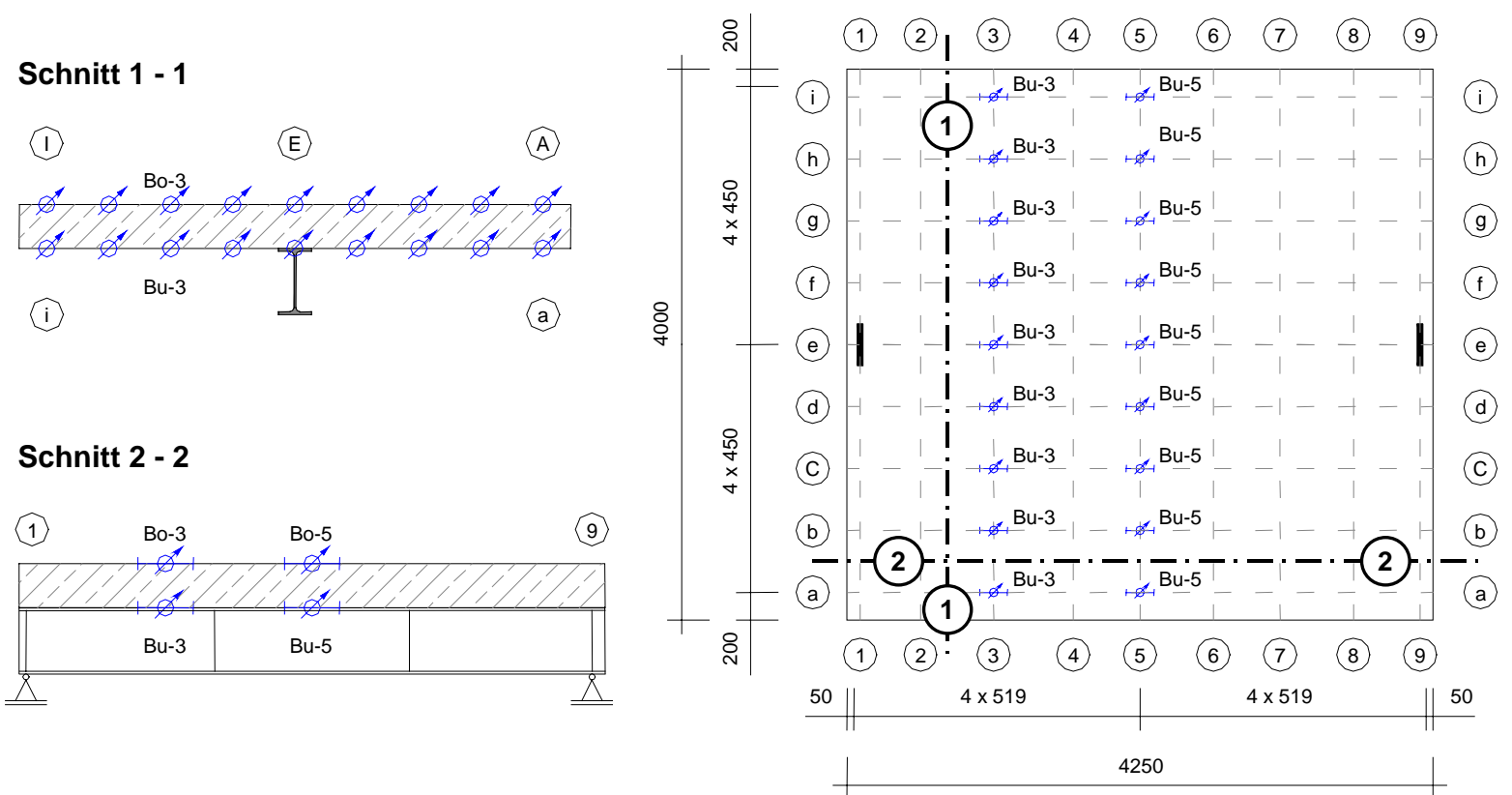

Abbildung 5-4: $\quad$ Messungen am Betongurt [2.27]

Tabelle 5-2: $\quad$ Werkstoffkennwerte des Betons [2.27]

\begin{tabular}{|c|c|c|c|c|c|c|c|c|}
\hline & \multirow{2}{*}{$\begin{array}{c}\text { Prüfalter } \\
\text { [Tage] }\end{array}$} & \multicolumn{3}{|c|}{ Istwerte $\left[\mathrm{N} / \mathrm{mm}^{2}\right]$} & \multicolumn{4}{|c|}{ Sollwerte $\left[\mathrm{N} / \mathrm{mm}^{2}\right]$} \\
\hline & & $f_{c}{ }^{1)}$ & $E_{c}{ }^{2)}$ & $f_{c t}{ }^{3)}$ & Güte & $f_{c}{ }^{1)}$ & $E_{c}{ }^{2)}$ & $f_{c t}{ }^{3)}$ \\
\hline VT 1 & 22 & 28,6 & 31529 & 2,0 & C 20/25 & 28 & 29000 & 2,2 \\
\hline VT 2 & 26 & 46,9 & 36108 & 3,1 & C $30 / 37$ & 38 & 32000 & 2,9 \\
\hline VT 3 & 49 & 36,8 & 33740 & 2,2 & C 20/25 & 28 & 29000 & 2,2 \\
\hline VT 4 & 26 & 31,0 & 32221 & 2,1 & C 20/25 & 28 & 29000 & 2,2 \\
\hline VT 5 & 36 & 39,2 & 34332 & 2,5 & C 20/25 & 28 & 29000 & 2,2 \\
\hline VT 6 & 40 & 39,2 & 34332 & 2,2 & C 30/37 & 38 & 32000 & 2,9 \\
\hline
\end{tabular}

\footnotetext{
${ }^{1)} f_{c} \quad$ Zylinderdruckfestigkeit des Betons

2) $E_{c} \quad$ Elastizitätsmodul des Betons

3) $\mathrm{f}_{\mathrm{ct}} \quad$ zentrische Zugfestigkeit des Betons
} 


\subsection{Werkstoffkennwerte}

Die am Tag der Versuchsdurchführung gemessenen Werkstoffwerte der Versuchsträger sind in Tabelle 5-2 und Tabelle 5-3 zusammengestellt. Die Prüfung der Betonprobewürfel (Kantenlänge $150 \mathrm{~mm})$ und Probeprismen $(100 \times 100 \times 500$ mm) erfolgte gemäß DIN 1048 [1.10]. Die Betonzugfestigkeit wurde anhand der Betonprismen mit einem Vierpunktbiegeversuch gemessen. Die Umrechnung der gemessenen Festigkeitswerte des Betons wurde nach [2.64] vorgenommen. Die Werkstoffkennwerte des Baustahls wurden gemäß DIN 10002-1 [1.11] ermittelt. Da alle Träger aus einer Charge stammten, stellen die Werte in Tabelle 5-3 die Mittelwerte der Proben dar. Entsprechend wurden die Werkstoffkennwerte der unteren Längsbewehrung bestimmt.

Tabelle 5-3: $\quad$ Werkstoffkennwerte des Baustahls und der Bewehrung [2.27]

\begin{tabular}{|c|c|c|c|c|c|c|c|c|c|}
\hline & \multicolumn{4}{|c|}{ Baustahl } & \multicolumn{5}{|c|}{ Bewehrung } \\
\hline & \multirow{2}{*}{ Güte } & $f_{y}$ & $E_{a}$ & $f_{u}$ & \multirow{2}{*}{ Güte } & $A_{s, 1}$ & $f_{p 02}$ & $E_{s}$ & $\mathbf{f}_{\mathrm{su}}$ \\
\hline & & {$\left[\mathrm{N} / \mathrm{mm}^{2}\right]$} & {$\left[\mathrm{N} / \mathrm{mm}^{2}\right]$} & {$\left[\mathrm{N} / \mathrm{mm}^{2}\right]$} & & {$\left[\mathrm{cm}^{2}\right]$} & {$\left[\mathrm{N} / \mathrm{mm}^{2}\right]$} & {$\left[\mathrm{N} / \mathrm{mm}^{2}\right]$} & {$\left[\mathrm{N} / \mathrm{mm}^{2}\right]$} \\
\hline VT 1 & \multirow{6}{*}{ S 235} & \multirow{6}{*}{334} & \multirow{6}{*}{204600} & \multirow{6}{*}{461} & \multirow{6}{*}{ S 500} & \multirow{3}{*}{10,05} & \multirow{3}{*}{547} & \multirow{3}{*}{198000} & \multirow{3}{*}{596} \\
\hline VT 2 & & & & & & & & & \\
\hline VT 3 & & & & & & & & & \\
\hline VT 4 & & & & & & 13,06 & 603 & 197000 & 648 \\
\hline VT 5 & & & & & & \multirow{2}{*}{15,71} & \multirow{2}{*}{562} & \multirow{2}{*}{203000} & \multirow{2}{*}{643} \\
\hline VT 5 & & & & & & & & & \\
\hline & $\begin{array}{l}f_{y} \\
E_{a} \\
f_{u}\end{array}$ & $\begin{array}{l}\text { Streckgren } \\
\text { Elastizitäts } \\
\text { Zugfestigk }\end{array}$ & $\begin{array}{l}\text { ze des Ba } \\
\text { nodul des } \\
\text { it des Bal }\end{array}$ & $\begin{array}{l}\text { stahls } \\
\text { 3austahls } \\
\text { tahls }\end{array}$ & $\begin{array}{l}A_{\mathrm{s}, 1} \\
f_{\mathrm{p} 02} \\
\mathrm{E}_{\mathrm{s}} \\
\mathrm{f}_{\mathrm{su}}\end{array}$ & $\begin{array}{l}\text { untere Län } \\
\text { Streckgrer } \\
\text { Elastizitäts } \\
\text { Zugfestigk }\end{array}$ & $\begin{array}{l}\text { sbewehru } \\
\text { e der Bew } \\
\text { odul der } \\
\text { t der Bew }\end{array}$ & $\begin{array}{l}\text { g } \\
\text { ehrung } \\
\text { ewehrung } \\
\text { hrung }\end{array}$ & \\
\hline
\end{tabular}

\subsection{Versuchsergebnisse}

\subsubsection{Trag- und Verformungsverhalten}

Alle Versuchsträger verhielten sich sehr duktil und das Versagen kündigte sich durch sehr große Verformungen an, siehe Abbildung 5-6 und Abbildung 5-7. Das Versagen trat bei allen Trägern im Bereich der Lasteinleitungspunkte auf.

Die Träger mit voller Verdübelung (das waren alle mit Ausnahme von VT 3) versagten durch Einschnüren der Betondruckzone und letztlich durch ein Druckversagen des Betons, siehe Abbildung 5-5. In Abbildung 5-6 bis Abbildung 5-9 sind die Last-Verformungs-Kurven der Versuchsträger dargestellt. In Tabelle 5-4 sind die Traglasten und die zugehörigen Mittendurchbiegungen der Träger sowie die Last-Verformungs-Werte im Grenzzustand der Gebrauchstauglichkeit angegeben. Ein Vergleich mit einer nichtlinearen Berechnung und der mittragenden Breite nach EN 1994-1-1 [1.1] zeigt, dass sich die Versuchsträger deutlich steifer verhielten als die Berechnung, siehe Abbildung 5-21 bis Abbildung 5-26. Die mittragende Breite ist somit größer als in EN 1994-1-1 angegeben. Die Berechnungen wurden mit

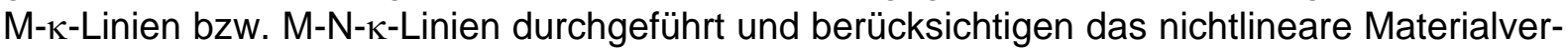
halten von Beton und Stahl, siehe Abschnitt 2.3.3. Der Endschlupf der Versuchsträger mit voller Verdübelung $(\eta=1,0)$ blieb bis zum Erreichen der Traglast kleiner als $0,8 \mathrm{~mm}$ und damit sehr klein, bis zum Erreichen der Streckgrenze des Baustahls kleiner als 0,02 mm. Es kann daher bei diesen Trägern von einem starren Verbund ausgegangen werden. 
Tabelle 5-4: $\quad$ Übersicht der wichtigsten Versuchsergebnisse

\begin{tabular}{|c|c|c|c|c|c|c|c|c|c|c|}
\hline & \multicolumn{5}{|c|}{ Kraftwerte } & \multicolumn{2}{|c|}{ Verformungen } & \multicolumn{2}{|c|}{$\mathbf{b}_{\mathbf{m}} / \mathbf{b}^{1)}$} & \multirow{2}{*}{\begin{tabular}{|c|}
$\mathbf{b}_{\mathbf{m}, \mathbf{v}} / \mathbf{b}^{2)}$ \\
$\mathbf{G Z G}{ }^{9)}$
\end{tabular}} \\
\hline & $\mathbf{P}_{\mathbf{u}}{ }^{3)}$ & $P_{G Z T}{ }^{4)}$ & $P_{G Z G}{ }^{5)}$ & $P_{\text {Riss }}{ }^{6)}$ & $\mathbf{P}_{\mathbf{y}}{ }^{7)}$ & $f\left(P_{u}\right)$ & $f\left(P_{G Z G}\right)$ & $\mathbf{G Z T}^{8)}$ & $\mathbf{G Z G}^{9)}$ & \\
\hline & {$[\mathrm{kN}]$} & {$\left[\cdot P_{u}\right]$} & {$\left[\cdot P_{\mathrm{u}}\right]$} & {$\left[\cdot P_{u}\right]$} & {$\left[\cdot P_{\mathrm{u}}\right]$} & [mm] & {$[\mathrm{mm}]$} & {$[-]$} & {$[-]$} & {$[-]$} \\
\hline VT 1 & 554,9 & 0,80 & 0,54 & 0,24 & 0,53 & 144,6 & 10,1 & 0,73 & 0,84 & 0,35 \\
\hline VT 2 & 586,9 & 0,77 & 0,52 & 0,12 & 0,64 & 121,6 & 8,6 & 0,75 & 0,78 & 0,44 \\
\hline VT 3 & 401,9 & 0,87 & 0,51 & 0,25 & 0,68 & 76,1 & 7,0 & 0,91 & 0,86 & 0,73 \\
\hline VT 4 & 648,2 & 0,73 & 0,48 & 0,09 & 0,53 & 156,0 & 8,9 & 0,76 & 0,81 & 0,61 \\
\hline VT 5 & 974,2 & 0,75 & 0,51 & 0,10 & 0,52 & 129,2 & 8,0 & 0,85 & 0,88 & 0,64 \\
\hline VT 6 & 992,9 & 0,74 & 0,50 & 0,07 & 0,52 & 128,4 & 8,0 & 0,81 & 0,81 & 0,57 \\
\hline
\end{tabular}

${ }^{1)} b_{m} / b$ bezogener Wert der spannungsbezogenen mittragenden Breite $b_{m}$

2) $b_{m, v} / b$ bezogener Wert der verformungsbezogenen mittragenden Breite $b_{m, v}$

3) $\mathrm{Pu}_{\mathrm{u}} \quad$ Bruchlast

4) $P_{\text {GZT }} \quad$ Last im Grenzzustand der Tragfähigkeit

5) PGzG Last im Grenzzustand der Gebrauchstauglichkeit, siehe Abschnitt 5.2

6) $P_{\text {Riss }}$ Last bei Rissbeginn im Beton

7) $P_{y} \quad$ Last bei beginnender Plastizierung des Stahlträgers

8) GZT Grenzzustand der Tragfähigkeit, siehe Abschnitt 5.2

9) GZG Grenzzustand der Gebrauchstauglichkeit, siehe Abschnitt 5.2

Beim Versuchsträger VT 3 mit einem planmäßigen Verdübelungsgrad $\eta=0,4$ wurde das Versagen durch ein Abscheren der Kopfbolzendübel an einem Auflager eingeleitet. Bei weiter steigender Verformung kam es dann zu einem Druckversagen des Betons im Bereich der Lasteinleitung. Der Endschlupf bei Erreichen der Last im Grenzzustand der Gebrauchstauglichkeit betrug ca. 0,4 mm. Bei Erreichen der Traglast betrug dieser ca. 8,3 mm.

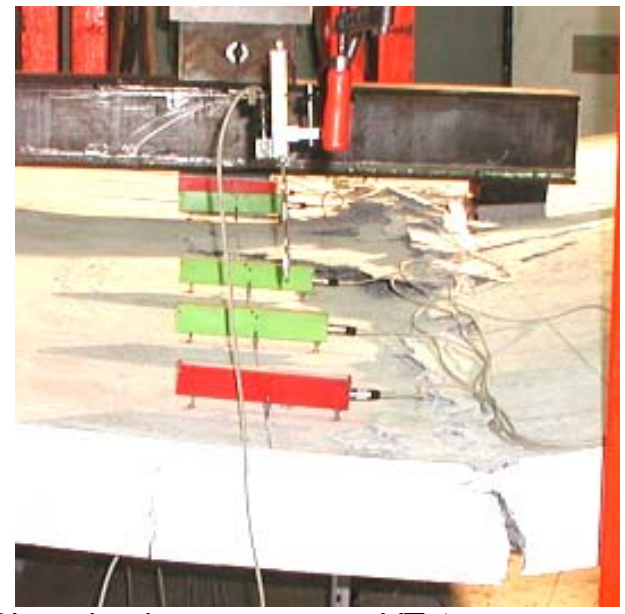

(a) Biegedruckversagen von VT 1

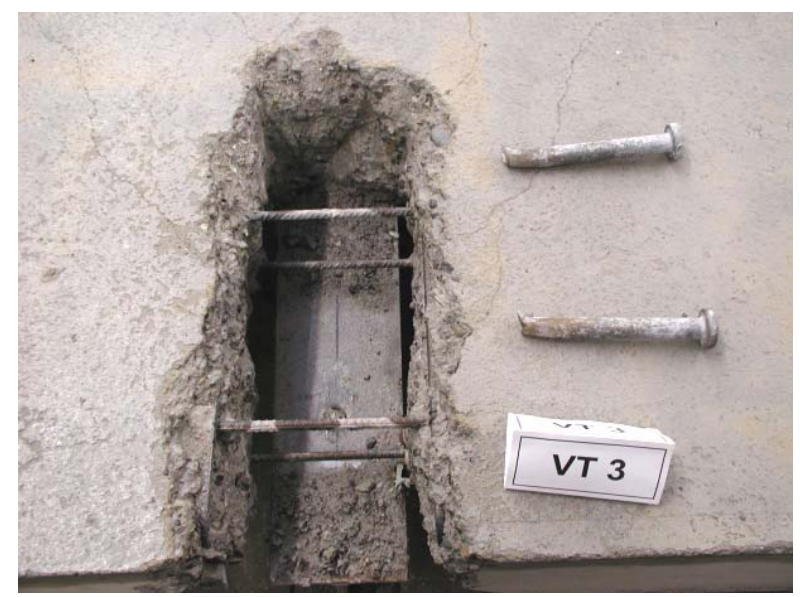

(b) abgescherte Dübel von VT 3

Abbildung 5-5: Versagen der Versuchsträger [2.27]

Bei allen Versuchsträgern nahm mit zunehmender Verformung des Trägers und zunehmender Rissbildung im Betongurt die Steifigkeit der Träger ab. Nach Erreichen der Streckgrenze des Stahlträgerobergurts bildeten sich ein oder zwei klaffende Querrisse in der Regel in der Achse der Lasteinleitungspunkte. In diesen Rissen kam es dann zum Abreißen von Stäben der unteren Längsbewehrung, was von einem deutlich hörbaren Knall begleitet wurde, und schließlich zum Bruch. 


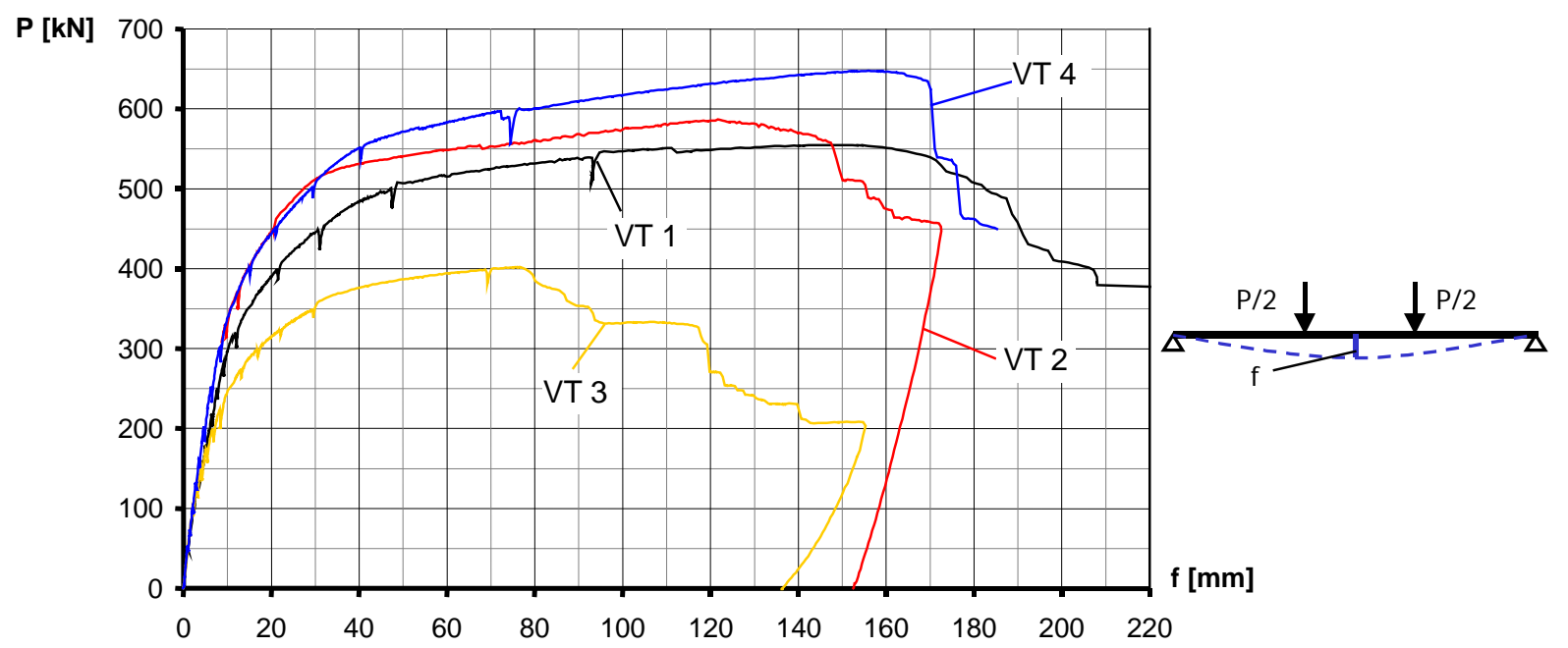

Abbildung 5-6: Last-Verformungs-Kurve der Versuchsträger VT 1, VT 2, VT 3 und VT 4 [2.27]

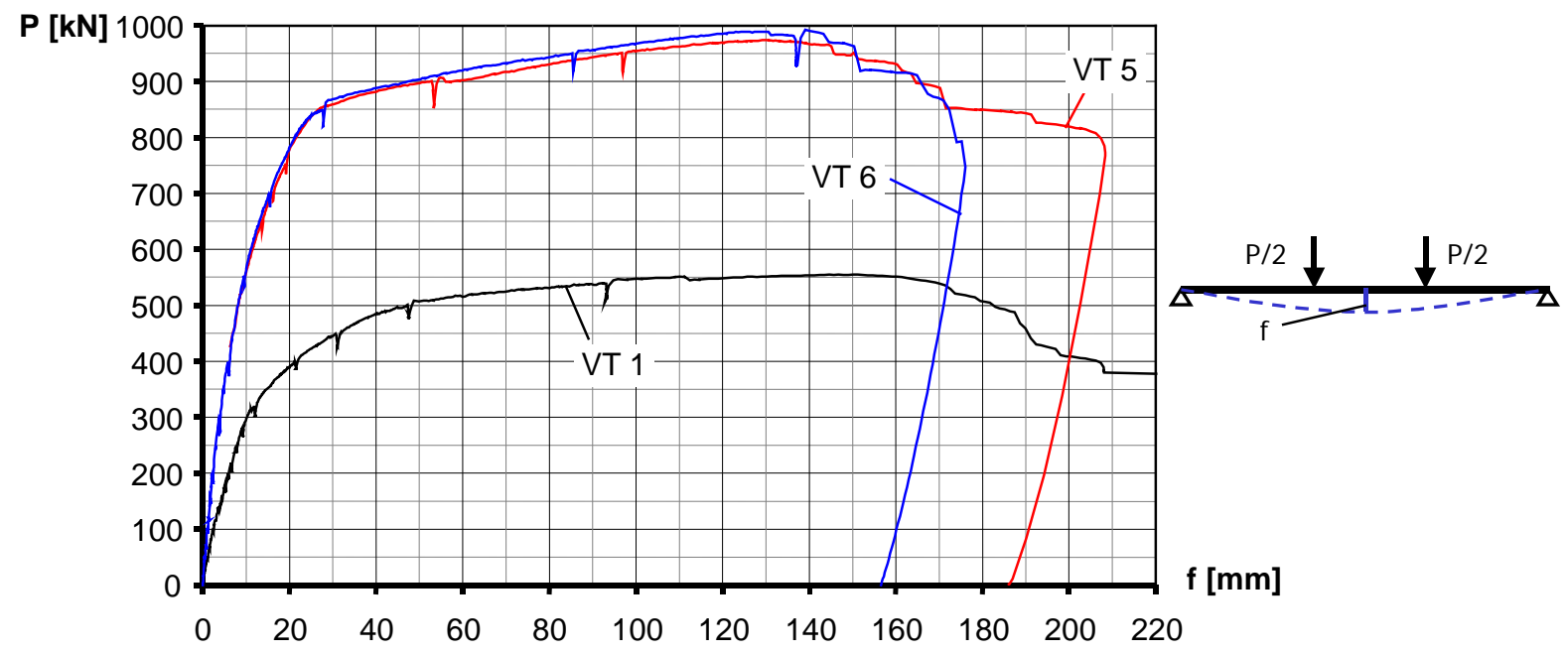

Abbildung 5-7: $\quad$ Last-Verformungs-Kurve der Versuchsträger VT 1, VT 5 und VT 6 [2.27]

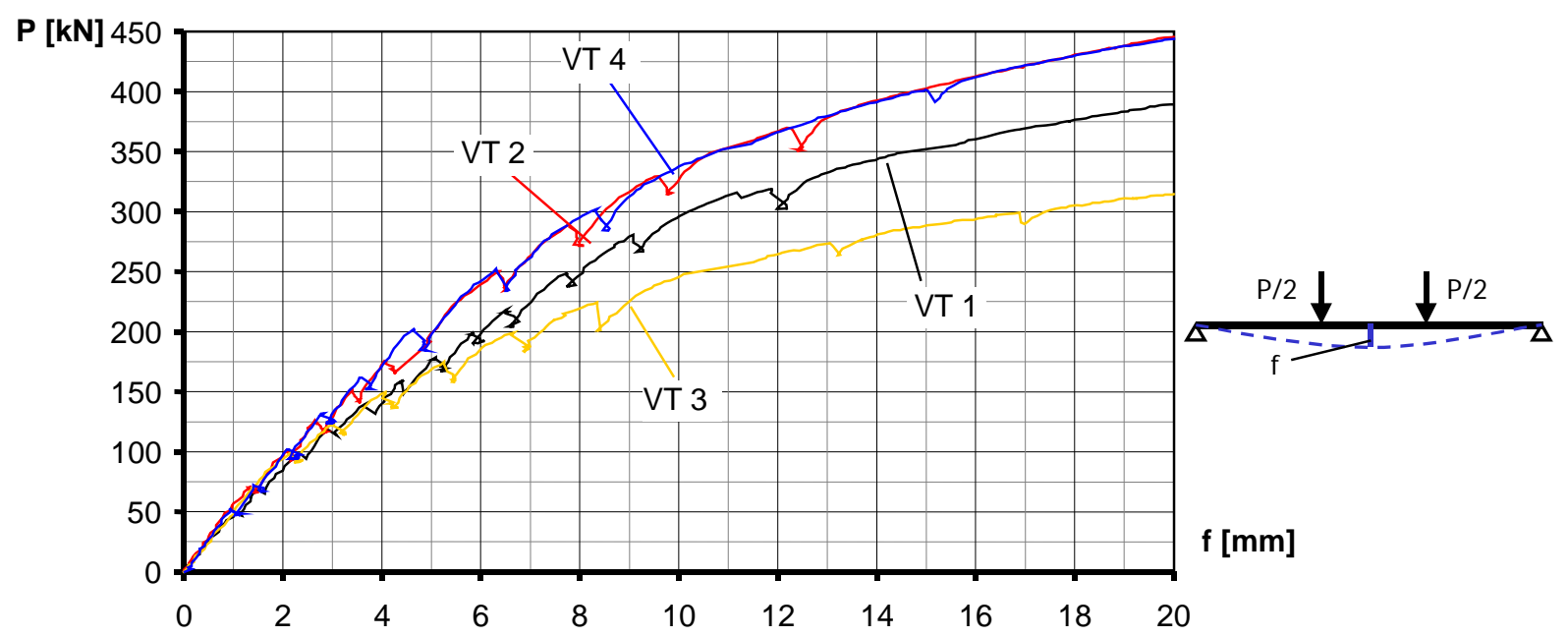

Abbildung 5-8: Last-Verformungs-Kurve der Versuchsträger VT 1, VT 2, VT 3 und VT 4 [2.27] (Anfangsbereich vergrößert dargestellt) 


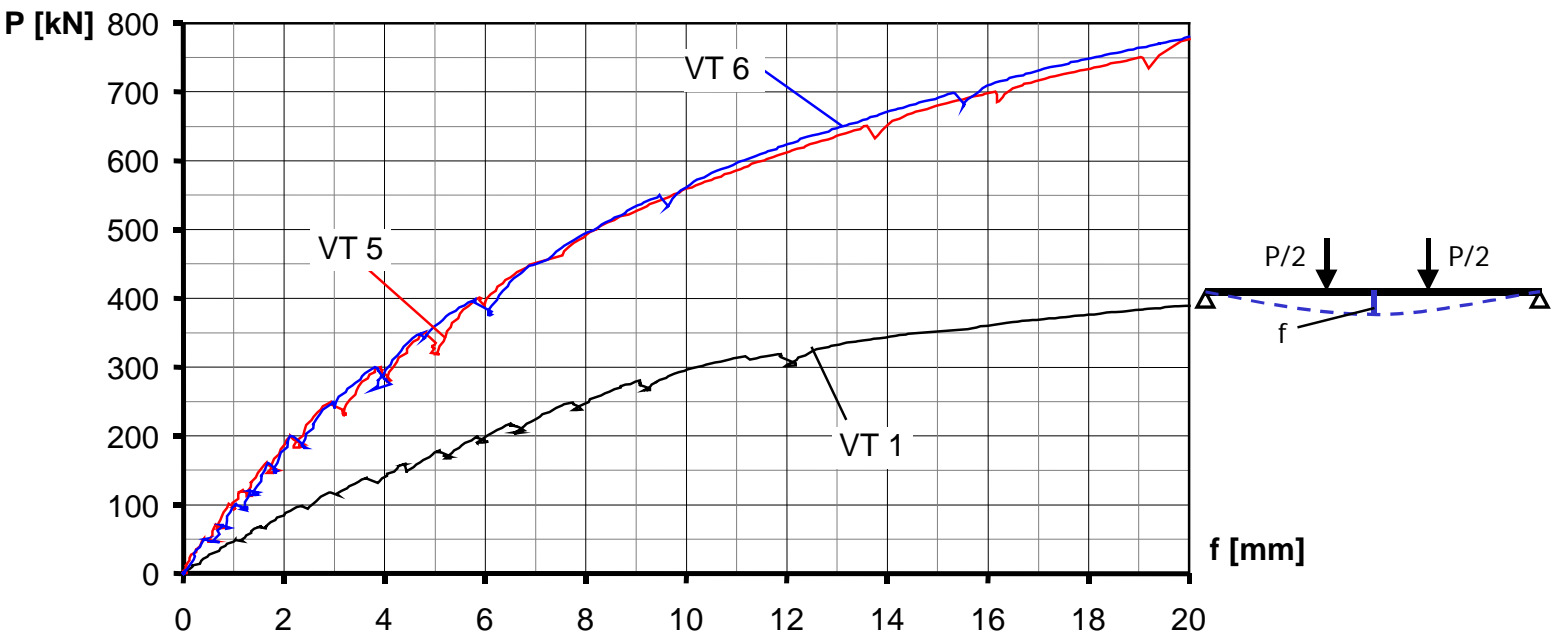

Abbildung 5-9: Last-Verformungs-Kurve der Versuchsträger VT 1, VT 5 und VT 6 [2.27] (Anfangsbereich vergrößert dargestellt)

Die in Abbildung 5-6 bis Abbildung 5-9 dargestellten Last-Verformungs-Kurven berücksichtigen nur die Pressenlast. Auf Grund der kurzen Versuchsdauer kann ein Einfluss von Kriechen und Schwinden des Betons auf das Last-Verformungs-Verhalten der Versuchsträger ausgeschlossen werden.

\subsubsection{Rissbildung im Betongurt}

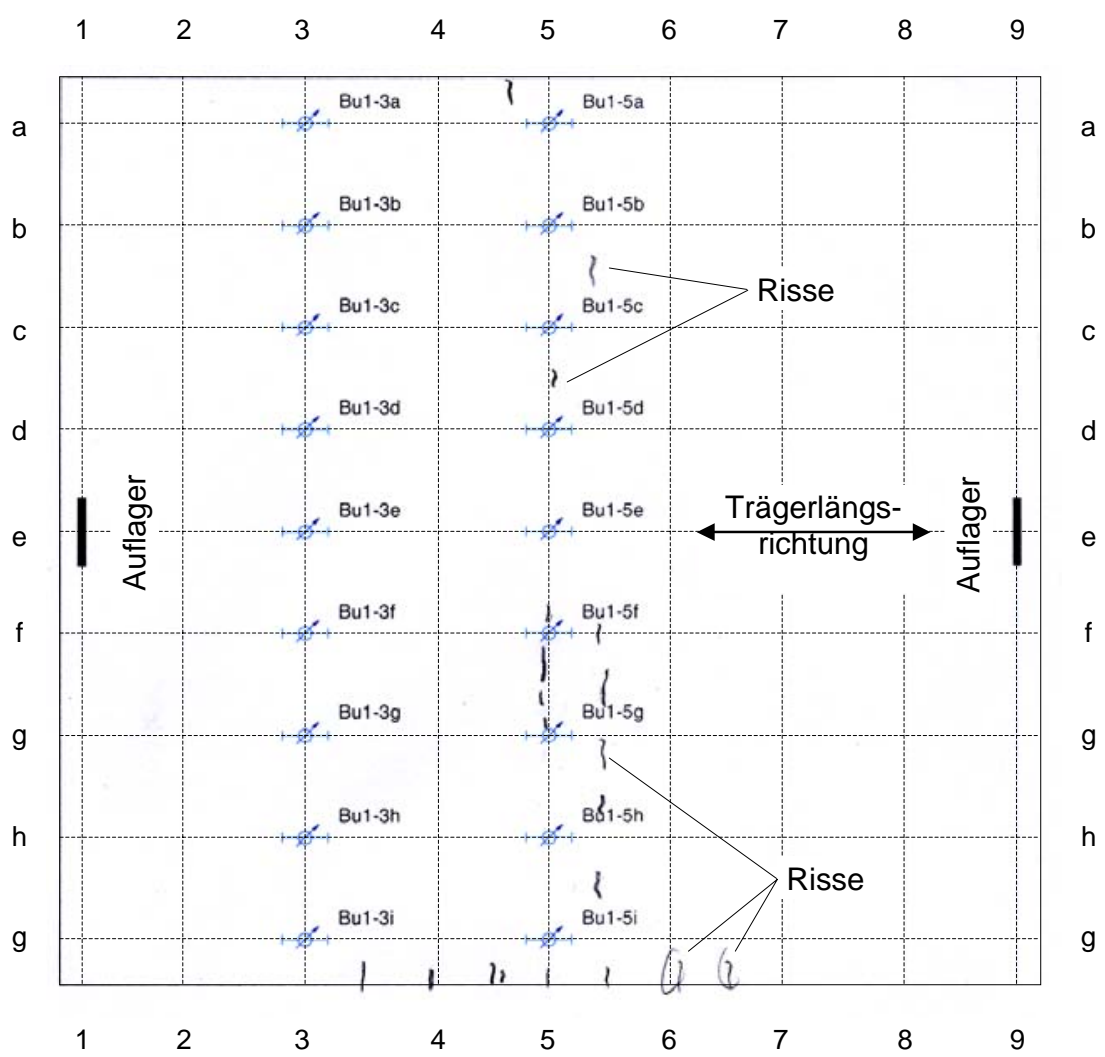

Abbildung 5-10: Beginnende Rissbildung an der Unterseite des Versuchsträgers VT 6 $\left(P \approx 0,07 \cdot P_{u}\right)[2.27]$ 
An den Unterseiten der Versuchsträger waren Risse schon bei sehr niedrigen Lasten $\left(0,07 \div 0,25 \cdot P_{u}\right)$ erkennbar. Bei den Versuchsträgern mit dickem Betongurt $h_{c}=26 \mathrm{~cm}$ startete die Rissbildung bei einer vergleichsweise geringeren Belastung $\left(P_{\text {Riss }} \approx 0,07 \cdot P_{u}\right)$ als bei den Trägern mit dünnem Gurt $h_{c}=16 \mathrm{~cm}\left(P_{\text {Riss }} \approx 0,13 \div 0,25 \cdot P_{u}\right)$.

Die Risse verliefen nahezu orthogonal zur Trägerlängsrichtung. Die ersten Anrisse zeigten sich bei fast allen Trägern am äußeren Rand des Betongurts. Diese waren jedoch nur wenige Zentimeter lang. Die weiteren Risse bildeten sich zwischen den Lasteinleitungspunkten (Querachse 4 bis 6) in der Regel zwischen dem Stahlträger und dem Rand des Betongurts. Mit zunehmender Laststeigerung bildeten sich weitere Risse etwa im Abstand der Querbewehrung. Im Bereich zwischen den Lasteinleitungspunkten lag bereits vor Erreichen der Last im Grenzzustand der Gebrauchstauglichkeit und der Streckgrenze des Baustahls ein vollständiges Rissbild vor. Nach Erreichen der Streckgrenze des Stahlträgerobergurts und der Abnahme der Trägersteifigkeit begannen sich die Risse sichtbar zu öffnen. Mit zunehmender Verformung des Trägers bildeten sich dann ein bis zwei klaffende Risse in der Achse eines der Lasteinleitungspunkte.

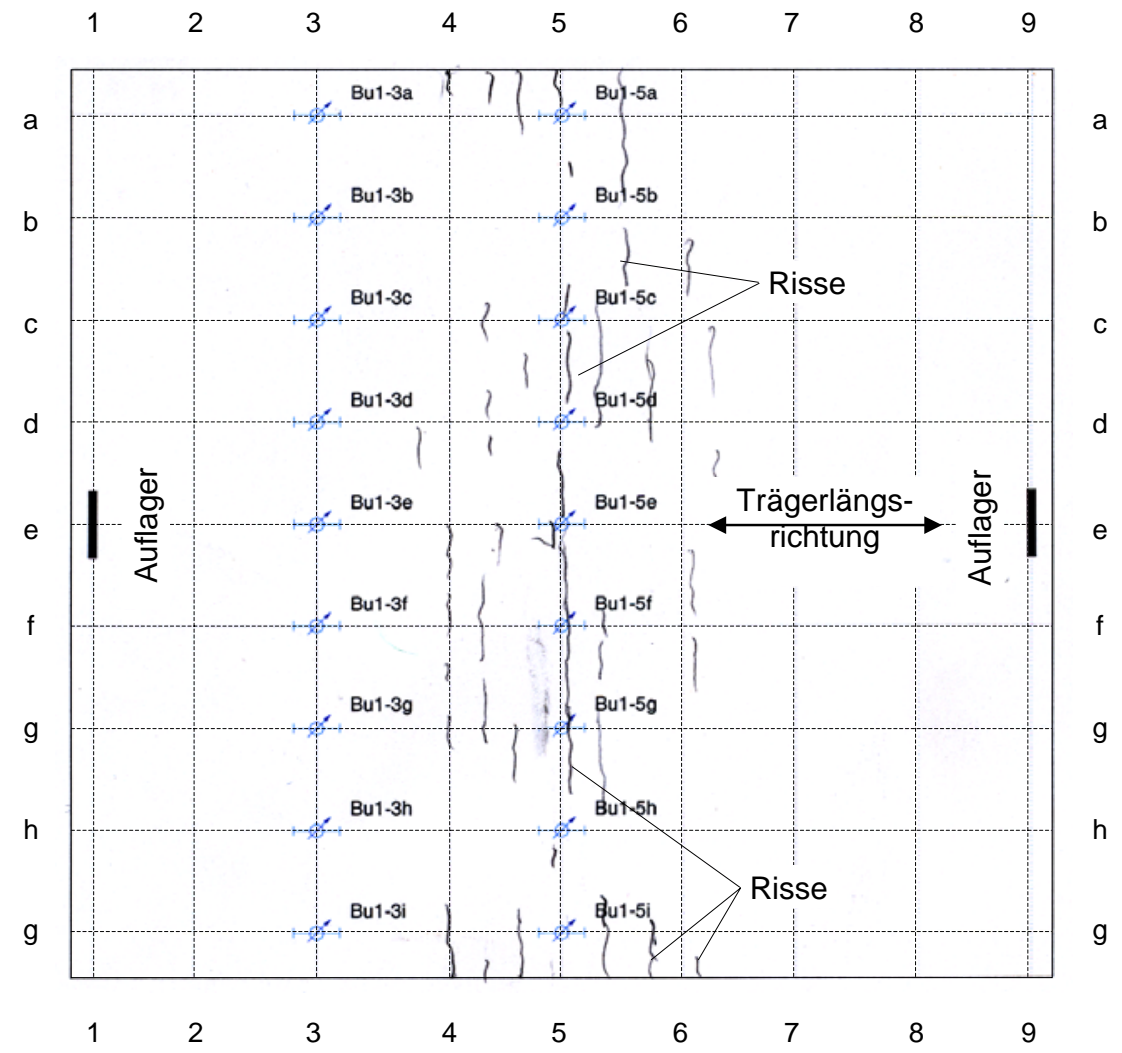

Abbildung 5-11: Rissbildung an der Unterseite des Versuchsträgers VT $6 \quad\left(P \approx 0,12 \cdot P_{u}\right)$ [2.27]

An den Trägerenden bildeten sich bei Lasten $P \approx 0,2 \div 0,4 \cdot P_{u}$ Schubrisse an den Auflagern, die durch die Einleitung der Längsschubkraft entstanden. Diese Risse verliefen an der Oberseite des Betongurts ausgehend vom Stahlträger diagonal in Richtung der Betondruckstreben.

\subsubsection{Betondehnungen}

Während der Versuchsdurchführung wurden die Betondehnungen an der Ober- und Unterseite des Betongurts sowie deren Verteilung in Querrichtung des Betongurts gemessen. Die 
Messungen wurden in Achse 5 (Trägermitte) und Achse 3 (Viertelpunkt) vorgenommen, siehe Abbildung 5-4.

Für die im weiteren diskutierten Betondehnungen gilt, dass es sich hierbei um über die Messlänge gemittelte Längenänderungen handelt und nicht um lokale Dehnungen. Im Druckbereich an der Oberseite des Betongurts kann mit ausreichender Näherung davon ausgegangen werden, dass die gemessene mittlere Längenänderung hinreichend genau der lokalen Dehnung entspricht. Dies gilt insbesondere deshalb, da zwischen den Lasteinleitungspunkten das äußere Moment nahezu konstant ist. Gleichermaßen gilt dies auch für den Zugbereich an der Unterseite des Betongurts im Zustand I. Im gerissenen Zustand hängt die gemessene mittlere Längenänderung an der Unterseite jedoch sehr stark davon ab, ob im Bereich der Messlänge ein oder evtl. mehrere Risse vorhanden sind. Die Bestimmung der lokalen Dehnung des Betons oder der unteren Längsbewehrung ist damit nicht möglich. Die Messung kann hier nur eine qualitative Aussage geben. Im Weiteren wird statt des Begriffs „mittlere Längenänderung“ der Begriff „Dehnung“ verwendet.

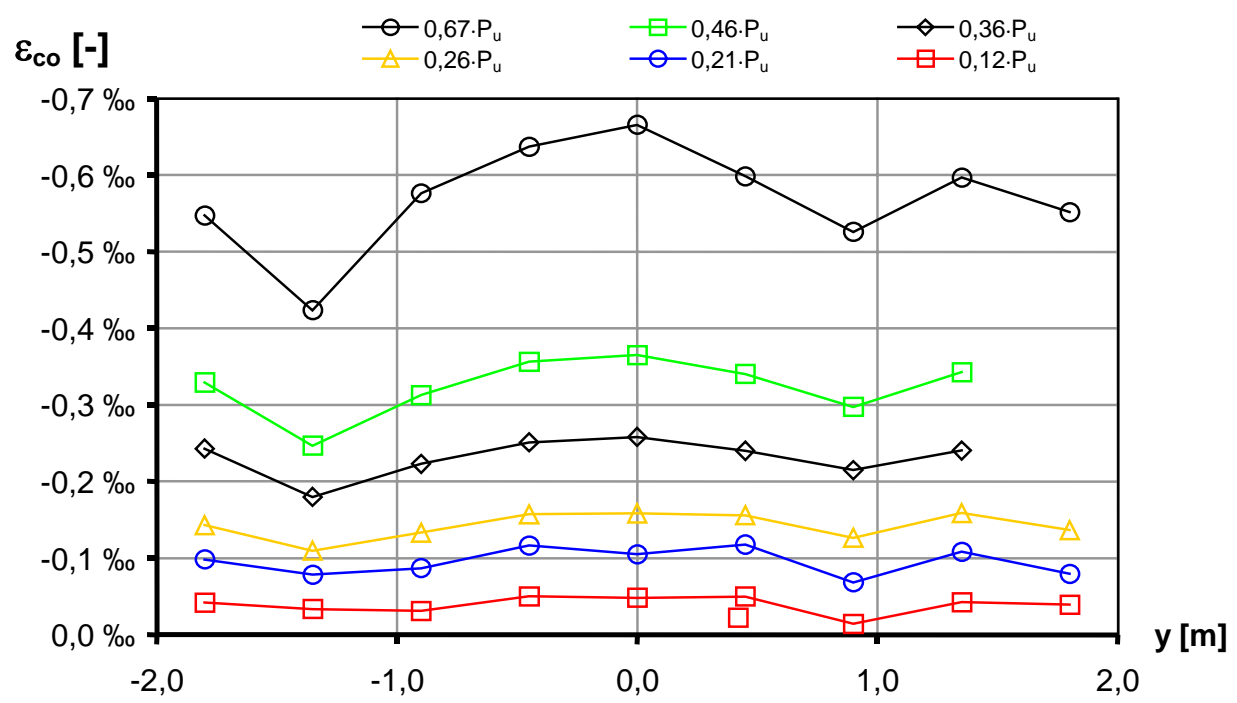

(a) Oberseite des Betongurts (Druckzone)

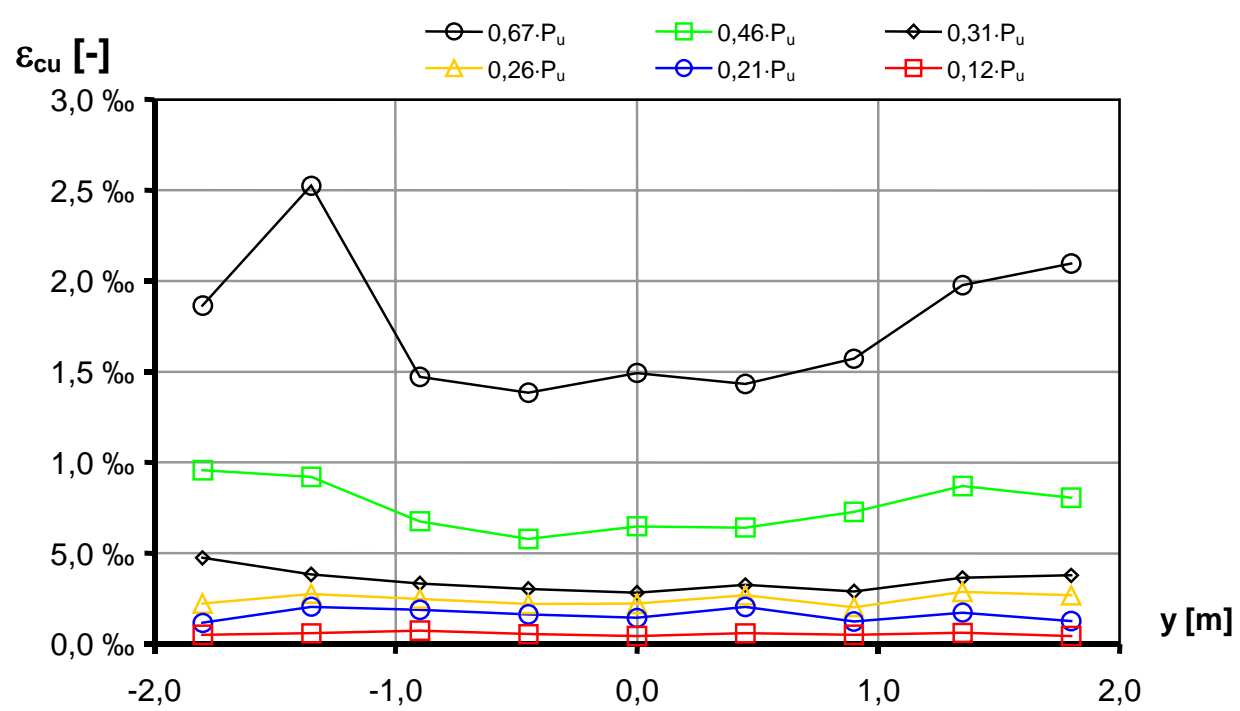

(b) Unterseite des Betongurts (Zugzone)

Abbildung 5-12: Qualitative Querverteilung der Betondehnungen des Versuchsträgers VT 5 in Trägermitte (Achse 5) [2.27] 
Abbildung 5-12 zeigt beispielhaft die Querverteilung der Betondehnungen des Versuchsträgers VT 5 an der Ober- und Unterseite in Trägermitte für verschiedene Laststufen. An der Oberseite des Betongurts ergibt sich eine glockenförmige Verteilung der Betondehnungen mit ihrem Maximalwert in der Stegebene des Stahlträgers. Der Abfall der Dehnungen zur Außenseite des Betongurts ist jedoch deutlich schwächer, als dies elastische Untersuchungen erwarten lassen. Insgesamt ist die Dehnungsverteilung an der Oberseite vergleichsweise ausgeglichen.

An der Betonunterseite zeigt sich ein anderes Bild, siehe Abbildung 5-12 (b). Für kleine Lasten ist die Dehnungsverteilung auch hier recht ausgeglichen. Mit zunehmender Belastung und fortschreitender Rissbildung weicht die Dehnungsverteilung jedoch stark von der an der Oberseite ab. Ausgehend vom Stahlträger werden die Dehnungen hin zur Außenseite des Betongurts größer. Dies ist eine grundlegend andere Verteilung als zunächst erwartet. Der Grund für diese Dehnungsverteilung ist, dass die Querverteilung des Betonmoments ausgeglichener ist als die der Betondruckkraft, siehe Kapitel 4. Dadurch werden die vorhandenen Querrisse nahe am Stahlträger stärker überdrückt als an der Außenseite und die Rissöffnung ist außen größer als am Stahlträger.

Aus der Betrachtung der Betondehnungen an der Ober- und Unterseite lässt sich schließen, dass die tatsächliche mittragende Breite des Betongurts nennenswert größer ist, als dies die aktuell gültigen Normen und in der Literatur beschriebene elastische Untersuchungen angeben. Dies zeigen auch die folgenden Auswertungen.

\subsubsection{Mittragende Breite des Betongurts}

\subsubsection{Grundsätzliches}

Im Folgenden wird für die Versuchsträger sowohl die spannungsbezogene mittragende Breite als auch die verformungsbezogene mittragende Breite ausgewertet.

\subsubsection{Spannungsbezogene mittragende Breite $b_{m}$}

Aus den gemessenen Betondehnungen kann mit Kenntnis der Spannungs-Dehnungs-Linie des Betons die spannungsbezogene mittragende Breite $b_{m}$ der Versuchsträger berechnet werden. Hierfür wird die in EN 1992-1-1, 4.2.1.3.2 [1.3] gegebene Spannungs-DehnungsBeziehung verwendet, siehe Abbildung 5-13. Für kleine Betonspannungen $\left(\sigma_{\mathrm{c}}<0,4 \cdot f_{\mathrm{ck}}\right)$ kann in ausreichender Genauigkeit von einem linearen Spannungs-Dehnungs-Verhalten des Betons im Druckbereich ausgegangen werden. In diesem Bereich können die gemessenen Betondehnungen direkt zur Bestimmung der mittragenden Breite verwendet werden.

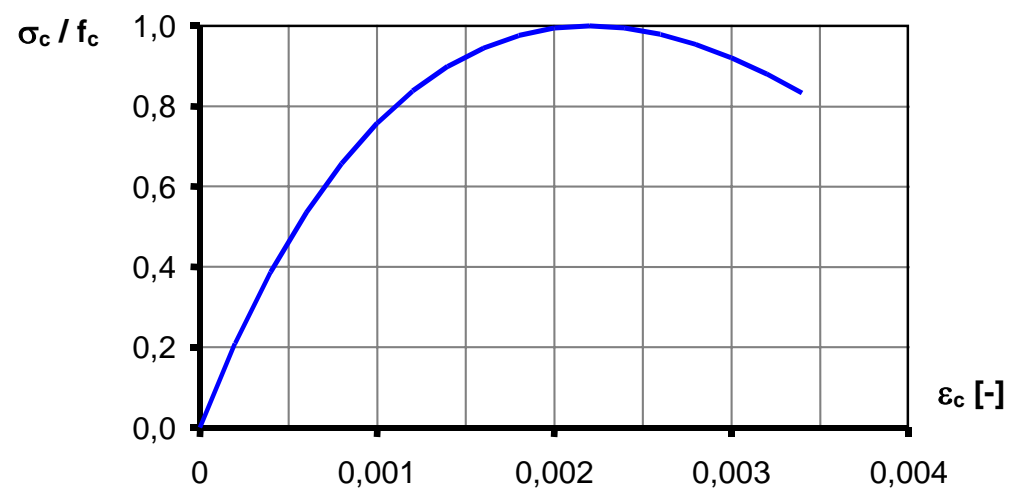

Abbildung 5-13: Idealisierte $\sigma-\varepsilon-L i n i e$ für Beton, EN 1992-1-1, 4.2.1.3.2 [1.3] 
Abbildung 5-14 bis Abbildung 5-16 zeigen die mittragende Breite der Betondruckspannungen an der Oberseite des Betongurts der Versuchsträger in Abhängigkeit der bezogenen Versuchslast $\mathrm{P} / \mathrm{P}_{\mathrm{u}}$. Im Bereich der Rissbildung zeigt sich eine Abhängigkeit der mittragenden Breite von der Belastung. Mit zunehmender Belastung und Rissbildung nimmt die mittragende Breite zu. Durch die Rissbildung kommt es zu einer Umverteilung der Spannungen im Beton und einer Vergrößerung der mittragenden Breite.

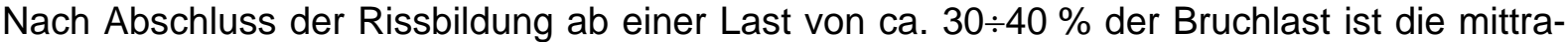
gende Breite nahezu unabhängig von der Belastungshöhe und pendelt sich bei allen Versuchsträgern bei ca. $b_{m} / b \approx 0,8 \div 0,9$ ein. Im Grenzzustand der Gebrauchstauglichkeit beträgt die bezogene mittragende Breite $b_{m} / b$ der Versuchsträger ca. 0,8 zu. Die mittragende Breite ist damit um ein Vielfaches größer als der Wert gemäß EN 1994-1-1 von 0,26 bzw. DIN 1045-1 von 0,41.

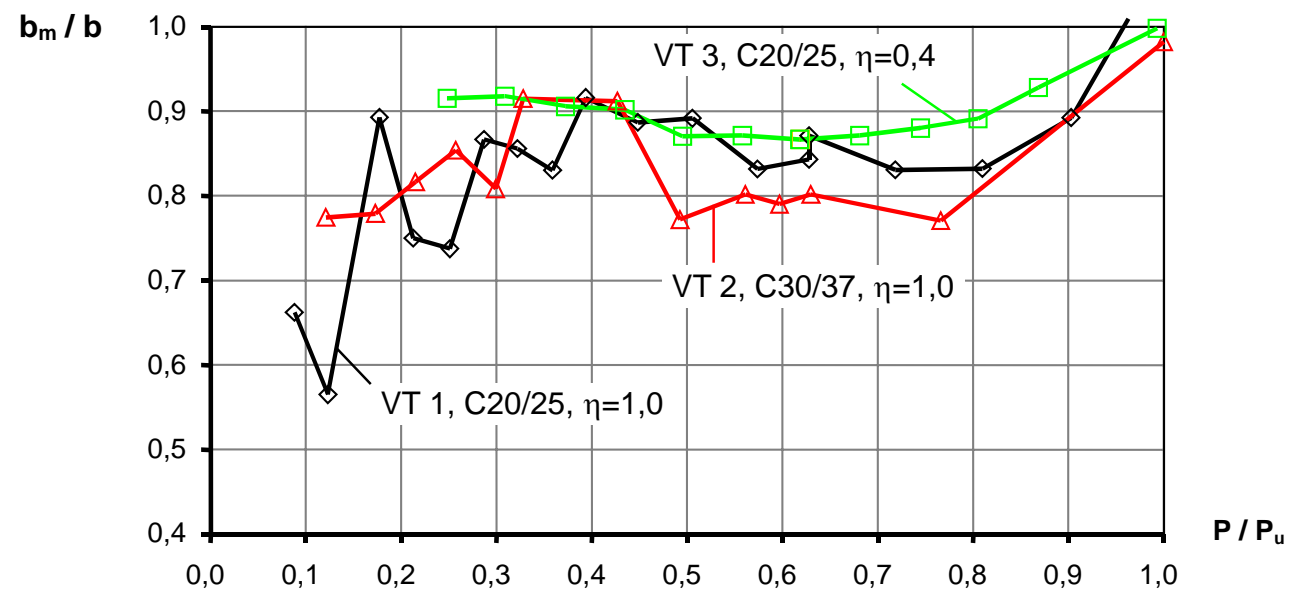

Abbildung 5-14: Mittragende Breite $b_{m}$ der Betondruckspannungen an der Oberseite des Betongurts in Feldmitte der Versuchsträger VT 1, VT 2, VT 3 [2.27]

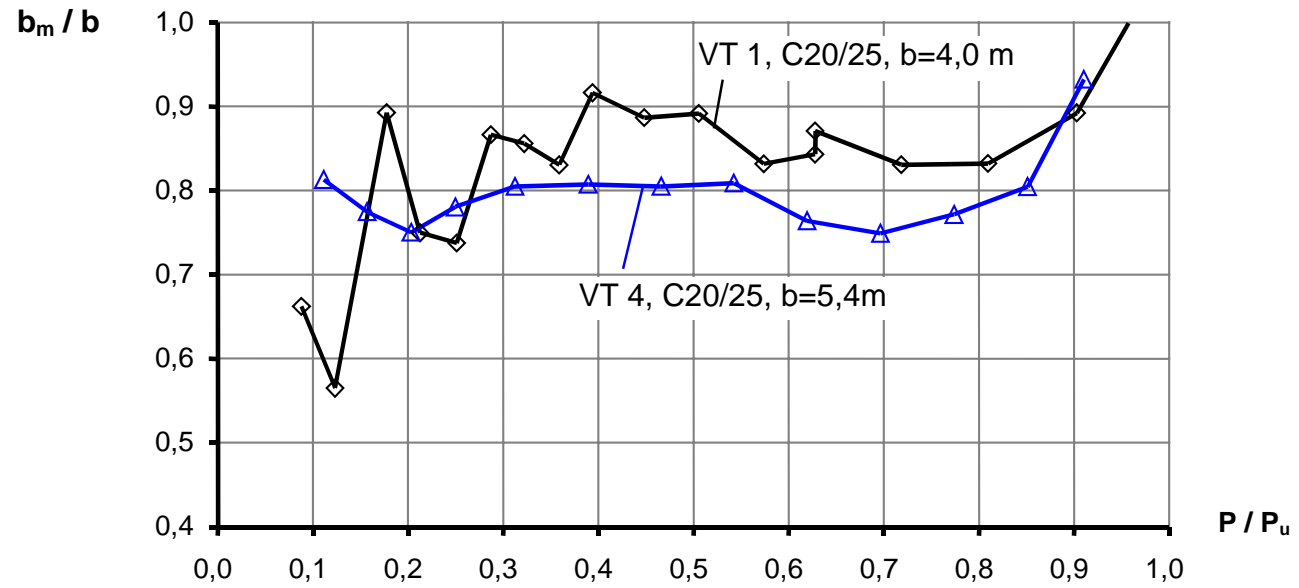

Abbildung 5-15: Mittragende Breite $b_{m}$ der Betondruckspannungen an der Oberseite des Betongurts in Feldmitte der Versuchsträger VT 1 und VT 4 [2.27]

Kurz vor Erreichen der Traglast erhöht sich die spannungsbezogene mittragende Breite bei allen Versuchsträgern durch die Einschnürung der Druckzone und erreicht nahezu die vorhandene Breite. 
Die Rissbildung führt zu einer Umverteilung der Spannungen im Beton, da das Biegemoment des Betongurts kleiner wird und sich das innere Kräftepaar im Verbundquerschnitt entsprechend vergrößert. Durch die größere Betondruckkraft und die infolge der Rissbildung niedrigere Druckzonenhöhe ergeben sich größere Betondruckspannungen. Da die SpannungsDehnungs-Linie des Betons für größere Spannungen flacher wird, ergibt sich für größere Betonspannungen eine ausgeglichenere Querverteilung der Spannungen, siehe Abbildung 5-13, und damit eine größere mittragende Breite. Im Bereich der abgeschlossenen Rissbildung zeigen die Versuchsträger folgendes Verhalten:

- Die mittragende Breite $b_{m}$ ist nahezu unabhängig von der Dicke des Betongurts $h_{c}$.

- Die Betongüte zeigt keinen einheitlichen Einfluss auf die mittragende Breite $b_{m}$.

- Der Verdübelungsgrad $\eta$ zeigt keinen Einfluss auf die mittragende Breite $b_{m}$.

Nach Abschluss der Rissbildung ist der Biegeanteil des Betongurts nahezu vollständig verschwunden, so dass der Betongurt „nur noch“ als Scheibe trägt. Wie bereits elastische Untersuchungen zeigen, hat die Dicke des Betongurt in diesem reinen Scheibenzustand keinen Einfluss auf die mittragende Breite, siehe Abschnitt 4.2. Dies wird bestätigt durch einen Vergleich der Ergebnisse des Versuchsträgers VT $1\left(C\right.$ 20/25, $\left.\mathrm{h}_{\mathrm{c}}=16 \mathrm{~cm}\right)$ mit VT 5 (C 20/25, $\mathrm{h}_{\mathrm{c}}=26 \mathrm{~cm}$ ), die bis auf die unterschiedliche Plattendicke identisch sind, bzw. durch Vergleich der Ergebnisse von VT $2\left(\mathrm{C} \mathrm{30/37,} \mathrm{h}_{\mathrm{c}}=16 \mathrm{~cm}\right)$ mit VT $6\left(\mathrm{C} \mathrm{30/37,} \mathrm{h}_{\mathrm{c}}=26 \mathrm{~cm}\right)$, siehe Abbildung 5-16. Im Bereich der abgeschlossenen Rissbildung ist die mittragende Breite dieser Träger etwa gleich groß. Im Bereich der Rissbildung zeigen die Ergebnisse jedoch, dass die größere Plattendicke der Träger VT 5 und VT 6 zu einer größeren mittragenden Breite führt.

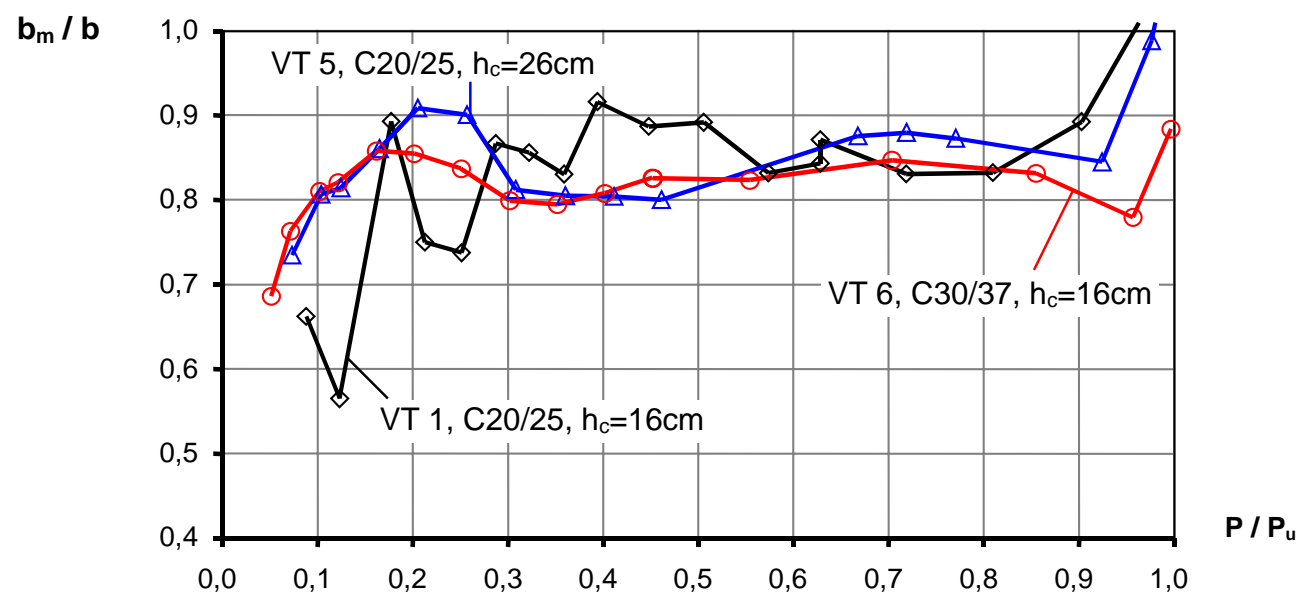

Abbildung 5-16: Mittragende Breite $b_{m}$ der Betondruckspannungen an der Oberseite des Betongurts in Feldmitte der Versuchsträger VT 1, VT 5, VT 6 [2.27]

Wider Erwarten zeigen die Betongüte und die Betonzugfestigkeit keinen eindeutigen Einfluss auf die mittragende Breite der Versuchsträger. Dies ist durch einen Vergleich der Ergebnisse des Versuchsträgers VT $1\left(C 20 / 25, h_{c}=16 \mathrm{~cm}\right)$ mit VT $2\left(C 30 / 37, h_{c}=16 \mathrm{~cm}\right)$, die bis auf eine unterschiedliche Betongüte völlig identisch sind, bzw. des Trägers VT 5 (C 20/25, $\left.\mathrm{h}_{\mathrm{c}}=26 \mathrm{~cm}\right)$ mit VT $6\left(\mathrm{C} \mathrm{30/37}, \mathrm{h}_{\mathrm{c}}=26 \mathrm{~cm}\right)$ zu erkennen, siehe Abbildung 5-14 und Abbildung 5-16. Dabei gleichen sich offensichtlich folgende beiden Einflüsse gegenseitig aus: Zum einen kommt es durch die geringere Betonzugfestigkeit bereits unter einer geringeren Belastung zur Rissbildung im Beton, wodurch die mittragende Breite gemäß den obigen Überlegungen größer werden sollte. Auf der anderen Seite führt die frühere Rissbildung zu einem früheren Verlust der Biegetragwirkung des Betongurts, was, gemäß elastischer Überlegungen, zu einer Abnahme der mittragenden Breite führt, siehe Abbildung 5-17 und Abschnitt 4.3. Durch die kleinere Dicke des Restquerschnitts am Riss nimmt die Biegetragwirkung des 
Betongurts im Vergleich zur Scheibentragwirkung überproportional ab. Dadurch ergibt sich bei einer rein elastischen Betrachtung eine geringere mittlere mittragende Breite, die sich mit zunehmender Risstiefe dem Grenzfall des reinen Scheibenzustands annähert.

Im Bereich der abgeschlossenen Rissbildung hat die Betonzugfestigkeit erwartungsgemäß keinen Einfluss auf das Tragverhalten des Betongurts und damit auf die mittragende Breite.

Der Einfluss des Verdübelungsgrads $\eta$ lässt sich in gleicher Weise erklären. Zu Beginn der Belastung ist die mittragende Breite des teilverdübelten Versuchsträgers VT 3 größer als des entsprechenden voll verdübelten Trägers VT 1, da durch den geringen Verdübelungsgrad die Biegetragwirkung des Betongurts einen relativ größeren Einfluss auf das Tragverhalten des Verbundträgers hat. Nach abgeschlossener Rissbildung trägt der Betongurt jedoch auch bei Trägern mit geringem Verdübelungsgrad nur im Scheibenzustand. Die mittragende Breite ist dann unabhängig vom Verdübelungsgrad.
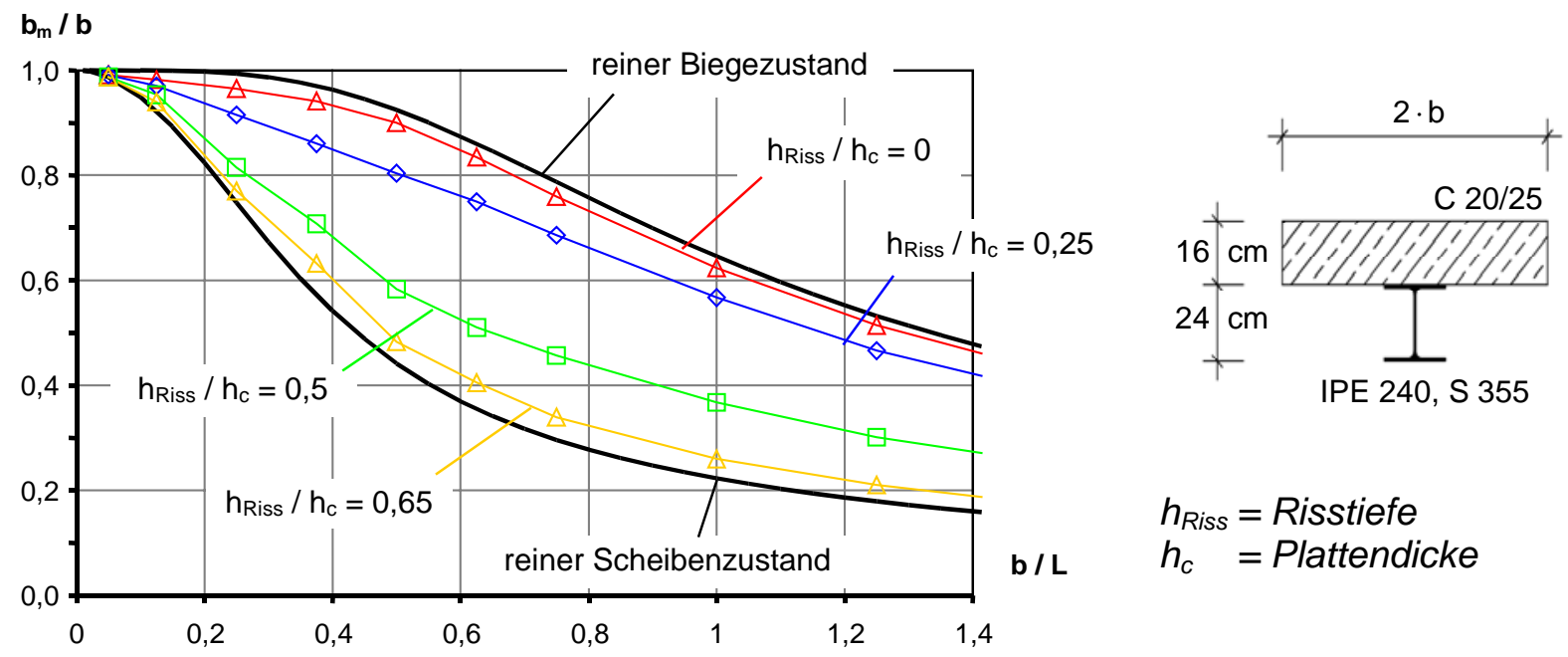

Abbildung 5-17: Einfluss der Risstiefe auf die mittragende Breite (Annahme: Der Restquerschnitt verhält sich linearelastisch.)

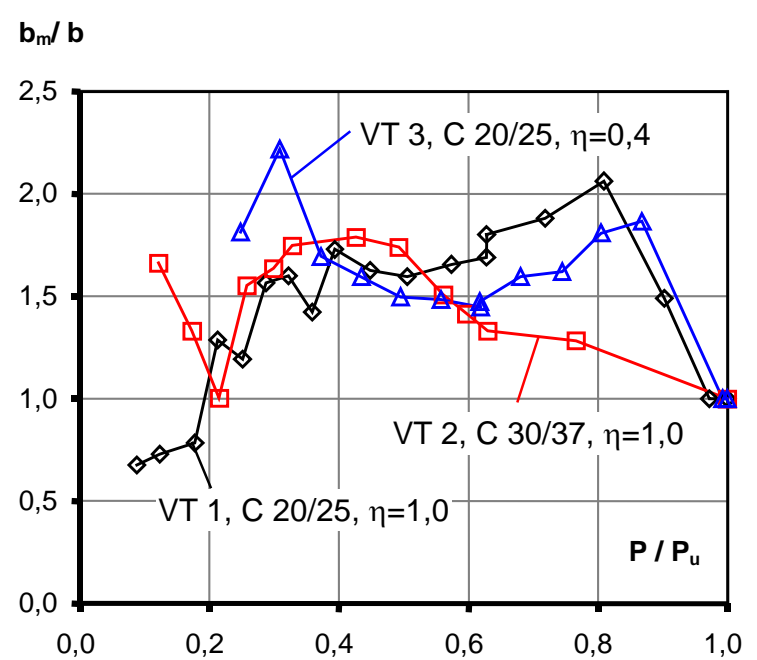

(a) bezogen auf die Spannung in Stegebene

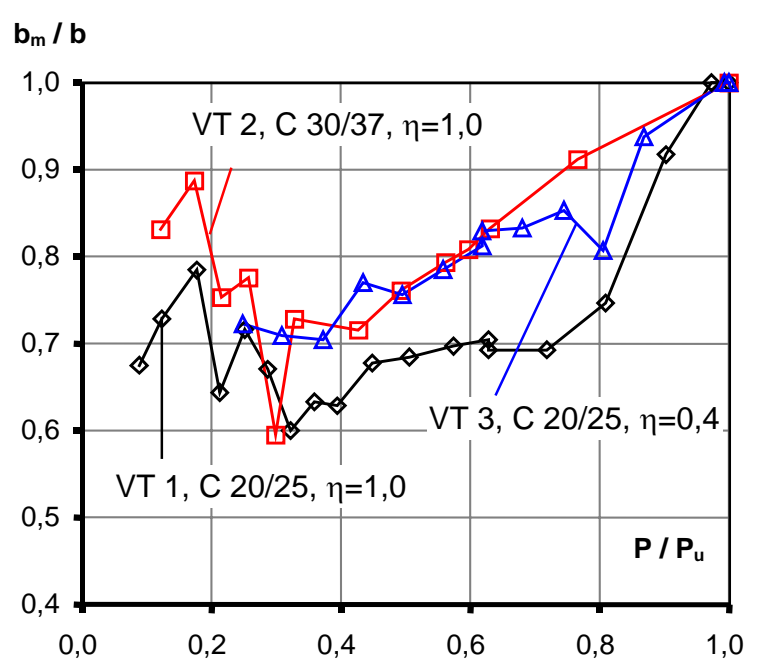

(b) bezogen auf die maximale Spannung

Abbildung 5-18: Mittragende Breite $b_{m}$ bezogen auf die Spannung der unteren Längsbewehrung in Feldmitte der Versuchsträger VT 1, VT 2 und VT 3 [2.27] 


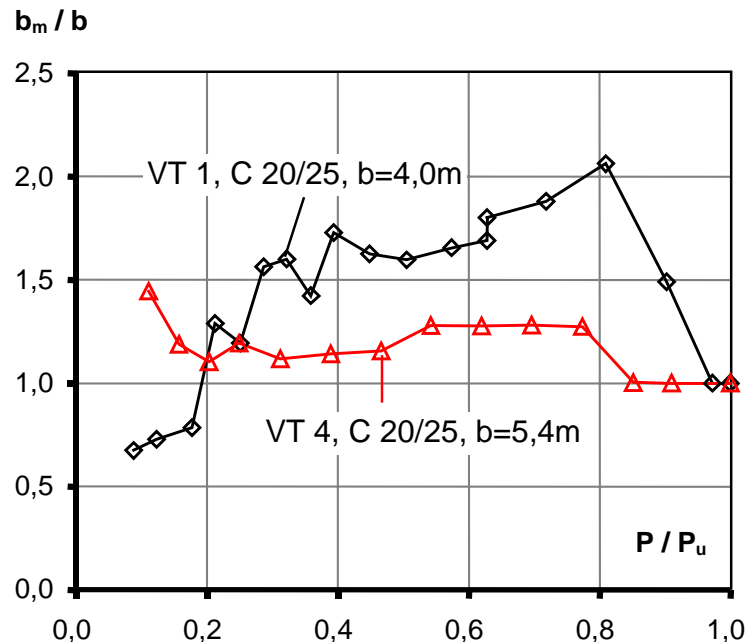

(a) bezogen auf die Spannung in Stegebene

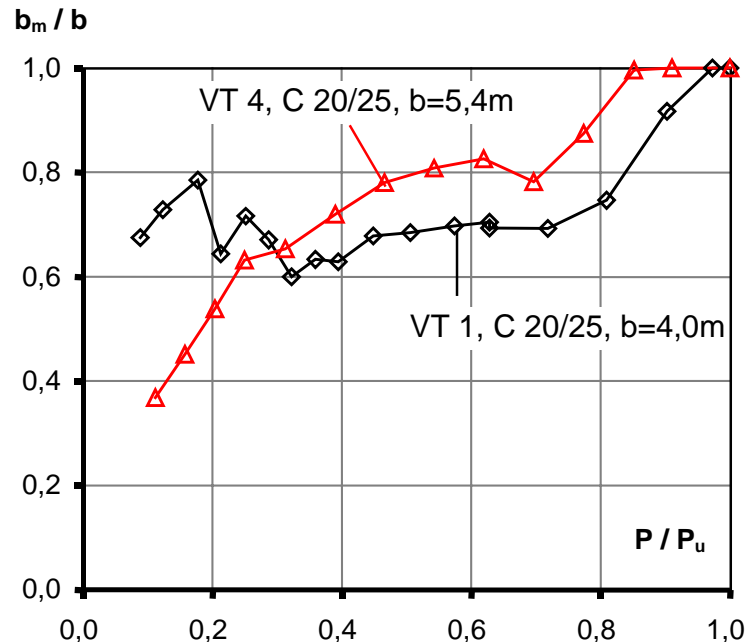

(b) bezogen auf die maximale Spannung

Abbildung 5-19: Mittragende Breite $b_{m}$ bezogen auf die Spannung der unteren Längsbewehrung in Feldmitte der Versuchsträger VT 1 und VT 4 [2.27]

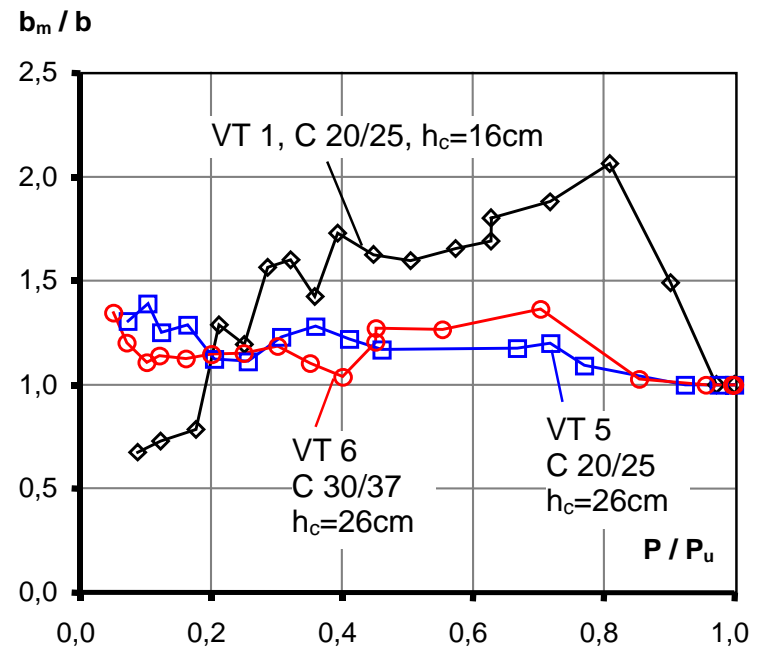

(a) bezogen auf die Spannung in Stegebene

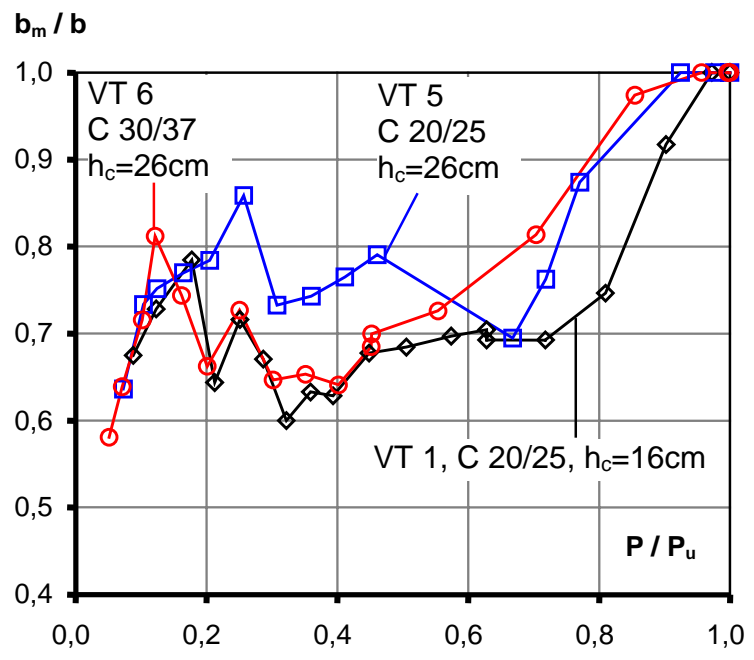

(b) bezogen auf die maximale Spannung

Abbildung 5-20: Mittragende Breite $b_{m}$ bezogen auf die Spannung der unteren Längsbewehrung in Feldmitte der Versuchsträger VT 1, VT 5 und VT 6 [2.27]

An der Unterseite des Betongurts ist es nur bedingt sinnvoll, die mittragende Breite auf die Betonspannungen zu beziehen, da im Zustand II die Betonspannungen nahezu null sind. Die mittragende Breite wird daher auf die Spannung der unteren Längsbewehrung bezogen, die unter Annahme eines schlupflosen Verbunds zwischen Bewehrung und Beton - die gleiche Dehnung erfährt wie der Beton. Bis zum Erreichen der Streckgrenze kann dabei von einer linearen Spannungs-Dehnungs-Beziehung der Längsbewehrung ausgegangen werden.

Abbildung 5-18 bis Abbildung 5-20 zeigen die mittragende Breite $b_{m}$ für die untere Längsbewehrung in Feldmitte. Diese Werte beruhen auf den gemessenen mittleren Betondehnungen, vgl. Abschnitt 5.5.3. Die mittragende Breite ist hierbei zum Einen wie gewöhnlich auf den Spannungswert in der Symmetrieachse am Steg des Stahlträgers bezogen. Die Werte sind größer eins, da die Dehnungen und Spannungen in den Außenbereichen des Betongurts 
größer sind als nahe der Symmetrieachse. Dies gilt vor allem für den Zustand II. Mit beginnendem Fließen der Bewehrung nimmt die mittragende Breite ab und erreicht im Bruchzustand bei allen Versuchsträgern die vorhandene Breite des Betongurts. Das heißt, die gesamte untere Längsbewehrung wirkt im Bruchzustand mit. Bezieht man die mittragende Breite auf die maximale Spannung, die an der Außenseite des Betongurts auftritt, so ergeben sich ähnliche Werte wie an der Oberseite des Betongurts.

Die Auswertung der gemessenen Betondehnungen lässt Folgendes schlussfolgern:

- Die spannungsbezogene mittragende Breite der Versuchsträger ist deutlich größer als gemäß den Regelungen in EN 1994-1-1 und DIN 1045-1.

- Im Bereich der Rissbildung zeigt sich eine deutliche Abhängigkeit der spannungsbezogenen mittragenden Breite $b_{m}$ von der Versuchslast und der Rissbildung. Mit zunehmender Versuchslast und Rissbildung nimmt die mittragende Breite zu.

- Nach abgeschlossener Rissbildung ist die spannungsbezogene mittragende Breite $b_{m}$ nahezu unabhängig von der Versuchslast.

- Die Betongüte und Betonzugfestigkeit zeigen keinen einheitlichen Einfluss auf die mittragende Breite der Versuchsträger.

- Die Dicke des Betongurts zeigt im Bereich der Rissbildung einen deutlichen Einfluss auf die mittragende Breite $b_{m}$. Mit zunehmender Dicke nimmt die mittragende Breite zu. Nach abgeschlossener Rissbildung geht dieser Einfluss weitestgehend verloren.

- An der Unterseite des Betongurts tritt die größte Spannung nicht direkt in der Symmetrieachse sondern im Außenbereich des Betongurts auf.

- Im Traglastbereich wirkte bei allen Versuchsträgern in Feldmitte die untere Längsbewehrung vollständig mit.

\subsubsection{Verformungsbezogene mittragende Breite $b_{m, v}$}

Grundsätzliches zur verformungsbezogenen mittragenden Breite $b_{m, v}$ ist in Abschnitt 3.3.3 gegeben. Ihre Definition lautet in Kurzform: Die Verformungsberechnung am Ersatzbalken mit konstantem Querschnitt und konstanter Breite $b_{m, v}$ ergibt die gleiche Verformung wie beim realen Verbundträger.

In Abbildung 5-21 bis Abbildung 5-26 ist für die Versuchsträger ein Vergleich der experimentellen Last-Verformungs-Kurve mit rechnerischen Kurven dargestellt. Die rechnerischen Kurven sind dabei für die mittragende Breite gemäß EN 1994-1-1 bzw. die volle Breite des Betongurts aufgetragen und stellen somit die Grenzwerte dar. Die Berechnungen berücksichtigen das nichtlineare Material- und Tragverhalten der Verbundträger mittels M- $\kappa$-Linien bzw. M-N-א-Linien, siehe Abschnitt 2.3.3. Der Vergleich zeigt, dass bei allen Versuchsträgern die experimentellen Last-Verformungs-Kurven zwischen den Werten für die volle Breite des Betongurts und der mittragenden Breite gemäß EN 1994-1-1 liegen. Die verformungsbezogene mittragende Breite $b_{m, v}$ der Versuchsträger liegt somit zwischen diesen beiden Grenzwerten und ist größer als der Wert nach EN 1994-1-1. Der Vergleich mit den rechnerischen Grenzkurven zeigt weiter, dass zu Belastungsbeginn die experimentellen Werte sehr nahe an der Kurve für die volle Plattenbreite liegen. Mit zunehmender Belastung wird der Abstand der experimentellen Kurven zu der oberen Grenzkurve größer und nähert sich der unteren Grenze an. Die verformungsbezogene mittragende Breite nimmt somit mit zunehmender Belastung ab.

Das Verformungsverhalten der Versuchsträger lässt sich anschaulich mit Abbildung 5-17 erklären. Darin dargestellt ist die mittragende Breite eines Betongurts in Abhängigkeit der 
Risstiefe zwischen den beiden Grenzkurven des reinen Scheibenzustands $b_{m, s}$ und des reinen Biegezustands $b_{m, B}$ für linearelastisches Materialverhalten. Zu Belastungsbeginn ist der Betongurt der Versuchsträger im ungerissenen Zustand und verhält sich in guter Näherung linearelastisch. Die mittragende Breite ist vergleichsweise groß und liegt nahe am Grenzwert des reinen Biegezustands. Mit der Bildung der ersten Risse beginnt der Traganteil des Betongurts auf Biegung abzunehmen. Die mittragende Breite wird kleiner. Mit weiter steigender Belastung und zunehmender Rissöffnung schnürt sich die Druckzone ein, so dass der Biegetraganteil des Betongurts verschwindet und dieser als reine Scheibe wirkt. Die mittragende Breite nähert sich somit mit zunehmender Rissbildung der Grenzkurve des reinen Scheibenzustands. Dieses Verhalten im höchstbeanspruchten Querschnitt breitet sich mit zunehmender Belastung und Rissbildung in Trägerlängsrichtung aus, so dass die über die Trägerlänge gemittelte verformungsbezogene mittragende Breite $b_{m, v}$ weiter abnimmt, siehe Abbildung 5-27 und Abbildung 5-28.

Abbildung 5-27 und Abbildung 5-28 zeigen auch, dass die verformungsbezogene mittragende Breite für höhere Lasten mit Erreichen der Streckgrenze im Baustahl wieder ansteigt. Durch das Plastizieren des Stahlträgers wird der innere Hebelarm im Verbundquerschnitt kleiner und das innere Kräftepaar größer. Durch die größere Betondruckkraft und die damit verbundenen größeren Betondehnungen wird die Querverteilung der Betondruckspannungen ausgeglichener und die mittragende Breite nimmt $z u$.

Durch Vergleich der mittragenden Breiten der Versuchsträger VT $1(b / L=0,48)$ und VT 4 $(b / L=0,65)$ zeigt sich, dass mit zunehmender Breite des Betongurts die mittragende Breite bei gleicher Stützweite zunimmt. Dies ist auch aus elastischen Berechnungen bekannt und kann hiermit auch für das nichtlineare Tragverhalten der Versuchsträger bestätigt werden. Der Vergleich der Ergebnisse der Versuchsträger VT 1 und VT $2\left(h_{c}=16 \mathrm{~cm}\right)$ mit den Trägern VT 5 und VT $6\left(h_{c}=26 \mathrm{~cm}\right)$ bestätigt die ebenfalls aus elastischen Berechnungen bekannte Tendenz, dass mit zunehmender Dicke des Betongurts die mittragende Breite größer wird, siehe Tabelle 5-5. Die Versuchsergebnisse zeigen weiter, dass die verformungsbezogene mittragende Breite $b_{m, v}$ bei einer größeren Betonzugfestigkeit zunimmt. Dies ist durch einen Vergleich der Ergebnisse von VT $1 \quad\left(f_{c t}=2,0 \mathrm{~N} / \mathrm{mm}^{2}, \quad h_{c}=16 \mathrm{~cm}\right)$ mit VT 2 $\left(f_{c t}=3,1 \mathrm{~N} / \mathrm{mm}^{2}, h_{c}=16 \mathrm{~cm}\right)$ bzw. der Ergebnisse von VT $5\left(f_{\mathrm{ct}}=2,5 \mathrm{~N} / \mathrm{mm}^{2}, \mathrm{~h}_{\mathrm{c}}=26 \mathrm{~cm}\right) \mathrm{mit}$ VT $6\left(f_{c t}=2,2 \mathrm{~N} / \mathrm{mm}^{2}, h_{c}=26 \mathrm{~cm}\right)$ zu erkennen, siehe Tabelle 5-5.

Tabelle 5-5: Verformungsbezogene mittragende Breite $b_{m, v}$ der Versuchsträger

\begin{tabular}{|c|c|c|c|c|c|}
\cline { 2 - 6 } \multicolumn{1}{c|}{} & VT 1 & VT 2 & VT 4 & VT 5 & VT 6 \\
\hline $\mathrm{b}_{\mathrm{m}, \mathrm{v}}[\mathrm{m}]^{1)}$ & 1,38 & 1,74 & 3,29 & 2,56 & 2,28 \\
\hline $\mathrm{b}_{\mathrm{m}, \mathrm{V}} / \mathrm{b}[-]^{2)}$ & 0,35 & 0,44 & 0,61 & 0,64 & 0,57 \\
\hline $\mathrm{b}_{\mathrm{m}, \mathrm{V}, \mathrm{el}}[\mathrm{m}]^{3)}$ & 3,51 & 3,54 & 4,25 & 3,60 & 3,60 \\
\hline $\mathrm{b}_{\mathrm{m}, \mathrm{EC} 4}[\mathrm{~m}]^{4)}$ & 1,04 & 1,04 & 1,04 & 1,04 & 1,04 \\
\hline
\end{tabular}

1) verformungsbezogene mittragende Breite

2) $b=$ Breite des Betongurts

3) mittragende Breite des Betongurts für elastisches Materialverhalten

4) mittragende Breite des Betongurts gemäß EN 1994-1-1 [1.1]

Die Auswertung der Versuche lässt folgende Schlussfolgerungen hinsichtlich der verformungsbezogenen mittragende Breite $b_{m, v} z u$ :

- Die verformungsbezogene mittragende Breite der Versuchsträger ist deutlich größer als der Wert in EN 1994-1-1. 
- Im ungerissenen Zustand ergibt sich eine sehr große verformungsbezogene mittragende Breite.

- Durch die Rissbildung im Betongurt nimmt die verformungsbezogene mittragende Breite ab.

- Durch das Plastizieren des Stahlträgers nimmt die verformungsbezogene mittragende Breite zu.

- Die verformungsbezogene mittragende Breite nimmt sowohl mit größer werdender Dicke als auch größer werdender Breite des Betongurts zu.

- Die verformungsbezogene mittragende Breite wird mit zunehmender Betonzugfestigkeit $\mathrm{f}_{\mathrm{ct}}$ größer.

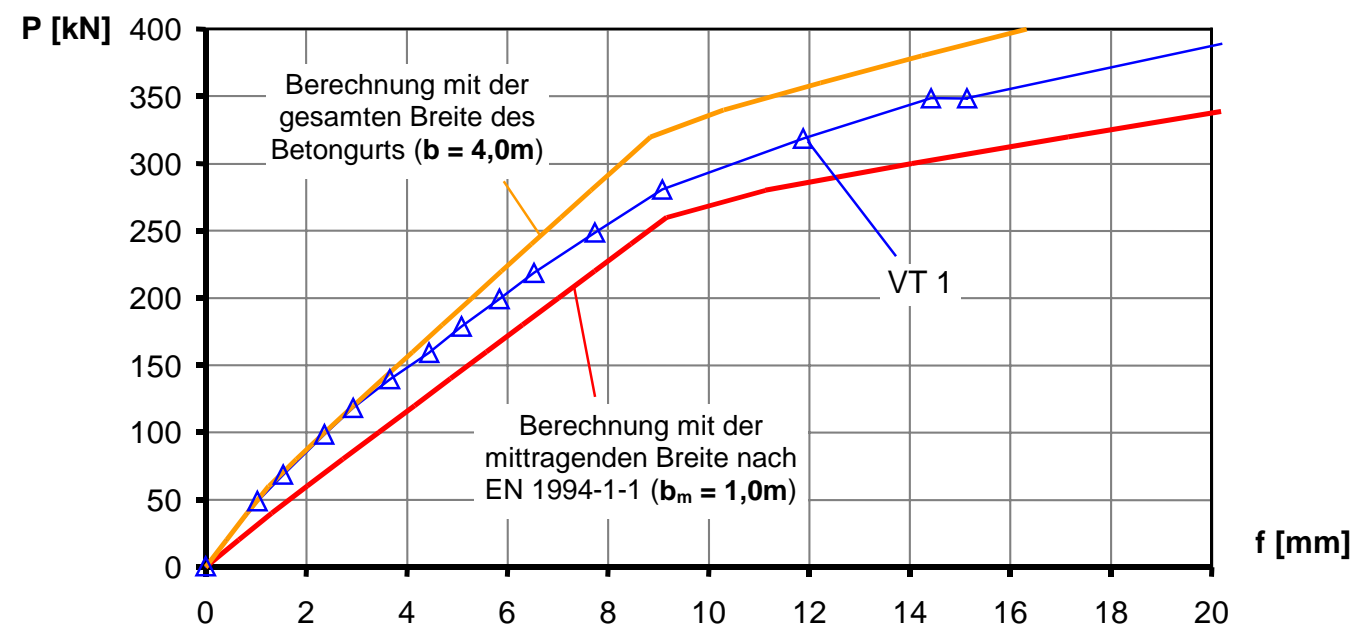

Abbildung 5-21: Vergleich der Last-Verformungs-Kurve von Versuchsträger VT 1 mit den rechnerischen Kurven [2.27]

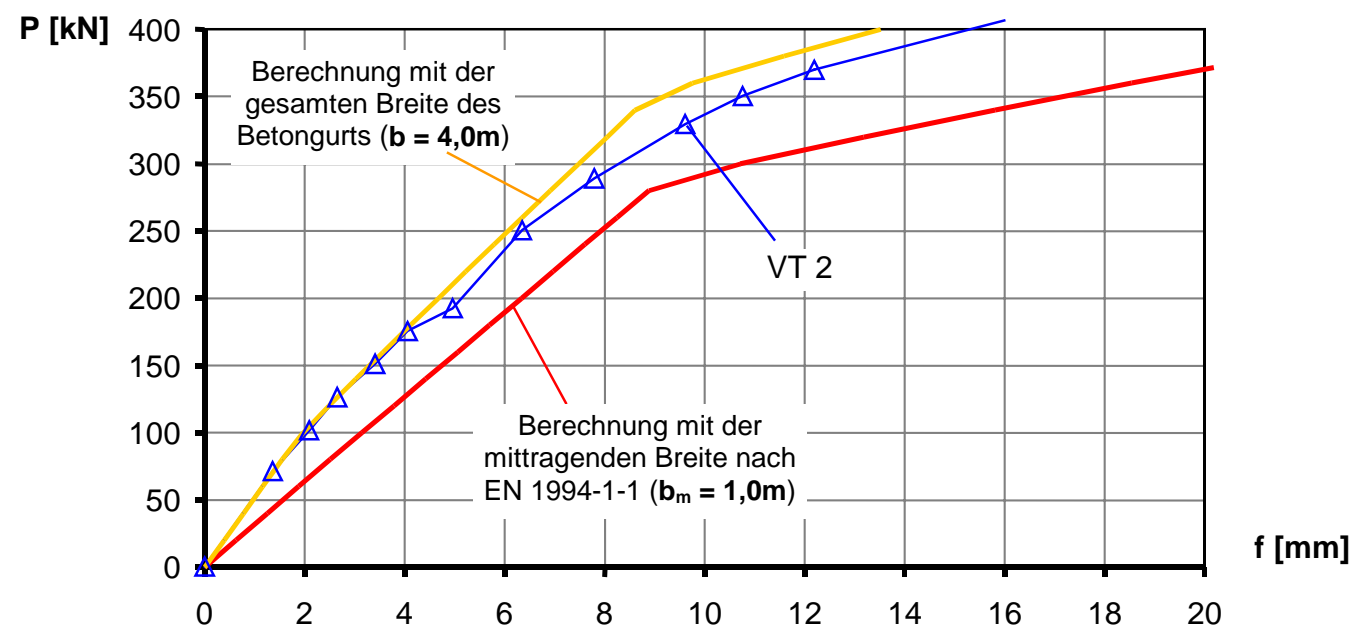

Abbildung 5-22: Vergleich der Last-Verformungs-Kurve von Versuchsträger VT 2 mit den rechnerischen Kurven [2.27] 


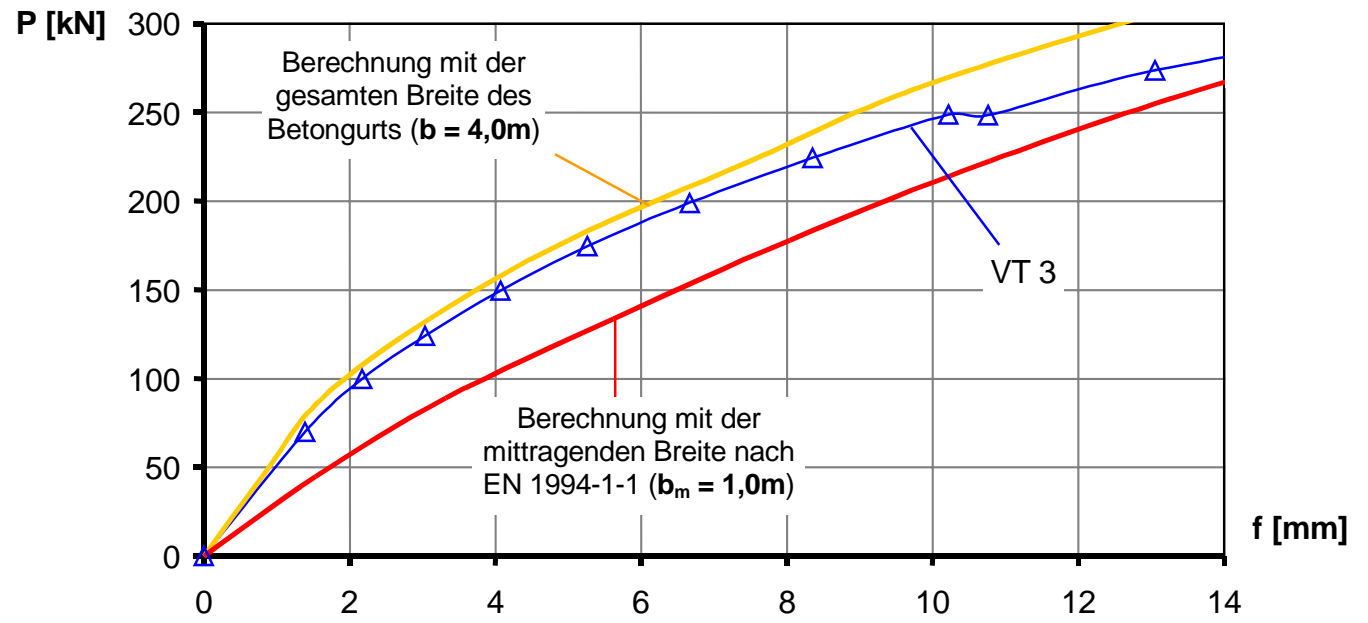

Abbildung 5-23: Vergleich der Last-Verformungs-Kurve von Versuchsträger VT 3 mit den rechnerischen Kurven [2.27]

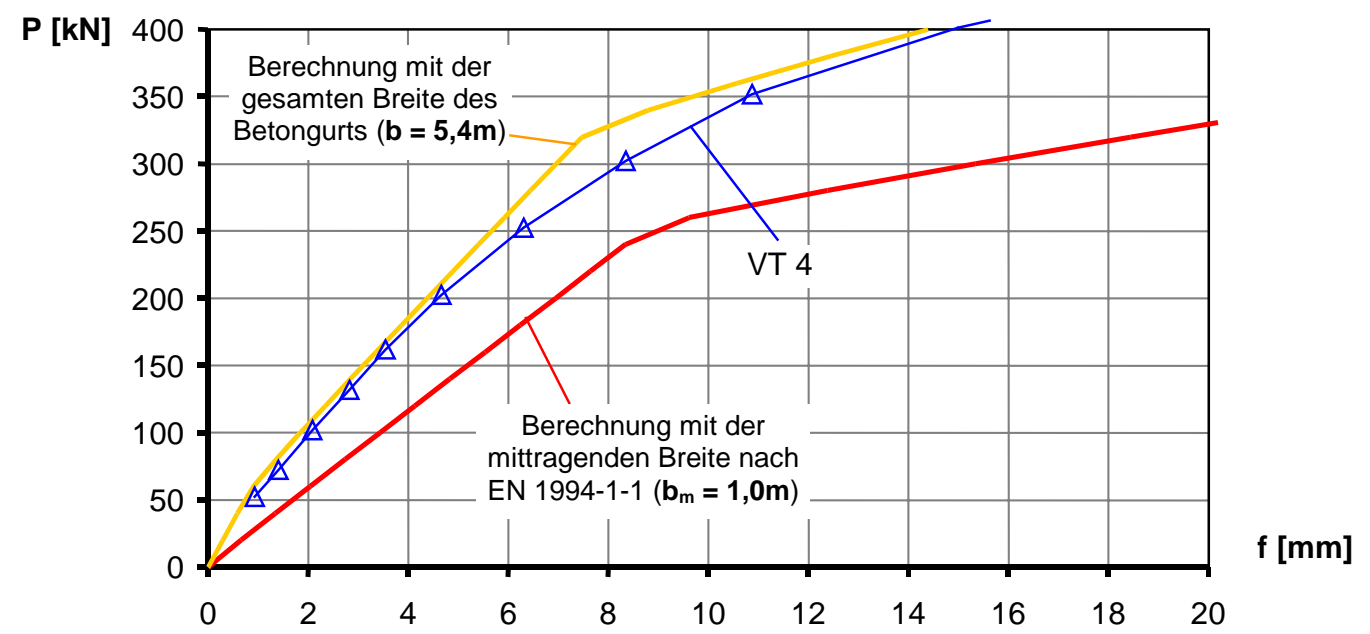

Abbildung 5-24: Vergleich der Last-Verformungs-Kurve von Versuchsträger VT 4 mit den rechnerischen Kurven [2.27]

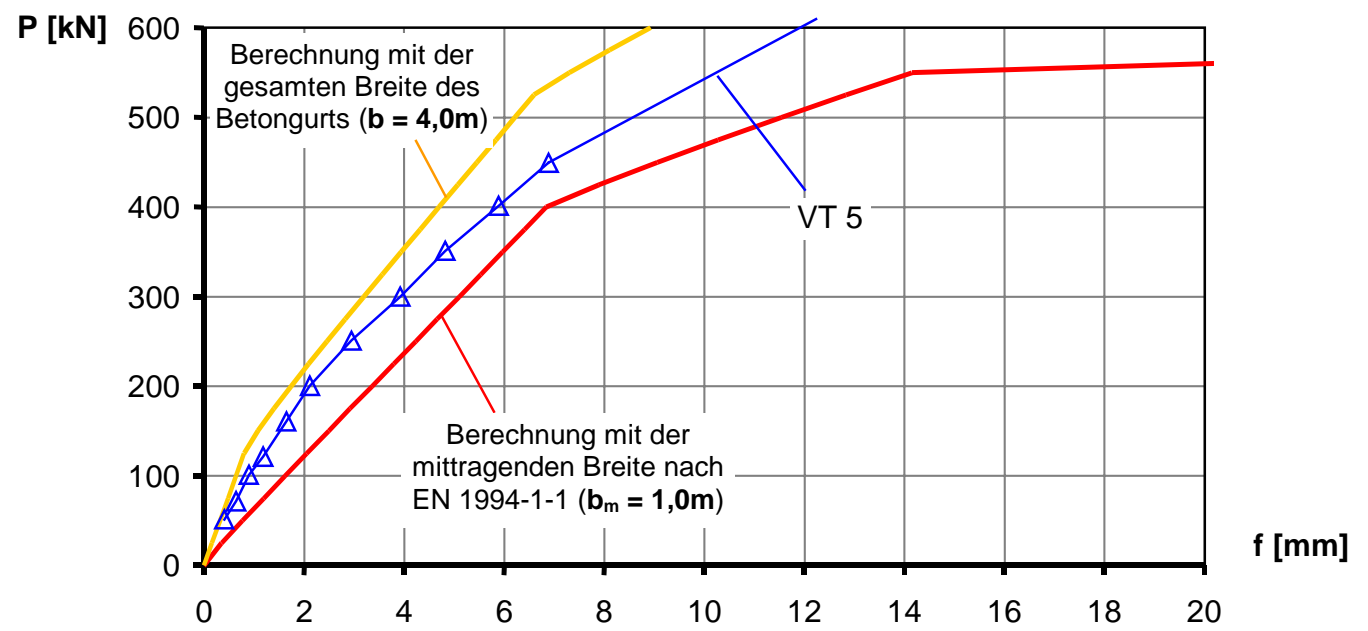

Abbildung 5-25: Vergleich der Last-Verformungs-Kurve von Versuchsträger VT 5 mit den rechnerischen Kurven [2.27] 


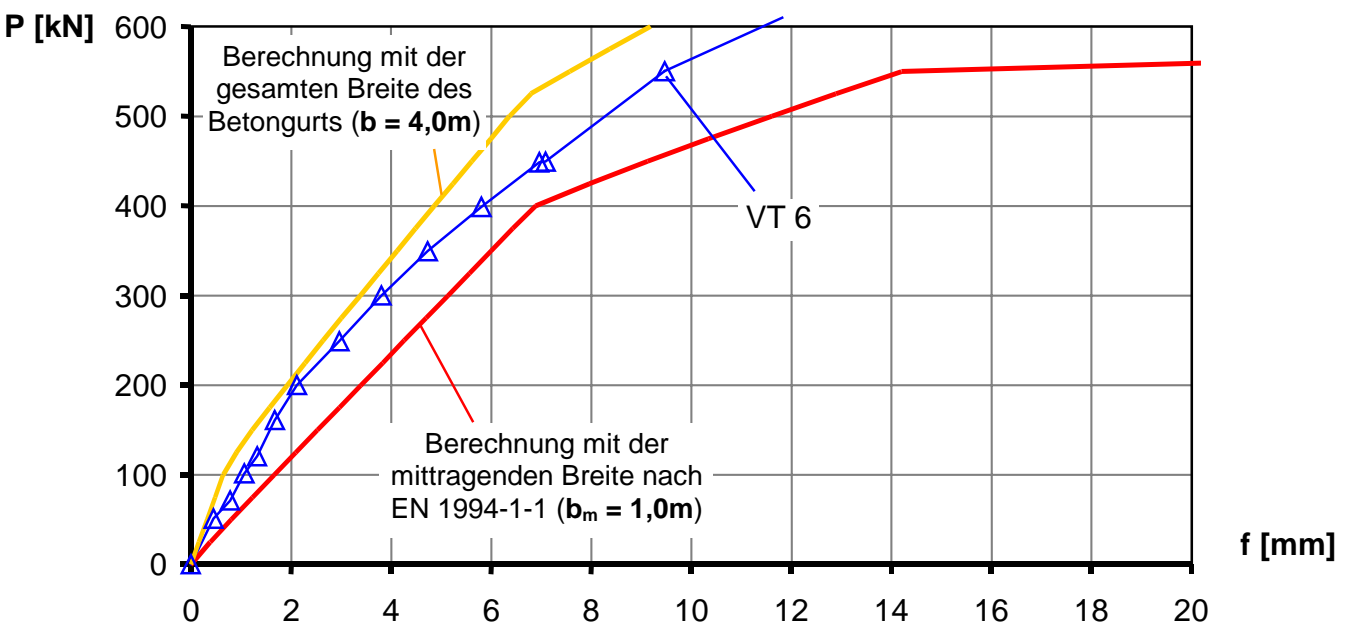

Abbildung 5-26: Vergleich der Last-Verformungs-Kurve von Versuchsträger VT 6 mit den rechnerischen Kurven [2.27]

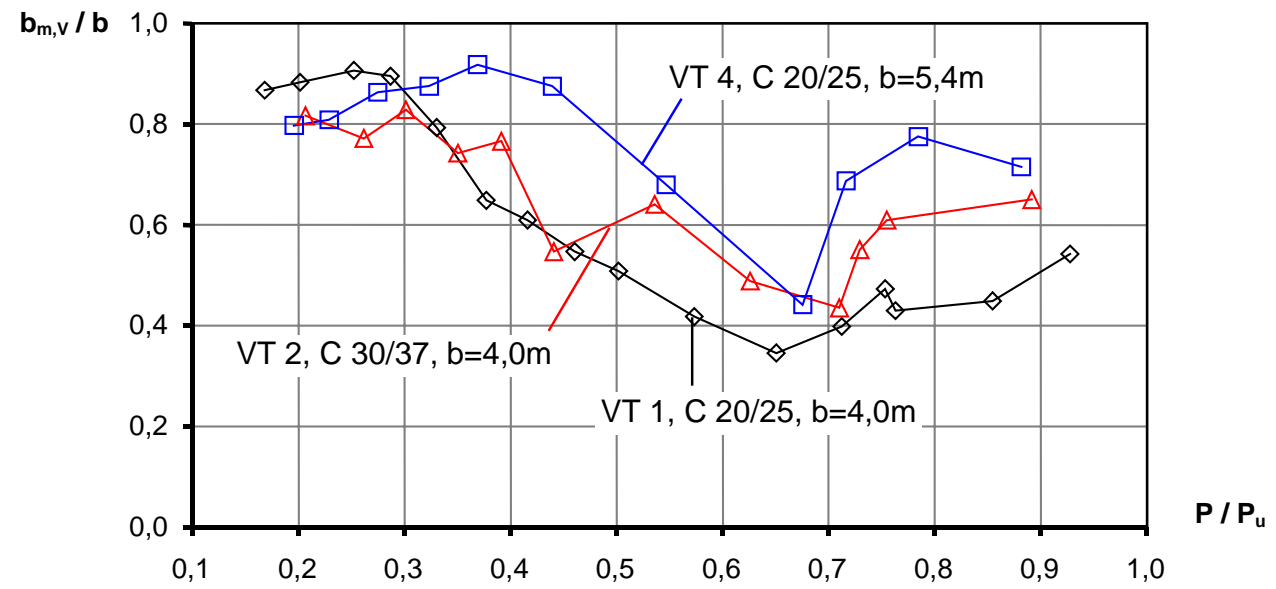

Abbildung 5-27: Verformungsbezogene mittragende Breite $b_{m, \vee}$ der Versuchsträger VT 1, VT 2 und VT 4 in Abhängigkeit der bezogenen Versuchslast P/Pu [2.27]

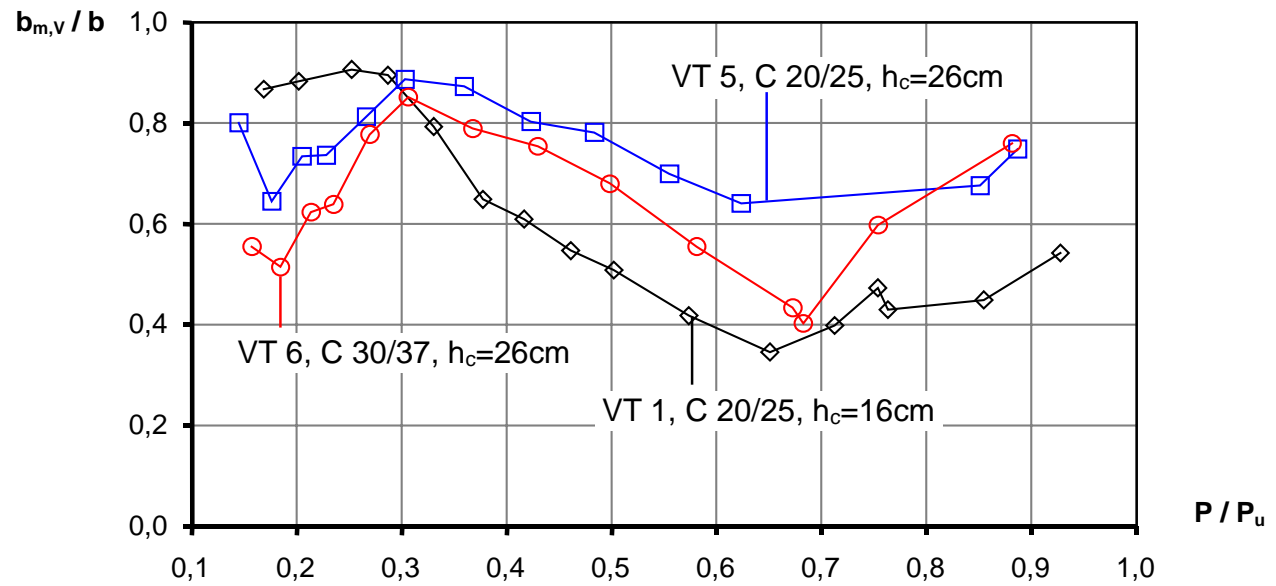

Abbildung 5-28: Verformungsbezogene mittragende Breite $b_{m, \vee}$ der Versuchsträger VT 1, VT 5 und VT 6 in Abhängigkeit der bezogenen Versuchslast P/P ${ }_{u}$ [2.27] 


\subsection{Zusammenfassung der wichtigsten Versuchsergebnisse}

Die experimentellen Untersuchungen umfassen sechs Versuchsträger niedriger Bauhöhe praxisüblicher Werkstoffe und Randbedingungen mit einem Verhältnis $b / L=0,48 \div 0,65$ und einem Verhältnis Plattendicke zu Stahlträgerhöhe $h_{c} / h_{a}=0,67 \div 1,08$. Die Versuchsträger verhielten sich sehr duktil. Das Versagen kündigte sich durch sehr große Verformungen zwischen 76 und $156 \mathrm{~mm}(=\mathrm{L} / 55 \div \mathrm{L} / 26)$ an. Bereits unter Gebrauchslasten kam es zu einer starken Rissbildung an der Unterseite der Betongurte. Die Risse bildeten sich erwartungsgemäß an den Stellen der unteren Querbewehrung und verliefen nahezu geradlinig in Querrichtung. Dabei bildeten sich die ersten Risse jedoch nicht am Stahlträger sondern zunächst am Rand des Betongurts bzw. zwischen Stahlträger und Außenseite des Betongurts.

Während der Versuchsdurchführung wurde die Querverteilung der Betondehnungen an der Ober- und Unterseite des Betongurts in Feldmitte und im Viertelspunkt gemessen. Die Auswertung der gemessenen Betondehnungen zeigt, dass die spannungsbezogene mittragende Breite an der Betonoberseite deutlich größer ist als im Vergleich zu den Regelungen in EN 1994-1-1 und DIN 1045-1. Die mittragende Breite der Versuchsträger nimmt dabei mit zunehmender Rissbildung zu. Im Bereich der Rissbildung nimmt die mittragende Breite mit der Belastungshöhe und der Plattendicke zu. Nach abgeschlossener Rissbildung verschwinden diese Einflüsse jedoch weitestgehend.

Die Messungen an der Unterseite des Betongurts zeigen, dass die maximale Dehnung nicht am Stahlträger sondern in einem größeren Abstand zu diesem auftritt. Bezieht man die mittragende Breite wie üblich auf die Spannung in der Symmetrieebene, so ergeben sich für die mittragende Breite an der Betonunterseite größere Werte als die vorhandene Breite. Bei Bezug auf die maximale Spannung stimmen die Werte der mittragende Breite an der Unterseite mit denen an der Oberseite weitestgehend überein.

Das Last-Verformungs-Verhalten der Versuchsträger ist deutlich steifer als nach Berechnungen auf Basis der mittragenden Breite gemäß EN 1994-1-1. Die verformungsbezogene mittragende Breite $b_{m, v}$ der Versuchsträger ist somit größer als nach EN 1994-1-1. Die Rückrechnung der verformungsbezogenen mittragenden Breite aus der Last-Verformungs-Kurve der Versuchsträger zeigt, dass diese bei Belastungsbeginn und im Zustand I deutlich größer ist als in EN 1994-1-1 gegeben. Diese Werte zeigen eine gute Übereinstimmung mit linearelastischen Berechnungen. Mit beginnender Rissbildung wird die mittragende Breite kleiner, da durch die Rissbildung der Biegezustand des Betongurts verschwindet. Durch das Plastizieren des Stahlträgers nimmt die verformungsbezogene mittragende Breite wieder zu. Die Auswertung der Versuche zeigt, dass die verformungsbezogene mittragende Breite mit der Plattendicke, Plattenbreite und Betonzugfestigkeit zunimmt. 


\section{Analytisches Berechnungsmodell zur Verformungsberechnung niedriger Verbundträger}

\subsection{Einführung}

\subsubsection{Allgemeines}

Wenn die Betongurte von Verbundträgern niedriger Bauhöhe breit sind, so zeigen diese aufgrund der Schubweichheit des Gurtes ein ausgeprägtes dreidimensionales Tragverhalten, siehe Abschnitt 3.2. Dabei entziehen sich Bereiche des Betongurts der Lastabtragung, die vom Stahlträger weiter entfernt sind. Das heißt, die Gurtspannungen nehmen mit zunehmendem Abstand vom Stahlträger ab und die Querverteilung der Gurtspannungen ist nicht konstant. Um diese Träger mit breiten Gurten dennoch rechnerisch wie stabförmige Bauteile behandeln zu können und eine aufwändige dreidimensionale Berechnung des Betongurts zu vermeiden, wurde die spannungsbezogene mittragende Breite $b_{m}$ eingeführt, siehe Abschnitt 3.3. Der Zusatz „spannungsbezogen“ deutet darauf hin, dass sich diese mittragende Breite aus der Querverteilung der Gurtspannungen bestimmt. Wie die in Abschnitt 3.5 dargestellten Untersuchungen in der Literatur und die in Kapitel 4 dargestellten elastischen Untersuchungen zeigen, verändert sich dabei die spannungsbezogene mittragende Breite im Allgemeinen in Trägerlängsrichtung und damit auch die Querschnittssteifigkeit.

Die elastischen Untersuchungen zeigen darüber hinaus, dass die rechnerische Steifigkeit niedriger Verbundträger sehr stark von der Biegetragwirkung des Betongurts abhängt. Zum Einen haben die Eigenbiegesteifigkeit des Betongurts bzw. dessen Trägheitsmoment $\mathrm{I}_{\mathrm{c}}$ einen großen Einfluss auf das Verbundträgheitsmoment $\mathrm{I}_{\mathrm{i}, 0}$. Zum anderen ist die zur Berechnung des Betonträgheitsmoments $I_{c} z u$ verwendende mittragende Breite des Biegezustands deutlich größer als die mittragende Breite des Scheibenzustands. Der Beitrag der Biegetragwirkung des Betongurts zur Steifigkeit des Verbundträgers wird jedoch durch die Rissbildung des Betongurts abgemindert und ist damit von der Belastungshöhe abhängig.

Für eine wirklichkeitsnahe Verformungsberechnung niedriger Verbundträger ist somit neben der Berücksichtigung der spannungsbezogenen mittragenden Breite des Scheiben- und Biegezustands sowie deren Verlauf in Trägerlängsrichtung auch die realistische Abbildung der Biegetragwirkung des Betongurts und dessen Rissbildung von großer Bedeutung. Um diese Einflüsse rechnerisch zu erfassen wurde ein analytisches Berechnungsmodell zur Verformungsberechnung niedriger Verbundträger entwickelt.

Dieses im Folgenden beschriebene Berechnungsmodell basiert dabei auf allgemein anerkannten mechanischen Grundlagen und vermeidet Konvergenzprobleme, wie diese bei Finite Element Modellierungen häufig auftreten. Die Verifikation des analytischen Modells wird dabei folgendermaßen vorgenommen:

- Nachvollziehbarkeit der mechanischen Grundlagen

- Vergleich mit den Ergebnissen der durchgeführten Versuche

- Plausibilitätsprüfung auf Basis der Erkenntnisse der elastischen Untersuchungen und der in der Literatur beschriebenen Untersuchungen, siehe Abschnitt 6.1.2

Im Vergleich zu einer Berechnung mit einem Finite Element Programm hat das entwickelte analytische Berechnungsmodell folgende Vorteile:

- einfache Nachvollziehbarkeit der mechanischen Grundlagen und der Berechnungsergebnisse 
- schnelle Rechenzeit und damit Möglichkeit zu einer umfangreichen Parameterstudie

- Trennung der unterschiedlichen Verformungseinflüsse, z. B. infolge Querkraft und Moment

- geringere Sensibilität der Berechnungsergebnisse bezüglich der Eingangsparameter, z. B. Lastschrittweite, Konvergenzmaß, etc.

Anhand des analytischen Berechnungsmodells wurde eine umfangreiche Parameterstudie durchgeführt, um die verschiedenen Einflüsse auf die verformungsbezogene mittragende Breite niedriger Verbundträger zu quantifizieren. Aufbauend auf den Ergebnissen dieser Parameterstudie wurde ein anwenderfreundlicher Berechnungsansatz für die verformungsbezogene mittragende Breite $b_{m, v}$ entwickelt, der eine wirklichkeitsnahe Verformungsberechnung niedriger Verbundträger mit vergleichsweise geringem Aufwand ermöglicht, siehe Kapitel 7.

Zum besseren Verständnis dieser Untersuchungen wird im Folgenden jedoch zunächst noch einmal kurz die Ausgangssituation dargestellt, die auf den Kenntnissen bei linearelastischem Materialverhalten, der in der Literatur beschriebenen Untersuchungen und den Erkenntnissen der durchgeführten Versuche basieren.

\subsubsection{Ausgangssituation}

Die im Weiteren beschriebenen Untersuchungen basieren auf den Erkenntnissen der bisherigen Untersuchungen. Zum besseren Verständnis werden diese hier noch einmal kurz zusammengefasst. Demnach stellt sich die Ausgangssituation folgendermaßen dar:

\section{(a) Elastische Untersuchungen zur mittragenden Breite}

(siehe Abschnitt 3.5 und Kapitel 4)

- Die mittragende Breite $b_{m}$ lässt sich in einen Wert des reinen Scheibenzustands $b_{m, s}$ und des reinen Biegezustands $b_{m, B}$ unterteilen, wobei der Wert des reinen Biegezustands $b_{m, B}$ deutlich größer ist als jener des reinen Scheibenzustands $b_{m, s}$.

- Die mittlere mittragende Breite $b_{m}$ ist dadurch sehr stark vom Verhältnis der Steifigkeitsverteilung innerhalb des Verbundträgers abhängig. Mit zunehmender Biegesteifigkeit des Betongurts im Verhältnis zur Gesamtsteifigkeit des Verbundträgers wird die mittragende Breite größer.

- Aus diesem Grund wird die mittragende Breite $b_{m}$ auch mit zunehmender Plattendicke $\mathrm{h}_{\mathrm{c}}$ und abnehmender Gesamthöhe des Trägers größer.

- Die mittragende Breite $b_{m}$ ist sehr stark vom Seitenverhältnis $b / L$ des Betongurts $a b$ hängig.

- Der Verlauf der mittragenden Breite $b_{m}$ in Trägerlängsrichtung ist im Allgemeinen nicht konstant und wird durch die Form der äußeren Belastung bestimmt.

\section{(b) Untersuchungen zur mittragenden Breite unter Berücksichtigung der Rissbildung} (siehe Abschnitt 3.5 und Kapitel 5)

- Mit Ausnahme der eigenen in Kapitel 5 beschriebenen experimentellen Untersuchungen liegen keine Arbeiten zur mittragenden Breite von Trägern mit großer Biegetragwirkung des Betongurts unter Berücksichtigung der Rissbildung vor. 
Die eigenen in Kapitel 5 beschriebenen experimentellen Untersuchungen an niedrigen Verbundträgern zeigen:

- Die verformungsbezogene mittragende Breite hängt sehr stark von der Rissbildung und der Belastungshöhe ab. Mit zunehmender Belastung und Rissbildung nimmt die verformungsbezogene mittragende Breite $b_{m, v}$ ab.

- Sowohl die verformungsbezogene als auch die spannungsbezogene mittragende Breite der Versuchsträger sind deutlich größer als die in den entsprechenden Normen angegebenen Werte.

- Sowohl die verformungsbezogene als auch die spannungsbezogene mittragende Breite werden mit zunehmender Dicke des Betongurts $h_{c}$ und zunehmender Breite des Betongurts größer.

- Die verformungsbezogene mittragende Breite $b_{m, v}$ wird mit zunehmender Betonzugfestigkeit $\mathrm{f}_{\mathrm{ct}}$ größer.

Die Untersuchungen von Amadio, et al. zur mittragenden Breite von Verbundträgern [2.63] zeigen an einem Versuchsträger mit vergleichsweise dünnem Betongurt, siehe Abschnitt 3.5, dass

- die spannungsbezogene mittragende Breite mit zunehmender Rissbildung und Belastungshöhe größer wird.

Die Arbeiten zur mittragenden Breite von Stahlbetonträgern [2.51], [2.52] zeigen, siehe Abschnitt 3.5, dass

- die Rissbildung einen nennenswerten Einfluss auf die spannungsbezogene mittragende Breite haben kann.

Mit Ausnahme der eigenen in Kapitel 5 beschriebenen experimentellen Untersuchungen liegen somit keine Arbeiten vor, die die verformungsbezogene mittragende Breite $b_{m, v}$ niedriger Verbundträger unter Berücksichtigung der Biegetragwirkung und der Rissbildung des Betongurts systematisch untersuchen. Für eine wirklichkeitsnahe Verformungsberechnung niedriger Verbundträger sind jedoch gerade diese Einflüsse maßgebend.

\subsection{Beschreibung des analytischen Berechnungsmodells}

\subsubsection{Grundsätzliches}

Das analytische Berechnungsmodell wurde zur Verformungsberechnung niedriger Verbundträger insbesondere im Bereich der Gebrauchslasten entwickelt. Die genaue Ermittlung der Spannungsverteilung im Betongurt und insbesondere der Querverteilung der Längsspannungen ist nicht möglich. In der vorliegenden Form ermöglicht dieses Berechnungsmodell die Verformungsberechnung von Einfeldträgern mit in Trägerlängsrichtung gleichbleibenden, symmetrischen Verbundquerschnitten nach Abbildung 6-1 unter Gleichlast oder mittiger Einzellast. Dies stellt jedoch keine prinzipielle Einschränkung des Berechnungsmodells dar. Für andere Querschnitts- und Belastungsformen sind lediglich wenige Änderungen des Programms notwendig. Das Berechnungsmodell orientiert sich dabei an Erkenntnissen der elastischen Untersuchungen, siehe Kapitel 4, und den nichtlinearen Berechnungsmethoden niedriger Verbundträger, siehe Abschnitt 2.3.3.

Als Eingabewerte für das Berechnungsmodell sind die folgenden Querschnitts-, Materialund Systemwerte erforderlich: 


\section{Querschnittswerte:}

- Abmessungen des Stahlprofil

- Dicke des Betongurts

- Querschnitt und Lage der Längsbewehrung

- evtl. Höhe eines Profilblechs

\section{Materialwerte:}

- Baustahl: Streckgrenze $f_{y}, E-M o d u l ~ E_{a}$

- Beton: Druckfestigkeit $f_{c}$, Zugfestigkeit $f_{c t}, E-M o d u l ~ E_{c m}$

- Bewehrung: Proportionalitätsgrenze $f_{s y}$, E-Modul $E_{S}$

\section{Systemwerte:}

- Stützweite $\mathrm{L}_{\mathrm{T}}$ des Einfeldträgers

- Gesamtbreite des Betongurts $b_{c}$

- Belastungsform und -höhe

Aus diesen Werten wird die Durchbiegung des Trägers in Feldmitte berechnet. Sie kann prinzipiell jedoch auch an anderer Stelle oder mehreren Stellen ermittelt werden.

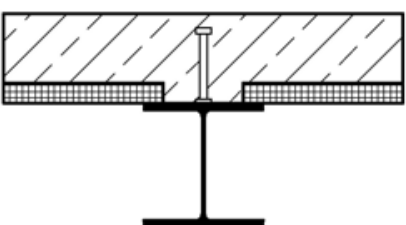

(a) Querschnitte herkömmlicher Bauart

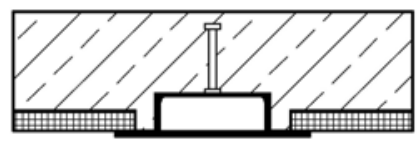

(b) Slim-Floor Querschnitte

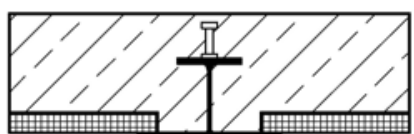

Abbildung 6-1: Im analytischen Berechnungsmodell implementierte Verbundquerschnitte

\subsubsection{System}

Das Modell basiert auf einer Diskretisierung des Trägers durch einzelne Trägerelemente, die das nichtlineare Tragverhalten des Verbundquerschnitts abbilden. Aus den Systemwerten des Trägers (Stützweite, Breite, Belastungsform, etc.) wird für jedes Element die spannungsbezogene mittragende Breite im reinen Scheibenzustand $b_{m, s}$ und reinen Biegezustand $b_{m, B}$ berechnet. Damit sind für jedes Element die rechnerischen Querschnittsabmessungen bestimmt und unter Berücksichtigung der Materialwerte kann für jedes Trägerelement die entsprechende $\mathrm{M}$-к-Linie berechnet werden. Dies gewährleistet eine wirklichkeitsnahe Steifigkeitsberechnung der Elemente. Parallel hierzu werden aus der äußeren Belastung die Schnittgrößen der einzelnen Trägerelemente berechnet. Bei den untersuchten statisch bestimmten Einfeldträgern ist dies unabhängig von der Steifigkeitsverteilung des Systems möglich. Mit Kenntnis der Belastung und des Tragverhaltens der Trägerelemente kann die Änderung der Verdrehung in jedem Element berechnet werden und daraus schließlich die Verformung des Trägers. Durch die wiederholte Berechnung für verschiedene Belastungsstufen kann die Last-Verformungs-Linie des Trägers ermittelt werden. Das Vorgehen ist schematisch in Abbildung 6-2 dargestellt.

Das Tragverhalten der Elemente wird dabei jeweils auf die Elementmitten bezogen. Hierbei wird unterstellt, dass sowohl der Querschnitt, die äußere Belastung als auch die Quer- 
schnittskrümmung entlang der Elemente konstant sind. Die Trägerelemente besitzen somit nur einen Mittelknoten, der das Verhalten der Elemente repräsentiert. Die einzelnen Elemente sind durch die Durchbiegungsgleichheit und die statischen Gleichgewichtsbedingungen an den Elementenden miteinander gekoppelt. Das Verformungsverhalten benachbarter Elemente hat jedoch keinen Einfluss auf das Tragverhalten des einzelnen Elements.

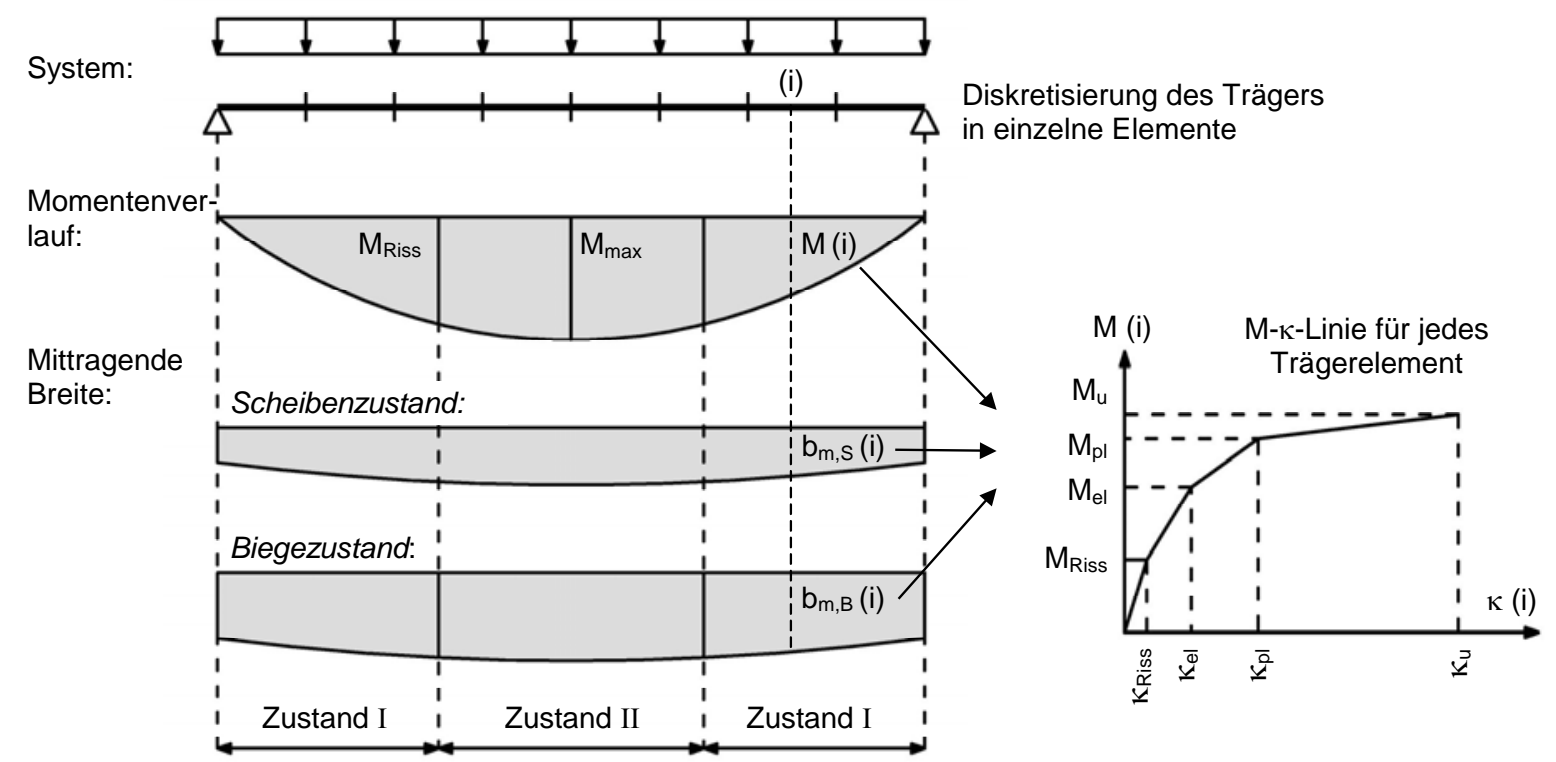

Abbildung 6-2: Schematische Darstellung des allgemeinen Berechnungsmodells

Die Breite des Betonquerschnitts der jeweiligen Trägerelemente wird entsprechend dem Verlauf der spannungsbezogenen mittragenden Breite in Trägerlängsrichtung angesetzt, der sich bei rein elastischen Systemverhalten ergibt. Da somit im Allgemeinen jedes Trägerelement eine andere Breite des Betongurts und damit einen anderen rechnerischen Querschnitt hat, weist jedes Trägerelemente ein geringfügig anderes Tragverhalten auf. Dadurch ist es notwendig, für jedes Element eine eigene M-к-Linie zu berechnen.

\subsubsection{Implementierte Materialmodelle}

Für eine wirklichkeitsnahe Berechnung der Steifigkeit und des Verformungsverhaltens der Verbundträger sind die verwendeten Materialmodelle von entscheidender Bedeutung. Im Gebrauchszustand der Träger hat dabei insbesondere das Verhalten des Betons auf Zug großen Einfluss, da mit Erreichen des Grenzzustands der Gebrauchstauglichkeit sich Stahlträger und Bewehrung im Allgemeinen noch elastisch verhalten. Bei höheren Belastungen ist hingegen die Beschreibung des Materialverhaltens des Stahls von großer Bedeutung. Die im entwickelten Berechnungsmodell verwendeten Materialmodelle tragen diesen beiden Umständen Rechnung.

Für das Materialmodell des Betons auf Druck wird das Parabel-Rechteck-Diagramm nach ENV 1992-1-1 [1.3], 4.2.1.3.3 (9) verwendet. Das Betonverhalten auf Zug wurde entsprechend Abbildung 6-3 (b) angesetzt. Bis zur Betonzugfestigkeit wird ein lineares Verhalten angenommen. Mit Erreichen der Rissdehnung $\varepsilon_{c t}$ fällt die Betonspannung auf $15 \%$ der Betonzugfestigkeit ab. Mit weiterer Steigerung der Dehnung nimmt die Spannung ab, bis mit Erreichen der Dehnung $\varepsilon_{\text {ctu }}$ die Spannung null wird. Dieser Verlauf ist an die von Hilfsdorf und Reinhardt in [2.64] beschriebene Spannungs-Rissöffnungs-Beziehung angelehnt.

Sowohl für den Stahlträger als auch die Bewehrung wird ein linearelastisch-idealplastisches Materialmodell verwendet, siehe Abbildung 6-4. Für die Berechnung der Verformungen im 
Gebrauchszustand der Verbundträger ist eine genauere Definition der Materialgesetze für Baustahl und Bewehrung nicht erforderlich, da bis zum Erreichen des Grenzzustands der Gebrauchstauglichkeit sowohl Baustahl als auch Bewehrung elastisch bleiben.

$\sigma_{\mathrm{c}} / \mathbf{f}_{\mathrm{c}}$

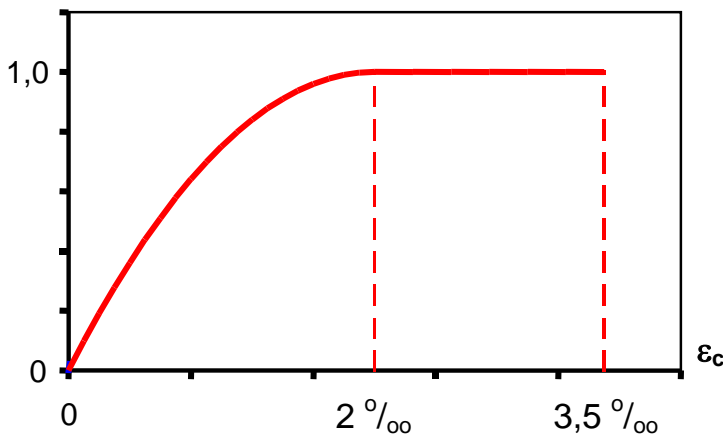

(a) Beton auf Druck

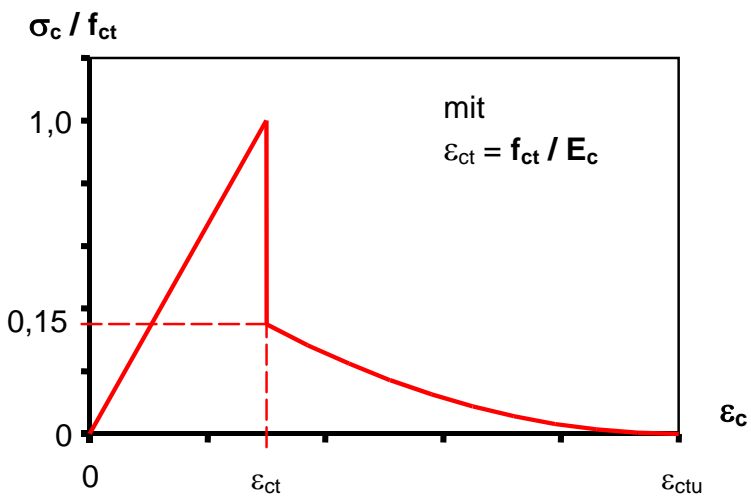

(b) Beton auf Zug

Abbildung 6-3: Verwendete Materialmodelle für den Beton

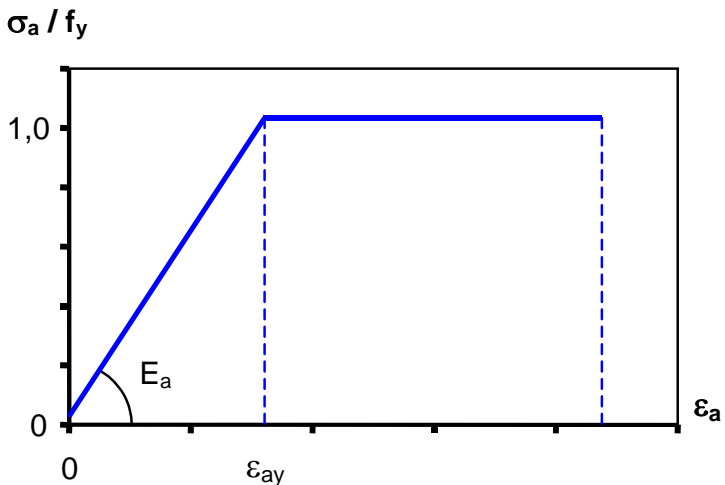

(a) Baustahl

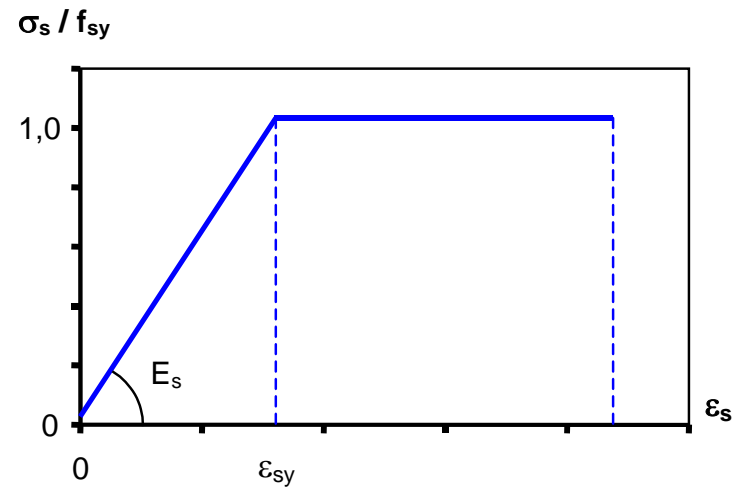

(b) Bewehrung

Abbildung 6-4: Verwendete Materialmodelle für Baustahl und Bewehrung

Da das Berechnungsmodell nur für Träger mit starrem Verbund entwickelt wurde, muss für die Verbundfuge kein Materialgesetz definiert werden.

\subsubsection{M- $\mathrm{K}$-Linien und Breite des Betongurts}

Für jedes Trägerelement wird eine individuelle $\mathrm{M}$-к-Linie berechnet. Bei den im Rahmen der vorliegenden Arbeit durchgeführten Untersuchungen wurden die M-к-Linien zwischen den charakteristischen Punkten linearisiert, siehe Abschnitt 2.3.3 und Abbildung 6-5. Als charakteristische Punkte dienen dabei:

- Rissbildung ( $\left.\mathrm{M}_{\text {Riss }}-\kappa_{\text {Riss }}\right)$ Im Beton wird erstmals die Rissdehnung $\varepsilon_{\mathrm{ct}}$ erreicht - im Allgemeinen an der Unterkante des Betonsgurts. Fortan ist der Beton im Zustand II. Die sich bildenden Risse werden dabei über die Länge der Trägerelemente verschmiert.

- Beginn des Plastizierens im Stahlträger $\left(\mathrm{M}_{\mathrm{el}}-\kappa_{\mathrm{el}}\right)$

An der höchstbelasteten Stelle im Stahlträger wird die Fließdehnung $\varepsilon_{a y}$ erreicht - im Allgemeinen an der Unterkante des Stahlträgers. Mit zunehmender Belastung durchläuft der Stahlträger teilplastische Zwischenzustände. 
- Stahlträger ist vollplastisch $\left(\mathrm{M}_{\mathrm{pl}}-\kappa_{\mathrm{pl}}\right)$

Mit Erreichen der Fließdehnung $\varepsilon_{a y}$ an der Stelle mit der geringsten Dehnung im Stahlträger, ist der Stahlträger vollplastisch. Bei weiterer Laststeigerung können zwar die Krümmung und die Dehnungen des Stahlträgers gesteigert werden, die Spannungen des Stahlträgers erhöhen sich dabei jedoch nicht mehr. Durch die zunehmende Querschnittskrümmung schnürt sich jedoch die Druckzone im Beton ein, die dadurch völliger wird. Dadurch kann der innere Hebelarm noch einmal gesteigert werden, was zu einer weiteren geringen Steigerung des aufnehmbaren Moments führt.

- Bruchzustand $\left(\mathrm{M}_{\mathrm{u}}-\kappa_{\mathrm{u}}\right)$

Wenn im Beton schließlich an der höchstbelasteten Stelle die Bruchdehnung $\varepsilon_{\mathrm{cu}}$ erreicht wird, kommt es rechnerisch zu einem Druckversagen des Betons. Eine weitere Laststeigerung ist dann nicht mehr möglich. Ein vorzeitiges Zugversagen des Stahlträgers wird bei der Berechnung zwar überprüft, wurde bei den durchgeführten Berechnungen jedoch nicht beobachtet.

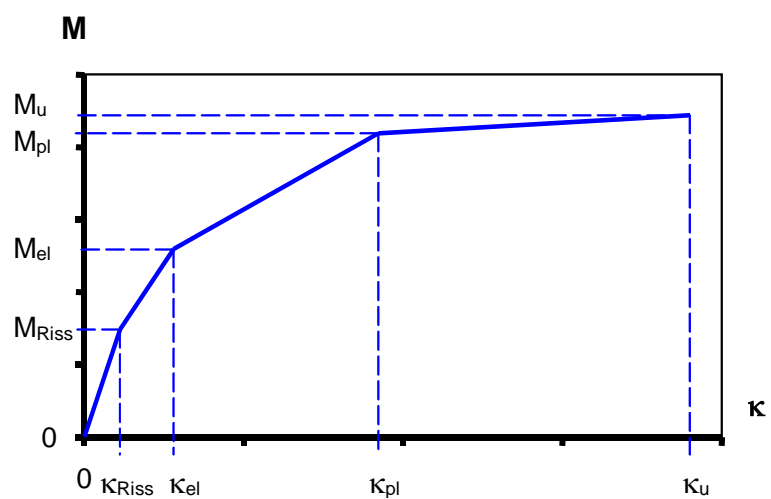

Abbildung 6-5: Linearisierte M-к-Linie

Bis zum Erreichen der Risskrümmung $\kappa_{\text {Riss }}$ verhält sich der Querschnitt elastisch. Der Risszustand kann somit nach den Regel der Elastizitätstheorie berechnet werden, siehe Kapitel 4. Die Bedingung für den Grenzzustand der Rissbildung ist, dass an der Stelle im Beton mit der größten Zugdehnung die Rissdehnung $\varepsilon_{\mathrm{ct}}$ erreicht wird [2.32]. Im Allgemeinen tritt dies an der Unterkante des Betons auf. Das effektive Trägheitsmoment des Verbundquerschnitts bis zum Erreichen des Rissmoments wird mit Gleichung (6-1) berechnet. Dabei wird für die Berechnung des Betonträgheitsmoments $I_{c, 0}$ als Breite des Betonquerschnitts die spannungsbezogene mittragende Breite $b_{m, B}$ des reinen Biegezustands verwendet. Für die Berechnung der Betonfläche $A_{c, 0}$ wird als Breite des Betonquerschnitts die spannungsbezogene mittragende Breite $b_{m, s}$ des reinen Scheibenzustands benutzt. Die Werte $b_{m, B}$ und $b_{m, S}$ werden dabei in Abhängigkeit der Belastungsfunktion, des Seitenverhältnisses $b / L$ des Betongurts und der Lage des Trägerelements im Träger verwendet.

$$
\begin{aligned}
I_{i, 0} & =I_{a}+I_{c, 0}+S_{i, 0} \cdot a_{S t} \\
& =I_{a}+\frac{b_{m, B} \cdot h_{c}^{3}}{12 \cdot n_{0}}+\frac{\frac{b_{m, S} \cdot h_{c}}{n_{0}} \cdot A_{a}}{\frac{b_{m, s} \cdot h_{c}}{n_{0}}+A_{a}} \cdot a_{S t}^{2}
\end{aligned}
$$

mit

$$
\begin{array}{ll}
\mathrm{I}_{\mathrm{i}, 0} & \text { elastisches Trägheitsmoment des Verbundquerschnitts } \\
\mathrm{I}_{\mathrm{a}} & \text { Trägheitsmoment des Stahlquerschnitts }
\end{array}
$$




$A_{a}$
$n_{0}=E_{a} / E_{c m}$
$b_{m, s}, b_{m, B}$
$h_{c}$
$a_{S t}$

$\mathrm{A}_{\mathrm{a}}$
Querschnittsfläche des Stahlquerschnitts

Reduktionszahl $\left(\mathrm{E}_{\mathrm{a}}=\mathrm{E}-\mathrm{Modul}\right.$ von Stahl, $\mathrm{E}_{\mathrm{cm}}=\mathrm{E}-$-Modul von Beton)

mittragende Breite des Betongurts für den reinen Scheiben- bzw. Biegezustand

Dicke des Betongurts

Schwerpunktabstand zwischen Beton- und Stahlquerschnitt

Bei weiterer Laststeigerung ist der Beton im Zustand II und die Berechnung kann daher nicht mehr linearelastisch erfolgen. Die weiteren Punkte der $\mathrm{M}$-к-Linie müssen durch Iteration bestimmt werden. Dabei wird die Dehnungsverteilung solange variiert, bis sich im Querschnitt ein Gleichgewichtszustands einstellt. Die Kräfte der Teilquerschnitte können mit Kenntnis der Materialmodelle aus der Dehnungsverteilung berechnet werden. Zweckmäßig wird der Betonquerschnitt rechnerisch in einen Druck- und einen Zugbereich unterteilt. Dem Betonquerschnitt wird dabei eine Druck- und eine Zugkraft zugewiesen.

Die Variation der Dehnungsverteilung wird bei der Berechnung der linearisierten M-к-Linie folgendermaßen vorgenommen: Da für die charakteristischen Punkte der M-к-Linie an einer Stelle die Dehnung bereits bekannt ist, wird die Variation der Dehnungsverteilung durch Variation der Querschnittskrümmung vorgenommen. Beim Beginn des Plastizierens des Stahlträgers $\left(\mathrm{Mel}_{\mathrm{el}}-\kappa_{\mathrm{el}}\right)$ ist zum Beispiel die Dehnung an der Unterkante des Stahlträgers, die gerade die Fließdehnung des Baustahls erreicht, bekannt.

Die Berechnung der Kräfte im Beton im Zustand II erfolgt unter der Verwendung folgender Breiten:

\section{- Betonzugkräfte}

Die Berechnung der Betonzugkräfte erfolgt unter Verwendung der spannungsbezogenen mittragenden Breite $b_{m, B}$ des reinen Biegezustands.

\section{- Betondruckkräfte}

Die Betondruckkräfte werden in einen Teil $\mathrm{N}_{\mathrm{c}, 1}$, der im Gleichgewicht mit der Zugkraft im Stahlträger steht, sowie einen Teil $\mathrm{N}_{\mathrm{c}, 2}$, der im Gleichgewicht mit der Betonzugkraft und der Zugkraft der Bewehrung steht, unterteilt. Die Betondruckkraft $\mathrm{N}_{c, 1}$, die im Gleichgewicht mit der Zugkraft im Stahlträger steht, wird mit der spannungsbezogenen mittragenden Breite $b_{m, s}$ des reinen Scheibenzustands berechnet. Die Betondruckkraft $\mathrm{N}_{c, 2}$, die im Gleichgewicht mit der Betonzugkraft und der Zugkraft der Bewehrung steht, wird mit der spannungsbezogenen mittragenden Breite $b_{m, B}$ des reinen Biegezustands berechnet.

Dieses Vorgehen entspricht demjenigen bei elastischem Materialverhalten. Hier wird der Betongurt durch eine Druckkraft $N_{c}$ und ein Betonmoment $M_{c}$ beansprucht. Das Betonmoment $M_{c}$ kann in ein Kräftepaar innerhalb des Betons aufgeteilt werden: eine Zugkraft $N_{c t, M}$ im unteren Teil des Betongurts und eine Druckkraft $N_{c, M}$ im oberen Teil. Die Betonzugkraft $N_{c t, M}$ und der Zugbereich im Beton resultieren folglich aus dem Biegezustand des Betongurts. Daher werden die Zugspannungen, die sich aus der Betonzugkraft $\mathrm{N}_{\mathrm{ct}, \mathrm{M}}$ ergeben, mit der mittragenden Breite $b_{m, B}$ des Biegezustands berechnet. Die Druckkraft im Beton setzt sich aus zwei Teilen zusammen: Zum einen der Druckkraft $N_{c, N}$, die sich aus dem inneren Kräftepaar im Verbundquerschnitt ergibt und im Gleichgewicht mit der Zugkraft $N_{a}$ im Stahlträger steht, und zum anderen der Druckkraft $\mathrm{N}_{\mathrm{c}, \mathrm{M}}$, die sich aus dem Betonmoment $\mathrm{M}_{\mathrm{c}}$ ergibt und im Gleichgewicht mit der Zugkraft $\mathrm{N}_{\mathrm{ct}, \mathrm{M}}$ im unteren Bereich des Betons steht. Der Druckkraftan- 
teil $N_{c, N}$ wird dabei mit der mittragenden Breite $b_{m, s}$ des Scheibenzustands berechnet und der Druckkraftanteil $\mathrm{N}_{\mathrm{c}, \mathrm{M}}$ mit der mittragenden Breite $\mathrm{b}_{\mathrm{m}, \mathrm{B}}$ des Biegezustands.

Wenn man dieses Vorgehen auf den Betonquerschnitt im Zustand II überträgt, bedeutet dies, dass Spannungsanteile des Betons, die aus einer Biegung des Betonquerschnitts resultieren, mit der mittragenden Breite $b_{m, B}$ des Biegezustands berechnet werden und Spannungsanteile des Betons, die im Gleichgewicht mit der Kraft im Stahlträger stehen, mit der mittragenden Breite $b_{m, s}$ des Scheibenzustands berechnet werden.

Weitere Hinweise zu M-к-Linien sind in [2.32], [2.37], [2.73]-[2.75] gegeben.

\subsubsection{Verformungsberechnung}

Nachdem für alle Trägerelemente die M-к-Linien bestimmt sind, wird aus der äußeren Belastung der Momentenverlauf berechnet. Im nächsten Schritt wird für jedes Element aus der entsprechenden $\mathrm{M}$ - $\kappa$-Linie die Krümmung im Elementmittelpunkt bestimmt. Dadurch ist der Krümmungsverlauf in Trägerlängsrichtung bekannt. Mit dem Prinzip der virtuellen Kräfte wird daraus die Durchbiegung des Trägers berechnet.

Grundsätzliche Hinweise zur Verformungsberechnung von Verbundträgern sind in Abschnitt 2.3 sowie [2.7], [2.32] gegeben.

\subsection{Voraussetzungen und Vereinfachungen}

Im Folgenden sollen die dem entwickelten Berechnungsmodell zugrunde liegenden Annahmen, die die Voraussetzungen und getroffenen Vereinfachungen erklären, kurz beschrieben werden:

- Der Betongurt weist eine konstante Dicke auf - sowohl in Längs- als auch in Querrichtung.

- Der Verbundträger wird als Einfeldträger ausgeführt.

Das Berechnungsmodell in der vorliegenden Form ermöglicht nur die Berechnung von Einfeldträgern. Eine Erweiterung auf Durchlaufträger ist jedoch grundsätzlich möglich.

- Die Träger weisen einen starren Verbund auf.

Zwischen Beton und Stahlträger entsteht kein nennenswerter Schlupf. Für Verbundträger mit voller Verdübelung stellt dies im Gebrauchszustand im Allgemeinen eine ausreichend genaue Vereinfachung dar.

- Ein Versagen der Verbundfuge wird ausgeschlossen.

- Die Bernoulli-Hypothese ist gültig.

Für Verbundträger mit starrem Verbund ist diese Annahme ausreichend genau.

- Die in Abschnitt 6.2.3 beschriebenen Materialmodelle beschreiben in ausreichender Genauigkeit das tatsächliche Verhalten der Teilquerschnitte.

- Risse im Beton werden über die Länge der Trägerelemente verschmiert und in eine mittlere Betonzugdehnung umgerechnet. Die Mitwirkung des Betons zwischen den Rissen wird durch eine effektive Betonzugspannung berücksichtigt.

Für die Betrachtung des globalen Verformungsverhaltens niedriger Verbundträger haben die lokalen Zusammenhänge im Rissquerschnitt keinen entscheidenden Ein- 
fluss. Die verschmierte Rissmethode ist für eine Verformungsberechnung daher ausreichend genau.

- M-к-Linien ermöglichen eine wirklichkeitsnahe Berechnung des nichtlinearen Tragund Verformungsverhaltens von Verbundträgern unter Berücksichtigung der Rissbildung des Betons und des Fließens von Stahl. Die abschnittsweise Linearisierung stellt dabei eine ausreichend genaue Vereinfachung dar.

- Im Berechnungsmodell wird nur eine Lage Längsbewehrung berücksichtigt. Eine eventuell vorhandene zweite obere Lage wird vernachlässigt. Es wird angenommen, dass die Längsbewehrung gleichmäßig verteilt über die Breite des Betongurts ist.

- Die Betonzugkraft $\mathrm{N}_{\mathrm{ct}}$ wird unter der Annahme berechnet, dass die Breite des Betonzugbereichs gleich der spannungsbezogenen mittragenden Breite $b_{m, B}$ des reinen Biegezustands ist, siehe Abschnitt 6.2.4.

- Die Betondruckkraft wird in zwei Teile unterteilt: den Druckkraftanteil $N_{c, N}$, der im Gleichgewicht mit der Zugkraft im Stahlträger steht, und den Druckkraftanteil $\mathrm{N}_{c, \mathrm{M}}$, der im Gleichgewicht mit der Betonzugkraft $N_{c t}$ und der Zugkraft in der Bewehrung $N_{s}$ steht, siehe Abschnitt 6.2.4.

- Der Betondruckkraftanteil $\mathrm{N}_{\mathrm{c}, \mathrm{N}}$, der im Gleichgewicht mit der Zugkraft im Stahlträger steht, wird unter der Annahme berechnet, dass die Breite des Betondruckbereichs gleich der spannungsbezogenen mittragenden Breite $b_{m, s}$ des reinen Scheibenzustands ist, siehe Abschnitt 6.2.4.

- Der Betondruckkraftanteil $\mathrm{N}_{\mathrm{c}, \mathrm{M}}$, der im Gleichgewicht mit der Betonzugkraft und Zugkraft der Bewehrung steht, wird unter der Annahme berechnet, dass die Breite des Betondruckbereichs gleich der spannungsbezogenen mittragenden Breite $b_{m, B}$ des reinen Biegezustands ist, siehe Abschnitt 6.2.4.

- Die Werte der spannungsbezogenen mittragenden Breite des Scheibenzustands $b_{m, s}$ und des Biegezustands $b_{m, B}$ werden entsprechend dem linearelastischen Fall angesetzt, siehe Kapitel 4. Es wird angenommen, dass diese Werte der mittragenden Breite auch im Zustand II gültig sind und die Rissbildung keinen Einfluss auf die Verteilung der spannungsbezogenen mittragenden Breiten hat bzw. dieser Einfluss vernachlässigbar ist.

Die mittragende Breite des Scheibenzustands ist im Wesentlichen von der Einleitung der Längsschubkräfte und die mittragende Breite des Biegezustands von den Plattenquerkräfte abhängig. Da diese durch die Rissbildung nur unwesentlich verändert werden, wird davon ausgegangen, dass die Rissbildung auch keinen wesentlichen Einfluss auf die Werte der spannungsbezogenen mittragenden Breite hat.

- Die Zunahme der mittragenden Breite bei großen Betondehnungen wird vernachlässigt.

Da mit zunehmenden Betondehnungen die Spannungs-Dehnungs-Linie des Betons im Druckbereich flacher wird, wird die Querverteilung der Betonlängsspannungen ausgeglichener als die zugehörige Dehnungsverteilung und die spannungsbezogene mittragende Breite wird größer.

Verformungen von niedrigen Verbundträgern werden im Allgemeinen im Gebrauchszustand berechnet. Da im Gebrauchszustand die Betondruckdehnungen noch vergleichsweise klein sind, kann hier die Vergrößerung der mittragenden Breite infolge der flacher werdenden Spannungs-Dehnungs-Beziehung vernachlässigt werden. 
- Ein Einfluss der Querbiegung auf das Trag- und Verformungsverhalten sowie die Verteilung der spannungsbezogenen mittragenden Breite wird vernachlässigt.

Bei elastischem Materialverhalten haben die Quer- und Längsbiegung des Betongurts aufgrund des Superpositionsprinzips keinen wechselseitigen Einfluss. Für den Ansatz der spannungsbezogenen mittragenden Breite im Zustand II ist dies eine Annahme, die durch weitere Untersuchungen bestätigt werden sollte.

- Die Durchbiegung des Verbundträgers infolge Querkraft wird elastisch am Stahlträger bestimmt. Hierfür muss die Aufteilung der Querkraft auf die Teilquerschnitte Beton und Stahlträger bekannt sein oder eine Annahme getroffen werden.

Die beschriebenen Annahmen wurden so getroffen, dass hiermit eine wirklichkeitsnahe Verformungsberechnung niedriger Verbundträger möglich ist. Dadurch unterliegt das analytische Berechnungsmodell jedoch unter Anderem folgenden Bedingungen:

- Eine Berechnung von Spannungen im Beton ist nur in der Symmetrieachse möglich. Insbesondere kann die Querverteilung der Betonspannungen nicht berechnet werden.

- Verbundträger mit ausgeprägtem Schlupf zwischen Beton und Stahlträger, zum Beispiel infolge einer teilweisen Verdübelung, können nicht berechnet werden.

- In der vorliegenden Form ermöglicht das Berechnungsmodell die Berechnung von Einfeldträgern. Eine Erweiterung auf Durchlaufträger ist jedoch ohne große Änderungen möglich.

\subsection{Verifikation des analytischen Berechnungsmodells}

\subsubsection{Grundsätzliches}

Der Nachweis, dass das entwickelte Berechnungsmodell für niedrige Verbundträger wirklichkeitsnahe Verformungen berechnet, erfolgt durch Vergleich mit vorhandenen Versuchsergebnissen. Eine sinnvolle Überprüfung des Berechnungsmodells setzt dabei voraus, dass die zum Vergleich herangezogenen Versuchsträger einen breiten Betongurt haben und das spezielle Tragverhalten niedriger Verbundträger aufweisen. Nur so kann überprüft werden, ob der Einfluss der Schubweichheit des Betongurts auf das Verformungsverhalten auch bei gleichzeitiger Rissbildung und großer Biegetragwirkung des Betongurts vom Berechnungsmodell ausreichend genau abgebildet wird. Somit sind für eine Überprüfung des Berechnungsmodell nur Versuche an niedrigen Verbundträgern mit breiten Gurten sinnvoll. Neben den eigenen in Kapitel 5 beschriebenen Versuchen sind dem Verfasser nur ein Versuch von Amadio, et. al. an einem niedrigen Verbundträger [2.63] und ein Versuch von Kuhlmann, Fries an einem Slim-Floor Träger [2.2], [2.4] bekannt, die für eine Überprüfung des Berechnungsmodells sinnvoll sind.

Auf die Nachrechnung von Versuchsträgern, deren Betongurt sehr schmal ist, wird an dieser Stelle verzichtet, da bei schmalen Gurten kein nennenswerter Einfluss der Schubweichheit des Betongurts besteht und somit hier nicht überprüft werden kann, ob vom Berechnungsmodell der Einfluss der kombinierten Wirkung aus Rissbildung und Schubweichheit des Betongurts richtig erfasst wird. Ein solcher Vergleich könnte nur die Methode der M-к-Linien zur prinzipiellen Beschreibung des nichtlinearen Verformungsverhaltens von Verbundquerschnitten bestätigen. Dies wird an dieser Stelle jedoch als allgemein anerkannt vorausgesetzt [2.32], [2.37], [2.73]-[2.75].

Auch werden keine Versuche mit hohen Verbundträgern nachgerechnet, da bei hohen Verbundträgern bis nahe an die Traglast keine Risse im Betongurt entstehen. 
Eine Überprüfung von im Versuch gemessenen Dehnungen und damit auch der spannungsbezogenen mittragenden Breite kann mit dem entwickelten Berechnungsmodell nicht erfolgen, siehe Abschnitt 6.2.

Über eine Verifikation des Berechnungsmodells durch Vergleich mit Versuchen hinaus kann das Berechnungsmodell durch Überprüfung der vergleichsweise einfachen mechanischen Grundlagen, siehe Abschnitt 6.2, nachvollzogen werden.

\subsubsection{Vergleich mit den eigenen experimentellen Untersuchungen}

Die Beschreibung der durchgeführten Versuche ist ausführlich in Kapitel 5 bzw. [2.27] gegeben. Da das Berechnungsmodell für Verbundträger mit starrem Verbund entwickelt wurde, kann der Versuchsträger VT 3 mit teilweiser Verdübelung zur Überprüfung des Berechnungsmodells nicht herangezogen werden.

Tabelle 6-1: Vergleich der rechnerischen Verformungen des analytischen Berechnungsmodells mit den experimentellen Verformungen der Versuchsträger im Bereich der Gebrauchslast

\begin{tabular}{|c|c|c|c|c|c|c|}
\hline & VT 1 & VT 2 & VT 4 & VT 5 & VT 6 & Mittelwert \\
\hline $\mathrm{P}_{\mathrm{GZG}}{ }^{1)}[\mathrm{kN}]$ & 298 & 305 & 308 & 493 & 493 & -- \\
\hline $\mathrm{f}_{\exp }{ }^{2)} \quad[\mathrm{mm}]$ & 10,1 & 8,6 & 8,9 & 8,0 & 8,0 & -- \\
\hline $\mathrm{f}_{\text {rechn }}-\mathrm{f}_{\exp }{ }^{3)}[\mathrm{mm}]$ & 0,29 & 0,23 & 0,78 & 0,12 & 0,10 & 0,30 \\
\hline $\mathrm{f}_{\text {rechn }} / \mathrm{f}_{\exp }-1$ & $3,0 \%$ & $2,8 \%$ & $9,0 \%$ & $2,0 \%$ & $1,2 \%$ & $3,6 \%$ \\
\hline
\end{tabular}

\footnotetext{
1) $P_{\text {GzG }} \quad$ Last im Grenzzustand der Gebrauchslast

2) $\mathrm{f}_{\exp } \quad$ Mittendurchbiegung der Versuchsträger

${ }^{3)} \mathrm{f}_{\text {rechn }} \quad$ rechnerische Mittendurchbiegung der Versuchsträger (analytisches Berechnungsmodell)
}

In Tabelle 6-1 ist ein Vergleich der Verformungen der Versuchsträger bei einer Berechnung mit dem analytischen Berechnungsmodell mit den experimentellen Werten im Grenzzustand der Gebrauchstauglichkeit dargestellt. Die Abweichungen der Ergebnisse des Berechnungsmodells zu den Versuchen betragen im Mittel 3,6\%. Abbildung 6-6 zeigt einen Vergleich der rechnerischen und experimentellen Last-Verformungs-Kurven, die sehr gut übereinstimmen. Die rechnerischen Werte berücksichtigen dabei die Querkraftverformung des Stahlträgers für die bei den Versuchen am Stahlträger gemessene Querkraft. 


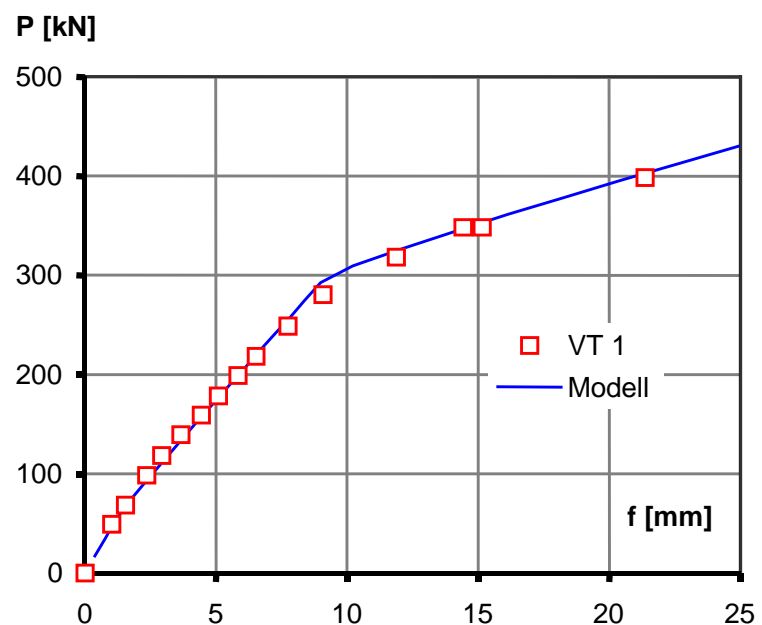

(a) VT 1

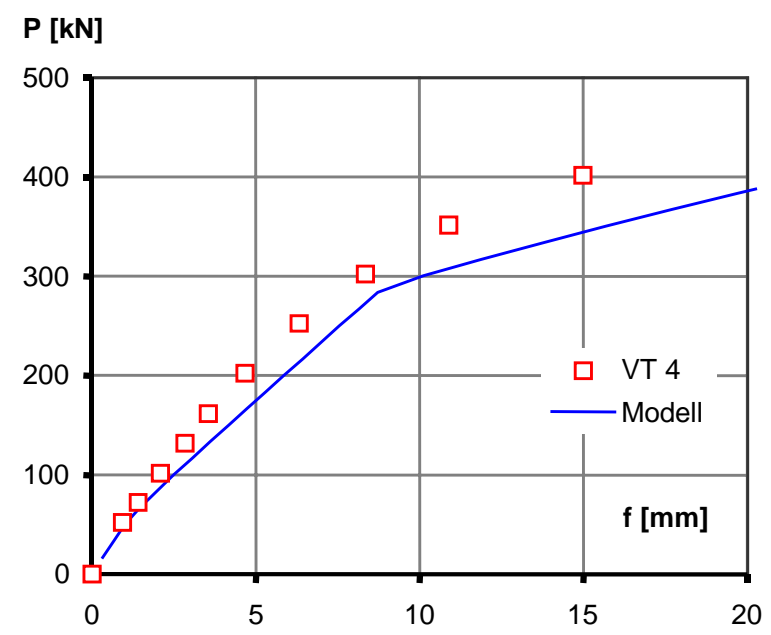

(c) VT 4

\section{$\mathrm{P}[\mathrm{kN}]$}

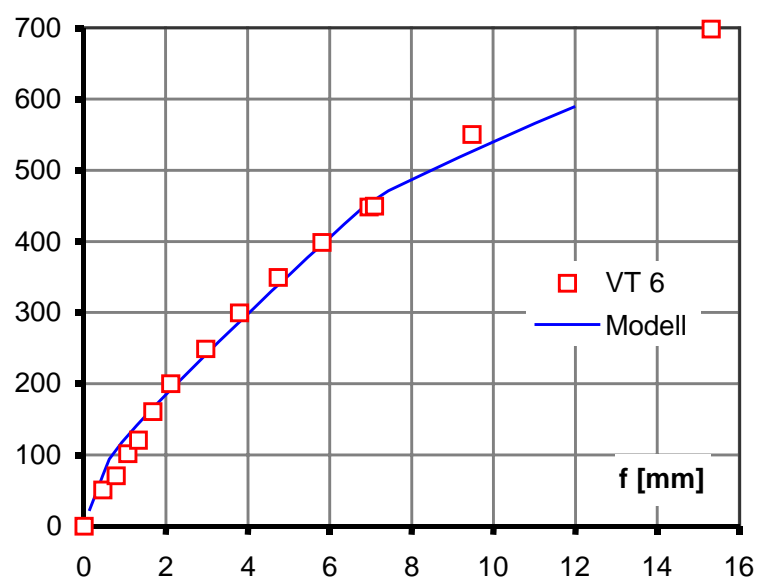

(e) $V T 6$

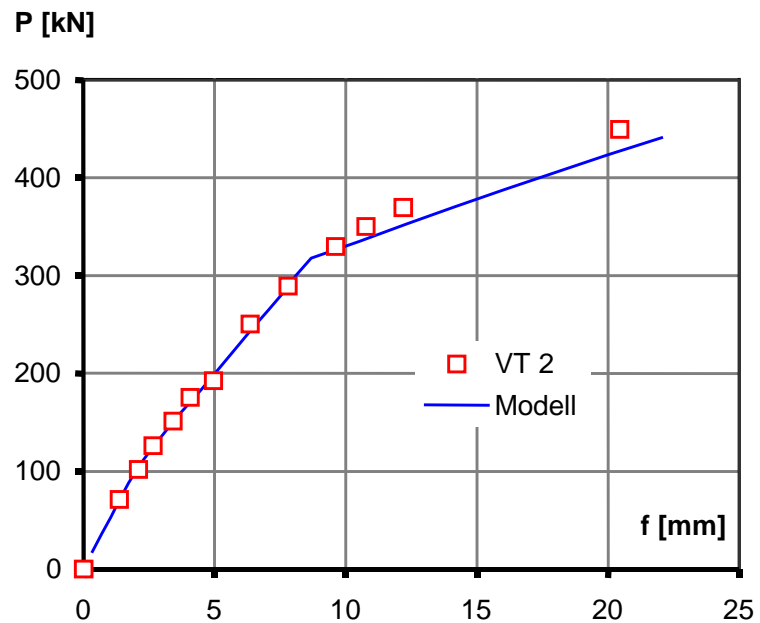

(b) $V T 2$

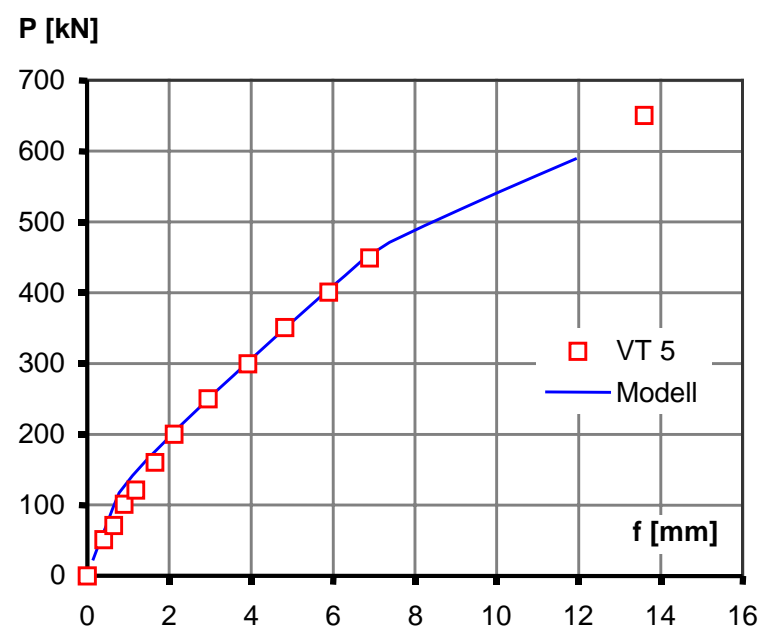

(d) VT 5

Abbildung 6-6: Vergleich der rechnerischen und experimentellen Last-Verformungs-Kurven der Versuchsträger [2.27] 


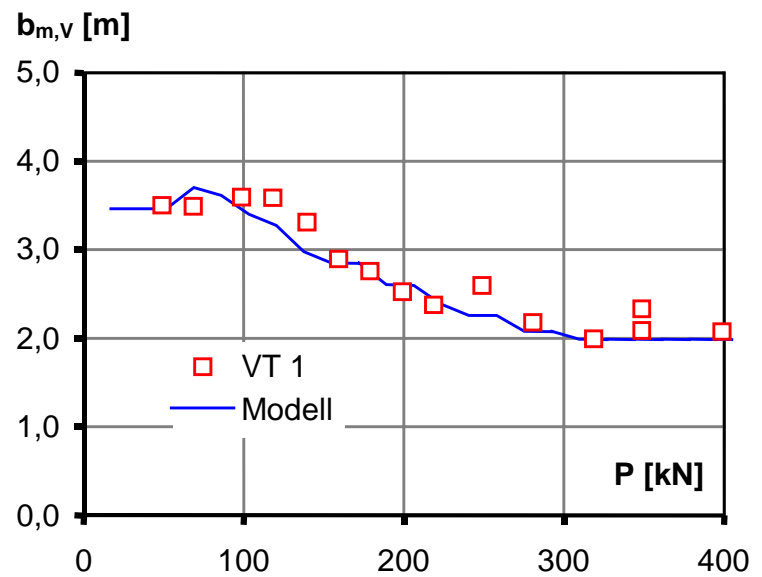

(a) $V T 1$

$\mathbf{b}_{\mathrm{m}, \mathrm{v}}[\mathrm{m}]$

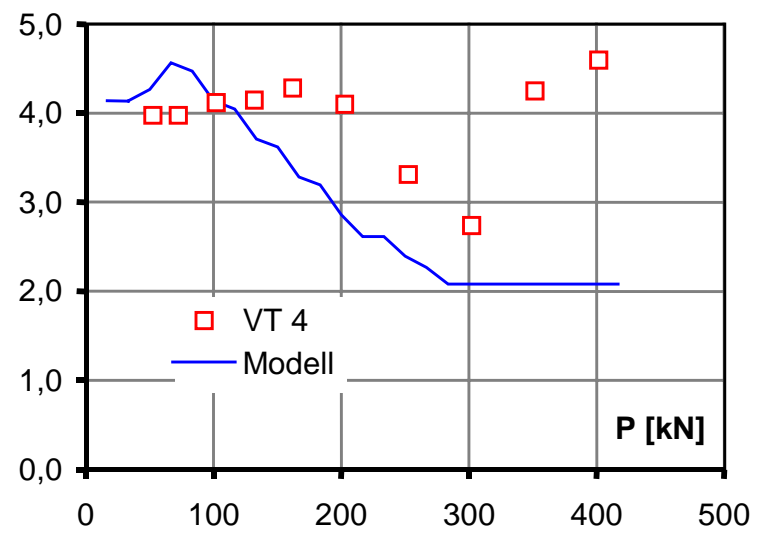

(c) VT 4

$\mathbf{b}_{\mathrm{m}, \mathrm{v}}[\mathrm{m}]$

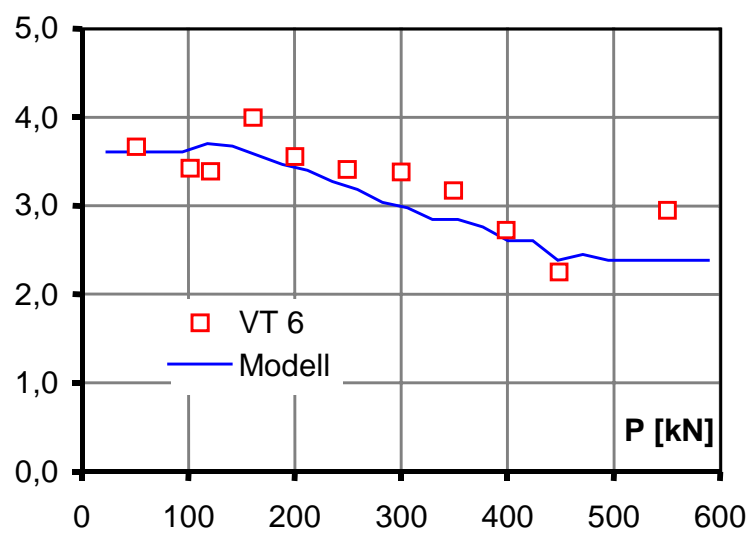

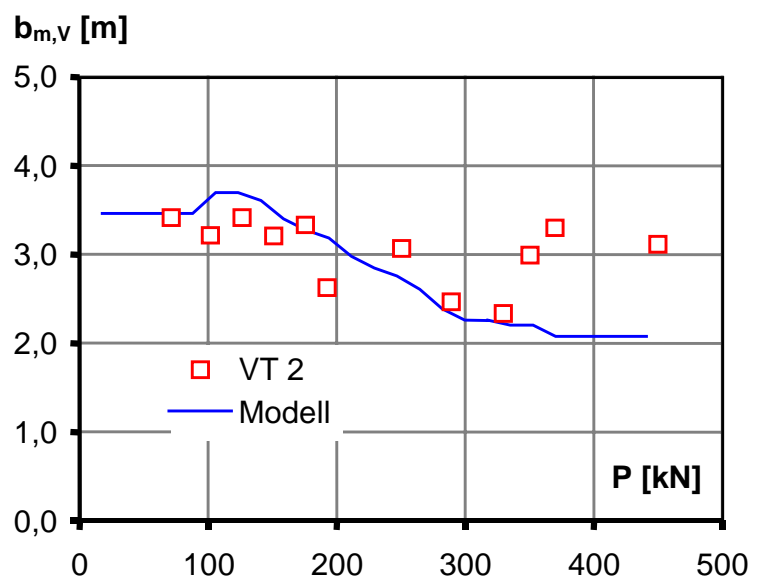

(b) $V T 2$

$\mathbf{b}_{\mathrm{m}, \mathrm{v}}[\mathrm{m}]$

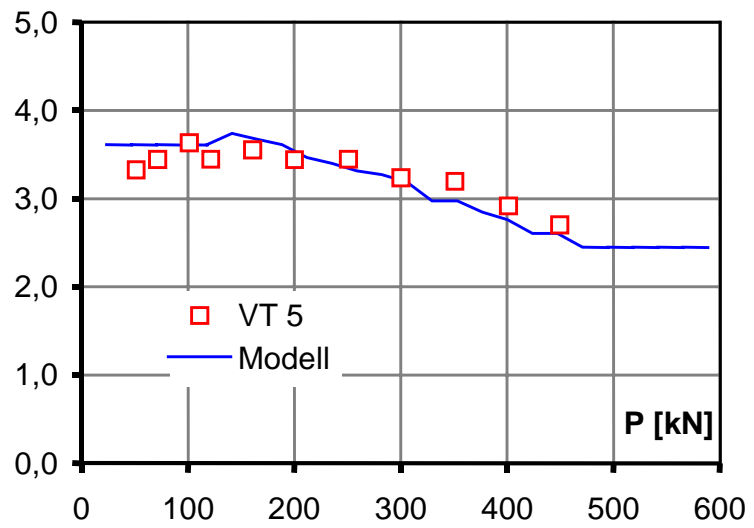

(d) VT 5

(e) $V T 6$

Abbildung 6-7: Vergleich der verformungsbezogenen mittragenden Breiten $b_{m, v}$ der Versuchsträger in Abhängigkeit der Versuchlast

$\mathrm{Da}$ dem analytischen Berechnungsmodell die elastischen Werte der spannungsbezogenen mittragenden Breite zugrunde liegen, wird bei diesem die Zunahme der spannungsbezogenen Breite bei großen Betondehnungen infolge der nichtlinearen Spannungs-DehnungsBeziehung des Betons nicht berücksichtigt. Deshalb nimmt die verformungsbezogene mittragende Breite des analytischen Berechnungsmodells stetig ab. 
Die beobachteten Abweichungen zwischen den experimentellen und den rechnerischen Verläufen der verformungsbezogenen mittragenden Breite $b_{m, v}$ haben auf eine praktische Verformungsberechnung jedoch keinen Einfluss, da Verformungen im Allgemeinen im Gebrauchszustand untersucht werden und die Verläufe hier eine gute Übereinstimmung aufweisen. Für höhere Belastungen liefert das Berechnungsmodell kleinere Werte für die verformungsbezogene mittragende Breite $b_{m, v}$ und liegt damit auf der sicheren Seite.

\subsubsection{Vergleich mit den experimentellen Untersuchungen von Amadio et al. [2.63]}

Von Amadio et al. wird in [2.63] von sechs Versuchen an Verbundträgern mit breiten Betongurten berichtet, siehe Abschnitt 3.5.3. Dabei wurde der Versuch B4 mit einer positiven Momentenbelastung entsprechend Abbildung 6-8 durchgeführt und kann somit zur Überprüfung des entwickelten Berechnungsmodells verwendet werden.
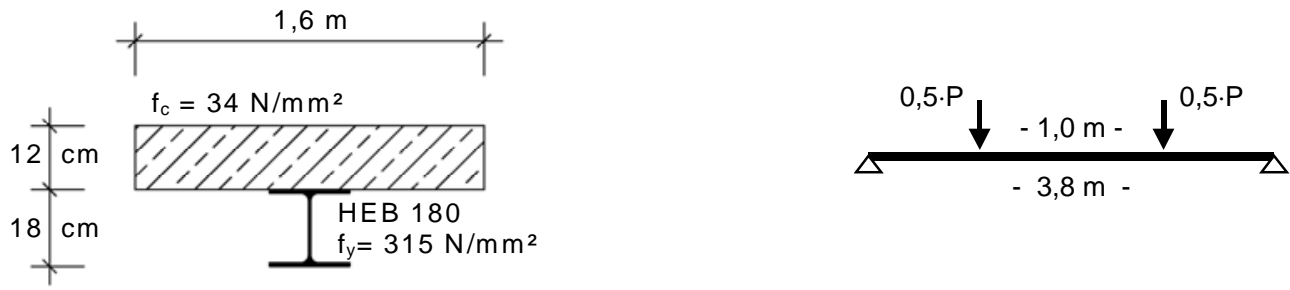

(a) Querschnitt

(b) System

Abbildung 6-8: System und Querschnitt des Versuchsträgers B4 nach Amadio et al. [2.63]

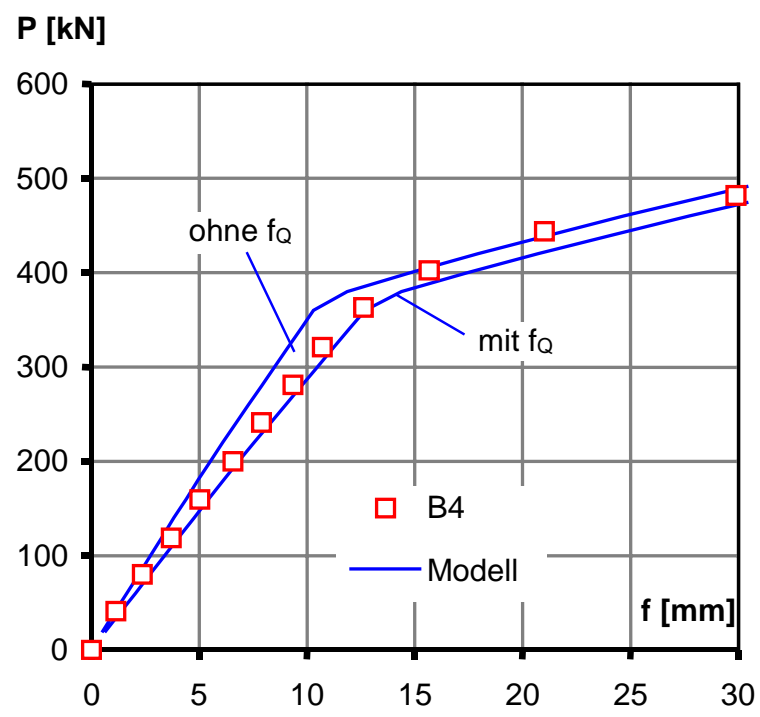

(a) Vergleich der Last-Verformungs-Kurven $\mathbf{b}_{\mathrm{m}, \mathrm{v}}[\mathrm{m}]$

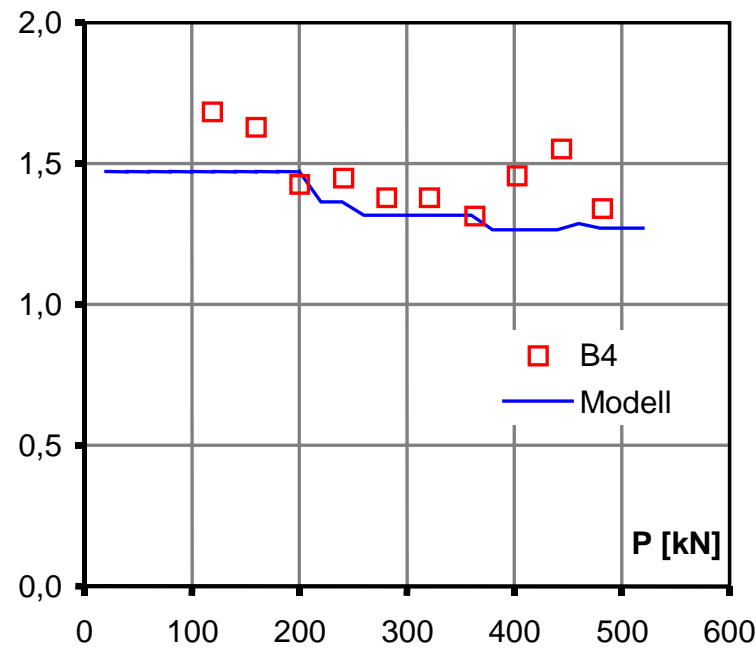

(b) Vergleich der verformungsbezogenen mittragenden Breite $b_{m, v}$

Abbildung 6-9: Vergleich der rechnerischen und experimentellen Ergebnisse des Versuchsträgers B4 nach Amadio et al. [2.63]

Der Vergleich der rechnerischen Last-Verformungs-Kurve mit der experimentellen zeigt eine gute Übereinstimmung, siehe Abbildung 6-9. Es sind zwei rechnerische Grenzkurven eingezeichnet: ohne Berücksichtigung einer Querkraftverformung (obere Grenze) und mit Berücksichtigung der Querkraftverformung unter der Annahme, dass die gesamte Querkraft vom Steg des Stahlträgers abgetragen wird (untere Grenzkurve). Die experimentellen Werte liegen zwischen diesen beiden rechnerischen Grenzkurven. Die Abweichung beträgt im 
Grenzzustand der Gebrauchsfähigkeit bei ca. 340 kN für die Rechnung mit Querkraftverformung nur ca. 3,4\%. Die rechnerischen Verformungen zeigen somit eine sehr gute Übereinstimmung mit den gemessenen Werten.

Für den Verlauf der verformungsbezogenen mittragenden Breite $b_{m, v}$ zeigt sich ein vergleichbarer Verlauf wie bei den eigenen experimentellen Untersuchungen, siehe Abschnitt 6.4.2. Dies gilt auch für die bei hoher Belastung vorliegenden Abweichungen. Insgesamt kann jedoch festgestellt werden, dass das entwickelte Berechnungsmodell in der Lage ist, die Verformungen des Versuchsträgers zuverlässig abzubilden.

\subsubsection{Vergleich mit den experimentellen Untersuchungen von Kuhlmann, Fries [2.4]}

Von Kuhlmann und Fries wird in [2.4] von neun Versuchen an Slim-Floor Trägern mit UPEProfilen berichtet. Versuchsträger VT 6 dieser Versuchsreihe wurde mit positiver Momentenbelastung und voller Verdübelung durchgeführt und kann somit mit dem entwickelten Berechnungsmodell berechnet werden. System und Abmessungen des Versuchsträger VT 6 kann Abbildung 6-10 entnommen werden.

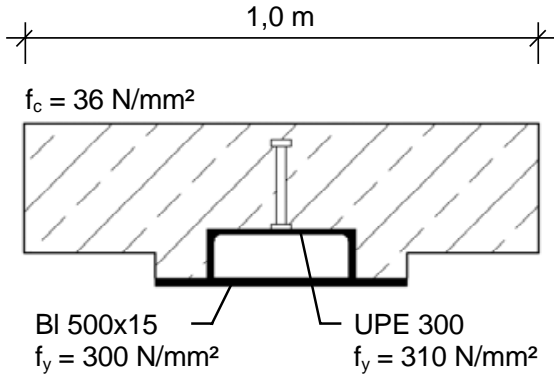

(a) Querschnitt

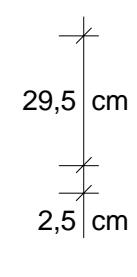

(b) System

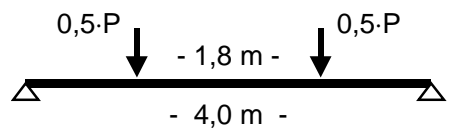

Abbildung 6-10: System und Querschnitt des Versuchsträgers VT 6 nach Kuhlmann, Fries [2.4]

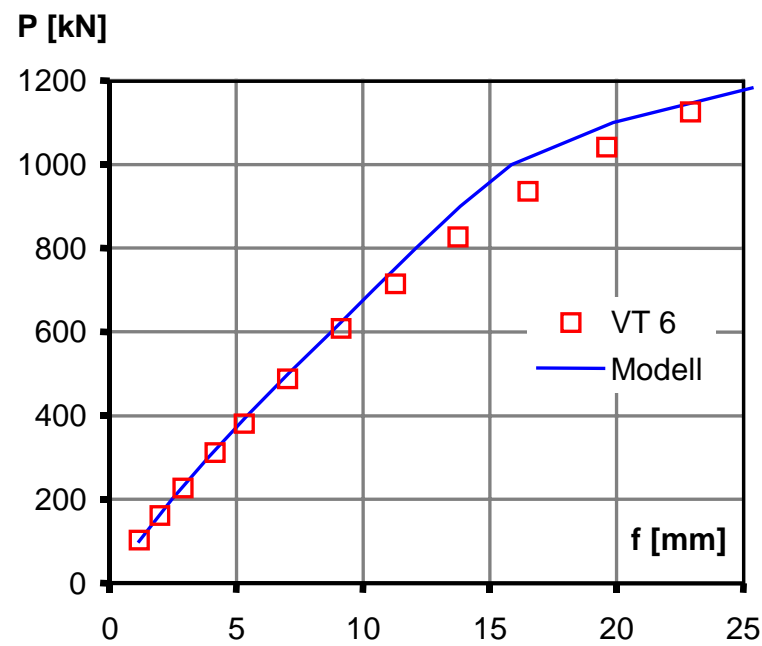

Abbildung 6-11: Vergleich der rechnerischen und experimentellen Last-Verformungs-Kurve des Versuchsträgers VT 6 nach Kuhlmann, Fries [2.4]

Der Vergleich der rechnerischen Last-Verformungs-Kurve mit der experimentellen zeigt eine gute Übereinstimmung, siehe Abbildung 6-11. Die mittlere Abweichung bis zum Erreichen 
des Grenzzustands der Gebrauchstauglichkeit bei ca. $900 \mathrm{kN}$ beträgt 3,2\%, im GZG ca. $11,0 \%$. Die rechnerischen Werte berücksichtigen dabei die Querkraftverformung des Stahlträgers für die bei den Versuchen gemessene Querkraft im Stahlträger.

Ein Vergleich der verformungsbezogenen mittragenden Breite $b_{m, v}$ macht für diesen Träger aufgrund der kleinen Breite des Betongurts und des kleinen Seitenverhältnissen keinen Sinn. Hier kann von einem Mitwirken der vollen Breite des Betongurts ausgegangen werden.

\subsubsection{Fazit der Vergleiche mit Versuchsergebnissen}

Der Vergleich der Last-Verformungs-Kurven und der verformungsbezogenen mittragenden Breite der vorliegenden experimentellen Untersuchungen mit den Ergebnissen des analytischen Berechnungsmodells zeigen eine gute Übereinstimmung. Die festgestellten Abweichungen liegen im üblichen Rahmen bei Nachrechnungen von Versuchsträgern. Darüber hinaus basiert das Berechnungsmodell auf nachvollziehbaren mechanischen Grundlagen, die die wesentlichen Effekte des Trag- und Verformungsverhalten niedriger Verbundträger beschreiben können. Es kann daher davon ausgegangen, dass mit dem entwickelten Berechnungsmodell wirklichkeitsnahe Verformungen niedriger Verbundträger berechnet werden können.

\subsection{Zusammenfassung}

Auf Basis der in Kapitel 4 beschriebenen elastischen Untersuchungen zur mittragenden Breite von Plattenbalken wurde ein analytisches Berechnungsmodell zur Verformungsberechnung niedriger Verbundträger entwickelt, das die maßgebenden Einflüsse auf das nichtlineare Trag- und Verformungsverhalten und die mittragende Breite dieser Träger berücksichtigt. Hierbei wurde vor allem Augenmerk auf den Einfluss des Biegezustands und der Rissbildung des Betongurts auf die mittragende Breite gelegt.

Die Anwendbarkeit des entwickelten Modells wurde durch Vergleich mit den Ergebnissen der eigenen durchgeführten Versuche und in der Literatur beschriebenen Versuchen verifiziert. Dabei zeigen die rechnerischen und experimentellen Last-Verschiebungs-Kurven eine sehr gute Übereinstimmung und der Vergleich der verformungsbezogenen mittragenden Breite eine gute Übereinstimmung.

Mit dem Berechnungsmodell kann somit das Verformungsverhalten niedriger Verbundträger wirklichkeitsnah simuliert werden. Dadurch können die Einflüsse auf die verformungsbezogene mittragende Breite anhand der in Kapitel 7 beschriebenen Parameterstudie auf Basis des beschriebenen analytischen Berechnungsmodells systematisch untersucht werden. 



\section{Herleitung eines Berechnungsansatzes für die verformungs- bezogene mittragende Breite niedriger Verbundträger}

\subsection{Einführung}

Aufgrund der Schubweichheit des Betongurtes entziehen sich Bereiche des Betongurts der Lastabtragung, die vom Stahlträger weiter entfernt sind. Bei der Spannungsberechnung wird dies durch die mittragende Breite berücksichtigt. In Trägerlängsrichtung ändert sich diese spannungsbezogene mittragende Breite und somit auch das Trägheitsmoment des Verbundträgers, siehe Abschnitt 3.5 und Kapitel 4. Da man in der Planungspraxis jedoch bestrebt ist, die Verformungsberechnung an einem Träger mit konstantem Querschnitt durchzuführen, wurde in Abschnitt 3.3.3 die verformungsbezogene mittragende Breite $b_{m, v}$ eingeführt. Der Zusatz "verformungsbezogen“ deutet dabei darauf hin, dass dies eine Größe zur Berechnung von Verformungen ist und nicht von Spannungen. Die zur Bestimmung der verformungsbezogenen mittragenden Breite durchgeführten Untersuchungen unter Beachtung des speziellen Trag- und Verformungsverhalten niedriger Verbundträger werden in diesem Kapitel beschrieben.

Hierfür wurde das in Abschnitt 6.2 beschriebene analytische Berechnungsmodell entwickelt, das sowohl das spezielle Trag- und Verformungsverhalten niedriger Verbundträger als auch alle wesentlichen Einflüsse auf die mittragende Breite abbildet. Auf Basis dieses Berechnungsmodells wurden die Einflüsse auf die verformungsbezogene mittragende Breite untersucht und im Rahmen einer umfangreichen Parameterstudie quantifiziert. Schließlich mündeten diese Erkenntnisse in der Entwicklung eines anwenderfreundlichen Berechnungsansatzes für die verformungsbezogene mittragende Breite, der eine wirklichkeitsnahe Verformungsberechnung niedriger Verbundträger bei vergleichsweise geringem Berechnungsaufwand ermöglicht. Darüber hinaus wird für weitere im Berechnungsansatz nicht berücksichtigte Parameter eine Abschätzung getroffen.

Die Ausgangssituation zur mittragenden Breite ist in Abschnitt 6.1.2 dargestellt. Mit Ausnahme der eigenen in Kapitel 5 beschriebenen experimentellen Untersuchungen liegen keine Arbeiten vor, die die verformungsbezogene mittragende Breite $b_{m, v}$ niedriger Verbundträger unter Berücksichtigung der Biegetragwirkung und der Rissbildung des Betongurts systematisch untersuchen. Für eine wirklichkeitsnahe Verformungsberechnung niedriger Verbundträger sind jedoch gerade diese Einflüsse maßgebend.

Zunächst wird zum besseren Verständnis die Definition der verformungsbezogenen mittragenden Breite $b_{m, v}$ kurz wiederholt, bevor die Einflüsse auf diese diskutiert werden und der entwickelte Berechnungsansatz vorgestellt wird.

\subsection{Bestimmung der verformungsbezogenen mittragenden Breite $b_{m, v}$}

Bei der verformungsbezogenen mittragenden Breite $b_{m, v}$ handelt es sich um einen ideellen Wert für die rechnerische Breite des Betongurts, der der vereinfachten Verformungsberechnung dient. Anstatt einer aufwändigen Berechnung des genauen dreidimensionalen Tragverhaltens des Verbundträgers mit breitem Betongurt wird eine vereinfachte Berechnung an einem Träger mit konstanter Breite und konstantem Querschnitt vorgenommen. Dieser Träger wird im Folgenden mit Ersatzbalken bezeichnet. Die Breite des Betongurts des Ersatzbalkens wird dabei so groß gewählt, dass der Wert der rechnerischen Verformung des Ersatzbalkens demjenigen des realen dreidimensionalen System entspricht. Diese Breite wird als verformungsbezogene mittragende Breite $b_{m, v}$ definiert, siehe Abschnitt 3.3.3. 


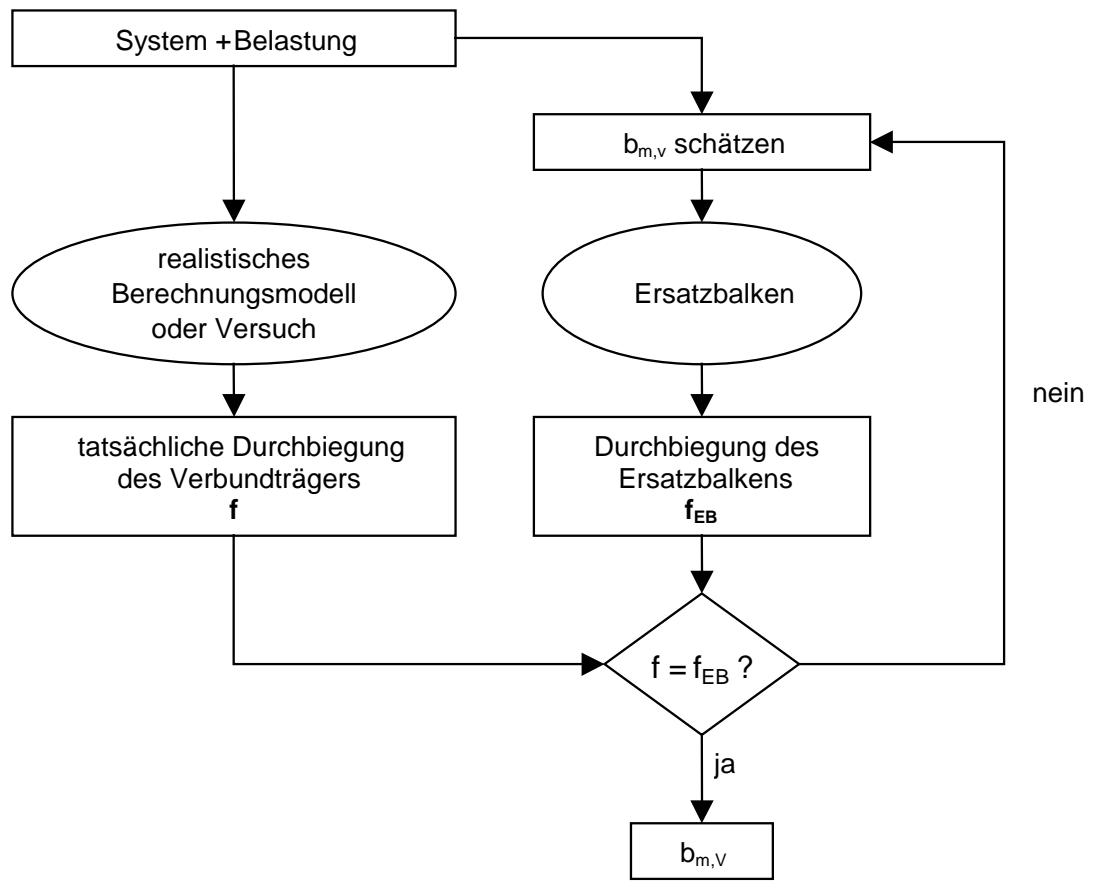

Abbildung 7-1: Berechnung der verformungsbezogenen mittragenden Breite $b_{m, v}$

Mit Kenntnis des tatsächlichen Last-Verformungs-Verhaltens eines Trägers, zum Beispiel aus Versuchen oder einer genauen Berechnung, kann die verformungsbezogene mittragende Breite $b_{m, v}$ für diesen Träger rückgerechnet werden. Dazu wird die rechnerische Breite des Ersatzbalkens solange variiert, bis die rechnerische Verformung des Ersatzbalkens mit der tatsächlichen Verformung des Verbundträgers übereinstimmt. Die so gefundene Breite des Ersatzbalkens wird als verformungsbezogene mittragende Breite $b_{m, v}$ definiert. Eine direkte Berechnung der verformungsbezogenen mittragenden Breite ist dabei aufgrund des nichtlinearen Material- und Tragverhaltens niedriger Verbundträger in der Regel nicht möglich. Abbildung 7-1 stellt dieses Vorgehen schematisch dar.

Da die verformungsbezogene mittragende Breite von der Rissbildung und damit auch von der Belastungshöhe des Trägers abhängt, ist sie kein Systemwert, der nur von den Abmessungen und Materialwerten abhängt, sondern auch von der Belastung. Aus diesem Grund wird die verformungsbezogene mittragende Breite in Abhängigkeit der Belastungshöhe bestimmt und kann in einem $b_{m, v}-M / M_{u}$-Diagramm dargestellt werden, siehe z. B. Abbildung 7-2. Die Belastungshöhe wird dabei als Verhältnis des maximalen Moments $M$ in Feldmitte zum Bruchmoment des Trägers $M_{u}$ dargestellt. Das Verhältnis $M / M_{u}$ kann auch als Ausnutzungsgrad des Trägers betrachtet werden. Bis zur Rissbildung $M_{R i s s} / M_{u}$ verhält sich der Träger elastisch und die verformungsbezogene mittragende Breite $b_{m, v}$ ist gleich dem elastischen Wert $b_{m, v, 0}$. Mit zunehmender Belastung und fortschreitender Rissbildung nimmt die verformungsbezogene mittragende Breite ab. Dies liegt daran, dass durch die Rissbildung die Biegesteifigkeit und Biegetragwirkung des Betongurts abnimmt. Da die mittragende Breite des Biegezustands $b_{m, B}$ deutlich größer ist als die des Scheibenzustands $b_{m, s}$, wird die verformungsbezogene mittragende Breite somit kleiner. Bei den durchgeführten Versuchen konnte beobachtet werden, dass die verformungsbezogene mittragende Breite für hohe Belastungen wieder zunimmt. Diese Umkehr tritt ungefähr auf dem Belastungsniveau ein, an dem der Stahlträger mit Plastizieren beginnt $\left(\rightarrow M_{e} / M_{u}\right)$. Dies liegt unter Anderem daran, dass mit zunehmender Belastung die Spannungs-DehnungsBeziehung des Betons flacher wird und dadurch die Querverteilung der Spannungen völliger wird als die der Dehnungen, was schließlich zu einer Vergrößerung der mittragenden Breite führt. Vereinfachend kann dieser Anstieg der verformungsbezogenen mittragenden Breite vernachlässigt werden. In diesem vereinfachten Fall nimmt die mittragende Breite stetig ab. Diese Vereinfachung ist im Allgemeinen ausreichend, da man sich in einer praktischen 
Allgemeinen ausreichend, da man sich in einer praktischen Berechnung nur für die Verformungen unter Gebrauchslasten interessiert und unter Gebrauchslasten in der Regel noch kein Plastizieren des Stahlträgers auftritt. Der untere Grenzwert für die verformungsbezogene mittragende Breite ist der Wert des reinen Scheibenzustands $b_{m, s}$, siehe Abbildung 7-2.

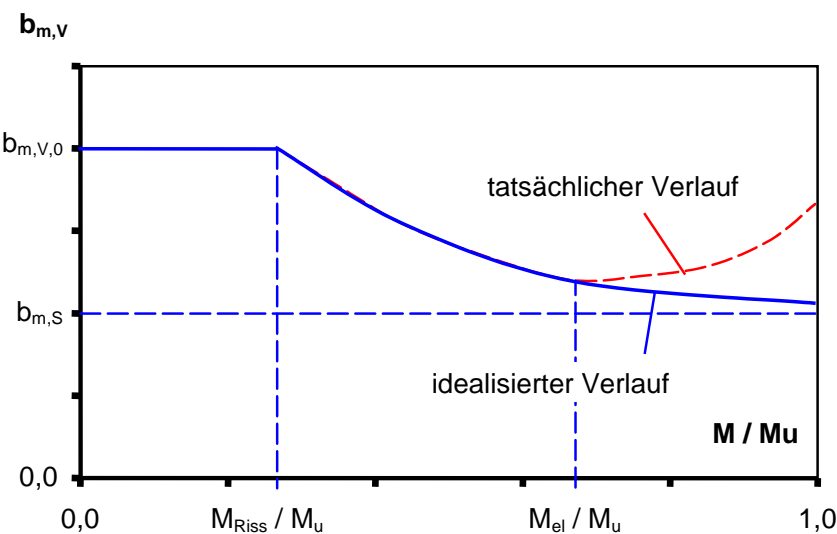

Querschnitt:

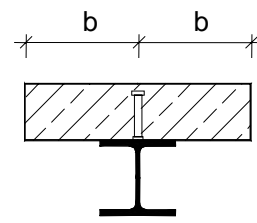

System:

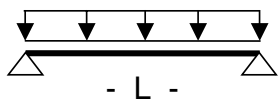

Abbildung 7-2: Prinzipieller Verlauf der verformungsbezogenen mittragende Breite $b_{m, v}$ niedriger Verbundträger in Abhängigkeit des Ausnutzungsgrads $M / M_{u}$

Für eine wirklichkeitsnahe Berechnung der Verformungen am Ersatzbalken muss das nichtlineare Trag- und Verformungsverhalten des Betons und insbesondere die Rissbildung bei der Berechnung berücksichtigt werden. Die Berechnung erfolgt deshalb zweckmäßig mit einer M-к-Linie, siehe Abschnitt 2.3.3.

\subsection{Parameterstudie im Anwendungsbereich des Hochbaus}

\subsubsection{Allgemeines}

Um die Einflüsse auf die verformungsbezogene mittragende Breite niedriger Verbundträger zu untersuchen, wurde im Rahmen der vorliegenden Arbeit eine Parameterstudie mit niedrigen Verbundträgern im Anwendungsbereich des Hoch- und Geschossbaus durchgeführt. Die Verformungen der untersuchten Träger wurden dabei mit dem in Abschnitt 6.2 beschriebenen Berechnungsmodell berechnet. Auf Basis der guten Übereinstimmung mit Versuchsträgern wird dabei davon ausgegangen, dass die so ermittelten Verformungen wirklichkeitsnahe Werte darstellen, siehe Abschnitt 6.4. Aus den so berechneten Verformungen wurde die verformungsbezogene mittragende Breite entsprechend Abschnitt 7.2 rückgerechnet.

Die Diskretisierung der berechneten Träger beim analytischen Berechnungsmodell wurde mit 50 Elementen vorgenommen.

Im Folgenden wird zunächst der Umfang der Parameterstudie beschrieben. Daran anschließend werden die einzelnen Einflüsse auf die verformungsbezogene mittragende Breite $b_{m, v}$ diskutiert.

\subsubsection{Untersuchter Parameterbereich}

Die Parameterstudie wurde im Rahmen der Anwendung von niedrigen Verbundträgern im normalen Hoch- und Geschossbau durchgeführt. Die variierten Parameter sind in Abbildung 7-3 zusammengefasst. 
Die Untersuchungen beziehen sich dabei auf Verbundeinfeldträger, die mit einer Gleichlast belastet werden und eine volle Verdübelung aufweisen. Es wird dabei angenommen, dass kein nennenswerter Schlupf zwischen Beton und Stahlträger auftritt. Für Verbundträger im Hochbau mit einer vollen Verdübelung mittels Kopfbolzendübel stellt dies jedoch im Gebrauchszustand keine Einschränkung dar.

\section{System und Querschnitt:}

$\begin{array}{ll}\text { Trägerstützweite: } & \mathrm{L}=5 \div 15 \mathrm{~m} \\ \text { Trägerbreite: } & \mathrm{b}=3 \div 9 \mathrm{~m} \\ \text { Plattendicke: } & \mathrm{h}_{\mathrm{c}}=16 \div 32 \mathrm{~cm} \\ \text { Höhe Stahlträger: } & \mathrm{h}_{\mathrm{a}}=200 \div 600 \mathrm{~mm} \\ \text { Profilreihen: } & \mathrm{HEA}, \mathrm{HEB}, \mathrm{IPE} \\ \text { Ausnutzungsgrad: } & \mathrm{M} / \mathrm{M}_{\mathrm{u}}=0 \div 1\end{array}$

Material:

Beton

Druckfestigkeit: $\quad \mathrm{f}_{\mathrm{c}}=18 \div 58 \mathrm{~N} / \mathrm{mm}^{2}$

Zugfestigkeit: $\quad \mathrm{f}_{\mathrm{ct}}=1,5 \div 4,5 \mathrm{~N} / \mathrm{mm}^{2}$

E-Modul: $\quad \mathrm{E}_{\mathrm{c}}=26000 \div 37000 \mathrm{~N} / \mathrm{mm}^{2}$

Baustahl

Streckgrenze:

Verdübelung:

Umfang:

(a) Abmessungen
System:

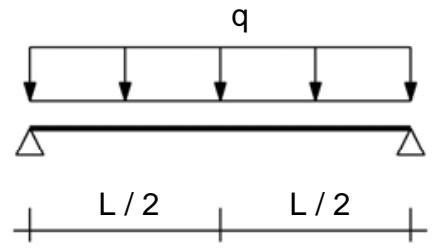

Querschnitt:

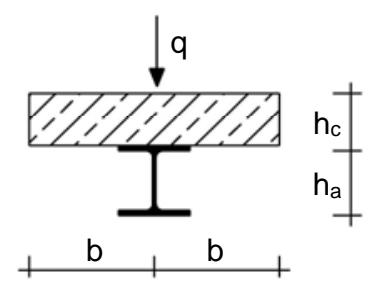

Abbildung 7-3: Umfang der Parameterstudie zur Entwicklung des Berechnungsansatzes für die verformungsbezogene mittragende Breite $b_{m, v}$

\subsubsection{Einflüsse auf die verformungsbezogene mittragende Breite $b_{m, v}$}

\subsubsection{Ausnutzungsgrad des Verbundträgers $M / M_{u}$}

Abbildung 7-4 zeigt den typischen Verlauf der verformungsbezogenen mittragenden Breite $b_{m, v}$ in Abhängigkeit des Ausnutzungsgrades $M / M_{u}$ eines Verbundträgers. Der Ausnutzungsgrad $M / M_{u}$ ist dabei als Verhältnis des maximalen Feldmoments $M$ in Trägermitte zum Bruchmoment $\mathrm{M}_{\mathrm{u}}$ des Verbundquerschnitts definiert. Die verformungsbezogene mittragende Breite ist dabei aus den Verformungen rückgerechnet, die mit dem analytischen Berechnungsmodell berechnet wurden.

Die verformungsbezogene mittragende Breite $b_{m, v}$ wird nach oben durch den elastischen Wert $b_{m, v, 0}$ und nach unten durch die mittragende Breite $b_{m, s}$ des reinen Scheibenzustands begrenzt. Der elastische Wert $b_{m, v, 0}$ der verformungsbezogenen mittragenden Breite kann aus den Werten des reinen Scheiben- und Biegezustands berechnet werden, siehe Abschnitt 4.3. Bis zum Erreichen des Rissmoments $M_{\text {Riss }}$ des Verbundträgers $\left(M<M_{\text {Riss }}\right)$ verhält sich der Verbundträger elastisch. Die verformungsbezogene mittragende Breite kann in diesem Bereich somit auf Basis der in Abschnitt 4.3 beschriebenen elastischen Untersuchungen bestimmt werden $\left(b_{m, v}=b_{m, v, 0}\right)$. Mit zunehmender Rissbildung $\left(M>M_{\text {Riss }}\right)$ nimmt die Biegetragwirkung des Betongurts ab und somit der Einfluss des Biegezustands auf die mittragende Breite. Die verformungsbezogene mittragende Breite nähert sich daher dem Grenzwert $b_{m, s}$ des reinen Scheibenzustands an. 
Dieser berechnete Verlauf der verformungsbezogenen mittragenden Breite entspricht im Gebrauchslastbereich den bei den Versuchsträgern beobachteten Zusammenhängen, siehe Abschnitt 5.5.4 und Abschnitt 6.4.

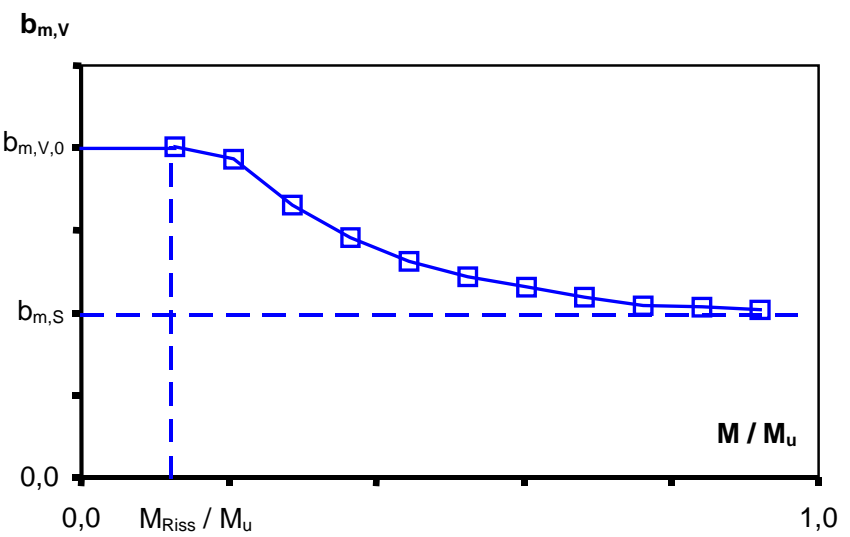

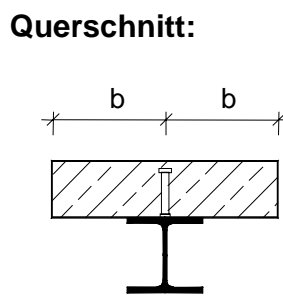

System:

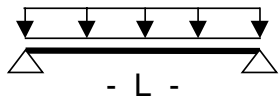

Abbildung 7-4: Typischer Verlauf der verformungsbezogenen mittragenden Breite $b_{m, v}$ niedriger Verbundträger in Abhängigkeit des Ausnutzungsgrads $M / M_{u}$

Der untere Grenzwert $b_{m, s}$ der verformungsbezogenen mittragenden Breite ist vom Seitenverhältnis des Betongurts b/L und dem Belastungstyp des Verbundträgers abhängig, siehe Abschnitt 4.2. Der Querschnitt und die Belastungshöhe haben hierauf keinen Einfluss. Der obere Grenzwert $b_{m, v, 0}$ ist vom Seitenverhältnis des Betongurts $b / L$, dem Belastungstyp und der Steifigkeitsverteilung im Verbundquerschnitt abhängig, siehe Abschnitt 4.3. Die Differenz zwischen dem oberen und unteren Grenzwert der verformungsbezogenen mittragenden Breite hängt somit wesentlich vom Querschnitt des Verbundträgers ab. Bei hohen Verbundträgern mit dünnem Betongurt hat der Biegezustand des Betongurts nur einen geringen Einfluss auf die verformungsbezogene mittragende Breite. Die Differenz $b_{m, v, 0}-b_{m, s}$ ist daher klein. Bei niedrigen Verbundträgern ist der Einfluss des Biegezustands auf die mittragende Breite jedoch sehr groß und daher auch auf die Differenz $b_{m, v, 0}-b_{m, s}$. Der Einfluss des Ausnutzungsgrades auf die verformungsbezogene mittragende Breite ist somit bei niedrigen Verbundträgern größer als bei hohen, siehe Abbildung 7-5.
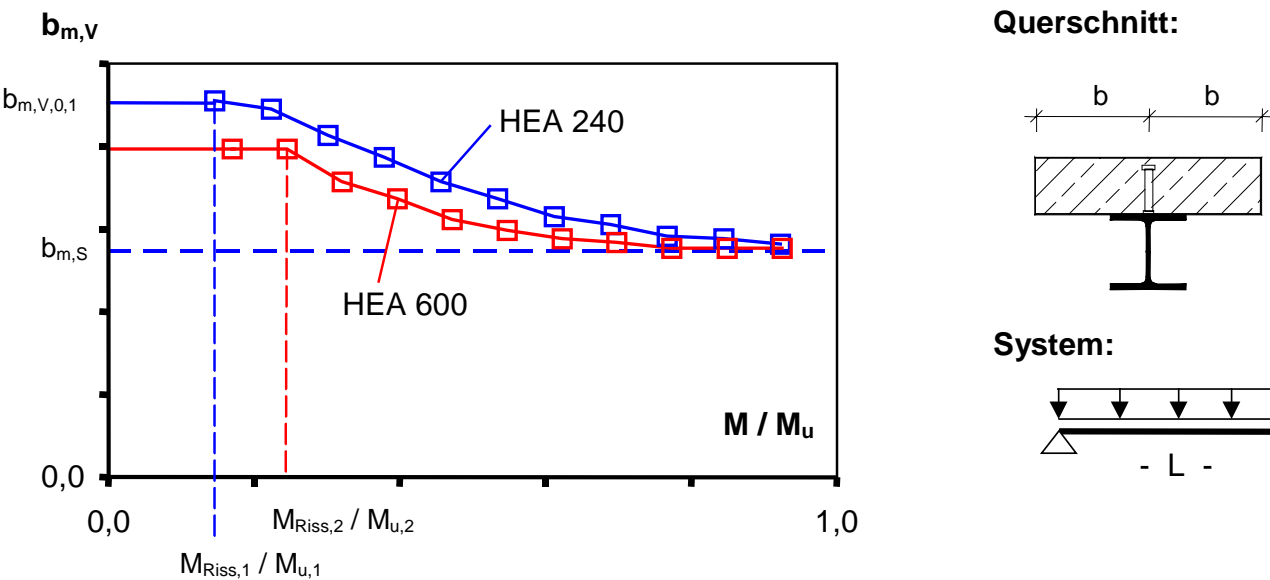

System:

Abbildung 7-5: Verformungsbezogene mittragende Breite $b_{m, v}$ bei hohen und niedrigen Verbundträger in Abhängigkeit des Ausnutzungsgrads $M / M_{u}$

Weitere Einflussfaktoren auf die verformungsbezogene mittragende Breite sind: Das Rissmoment $M_{\text {Riss }}$ des Trägers hängt neben den Querschnittswerten wesentlich von der Beton- 
zugfestigkeit $\mathrm{f}_{\mathrm{ct}}$ ab. Das Bruchmoment $\mathrm{M}_{\mathrm{u}}$ wird maßgebend vom Querschnitt und der Streckgrenze des Stahlträgers bestimmt.

Der bei manchen Trägern zu beobachtende Anstieg der verformungsbezogenen mittragenden Breite nach Erreichen des Rissmoments lässt sich wie folgt erklären, siehe z. B. Abbildung 7-6: Die Rissbildung beginnt im Allgemeinen im höchstbelasteten Querschnitt, in der Regel in Feldmitte. Beim analytischen Modell ist die mittragende Breite an dieser Stelle am größten und somit auch das Rissmoment. Da die verformungsbezogene mittragende Breite des Ersatzbalkens im elastischen Bereich kleiner ist als die spannungsbezogene mittragende Breite in Feldmitte des analytischen Berechnungsmodells, ist hier auch das rechnerische Rissmoment kleiner. Deshalb beginnt die Rissbildung am Ersatzbalken rechnerisch bei einer geringeren Belastung als beim analytischen Modell. Um am Ersatzbalken, der bei dieser Last bereits im Zustand II ist, die gleiche rechnerische Steifigkeit wie am ungerissenen analytischen Modell zu erhalten, muss die verformungsbezogene mittragende Breite des Ersatzbalkens vergrößert werden.

\subsubsection{Betonzugfestigkeit $f_{c t}$}

Wie oben bereits erwähnt, vergrößert sich die verformungsbezogene mittragende Breite $b_{m, v}$ mit zunehmender Betonzugfestigkeit $f_{c t}$, siehe Abbildung 7-6. Dieser Effekt wird auch bei numerischen Untersuchungen in [2.35], [2.65], [2.66] beobachtet.

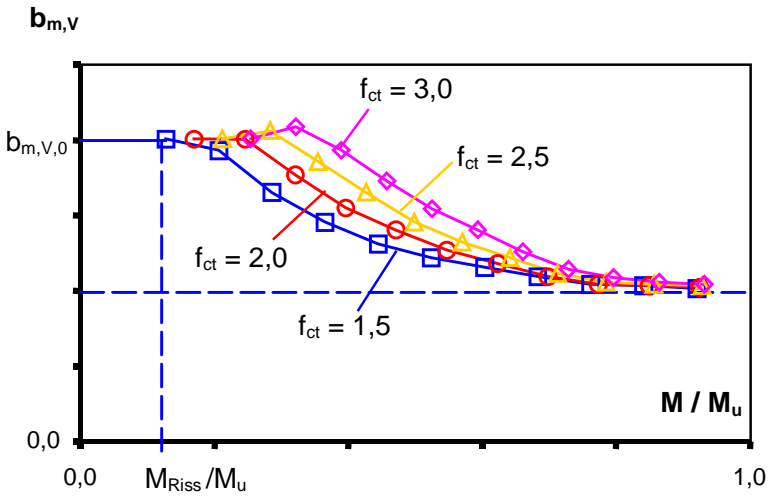

(a) berechnete Werte

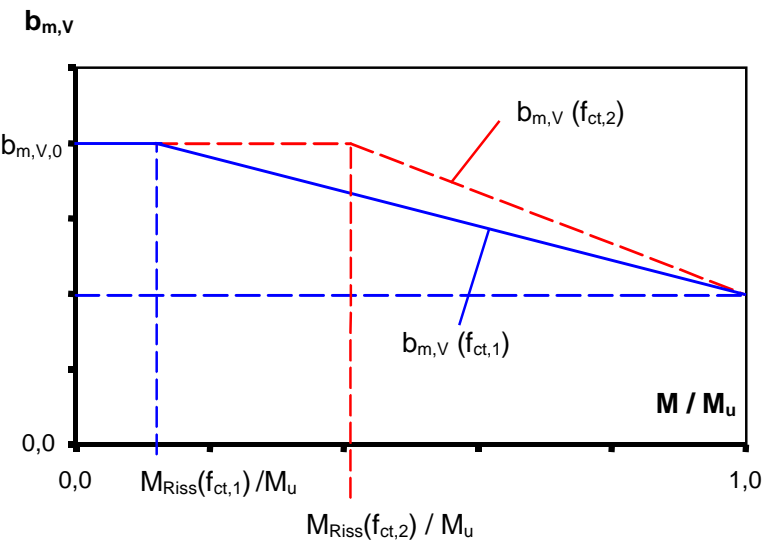

(b) schematische Darstellung $\left(f_{c t, 1}<f_{c t, 2}\right)$

Abbildung 7-6: Einfluss der Betonzugfestigkeit $f_{c t}$ auf die verformungsbezogene mittragende Breite $b_{m, v}$ niedriger Verbundträger $\left(f_{c t}\right.$ in $\left.\left[\mathrm{N} / \mathrm{mm}^{2}\right]\right)$

Die Gründe hierfür sind:

- Mit zunehmender Betonzugfestigkeit $f_{c t}$ nimmt das Rissmoment $M_{\text {Riss }}$ des Verbundträgers zu - bei gleichbleibendem Bruchmoment $M_{u}$. Dies führt dazu, dass der Verlauf der mittragenden Breite $b_{m, v}$ im Bereich der Rissbildung steiler wird und deshalb $b_{m, v}$ größere Werte annimmt, siehe Abbildung 7-6 (b).

- Durch die höhere Betonzugfestigkeit ergibt sich eine größere Mitwirkung des Betons auf Zug im Rissquerschnitt und zwischen den Rissen. Dadurch ergibt sich eine größere Biegetragwirkung des Betongurts und somit eine größere mittragende Breite.

- Durch das größere Rissmoment bei höherer Betonzugfestigkeit ist bei gleicher Belastung die Ausdehnung des gerissenen Bereichs in Trägerlängsrichtung kleiner und größere Bereiche des Trägers verhalten sich noch elastisch. Dadurch ist die verformungsbezogene mittragende Breite größer. 


\subsubsection{Streckgrenze des Baustahls}

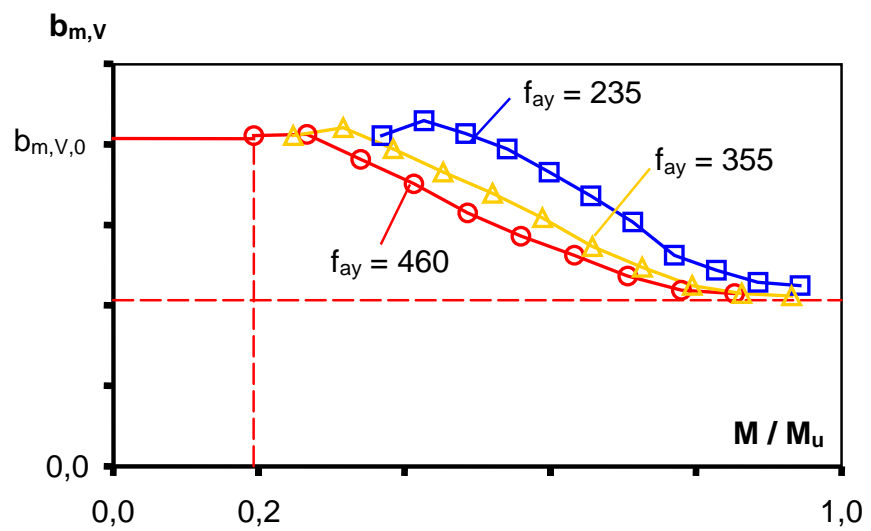

Querschnitt:

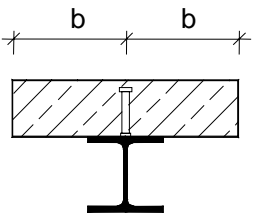

System:

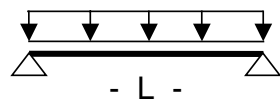

Abbildung 7-7: Einfluss der Streckgrenze $f_{y}$ des Baustahls auf die verformungsbezogene mittragende Breite $b_{m, v}\left(f_{y}\right.$ in $\left.\left[N / m^{2}\right]\right)$

Das Bruchmoment $M_{u}$ von Verbundquerschnitten wird maßgeblich von der Streckgrenze des Stahlträgers bestimmt. Durch Erhöhung der Streckgrenze nimmt das Bruchmoment zu und das bezogene Rissmoment $M_{\text {Riss }} / M_{u}$ des Trägers ab. Dadurch nimmt die verformungsbezogene mittragende Breite $b_{m, v}$ bei gleichem Ausnutzungsgrad $M / M_{u}$ des Trägers ab, siehe Abbildung 7-7. Dies ist in der schematischen Darstellung in Abbildung 7-8 (a) gut zu erkennen.

Wird das äußere Moment M absolut konstant gehalten, ergibt die Erhöhung der Streckgrenze hingegen eine größere verformungsbezogene mittragende Breite $b_{m, v}$, siehe Abbildung 7-8 (b).

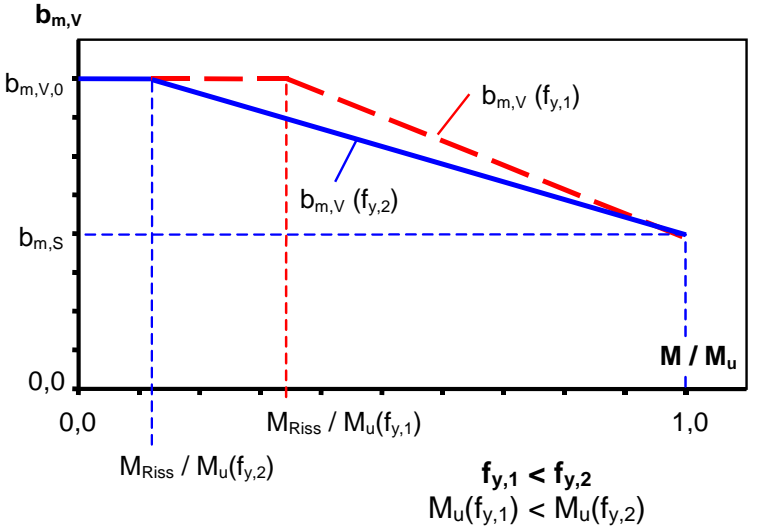

(a) Darstellung mit bezogenen Momenten

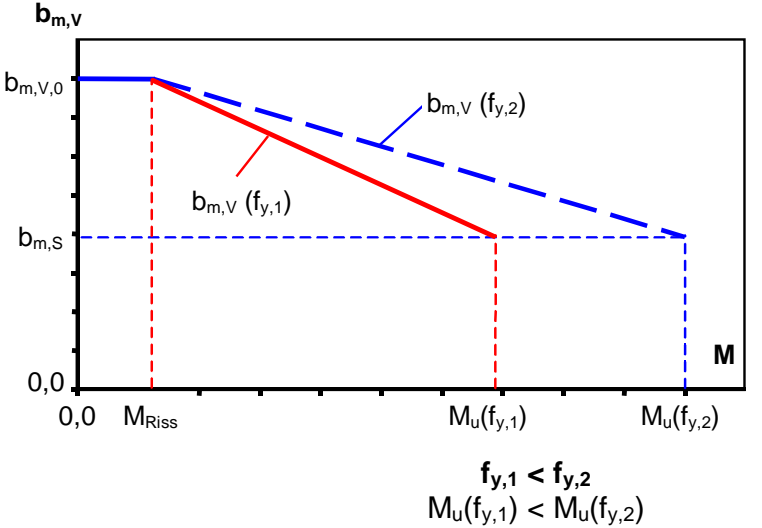

(b) Darstellung mit absoluten Momenten

Abbildung 7-8: Schematische Darstellung des Einflusses der Streckgrenze $f_{y}$ des Baustahls auf die verformungsbezogene mittragende Breite $b_{m, v}$

\subsubsection{Verhältnis Gurtdicke zu Trägerhöhe $h_{d} / h$}

Die Biegetragwirkung des Betongurts nimmt mit zunehmender Dicke des Betongurts im Verhältnis zur Gesamthöhe des Trägers zu. Dies liegt daran, dass die Eigenbiegesteifigkeit $\mathrm{I}_{c}$ des Betongurts in dritter Potenz der Gurtdicke $h_{c}$ ansteigt, während der Steifigkeitsanteil des inneren Kräftepaars im Verbundquerschnitt (Steiner-Anteil) langsamer wächst (Potenz < 2), 
siehe Gleichung (6-1). Eine größere Biegetragwirkung führt auf eine größere verformungsbezogene mittragende Breite $b_{m, v}$, siehe Abbildung 7-9. Dieser Effekt wurde auch bei den durchgeführten Versuchen, siehe Abschnitt 5.5.4, den elastischen Untersuchungen, siehe Abschnitt 4.3, und den in der Literatur beschriebenen Untersuchungen, siehe Abschnitt 3.5.3, beobachtet.

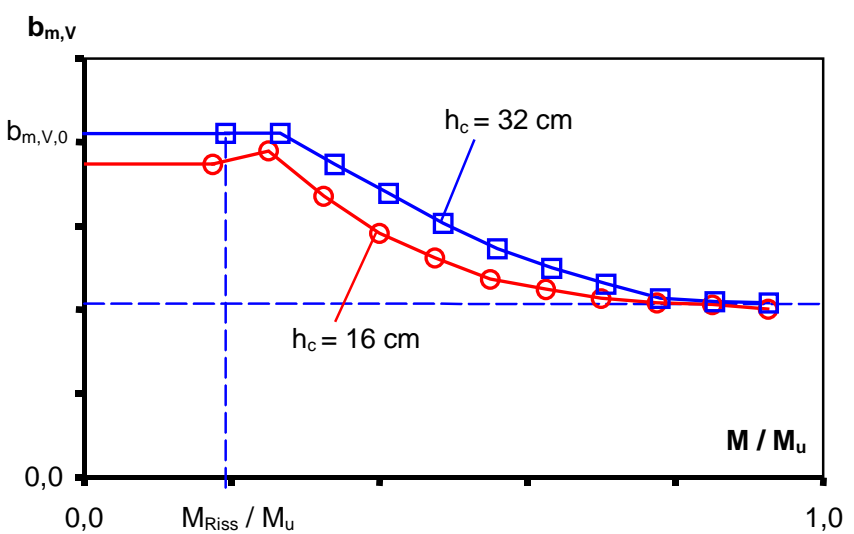

\section{Querschnitt:}

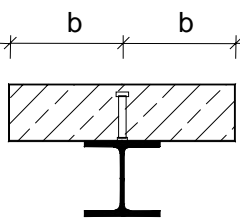

System:

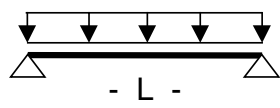

Abbildung 7-9: Einfluss der Gurtdicke $h_{c}$ auf die verformungsbezogene mittragende Breite $b_{m, v}$ niedriger Verbundträger

\subsubsection{Seitenverhältnis des Betongurts $b / L$}

Der Unterschied zwischen der mittragenden Breite des reinen Scheibenzustands $b_{m, s}$ und des reinen Biegezustands $b_{m, B}$ ist abhängig vom Seitenverhältnis des Betongurts $b / L$, siehe Abbildung 7-11. Für kleine Verhältnisse $\mathrm{b} / \mathrm{L}$ ist der Unterschied vergleichsweise gering. Für mittlere und große Verhältnisse $\mathrm{b} / \mathrm{L}$ ist der Unterschied dagegen groß. Entsprechend ist der Einfluss der mittragenden Breite $b_{m, B}$ des Biegezustands auf die verformungsbezogene mittragende Breite $b_{m, v}$ bei mittleren und großen Seitenverhältnissen $b / L$ größer als bei kleinen, siehe Abbildung 7-10. Dieser Einfluss zeigt sich qualitativ auch bei Vergleich der eigenen Versuchsträger VT 2 und VT 4 mit dem Versuchsträger B4 von Amadio et al., siehe Abschnitt 6.4.

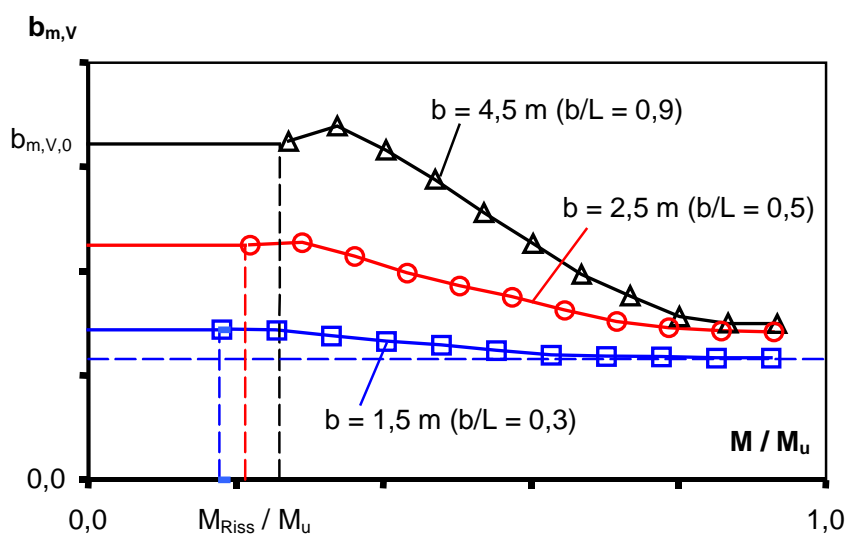

\section{Querschnitt:}

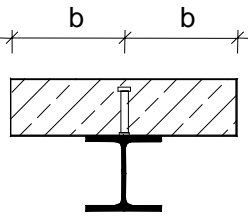

System:

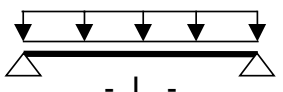

Abbildung 7-10: Verformungsbezogene mittragende Breite $b_{m, v}$ in Abhängigkeit des Seitenverhältnisses b/L 


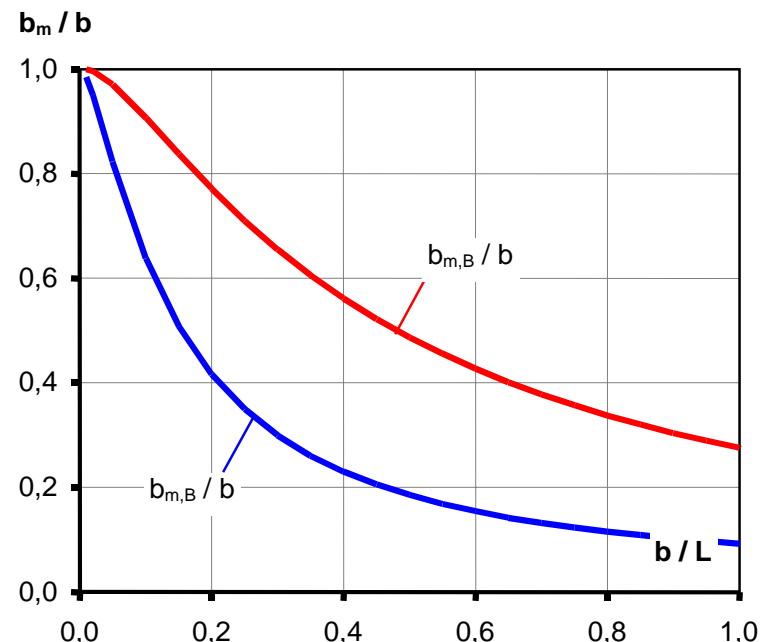

(a) Verlauf in Abhängigkeit von $b / L$

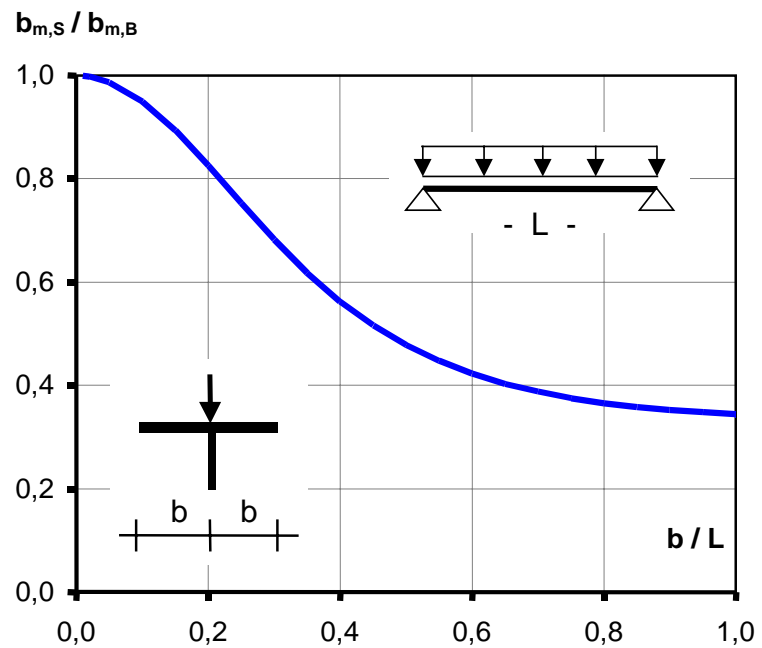

(b) Verhältnis $b_{m, s} / b_{m, B}$

Abbildung 7-11: Mittragende Breite des reinen Scheibenzustands $b_{m, s}$ und des reinen Biegezustands $b_{m, B}$ in Feldmitte eines einstegigen Verbundträgers unter Gleichlast

\subsubsection{Querschnitt des Baustahlträgers}

Die Querschnittsform des Stahlträgers beeinflusst die Steifigkeitsverteilung im Verbundquerschnitt und somit die verformungsbezogene mittragende Breite $b_{m, v}$. So wird $z$. B. bei Verwendung eines Trägers der HEB-Reihe statt der IPE-Reihe zum einen die Eigenbiegesteifigkeit $I_{a}$ des Stahlträgers und zum anderen der Steiner-Anteil der Teilflächen erhöht - bei gleichbleibender Biegesteifigkeit $\mathrm{I}_{\mathrm{c}}$ des Betongurts. Dadurch wird der Einfluss des Biegezustands des Betongurts auf die mittragende Breite geschwächt. Es ergibt sich eine kleinere verformungsbezogene mittragende Breite $b_{m, v}$, siehe Abbildung 7-12.

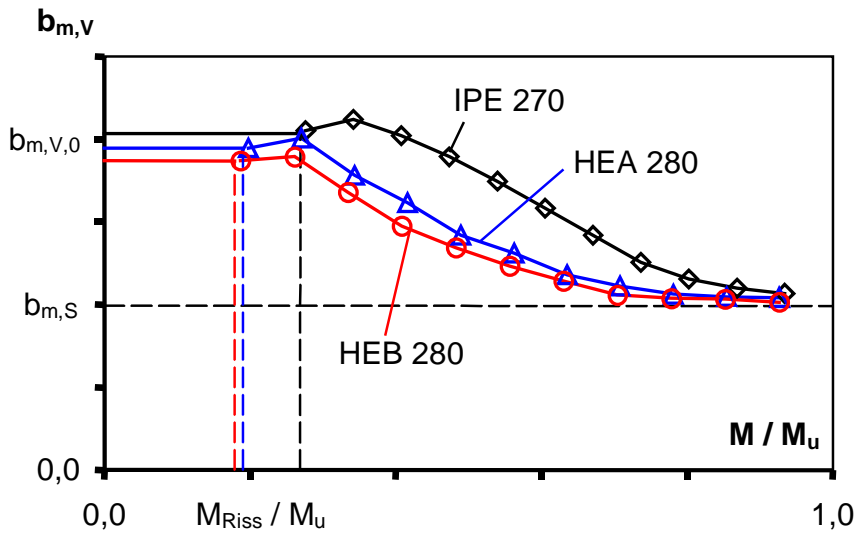

Querschnitt:

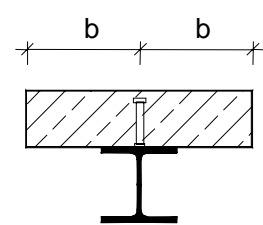

System:

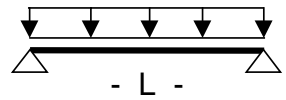

Abbildung 7-12: Einfluss der Querschnittsform des Stahlträgers auf die verformungsbezogene mittragende Breite $b_{m, v}$

Ein weiterer Grund ist, dass mit zunehmender Schlankheit des Stahlprofils dessen Querschnittsfläche $A_{a}$ und damit auch das Bruchmoment $M_{u}$ kleiner wird. Wohingegen das Rissmoment $M_{\text {Riss }}$ nur wenig beeinflusst wird. Insofern nimmt die verformungsbezogene mittragende Breite ab, siehe Abschnitt 7.3.3.3. 
Dieser Einfluss ist um so schwächer ausgeprägt, je dicker und größer der Betongurt im Vergleich zum Querschnitt des Stahlträgers ist. Bei niedrigen Verbundträger, deren Steifigkeit und damit auch deren mittragende Breite überwiegend vom Biegezustand des Betongurts bestimmt werden, ist dieser Effekt im Vergleich zu weiteren Einflüsse deshalb klein.

\subsubsection{Druckfestigkeit des Betongurts}

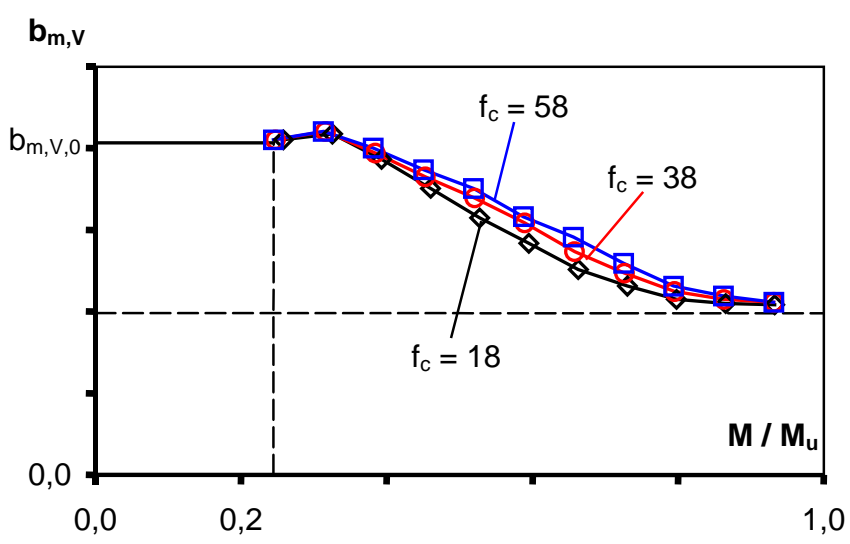

Querschnitt:

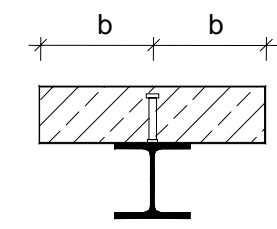

System:

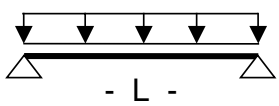

Abbildung 7-13: Einfluss der Betondruckfestigkeit $f_{c}$ auf die verformungsbezogene mittragende Breite $b_{m, v}\left(f_{c}\right.$ in $\left.\left[N / m^{2}\right]\right)$

Die Druckfestigkeit $f_{c}$ des Betongurts hat nur einen geringen Einfluss auf die verformungsbezogene mittragende Breite $b_{m, v}$ von Verbundträgern, siehe Abbildung 7-13. Grund hierfür ist, dass die Druckfestigkeit des Betons weder das Rissverhalten noch das Bruchmoment eines Verbundträgers wesentlich beeinflusst.

\subsubsection{Vergleich mit bestehenden Normregelungen}

\subsubsection{Grundsätzliches}

Ein Vergleich mit den bestehenden Regelungen zur mittragenden Breite der zur Zeit geltenden Normen EN 1994-1-1 [1.1], DIN 18800-5 [1.5] und DIN 1045-1 [1.8] soll zeigen, in wieweit diese Regelungen als Näherung für die verformungsbezogene mittragende Breite $b_{m, v}$ und zur Berechnung der Verformungen niedriger Verbundträger verwendet werden können. Dieser Vergleich wird an den im Rahmen der durchgeführten Parameterstudie berechneten Verbundträger vorgenommen. Die Randbedingungen und der Umfang dieser Parameterstudie sind in Abschnitt 7.3.2 beschrieben.

Grundsätzlich ist festzustellen, dass keine der angesprochenen Normen eine spezielle Regel für die verformungsbezogene mittragende Breite $b_{m, v}$ enthalten. Diese Regelungen gelten gleichermaßen für die spannungsbezogene als auch die verformungsbezogene mittragende Breite.

Die im folgenden beschriebenen Vergleiche basieren auf der Annahme, dass mit dem analytischen Berechnungsmodell wirklichkeitsnahe Werte für die Verformungen und die verformungsbezogene mittragende Breite ermittelt werden, siehe Abschnitt 6.4.

\subsubsection{Vergleich mit EN 1994-1-1 und DIN 18800-5}

Die Regeln zur mittragenden Breite in EN 1994-1-1 [1.1] und DIN 18800-5 [1.5] sind identisch. In Abbildung 7-14 ist ein Vergleich der rechnerischen Verformungen des analytischen 
Modells und EN 1994-1-1 [1.1] dargestellt. Die Berechnungen nach EN 1994-1-1 berücksichtigen dabei das nichtlineare Materialverhalten der Verbundträger mittels M-к-Linien und verwenden als Trägerbreite die mittragende Breite nach EN 1994-1-1. Im Vergleich zum analytischen Modell zeigen die Werte nach EN 1994-1-1 eine sehr große Streuung mit einer mittleren Abweichung von $36 \%$ bei einer Standardabweichung von $60 \%$. Im Allgemeinen überschätzen die Berechnungen nach EN 1994-1-1 die Verformungen hierbei. In besonderen Fällen erreicht die Abweichung mehr als $300 \%$. Die Abweichungen sind dabei besonders bei niedrigen Verbundträgern mit ausgeprägter Rissbildung und vergleichsweise großem Seitenverhältnis b/L des Betongurts groß.

rel. Häufigkeit

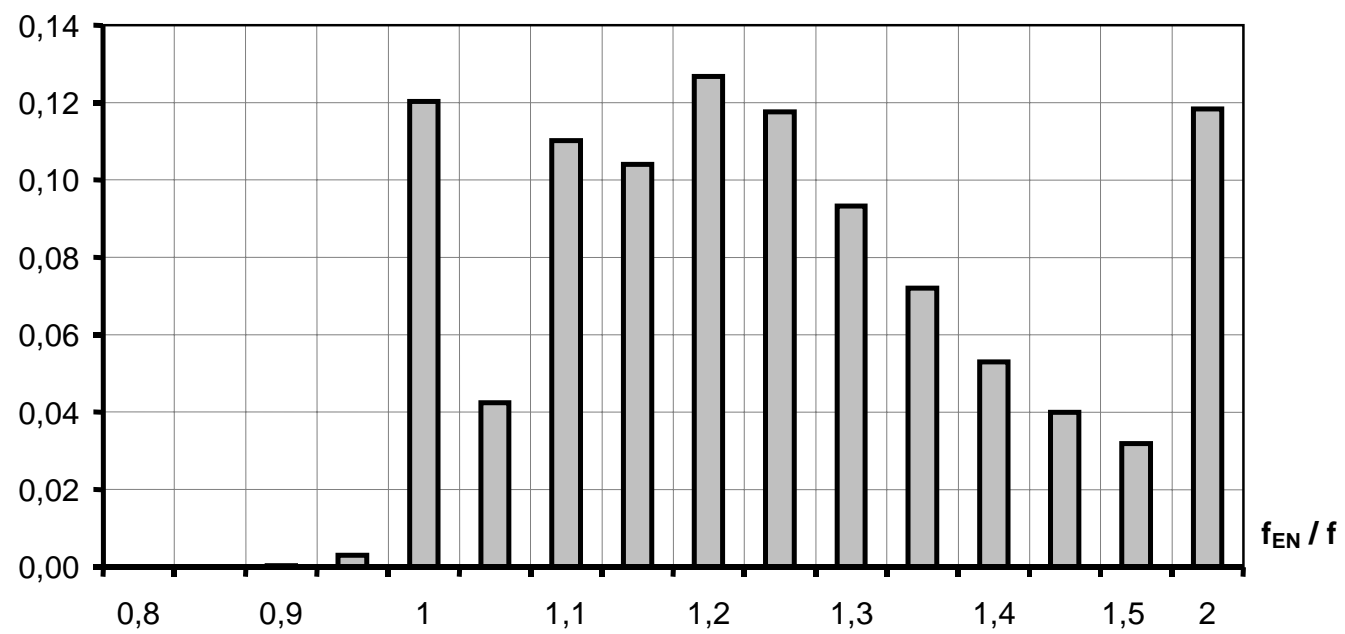

Abbildung 7-14: Vergleich der rechnerischen Verformungen des analytischen Berechnungsmodells $f$ mit der Berechnung nach EN 1994-1-1 $f_{E N}$

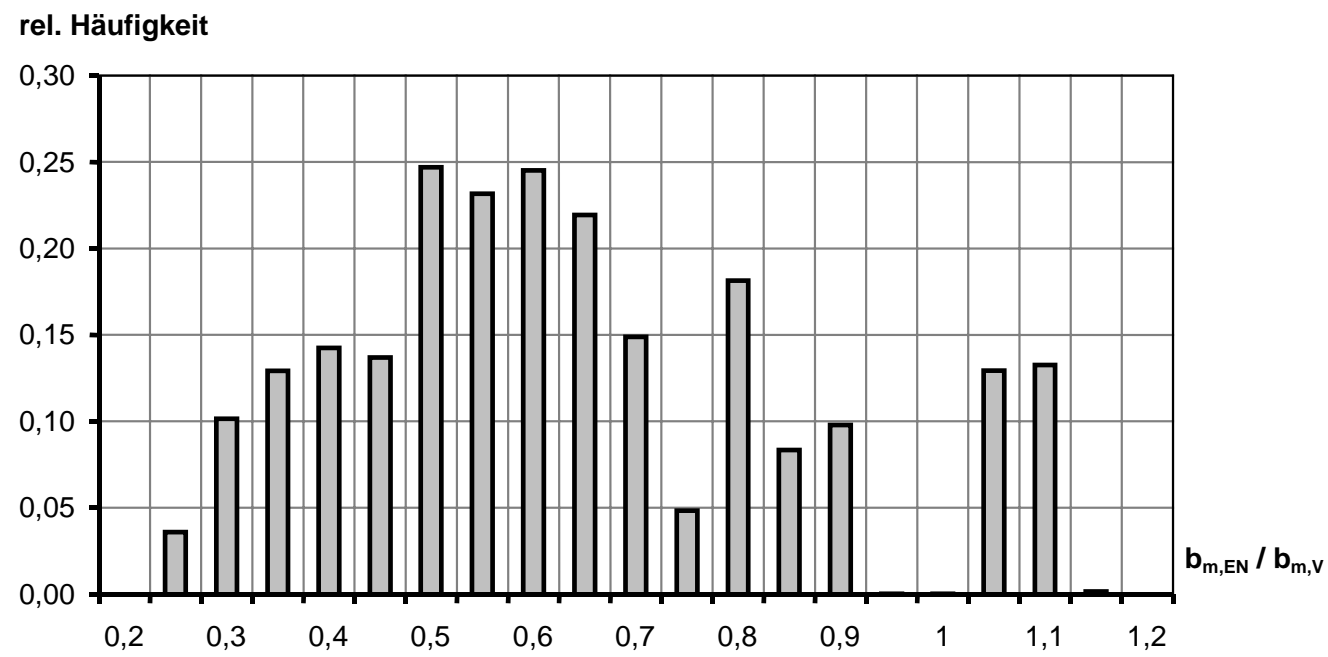

Abbildung 7-15: Vergleich der verformungsbezogenen mittragenden Breite $b_{m, v}$ des analytischen Berechnungsmodells mit der mittragende Breite nach EN 1994-1-1 $b_{m, E N}$

Abbildung 7-15 zeigt den Vergleich der mit dem analytischen Berechnungsmodell ermittelten verformungsbezogenen mittragenden Breite und der mittragenden Breite nach EN 1994-1-1 im Rahmen der Parameterstudie. Die Werte nach EN 1994-1-1 streuen im Vergleich zur genaueren Rechnung mit dem analytischen Berechnungsmodell in einem Bereich von $-80 \%$ bis $+20 \%$ bei einer mittleren Abweichung von $41 \%$. 
Auf Grund der festgestellten großen Abweichungen ist die Anwendbarkeit der Regeln für die mittragende Breite in EN 1994-1-1 und DIN 18800-5 zur Verformungsberechnung niedriger Verbundträger ungeeignet. Im Allgemeinen werden dadurch die Verformungen überschätzt, was in vielen Fällen zu einer unwirtschaftlichen Dimensionierung führt.

\subsubsection{Vergleich mit DIN 1045-1}

In Abbildung 7-16 ist ein Vergleich der rechnerischen Verformungen des analytischen Modells und der Berechnung nach DIN 1045-1 [1.8] dargestellt. Die Berechnungen nach DIN 1045-1 berücksichtigen dabei das nichtlineare Materialverhalten der Verbundträger mittels M-к-Linien und verwenden als Trägerbreite die mittragende Breite nach DIN 1045-1. Im Vergleich zum analytischen Modell zeigen die Werte nach DIN 1045-1 eine große Streuung mit einer mittleren Abweichung von $7,4 \%$ bei einer Standardabweichung von 12,5\%. In besonderen Fällen erreicht die Abweichung mehr als $100 \%$. Die Abweichungen sind deutlich kleiner als bei der Berechnung nach EN 1994-1-1 bzw. DIN 18800-5. Die Regel zur mittragenden Breite in DIN 1045-1 ist somit wesentlich besser dazu geeignet, die Verformungen von niedrigen Verbundträgern zu berechnen als jene der Verbundbaunormen. Dies gilt jedoch nur, wenn die Verformungen unter direkter Berücksichtigung des nichtlinearen Material- und Tragverhaltens der Verbundträger berechnet werden, siehe Abschnitt 8.4.4.

\section{rel. Häufigkeit}

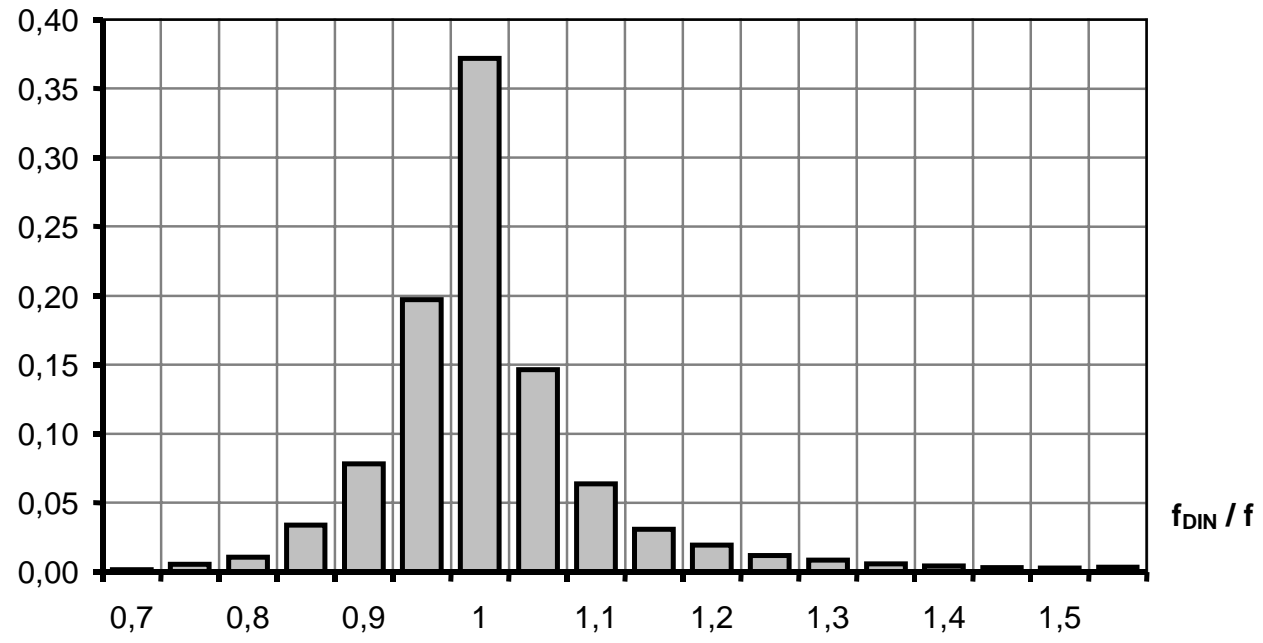

Abbildung 7-16: Vergleich der rechnerischen Verformungen des analytischen Berechnungsmodells $f$ mit der Berechnung nach DIN 1045-1 $f_{\text {DIN }}$

Abbildung 7-17 zeigt den Vergleich der mit dem analytischen Berechnungsmodell berechneten verformungsbezogenen mittragenden Breite und der mittragenden Breite nach DIN 10451 [1.8] im Rahmen der durchgeführten Parameterstudie. Die Werte nach DIN1045-1 streuen dabei im Vergleich zur genaueren Rechnung mit dem analytischen Berechnungsmodell in einem Bereich von $-50 \%$ bis $+100 \%$ bei einer mittleren Abweichung von $21 \%$. Die Abweichungen sind somit zwar wesentlich kleiner als nach EN 1994-1-1 bzw. DIN 18800-5 aber immer noch sehr groß. 
rel. Häufigkeit

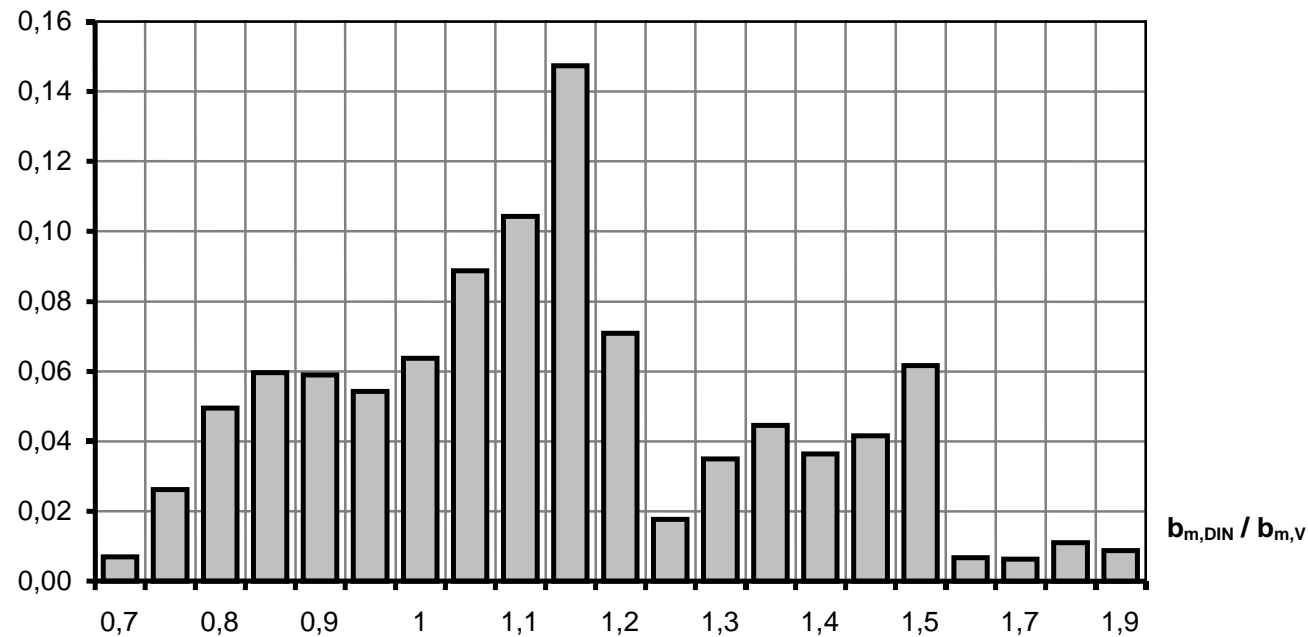

Abbildung 7-17: Vergleich der verformungsbezogenen mittragenden Breite $b_{m, v}$ des analytischen Berechnungsmodells mit der mittragende Breite nach DIN 1045-1 $\mathrm{b}_{\mathrm{m}, \mathrm{DIN}}$

\subsubsection{Schlussfolgerung aus dem Vergleich mit den bestehenden Normregelungen}

Die vorangegangen Vergleiche zeigen, dass die Regelungen zur mittragenden Breite in EN 1994-1-1 und DIN 18800-5 von der genauen Berechnung der verformungsbezogenen mittragenden Breite niedriger Verbundträger sehr stark abweichen und eine große Streuung aufweisen. Auf Basis dieser Regeln berechnete Verformungen niedriger Verbundträger überschätzen die tatsächlichen Verformungen dieser Träger in der Regel sehr stark und streuen in erheblichem Maß. Diese Regeln zur mittragenden Breite sind zur Verformungsberechnung niedriger Verbundträger somit nicht geeignet.

Eine bessere Näherung für die verformungsbezogene mittragende Breite stellt die Regel zur mittragenden Breite in DIN 1045-1 dar. Im Vergleich zu dem in dieser Arbeit entwickelten Berechnungsansatz sind die festgestellten Abweichungen dennoch deutlich größer, siehe Abschnitt 7.4.

\subsection{Berechnungsansatz für die verformungsbezogene mittragende Breite niedriger Verbundträger}

\subsubsection{Grundsätzliches}

Wie der Vergleich mit den bestehenden Normregelungen im vorangegangen Abschnitt zeigt, werden die Verformungen niedriger Verbundträger auf Basis dieser Regelungen im Allgemeinen überschätzt, was zu einer unwirtschaftlichen Dimensionierung dieser Träger führen kann. Ziel der vorliegenden Arbeit ist es daher, durch die Entwicklung eines realistischeren Berechnungsansatzes für die verformungsbezogene mittragende Breite eine wirklichkeitsnahe Verformungsberechnung und damit eine wirtschaftlichere Dimensionierung niedriger Verbundträger zu ermöglichen.

Da niedrige Verbundträger bereits unter Gebrauchslasten eine ausgeprägte Rissbildung aufweisen, muss diese für eine wirklichkeitsnahe Verformungsberechnung in der Rechnung berücksichtigt werden. Im Rahmen des vorgestellten Berechnungsansatzes wird dies mittels einer $\mathrm{M- \kappa -Linie} \mathrm{vorgenommen.} \mathrm{Abbildung} \mathrm{7-18} \mathrm{stellt} \mathrm{dieses} \mathrm{Vorgehen} \mathrm{schematisch} \mathrm{dar.} \mathrm{In}$ 
Abhängigkeit der System- und Belastungswerte wird zunächst die verformungsbezogene mittragende Breite $b_{m, v}$ berechnet. Mit deren Hilfe wird die M- $\kappa$-Linie des Verbundquerschnitts in Abhängigkeit der Querschnitts- und Materialwerte des Verbundträgers berechnet. Damit kann schließlich mit dem Prinzip der virtuellen Kräfte die Verformung des Trägers bestimmt werden.

Der Berechnungsansatz wurde auf Basis der in Abschnitt 7.3 beschriebenen Parameterstudie entwickelt. Er unterliegt daher folgenden Einschränkungen, kann jedoch auch bei vergleichbaren Querschnitten und Systemen näherungsweise angewendet werden:

- Der Querschnitt des Verbundträgers ist in Trägerlängsrichtung konstant.

- Der Betongurt weist eine konstante Dicke auf.

- Der Verbundträger wird als Einfeldträger ausgeführt.

- Der Verbundträger weist einen starren Verbund auf.

- Ein Versagen der Verbundfuge wird ausgeschlossen.

- Die Durchbiegung des Verbundträgers infolge Querkraft wird elastisch am Stahlträger bestimmt.

- Eigenspannungen im Stahlträger haben keinen nennenswerten Einfluss auf das Verformungsverhalten des Verbundträgers.

- Die Abmessungen des Verbundträgers liegen im Rahmen der durchgeführten Parameterstudie, siehe Abbildung 7-3.

- Die Werkstoffkennwerte des Verbundträgers liegen im Rahmen der durchgeführten Parameterstudie, siehe Abbildung 7-3.

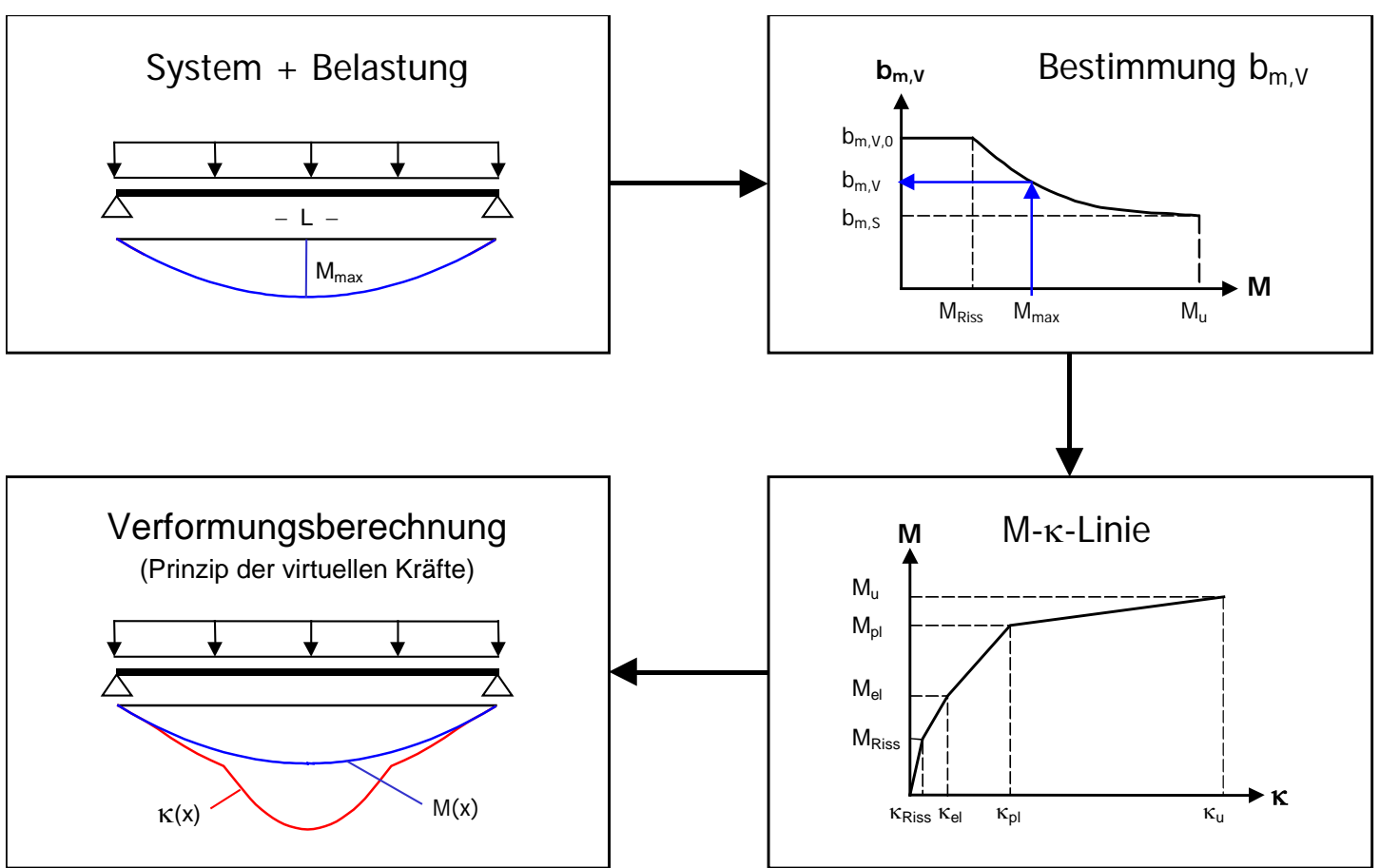

Abbildung 7-18: Verformungsberechnung niedriger Verbundträger auf Basis der verformungsbezogenen mittragenden Breite $b_{m, v}$ 
Der Berechnungsansatz wurde durch systematische Auswertung der durchgeführten Parameterstudie entwickelt. Dabei wurde versucht, für die genauen Werte der verformungsbezogene mittragende Breite $b_{m, v}$ eine gute Näherungsfunktion über die Minimierung der Fehlerquadrate zu finden.

Der Berechnungsansatz wurde in zwei Genauigkeitsstufen ausgearbeitet.

\section{Stufe 1 - Genauer Berechnungsansatz}

Der genaue Berechnungsansatz berücksichtigt die maßgebenden Einflüsse und liefert einen realistischen Wert für die verformungsbezogene mittragende Breite $b_{m, v}$ niedriger Verbundträger, siehe Abschnitt 7.4.2. Die damit berechneten Verformungen stellen im Allgemeinen sehr realistische Werte bei einem mittleren Berechnungsaufwand dar.

Stufe 2 - Vereinfachter Berechnungsansatz

Der vereinfachte Berechnungsansatz stellt eine Vereinfachung des genauen Berechnungsansatzes dar, siehe Abschnitt 7.4.3. Der Ansatz berücksichtigt im Unterschied zur Stufe 1 pauschal nur den maßgebenden Einfluss des Ausnutzungsgrads und damit implizit der Rissbildung. Die Genauigkeit der damit erzielten Ergebnisse ist in der Regel im Vergleich zur Zuverlässigkeit weiterer Einflüsse auf die Verformung niedriger Verbundträger ausreichend.

\subsubsection{Genauer Berechnungsansatz (Stufe 1)}

Abbildung 7-19 zeigt den idealisierten Verlauf der verformungsbezogenen mittragenden Breite $b_{m, v}$ in Abhängigkeit des Ausnutzungsgrads $M / M_{u}$ des Verbundträgers. Gleichung (7-1) und (7-2) geben den genauen Berechnungsansatz für die verformungsbezogene mittragende Breite $b_{m, v}$ in Abhängigkeit der maßgebenden Parameter an. Mit der so bestimmten mittragenden Breite ist die wirklichkeitsnahe Verformungsberechnung niedriger Verbundträger möglich.

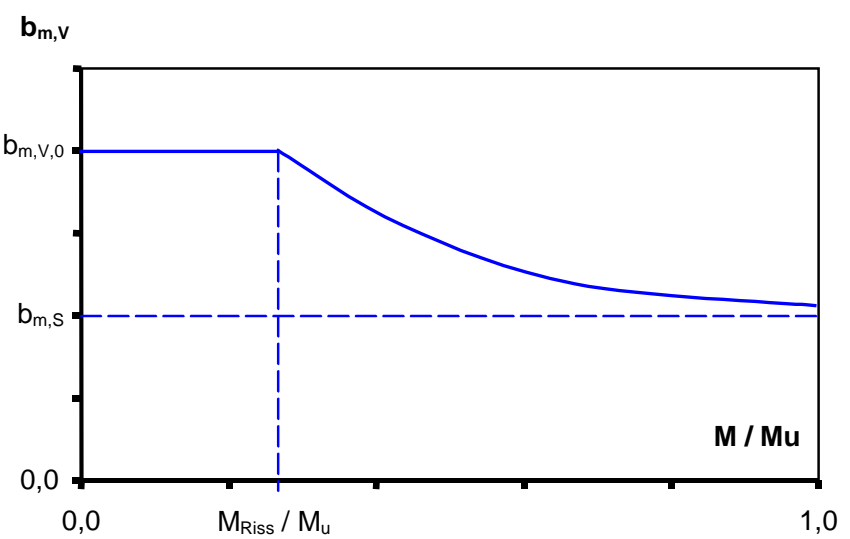

Querschnitt:

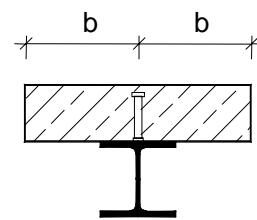

System:

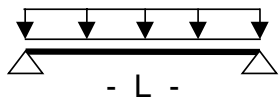

Abbildung 7-19: Idealisierter Verlauf der verformungsbezogenen mittragende Breite $b_{m, v}$ in Abhängigkeit des Ausnutzungsgrads $M / M_{u}$

$$
\mathrm{b}_{\mathrm{m}, \mathrm{v}}=\mathrm{b}_{\mathrm{m}, \mathrm{S}}+\left(\mathrm{b}_{\mathrm{m}, \mathrm{v}, 0}-\mathrm{b}_{\mathrm{m}, \mathrm{s}}\right) \cdot \beta_{\mathrm{m}, \mathrm{v}}
$$

mit

$$
\begin{aligned}
& b_{m, v} \\
& b_{m, s} \\
& b_{m, v, 0}
\end{aligned}
$$$$
\text { verformungsbezogene mittragende Breite }
$$
mittragende Breite des reinen Scheibenzustands nach Abbildung 7-20 elastischer Wert der verformungsbezogenen mittragenden Breite 
$\beta_{\mathrm{m}, \mathrm{V}}$

Abminderungsfaktor für die verformungsbezogene mittragende Breite nach Gleichung (7-2)

$$
\beta_{m, V}=0,83 \cdot\left(1-k_{M}\right)^{1,49} \cdot\left(\frac{f_{c t}}{f_{c t, 0}}\right)^{0,4} \cdot\left(\frac{h_{c}}{h}\right)^{0,77} \cdot\left(\frac{f_{y}}{f_{y, 0}}\right)^{-0,39} \cdot\left(\frac{A_{a}}{h_{a}^{2}}\right)^{-0,28} \leq 1
$$

mit

$\beta_{\mathrm{m}, \mathrm{V}}$

$$
\mathrm{k}_{\mathrm{M}}=\frac{\mathrm{M}-\mathrm{M}_{\text {Riss }}}{\mathrm{M}_{\mathrm{u}}-\mathrm{M}_{\text {Riss }}}
$$

M

M Riss

$M_{u}$

$\mathrm{f}_{\mathrm{ct}}$

$\mathrm{f}_{\mathrm{ct}, 0}=1,0 \mathrm{~N} / \mathrm{mm}^{2}$

$\mathrm{h}_{\mathrm{c}}$

$\mathrm{h}$

$f_{y}$

$\mathrm{f}_{\mathrm{y}, 0}=355 \mathrm{~N} / \mathrm{mm}^{2}$

$A_{a}$

$\mathrm{h}_{\mathrm{a}}$
Abminderungsfaktor für die verformungsbezogene mittragende Breite

maximales Moment des Verbundträgers in Feldmitte

Rissmoment des Verbundträgers

Bruchmoment des Verbundträgers

Betonzugfestigkeit

$1,5 \mathrm{~N} / \mathrm{mm}^{2} \leq \mathrm{f}_{\mathrm{ct}} \leq 4,5 \mathrm{~N} / \mathrm{mm}^{2}$

Bezugswert für die Betonzugfestigkeit

Dicke des Betongurts

$16 \mathrm{~cm} \leq \mathrm{h}_{\mathrm{c}} \leq 32 \mathrm{~cm}$

Gesamthöhe des Verbundträgers

Streckgrenze des Baustahls

$235 \mathrm{~N} / \mathrm{mm}^{2} \leq \mathrm{f}_{\mathrm{y}} \leq 460 \mathrm{~N} / \mathrm{mm}^{2}$

Bezugswert für die Streckgrenze des Baustahls

Querschnittsfläche des Stahlträgers

Höhe des Stahlträgers $b_{m} / \mathbf{b}$

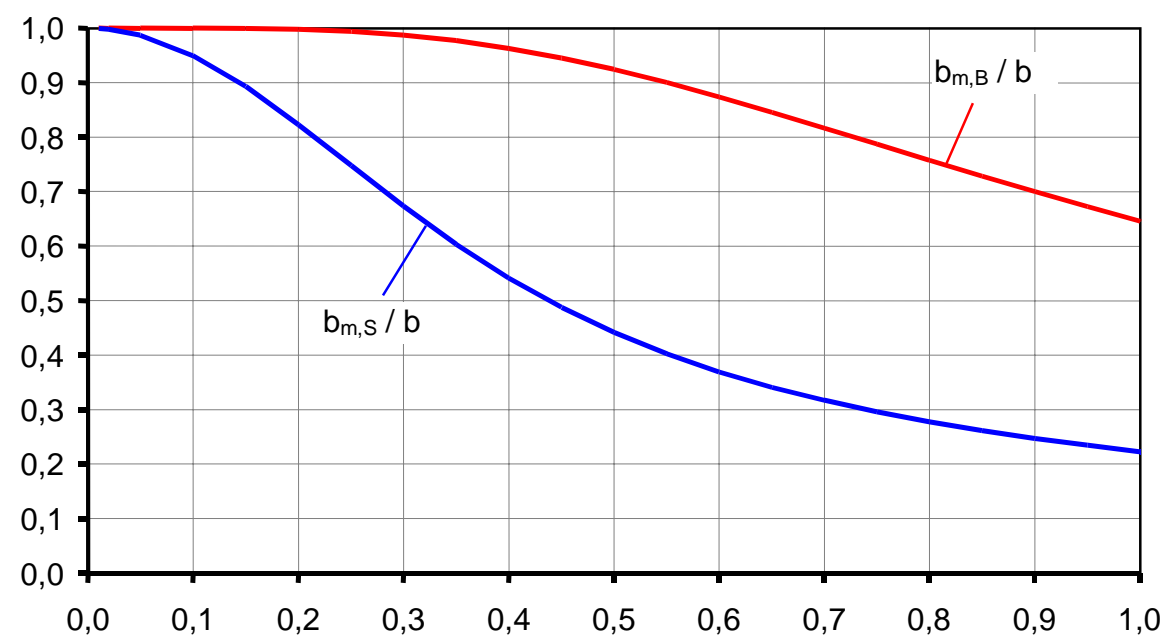

Querschnitt:

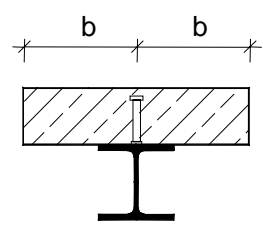

System:

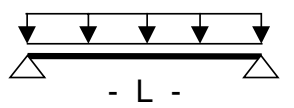

b / L

Abbildung 7-20: Mittragende Breite $b_{m, S}$ des reinen Scheibenzustands und $b_{m, B}$ des reinen Biegezustands in Feldmitte für Träger mit einer Gleichlast 


\section{rel. Häufigkeit}

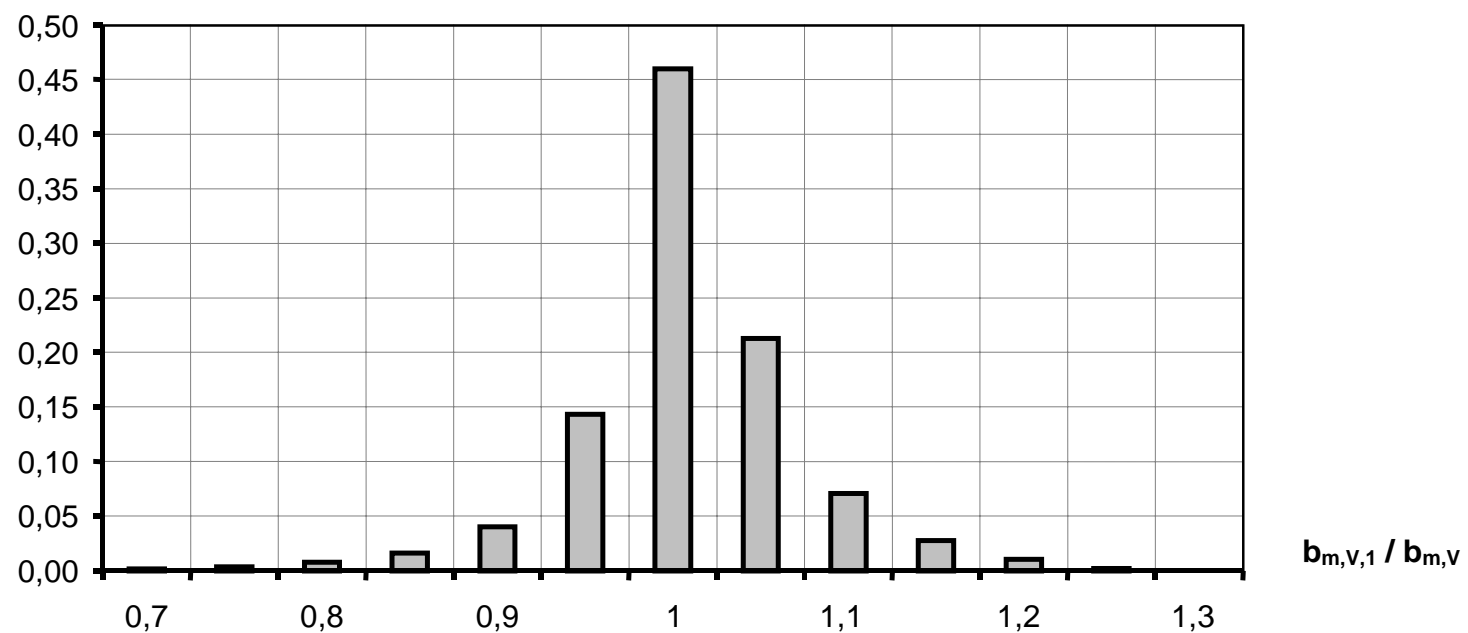

Abbildung 7-21: Abweichungen der verformungsbezogenen mittragenden Breite nach Gleichung (7-1) $b_{m, v, 1}$ zum analytischen Berechnungsmodell $b_{m, v}$

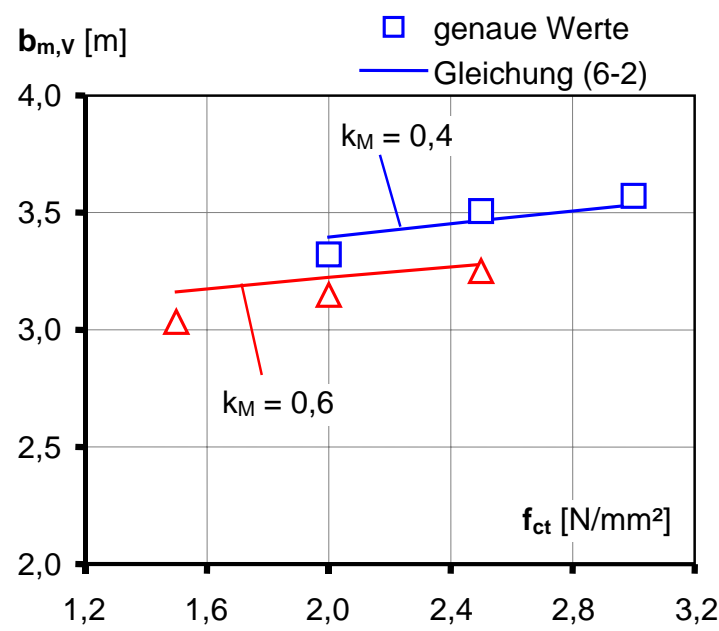

(a) in Abhängigkeit der Betonzugfestigkeit $f_{c t}$

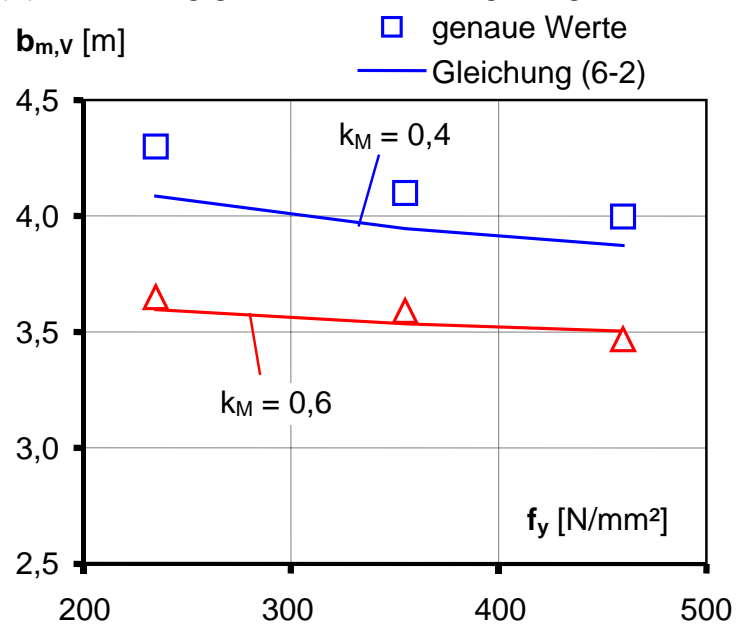

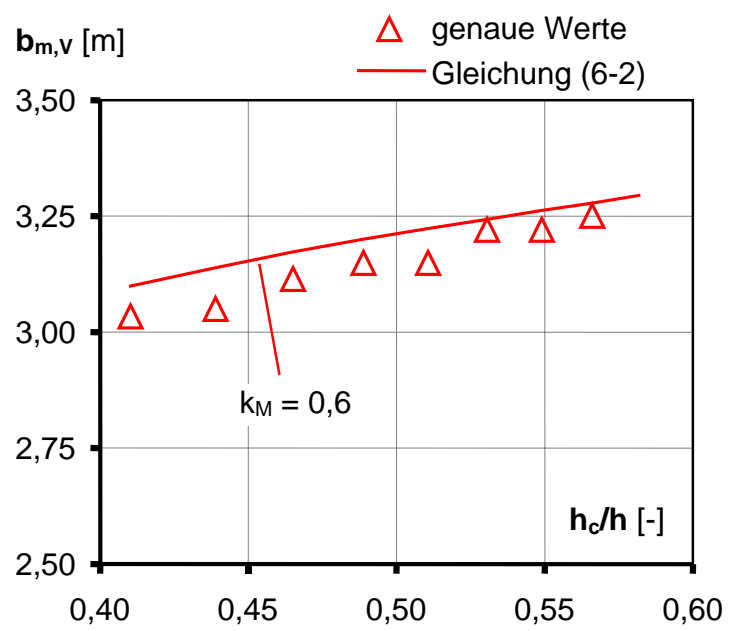

(b) in Abhängigkeit des Verhältnisses $h_{d} / h$

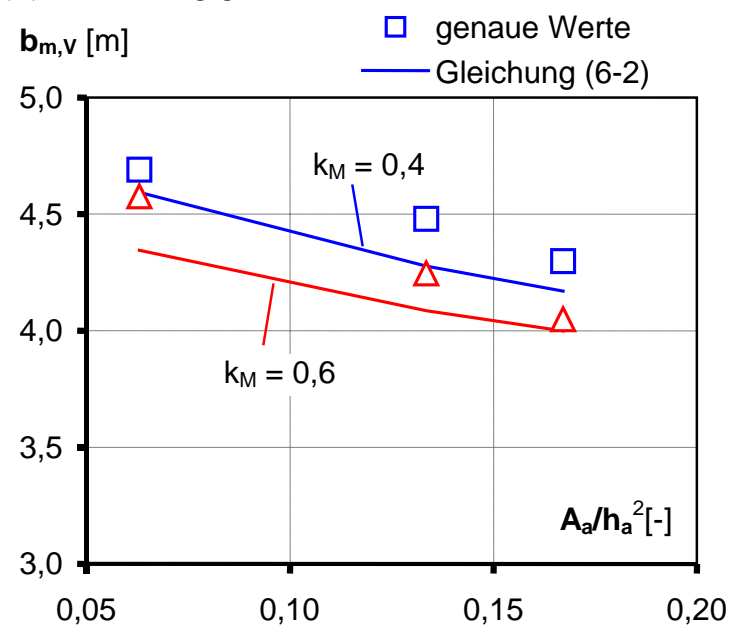

(c) in Abhängigkeit der Streckgrenze des Stahlträ- (d) in Abhängigkeit des Verhältnisses $A_{a} / h_{a}{ }^{2}$ gers $f_{y}$

Abbildung 7-22: Vergleich des Berechnungsansatzes für die verformungsbezogene mittragende Breite Gleichung (7-1) mit den genauen Werten 
Die Abweichungen der mit Gleichung (7-1) ermittelten Werte für die verformungsbezogene mittragende Breite $b_{m, v}$ im Vergleich zur genaueren Berechnung mit dem analytischen Berechnungsmodell liegen im Mittel bei ca. 4,3\%, siehe Abbildung 7-21. Die Standardabweichung beträgt 6,1\%. Der 5\%-Quantilwert liegt bei 0,89 und der 95\%-Quantilwert bei 1,09. Im Grenzzustand der Gebrauchstauglichkeit liegen die Abweichungen im Mittel bei 4,0\%, die Standardabweichung bei 5,3\%. Da der Einfluss der verformungsbezogenen mittragenden Breite auf die Durchbiegung der Verbundträger unterproportional ist, zeigen die Verformungen, die mit Gleichung (7-1) berechnet werden, eine noch bessere Übereinstimmung mit denen des analytischen Berechnungsmodells als die verformungsbezogene mittragende Breite. Der Berechnungsansatz zeigt somit eine sehr gute Übereinstimmung mit den genauen Werten bei einer Berechnung mit dem analytischen Berechnungsmodell und liefert realistische Werte für die verformungsbezogene mittragende Breite sowie die rechnerischen Verformungen niedriger Verbundträger. Dies gilt besonders im Vergleich zu den bestehenden Regelungen und vor allem zu EN 1994-1-1 und DIN 18800-5, siehe Abschnitt 7.3.4.

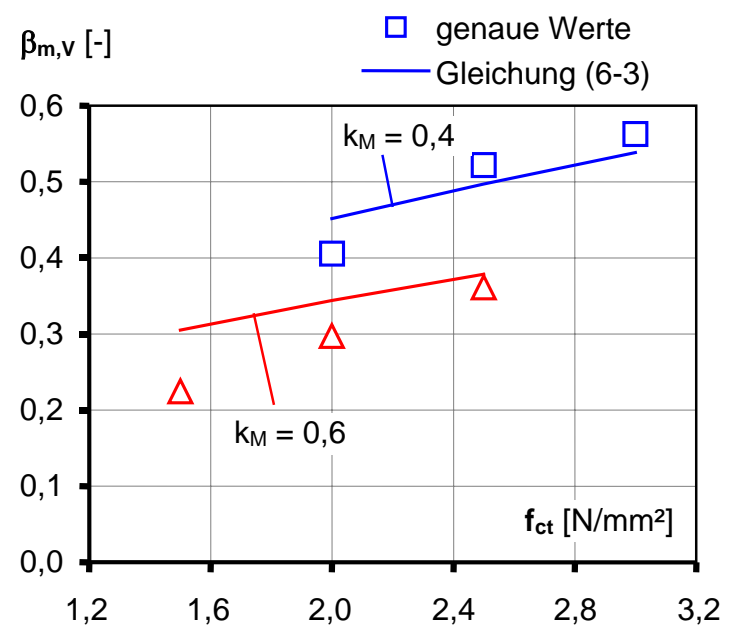

(a) in Abhängigkeit der Betonzugfestigkeit $f_{c t}$

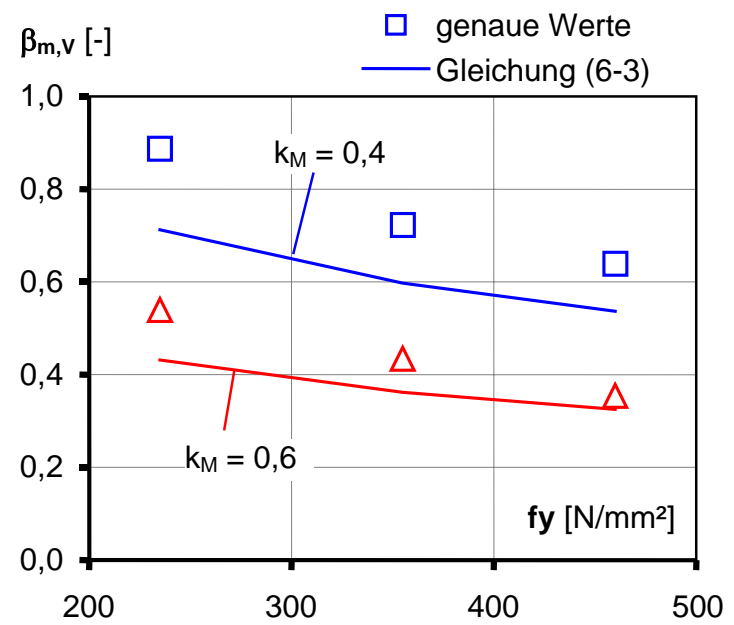

(c) in Abhängigkeit der Streckgrenze des Stahlträgers $f_{y}$

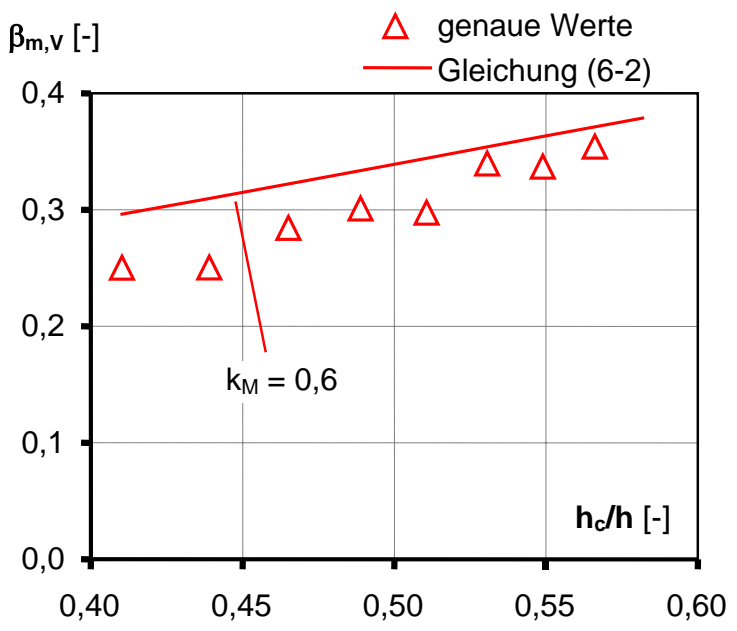

(b) in Abhängigkeit des Verhältnisses $h_{d} / h$

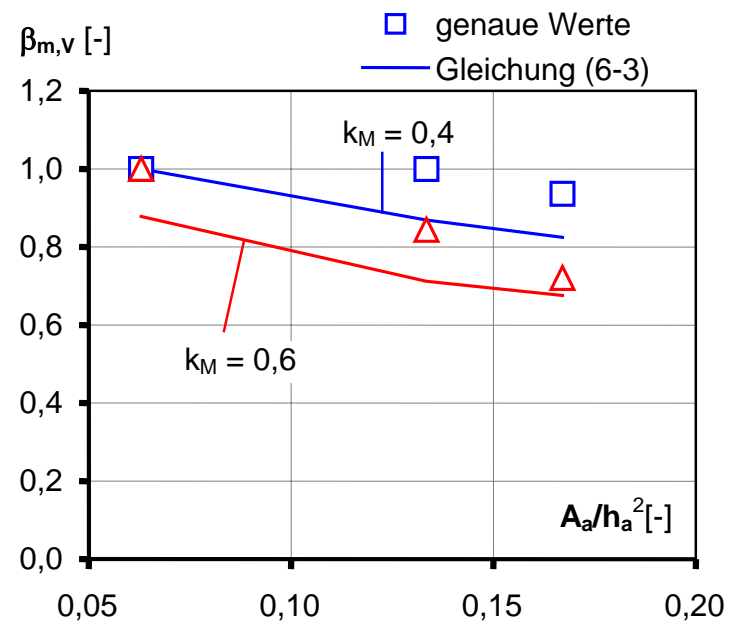

Abbildung 7-23: Vergleich des Berechnungsansatzes für den Abminderungsfaktor Gleichung (7-2) mit den genauen Werten

Die Exponenten und Koeffizienten von Gleichung (7-2) wurden durch eine Regression über die Minimierung der Fehlerquadrate mit Hilfe des in Microsoft Excel implementierten Solver bestimmt. Dabei wurde berücksichtigt, dass die einzelnen Parameter unter einander abhän- 
gig sein können. In Abbildung 7-22 und Abbildung 7-23 sind exemplarische Vergleiche des Berechnungsansatzes Gleichungen (7-1) und (7-2) mit den genauen Werten für die im Berechnungsansatz berücksichtigten Parameter des in Abbildung 7-24 gegebenen Trägers dargestellt. Diese dienen jedoch nur zur Veranschaulichung des Berechnungsansatzes und nicht zu dessen Herleitung.

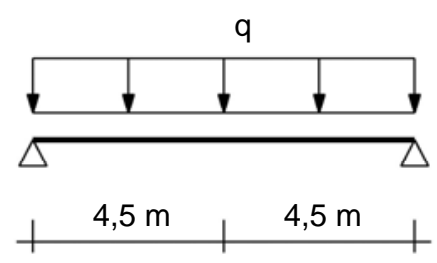

(a) System

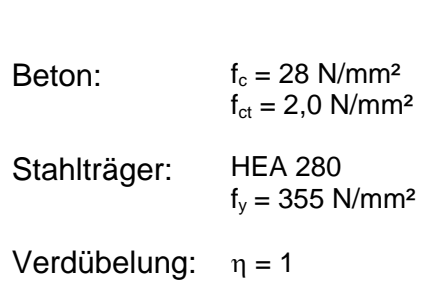

(b) Querschnitt und Materialwerte

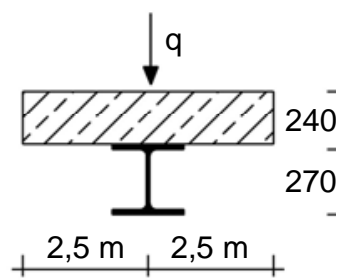

Abbildung 7-24: System und Querschnitt des Basisträgers

\subsubsection{Vereinfachter Berechnungsansatz (Stufe 2)}

Eine Vereinfachung des im vorangegangenen Abschnitts beschriebenen genauen Berechnungsansatzes für die verformungsbezogene mittragende Breite $b_{m, v}$ stellen Gleichung (7-3) und (7-4) dar. Im Vergleich zum genauen Ansatz wird hierbei nur der maßgebende Einfluss des Ausnutzungsgrads $M / M_{u}$ des Verbundträgers sowie implizit der Rissbildung des Betongurts berücksichtigt.

$$
\begin{aligned}
& b_{m, v}=b_{m, S}+\left(b_{m, v, 0}-b_{m, s}\right) \cdot \beta_{m, v} \\
& \beta_{m, v}=\left(1-k_{M}\right)^{2} \leq 1
\end{aligned}
$$

$$
\begin{array}{ll}
b_{m, V} & \text { verformungsbezogene mittragende Breite } \\
k_{M}=\frac{M-M_{\text {Riss }}}{M_{u}-M_{\text {Riss }}} & \\
M & \text { maximales Moment des Verbundträgers in Feldmitte } \\
M_{\text {Riss }} & \text { Rissmoment des Verbundträgers } \\
M_{u} & \text { Bruchmoment des Verbundträgers }
\end{array}
$$

Die Abweichungen dieses vereinfachten Berechnungsansatzes im Vergleich zur genaueren Berechnung mit dem analytischen Berechnungsmodell betragen im Mittel 8,7\%, siehe Abbildung 7-25. Die Standardabweichung beträgt 11,2\%. Der Mittelwert liegt bei 0,9. Somit wird die verformungsbezogene mittragende Breite tendenziell etwas unterschätzt und die damit berechneten Verformung folglich eher etwas überschätzt. Nach Meinung des Verfassers ist dies jedoch durchaus gewünscht. Der 5\%-Quantilwert beträgt 0,76 und der 95\%Quantilwert 0,99. Die Abweichungen der mit diesem vereinfachten Berechnungsansatz berechneten Verformungen betragen im Mittel nur 2,2\%, siehe Abbildung 7-26. Der Mittelwert liegt hier bei 1,0. Die Standardabweichung beträgt 7,4\%. Der 5\%-Quantilwert beträgt 0,95 und der 95\%-Quantilwert 1,04. Die Verformungen der berechneten Träger können somit mit dem vereinfachten Berechnungsansatz für die verformungsbezogene mittragende Breite $b_{m, v}$ nach Gleichung (7-3) und (7-4) sehr realistisch bestimmt werden. Dies gilt vor allem im Vergleich zu den bestehenden Normregelungen und insbesondere im Vergleich zu EN 19941-1 und DIN 18800-5, siehe Abschnitt 7.3.4. 
rel. Häufigkeit

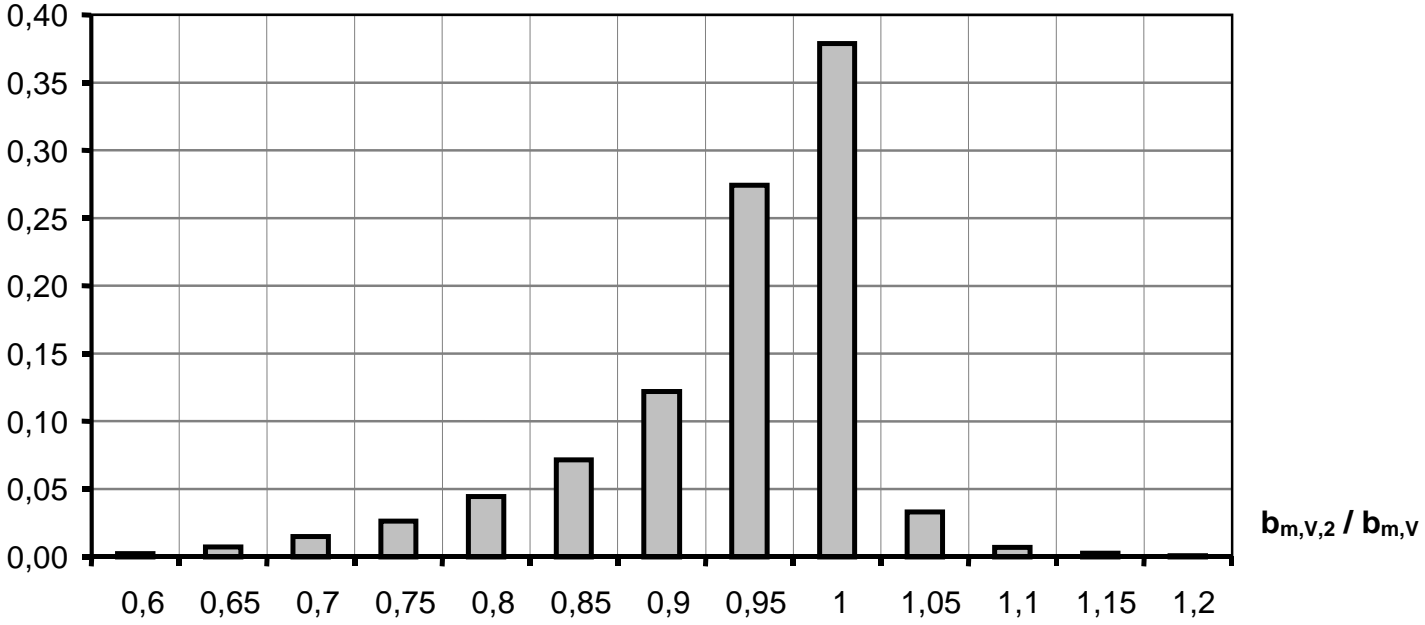

Abbildung 7-25: Abweichung des vereinfachten Berechnungsansatz für die verformungsbezogene mittragende Breite $b_{m, v, 2}$ im Vergleich zum analytischen Berechnungsmodell $b_{m, v}$

rel. Häufigkeit

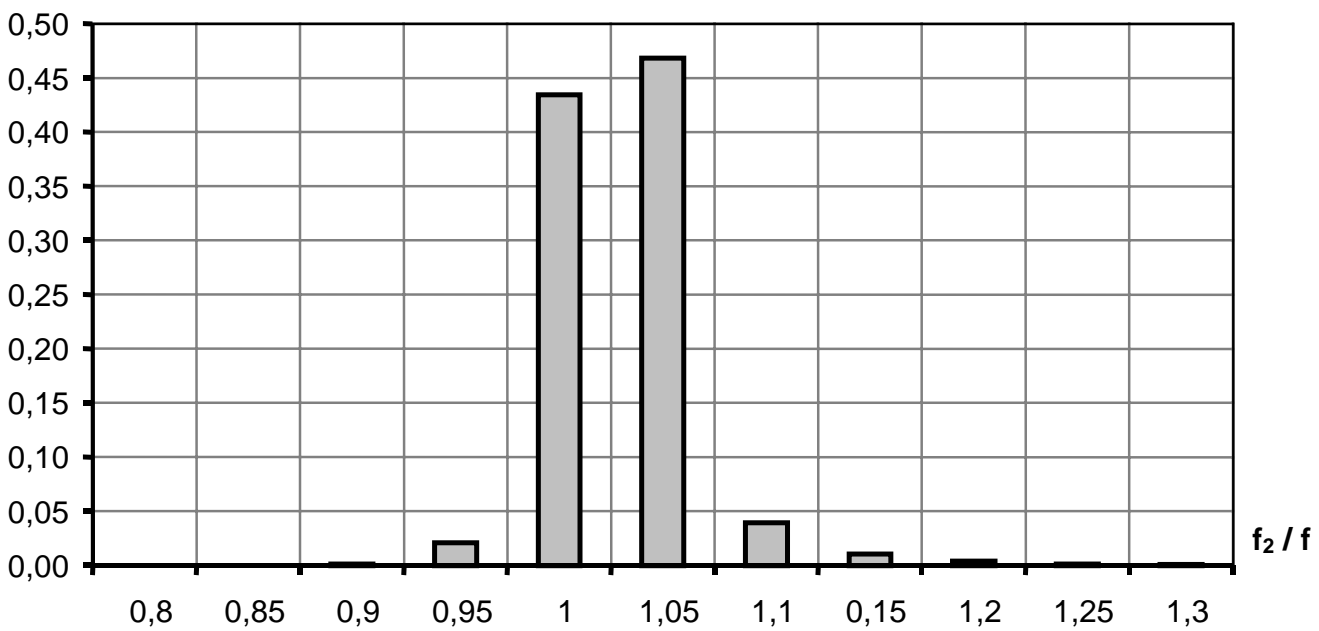

Abbildung 7-26: Abweichungen der mit dem vereinfachten Berechnungsansatz Gleichung (7-3) berechneten Verformungen $\mathrm{f}_{2}$ im Vergleich zum analytischen Berechnungsansatz $\mathrm{f}$

\subsubsection{Schlussfolgerung aus dem entwickelten Berechnungsansatz}

Durch die systematische Auswertung der durchgeführten Parameterstudie mit typischen niedrigen Verbundträgern des Hoch- und Geschossbaus konnte für die verformungsbezogene mittragende Breite ein Berechungsansatz entwickelt werden, der eine wirklichkeitsnahe Verformungsberechnung und eine wirtschaftliche Dimensionierung dieser Träger ermöglicht. Dies gilt insbesondere im Vergleich zu den bestehenden Normregelungen. 


\subsection{Abschätzung weiterer Einflüsse}

\subsubsection{Allgemeines}

Die Herleitung der oben beschriebenen Berechnungsansätze konnte nicht alle möglichen Konstellationen und Einflüsse von Verbundträgern in realen Hochbauten miteinbeziehen. Im folgenden werden daher die Einflüsse weiterer, im Berechnungsansatz nicht explizit berücksichtigter Parameter auf die verformungsbezogene mittragende Breite abgeschätzt.

\subsubsection{Belastungstyp}

Die Herleitung des in den voranstehenden Abschnitten beschriebenen Berechnungsansatz für die verformungsbezogene mittragende Breite $b_{m, v}$ bezieht sich strenggenommen ausschließlich auf eine Belastung des Trägers mit einer Gleichlast, siehe Abschnitt 7.3.2. Wie die in Abschnitt 4.3.3 beschriebenen Untersuchungen jedoch nahe legen, ist der Einfluss des Belastungstyps auf die verformungsbezogene mittragende Breite vergleichsweise gering, siehe Abbildung 4-14. Dies kann anhand von Vergleichsrechnungen an niedrigen Verbundträgern unter einer mittigen Einzellast gezeigt werden.

Die Belastung mit einer mittigen Einzellast stellt wegen der ausgeprägten Einschnürung der mittragenden Breite im Bereich der Einzellast den ungünstigsten Fall für die üblichen Belastungstypen von Trägern im Hochbau dar. Um den Einfluss des Belastungstyps auf die verformungsbezogene mittragende Breite größtmöglich abzuschätzen, wurden im Rahmen der durchgeführten Vergleichsrechnungen die Träger ausschließlich mit einer mittigen Einzellast belastet. Eine immer vorhandene mehr oder minder gleichmäßig verteilte Eigengewichtsbelastung wurde in die mittige Einzellast eingerechnet.

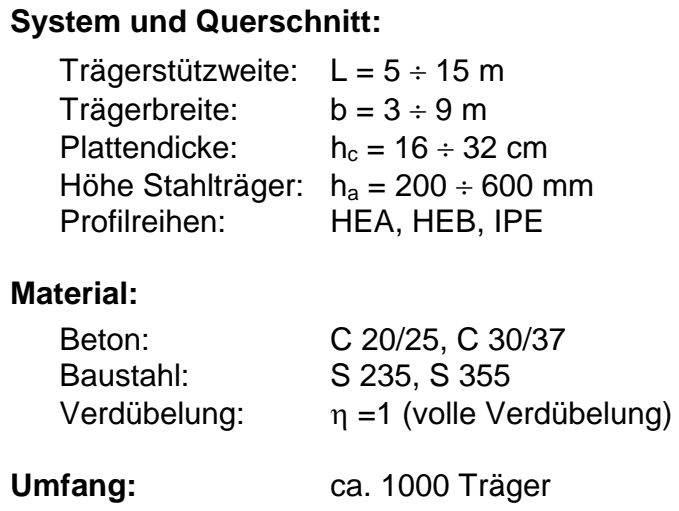

(a) Abmessungen
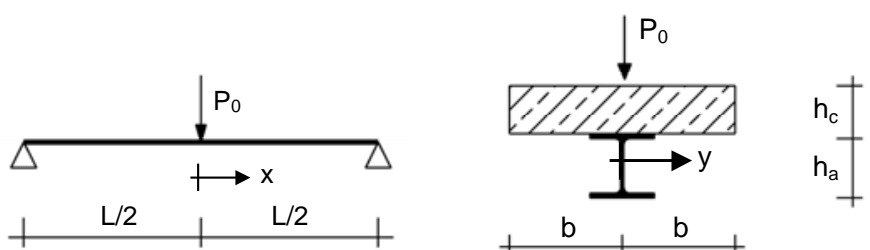

(b) Bezeichnungen

Abbildung 7-27: Umfang der Vergleichsrechnungen niedriger Verbundträger mit mittiger Einzellast

Einzellasten können in baupraktischen Fällen zum Beispiel durch das Abfangen von Stützen, konzentrierte Maschinenlasten oder die indirekte Lagerung von Nebenträgern auf Hauptträgern entstehen. Aufgrund der begrenzten Tragfähigkeit ist die Belastung niedriger Verbundträger mit vergleichsweise großen Einzellasten jedoch eher selten.

Der Umfang der durchgeführten Vergleichsrechnungen ist in Abbildung 7-27 dargestellt. Die Vorgehensweise bei der Auswertung der verformungsbezogenen mittragenden Breite $b_{m, v}$ entspricht derjenigen für Gleichlast, siehe Abschnitt 7.2. 
rel. Häufigkeit

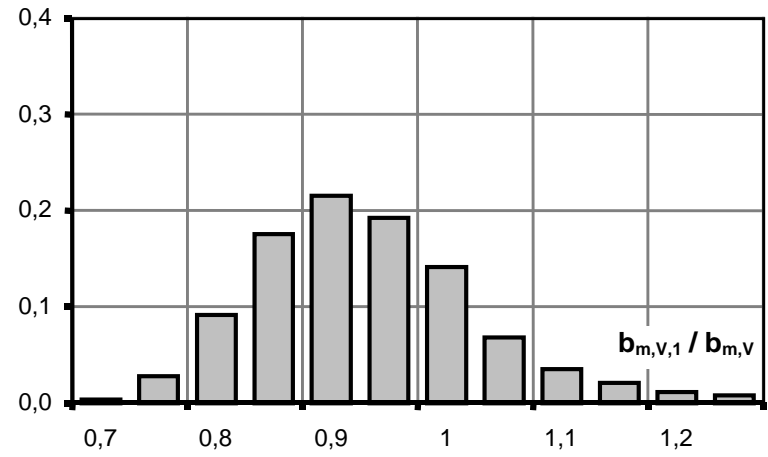

(a) für Ausnutzungen $M / M_{R d}=0 \div 0,8$

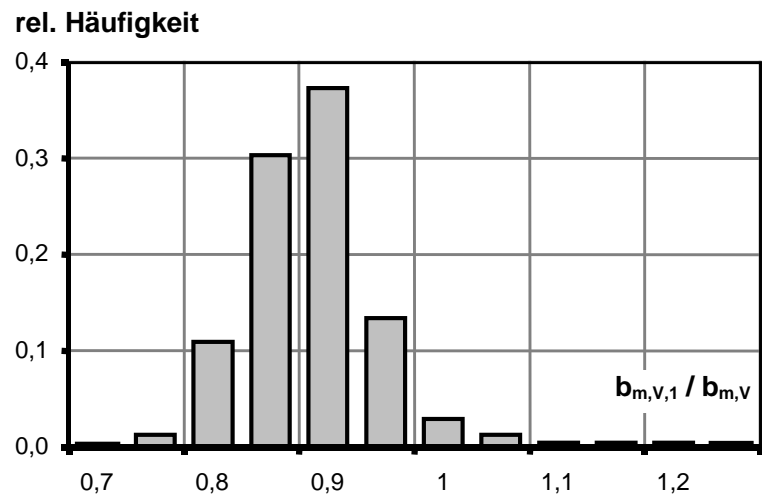

(b) im GZG $\left(M / M_{R d} \approx 0,65\right)$

Abbildung 7-28: Abweichungen der verformungsbezogenen mittragenden Breite nach Gleichung (7-1) $b_{m, v, 1}$ zum analytischen Berechnungsmodell $b_{m, v}$ bei mittiger Einzellast

In Abbildung 7-28 sind die Abweichungen der mit dem analytischen Berechnungsmodell mit mittiger Einzellast ermittelten Werte der verformungsbezogenen mittragenden Breite $b_{m, v}$ und dem genauen Berechnungsansatz nach Gleichung (7-1) dargestellt. Die Abweichungen liegen im Mittel bei ca. $7 \%$ und die Standardabweichung bei ca. 12,2 \%. Der 5\%-Quantilwert liegt bei 0,76 und der 95\%-Quantilwert bei 1,10. Im Grenzzustand der Gebrauchstauglichkeit liegen die Abweichungen im Mittel bei $11 \%$, die Standardabweichung bei 13,2\%. Die Abweichungen sind somit etwas größer als bei den untersuchten Trägern mit Gleichlast, siehe Abschnitt 7.4.2. Der Berechnungsansatz nach Gleichung (7-1) unterschätzt dabei die verformungsbezogene mittragende Breite in der Regel. Dennoch kann die Anwendung von Gleichung (7-1) auch bei Belastung mit mittiger Einzellast aus folgenden Gründen empfohlen werden:

- Da der Einfluss der verformungsbezogenen mittragenden Breite auf die Durchbiegung der Verbundträger unterproportional ist, zeigen die Verformungen, die mit Gleichung (7-1) berechnet werden, eine bessere Übereinstimmung mit denen des analytischen Berechnungsmodells als die verformungsbezogene mittragende Breite, siehe Abbildung 7-29.

- Bei realen Verbundträgern ist immer ein vergleichsweise hoher Belastungsanteil infolge Eigengewicht vorhanden, der gleichförmig verteilt ist. Die mittragende Breite aus der kombinierten Wirkung dieser Lasten ist größer als bei ausschließlicher Betrachtung der Einzellast.

- Einzellasten in realen Bauwerken besitzen eine endliche Lastverteilungsfläche. Im Vergleich zur theoretischen Punktlast wird dadurch die Spannungsspitze im Bereich der Einzellast sehr stark abgeschwächt und die mittragende Breite größer.

In Abbildung 7-29 ist ein Vergleich der rechnerischen Verformungen der durchgeführten Vergleichsrechnungen bei Ansatz der verformungsbezogenen mittragenden Breite nach Gleichung (7-1) und bei Berechnung mit dem analytischen Berechnungsmodell dargestellt. Die Verformungen werden dabei mit Gleichung (7-1) im Grenzzustand der Gebrauchstauglichkeit im Mittel um $9 \%$ überschätzt. Die Standardabweichung liegt bei 8,8\%. Gleichung (7-1) liefert somit für die Verformungsberechnung niedriger Verbundträger mit mittiger Einzellast eine ausreichend genau Näherung der verformungsbezogenen mittragenden Breite. Dies gilt insbesondere unter Beachtung der oben genannten Punkte. 


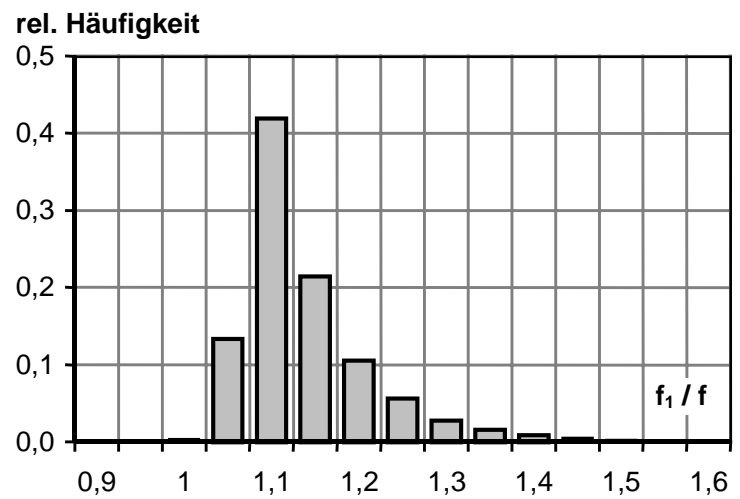

(a) für Ausnutzungen $M / M_{R d}=0 \div 0,8$

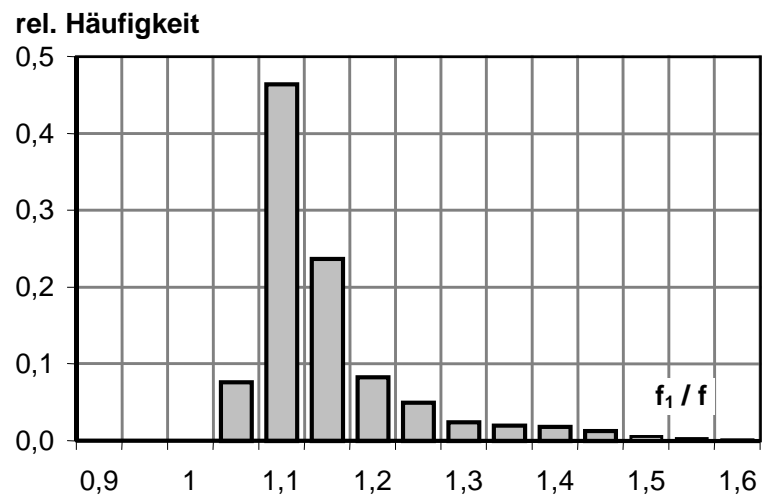

(b) im $G Z G\left(M / M_{R d} \approx 0,65\right)$

Abbildung 7-29: Vergleich der rechnerischen Verformungen niedriger Verbundträger mit mittiger Einzellast bei Verwendung von Gleichung (7-1) $f_{1}$ und dem analytischen Berechnungsmodell $f$

\subsubsection{Eigenspannungen im Stahlprofil}

In Stahlträgern sind in der Regel immer Eigenspannungen vorhanden - entweder infolge des Walzvorgangs oder bei geschweißten Trägern infolge der ungleichmäßigen Erwärmung bzw. Abkühlung beim Schweißvorgang. Die Eigenspannungen stehen dabei verteilt über den Stahlquerschnitt im Gleichgewicht. Daher treten in den Gurten sowohl Zug- als auch Druckeigenspannungen auf. Die Zugeigenspannungen führen bei Verbundträgern unter positiven Momenten bei niedrigeren Lasten zu Plastizierungen als bei vergleichbaren Trägern ohne Eigenspannungen und zu größeren Verformungen. Die Auswirkungen von Eigenspannungen können rechnerisch als lokale Absenkung bzw. Erhöhung der Streckgrenze berücksichtigt werden.

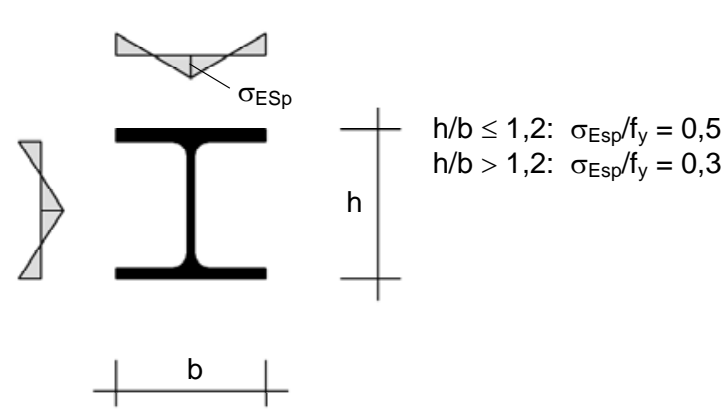

(a) Eigenspannungsverteilung nach [2.69][2.71]
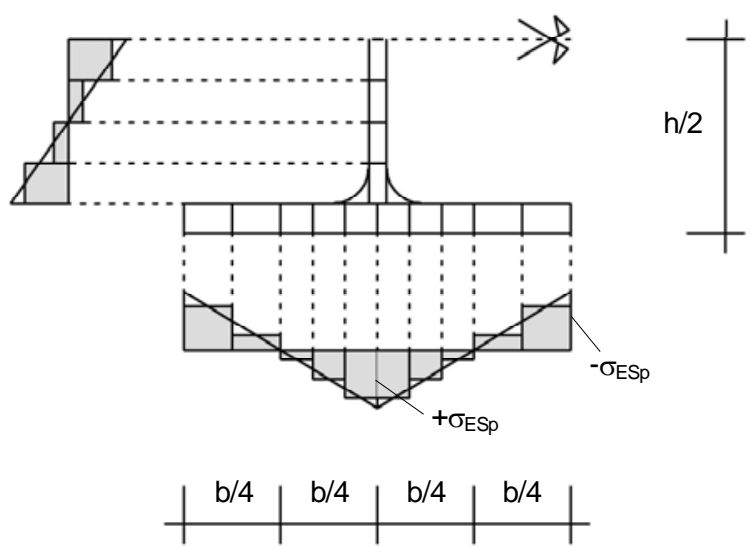

(b) Ansatz der Eigenspannungsverteilung beim erweiterten analytischen Berechnungsmodell

Abbildung 7-30: Gewählte Eigenspannungsverteilung in Stahlträgern mit I-Profil

Zur qualitativen Abschätzung dieses Einflusses, wurde das analytische Berechnungsmodell zur Berücksichtigung des Eigenspannungszustands erweitert. Die Verteilung der Eigenspannungen im Querschnitt wurde gemäß [2.69]-[2.71] berücksichtigt, siehe Abbildung 7-30. Beim analytischen Berechnungsmodell wurden die Gurte dabei mit zehn, der Steg des Stahlträgerquerschnitts mit acht Elementen diskretisiert. Jedem Element wurde eine modifizierte Streckgrenze entsprechend der Eigenspannungsverteilung zugeordnet. 
System und Querschnitt:

Trägerstützweite: $L=5 \div 15 \mathrm{~m}$

Trägerbreite: $\quad b=3 \div 9 \mathrm{~m}$

Plattendicke: $\quad \mathrm{h}_{\mathrm{c}}=16 \div 32 \mathrm{~cm}$

Höhe Stahlträger: $h_{a}=200 \div 600 \mathrm{~mm}$

Profilreihen: $\quad$ HEA, HEB, IPE

Material:

Beton:

C 20/25, C 30/37

Baustahl:

S 235, S 355

Verdübelung:

$\eta=1$ (volle Verdübelung)
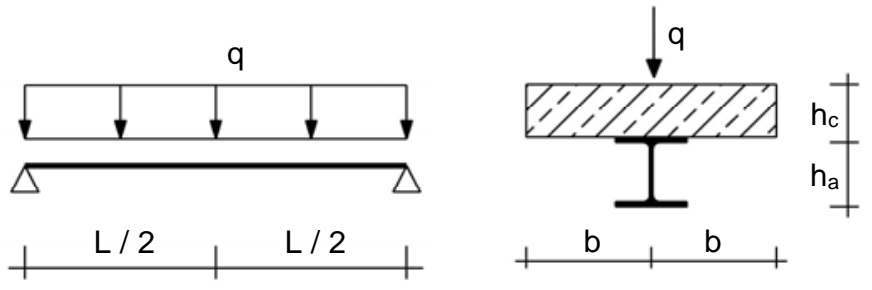

Umfang:

ca. 1000 Träger

(a) Abmessungen

(b) Bezeichnungen

Abbildung 7-31: Umfang der Vergleichsrechnungen zur Berücksichtigung von Eigenspannungen im Stahlträger

Zur Untersuchung des Einflusses von Eigenspannungen auf die Verformungen von Verbundträgern niedriger Bauhöhe wurden Vergleichsrechnungen an Trägern mit und ohne Eigenspannungen durchgeführt, deren Umfang in Abbildung 7-31 dargestellt ist. Im Grenzzustand der Gebrauchstauglichkeit ergeben sich durch die Eigenspannungen für die untersuchten Träger eine Erhöhung der Verformungen von im Mittel ca. 3 $\div 10 \%$ und maximal bis zu $20 \%$ je nach Höhe der Eigenspannungen und System, siehe Tabelle 7-1 und Abbildung 7-32.

Die Vergleichsrechnungen zeigen, dass in vielen Fällen der Einfluss der Eigenspannungen auf die Verformung niedriger Verbundträger im Grenzzustand der Gebrauchstauglichkeit im Vergleich zu den weiteren Einflüssen vernachlässigt werden kann. Dies gilt insbesondere für Eigenspannungsverteilungen mit $\sigma_{E S p} / f_{y} \leq 0,3$. In besonderen Fällen, wenn eine größere Genauigkeit gefordert ist, sollten die Eigenspannungen aber bei der Verformungsberechnung explizit berücksichtigt werden, gegebenenfalls durch Abschätzung mit den oben genannten Abweichungen.

rel. Häufigkeit

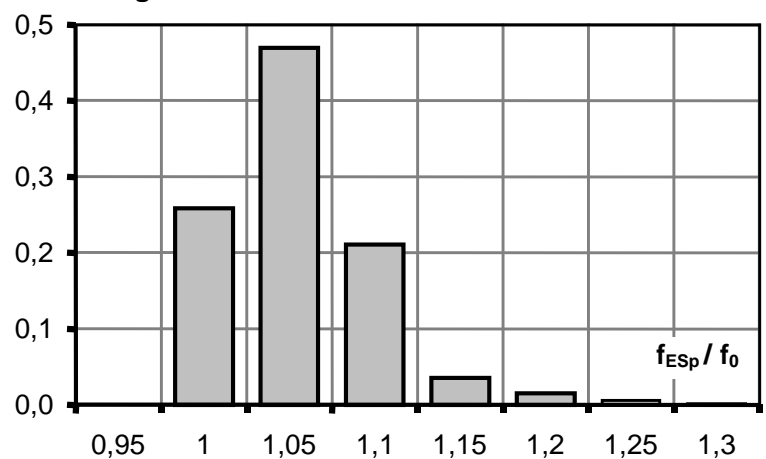

(a) $\sigma_{E S p} / f_{y}=0,3 \div 0,5$

$f_{0} \quad$ Verformung ohne Eigenspannungen

$\mathrm{f}_{\mathrm{ESp}} \quad$ Verformung mit Eigenspannungen rel. Häufigkeit

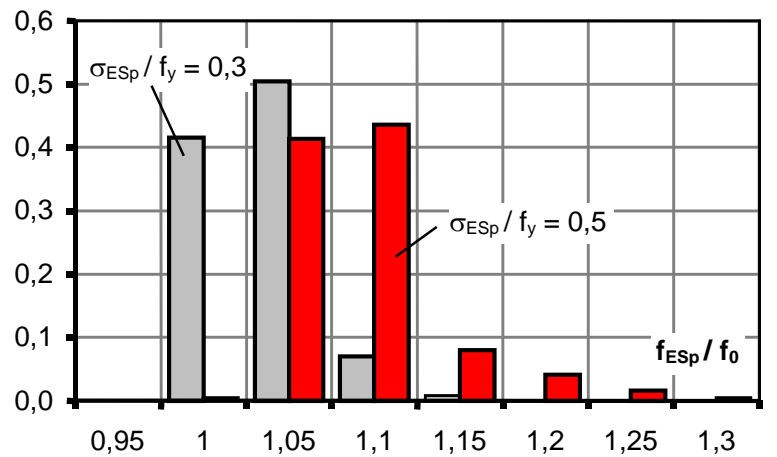

(b) $\sigma_{E S p} / f_{y}=0,3$ bzW. $\sigma_{E S p} / f_{y}=0,5$

Abbildung 7-32: Verformungseinfluss von Eigenspannungen im Stahlträger niedriger Verbundträger im Grenzzustand der Gebrauchstauglichkeit 
Tabelle 7-1: Vergleich der Verformungen niedriger Verbundträger im Grenzzustand der Gebrauchstauglichkeit mit und ohne Berücksichtigung von Eigenspannungen im Stahlträger

\begin{tabular}{|c|c|c|c|c|c|c|c|c|c|}
\hline \multirow{2}{*}{$\frac{\sigma_{\mathrm{ESP}} / \mathbf{f}_{\mathrm{y}}}{\text { Profil }}$} & \multicolumn{4}{|c|}{0,3} & \multicolumn{4}{|c|}{0,5} & \multirow{2}{*}{$\frac{\mathbf{0 , 3} \div \mathbf{0 , 5}}{\text { alle }}$} \\
\hline & alle & IPE & HEA & HEB & alle & IPE & HEA & HEB & \\
\hline $\mathrm{MW}^{1)}$ & 1,03 & 1,04 & 1,03 & 1,03 & 1,09 & - & 1,11 & 1,08 & 1,06 \\
\hline$\delta_{\mathrm{m}}{ }^{2)}[\%]$ & 1,7 & 2,0 & 1,3 & 1,6 & 2,8 & - & 3,8 & 1,9 & 2,9 \\
\hline $\mathrm{s}^{3)}[\%]$ & 2,4 & 2,7 & 1,7 & 2,2 & 4,2 & - & 5,3 & 2,8 & 4,1 \\
\hline$q_{0,05}{ }^{4)}$ & 1,01 & 1,01 & 1,01 & 1,01 & 1,01 & - & 1,06 & 1,05 & 1,01 \\
\hline$q_{0,95}{ }^{5)}$ & 1,07 & 1,08 & 1,05 & 1,07 & 1,17 & - & 1,20 & 1,13 & 1,11 \\
\hline 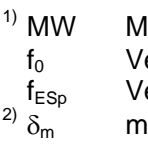 & $\begin{array}{l}\text { Nert } f_{E S} \\
\text { mung } \\
\text { mung } \\
\text { e Abw }\end{array}$ & $\begin{array}{l}\text { e Eige } \\
\text { Eigens } \\
\text { ung } \mathrm{f}_{\mathrm{E}}\end{array}$ & $\begin{array}{l}\text { annung } \\
\text { nungen }\end{array}$ & & $\begin{array}{l}s \\
q_{0,95} \\
q_{0,95}\end{array}$ & $\begin{array}{l}\text { Standar } \\
\text { 95\%-QL } \\
95 \%-Q \mathrm{~L}\end{array}$ & & & \\
\hline
\end{tabular}

\subsubsection{Querbiegung im Betongurt}

Da bei Verbundträgern in realen Bauwerken die Belastung im Allgemeinen nicht ausschließlich in der Achse der Verbundträger aufgebracht wird, entsteht im Betongurt nicht zuletzt infolge des Eigengewichts eine Querbiegung. Solange der Betongurt im Zustand I ist, hat diese Querbiegung keinen Einfluss auf das Tragverhalten des Verbundträgers und die mittragende Breite des Betongurts (Superpositionsprinzip), siehe Kapitel 4. Infolge der Querbiegung können an den Stahlträgern, die als Auflager für die quergespannte Decke dienen, jedoch Längsrisse im oberen Bereich des Betongurts entstehen, die die Verhältnisse zwischen Längs-, Quer- und Schubsteifigkeit des Betongurts verändern. Diese Veränderung der Steifigkeitsverhältnisse ist dabei unter anderem von der Intensität der Rissbildung und somit von der Belastungshöhe abhängig. Eine exakte Berücksichtigung dieser Einflüsse ist sehr aufwändig. Eine Abschätzung des Einflusses der veränderten Steifigkeitsverhältnisse im Betongurt kann durch Betrachtung des Betongurts als orthotrope Scheibe bzw. Platte erfolgen. Für eine ausführliche Darstellung dieser Vergleichsrechnung wird an dieser Stelle auf [2.77] verwiesen.

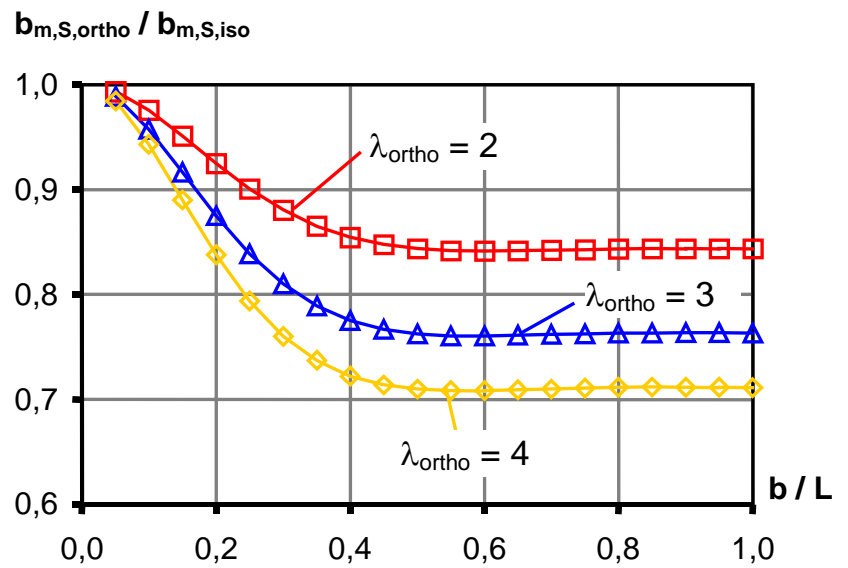

Steifigkeitsverhältnisse:

$$
\begin{aligned}
& D_{x}=\lambda_{\text {ortho }} \cdot D_{y} \\
& D_{x y}=\sqrt{\lambda_{\text {ortho }}} \cdot D_{y}
\end{aligned}
$$

mit $D_{x}$ Dehnsteifigkeit in Längsrichtung $D_{y}$ Dehnsteifigkeit in Querrichtung $D_{\text {xy }}$ Schubsteifigkeit

$$
\begin{array}{ll}
\mathrm{b}_{\mathrm{m}, \mathrm{s}, \text { ortho }} & \begin{array}{l}
\text { mittragende Breite der } \\
\text { orthotropen Scheibe }
\end{array} \\
\mathrm{b}_{\mathrm{m}, \mathrm{S}, \text { iso }} & \begin{array}{l}
\text { mittragende Breite der } \\
\text { isotropen Scheibe }
\end{array}
\end{array}
$$

Abbildung 7-33: Mittragende Breite der orthotropen Scheibe $b_{m, s, o r t h o}$ im Vergleich zur isotropen Scheibe $b_{m, S \text {,iso }}$ in Abhängigkeit des Orthotropiefaktors $\lambda_{\text {ortho }}$ 
$b_{m, B, o r t h o} / b_{m, B, i s o}$

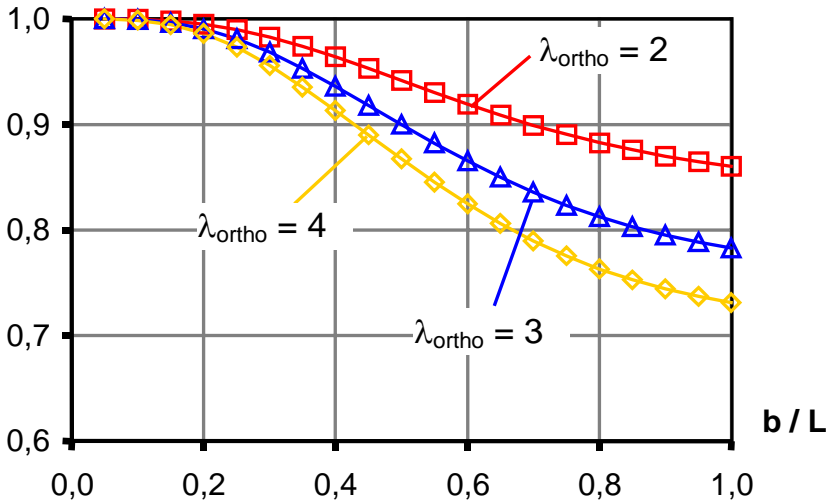

Steifigkeitsverhältnisse:

$\mathrm{K}_{\mathrm{x}}=\lambda_{\text {ortho }} \cdot \mathrm{K}_{\mathrm{y}}$

$\mathrm{K}_{\mathrm{xy}}=\sqrt{\lambda_{\text {ortho }}} \cdot \mathrm{K}_{\mathrm{y}}$

mit $K_{x}$ Biegesteifigkeit in Längsrichtung $\mathrm{K}_{\mathrm{y}}$ Biegesteifigkeit in Querrichtung $\mathrm{K}_{\mathrm{xy}}$ Drillsteifigkeit

$b_{m, B, o r t h o}$ mittragende Breite der orthotropen Platte

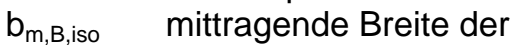
isotropen Platte

Abbildung 7-34: Mittragende Breite der orthotropen Platte $b_{m, B, o r t h o}$ im Vergleich zur isotropen Platte $b_{m, B, i s o}$ in Abhängigkeit des Orthotropiefaktors $\lambda_{\text {ortho }}$

Gleichungen (7-5) und (7-6) zeigen die Differentialgleichungen der orthotropen Scheibe und Platte. In Abbildung 7-33 und Abbildung 7-34 sind die Werte der mittragenden Breite für verschiedene Orthotropiefaktoren auf Basis der Elastizitätstheorie im Vergleich zu den entsprechenden Werte der isotropen Scheibe und Platte dargestellt. Wie zu erkennen ist, ergibt sich bei geringerer Steifigkeit des Gurtes in Querrichtung $\left(\lambda_{\text {ortho }}>1\right)$ eine geringere mittragende Breite. Die Abweichungen sind sehr stark vom Orthotropiefaktor $\lambda_{\text {ortho }}$ und dem Seitenverhältnis $b / L$ des Betongurts abhängig. Die Abnahme der mittragenden Breite infolge der Orthotropie des Gurtes liegt dabei im Hauptanwendungsgebiet bei ca. $10 \div 25 \%$ (elastischer Fall).

$$
\frac{1}{D_{y}} \cdot \frac{\partial^{4} \Phi}{\partial y^{4}}+\frac{2}{D_{x y}} \cdot \frac{\partial^{4} \Phi}{\partial x^{2} \partial y^{2}}+\frac{1}{D_{x}} \cdot \frac{\partial^{4} \Phi}{\partial x^{4}}=0
$$

mit

$\begin{array}{ll}\Phi & \text { Airysche Spannungsfunktion } \\ x & \text { Koordinate der Gurtscheibe in Längsrichtung des Trägers } \\ y & \text { Koordinate der Gurtscheibe in Querrichtung des Trägers } \\ D_{x} & \text { Dehnsteifigkeit der Scheibe in x-Richtung } \\ D_{y} & \text { Dehnsteifigkeit der Scheibe in y-Richtung } \\ D_{x y} & \text { Schubsteifigkeit der Scheibe }\end{array}$

$$
K_{x} \cdot \frac{\partial^{4} W}{\partial y^{4}}+2 \cdot K_{x y} \cdot \frac{\partial^{4} w}{\partial x^{2} \partial y^{2}}+K_{y} \cdot \frac{\partial^{4} w}{\partial x^{4}}=0
$$

mit

$\mathrm{w}$
$\mathrm{x}$
$\mathrm{y}$
$\mathrm{K}_{\mathrm{x}}$
$\mathrm{K}_{\mathrm{y}}$
$\mathrm{K}_{\mathrm{xy}}$

Durchbiegung der Gurtplatte

Koordinate der Gurtplatte in Längsrichtung des Trägers Koordinate der Gurtplatte in Querrichtung des Trägers Biegesteifigkeit der Platte in X-Richtung Biegesteifigkeit der Platte in y-Richtung Drillsteifigkeit der Platte

Wenn man beim analytischen Berechnungsmodell in einem weiteren Schritt anstatt der üblicherweise verwendeten isotropen Werte für die spannungsbezogene mittragende Breite die orthotropen Werte ansetzt, kann eine Abschätzung des Einflusses der Orthotropie des Gurtes auf das Verformungsverhalten niedriger Verbundträger unter Beachtung der Rissbildung im Beton bestimmt werden. Für die in Abbildung 7-35 dargestellten Träger wurden 
hierfür die Mittendurchbiegungen im isotropen und orthotropen Fall mit Hilfe des analytischen Berechungsmodells bestimmt. Im Grenzzustand der Gebrauchstauglichkeit ergeben sich bei den berechneten Trägern für Orthotropiefaktoren $\lambda_{\text {ortho }}=2 \div 4$ im Mittel ca. $3 \div 8 \%$ größere Verformungen. Der Einfluss der Orthotropie des Betongurts auf die Verformung ist somit vergleichsweise gering.

Die Ergebnisse dieser Vergleichsrechnungen können allerdings nur als grobe Anhaltswerte dienen, da sich das Steifigkeitsverhältnis im Betongurt durch die Rissbildung mit zunehmender Belastung ändert (die Rissbildung führt dabei sowohl in Längs- als auch in Querrichtung zu einer Steifigkeitsänderung). Für eine genauere Abschätzung des Einflusses der Querbiegung auf die mittragende Breite sind weitere Untersuchungen z. B. mit Finite-ElementModellen notwendig, die das nichtlineare Tragverhalten von Beton und vor allem den Einfluss der Rissbildung auf die Steifigkeit abbilden können. Dies ist Gegenstand zur Zeit laufender Untersuchungen [2.77].

\section{System und Querschnitt:}

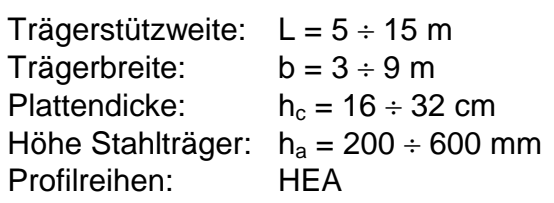

Material:

$\begin{array}{ll}\text { Beton: } & \text { C 20/25 } \\ \text { Baustahl: } & \text { S 355 } \\ \text { Verdübelung: } & \eta=1 \text { (volle Verdübelung) }\end{array}$

(a) Abmessungen
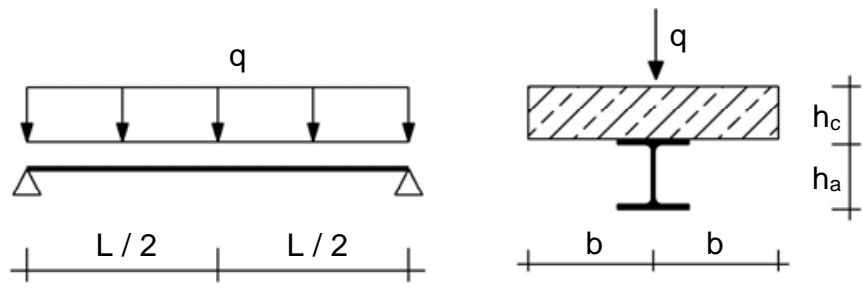

(b) Bezeichnungen

Abbildung 7-35: Umfang der Vergleichsrechnungen der Einflusses der Orthotropie des Betongurts auf die mittragende Breite

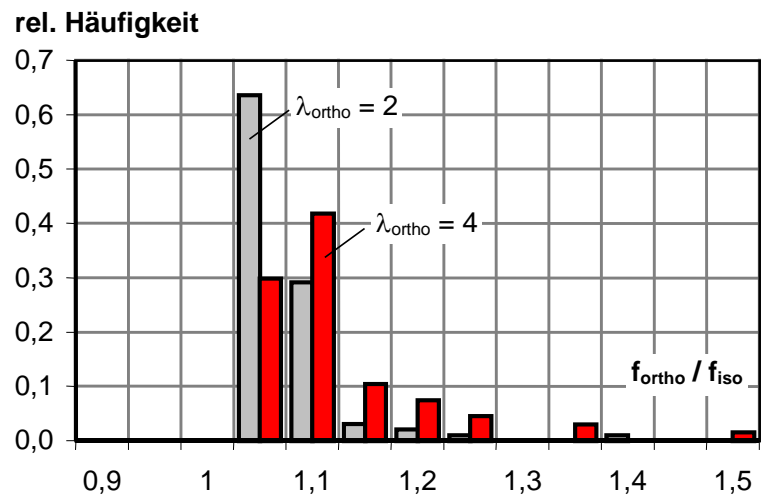

(a) Häufigkeitsverteilung

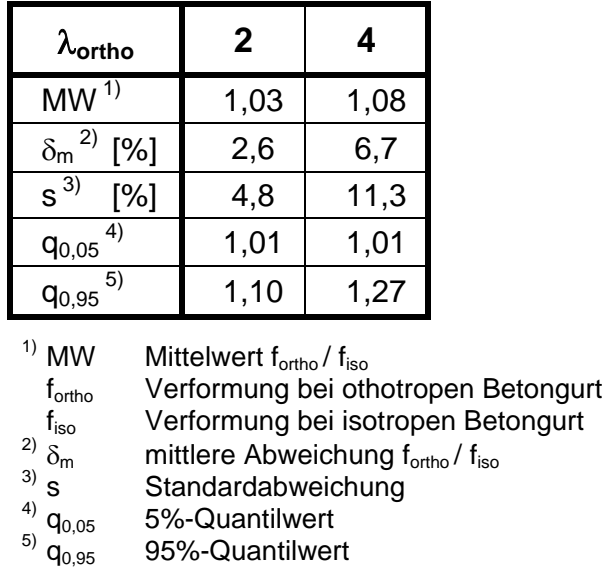

(b) mittlere Abweichungen

Abbildung 7-36: Vergleich der rechnerischen Verformungen für verschiedene Orthotropiefaktoren $\lambda_{\text {ortho }}$ 


\subsection{Zusammenfassung}

Anhand einer umfangreichen Parameterstudie mit dem entwickelten analytischen Berechnungsmodell wurden die Einflüsse auf die verformungsbezogene mittragende Breite systematisch untersucht und quantifiziert. Dies führte zur Entwicklung eines realistischen Berechnungsansatzes für die verformungsbezogene mittragende Breite $b_{m, v}$, der die maßgebenden Einflüsse berücksichtigt. Unter anderen haben der Ausnutzungsgrad des Trägers, die Betonzugfestigkeit, die Streckgrenze des Stahlträgers und das Verhältnis Gurtdicke zu Gesamthöhe des Trägers einen wesentlichen Einfluss. Die mit diesem Ansatz ermittelten Werte für die verformungsbezogene mittragende Breite haben im Mittel eine Abweichung von 4,3\% im Vergleich zur genaueren Berechnung mit dem analytischen Berechnungsmodell. Sie liefern somit eine sehr gute Übereinstimmung. Mit dem vorgestellten Berechnungsansatz können somit wirklichkeitsnahe Verformungswerte niedriger Verbundträger berechnet werden. Dies stellt im Vergleich zu den bestehenden Regelungen und insbesondere zu EN 1994-1-1 und DIN 18800-5 eine wesentliche Verbesserung dar.

Wenn eine geringere Genauigkeit für die Verformungsberechnung ausreichend ist, wird ein vereinfachter Berechnungsansatz für die verformungsbezogene mittragende Breite vorgeschlagen. Die Abweichungen im Vergleich zur genaueren Berechnung mit dem analytischen Berechnungsmodell betragen für die verformungsbezogene mittragende Breite im Mittel $8,7 \%$ und für die damit berechneten Verformungen 2,2\%. Auch mit diesem vereinfachten Ansatz ist somit eine deutlich realistischere Verformungsberechnung niedriger Verbundträger möglich als mit den bestehenden Regelungen.

Durch Vergleichsrechnungen konnte gezeigt werden, dass der entwickelte Berechnungsansatz auch zur Verformungsberechung bei von einer gleichmäßigen Belastung abweichenden Laststellungen angewendet werden kann. Vergleichsrechnungen, die die Eigenspannungen im Stahlträger berücksichtigen, zeigen, dass die Eigenspannungen bei Stahlquerschnitten mit b/h > 1,2 für die Verformungen im Grenzzustand der Gebrauchstauglichkeit vernachlässigt werden können. Bei Querschnitten mit b/h $<1,2$ können diese jedoch zu einer Erhöhung der Verformungen von ca. 10 \% führen. Wenn eine hohe Genauigkeit der Verformungsberechnung gefordert wird, sollten die Eigenspannungen somit bei diesen Querschnitten in der Berechnung explizit berücksichtigt werden. Für den Einfluss einer Längsrissbildung des Betongurts am Stahlträger infolge einer Querbiegung im Betongurt wurde eine Abschätzung durch Betrachtung des Betongurts als orthotrope Scheibe bzw. Platte gemacht. Es zeigte sich, dass die Orthotropie des Gurtes im Hauptanwendungsgebiet niedriger Verbundträger einen Einfluss von ca. 10 $\div 25 \%$ auf die spannungsbezogene mittragende Breite haben kann. Durch Vergleichsrechnung konnte gezeigt werden, dass dies die Verformungen von niedrigen Verbundträgern nur in einer Größenordnung von ca. $5 \%$ beeinflusst und somit in vielen Fällen vernachlässigt werden kann. Für eine genauere Abschätzung dieses Einflusses sind jedoch weitere Untersuchungen notwendig.

Die hier vorgestellten Berechnungsansätze ermöglichen eine realistische Ermittlung der verformungsbezogenen mittragenden Breite niedriger Verbundträger und die wirklichkeitsnahe Berechnung deren Durchbiegung. Im Vergleich zu den bestehenden Regelungen ergeben sich hierbei im Allgemeinen kleinere Verformungswerte, wodurch eine wirtschaftlichere Dimensionierung dieser Träger möglich ist. 


\section{Vorschlag zur quasi-elastischen Verformungsberechnung niedriger Verbundträger}

\subsection{Grundsätzliches}

Verbundträger niedriger Bauhöhe weisen bereits unter Gebrauchslasten eine starke Rissbildung und ein ausgeprägtes nichtlineares Trag- und Verformungsverhalten auf. Eine rein elastische Verformungsberechnung unterschätzt daher im Allgemeinen die tatsächlichen Verformungen und stellt in vielen Fällen eine unzureichende Näherung dar. Eine wirklichkeitsnahe Verformungsberechnung muss daher das nichtlineare Tragverhalten dieser Träger berücksichtigen. Die Berechnung von Verformungen unter expliziter Berücksichtigung des

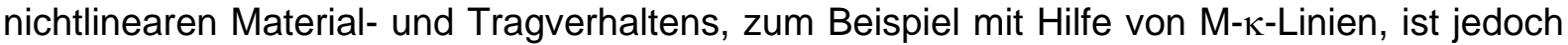
vergleichsweise aufwändig. Für eine Berechnung in der Planungspraxis ist daher ein vereinfachtes Verfahren auf Basis des bekannten elastischen Berechnungsverfahren von Vorteil und wünschenswert. Um zum einen den Einfluss des nichtlinearen Tragverhaltens niedriger Verbundträger bei der Verformungsberechnung zu berücksichtigen und zum Anderen für die Planungspraxis ein handhabbares Verfahren zu gewährleisten, wird im Folgenden ein Vorschlag zur Modifikation des bekannten Gesamtquerschnittsverfahrens [2.29], [2.30] gemacht, der diese Aspekte berücksichtigt.

Kernstück der Modifikation ist die Bestimmung eines effektiven Trägheitsmoments $\mathrm{I}_{\mathrm{i}, \text { eff }}$ des Verbundträgers, mit dem die Verformungen quasi-elastisch berechnet werden können. Das effektive Trägheitsmoment $\mathrm{I}_{\mathrm{i}, \text { eff }}$ berücksichtigt dabei das nichtlineare Material- und Tragverhalten niedriger Verbundträger implizit durch Reduktion der elastischen Steifigkeitsanteile des Betons mit einem Abminderungsfaktor $\alpha_{c}$. Anhand einer umfangreichen Parameterstudie wurden im Rahmen der vorliegenden Arbeit die Einflussfaktoren auf den Abminderungsfaktor $\alpha_{c}$ untersucht und eine Näherungsfunktion hierfür entwickelt, die eine schnelle und wirklichkeitsnahe Verformungsberechnung dieser Träger ermöglicht.

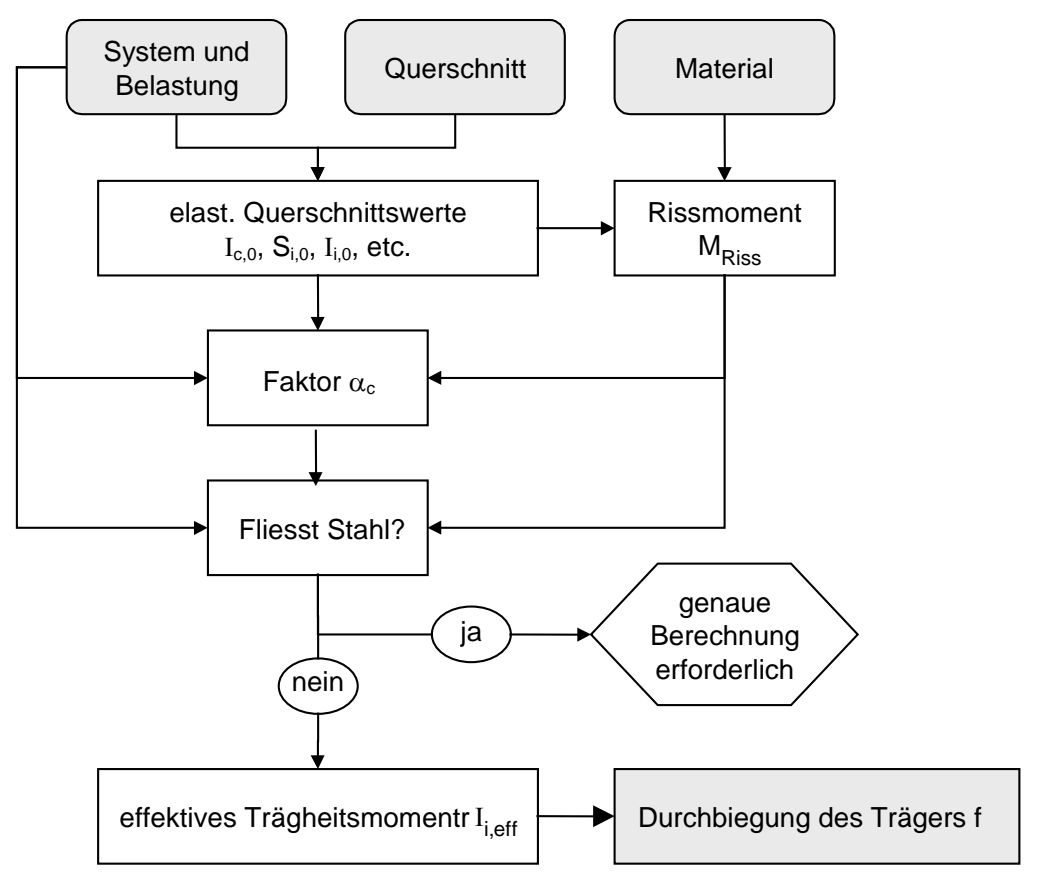

Abbildung 8-1: Vorgehen bei der quasi-elastischen Verformungsberechnung 


\subsection{Beschreibung des quasi-elastischen Berechnungsvorschlags}

\subsubsection{Allgemeines}

Das grundsätzliche Vorgehen bei der vorgeschlagenen quasi-elastischen Verformungsberechnung ist in Abbildung 8-1 dargestellt:

1. Berechnung der elastischen Steifigkeitswerte und Trägheitsmomente $\left(\mathrm{I}_{i, 0}, \mathrm{I}_{\mathrm{c}, 0}, \mathrm{~S}_{\mathrm{i}, 0}\right.$, etc.) und des Rissmoments $M_{\text {Riss }}$ des Verbundquerschnitts

2. Überprüfung der Rissbildung des Betongurts und des Spannungszustands des Stahlträgers

3. Berechnung des Abminderungsfaktors $\alpha_{c}$

4. Berechnung des effektiven Trägheitsmoments $\mathrm{I}_{\mathrm{i}, \mathrm{eff}}$ des Verbundträgers

5. Quasi-elastische Berechnung der Verformung mit Hilfe des effektiven Trägheitsmoments $\mathrm{I}_{\mathrm{i}, \mathrm{eff}}$

\subsubsection{Effektives Trägheitsmoment $\mathrm{I}_{\mathrm{i}, \mathrm{eff}}$}

Der Grundgedanke des quasi-elastischen Berechnungsvorschlags ist, die Verformungen niedriger Verbundträger wie beim Gesamtquerschnittsverfahren [2.29], [2.30] zu berechnen und dabei den Einfluss der Rissbildung und des nichtlinearen Tragverhaltens durch die Reduktion des Trägheitsmoments des Verbundträgers zu berücksichtigen. Hierzu werden die Steifigkeitsanteile des Betons mit den Abminderungsfaktor $\alpha_{c}$ reduziert, siehe Gleichung (8-1) bis (8-8). Das Trägheitsmoment $I_{c}$ des Betons wird dabei mit der mittragenden Breite $b_{m, B}$ des Biegezustands in Trägermitte berechnet, die Betonfläche $A_{c}$ mit der mittragenden Breite $b_{m, s}$ des Scheibenzustands in Trägermitte.

$$
\begin{aligned}
& I_{i, e f f}=I_{a}+\alpha_{c} \cdot\left(I_{c, 0}+S_{i, 0} \cdot a_{S t}\right) \\
& I_{i, 0}=I_{a}+I_{c, 0}+S_{i, 0} \cdot a_{S t} \\
& S_{i, 0}=A_{c, 0} \cdot Z_{i, 0} \\
& Z_{i, 0}=\frac{A_{a} \cdot a_{S t}}{A_{i, 0}} \\
& A_{i, 0}=A_{a}+A_{c, 0} \\
& I_{c, 0}=\frac{b_{m, B} \cdot h_{c}^{3}}{12 \cdot n_{0}} \\
& A_{c, 0}=\frac{b_{m, S} \cdot h_{c}}{n_{0}} \\
& n_{0}=\frac{E_{a}}{E_{c m}}
\end{aligned}
$$

mit

$$
\begin{aligned}
& I_{i, \text { eff }} \\
& I_{i, 0}, I_{a}, I_{c, 0} \\
& A_{i, 0}, A_{a}, A_{c, 0}
\end{aligned}
$$

effektives Trägheitsmoment des Verbundträgers elastische Trägheitsmomente des Verbund-, Stahl- und Betonquerschnitts

Querschnittsflächen des Verbund-, Stahl- und Betonquerschnitts 


$a_{s t}$
$n_{0}$
$E_{a}, E_{c m}$
$b_{m, s}, b_{m, B}$
$\alpha_{c}$

$\mathrm{a}_{\mathrm{St}}$
Schwerpunktsabstand zw. Stahl- und Betonquerschnitt Reduktionszahl für den Betonquerschnitt

E-Modul des Stahl- und Betonquerschnitts

mittragende Breite des Scheiben- und Biegezustands nach Abschnitt 4.2

Abminderungsfaktor, siehe Abschnitt 8.3

\subsubsection{Quasi-elastische Verformungsberechnung}

Nachdem das effektive Trägheitsmoment $\mathrm{I}_{\mathrm{i}, \text { eff }}$ des Verbundträgers bestimmt wurde, kann die Verformung quasi-elastisch am Ersatzbalken mit konstanter Steifigkeit $\mathrm{I}_{\mathrm{i}, \text { eff }}$ gemäß den Regeln der technischen Biegelehre berechnet werden. Abschließend muss kontrolliert werden, ob der Stahlträger unter der gegebenen Belastung bereits plastiziert. Sollte dies der Fall sein, so ist eine genauere Verformungsberechnung unter expliziter Berücksichtigung des nichtlinearen Materialverhaltens erforderlich.

\subsubsection{Voraussetzungen und Annahmen}

In der vorliegenden Arbeit wurde der Abminderungsfaktor $\alpha_{c}$ für Verbundeinfeldträger mit einer Gleichlast unter hochbautypischen Randbedingungen bestimmt. Der Abminderungsfaktor $\alpha_{c}$ wurde dabei aus den Ergebnissen der in Abschnitt 7.3 dargestellten Parameterstudie mit dem in Abschnitt 6.2 beschriebenen analytischen Berechnungsmodell rückgerechnet. Für den in Abschnitt 8.4 entwickelten Berechnungsansatz treffen somit ebenfalls die in Abschnitt 6.3 und 7.3.2 genannten Voraussetzungen und Annahmen zu. Die Wichtigsten sind:

- Der Querschnitt des Verbundträgers ist in Trägerlängsrichtung konstant.

- Der Verbundträger wird als Einfeldträger ausgeführt.

- Die Träger weisen einen starren Verbund auf.

- Ein Versagen der Verbundfuge wird ausgeschlossen.

- Der Stahlträger bleibt elastisch.

- Der Einfluss der Querbiegung auf das Trag- und Verformungsverhalten sowie die Verteilung der spannungsbezogenen mittragenden Breite kann vernachlässigt werden.

- Die System- und Materialwerte liegen im Rahmen des in Abbildung 7-3 dargestellten Umfangs.

- Eigenspannungen im Stahlträger haben keinen nennenswerten Einfluss auf das Verformungsverhalten des Verbundträgers.

- Die Durchbiegung des Verbundträgers infolge Querkraft wird elastisch am Stahlträger bestimmt.

- Mit dem analytischen Berechnungsmodell kann die Durchbiegung von niedrigen Verbundträgern in ausreichender Genauigkeit berechnet werden, siehe Abschnitt 6.2 und Abschnitt 6.3.

Die beschriebenen Annahmen wurden so getroffen, dass hiermit eine wirklichkeitsnahe Verformungsberechnung niedriger Verbundträger möglich ist. Dadurch unterliegt der Vorschlag zur quasi-elastische Verformungsberechnung jedoch unter Anderem folgenden Einschränkungen: 
- Eine Berechnung von Spannungen im Beton ist nicht möglich.

- Verbundträger mit ausgeprägtem Schlupf zwischen Beton und Stahlträger, zum Beispiel infolge einer teilweisen Verdübelung, können nicht berechnet werden.

\subsection{Abminderungsfaktor $\alpha_{c}$ zur quasi-elastischen Verformungsberechnung niedriger Verbundträger}

\subsubsection{Allgemeines}

Niedrige Verbundträger weisen bereits unter Gebrauchlasten eine ausgeprägte Rissbildung auf. Bei dem vorgeschlagenen quasi-elastischen Berechnungsvorschlag wird der Einfluss der Rissbildung auf die Verformung rechnerisch durch Reduktion der elastischen Steifigkeitsanteile des Betons mit dem Abminderungsfaktor $\alpha_{c}$ berücksichtigt, siehe Abschnitt 8.1. An Trägern, deren tatsächliche Verformungen $\mathrm{f}_{0}$ bekannt sind, kann der Abminderungsfaktor $\alpha_{c}$ durch Vergleich mit der rein elastischen Verformung $f_{e l}$ rückgerechnet werden, siehe Gleichung (8-9) bis (8-11). Die elastische Verformung $f_{\mathrm{el}}$ wird dabei am Ersatzbalken mit konstanter Steifigkeit $\mathrm{I}_{\mathrm{i}, \text { eff }}$ berechnet, siehe Abschnitt 8.2.

$$
\begin{aligned}
& \frac{f_{0}}{f_{e l}}=\frac{I_{i, 0}}{I_{i, e f f}} \\
& I_{i, \text { eff }}=\frac{f_{e l}}{f_{I I}} \cdot I_{i, 0}
\end{aligned}
$$

mit

$$
\begin{aligned}
& I_{i, e f f} \\
& I_{i, 0} \\
& f_{e l} \\
& \alpha_{c}=\frac{I_{i, 0} \cdot \frac{f_{e l}}{f_{0}}-I_{a}}{I_{c, 0}+S_{i, 0} \cdot a_{S t}}
\end{aligned}
$$

mit

$$
\alpha_{c}
$$

Abminderungsfaktor zur Berücksichtigung des nichtlinearen Material- und Tragverhaltens niedriger Verbundträger

Da die Rissbildung sehr stark von der Belastungshöhe abhängt, sind in gleicher Weise auch das effektive Trägheitsmoment $\mathrm{I}_{\mathrm{i}, \text { eff }}$ und der Abminderungsfaktor $\alpha_{\mathrm{c}}$ von der Belastungshöhe abhängig. Die typischen Verläufe des effektiven Trägheitsmoments und des Abminderungsfaktors in Abhängigkeit des Ausnutzungsgrads $M / M_{u}$ des Trägers sind in Abbildung 8-2 dargestellt. Der Ausnutzungsgrad stellt dabei das Verhältnis des maximalen Moments $M$ in Trägermitte zum Bruchmoment $\mathrm{M}_{\mathrm{u}}$ des Trägers dar. Die Verläufe lassen sich in drei Bereiche unterteilen: 
- Bereich 1 - Elastischer Bereich

Der Träger verhält sich elastisch und ist im Zustand I. Die Belastung ist kleiner als die Risslast $\left(M / M_{u}<M_{\text {Riss }} / M_{u}\right)$. Das effektive Trägheitsmoment $I_{i, \text { eff }}$ ist gleich dem elastischen $\mathrm{I}_{\mathrm{i}, 0}$, der Abminderungsfaktor $\alpha_{\mathrm{c}}$ ist eins.

- Bereich 2 - Zustand II, Rissbildung

Die Belastung ist größer als die Risslast $\left(M / M_{u}>M_{\text {Riss }} / M_{u}\right)$, der Träger ist gerissen und teilweise oder vollständig im Zustand II. Die Steifigkeit und damit das effektive Trägheitsmoment $\mathrm{I}_{\mathrm{i}, \text { eff }}$ nehmen ab, der Abminderungsfaktor $\alpha_{\mathrm{c}}$ wird kleiner eins.

- Bereich 3 - Fließen des Stahlträgers

Mit beginnender Plastizierung des Stahlträgers $\left(M / M_{u}>M_{e} / M_{u}\right)$ nehmen die Steifigkeit des Trägers und der Abminderungsfaktor $\alpha_{c}$ rasch ab. Die Definition des Abminderungsfaktor $\alpha_{c}$ ist hier jedoch nicht weiter sinnvoll, da ein zunehmender Anteil der Verformung aus dem Fließen des Stahlträgers und nicht aus der Rissbildung des Betons resultiert.

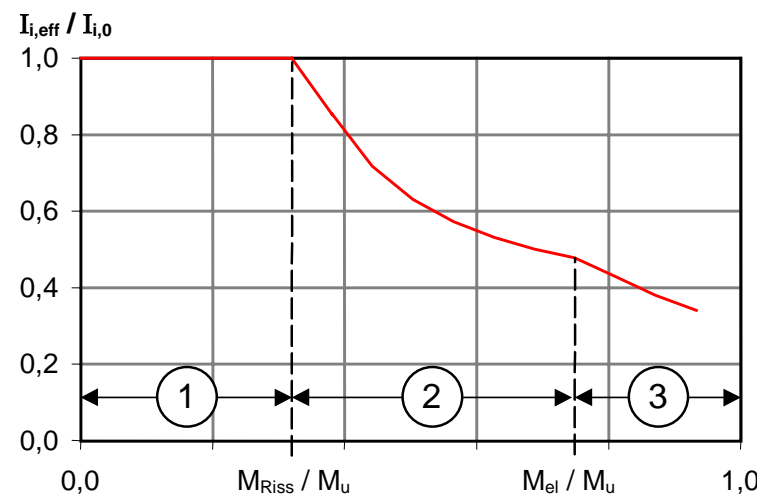

(a) effektives Trägheitsmoment $I_{i, \text { eff }}$

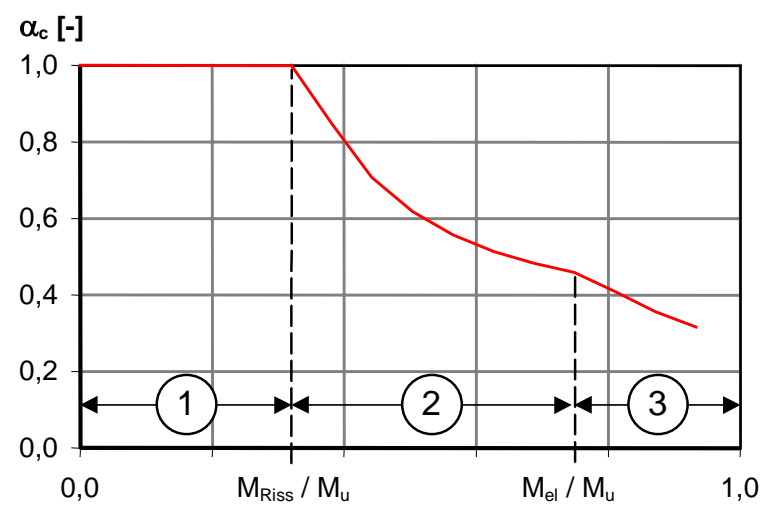

(b) Abminderungsfaktor $\alpha_{c}$

Abbildung 8-2: Typische Verläufe des effektiven Trägheitsmoments $\mathrm{I}_{\mathrm{i}, \text { eff }}$ und des Abminderungsfaktors $\alpha_{c}$ in Abhängigkeit des Ausnutzungsgrads $M / M_{u}$

Im Zuge der in Abschnitt 7.3 dargestellten Parameterstudie an Verbundträgern niedriger Bauhöhe wurde der Abminderungsfaktor $\alpha_{c}$ systematisch untersucht. Innerhalb der Parameterstudie wurden die Verformungen der untersuchten Verbundträger mit dem in Abschnitt 6.2 beschriebenen analytischen Berechnungsmodell berechnet und daraus der Abminderungsfaktor $\alpha_{c}$ rückgerechnet. Die systematische Untersuchung der Einflüsse auf den Abminderungsfaktor $\alpha_{c}$ mündete in die Entwicklung eines einfachen Berechnungsansatzes für den Abminderungsfaktor $\alpha_{c}$, der eine wirklichkeitsnahe Verformungsberechnung niedriger Verbundträger ermöglicht.

Im Folgenden werden die maßgebenden Einflüsse auf den Abminderungsfaktor $\alpha_{c}$ diskutiert und der entwickelte Berechnungsvorschlag vorgestellt. Ein Vergleich mit den genauen Verformungswerten, die sich bei einer Berechnung mit dem analytischen Berechnungsmodell ergeben, und mit einer Berechnung nach EN 1994-1-1 bzw. DIN 18800-5 zeigt, dass der entwickelte Berechnungsvorschlag eine gute Näherung für die Verformungsberechnung niedriger Verbundträger darstellt. 


\subsubsection{Einflüsse auf den Abminderungsfaktor $\alpha_{c}$}

\subsubsection{Grundsätzliches}

Da die Rissbildung vor allem zu einer Abnahme der Biegesteifigkeit des Betongurts führt, ist bei Trägern mit großer Biegetragwirkung des Betongurts der Einfluss der Rissbildung auf die effektive Steifigkeit des Verbundträgers und den Abminderungsfaktor $\alpha_{c}$ generell größer als bei Trägern mit geringer Biegetragwirkung des Betongurts.

\subsubsection{Ausnutzungsgrad $M / M_{u}$}

Wie oben bereits dargestellt, ist das Verformungsverhalten niedriger Verbundträger sehr stark von der Rissbildung und damit von der Belastungshöhe abhängig, siehe Abbildung 8-2. Die Belastungshöhe lässt sich dabei als Ausnutzungsgrad darstellen, der als Verhältnis des maximalen Moments $M$ in Trägermitte zum Bruchmoment $M_{u}$ des Verbundträgers definiert ist. Eine genaue Beschreibung des Verhaltens ist in Abschnitt 8.3.1 gegeben.

Mit dem Fließen des Stahlträgers ist die Verwendung des Abminderungsfaktors $\alpha_{c}$ nicht mehr sinnvoll, da dieser als Reduktionsfaktor für die Steifigkeitsanteile des Betongurts definiert ist. Durch das Fließen des Stahlträgers ergibt sich jedoch ein zunehmend größerer Teil der Verformungen aus dem Plastizieren des Stahls.

\subsubsection{Betonzugfestigkeit $f_{c t}$}

Einen großen Einfluss auf das Rissmoment und das Rissverhalten des Verbundträgers und somit auch auf die effektive Steifigkeit und den Abminderungsfaktor $\alpha_{c}$ hat die Betonzugfestigkeit $\mathrm{f}_{\mathrm{ct}}$, siehe Abbildung 8-3.

Dieser Einfluss ist vor allem im Bereich 2 des Ausnutzungsgrads ausgeprägt, siehe Abschnitt 8.3.1. Bei größerer Betonzugfestigkeit ist das Rissmoment des Träger größer und die Rissbildung setzt später ein. Bei gleicher Belastungshöhe ist somit die Risstiefe bei großer Betonzugfestigkeit kleiner und der gerissene Bereich des Betongurts in Trägerlängsrichtung weniger ausgedehnt.

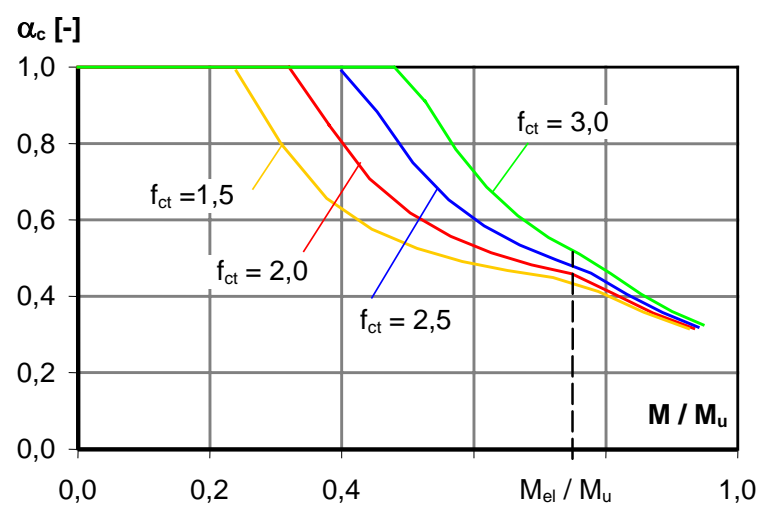

Abbildung 8-3: Typische Verläufe des Abminderungsfaktors $\alpha_{c}$ in Abhängigkeit der Betonzugfestigkeit $\mathrm{f}_{\mathrm{ct}}\left[\mathrm{N} / \mathrm{mm}^{2}\right]$

Mit dem Fließen des Stahlträgers $\left(M / M_{u}>M_{e} / M_{u}\right)$ geht der Einfluss der Betonzugfestigkeit jedoch weitestgehend verloren. Dies liegt unter Anderem daran, dass in diesem Bereich zunehmende Verformungsanteile aus dem Fließen des Stahlträgers entstehen und im Betongurt bereits ein weitestgehend abgeschlossenes Rissbild vorliegt. Dadurch ist der Beitrag 
des Biegezustands des Betongurts zur Steifigkeit des Verbundträgers vergleichsweise gering.

\subsubsection{Streckgrenze $f_{y}$ des Stahlträgers}

Mit zunehmender Streckgrenze $f_{y}$ des Stahlträgers nimmt das Bruchmoment $M_{u}$ des Stahlträgers zu und das Verhältnis zwischen Rissmoment $M_{\text {Riss }}$ und Bruchmoment $M_{u}$ verändert sich entsprechend, da das Rissmoment durch die Streckgrenze des Stahlträgers nicht beeinflusst wird.

Wird der Abminderungsfaktor $\alpha_{c}$ in Abhängigkeit des Ausnutzungsgrads $M / M_{u}$ betrachtet, so ergibt sich bei größerer Streckgrenze ein kleinerer Abminderungsfaktor $\alpha_{c}$, da dadurch das bezogene Rissmoment $M_{\text {Riss }} / M_{u}$ kleiner wird und der Träger früher vom Zustand $I$ in den Zustand II wechselt, siehe Abbildung 8-4 (a).

Wird der Abminderungsfaktor $\alpha_{c}$ jedoch in Abhängigkeit des Absolutwertes des Belastungsmoments $M$ betrachtet, so ergibt sich bei größerer Streckgrenze und gleichem Belastungsmoment $M$ ein größerer Abminderungsfaktor $\alpha_{c}$, siehe Abbildung 8-4 (b).

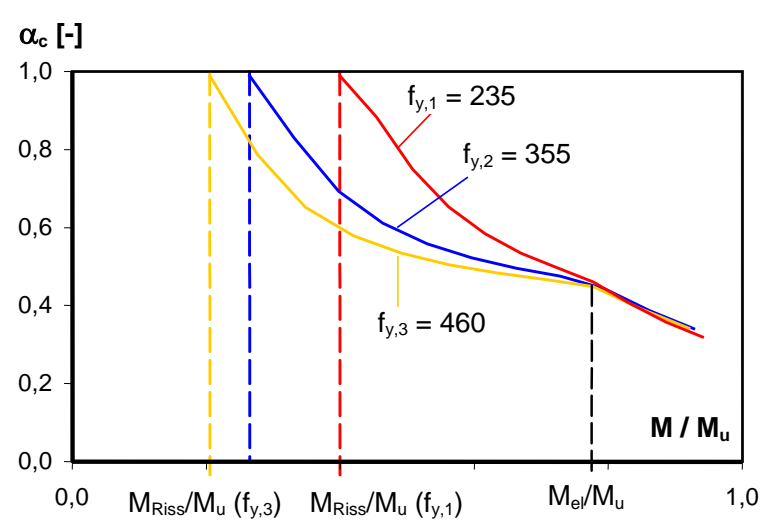

(a) Darstellung mit bezogenen Momenten

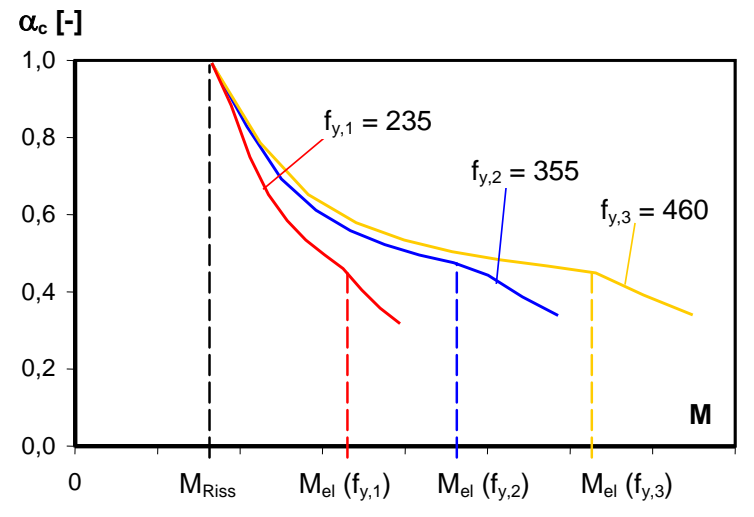

(b) Darstellung mit absoluten Momenten

Abbildung 8-4: Typische Verläufe des Abminderungsfaktors $\alpha_{c}$ in Abhängigkeit der Streckgrenze des Stahlträgers $\mathrm{f}_{\mathrm{y}}\left[\mathrm{N} / \mathrm{mm}^{2}\right]$

\subsubsection{Dicke des Betongurts $h_{c}$ und Trägerhöhe}

Abbildung 8-5 zeigt den Einfluss der Gurtdicke $h_{c}$ auf den Abminderungsfaktor $\alpha_{c}$. Es ist zu erkennen, dass das bezogene Rissmoment $M_{\text {Riss }} / M_{u}$ bei dickerem Gurt größer ist und die Rissbildung erst bei einer höheren Belastung beginnt. Da bei Verbundträgern mit dickem Betongurt der Biegeanteil des Betongurts und dessen Biegesteifigkeit vergleichsweise größer sind als bei Trägern mit dünnem Gurt, hat bei diesen Trägern auch die Rissbildung einen größeren Einfluss auf die effektive Steifigkeit des Trägers. Das heißt, durch die Rissbildung nimmt die effektive Steifigkeit und der Abminderungsfaktor $\alpha_{c}$ bei Trägern mit dickem Betongurt stärker ab als bei Trägern mit dünnem Gurt, siehe Abbildung 8-5 (a).

Entsprechend ist auch der Einfluss der Stahlträgerhöhe $h_{a}$. Bei niedrigen Trägern sind die Biegetragwirkung und die Biegesteifigkeit des Betongurts vergleichsweise größer als bei hohen Trägern. Da die Biegesteifigkeit des Betongurts durch die Rissbildung des Betongurts sehr stark reduziert wird, ist der Abminderungsfaktor $\alpha_{c}$ bei niedrigen Trägern kleiner als bei hohen, wenn die Belastung größer ist als die Risslast. Da bei hohen Trägern ein großer Anteil der Steifigkeit aus dem inneren Kräftepaar im Verbundquerschnitt und der reinen 
Steifigkeit des Stahlträgers resultiert, diese jedoch von der Rissbildung vergleichsweise wenig beeinflusst werden bzw. unabhängig sind, ist der Abminderungsfaktor $\alpha_{c}$ bei hohen Trägern größer als bei niedrigen, siehe Abbildung 8-5 (b).

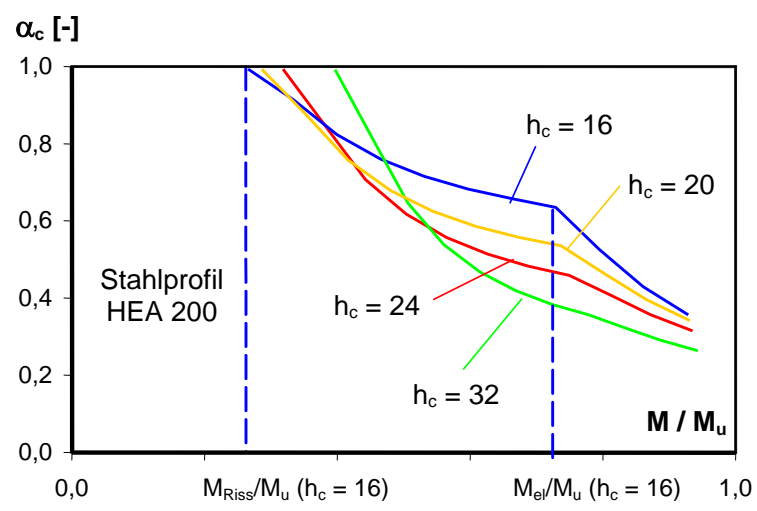

(a) Einfluss der Gurtdicke $h_{c}[\mathrm{~cm}]$

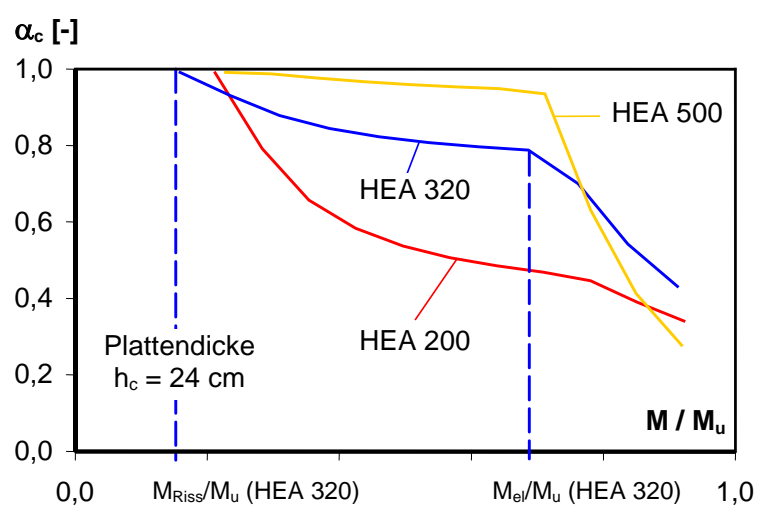

(b) Einfluss der Trägerhöhe $h_{a}[\mathrm{~mm}]$

Abbildung 8-5: Typische Verläufe des Abminderungsfaktors $\alpha_{c}$ in Abhängigkeit der Gurtdicke und der Stahlträgerhöhe

\subsubsection{Seitenverhältnis des Betongurts $b / L$}

Mit zunehmendem Seitenverhältnis b/L wird der Einfluss der Biegetragwirkung des Betongurts auf die Steifigkeit des Verbundträgers größer. Dies liegt daran, dass mit zunehmendem Verhältnis $b / L$ die mittragende Breite des Biegezustands $b_{m, B}$ im Vergleich zur mittragenden Breite des Scheibenzustands überproportional zunimmt und damit das Verhältnis $b_{m, S} / b_{m, B}$ kleiner wird, siehe Abbildung 8-6 (b). Dadurch ist bei großen Seitenverhältnissen b/L auch der Einfluss der Rissbildung auf die effektive Trägersteifigkeit und den Abminderungsfaktor $\alpha_{c}$ größer, siehe Abbildung 8-6 (a).

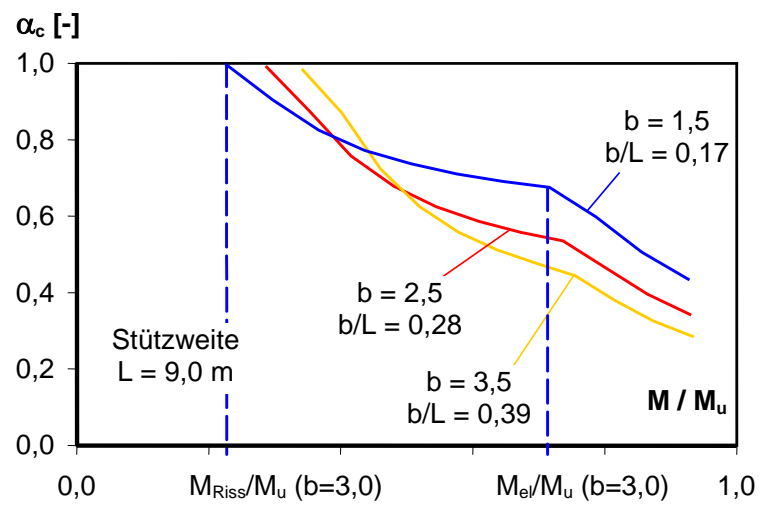

(a) Einfluss der Gurtbreite $b$ [m]

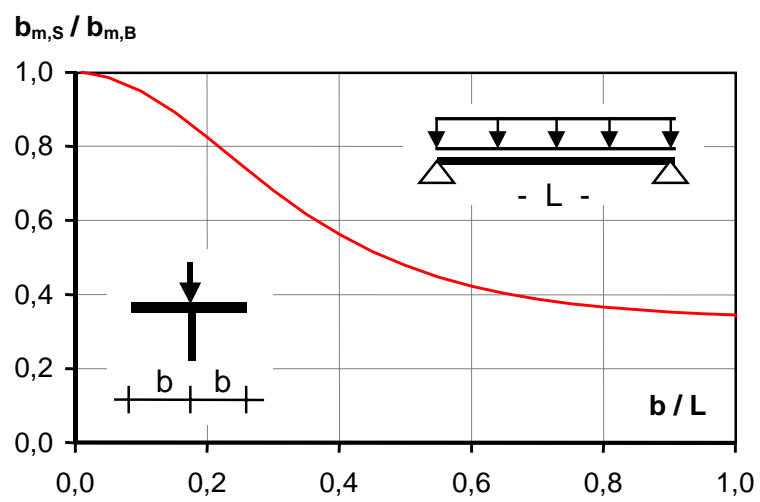

(b) Verhältnis $b_{m, s} / b_{m, B}$

Abbildung 8-6: (a) Typische Verläufe des Abminderungsfaktors $\alpha_{c}$ in Abhängigkeit des Seitenverhältnisses b/L des Betongurts,

(b) Verhältnis der mittragenden Breite des reinen Scheibenzustands $b_{m, s}$ zum reinen Biegezustand $b_{m, B}$ 


\subsection{Berechnungsansatz für den Abminderungsfaktor $\alpha_{c}$}

\subsubsection{Herleitung des Berechnungsansatzes}

Der Berechnungsansatz für den Abminderungsfaktor $\alpha_{c}$ wurde auf Basis der in Abschnitt 7.3 beschriebenen Parameterstudie für Verbundeinfeldträger mit einer Gleichlast unter hochbautypischen Randbedingungen entwickelt. Die genauen Werte des Abminderungsfaktors $\alpha_{c}$ wurden dabei aus den Ergebnissen der Parameterstudie mit dem in Abschnitt 6.2 beschriebenen analytischen Berechnungsmodell rückgerechnet. Der Berechnungsansatz unterliegt daher den in Abschnitt 6.3 und 7.3.2 beschriebenen Einschränkungen, kann jedoch auch bei vergleichbaren Querschnitten und Systemen näherungsweise angewendet werden, siehe Abschnitt 8.2.4.

Der Berechnungsansatz wurde als Näherungsfunktion für die genauen Werte des Abminderungsfaktors $\alpha_{c}$ über die Minimierung der Fehlerquadrate entwickelt. Er basiert auf der Auswertung von ca. 7000 Trägern.

Gleichung (8-12) stellt den Berechnungsansatz für den Abminderungsfaktor $\alpha_{c}$ dar. Durch den zunehmenden Steifigkeitsverlust des Verbundträgers mit zunehmender Rissbildung bei höherer Belastung ist der Abminderungsfaktor wesentlich von der Belastungshöhe des Verbundträgers und der Betonzugfestigkeit $f_{c t}$ abhängig. Der Abminderungsfaktor $\alpha_{c}$ wird dabei in drei Faktoren unterteilt: In $\alpha_{M a t}$ sind die Materialeinflüsse zusammengefasst, in $\alpha_{\mathrm{QS}}$ die Einflüsse aus den Querschnittswerte und in $\alpha_{M}$ der Belastungseinfluss, siehe Gleichungen (8-13) bis (8-15).

$$
\alpha_{\mathrm{c}}=\alpha_{\mathrm{Mat}} \cdot \alpha_{\mathrm{QS}} \cdot \alpha_{\mathrm{M}}
$$

mit

\begin{tabular}{|c|c|}
\hline$\alpha_{c}$ & $\begin{array}{l}\text { Abminderungsfaktor zur Berücksichtigung des nicht- } \\
\text { linearen Material- und Tragverhaltens niedriger Verbund- } \\
\text { träger }\end{array}$ \\
\hline$\alpha_{\text {Mat }}$ & $\begin{array}{l}\text { Faktor für den Einfluss der Materialkennwerte, siehe } \\
\text { Gleichung (8-13) }\end{array}$ \\
\hline$\alpha_{\mathrm{QS}}$ & $\begin{array}{l}\text { Faktor für den Einfluss der Querschnittswerte, siehe } \\
\text { Gleichung (8-14) }\end{array}$ \\
\hline$\alpha_{M}$ & $\begin{array}{l}\text { Faktor für den Einfluss der Belastung siehe Gleichung } \\
(8-15)\end{array}$ \\
\hline
\end{tabular}

$\alpha_{\text {Mat }}=\left(\frac{f_{c t}}{f_{c t, 0}}\right)^{-0,154} \cdot\left(\frac{f_{c}}{f_{c, 0}}\right)^{0,146} \cdot\left(\frac{f_{a y}}{f_{a y, 0}}\right)^{0,152}$

$\alpha_{Q S}=\left(\frac{I_{c, 0}}{I_{i, 0}}\right)^{0,06} \cdot\left(\frac{S_{i, 0} \cdot a_{S t}}{I_{i, 0}}\right)^{0,565} \cdot\left(\frac{z_{i, 0}}{a_{S t}}\right)^{0,15} \cdot\left(\frac{b}{L}\right)^{-0,125}$.

$\alpha_{M}=1,357 \cdot\left(1-\frac{M_{\text {Riss }}}{M}\right)^{-0,493}$

mit

$\begin{array}{ll}M & \text { Moment in Trägermitte } \\ M_{\text {Riss }} & \text { Rissmoment des Verbundträgers } \\ I_{i, 0}, I_{c, 0}, S_{i, 0}, Z_{i, 0}, a_{S t} & \begin{array}{l}\text { elastische Querschnittswerte des Verbund-, Stahl- und } \\ \text { Betonquerschnitts nach Gleichung (8-2) bis (8-8) }\end{array}\end{array}$




$\begin{array}{ll}f_{c t} & \text { Betonzugfestigkeit } \\ f_{c t, 0}=1,0 \mathrm{~N} / \mathrm{mm}^{2} & \text { Bezugswert der Betonzugfestigkeit } \\ f_{c} & \text { Betondruckfestigkeit } \\ f_{c, 0}=30 \mathrm{~N} / \mathrm{mm}^{2} & \text { Bezugswert der Betondruckfestigkeit } \\ \mathrm{f}_{\mathrm{ay}} & \text { Streckgrenze des Stahlträgers } \\ \mathrm{f}_{\mathrm{ay}, 0}=355 \mathrm{~N} / \mathrm{mm}^{2} & \text { Bezugswert der Streckgrenze des Stahlträgers }\end{array}$

Die Exponenten und Koeffizienten der Gleichungen (8-12) bis (8-15) wurden durch eine Regression über die Minimierung der Fehlerquadrate mit Hilfe des in Microsoft Excel implementierten Solver bestimmt. Dabei wurde berücksichtigt, dass die einzelnen Parameter unter einander abhängig sein können. In Abbildung 8-7 bis Abbildung 8-13 sind exemplarische Vergleiche des Berechnungsansatzes Gleichungen (8-1) und (8-12) mit den genauen Werten für die im Berechnungsansatz berücksichtigten Parameter des in Abbildung 8-14 gegebenen Trägers dargestellt. Diese dienen jedoch nur zur Veranschaulichung des Berechnungsansatzes und nicht zu dessen Herleitung.

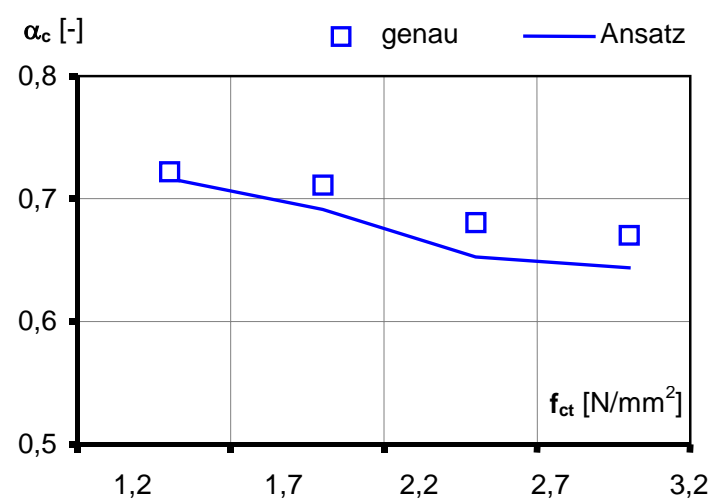

(a) $\alpha_{c}$ nach Gleichung (8-12)

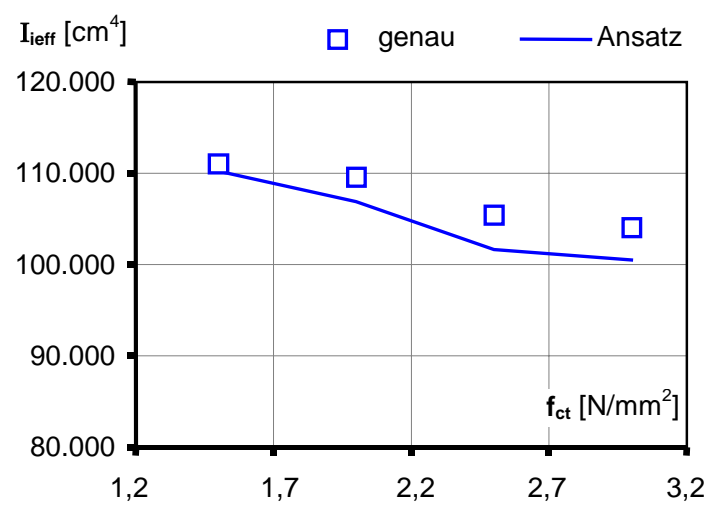

(b) $I_{i, \text { eff }}$ nach Gleichung (8-1)

Abbildung 8-7: Vergleich des quasi-elastischen Berechnungsansatzes mit den genauen Werten in Abhängigkeit der Betonzugfestigkeit $\mathrm{f}_{\mathrm{ct}}$

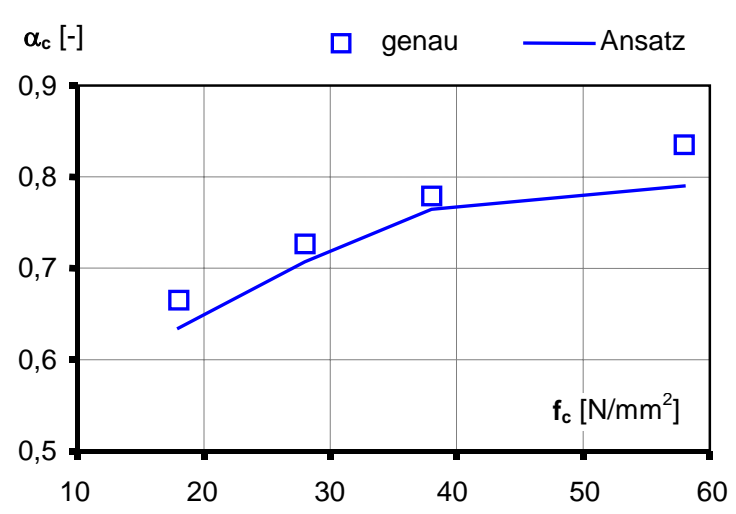

(a) $\alpha_{c}$ nach Gleichung (8-12)

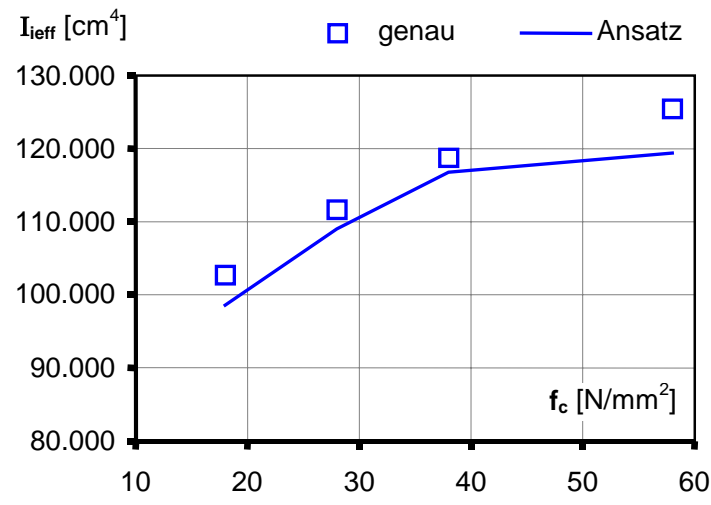

(b) $I_{i, \text { eff }}$ nach Gleichung (8-1)

Abbildung 8-8: Vergleich des quasi-elastischen Berechnungsansatzes mit den genauen Werten in Abhängigkeit der Betondruckfestigkeit $\mathrm{f}_{\mathrm{c}}$ 


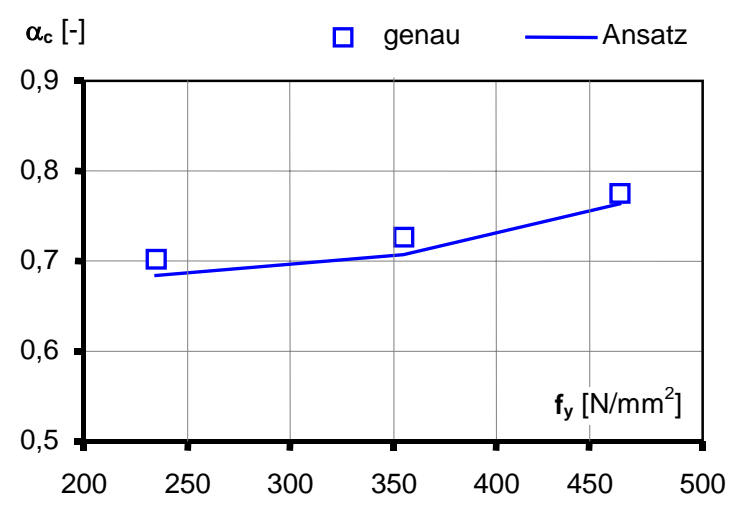

(a) $\alpha_{c}$ nach Gleichung (8-12)

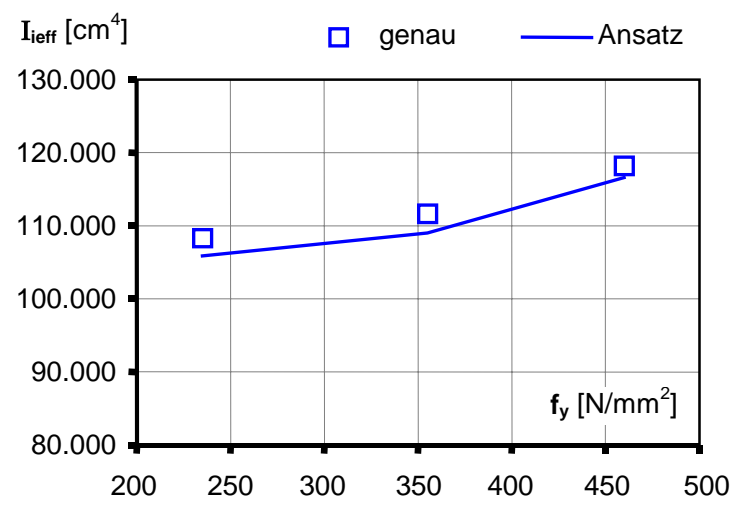

(b) $I_{i, \text { eff }}$ nach Gleichung (8-1)

Abbildung 8-9: Vergleich des quasi-elastischen Berechnungsansatzes mit den genauen Werten in Abhängigkeit der Streckgrenze des Stahlträgers $\mathrm{f}_{\mathrm{y}}$

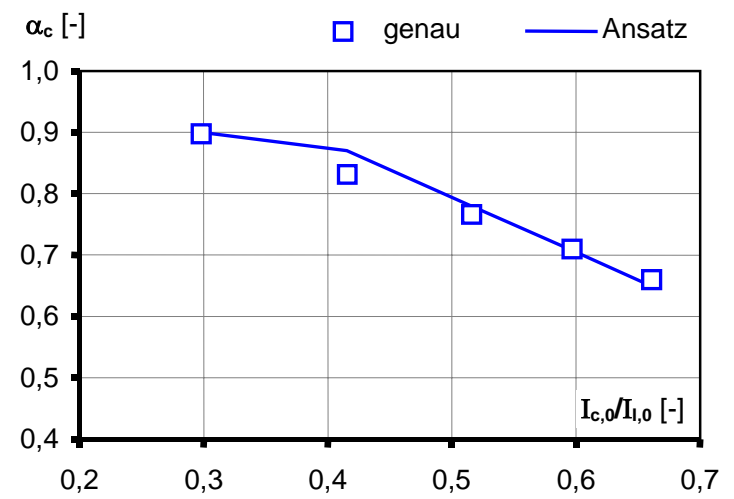

(a) $\alpha_{c}$ nach Gleichung (8-12)

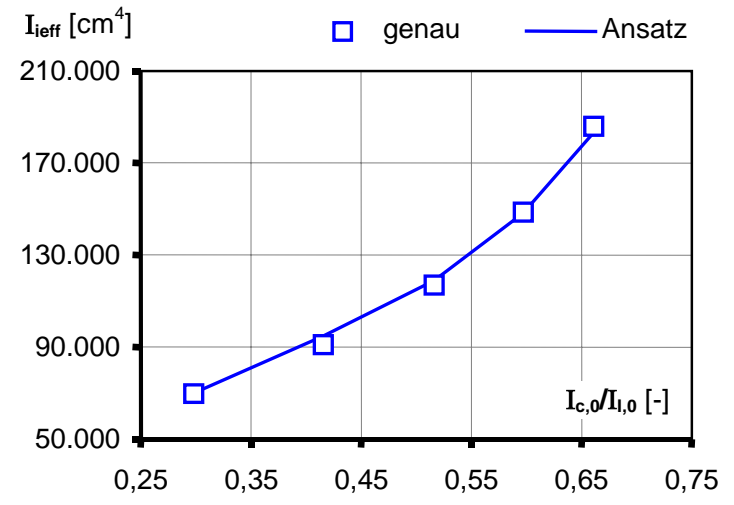

(b) $I_{i, \text { eff }}$ nach Gleichung (8-1)

Abbildung 8-10: Vergleich des quasi-elastischen Berechnungsansatzes mit den genauen Werten in Abhängigkeit des Verhältnisses $\mathrm{I}_{\mathrm{c}, 0} / \mathrm{I}_{\mathrm{i}, 0}$

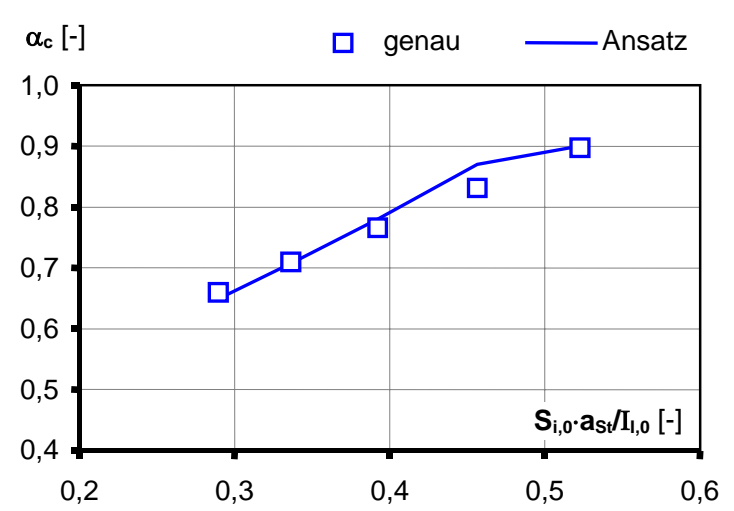

(a) $\alpha_{c}$ nach Gleichung (8-12)

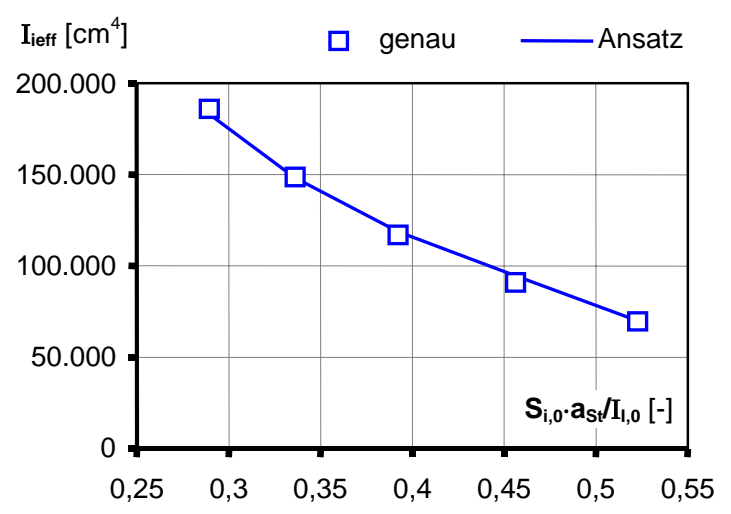

(b) $I_{i, \text { eff }}$ nach Gleichung (8-1)

Abbildung 8-11: Vergleich des quasi-elastischen Berechnungsansatzes mit den genauen Werten in Abhängigkeit des Verhältnisses $S_{i, 0} \cdot a_{S t} / I_{i, 0}$ 


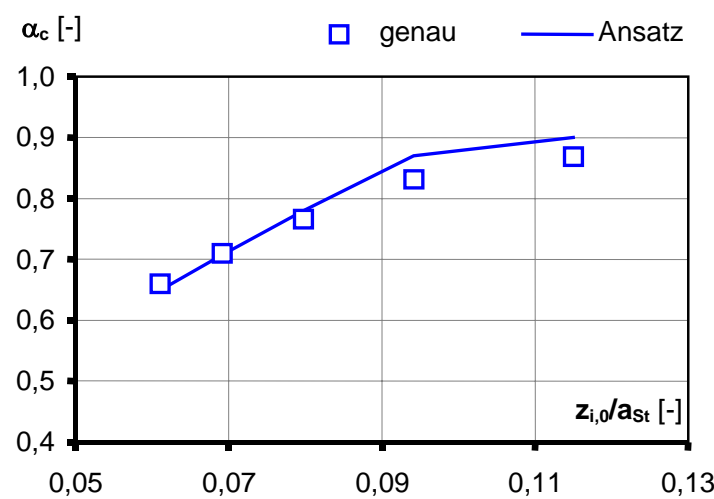

(a) $\alpha_{c}$ nach Gleichung (8-12)

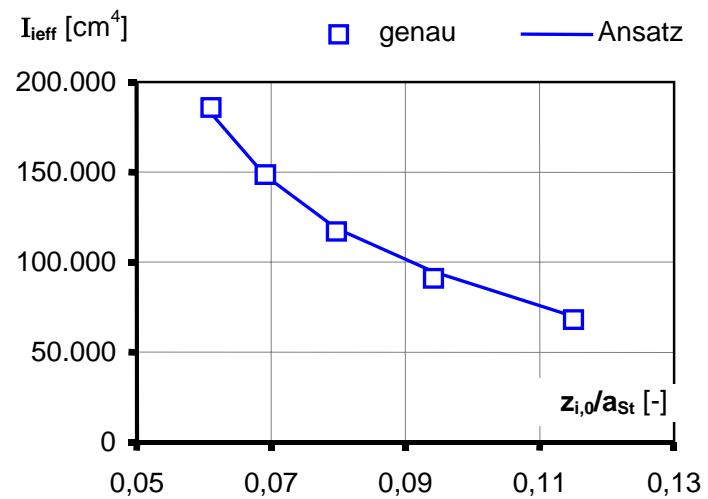

(b) $I_{i, \text { eff }}$ nach Gleichung (8-1)

Abbildung 8-12: Vergleich des quasi-elastischen Berechnungsansatzes mit den genauen Werten in Abhängigkeit des Verhältnisses $z_{i, 0} / a_{S t}$

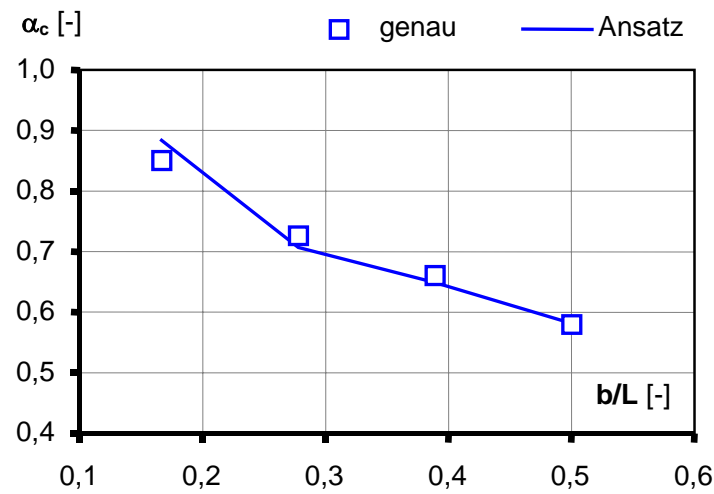

(a) $\alpha_{c}$ nach Gleichung (8-12)

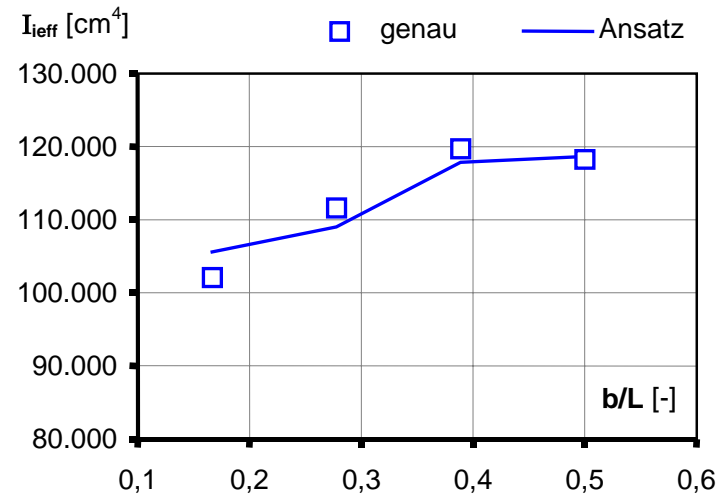

(b) $I_{i, \text { eff }}$ nach Gleichung (8-1)

Abbildung 8-13: Vergleich des quasi-elastischen Berechnungsansatzes mit den genauen Werten in Abhängigkeit des Verhältnisses b/L

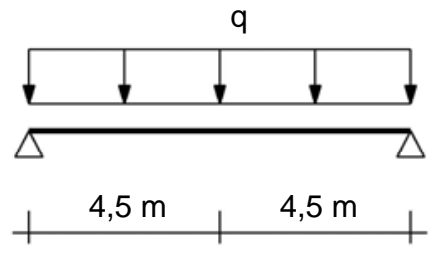

(a) System

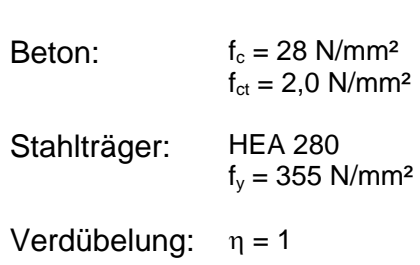

(b) Querschnitt und Materialwerte
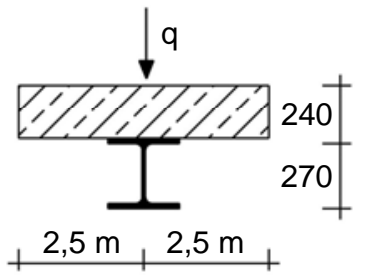

Abbildung 8-14: System und Querschnitt des Basisträgers

\subsubsection{Vergleich mit Versuchsergebnissen}

In Abbildung 8-15 bis Abbildung 8-20 sind die Vergleiche der experimentellen LastVerformungs-Kurven der eigenen Versuchsträger und des Versuchsträgers B4 von Amadio et al. mit den rechnerischen Verläufen, die mit Gleichungen (8-1) und (8-12) berechnet werden, dargestellt. Eine Beschreibung der Versuche ist in Kapitel 5 und Abschnitt 6.4 gegeben. Die Verläufe zeigen bis zum Erreichen des Grenzzustands der Gebrauchstauglichkeit eine 
sehr gute Übereinstimmung. Die Abweichungen betragen im Grenzzustand der Gebrauchstauglichkeit ca. 4,7\%, siehe Tabelle 8-1.

Tabelle 8-1: $\quad$ Vergleich der experimentellen und rechnerischen Verformungen der Versuchsträger im Grenzzustand der Gebrauchstauglichkeit

\begin{tabular}{|r|c|c|c|c|c|c|}
\cline { 2 - 7 } \multicolumn{1}{c|}{} & VT 1 & VT 2 & VT 4 & VT 5 & VT 6 & B4 \\
\hline $\mathrm{P}_{\mathrm{GZG}}{ }^{1)}[\mathrm{kN}]$ & 298 & 305 & 308 & 493 & 493 & 340 \\
\hline $\mathrm{f}_{\exp }{ }^{2)}[\mathrm{mm}]$ & 10,1 & 8,6 & 8,9 & 8,0 & 8,0 & 11,6 \\
\hline $\mathrm{f}_{\text {rechn }}-\mathrm{f}_{\exp }{ }^{3)}[\mathrm{mm}]$ & $-0,9$ & $-0,2$ & 0,3 & $-0,5$ & $-0,4$ & 0,3 \\
\hline $\mathrm{f}_{\mathrm{rechn}} / \mathrm{f}_{\exp }-1 \quad[-]$ & $8,9 \%$ & $2,3 \%$ & $3,4 \%$ & $6,2 \%$ & $5,0 \%$ & $2,1 \%$ \\
\hline
\end{tabular}

1) $P_{\text {GzG }} \quad$ Last im Grenzzustand der Gebrauchslast

2) $f_{\exp } \quad$ Mittendurchbiegung der Versuchsträger

3) $\mathrm{f}_{\text {rechn }}$ rechnerische Mittendurchbiegung der Versuchsträger nach Gleichungen (8-1) und (8-12)

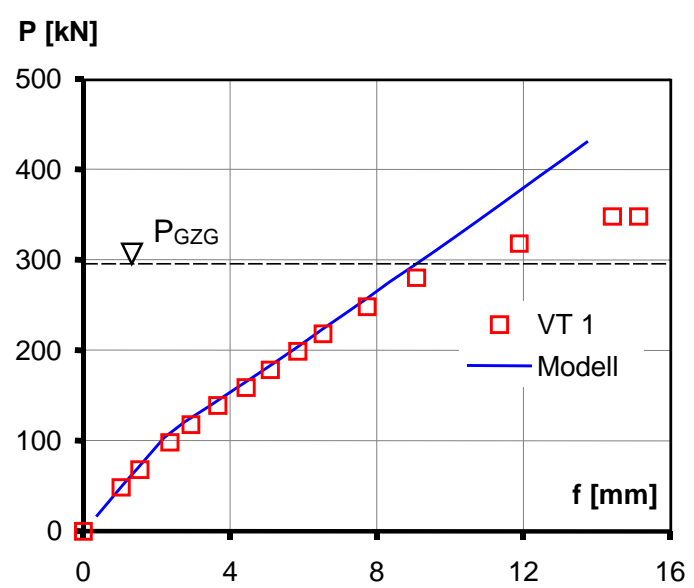

(a) Last-Verformungs-Kurven

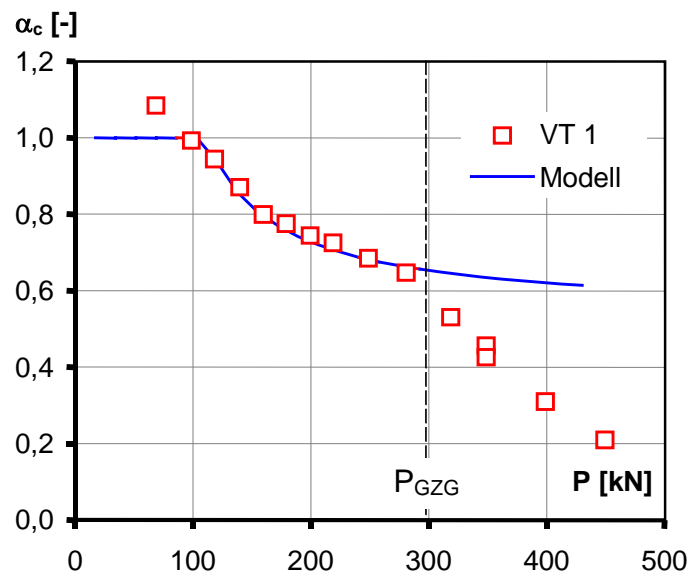

(b) Abminderungsfaktoren $\alpha_{c}$

Abbildung 8-15: Versuchsträger VT 1 [2.27]: Vergleich der rechnerischen und experimentellen Last-Verformungs-Kurven (a) und Abminderungsfaktoren $\alpha_{c}$ (b)

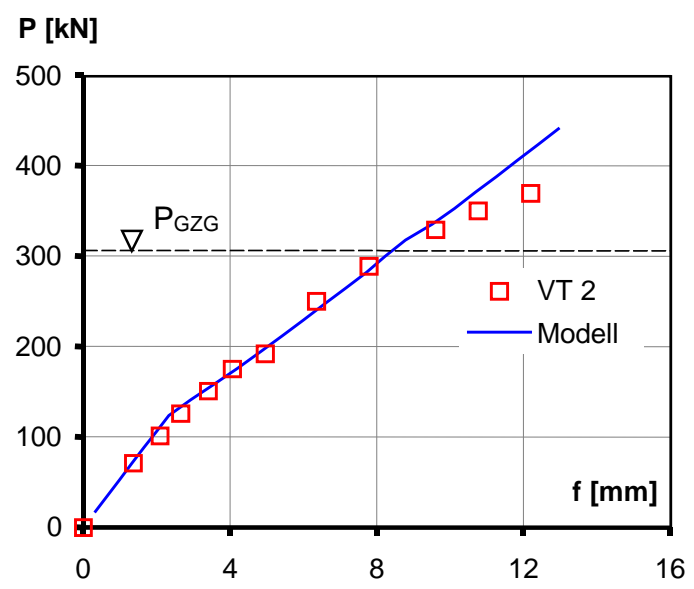

(a) Last-Verformungs-Kurven

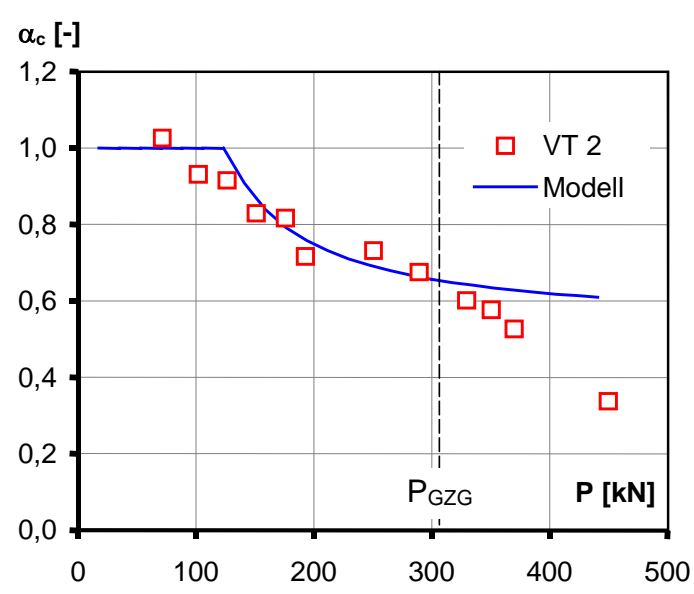

(b) Abminderungsfaktoren $\alpha_{c}$

Abbildung 8-16: Versuchsträger VT 2 [2.27]: Vergleich der rechnerischen und experimentellen Last-Verformungs-Kurven (a) und Abminderungsfaktoren $\alpha_{c}(b)$ 


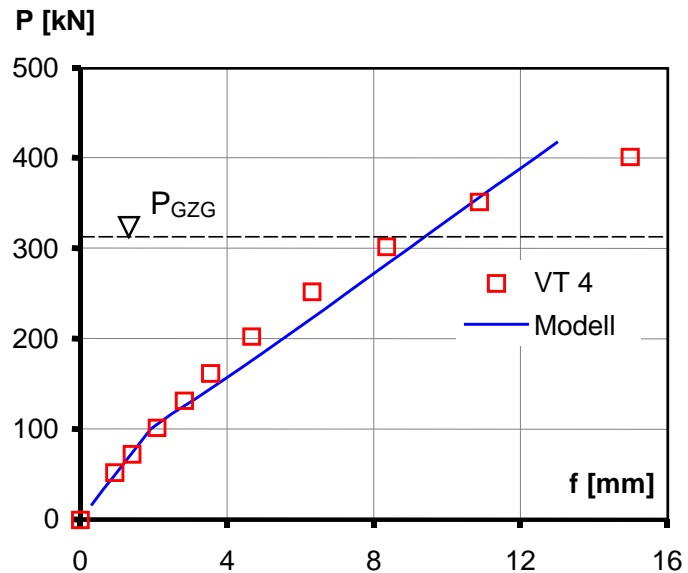

(a) Last-Verformungs-Kurven

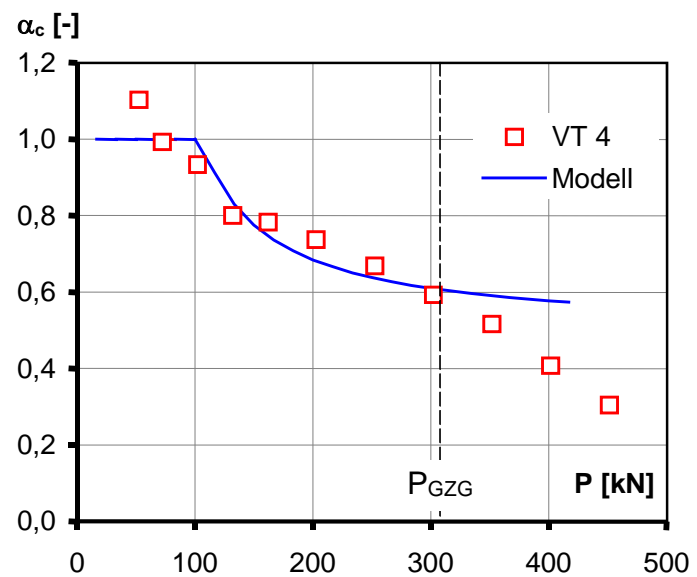

(b) Abminderungsfaktoren $\alpha_{c}$

Abbildung 8-17: Versuchsträger VT 4 [2.27]: Vergleich der rechnerischen und experimentellen Last-Verformungs-Kurven (a) und Abminderungsfaktoren $\alpha_{c}$ (b)

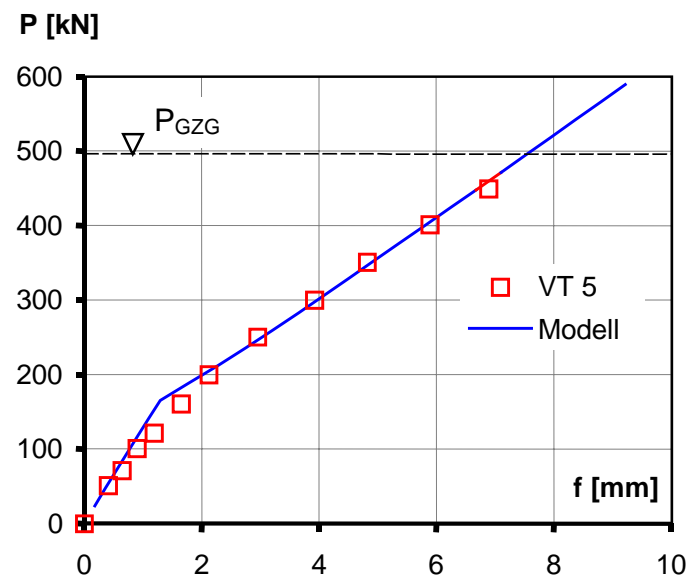

(a) Last-Verformungs-Kurven

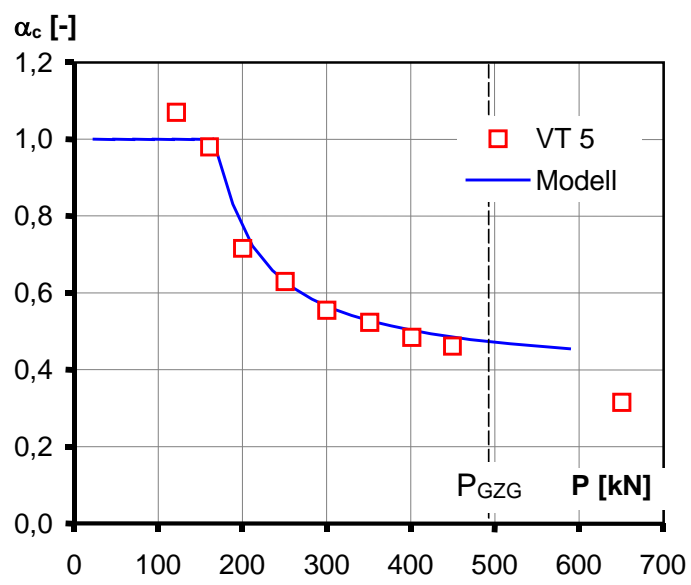

(b) Abminderungsfaktoren $\alpha_{c}$

Abbildung 8-18: Versuchsträger VT 5 [2.27]: Vergleich der rechnerischen und experimentellen Last-Verformungs-Kurven (a) und Abminderungsfaktoren $\alpha_{c}$ (b)

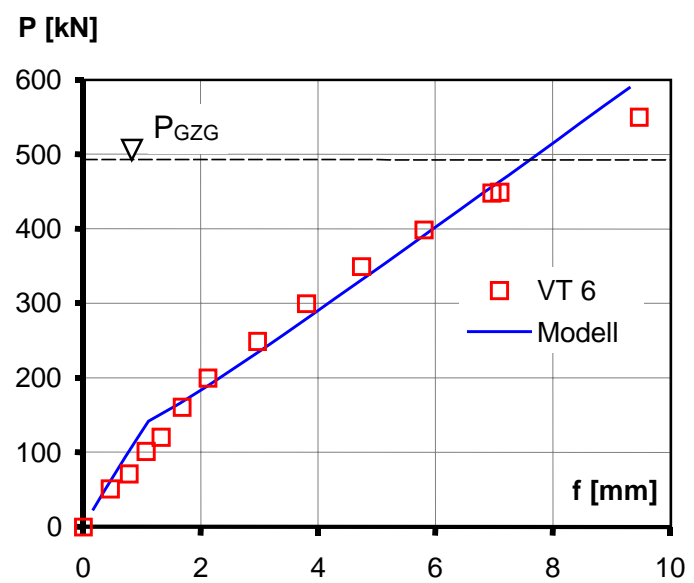

(a) Last-Verformungs-Kurven

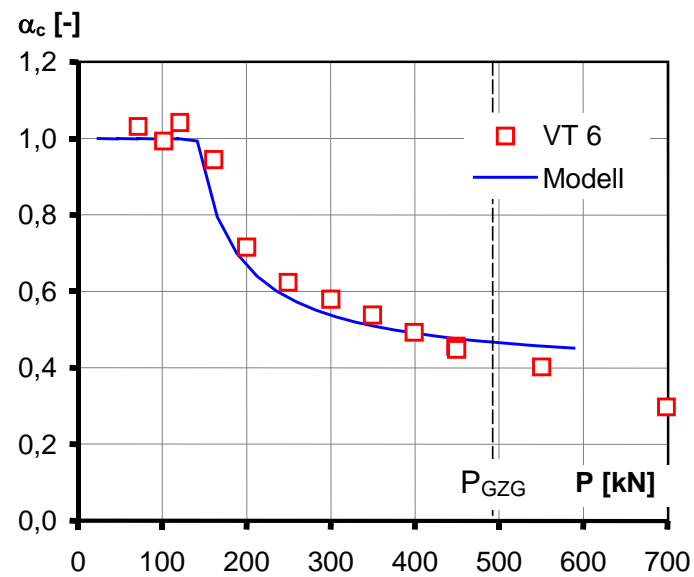

(b) Abminderungsfaktoren $\alpha_{c}$

Abbildung 8-19: Versuchsträger VT 6 [2.27]: Vergleich der rechnerischen und experimentellen Last-Verformungs-Kurven (a) und Abminderungsfaktoren $\alpha_{c}$ (b) 


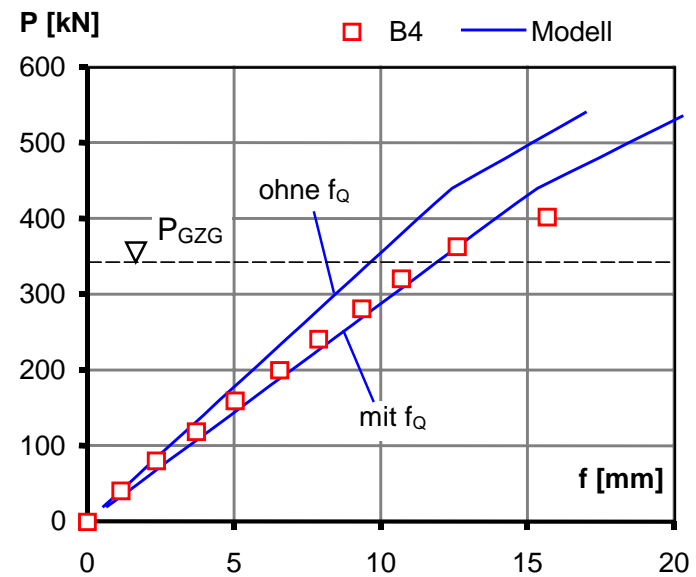

(a) Last-Verformungs-Kurven ( $f_{Q}=$ Querkraftverformung)

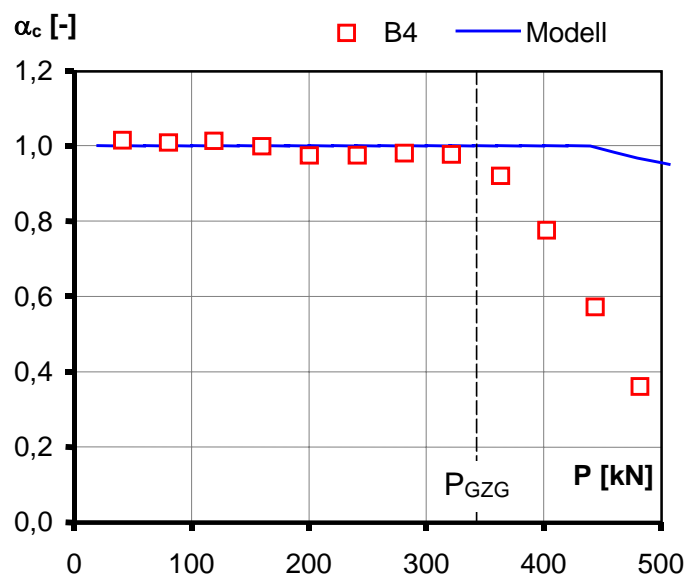

(b) Abminderungsfaktoren $\alpha_{c}$

Abbildung 8-20: Versuchsträger B4 [2.63]: Vergleich der rechnerischen und experimentellen Last-Verformungs-Kurven (a) und Abminderungsfaktoren $\alpha_{c}(b)$

Auch ein Vergleich der Abminderungsfaktor $\alpha_{c}$, die aus den experimentellen Verformungswerten rückrechnet werden, mit dem Berechnungsansatz Gleichung (8-12) zeigt eine sehr gute Übereinstimmung, siehe Abbildung 8-15 bis Abbildung 8-20.

\subsubsection{Vergleich mit dem analytischen Berechnungsmodell}

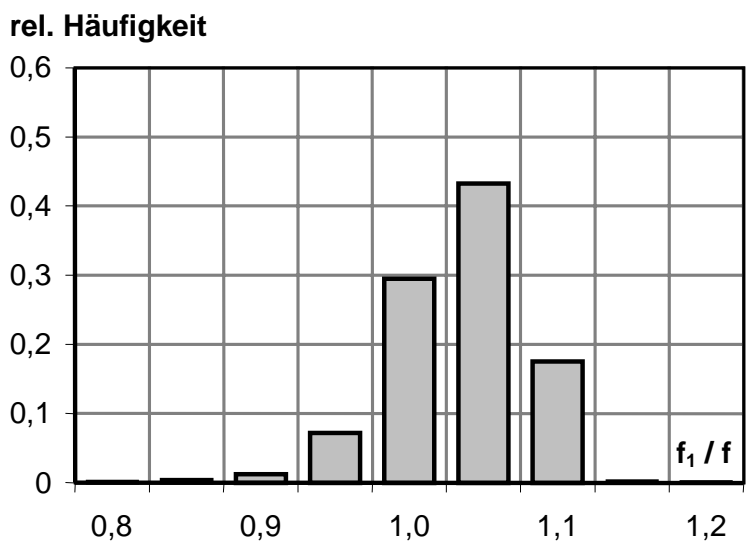

(a) Ausnutzungen $M / M_{u}=0 \div 0,8$

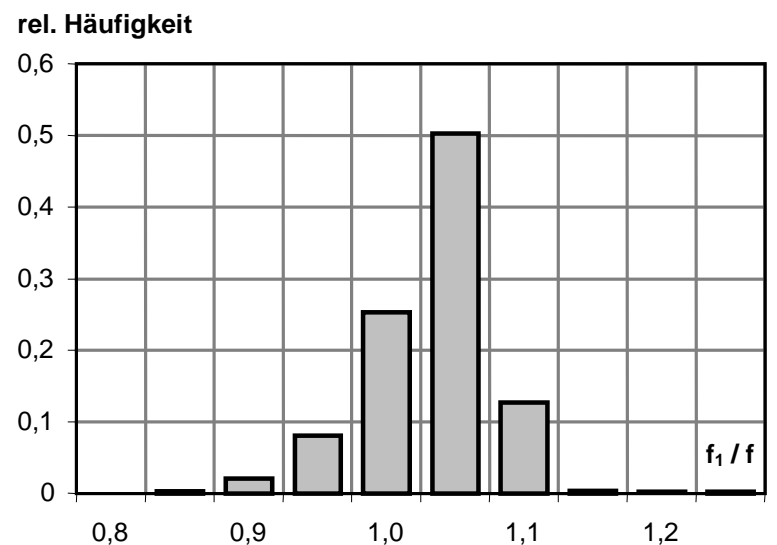

(b) $\operatorname{im} G Z G\left(M / M_{u} \approx 0,65\right)$

Abbildung 8-21: Vergleich der rechnerischen Verformungen $f_{1}$ des quasi-elastischen Berechnungsvorschlags Gleichungen (8-1) und (8-12) und denen des analytischen Berechnungsmodell f nach Abschnitt 7.3

Die Anwendbarkeit des oben beschriebenen Berechnungsansatz für das effektive Trägheitsmoment $\mathrm{I}_{\mathrm{i}, \text { eff }}$ zur Verformungsberechnung niedriger Verbundträger kann durch einen Vergleich der rechnerischen Verformungen mit dem in Abschnitt 6.2 beschriebenen analytischen Berechnungsmodell gezeigt werden, siehe Abbildung 8-21. Die Abweichungen der mit Gleichungen (8-1) und (8-12) berechneten Verformungen zum analytischen Berechnungsmodell betragen für die Ausnutzungsgrade $M / M_{u}=0 \div 0,8 \mathrm{im}$ Mittel 3,5\% und die Standardabweichung liegt bei 4,5\%. Der $5 \%$-Quantilwert liegt bei 0,92 der $95 \%$-Quantilwert bei 1,09. Im Grenzzustand der Gebrauchstauglichkeit $\left(M / M_{u} \approx 0,65\right)$ beträgt die mittlere Abweichung 3,6 \% und die Standardabweichung 5,3 \%. In Tabelle 8-2 und Tabelle 8-3 sind die ermittelten Abweichungen differenzierter aufgeführt. Die Übereinstimmung der mit Gleichungen (8-1) 
und (8-12) berechneten Verformungen mit den genaueren Werten nach Abschnitt 7.3 ist somit sehr gut. Mit dem Konzept des effektiven Trägheitsmoments $\mathrm{I}_{\mathrm{i}, \text { eff }}$ können folglich die Verformungen niedriger Verbundträger wirklichkeitsnah berechnet werden.

Tabelle 8-2: $\quad$ Abweichungen der rechnerischen Verformungen des quasi-elastischen Berechnungsvorschlags Gleichungen (8-1) und (8-12) zum analytischen Berechnungsmodell (Ausnutzungsgrade $M / M_{u}=0 \div 0,8$ )

\begin{tabular}{|c|c|c|c|c|c|c|c|c|c|c|}
\hline \multirow{2}{*}{\multicolumn{2}{|c|}{$\begin{array}{l}\text { Profilreihe } \\
\text { Profilhöhe }\end{array}$}} & HEA & HEB & IPE & \multicolumn{5}{|c|}{ HEA, HEB, IPE } & \multirow{2}{*}{$\begin{array}{l}\text { alle } \\
\text { alle }\end{array}$} \\
\hline & & \multicolumn{3}{|c|}{$200 \div 500$} & 200 & $280^{1)}$ & $320^{2)}$ & 400 & 500 & \\
\hline$\delta_{\mathrm{m}}[\%]$ & $\begin{array}{l}\text { mittlere } \\
\text { Abweichung }\end{array}$ & 3,5 & 3,7 & 3,3 & 3,0 & 3,1 & 3,4 & 3,8 & 4,4 & 3,5 \\
\hline S [\%] & $\begin{array}{l}\text { Standardab- } \\
\text { weichung }\end{array}$ & 4,4 & 4,7 & 4,3 & 3,9 & 3,9 & 4,4 & 4,8 & 5,3 & 4,5 \\
\hline$q_{0,05} \quad[-]$ & 5\%-Quantil & 0,93 & 0,94 & 0,90 & 0,88 & 0,92 & 0,93 & 0,94 & 0,95 & 0,92 \\
\hline $\mathbf{q}_{0,95} \quad[-]$ & 95\%-Quantil & 1,09 & 1,09 & 1,08 & 1,06 & 1,08 & 1,09 & 1,09 & 1,09 & 1,09 \\
\hline
\end{tabular}

1) HEA 280, HEB 280, IPE 270

2) HEA 320, HEB 320, IPE 330

Tabelle 8-3: $\quad$ Abweichungen der rechnerischen Verformungen des quasi-elastischen Berechnungsvorschlags Gleichungen (8-1) und (8-12) zum analytischen Berechnungsmodell im Grenzzustand der Gebrauchstauglichkeit

\begin{tabular}{|c|c|c|c|c|c|c|c|c|c|c|}
\hline \multirow{2}{*}{\multicolumn{2}{|c|}{$\begin{array}{l}\text { Profilreihe } \\
\text { Profilhöhe }\end{array}$}} & HEA & HEB & IPE & \multicolumn{5}{|c|}{ HEA, HEB, IPE } & \multirow{2}{*}{$\begin{array}{l}\text { alle } \\
\text { alle } \\
\end{array}$} \\
\hline & & \multicolumn{3}{|c|}{$200 \div 500$} & 200 & $280^{1)}$ & $320^{2)}$ & 400 & 500 & \\
\hline$\delta_{\mathrm{m}}[\%]$ & $\begin{array}{l}\text { mittlere } \\
\text { Abweichung }\end{array}$ & 3,5 & 3,6 & 3,7 & 3,0 & 3,4 & 3,6 & 3,9 & 4,1 & 3,6 \\
\hline $\mathbf{S}[\%]$ & $\begin{array}{l}\text { Standardab- } \\
\text { weichung }\end{array}$ & 4,8 & 6,1 & 4,9 & 4,0 & 4,6 & 6,4 & 5,4 & 5,5 & 5,3 \\
\hline$q_{0,05} \quad[-]$ & 5\%-Quantil & 0,93 & 0,94 & 0,90 & 0,88 & 0,92 & 0,93 & 0,94 & 0,95 & 0,92 \\
\hline $\mathbf{q}_{0,95}[-]$ & 95\%-Quantil & 1,09 & 1,09 & 1,07 & 1,05 & 1,08 & 1,08 & 1,09 & 1,09 & 1,09 \\
\hline
\end{tabular}

1) HEA 280, HEB 280, IPE 270

2) HEA 320, HEB 320, IPE 330

Dass sich für höhere Belastungen und Ausnutzungsgrade $M / M_{u}$ mit dem vorgestellten Berechnungsansatz größere Abweichungen ergeben, liegt an der zunehmenden Rissbildung der Träger, deren Auswirkung auf die Verformung durch den vergleichsweise einfachen Ansatz Gleichungen (8-1) und (8-12) nicht exakt quantifiziert werden kann. Dies ist auch in Tabelle 8-2 und Tabelle 8-3 zu erkennen: Je niedriger der Verbundträger ist, desto größer ist der Einfluss der Rissbildung auf die Verformung und um so größer sind die Abweichungen.

\subsubsection{Vergleich mit bestehenden Regelungen}

\subsubsection{EN 1994-1-1 und DIN 18800-5}

Zur Einordnung der Ergebnisse des oben beschriebenen Berechnungsansatzes wird ein Vergleich mit dem zur Zeit üblichen Vorgehen durchgeführt. Dabei werden die Verformungen in der Regel mit dem Gesamtquerschnittsverfahren [2.29], [2.30] berechnet. Diese wurden für die untersuchten Träger mit der mittragenden Breite nach EN 1994-1-1 [1.1] bestimmt. Nach DIN 18800-5 [1.5] ergeben sich dabei die gleichen Werte wie nach EN 1994-1-1, da in beiden Normen die Regeln zur mittragenden Breite identisch sind. In Abbildung 8-22 (a) und Tabelle 8-4 sind die Abweichungen der Verformungen, die mit EN 1994-1-1 berechnet werden, im Vergleich zu den Werten der genauen Berechnung mit dem analytischen Berech- 
nungsmodell, siehe Abschnitt 6.2, im Grenzzustand der Gebrauchstauglichkeit $\left(M / M_{u} \approx 0,65\right)$ dargestellt. Wie zu erkennen ist, streuen die rechnerischen Verformungen nach EN 1994-1-1 in erheblichem Maß um die genauen Werte. Im Grenzzustand der Gebrauchstauglichkeit beträgt die mittlere Abweichung 13,1\%, die Standardabweichung 19,7\%, der 5\%Quantilwert 0,93 und der 95\%-Quantilwert 1,45. Für Ausnutzungsgrade $M / M_{u}=0 \div 0,8$ beträgt die mittlere Abweichung 18,9\%, die Standardabweichung 28,6\%, der 5\%-Quantilwert 0,98 und der 95\%-Quantilwert 1,63. Aufgrund der großen Abweichungen sind die Regeln zur mittragenden Breite in EN 1994-1-1 und DIN 18800-5 zur elastischen Verformungsberechnung niedriger Verbundträger nicht geeignet.

rel. Häufigkeit

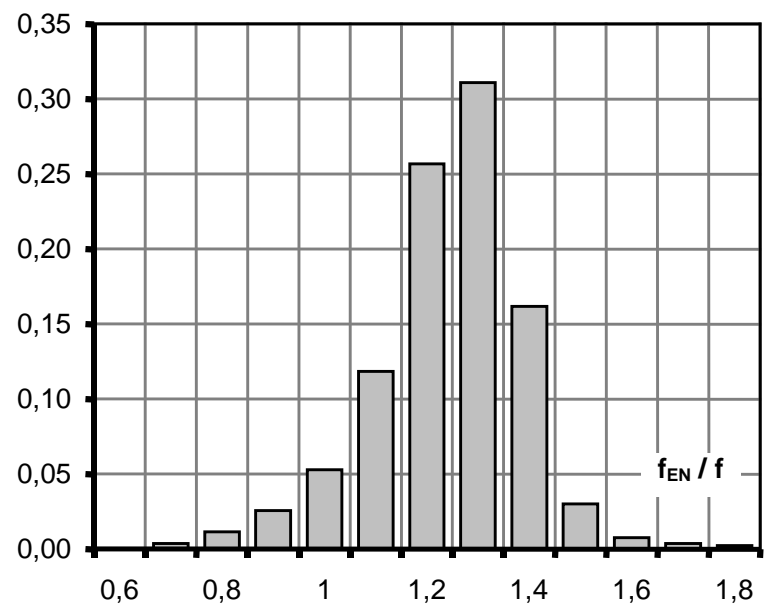

(a) Verformung $f_{E N}$ mit $b_{m}$ nach EN 1994-1-

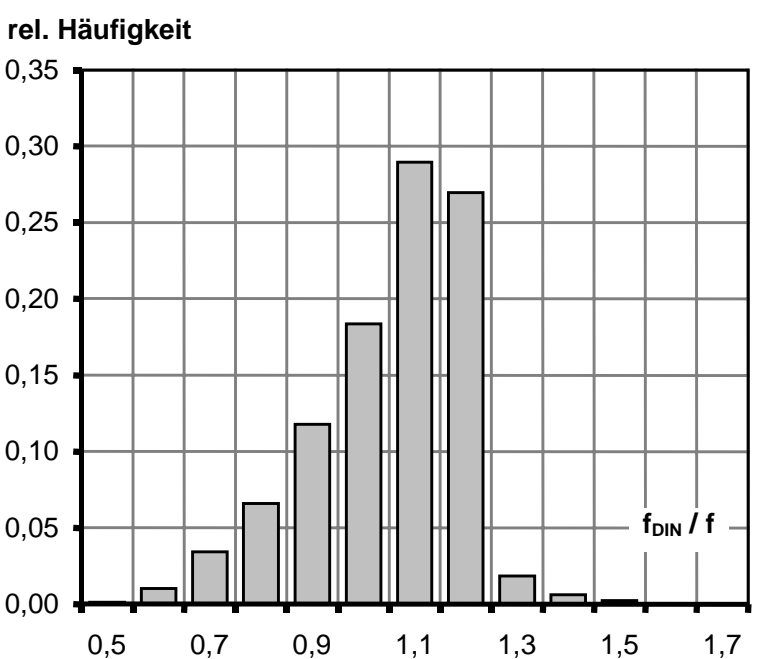

(b) Verformung $f_{D I N}$ mit $b_{m}$ nach DIN 1045-

Abbildung 8-22: Vergleich der rechnerischen Verformungen mit dem Gesamtquerschnittsverfahren [2.30] bei Ansatz der mittragenden Breite nach Norm und denen des analytischen Berechnungsmodell $\mathrm{f}$ im $\mathrm{GZG}\left(\mathrm{M} / \mathrm{M}_{\mathrm{u}} \approx 0,65\right)$

Die Abweichungen der rechnerischen Verformungen auf Basis des vorgeschlagenen Berechnungsansatzes Gleichungen (8-1) und (8-12) sind im Vergleich dazu um ein vielfaches geringer, siehe Tabelle 8-4. Der vorgeschlagene Berechnungsansatz ermöglicht somit eine genauere Ermittlung der Verformungen niedriger Verbundträger.

Tabelle 8-4: $\quad$ Abweichungen der berechneten Verformungswerte zur genaueren Berechnung mit analytischen Berechnungsmodell im $G Z G\left(M / M_{u} \approx 0,65\right)$

\begin{tabular}{|r|l|c|c|c|}
\hline \multicolumn{2}{|c|}{ Berechnungsmethode } & Vorschlag $^{\text {1) }}$ & EN 1994-1-1 $^{\text {2) }}$ & DIN 1045-1 $^{\text {3) }}$ \\
\hline$\delta_{\mathbf{m}}[\%]$ & $\begin{array}{l}\text { mittlere } \\
\text { Abweichung }\end{array}$ & 3,6 & 13,1 & 18,1 \\
\hline $\mathbf{S}[\%]$ & $\begin{array}{l}\text { Standardab- } \\
\text { weichung }\end{array}$ & 5,3 & 19,7 & 23,2 \\
\hline $\mathbf{q}_{0,05}[-]$ & $5 \%-Q u a n t i l$ & 0,92 & 0,93 & 0,73 \\
\hline $\mathbf{q}_{0,95}[-]$ & $95 \%-Q u a n t i l$ & 1,09 & 1,45 & 1,20 \\
\hline
\end{tabular}

1) Berechnung nach Gleichung (8-1) und (8-12)

2) elastische Berechnung mit der mittragenden Breite nach EN 1994-1-1 [1.1]

3) elastische Berechnung mit der mittragenden Breite nach DIN 1045-1 [1.8]

\subsubsection{DIN 1045-1}

In Abbildung 8-22 (b) und Tabelle 8-4 sind die Abweichungen der elastischen Verformungsberechnung mit der mittragenden Breite nach DIN 1045-1 [1.8] im Vergleich zur genauen Berechnung mit dem analytischen Berechnungsmodell, siehe Abschnitt 6.2, dargestellt. Wie 
zu erkennen ist, streuen auch die Verformungen nach DIN 1045-1 in erheblichem Maß um die genauen Werte. Im Grenzzustand der Gebrauchstauglichkeit $\left(M / M_{u} \approx 0,65\right)$ beträgt die mittlere Abweichung 18,1\%, die Standardabweichung 23,2\%, der 5\%-Quantilwert 0,73 und der 95\%-Quantilwert 1,2. Für Ausnutzungsgrade $M / M_{u}=0 \div 0,8$ beträgt die mittlere Abweichung 12,0\%, die Standardabweichung $16,9 \%$, der 5\%-Quantilwert 0,8 und der 95\%Quantilwert 1,3. Die rechnerischen Verformungswerte nach DIN 1045-1 unterschätzen dabei die genaueren im Allgemeinen im Mittel um $18 \%$. Aufgrund der großen Abweichungen und der tendenziell großen Unterschätzung der Verformungen ist die Regel zur mittragenden Breite in DIN 1045-1 zur elastischen Verformungsberechnung niedriger Verbundträger somit nicht geeignet.

Die Abweichungen der rechnerischen Verformungen auf Basis des vorgeschlagenen Berechnungsansatzes Gleichungen (8-1) und (8-12) sind im Vergleich dazu um ein vielfaches geringer, siehe Tabelle 8-4. Der vorgeschlagene Berechnungsansatz ermöglicht somit eine genauere Ermittlung der Verformungen niedriger Verbundträger.

\subsubsection{Schlussfolgerungen aus dem quasi-elastischen Berechnungsansatz}

Durch die systematische Auswertung der durchgeführten Parameterstudie konnte für den Abminderungsfaktor $\alpha_{c}$ ein einfacher Berechnungsansatz entwickelt werden, der eine wirklichkeitsnahe Verformungsberechung niedriger Verbundträger ermöglicht. Ein Vergleich der quasi-elastischen Verformungsberechnung mit dem entwickelten Berechnungsansatz und der genaueren Berechnung mit dem analytischen Berechnungsmodell zeigt eine gute Übereinstimmung.

Der Vergleich der elastischen Verformungsberechung auf Basis der Regelungen zur mittragenden Breite der bestehenden Normen mit der genaueren Berechnung mit dem analytischen Berechnungsmodell zeigt, dass diese für eine elastische Verformungsberechnung niedriger Verbundträger ungeeignet sind. Die rechnerischen Verformungswerte weichen in erheblichem Maß von der genaueren Berechnung ab. Mit dem vorgeschlagenen Berechnungsansatz zur quasi-elastischen Verformungsberechnung niedriger Verbundträger ist somit besonders im Vergleich zu den Normregelungen eine genauere Verformungsberechnung möglich.

\subsection{Abschätzung weiterer Einflüsse}

\subsubsection{Allgemeines}

Die Herleitung des im voranstehenden Abschnitt beschriebenen Berechnungsansatzes konnte nicht alle möglichen Einflüsse auf Verbundträger in realen Hochbauten miteinbeziehen. Im Folgenden werden daher die Einflüsse weiterer, im Berechnungsansatz nicht explizit berücksichtigter Parameter auf das effektive Trägheitsmoment $\mathrm{I}_{\mathrm{i}, \text { eff }}$ niedriger Verbundträger abgeschätzt.

\subsubsection{Belastungstyp}

Die Herleitung des entwickelten Berechnungsansatzes für das effektive Trägheitsmoment $\mathrm{I}_{\mathrm{i}, \text { eff }}$ niedriger Verbundträger ist strenggenommen ausschließlich für eine Belastung des Trägers mit einer Gleichlast gültig, siehe Abschnitt 8.3. Wie die in Abschnitt 4.3.3 beschriebenen Untersuchungen jedoch vermuten lassen, ist der Einfluss des Belastungstyps auf das effektive Trägheitsmoment vergleichsweise gering. Dies kann anhand von Vergleichsrechnungen mit niedrigen Verbundträgern unter einer mittigen Einzellast gezeigt werden. Die Belastung mit einer mittigen Einzellast stellt wegen der ausgeprägten Einschnürung der mittragenden 
Breite im Bereich der Einzellast den ungünstigsten Fall für die üblichen Belastungsformen von Trägern im Hochbau dar. Um den Einfluss des Belastungstyps größtmöglich abzuschätzen, wurden im Rahmen dieser Vergleichsberechnungen die Träger ausschließlich mit einer mittigen Einzellast belastet. Eine immer vorhandene mehr oder minder gleichmäßig verteilte Eigengewichtsbelastung wurde in die mittige Einzellast eingerechnet.

Der Umfang der durchgeführten Vergleichsrechnungen ist in Abbildung 8-23 dargestellt.

\section{System und Querschnitt:}

$$
\begin{array}{ll}
\text { Trägerstützweite: } & \mathrm{L}=5 \div 15 \mathrm{~m} \\
\text { Trägerbreite: } & \mathrm{b}=3 \div 9 \mathrm{~m} \\
\text { Plattendicke: } & \mathrm{h}_{\mathrm{c}}=16 \div 32 \mathrm{~cm} \\
\text { Höhe Stahlträger: } & \mathrm{h}_{\mathrm{a}}=200 \div 600 \mathrm{~mm} \\
\text { Profilreihen: } & \mathrm{HEA}, \mathrm{HEB}, \mathrm{IPE}
\end{array}
$$

Material:

Beton:

Baustahl:

Verdübelung:

Umfang:

C $20 / 25$, C $30 / 37$

\section{$\mathrm{S} 235, \mathrm{~S} 355$}

$\eta=1$ (volle Verdübelung)
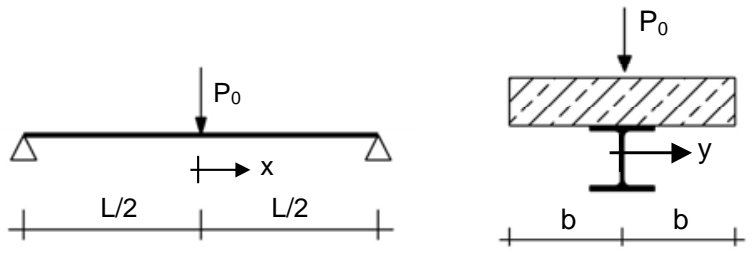

$\mathrm{h}_{\mathrm{c}}+$
$\mathrm{h}_{\mathrm{a}}+$

ca. 900 Träger (a) Abmessungen

Abbildung 8-23: (b) Bezeichnungen

\section{Umfang der Vergleichsrechnungen niedriger Verbundträger mit mittiger Einzellast}

In Abbildung 8-24 sind die Abweichungen der mit dem analytischen Berechnungsmodell mit mittiger Einzellast ermittelten Verformungswerte und den mit Gleichungen (8-1) und (8-12) ermittelten dargestellt. Die Abweichungen liegen im Mittel bei ca. 3,8 \% und die Standardabweichung bei ca. 5,4\%. Im Grenzzustand der Gebrauchstauglichkeit liegen die Abweichungen im Mittel bei 3,3\%, die Standardabweichung bei 4,9\%. Der 5\%-Quantilwert liegt bei 0,90 und der 95\%-Quantilwert bei 1,04. Die Abweichungen sind somit etwas größer als bei den untersuchten Trägern mit Gleichlast jedoch auch bei Belastung mit mittiger Einzellast vergleichsweise gering, siehe Abschnitt 8.3. Die Anwendung von Gleichungen (8-1) und (8-12) kann somit auch bei Belastung mit mittiger Einzellast empfohlen werden.

\section{rel. Häufigkeit}

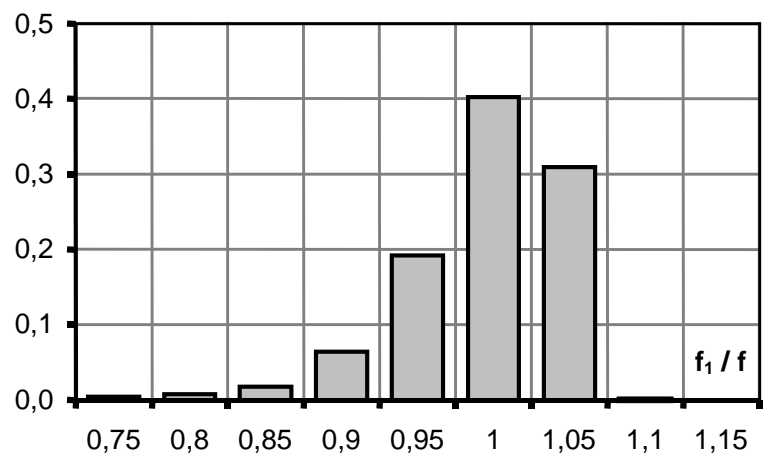

(a) für Ausnutzungen $M / M_{u}=0 \div 0,8$

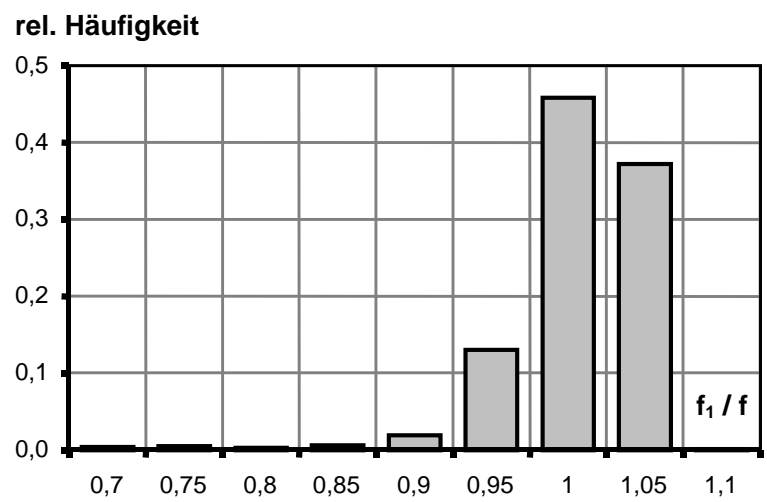

(b) $\operatorname{im} G Z G\left(M / M_{u} \approx 0,65\right)$

Abbildung 8-24: Abweichungen der Verformungen $\mathrm{f}_{1}$ des quasi-elastischen Berechnungsansatzes Gleichungen (8-1) und (8-12) zum analytischen Berechnungsmodell $\mathrm{f}$ bei mittiger Einzellast 


\subsubsection{Eigenspannungen im Stahlprofil}

Wie in Abschnitt 7.5.3 dargelegt, können Eigenspannungen im Stahlprofil zu einer Vergrößerung der Verformungen niedriger Verbundträger führen. Wie groß dieser Einfluss ist, hängt maßgeblich von der Höhe der Eigenspannungen ab. Die in Abschnitt 7.5.3 beschriebenen Vergleichsrechnungen zeigen, dass dieser Einfluss bei Ansatz der Eigenspannungen nach [2.69]-[2.71] im Grenzzustand der Gebrauchstauglichkeit für Stahlträgerquerschnitte mit $\mathrm{b} / \mathrm{h}>1,2$ vernachlässigbar ist. Bei Querschnitten mit $\mathrm{b} / \mathrm{h}<1,2$ erhöhen sich die rechnerischen Verformungen jedoch um ca. 3 $\div 10 \%$. Wenn eine hohe Genauigkeit der Verformungsberechnung notwendig ist, sollten die Eigenspannungen daher bei der Berechnung explizit berücksichtigt werden. In anderen Fällen kann eine Abschätzung der Verformungen durch Erhöhung der Verformungen um die genannten $3 \div 10 \%$ erfolgen. Für eine genauere Untersuchung dieses Einflusses sind jedoch weitere Untersuchungen notwendig.

Eine Möglichkeit wäre, die Bereiche des Stahlträgers mit Zugeigenspannungen im Untergurt und im unteren Stegabschnitt bei der Berechnung der Querschnittswerte und Verformungen proportional zur Eigenspannungsausnutzung zu reduzieren. Hier besteht jedoch die Notwendigkeit weiterer Vergleichsrechnungen

\subsubsection{Querbiegung im Betongurt}

Wie in Abschnitt 7.5.4 dargelegt, ergibt sich bei Verbundträgern in realen Bauwerken immer eine Querbiegung im Betongurt infolge der Exzentrizität der Belastung zur Achse des Trägers, die zu Längsrissen im oberen Bereich des Betongurts am Stahlträger führen kann. Die Rissbildung im Betongurt führt dazu, dass sich die Verhältnisse zwischen Längs-, Quer- und Schubsteifigkeit des Betongurts verändern. Diese Veränderung der Steifigkeitsverhältnisse ist dabei maßgeblich von der Intensität der Rissbildung und somit von der Belastungshöhe abhängig. Eine genaue Berücksichtigung dieser Einflüsse ist sehr aufwändig. Eine Abschätzung des Einflusses der veränderten Steifigkeitsverhältnisse im Betongurt wurde in Abschnitt 7.5.4 durch Betrachtung des Betongurts als orthotrope Scheibe bzw. Platte vorgenommen [2.77].

Diese Untersuchungen zeigen, dass die Orthotropie des Betongurts mit geringerer Steifigkeit in Querrichtung zu einer Vergrößerung der rechnerischen Verformungen im Grenzzustand

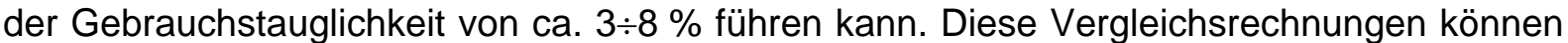
jedoch nur als grobe Abschätzung für das tatsächliche Verformungsverhalten bei Trägern mit Querbiegung und Längsrissen im Betongurt dienen. Für eine genauere Abschätzung des Einflusses der Querbiegung auf die mittragende Breite sind weitere Untersuchungen z. B. mit Finite-Element-Modellen notwendig, die das nichtlineare Tragverhalten von Beton und vor allem den Einfluss der Rissbildung auf die Steifigkeit abbilden können [2.77].

\subsubsection{Slim-Floor Träger}

Slim-Floor Träger stellen in ihrem Trag- und Verformungsverhalten sowie ihrer Querschnittscharakteristik in gewisser Weise den Extremfall niedriger Verbundträger dar, siehe Abschnitt 1.1 und 2.2. Damit sind diese Träger bestens geeignet, die Brauchbarkeit des in Abschnitt 8.3 vorgeschlagenen Berechnungsansatzes für die quasi-elastische Verformungsberechnung niedriger Verbundträger in einer Grenzwertbetrachtung zu überprüfen. Hierfür wurden die in Abbildung 8-25 dargestellten 116 Slim-Floor Systeme untersucht. Die untersuchten Träger entsprechen dabei im Wesentlichen dem Umfang der bauaufsichtlichen Zulassung der Slim-Floor Träger mit UPE-Profilen [2.72].

In Abbildung 8-26 sind die Abweichungen der rechnerischen Verformungen mit dem quasielastischen Berechnungsansatz der Gleichungen (8-1) und (8-12) sowie der Berechnung mit 
dem analytischen Berechnungsmodell der untersuchten Slim-Floor Träger dargestellt. Die Abweichungen im Grenzzustand der Gebrauchstauglichkeit betragen im Mittel ca. 7,8 \%, die Standardabweichung 8,7\%. Die Verformungen werden dabei mit dem vorgeschlagenen Berechnungsansatz in der Regel geringfügig überschätzt. Im Vergleich dazu führt eine elastische Berechnung mit dem Gesamtquerschnittsverfahren [2.30] und einer mittragenden Breite nach EN 1994-1-1 [1.1] zu sehr großen Abweichungen (im Mittel ca. 16,6 \%) mit einer sehr großen Streuung von ca. $-40 \%$ bis $+10 \%$. Die Verformungen werden dabei im Allgemeinen sehr stark unterschätzt. Mit dem in Abschnitt 8.3 vorgeschlagenen Berechnungsansatz für die quasi-elastische Verformungsberechnung ist also eine deutlich realistischere Verformungsberechnung von Slim-Floor Trägern möglich. Die Anwendung dieses Berechnungsansatzes kann somit auch für Slim-Floor Träger nach Abbildung 8-25 und vergleichbare Systeme empfohlen werden.

\section{System und Querschnitt:}

$\begin{array}{ll}\text { Trägerstützweite: } & \mathrm{L}=4 \div 10 \mathrm{~m} \\ \text { Trägerbreite: } & \mathrm{b}=4 \div 10 \mathrm{~m} \\ \text { Plattendicke: } & \mathrm{h}_{\mathrm{c}}=16 \div 32 \mathrm{~cm} \\ \text { UPE-Profil: } & \text { UPE } 160 \div 400\end{array}$

Material:

Beton:

Baustahl:

Verdübelung:

Umfang:

(a) Abmessungen
C $20 / 25$, C $30 / 37$

S 235, S 355

$\eta=1$ (volle Verdübelung)

116 Träger

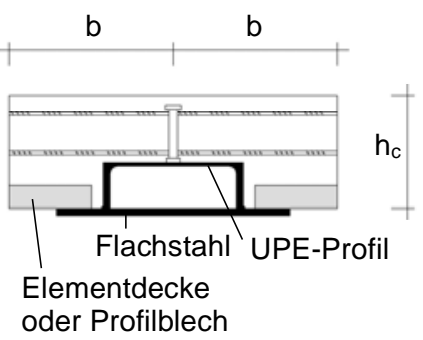

(b) Bezeichnungen

Abbildung 8-25: Umfang der Vergleichsrechnungen an Slim-Floor Trägern mit UPE-Profilen

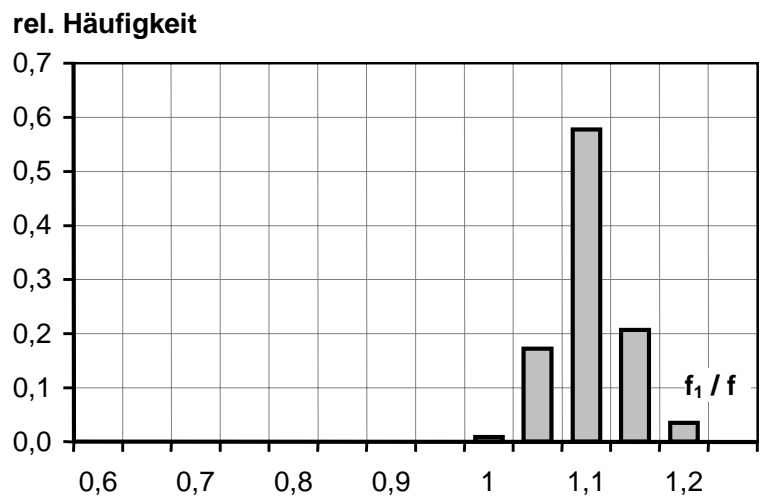

(a) Verformung $f_{1}$ nach Gleichungen (8-1) und (8-12)

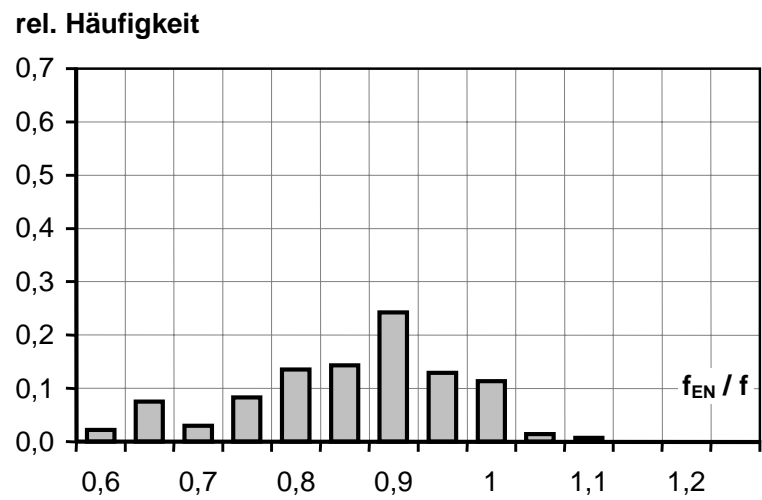

(b) Verformung $f_{E N}$ mit $b_{m}$ nach EN 1994-1-1

Abbildung 8-26: Abweichungen der Verformungen $f_{1}$ mit dem quasi-elastischen Berechnungsansatz Gleichungen (8-1) und (8-12) bzw. mit dem Gesamtquerschnittsverfahren [2.30] und $b_{m}$ nach EN 1994-1-1 zum analytischen Berechnungsmodell $f$ bei Slim-Floor Trägern mit UPE-Profilen im Grenzzustand der Gebrauchstauglichkeit 


\subsection{Zusammenfassung}

Die Berechnung von Verformungen unter expliziter Berücksichtigung des nichtlinearen Mate-

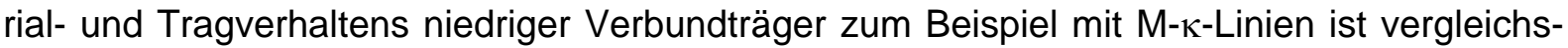
weise aufwändig. Für eine Berechnung in der Planungspraxis ist daher ein vereinfachtes Verfahren auf Basis der bekannten elastischen Berechnungsverfahren von Vorteil und wünschenswert. Um zum Einen das nichtlineare Tragverhalten niedriger Verbundträger bei der Verformungsberechnung zu berücksichtigen und zum Anderen für die Planungspraxis ein handhabbares Vorgehen zu gewährleisten, wurde ein Vorschlag zur quasi-elastischen Verformungsberechnung vorgestellt, der diese Aspekte berücksichtigt.

Kernstück dieses quasi-elastischen Berechnungsvorschlags ist die Bestimmung eines effektiven Trägheitsmoments $I_{i, \text { eff }}$ des Verbundquerschnitts, mit dem die Verformungen niedriger Verbundträger quasi-elastisch berechnet werden können. Das effektive Trägheitsmoment $\mathrm{I}_{\mathrm{i}, \text { eff }}$ basiert dabei auf den elastischen Querschnittswerten des Verbundquerschnitts und berücksichtigt das nichtlineare Material- und Tragverhalten niedriger Verbundträger implizit über den Abminderungsfaktor $\alpha_{c}$. Durch eine umfangreiche Parameterstudie anhand des analytischen Berechnungsmodell konnten die Haupteinflussgrößen auf das effektive Trägheitsmoment identifiziert und in einen Berechnungsansatz umgesetzt werden, der im Rahmen der Randbedingungen des normalen Hoch- und Geschossbaus gültig ist.

Während eine elastische Verformungsberechnung mit dem Gesamtquerschnittsverfahren und der mittragenden Breite nach EN 1994-1-1 bzw. DIN 1045-1 zu großen Abweichungen gegenüber den genauen Werten führt, ist mit dem vorgeschlagenen Berechnungsansatz eine wirklichkeitsnahe Verformungsberechnung möglich. Anhand des entwickelten quasielastischen Berechnungsansatzes ist somit die zuverlässige Ermittlung der Verformungen niedriger Verbundträger in einfacher Weise möglich. 


\section{Einfluss von Kriechen und Schwinden des Betons}

\subsection{Grundsätzliches}

Durch Kriechen und Schwinden des Betons kommt es in Verbundträgern im Laufe der Zeit zu einer Zunahme der Verformungen. Diese Langzeitverformungen stellen dabei einen Großteil der Gesamtverformungen des Verbundträgers dar. Unter Kriechen versteht man dabei, dass bei andauernder Belastung des Betons die Betondehnungen und -verformungen stetig zunehmen. Mit Schwinden wird dagegen die spannungslose zunehmende Stauchung des Betons infolge Austrocknung bezeichnet [2.37], [2.64], [2.68].

Durch die Verdübelung setzt der Stahlträger in Verbundträgern der Kriech- und Schwindverformung des Betons einen elastischen Widerstand entgegen [2.67], [2.68]. Dadurch entstehen im Verbundquerschnitt Teilschnittgrößen, die im Gleichgewicht stehen und einen Eigenspannungszustand darstellen. Aus diesen Eigenspannungen entstehen Verformungen des Verbundträgers, die in statisch unbestimmten Systemen Auflagerreaktionen und Schnittgrößen erzeugen. In gerissenen Bereichen wird der Eigenspannungszustand aus Kriechen und Schwinden mit fortschreitender Rissbildung deutlich reduziert und verschwindet nahezu völlig mit abgeschlossener Rissbildung [2.73]. In den gerissenen Bereichen kann sich über die Risse keine Zwängung infolge Schwinden aufbauen. Schwinden führt hier lediglich zu einer Vergrößerung der Rissöffnung. Dort wo die Risse über die gesamte Höhe des Betongurts verlaufen, bedeutet dies, dass auch die Schwindnormalkraft und das zugehörige Schwindmoment verschwinden. Folglich wird im Allgemeinen bei der Berechnung von durchlaufenden Verbundträgern im Stützbereich keine Schwindnormalkraft und kein (primäres) Schwindmoment berücksichtigt.

Im Feldbereich von Verbundträgern niedriger Bauhöhe sind die Zusammenhänge infolge der Rissbildung komplizierter: Im Druckbereich des Betongurts ist der Beton ungerissen und verhält sich unter Gebrauchslasten näherungsweise elastisch. Im Zugbereich ist der Beton in der Regel bereits unter Gebrauchslasten gerissen. Die Zwängungen aus dem Schwinden des Betons werden hier durch die Risse abgebaut. Durch die Nichtlinearität infolge der Rissbildung kann das Schwinden des Betons jedoch nicht unabhängig von den belastungsabhängigen Dehnungen und Spannungen des Verbundträgers betrachtet werden.

Die vorgeschlagenen Berechnungsansätze für die verformungsbezogene mittragende Breite niedriger Verbundträger, siehe Abschnitt 7.4, und für die quasi-elastische Verformungsberechnung, siehe Abschnitt 8.4, wurden für kurzzeitig wirkende Lasten entwickelt. Ein Großteil der Verformungen von Verbundträgern in realen Bauwerken entsteht jedoch durch Kriechen und Schwinden des Betons. Eine explizite Berücksichtigung dieser Einflüsse bei der Entwicklung der Berechnungsansätze hätte den Rahmen dieser Arbeit überschritten. Anhand der im Folgenden dargestellten Vergleichsrechnungen kann jedoch gezeigt werden, dass mit den entwickelten Berechnungsansätze die Langzeitverformungen der hier betrachteten niedrigen Verbundträger ausreichend genau bestimmt werden können.

Im Folgenden wird zunächst die Erweiterung des analytischen Berechnungsmodell zur Berücksichtigung von Kriechen und Schwinden des Betons erläutert, das zur Berechnung der "genauen" Verformungswerte verwendet wird, siehe Abschnitt 9.2. Daran anschließend werden die Vergleichsrechnungen zum Berechnungsansatz für die verformungsbezogene mittragende Breite und für den quasi-elastischen Berechungsansatz diskutiert. Die Auswertung dieser Vergleichsrechnungen zeigt, dass die entwickelten Berechnungsansätze in guter Näherung auch bei Kriechen und Schwinden des Betons zur wirklichkeitsnahen Verformungsberechnung niedriger Verbundträger verwendet werden können. 


\subsection{Berücksichtigung von Kriechen und Schwinden beim analytischen Berechnungsmodell}

\subsubsection{Allgemeines}

Für die "genaue" Berechnung der Verformungen unter Berücksichtigung von Kriechen und Schwinden muss das in Abschnitt 6.2 beschriebene analytische Berechnungsmodell erweitert werden. Hierfür wurden die implementierten Materialmodelle des Betons erweitert, siehe Abschnitt 9.2.2. Die mit dem so erweiterten Berechnungsmodell ermittelten Verformungswerte werden als Referenzwerte für die Überprüfung der entwickelten Berechnungsansätze für die verformungsbezogene mittragende Breite und den quasi-elastischen Berechnungsansatz verwendet, siehe Abschnitt 9.4 und Abschnitt 9.5.

\subsubsection{Materialmodelle für den Beton unter Berücksichtigung von Kriechen und Schwinden}

Bei elastischem Materialverhalten kann Kriechen und Schwinden des Betons durch die algebraische Spannungs-Dehnungs-Beziehung vergleichsweise einfach durch Superposition der einzelnen Dehnungs- und Spannungsanteile des Betons berücksichtigt werden, siehe Gleichung (9-1) [2.29], [2.30], [2.33]. In der Regel werden Kriechen und Schwinden daher als eigenständige und unabhängige Lastfälle betrachtet.

$$
\varepsilon_{\mathrm{c}}(\mathrm{t})=\varepsilon_{\mathrm{cs}}(\mathrm{t})+\frac{\sigma_{\mathrm{c}}(0)}{\mathrm{E}_{\mathrm{cm}}} \cdot(1+\varphi)+\frac{\sigma_{\mathrm{c}}(\mathrm{t})-\sigma_{\mathrm{c}}(0)}{\mathrm{E}_{\mathrm{cm}}} \cdot(1+\rho \cdot \varphi)
$$

mit

$$
\begin{aligned}
& \varepsilon_{c}(t) \\
& \varepsilon_{c s}(t) \\
& \sigma_{c}(t), \sigma_{c}(0) \\
& E_{c m}
\end{aligned}
$$$$
\varphi
$$$$
\rho
$$

\author{
Gesamtdehnung des Betons zum Zeitpunkt t \\ Schwinddehnung des Betons zum Zeitpunkt t \\ Betonspannung zum Zeitpunkt t bzw. Belastungsbeginn \\ E-Modul des Betons
}

Kriechzahl zum Zeitpunkt t

Relaxationskennwert zur Berücksichtigung des abnehmenden Kriechverhaltens des Betons mit zunehmender Betonalterung

Wenn im Beton Risse auftreten, ist die Superposition einzelner Lastfälle nicht weiter möglich. Durch diese Nichtlinearität kann das lastunabhängige Schwinden des Betons nicht unabhängig von den belastungsabhängigen Dehnungen und Spannungen des Verbundträgers betrachtet werden. Kriechen und Schwinden müssen in diesem Fall gemeinsam mit den weiteren Lastfällen betrachtet werden. Deshalb werden zur Berücksichtigung von Kriechen und Schwinden des Betons die Spannungs-Dehnungs-Beziehungen des Betons in Anlehnung an die algebraische Spannungs-Dehnungs-Beziehung modifiziert, siehe Abbildung 9-1.

Die Gesamtdehnung des Betons wird in einen lastunabhängigen und einen spannungserzeugenden Teil unterteilt, siehe Gleichung (9-2), wie dies letztlich auch durch die algebraische Spannungs-Dehnungs-Beziehung geschieht. Die lastunabhängige Dehnung $\varepsilon_{c s}$ ist dabei gleich der Schwinddehnung und erzeugt keine Spannung im Beton. Für die spannungserzeugende Dehnung $\varepsilon_{c, \sigma}$ des Betons wird das in Abbildung 9-1 dargestellte Materialmodell verwendet. Im Vergleich zum Zeitpunkt $\mathrm{t}=0$ werden die spannungserzeugenden Dehnungen dabei um den Faktor $1+\varphi$ gestreckt. Im Beton entstehen rechnerisch somit nur dann Spannungen, wenn die Betondehnung $\varepsilon_{\mathrm{c}}$ von der Schwinddehnung $\varepsilon_{\mathrm{cs}}$ abweicht. In 
gerissenen Betonbereichen ergeben sich infolge Schwinden somit rechnerisch keine Spannungen.

$$
\varepsilon_{\mathrm{c}}=\varepsilon_{\mathrm{cS}}+\varepsilon_{\mathrm{c}, \sigma}
$$

mit

$$
\begin{aligned}
& \varepsilon_{\mathrm{c}} \\
& \varepsilon_{\mathrm{cS}} \\
& \varepsilon_{\mathrm{c}, \sigma}
\end{aligned}
$$

Gesamtdehnung des Betons zum Zeitpunkt $\mathrm{t}=\propto$ spannungslose Dehnung des Betons zum Zeitpunkt $\mathrm{t}=\propto$ spannungserzeugende Dehnung des Betons zum Zeitpunkt $\mathrm{t}=\propto$

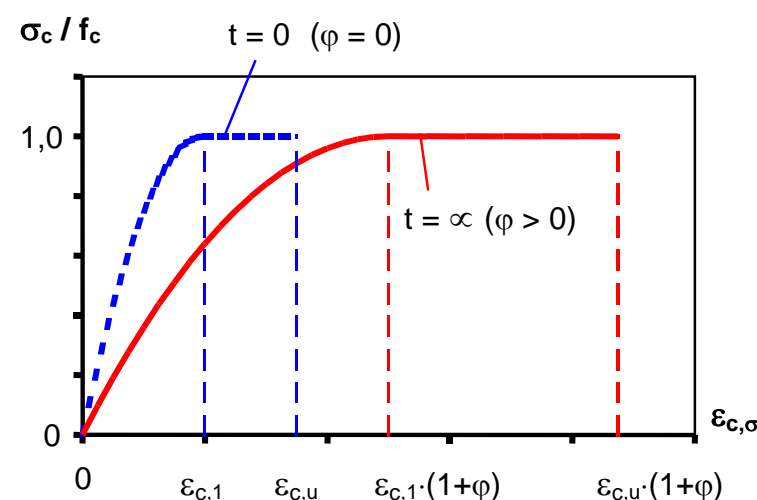

(a) Druckbereich (mit $\varepsilon_{\mathrm{c}, \sigma}=\varepsilon_{\mathrm{c}}-\varepsilon_{\mathrm{cs}}$ )

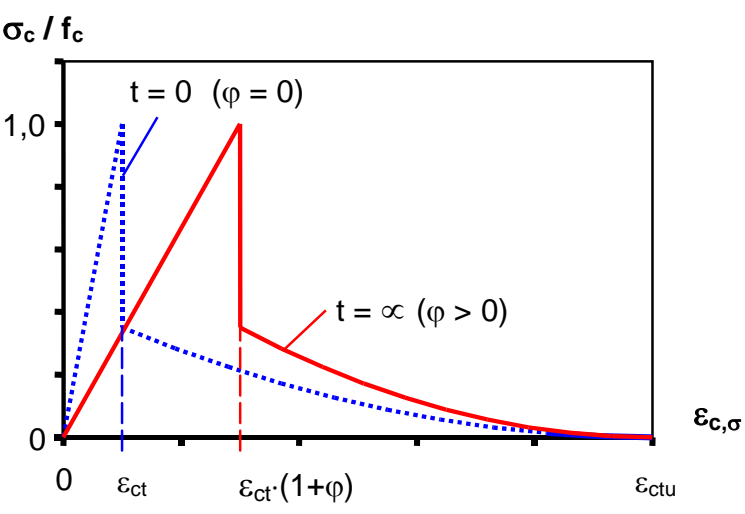

(b) Zugbereich (mit $\varepsilon_{\mathrm{c}, \sigma}=\varepsilon_{\mathrm{c}}-\varepsilon_{\mathrm{cs}}$ )

Abbildung 9-1: Modifizierte Spannungs-Dehnungs-Linie des Betons zur Berücksichtigung von Kriechen und Schwinden [2.32]

Die Vergrößerung der lastabhängigen Betondehnungen durch Kriechen um den Faktor $1+\varphi$ ist strenggenommen nur für ständige Lasten richtig und unterstellt, dass sich das Kriechverhalten des Betons im Laufe der Zeit nicht verändert. Da bei Verbundträgern im Hochbau der Großteil der Belastung jedoch ständig wirkt, ist die Vernachlässigung des abnehmenden Kriechverhaltens später einsetzender Belastungen mit zunehmender Betonalterung eine ausreichend genaue Näherung. Dies gilt besonders im Vergleich zu den weiteren Unsicherheiten der Modellbildung und der Eingangsgrößen der Berechnung, zum Beispiel der Größe der Kriechzahl oder des Schwindmaßes.

\subsubsection{Erweiterung des analytischen Berechnungsmodells für Kriechen und Schwin- den des Betons}

Mit den so definierten Materialgesetzen für den Beton können die Momenten-KrümmungsBeziehungen von Verbundquerschnitten sinngemäß entsprechend dem Fall ohne Kriechen und Schwinden berechnet werden, siehe Abschnitt 2.3.3 und 6.2. Das Rissmoment und die zugehörige Querschnittskrümmung werden dabei gemäß dem Gesamtquerschnittsverfahren [2.29], [2.30] berechnet.

Die Werte für die spannungsbezogene mittragende Breite des Scheiben- und Biegezustands des Betongurts werden wie zum Zeitpunkt $t=0$ in der Rechnung berücksichtigt. Dies stellt nach Auffassung des Verfassers eine ausreichend genaue Näherung dar, da diese Grundwerte der mittragende Breite nicht durch die absolute Größe der Gurtsteifigkeiten sondern nur vom den Verhältnis zwischen Längs-, Quer- und Schubsteifigkeit des Betongurts abhängen. Diese Verhältnisse werden jedoch durch Kriechen und Schwinden des Betons nur wenig beeinflusst. 
Nachteilig bei dem beschriebenen Vorgehen ist, dass für Kriechen und Schwinden des Betons eine gemeinsame Kriechzahl $\varphi$ berücksichtigt werden muss. Dies bedeutet, dass rechnerisch Kriechen und Schwinden des Betons zum gleichen Zeitpunkt beginnen.

Eine Verifikation des analytischen Berechnungsmodell für Kriechen und Schwinden kann anhand von Versuchsergebnisse nicht vorgenommen werden, da diese nicht vorliegen. Das Vorgehen ist jedoch dahingehend plausibel, dass das Vorgehen beim Übergang auf Träger ohne Rissbildung dem elastischen Vorgehen entspricht. Die Dehnungen des Betons werden im Vergleich zu denen beim Zeitpunkt $t=0$ um den Faktor $1+\varphi$ vergrößert. Dies entspricht der algebraischen Spannungs-Dehnungs-Beziehung, wenn der Relaxationskennwert $\rho$ näherungsweise gleich 1 gesetzt wird.

Die getroffenen ingenieurmäßigen Annahmen sind nach Auffassung des Verfassers im Vergleich zu den sonstigen Unsicherheiten bei der rechnerischen Erfassung der Einflüsse aus Kriechen und Schwinden (Kriechzahl, Schwindmaß, Witterungseinflüsse, etc.) somit ausreichend genau. Es kann daher angenommen werden, dass mit dem so erweiterten analytischen Berechnungsmodell eine wirklichkeitsnahe Berechnung der Verformungen niedriger Verbundträger unter Berücksichtigung von Kriechen und Schwinden möglich ist.

\subsection{Umfang der Vergleichsrechnungen}

Der Umfang der durchgeführten Vergleichsrechnungen zur Abschätzung der Einflüsse von Kriechen und Schwinden auf die entwickelten Berechnungsansätze ist in Abbildung 9-2 dargestellt. Die Kriechzahl $\varphi$ und das Schwindmaß $\varepsilon_{\text {cs }}$ wurden dabei nach ENV 1992-1-1 [1.3] für eine trockene Umgebungsbedingung und ein Belastungsalter von 28 Tagen angesetzt. Die untersuchten Randbedingungen entsprechen dabei denjenigen des normalen Hoch- und Geschossbaus.

\section{System und Querschnitt:}

$$
\begin{array}{ll}
\text { Trägerstützweite: } & \mathrm{L}=5 \div 15 \mathrm{~m} \\
\text { Trägerbreite: } & \mathrm{b}=3 \div 9 \mathrm{~m} \\
\text { Plattendicke: } & \mathrm{h}_{\mathrm{c}}=16 \div 32 \mathrm{~cm} \\
\text { Höhe Stahlträger: } & \mathrm{h}_{\mathrm{a}}=200 \div 600 \mathrm{~mm} \\
\text { Profilreihen: } & \mathrm{HEA}, \mathrm{HEB}, \mathrm{IPE}
\end{array}
$$

Material:

Beton:

Baustahl:

Verdübelung:

Umfang:

(a) Abmessungen

C $20 / 25$, C $30 / 37$

S 235, S 355

$\eta=1$ (volle Verdübelung)
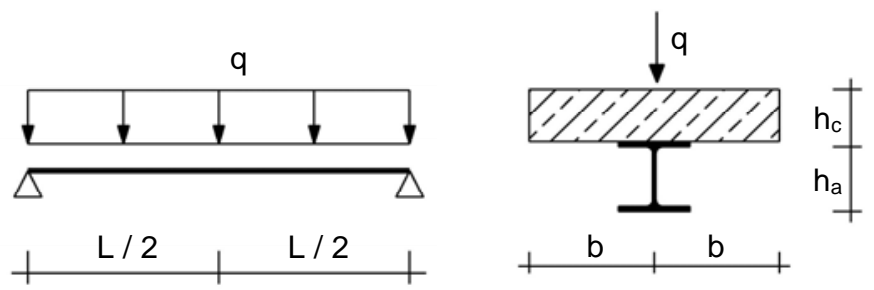

ca. 500 Träger

Abbildung 9-2: Umfang der Vergleichsrechnungen zur Berücksichtigung von Kriechen und Schwinden

\subsection{Verformungsbezogene mittragende Breite}

\subsubsection{Allgemeines}

Der Berechnungsansatz für die verformungsbezogene mittragende Breite niedriger Verbundträger in Abschnitt 7.4 wurde für kurzzeitig wirkende Lasten entwickelt und gilt somit streng- 
genommen bei Kriechen und Schwinden des Betons nicht. Durch Vergleichsrechnungen kann jedoch gezeigt werden, dass der mit Gleichung (7-1) beschriebene Berechnungsansatz in guter Näherung auch bei Berücksichtigung von Kriechen und Schwinden des Betons angewendet werden kann.

Bei den Vergleichsrechnungen werden die genauen Verformungen zum Zeitpunkt $t=\propto$, die mit dem für Kriechen und Schwinden erweiterten analytischen Berechnungsmodell berechnet werden, siehe Abschnitt 9.2, mit den rechnerischen Verformungen des Ersatzbalkens unter Ansatz der mittragenden Breite nach Gleichung (7-1) verglichen, siehe Abschnitt 7.2. Die M-к-Linie des Ersatzbalkens wird dabei mit den in Abschnitt 9.2.2 beschriebenen Materialmodellen des Betons berechnet.

Der Umfang der durchgeführten Vergleichsrechnungen ist in Abbildung 9-2 dargestellt.

\subsubsection{Vergleich des Berechnungsansatzes für Kriechen}

Wenn man die Verformungen niedriger Verbundträger unter Berücksichtigung von Kriechen mit den in Abschnitt 9.2.2 beschriebenen Materialmodellen des Betons und Gleichung (7-1) für die verformungsbezogene mittragende Breite berechnet und diese mit der genaueren Berechnung des analytischen Berechnungsmodells vergleicht, so zeigt sich, dass diese sehr gut übereinstimmen, siehe Abbildung 9-3. Die mittlere Abweichung der ca. 500 Vergleichsrechnungen im Grenzzustand der Gebrauchstauglichkeit $\left(M / M_{u} \approx 0,65\right)$ beträgt $1,4 \%$, die Standardabweichung 6,2\%.

\section{rel. Häufigkeit}

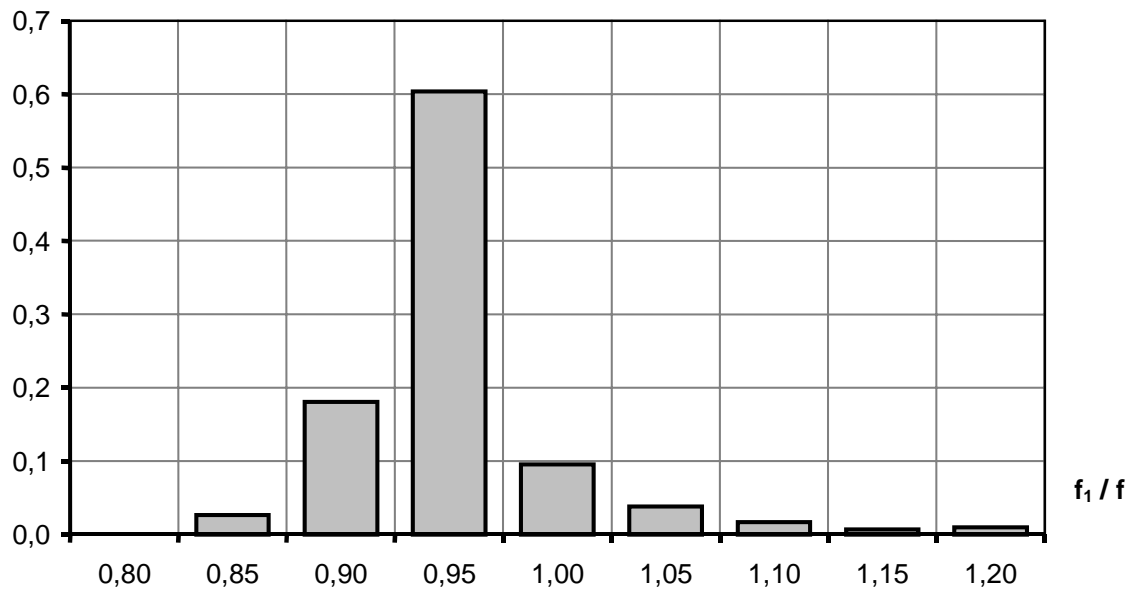

Abbildung 9-3: Vergleich der Verformungen des analytischen Berechnungsmodells $f$ und der Berechnung mit Gleichung (7-1) unter Berücksichtigung von Kriechen $\mathrm{f}_{1}$ zum Zeitpunkt $\mathrm{t}=\propto$ im $\mathrm{GZG}\left(\mathrm{M} / \mathrm{M}_{\mathrm{u}} \approx 0,65\right)$

Prinzipiell nimmt durch das Kriechen die Steifigkeit des Betongurts ab und es kommt folglich zu einer Änderung der Steifigkeitsverteilung im Querschnitt. Dadurch wird der Einfluss der mittragenden Breite des Biegezustands auf die verformungsbezogene mittragende Breite geringer, siehe Abschnitt 4.3. Die verformungsbezogene mittragende Breite ist unter Beachtung des Kriechens somit prinzipiell kleiner als zum Zeitpunkt $t=0$ und Gleichung (7-1) unterschätzt die tatsächlichen Werte geringfügig. Auf die Verformungen hat dies jedoch einen vernachlässigbaren Einfluss, wie die Vergleichsrechnungen zeigen. Die Verwendung von Gleichung (7-1) kann somit in ausreichender Näherung auch für die Verformungsberechnung unter Berücksichtigung des Kriechens von Beton empfohlen werden. 


\subsubsection{Vergleich des Berechnungsansatzes für Schwinden}

Wenn man die Verformungen niedriger Verbundträger unter Berücksichtigung von Kriechen und Schwinden mit den in Abschnitt 9.2.2 beschriebenen Materialmodellen des Betons und Gleichung (7-1) für die verformungsbezogene mittragende Breite berechnet und diese mit der genaueren Berechnung des analytischen Berechnungsmodells vergleicht, so zeigt sich, dass diese sehr gut übereinstimmen, siehe Abbildung 9-4. Die mittlere Abweichung der ca. 500 Vergleichsrechnungen im Grenzzustand der Gebrauchstauglichkeit $\left(M / M_{u} \approx 0,65\right)$ beträgt 2,5\%, die Standardabweichung 4,4\%. Die Verwendung von Gleichung (7-1) kann somit in ausreichender Näherung auch für die Verformungsberechnung unter Berücksichtigung von Kriechen und Schwinden des Betons empfohlen werden.

rel. Häufigkeit

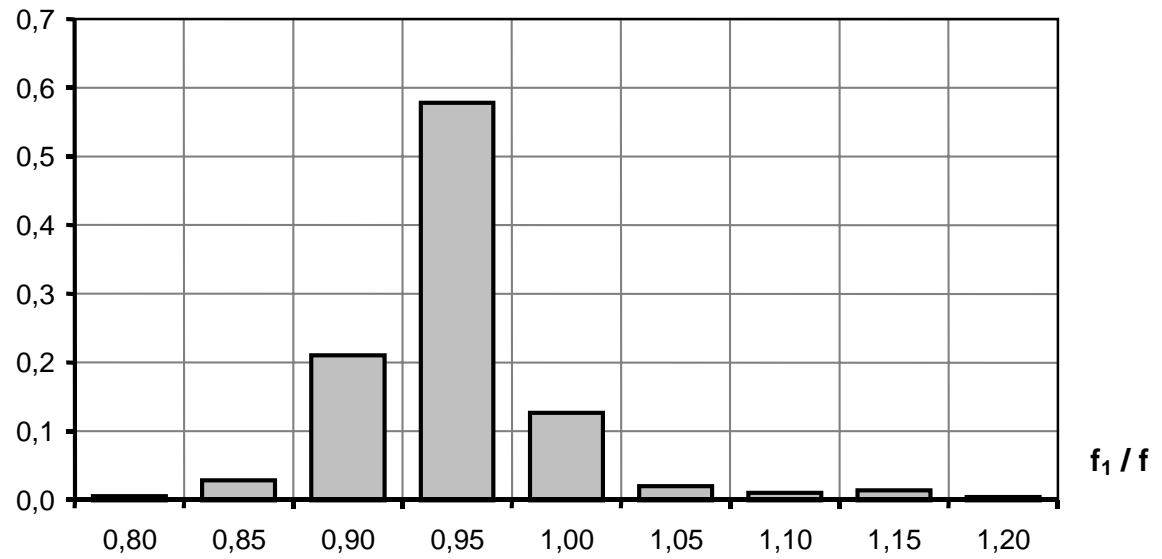

Abbildung 9-4: Vergleich der Verformungen des analytischen Berechnungsmodells $f$ und der Berechnung mit Gleichung (7-1) unter Berücksichtigung von Kriechen und Schwinden $\mathrm{f}_{1}$ zum Zeitpunkt $\mathrm{t}=\propto$ im GZG $\left(\mathrm{M} / \mathrm{M}_{\mathrm{u}} \approx 0,65\right)$

\subsection{Elastische Verformungsberechnung}

\subsubsection{Allgemeines}

Der vorgeschlagene Berechnungsansatz zur quasi-elastischen Verformungsberechnung niedriger Verbundträger in Abschnitt 8.4 wurde für kurzzeitig wirkende Lasten entwickelt. Ein Großteil der Verformungen von Verbundträgern in realen Bauwerken entsteht jedoch durch Kriechen und Schwinden des Betons. Da der Einfluss der Rissbildung auf die Kriech- und Schwindverformungen niedriger Verbundträger geringer ist als auf die Kurzzeitverformung, wird vorgeschlagen die Kriech- und Schwindverformungen rein elastisch nach Gleichung (9-3) zu berechnen. Anhand von Vergleichsrechnungen kann gezeigt werden, dass hiermit die Langzeitverformungen niedriger Verbundträger ausreichend genau abgeschätzt werden.

Die Gesamtverformung $f_{\infty}$ der berechneten Verbundeinfeldträger unter Gleichlast setzt sich somit aus den Teilen $f_{0}$ (quasi-elastische Anfangsverformung), $f_{B, e l}$ (elastische Kriechverformung) und $\mathrm{f}_{\mathrm{S}, \mathrm{el}}$ (elastische Schwindverformung) zusammen, siehe Gleichung (9-3).

$$
\mathrm{f}_{\infty}=\mathrm{f}_{0}+\mathrm{f}_{\mathrm{B}, \mathrm{el}}+\mathrm{f}_{\mathrm{S}, \mathrm{el}}
$$

mit

$f_{\infty}$

Gesamtverformung zum Zeitpunkt $t=\propto$ 
$f_{0}$

$f_{B, e l}$

$\mathrm{f}_{\mathrm{S}, \mathrm{el}}$

$\mathrm{L}$

q
Anfangsverformung zum Zeitpunkt $\mathrm{t}=0$ nach Gleichung (9-4)

Kriechverformung zum Zeitpunkt $t=\propto$ nach Gleichung (9-6)

Schwindverformung zum Zeitpunkt $\mathrm{t}=\propto$ nach Gleichung (9-13)

Spannweite der Verbundeinfeldträger

ständig wirkender Anteil der Gleichlast

Quasi-elastische Anfangsverformung $\mathrm{f}_{0}$ mit effektiven Trägheitsmoment $\mathrm{I}_{\mathrm{i}, \text { eff }}$ nach Gleichung (8-1) und Abminderungsfaktor $\alpha_{c}$ nach Gleichung (8-12):

$$
\begin{aligned}
& \mathrm{f}_{0}=\frac{5}{384} \cdot \frac{\mathrm{q} \cdot \mathrm{L}^{4}}{\mathrm{E}_{\mathrm{a}} \cdot \mathrm{I}_{\mathrm{i}, \text { eff }}} \\
& \mathrm{I}_{\mathrm{i}, \text { eff }}=\mathrm{I}_{\mathrm{a}}+\alpha_{\mathrm{c}} \cdot\left(\mathrm{I}_{\mathrm{c}, 0}+\mathrm{S}_{\mathrm{i}, 0} \cdot \mathrm{a}_{\mathrm{St}}\right)
\end{aligned}
$$

Elastische Kriechverformung $\mathrm{f}_{\mathrm{B}, \mathrm{e}}$ :

$$
\begin{aligned}
& \mathrm{f}_{\mathrm{B}, \mathrm{el}}=\frac{5}{384} \cdot \frac{\mathrm{q} \cdot \mathrm{L}^{4}}{\mathrm{E}_{\mathrm{a}} \cdot \mathrm{I}_{\mathrm{i}, \mathrm{B}}} \\
& \mathrm{I}_{\mathrm{i}, \mathrm{B}}=\mathrm{I}_{\mathrm{a}}+\mathrm{I}_{\mathrm{c}, \mathrm{B}}+\mathrm{S}_{\mathrm{i}, \mathrm{B}} \cdot \mathrm{a}_{\mathrm{St}} \\
& \mathrm{I}_{\mathrm{c}, \mathrm{B}}=\frac{\mathrm{b}_{\mathrm{m}, \mathrm{B}} \cdot \mathrm{h}_{\mathrm{c}}^{3}}{12 \cdot \mathrm{n}_{\mathrm{I}, \mathrm{B}}} \\
& \mathrm{A}_{\mathrm{c}, \mathrm{B}}=\frac{\mathrm{b}_{\mathrm{m}, \mathrm{S}} \cdot \mathrm{h}_{\mathrm{c}}}{\mathrm{n}_{\mathrm{F}, \mathrm{B}}} \\
& \mathrm{n}_{\mathrm{I}, \mathrm{B}}=\mathrm{n}_{0} \cdot\left(1+\psi_{\mathrm{I}, \mathrm{B}} \cdot \varphi\right) \\
& \mathrm{n}_{\mathrm{F}, \mathrm{B}}=\mathrm{n}_{0} \cdot\left(1+\psi_{\mathrm{F}, \mathrm{B}} \cdot \varphi\right) \\
& \mathrm{n}_{0}=\mathrm{E}_{\mathrm{a}} / \mathrm{E}_{\mathrm{cm}}
\end{aligned}
$$

mit

$\mathrm{I}_{i, B}, \mathrm{~S}_{\mathrm{i}, \mathrm{B}}, \mathrm{A}_{\mathrm{i}, \mathrm{B}}$
$\mathrm{I}_{\mathrm{c}, \mathrm{B}}, \mathrm{A}_{\mathrm{c}, \mathrm{B}}$
$\mathrm{I}_{\mathrm{a}}, \mathrm{A}_{\mathrm{a}}$
$\varphi$
$\mathrm{n}_{\mathrm{F}, \mathrm{B}}, \mathrm{n}_{\mathrm{I}, \mathrm{B}}$

elastische Querschnittswerte des Verbundquerschnitts für ständige Einwirkungen

elastische Querschnittswerte des Betonquerschnitts für ständige Einwirkungen

elastische Querschnittswerte des Stahlquerschnitts

Kriechzahl des Betons

Reduktionszahlen der Betonfläche bzw. des Betonträgheitsmoments für ständige Einwirkungen

$\psi_{\mathrm{F}, \mathrm{B}}, \psi_{\mathrm{I}, \mathrm{B}}$

Kriechbeiwerte der Betonfläche bzw. des Betonträgheitsmoments für ständige Einwirkungen

$E_{a}, E_{c m}$

E-Modul des Stahl- und Betonquerschnitts

$b_{m, s}, b_{m, B}$

mittragende Breite des Scheiben- und Biegezustands nach Abschnitt 4.2 
Elastische Schwindverformung $\mathrm{f}_{\mathrm{S}, \mathrm{e}}$ :

$$
\begin{aligned}
& \mathrm{f}_{\mathrm{S}, \mathrm{el}}=\frac{1}{8} \cdot \frac{\mathrm{M}_{\mathrm{s}} \cdot \mathrm{L}^{2}}{\mathrm{E}_{\mathrm{a}} \cdot \mathrm{I}_{\mathrm{i}, \mathrm{S}}} \\
& \mathrm{I}_{\mathrm{i}, \mathrm{S}}=\mathrm{I}_{\mathrm{a}}+\mathrm{I}_{\mathrm{c}, \mathrm{S}}+\mathrm{S}_{\mathrm{i}, \mathrm{S}} \cdot \mathrm{a}_{\mathrm{St}} \\
& \mathrm{I}_{\mathrm{c}, \mathrm{S}}=\frac{\mathrm{b}_{\mathrm{m}, \mathrm{B}} \cdot \mathrm{h}_{\mathrm{c}}^{3}}{12 \cdot \mathrm{n}_{\mathrm{I}, \mathrm{S}}} \\
& \mathrm{A}_{\mathrm{c}, \mathrm{S}}=\frac{\mathrm{b}_{\mathrm{m}, \mathrm{S}} \cdot \mathrm{h}_{\mathrm{c}}}{\mathrm{n}_{\mathrm{F}, \mathrm{S}}} \\
& \mathrm{n}_{\mathrm{I}, \mathrm{S}}=\mathrm{n}_{0} \cdot\left(1+\psi_{\mathrm{I}, \mathrm{S}} \cdot \varphi\right) \\
& \mathrm{n}_{\mathrm{F}, \mathrm{S}}=\mathrm{n}_{0} \cdot\left(1+\psi_{\mathrm{F}, \mathrm{S}} \cdot \varphi\right)
\end{aligned}
$$

mit

$$
I_{i, S}, S_{i, S}, A_{i, S}
$$

elastische Querschnittswerte des Verbundträgers für Schwinden

$I_{c, S}, A_{c, S}$

$\mathrm{n}_{\mathrm{F}, \mathrm{S}}, \mathrm{n}_{\mathrm{I}, \mathrm{S}}$

$\psi_{\mathrm{F}, \mathrm{S}}, \psi_{\mathrm{I}, \mathrm{S}}$

$\mathrm{M}_{\mathrm{S}}$

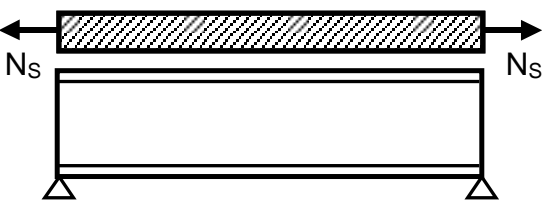

(a) Betongurt vom Stahlträger losgelöst elastische Querschnittswerte des Betonquerschnitts für Schwinden

Reduktionszahlen der Betonfläche bzw. des Betonträgheitsmoments für Schwinden

Kriechbeiwerte der Betonfläche bzw. des Betonträgheitsmoments für Schwinden

elastisches Schwindmoment nach Abbildung 9-5

Abbildung 9-5: Hilfsvorstellung für den Lastfall Schwinden [2.30]

Bei den Vergleichsrechnungen werden die genauen Verformungen zum Zeitpunkt $t=\propto$, die mit dem für Kriechen und Schwinden erweiterten analytischen Berechnungsmodell berechnet werden, siehe Abschnitt 9.2, mit den quasi-elastischen Verformungen $f_{\propto}$ nach Gleichung (9-3) verglichen.

Der Umfang der durchgeführten Vergleichsrechnungen ist in Abbildung 9-2 dargestellt.

Näherungsweise wird bei dem hier vorgeschlagenen Berechnungsansatz Gleichung (9-3) die Zwangskraft $\mathrm{N}_{\mathrm{S}}$ in voller Höhe angesetzt, obwohl die Rissbildung im Betongurt diese reduziert, siehe Abschnitt 9.1. Für eine weitergehende Berücksichtigung dieses Verhaltens sind jedoch weitere Untersuchungen notwendig. 


\subsubsection{Vergleich des quasi-elastischen Berechnungsansatzes für Kriechen}

Vergleicht man die mit Gleichung (9-3) ermittelten Verformungen mit denen des analytischen Berechnungsmodells so ergeben sich für die durchgeführten Vergleichsrechnungen im Grenzzustand der Gebrauchstauglichkeit $\left(M / M_{u} \approx 0,65\right)$ die in Abbildung 9-6 (a) dargestellten Abweichungen. Die Abweichungen sind erwartungsgemäß größer als für kurzzeitige Einwirkungen, für die der Berechnungsansatz hergeleitet wurde. Im Mittel betragen die Abweichungen $13 \%$ und die Standardabweichung 7,7\%. Der 5\%-Quantilwert beträgt 0,98 und der 95\%-Quantilwert 1,30.

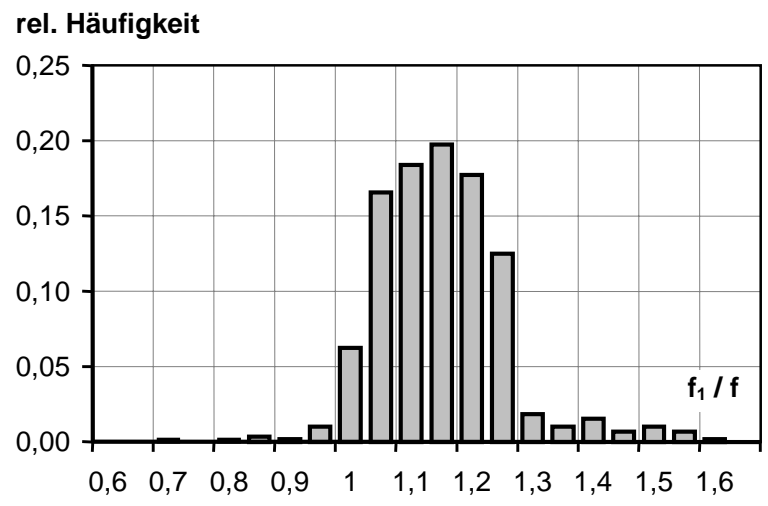

(a) Verformung $f_{1}$ nach Gleichung (9-3)

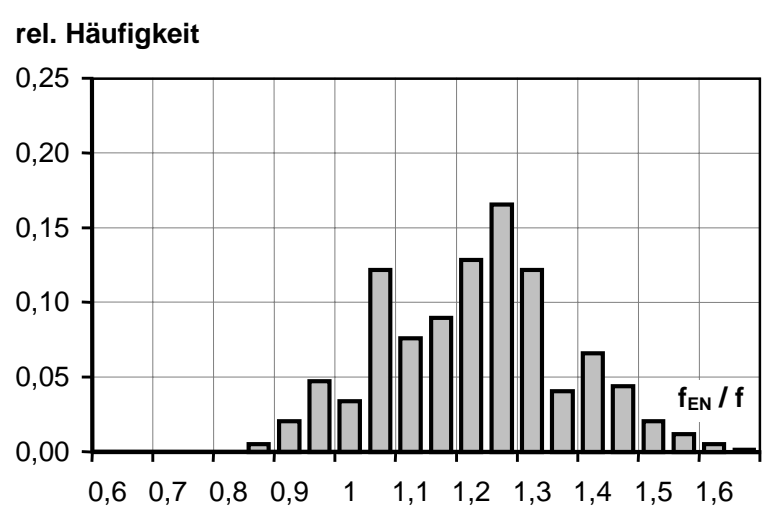

(b) Verformung $f_{E N}$ mit $b_{m}$ nach EN 1994-1-1

Abbildung 9-6: Vergleich der rechnerischen Verformungen $f_{1}$ des vorgeschlagenen Berechnungsansatzes nach Gleichung (9-3) bzw. $f_{E N}$ bei Berechnung mit dem Gesamtquerschnittsverfahren [2.30] und der mittragenden Breite nach EN 1994-1-1 mit denen des analytischen Berechnungsmodell $f$ für ständige Belastungen unter Berücksichtigung von Kriechen im GZG $\left(M / M_{u} \approx 0,65\right)$

Gleichung (9-3) überschätzt die Kriechverformungen dabei im Allgemeinen. Dies liegt daran, dass beim analytischen Berechnungsmodell durch die Rissbildung der Beton nur auf Druck kriecht und daher der Steifigkeitsverlust geringer ist als bei kurzzeitig wirkender Belastung. Die festgestellten, vergleichsweise großen Abweichungen relativieren sich jedoch, wenn in der Berechnung die Einflüsse des Schwindens berücksichtigt werden. In diesem Fall zeigt der vorgeschlagene Berechnungsansatz eine sehr gut Übereinstimmung mit der genaueren Berechnung des analytischen Berechnungsmodell, siehe Abschnitt 9.5.3.

Der Vergleich mit einer Berechnung mit dem Gesamtquerschnittsverfahren [2.30] und der mittragenden Breite nach EN 1994-1-1 [1.1] zeigt darüber hinaus, dass durch den vorgeschlagenen Berechnungsansatz die Genauigkeit der Verformungsberechnung deutlich verbessert wird. Die mittlere Abweichung bei der Berechnung nach EN 1994-1-1 beträgt 21 \% und die Standardabweichung $15 \%$, siehe Abbildung 9-6 (b). Der vorgeschlagene Berechnungsansatz ermöglicht somit eine realistischere Verformungsberechnung niedriger Verbundträger als mit dem Gesamtquerschnittsverfahren und einer mittragenden Breite nach EN 1994-1-1.

\subsubsection{Vergleich des quasi-elastischen Berechnungsansatzes für Schwinden}

Vergleicht man die mit Gleichung (9-3) berechneten Verformungen mit denen des analytischen Berechnungsmodells, so ergeben sich für die durchgeführten Vergleichsrechnungen im Grenzzustand der Gebrauchstauglichkeit $\left(M / M_{u} \approx 0,65\right)$ die in Abbildung 9-7 (a) dargestellten Abweichungen. Die Abweichungen sind erwartungsgemäß größer als für kurzzeitige Einwirkungen, für die der Berechnungsansatz hergeleitet wurde. Im Mittel betragen die Abweichungen im Grenzzustand der Gebrauchstauglichkeit 4,5\% und die Standardabweichung 
7,9 \%. Der 5\%-Quantilwert beträgt 0,85 und der 95\%-Quantilwert 1,14. Damit ist der vorgeschlagene Berechnungsansatz im Vergleich zu den Unsicherheiten weiterer Einflüsse auf das Schwindverhalten (z. B. Kriechzahl, Schwindmaß, Einfluss der Rissbildung, etc.) nach Auffassung des Verfassers ausreichend genau. Die Abweichungen bei einer elastischen Berechnung mit dem Gesamtquerschnittsverfahren [2.30] mit der mittragenden Breite nach EN 1994-1-1 betragen im Vergleich dazu im Mittel 16,6 \%, die Standardabweichung 20,0 \%. Der vorgeschlagene quasi-elastische Berechnungsansatz ermöglicht somit eine deutlich genauere Berechnung der Verformungen von niedrigen Verbundträgern.

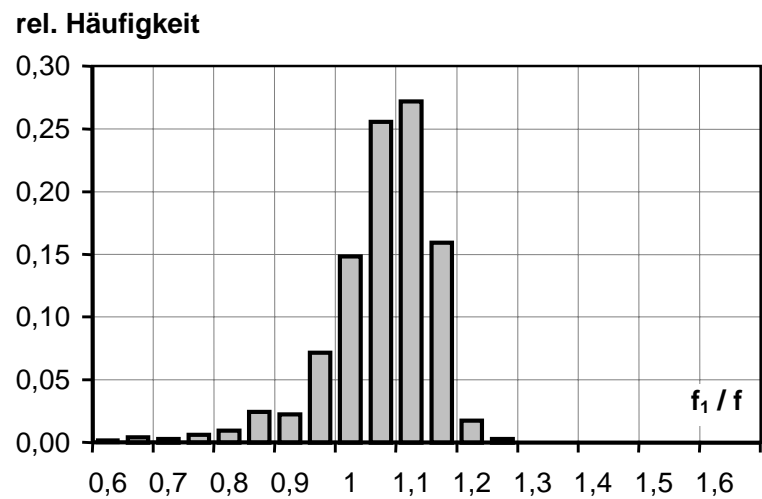

(a) Verformung $f_{1}$ nach Gleichung (9-3)

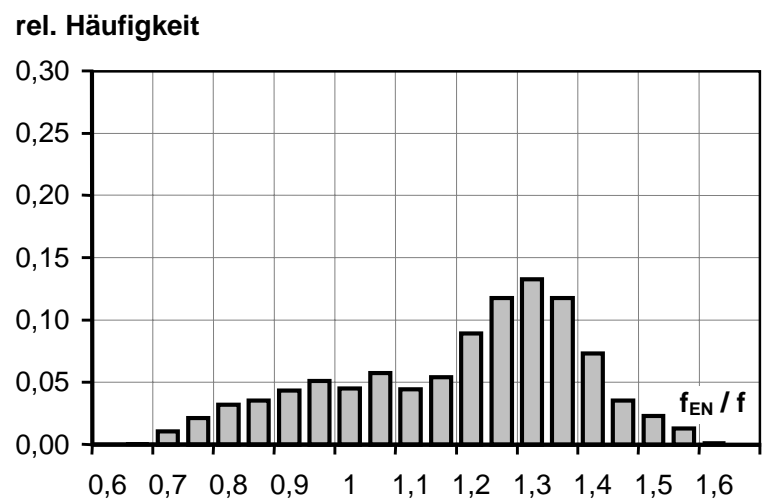

(b) Verformung $f_{E N}$ mit $b_{m}$ nach EN 1994-1-1

Abbildung 9-7: Vergleich der rechnerischen Verformungen $f_{1}$ des quasi-elastischen Berechnungsvorschlags nach Gleichung (9-3) bzw. $f_{E N}$ bei Berechnung mit dem Gesamtquerschnittsverfahren [2.30] und der mittragenden Breite nach EN 1994-1-1 mit denen des analytischen Berechnungsmodell f für ständige Belastungen unter Berücksichtigung von Kriechen und Schwinden im GZG $\left(\mathrm{M} / \mathrm{M}_{\mathrm{u}} \approx 0,65\right)$

\subsection{Schlussfolgerung}

Kriechen und Schwinden des Betons stellen für die Verformungsberechnung von Verbundträgern niedriger Bauhöhe wichtige Einflüsse dar. Die genaue Berücksichtigung dieser Einflüsse bei Verbundträgern mit ausgeprägter Rissbildung ist schwierig. Die voran beschriebenen Vergleichsrechnungen zeigen jedoch, dass der für kurzzeitig wirkende Belastungen entwickelte Berechnungsansatz für die verformungsbezogene mittragende Breite auch zur Berechnung der Langzeitverformungen niedriger Verbundträger verwendet werden kann, wenn man bei der Verformungsberechnung am Ersatzbalken die M- $\kappa$-Linie mit den modifizierten Materialmodellen des Betons berechnet. Des weiteren zeigen die Vergleichsrechnungen, dass die elastische Berechnung der Kriech- und Schwindverformungen zusammen mit der quasi-elastischen Berechnung der kurzzeitigen Verformungen nach Gleichung (9-3) eine gute Näherung der tatsächlichen Verformungen darstellt.

Die im Vergleich zum genaueren analytischen Berechnungsmodell vorhandenen Abweichungen sind im Vergleich zu den sonstigen Unsicherheiten bei der rechnerischen Erfassung von Kriechen und Schwinden des Betons gering. Somit können die entwickelten Berechnungsansätze auch zur Verformungsberechung unter Berücksichtigung von Kriechen und Schwinden des Betons empfohlen werden. 


\section{Zusammenfassung und Ausblick}

\subsection{Zusammenfassung}

Aufgrund der vergleichsweise großen Schlankheit von Verbundträgern niedriger Bauhöhe sind bei diesen Trägern die Verformungen für die Nutzung und die Dimensionierung sehr wichtig. Im Gegensatz zu hohen Verbundträgern wird bei diesen Trägern in sehr vielen Fällen die Einhaltung von Verformungsgrenzwerten unter Gebrauchslasten zum bemessungsrelevanten Aspekt. Dies liegt daran, dass durch die geringere Bauhöhe die Trägersteifigkeit im Vergleich zur Tragfähigkeit überproportional abnimmt. Das Verformungsverhalten der betrachteten niedrigen Verbundträger wird dabei maßgeblich durch die große Biegesteifigkeit des Betongurts und dessen Rissbildung, die bereits unter vergleichsweise geringen $\mathrm{Ge}$ brauchslasten auch im Bereich positiver Momente einsetzt, bestimmt. Die bestehenden Regeln zur Verformungsberechnung von Verbundträgern in den einschlägigen Normen tragen diesem Zustand jedoch nicht bzw. nur eingeschränkt Rechnung. Einer dieser Einflüsse auf die rechnerischen Verformungen ist die mittragende Breite des Betongurts, dessen Wert durch die bestehenden Normregeln pauschal und ohne Berücksichtigung des speziellen Trag- und Verformungsverhaltens niedriger Verbundträger abgeschätzt wird. Auf die rechnerische Tragfähigkeit von Verbundträgern hat dies zwar keinen nennenswerten Einfluss, für die rechnerische Steifigkeit stellt diese jedoch einen wesentlichen Einfluss dar. Im Rahmen der vorliegenden Arbeit wurden daher die mittragende Breite niedriger Verbundträger und deren Einflussfaktoren untersucht.

Die systematische Aufarbeitung der bisherigen Arbeiten zur mittragenden Breite von Plattenbalken des Verbund-, Stahl- und Massivbaus und der Vergleich der bestehenden Normregelungen in Kapitel 3 zeigen eine große Bandbreite an möglichen Werten für die mittragende Breite. Dabei konnte gezeigt werden, dass bei der Entwicklung der bestehenden Ansätze die besonderen Aspekte des Trag- und Verformungsverhalten niedriger Verbundträger und hierbei insbesondere die kombinierte Wirkung aus der großen Biegesteifigkeit des Betongurts sowie dessen Rissbildung nicht berücksichtigt wurden. Diese Untersuchungen zeigen jedoch, dass die mittragende Breite neben den bekannten Einflüssen wie z. B. dem Seitenverhältnis des Betongurts, der Lagerungs- und Belastungsbedingungen vor allem vom Steifigkeitsverhältnis der Teilquerschnitte und der Rissbildung im Betongurt beeinflusst werden. Für den elastischen Fall kann das Tragverhalten des Betongurts in einen Scheiben- und einen Biegezustand getrennt werden und hierfür jeweils ein Wert für die mittragende Breite bestimmt werden. Die mittragende Breite des Biegezustands ist dabei wesentlich größer als die des Scheibenzustands. Die Berücksichtigung des Biegezustands führt damit im Vergleich zum derzeit üblichen Vorgehen auf eine größere mittragende Breite. Da die Regelungen in den derzeit gültigen Verbundbaunormen den Einfluss des Biegezustands auf die mittragende Breite nicht berücksichtigen, ergeben sich hierbei geringere Werte für die mittragende Breite. Es konnte gezeigt werden, dass dieses Vorgehen zwar für hohe Verbundträger aufgrund deren vergleichsweise geringen Biegesteifigkeit des Betongurts und zur Ermittlung der Querschnittstragfähigkeit im Grenzzustand der Tragfähigkeit eine angemessene Näherung darstellt, für niedrige Verbundträger jedoch eine zu große Vereinfachung des tatsächlichen Tragverhaltens ist. Die Untersuchungen zeigen darüber hinaus, dass zwischen der (spannungsbezogenen) mittragenden Breite, die der Querverteilung der Längsspannungen im Querschnitt Rechnung trägt und in Trägerlängsrichtung im Allgemeinen variiert, und der verformungsbezogenen mittragenden Breite, die für eine vereinfachte Verformungsberechnung in Trägerlängsrichtung konstant ist, unterschieden werden muss.

Die im Rahmen eines AiF-Forschungsvorhabens durchgeführten und in Kapitel 5 beschriebenen Versuche an niedrigen Verbundträgern mit breiten Gurten bestätigen dies. Die Versuchsträger waren so konzipiert, dass sie der Anwendung im normalen Hoch- und Ge- 
schossbau entsprechen, das spezielle Tragverhalten niedriger Verbundträger aufweisen und so breit sind, dass ein deutlicher Einfluss der Schubweichheit des Betongurts zu erkennen ist. Die variierten Versuchsparameter sind: Dicke des Betongurts, Betongüte, Breite des Betongurts und der Verdübelungsgrad. Die Messung der Querverteilung der Betondehnungen zeigt, dass diese im Vergleich zu elastischen Berechnungen am Druckrand des Betongurts deutlich ausgeglichener sind und im Bereich der Rissbildung von der Belastungshöhe abhängen. Im Rissbildungsbereich ist die (spannungsbezogene) mittragende Breite bei dickeren Betongurten und breiterem Betongurt größer. Die Betongüte zeigt dabei keinen einheitlichen Einfluss. Die verformungsbezogene mittragende Breite der Versuchsträger ist im ungerissenen Bereich sehr groß und nimmt mit zunehmender Rissbildung aufgrund der dadurch kleiner werdenden Biegesteifigkeit des Betongurts ab. Durch das Plastizieren im Stahlträger steigt sie wieder an. Sowohl bei größerer Dicke und Breite des Betongurts als auch höherer Betonzugfestigkeit ergibt sich eine größere verformungsbezogene mittragende Breite.

Zur Untersuchung der wesentlichen Einflussparameter auf die verformungsbezogene mittragende Breite niedriger Verbundträger wurde das in Kapitel 6 beschriebene analytische Berechnungsmodell entwickelt, das das nichtlineare Material- und Tragverhalten niedriger Verbundträger berücksichtigt. Durch Vergleich mit den Ergebnissen der experimentellen Untersuchungen konnte gezeigt werden, dass das entwickelte Modell zur wirklichkeitsnahen Verformungsberechnung niedriger Verbundträger verwendet werden kann. Anhand einer umfangreichen Parameterstudie konnten sodann die wesentlichen Einflüsse auf die verformungsbezogene mittragende Breite identifiziert und quantifiziert werden. Neben den bekannten Einflüssen auf den elastischen Wert der mittragenden Breite, z. B. das Seitenverhältnis des Betongurts, haben vor allem der Ausnutzungsgrad des Verbundträgers, die Rissbildung und Biegetragwirkung des Betongurts und somit auch die Betonzugfestigkeit einen wesentlichen Einfluss. Ein Vergleich der rechnerischen Verformungswerte mit den Regeln zur mittragenden Breite in EN 1994-1-1 und DIN 18800-5 zeigt, dass diese in erheblichem Maß von den genaueren Werten abweichen und die tatsächlichen Verformungen im Allgemeinen deutlich überschätzen. Die in DIN 1045-1 enthaltene Regel zur mittragenden Breite stellt dabei eine bessere Näherung dar, wenn bei Anwendung von DIN 1045-1 die Verformungen unter Berücksichtigung des nichtlinearen Tragverhaltens berechnet werden.

Durch die systematische Auswertung der Parameterstudie konnte ein handhabbarer Berechnungsansatz für die verformungsbezogene mittragende Breite abgeleitet werden, der eine wirklichkeitsnahe Verformungsberechnung niedriger Verbundträger ermöglicht. Die so ermittelten Verformungswerte sind im Allgemeinen kleiner als die auf Basis der bestehenden Normregelungen zur mittragenden Breite berechneten Verformungen und erlauben eine wirtschaftlichere Dimensionierung dieser Trägerformen.

Da eine Verformungsberechnung unter direkter Berücksichtigung des nichtlinearen Materialund Tragverhaltens niedriger Verbundträger vergleichsweise aufwändig ist, wurde in Kapitel 8 für die Verformungsberechnung in der Ingenieurpraxis ein effektives Trägheitsmoment abgeleitet. Hierfür wurde durch systematische Auswertung der Parameterstudie und Vergleich mit den elastischen Verformungen ein Berechnungsansatz entwickelt, mit dem die Verformungen niedriger Verbundträger quasi-elastisch berechnet werden können. Das effektive Trägheitsmoment setzt sich dabei aus den elastischen Querschnittswerten des Verbundquerschnitts und einem Abminderungsfaktor $\alpha_{c}$ zusammen, mit dem die Steifigkeitsanteile des Betongurts entsprechend dessen Rissbildung reduziert werden. Ein Vergleich mit der elastischen Berechnung anhand des Gesamtquerschnittsverfahrens und der mittragenden Breite nach EN 1994-1-1, DIN 18800-5 und DIN 1045-1 zeigte, dass diese im Vergleich zur genaueren Berechnung sehr stark abweichen und die Abweichungen in erheblichem Maß streuen. Vor allem die elastische Berechnung mit der mittragenden Breite nach DIN 1045-1 unterschätzt dabei die tatsächlichen Verformungen in der Regel deutlich. 
Abschließend kann festgehalten werden, dass die verformungsbezogene mittragende Breite von Verbundträgern wesentlich von der Biegetragwirkung und der Rissbildung des Betongurts abhängig ist. Mit den entwickelten Berechnungsansätzen für die verformungsbezogene mittragende Breite und für das effektive Trägheitsmoment niedriger Verbundträger ist die wirklichkeitsnahe Verformungsberechnung und damit eine wirtschaftlichere Dimensionierung dieser Träger möglich.

\subsection{Ausblick}

Das Verformungsverhalten niedriger Verbundträger wird von vielen Faktoren beeinflusst, die derzeit zum Teil nur unzureichend erforscht sind. Die Untersuchungen zur mittragenden Breite im Rahmen der vorliegenden Arbeit haben hierfür einen Beitrag geleistet. Die Untersuchungen beschränkten sich jedoch auf Verbundträger herkömmlicher Bauart im positiven Momentenbereich. Für den Bereich negativer Momente in durchlaufenden Trägersystemen und für Slim-Floor Träger sind weitere Untersuchungen notwendig. Das entwickelte analytische Berechnungsmodell ist nach Meinung des Verfassers hierfür anwendbar, muss dafür jedoch an weiteren experimentellen und numerischen Untersuchungen verifiziert werden. Es ist zu vermuten, dass die Rissbildung des Betongurts hier einen noch größeren Einfluss auf die mittragende Breite hat.

Im Rahmen der in der vorliegenden Arbeit durchgeführten Versuche wurde der Einfluss einer Querbiegung im Betongurt und daraus resultierender Längsrisse nicht untersucht. Vergleichsrechnungen in dieser Arbeit zur groben Abschätzung des Einflusses der Längsrissbildung im Betongurt infolge Querbiegung zeigen, dass zwar die mittragende Breite durch diese sehr stark beeinflusst wird, der Einfluss auf die Verformungen jedoch deutlich geringer ist. Bei den durchgeführten Vergleichsrechnungen ergibt sich im Mittel eine Erhöhung der

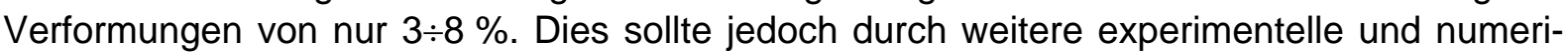
sche Untersuchungen bestätigt werden.

Für den praktisch tätigen Ingenieur wäre die Entwicklung von Anwendungsregeln oder -tabellen, durch deren Einhaltung ein impliziter Nachweis geforderter Verformungsgrenzwerte sichergestellt ist, hilfreich und nützlich.

Die durchgeführten experimentellen Untersuchungen im Rahmen der vorliegenden Arbeit zeigen, dass auch im Bruchzustand des Trägers ein deutlich größerer Bereich des Betongurts mitwirkt, als die bestehenden Regeln und Untersuchungen angeben, siehe auch [2.2], [2.4], [2.76]. Zur Aktivierung dieser Reserven bei der praktischen Bemessung sind weitere Untersuchungen notwendig. 



\section{Literatur}

\subsection{Normen und Richtlinien}

[1.1] EN 1994-1-1: Eurocode 4, Teil 1-1: Bemessung und Konstruktion von Verbundtragwerken aus Stahl und Beton; Allgemeine Bemessungsregeln, Bemessungsregeln für den Hochbau, 3. September 2004.

[1.2] prEN 1994-2: Eurocode 4, Teil 2: Bemessung und Konstruktion von Verbundtragwerken aus Stahl und Beton; Allgemeine Bemessungsregeln und Anwendungsregeln für Brücken, 10. November 2004.

[1.3] DIN V ENV 1992-1-1: Eurocode 2, Teil 1-1: Planung von Stahlbeton- und Spannbetontragwerken; Grundlagen und Anwendungsregeln für den Hochbau, Juni 1992.

[1.4] DIN V ENV 1992-2: Eurocode 2, Teil 2: Planung von Stahlbeton- und Spannbetontragwerken; Betonbrücken, Oktober 1997.

[1.5] DIN V 18800, Teil 5: Stahlbauten; Verbundtragwerke aus Stahl und Beton, Bemessung und Konstruktion, August 2004.

[1.6] DIN 1075: Betonbrücken; Bemessung und Ausführung, April 1981.

[1.7] Richtlinien für Stahlverbundträger: Richtlinie für die Bemessung und Ausführung von Stahlverbundträgern, März 1981.

[1.8] DIN 1045-1: Tragwerke aus Beton, Stahlbeton und Spannbeton; Teil 1: Bemessung und Konstruktion, Juli 2001.

[1.9] BS 5400-5: Steel, concrete and composite bridges; Part 5: Code of practice for design of composite bridges. British Standards Institution, 1979.

[1.10] DIN 1048, Teil 1: Prüfverfahren für Beton; Frischbeton, Festbeton gesondert hergestellter Probekörper, Dezember 1978.

[1.11] DIN EN 10002-1: Prüfung metallischer Werkstoffe, Zugversuch. Teil 1: Prüfverfahren (bei Raumtemperatur), Juli 1998.

[1.12] DIN EN 1993-1-1: Eurocode 3, Teil 1-1: Bemessung und Konstruktion von Stahlbauten; Allgemeine Bemessungsregeln und Regeln für den Hochbau; Juli 2005.

\subsection{Veröffentlichungen}

[2.1] Kuhlmann, U.; Rieg, A.: Flachdecken mit Slim-Floor Profilen. In: VDI-Gesellschaft Bautechnik (Hrsg.), Stahlbetondecken mit Vorspannhilfe nach DIN 1045-1, Fachseminar 21. Oktober 2003, Stuttgart.

[2.2] Kuhlmann, U., Fries, J.: Optimierung der Bemessung von deckengleichen Verbundträgern in Hutform. Forschungsvereinigung Stahlanwendung e.V. -FOSTA- (Hrsg.), Verlag und Vertriebsgesellschaft, 2004.

[2.3] Kuhlmann, U., Fries, J., Rieg, A.: Entwicklung von Bemessungstabellen für SlimFloor Träger mit UPE-Profilen. Forschungsarbeit, Institut für Konstruktion und Entwurf I, Universität Stuttgart, Förderung durch Salzgitter AG, 2002. 
[2.4] Fries, J.: Tragverhalten von Flachdecken mit Hutprofilen. Mitteilungen des Instituts für Konstruktion und Entwurf, Nr. 2002-1, Dissertation, Institut für Konstruktion und Entwurf, Universität Stuttgart, 2001.

[2.5] Kuhlmann, U., Fries, J., Leukart, M.: Bemessung von Flachdecken mit Hutprofilen. Stahlbau-Kalender 2000, Verlag Ernst \& Sohn, 2000.

[2.6] Kuhlmann, U., Fries, J., Rieg, A.: Composite Girders with Reduced Height. In: Connections between Steel and Concrete, RILEM Proceedings PRO 21, RILEM Publications S.A.R.L., Page 1371-1381, 2001.

[2.7] Kuhlmann, U., Fries, J.: Slim-Floor Deckenträger mit Hutprofil. Fachseminar und Workshop, Verbundbau 2, FH München und Bauen mit Stahl e.V., München, 1998.

[2.8] Kuhlmann, U.: Neues aus der Verbundbaunormung. VPI-ArbeitstagungFreudenstadt, Freudenstadt, Juni 1998.

[2.9] Lenzen, K., Kuhlmann, U., Fries, J.: Slim-Floor Deckenträger mit UPE-Profilen. FRILO-Magazin 1/99, Friedrich \& Lochner GmbH, Seite 53-56.

[2.10] Kuhlmann, U., Fries, J.: Zur Anwendung von Hutprofilen mit Stanzdübeln. Festschrift 60. Geburtstag Prof. Tschemmernegg, Institut für Stahlbau, Holzbau und Mischbautechnologie, Leopold-Franzens-Universität Innsbruck, Seite 491-507, 1999.

[2.11] Bauberatung Stahl: Dokumentation 605: Geschoßbau in Stahl, Flachdeckensysteme. 1996.

[2.12] Muess, H.: Interessante Tragwerkslösung im Verbund. Stahlbau 65 (1996), Heft 10, Seite 349-355.

[2.13] Tschemmernegg, F., Huber, G.: Flachdecken mit Stanzdübeln. Bauingenieur 71 (1996), Heft 10, Seite 425-436.

[2.14] Baehre, R., Pepin, R.: Flachdecken mit Stahlträgern in Skelettbauten. Bauingenieur 70 (1995), Heft 2, Seite 65-71.

[2.15] Lu, M., Mäkeläinen, P.: Slim Floor Developments in Sweden and Finland. Structural Engineering International, 1996, Heft 2, Seite 127-129.

[2.16] Müller, C., Feldmann, M.: Flachdecken in Stahl- und Stahlverbundbauweise. 10. DAST-Forschungskolloqium Stahlbau, RWTH Aachen, 4/1997, Seite 9-14.

[2.17] Huber, G., Obholzer, A.: Verbundflachdecken beim Millenium Tower. Stahlbau 68 (1999), Heft 8, Seite 623-630.

[2.18] Arbed: Vorbemessungstabellen IFB- bzw. SFB-Träger. Esch-sur-Alzette, Luxemburg, Firmenschrift, 1997.

[2.19] Baumgärtner, H., Krampe, A., Muess, H., Sauerborn, N.: Die Stahlverbundbauweise - Erfolgreiche Anwendung bei der Goethe Galerie in Jena. Bauingenieur 72 (1997), Seite 67-74.

[2.20] Lange, J.: Flachdecken in Stahlbauweise - Bemessung von Randträgern. Stahlbau 74 (2005), Seite 580-586.

[2.21] Grages, H., Lange, J.: Deflection of Steel Composite Beams in real Structures as basis for the Calculation of the Serviceability of Buildings. Composite Construction V, July 18-23, 2004, Kruger National Park, South Africa.

[2.22] Feldmann, M., Müller, C., Stengel, J.: Zum Tragverhalten von Stahlflachdecken. Bauingenieur 73 (1998), Seite 427-434. 
[2.23] Leskelä, M.: Shallow Floor Integrated Beams and their Components: Comparison of Behavior. In: Hajjar, J., Hosain, M., Easterling, W., Shahrooz, B. (Eds.): Proceedings of the conference "Composite Construction in Steel and Concrete IV“, May 28 June 2, 2000, Banff, Canada.

[2.24] Lawson, R., Leskelä, M.: Slim Floor Construction. Structural Engineering International1996, Heft 2, Seite 122-126.

[2.25] Leskelä, M.: Slim Floors - The Scandinavian Approach. In: Bode, H., Ramm, W., Wittek, U., Schmackpfeffer, H.: Beiträge zur Fachtagung „Verbundkonstruktionen. Neues aus Forschung, Entwicklung und Normung“. 24.-25.07.1997, Universität Kaiserslautern.

[2.26] Haßlacher, S.: Die mittragende Breite von Betongurten für Biegebeanspruchung. Diplomarbeit, Nr. 2001-38X, Institut für Konstruktion und Entwurf, Universität Stuttgart, 2001.

[2.27] Kuhlmann, U., Rieg, A.: Mittragende Betongurtbreite niedriger Verbundträger. AiFForschungsvorhaben Nr. 13460 N/1, Schlussbericht, Nr. 2004-46X Institut für Konstruktion und Entwurf, Universität Stuttgart, 2004.

[2.28] Haas, U.: Wirtschaftlichkeitsvergleich von Slim-Floor Decken mit konventionellen Flachdecken. Diplomarbeit, Nr. 1999-12X, Institut für Konstruktion und Entwurf I, Universität Stuttgart, 1999.

[2.29] Haensel, J.: Praktische Berechnungsverfahren für Stahlträgerverbundkonstruktionen unter der Berücksichtigung neuerer Erkenntnisse zum Betonzeitverhalten. Technisch-wissenschaftliche Mitteilung Nr. 75-2, Institut für konstruktiven Ingenieurbau, Ruhr-Universität Bochum, 1975.

[2.30] Roik, K., Bergmann, R., Haensel, J., Hanswille, G.: Verbundkonstruktionen - Bemessung auf der Grundlage des Eurocode 4 Teil 1. Betonkalender 1999, Verlag Ernst \& Sohn, 1999.

[2.31] Kindmann, R., Xia, G.: Erweiterung der Berechnungsverfahren für Verbundträger. Stahlbau 69 (2000), Seite 170-183.

[2.32] Rieg, A.: Kriechen und Schwinden bei Verbundträgern mit niedriger Bauhöhe. Diplomarbeit, Nr. 1998-16X, Institut für Konstruktion und Entwurf I, Universität Stuttgart, 1998.

[2.33] Trost, H.: Zur Berechnung von Stahlverbundträgern im Gebrauchszustand auf Grund neuerer Erkenntnisse des viskoelastischen Verhaltens des Betons. Stahlbau 37 (1968), Seite 321-331.

[2.34] Sattler, K.: Theorie der Verbundkonstruktionen. Verlag W. Ernst \& Sohn, Berlin 1959, Band I und II.

[2.35] Rieg, A.: Effective Width of Composite Girders with Reduced Height. In: Walraven, J., Blaauwendraad, J., Scarpas, T., Snijder, B. (Eds.): Proceedings of the $5^{\text {th }}$ International PhD Symposium in Civil Engineering, 16-19 June 2004, Delft, The Netherlands.

[2.36] Rybinski, M.: Erfassung der materiellen Nichtlinearität von Stahlbetongurten bei Verbundträgern. Diplomarbeit, Nr. 2000-25X, Institut für Konstruktion und Entwurf, Universität Stuttgart, 2000.

[2.37] Schlaich, J., Schäfer, K.: Bemessen und Konstruieren im Stahlbetonbau. Vorlesungsmanuskript, Institut für Konstruktion und Entwurf II, Universität Stuttgart, 1997, unveröffentlicht. 
[2.38] Schmidt, H., Peil, U.: Berechnung von Balken mit breiten Gurten. Springer-Verlag, Berlin, Heidelberg, New York, 1976.

[2.39] von Karman, Th.: Die mittragende Breite. August-Föppel-Festschrift, Springer Verlag, Berlin, 1924, S. 114-127.

[2.40] Metzer, W.: Die mittragende Breite. Luftfahrtforschung, Heft 4, 1929, Seite 1-20.

[2.41] Chwalla, E.: Die Formeln zur Berechnung der „voll mittragenden Breite“ dünner Gurtund Rippenplatten. Stahlbau 9 (1936), Heft 10, Seite 73-78.

[2.42] Marguerre, K.: Über die Beanspruchung von Plattenträgern. Stahlbau 61 (1952), Seite 129-132.

[2.43] van Langendock, T.: Die Berechnung der Plattenbalken und Verbundträger. Die Bautechnik 35 (1958), Heft 12, S. 467-474.

[2.44] Brendel, G.: Die mitwirkende Plattenbreite nach Theorie und Versuch. Beton- und Stahlbetonbau 55 (1960), Seite 177-185.

[2.45] Severn, R. T.: The effective width of T-beams. Magazine of Concrete Research (Great Britian), Band 16 (1964), Heft 47, Seite 99-102.

[2.46] Rose, E. A.: Ein weiterer Beitrag zur Berechnung der mitwirkenden Breite bei Plattenbalken. Bautechnik 42 (1965), Seite 65-71.

[2.47] Koepcke, W., Denecke, G.: Die mitwirkende Breite der Gurte von Plattenbalken. Deutscher Ausschuß für Stahlbeton, Heft 192, Ernst Verlag, Berlin, 1967.

[2.48] Albrecht, G.: Beitrag zur mittragenden Breite von Plattenbalken im elasto-plastischen Bereich. Technisch-wissenschaftliche Mitteilung Nr. 76-7, Institut für Konstruktiven Ingenieurbau, Universität Bochum, 1976.

[2.49] Schmidt, H., Born, W.: Die Mitwirkung breiter Gurte in Balkenbrücken mit veränderlichem Querschnitt. Verlag Ernst \& Sohn, Berlin, 1978.

[2.50] Schröder, S., Stritzke, J.: Neue Untersuchungen zur mittragenden Breite von Plattenbalken. Bauplanung - Bautechnik, Band 32 (1978), Heft 4, Seite 176-180.

[2.51] Stegbauer, A.: Untersuchungen des Grenztragverhaltens von symmetrischen Stahlbetonplattenbalken mit Hilfe der Methode der finiten Elemente. Bauingenieur 53 (1978), Seite 51-54.

[2.52] Grünberg, J.: Spannungszustände und mitwirkende Plattenbreite eines Stahl-BetonPlattentragwerks im gerissenen Zustand. Beton- und Stahlbetonbau 53 (1978), Seite 177-181.

[2.53] Grasser, E.: Bemessung von Beton- und Stahlbetonbauteilen nach DIN 1045 (Ausgabe Dez. 1978), Teil 1: Biegung mit Längskraft, Schub, Torsion. Deutscher Ausschuß für Stahlbeton, Heft 220, 2. Auflage, Beuth Verlag, Berlin, 1979.

[2.54] Grasser, E., Moosecker, W.: Hilfsmittel zur näherungsweisen Bestimmung der mitwirkenden Breite von Plattenbalken im Stahlbetonbau. Beton- und Stahlbetonbau, Band 77 (1982), Heft 6, Seite 164-167.

[2.55] Loo-Yew-Chaye: Effective Flange Width Formulas fot T-Beams. Concrete International, Design and Construction, Band 8 (1986), Heft 2, Seite 40-45.

[2.56] Sedlacek, G., Bild, S.: Vorgehensweise bei der Ermittlung der mittragenden Breite. Bauingenieur 65 (1990), Seite 551-562.

[2.57] Holtkamp, H. J.: Zur mittragenden Breite von Verbundträgern im Bereich negativer Momente. Dissertation, Mitteilung Nr. 91-3, Institut für Konstruktiven Ingenieurbau, Ruhr-Universität Bochum, 1991. 
[2.58] Grasser, E., Thielen, G.: Hilfsmittel zur Berechnung der Schnittgrößen und Formänderungen von Stahlbetontragwerken. Deutscher Ausschuß für Stahlbeton, Heft 240, 3. Auflage, Beuth Verlag, Berlin, 1991.

[2.59] Hofmann, T. J., Ramm, E.: Erfassung der mittragenden Breite durch eine verfeinerte Balkentheorie. Festschrift Udo Vogel, Institut für Baustatik, Universität Karlsruhe, Seite 107-125, 1993.

[2.60] Weyer, U., Uhlendahl, J.: Rissesicherung schlaff bewehrter Verbundbrücken. Bauingenieur 70 (1995), Seite 331-341.

[2.61] Weyer, U., Uhlendahl, J.: Verbundträger im Brückenbau; Rissesicherung schlaff bewehrter durchlaufender Verbundbrücken. Studiengesellschaft Stahlanwendung e.V. (Hrsg.), Verlag und Vertriebsgesellschaft, 1995.

[2.62] Amadio, C., Fragiacomo, M.: Effective Width Evaluation for Steel-Concrete Composite Beams. Journal of Constructional Steel Research 58 (2002), Page 373-388.

[2.63] Amadio, C., Fedrigo, C., Fragiacomo, M., Macorini, L.: Experimental evaluation of effective width in steel-concrete composite beams. Journal of Constructional Steel Research 60 (2004), pp 199-220.

[2.64] Hilsdorf, H. K., Reinhardt, H.-W.: Beton. Betonkalender 1998, Verlag Ernst \& Sohn, 1998.

[2.65] Kuhlmann, U., Rieg, A., Nakajima, S.: Numerical Studies on the Effective Widths of Composite Girders Considering Cracking Behaviour. In: Matsui, S., Nakai, H. (Eds.): Proceedings of the $5^{\text {th }}$ Japanese-German Joint Symposium on Steel and Composite Bridges, 9-12 September 2003, Osaka University, Japan.

[2.66] Nakajima, S.: Definition of Effective Widths for Composite Girders based on Numerical Studies considering the Cracking Behaviour. Master Thesis, Nr. 2003-32X, Institut für Konstruktion und Entwurf, Universität Stuttgart, 2003.

[2.67] Hanswille, G., Schäfer, M.: Verbundtragwerke aus Stahl und Beton, Bemessung Konstruktion - Kommentar zu DIN V 18800-5, Ausgabe November 2004. Stahlbaukalender 2005, Verlag Ernst \& Sohn, 2005.

[2.68] Bode, H.: Euro-Verbundbau, Konstruktion und Berechnung. 2. Auflage, Werner Verlag, 1998.

[2.69] Petersen, C.: Stahlbau; Grundlagen der Berechnung und baulichen Ausbildung von Stahlbauten. 3. Auflage, Verlag Friedr. Vieweg \& Sohn, 2003.

[2.70] ECCS European Convention of Constructural Steelwork: Stability of Steel Structures. Final Report, Second International Colloquium, Liège 13th - 15th April 1977.

[2.71] ECCS European Convention for Constructural Steelwork COMMITTEE 8: Manual on Stability of Steel Structures. June 1976.

[2.72] Deutsches Institut für Bautechnik: Zulassungsbescheid Z-26.2-48; Slim-Floor Träger mit UPE-Profilen, Peiner Träger GmbH, 27. Juli 2005.

[2.73] Hanswille, G.: Zur Rissbreitenbeschränkung von Verbundträgern. Technischwissenschaftliche Mitteilung Nr. 86-1, Institut für konstruktiven Ingenieurbau, RuhrUniversität Bochum, 1986.

[2.74] Manleitner, S.: Bestimmungen; DIN 1045-1 Tragwerke aus Beton, Stahlbeton und Spannbeton; Teil 1: Bemessung und Konstruktion. Betonkalender 2000, Verlag Ernst \& Sohn, 2000.

[2.75] Deutscher Ausschuss für Stahlbeton: Erläuterungen zu DIN 1045-1. Heft 525, 1. Auflage 2003, Beuth Verlag GmbH, 2003. 
[2.76] Schäfer, M.: Zum Rotationsnachweis teiltragfähiger Verbundknoten in verschieblichen Verbundrahmen. Mitteilungen des Instituts für Konstruktion und Entwurf, Nr. 2005-1, Dissertation, Institut für Konstruktion und Entwurf, Universität Stuttgart, 2005.

[2.77] Kuhlmann, U., Hauf, G., Rieg, A.: Effiziente Dimensionierung niedriger Verbundträger. Forschungsvorhaben gefördert durch Stiftung Industrieforschung, Köln, voraussichtlich Ende 2006. 


\section{Anhang A}

\section{Berechnungsbeispiel}





\section{A Berechnungsbeispiel}

\section{A 1 System und Querschnitt des Beispielträgers}

In Abbildung A-1 sind System und Querschnitt des gewählten Beispielträgers dargestellt. Die Materialwerte und die Belastung des Trägers betragen:

\section{(a) Materialwerte}

\section{Beton}

Druckfestigkeit:

Zugfestigkeit:

E-Modul:

Teilsicherheitsbeiwert: $\mathrm{f}_{\mathrm{ck}}=30 \mathrm{~N} / \mathrm{mm}^{2}$

$\mathrm{f}_{\mathrm{ctm}}=0,29 \mathrm{~N} / \mathrm{mm}^{2}$

$\mathrm{E}_{\mathrm{cm}}=3.200 \mathrm{~N} / \mathrm{mm}^{2}$

$\gamma_{\mathrm{c}}=1,5$

\section{Baustahl}

Streckgrenze:

Zugfestigkeit:

E-Modul:

Teilsicherheitsbeiwert: $\mathrm{f}_{\mathrm{yk}}=355 \mathrm{~N} / \mathrm{mm}^{2}$

$\mathrm{f}_{\mathrm{uk}}=510 \mathrm{~N} / \mathrm{mm}^{2}$

$E_{a}=210.000 \mathrm{~N} / \mathrm{mm}^{2}$

$\gamma_{a}=1,15$

\section{(b) Belastung}

Eigengewicht:

$\mathrm{g}_{1}=25 \cdot 0,28 \cdot 1,13 \cdot 7+0,9=56,3 \mathrm{kN} / \mathrm{m}$

Ausbaulasten:

$\mathrm{g}_{2}=1,0 \cdot 1,25 \cdot 7=8,7 \mathrm{kN} / \mathrm{m}$

Nutzlasten:

$$
\mathrm{p}=3,5 \cdot 1,25 \cdot 7=30,6 \mathrm{kN} / \mathrm{m}
$$

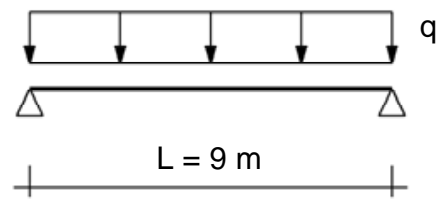

(a) System
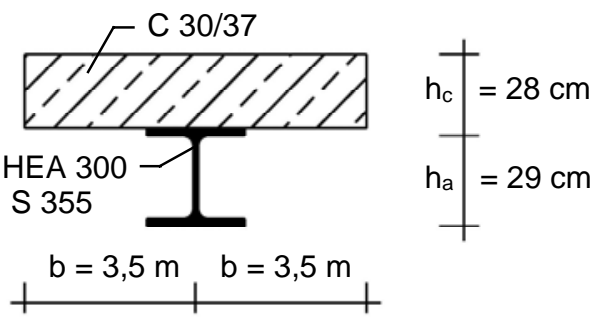

Abbildung A-1: System und Querschnitt des Beispielträgers

\section{A 2 Nachweis der Tragfähigkeit}

Zunächst werden für den gewählten Beispielträger die wesentlichen Nachweise im Grenzzustand der Tragfähigkeit in der Form einer Vorbemessung geführt.

\section{(a) Bemessungsschnittgrößen}

Bemessungswert der Belastung:

$$
\mathrm{q}_{\mathrm{sd}}=1,35 \cdot \mathrm{g}_{\mathrm{k}}+1,5 \cdot \mathrm{p}_{\mathrm{k}}=1,35 \cdot(56,3+8,7)+1,5 \cdot 30,6=133,7 \mathrm{kN} / \mathrm{m}
$$

Schnittgrößen:

$$
\begin{aligned}
& M_{S d}=q_{S d} \cdot L^{2} / 8=133,7 \cdot 9^{2} / 8=1353 k N m \\
& V_{S d}=q_{S d} \cdot L / 2=133,7 \cdot 9 / 2=601,7 k N
\end{aligned}
$$

\section{(b) Momententragfähigkeit des Querschnitts}

Die mittragende Breite im Grenzzustand der Tragfähigkeit wird gemäß EN 1994-1-1 [1.1], 4.2.2.1 bestimmt.

$$
\begin{aligned}
& \mathrm{b}_{\mathrm{m}}=2 \cdot \mathrm{L} / 8=2 \cdot 9 / 8=2,25 \mathrm{~m} \\
& \mathrm{~N}_{\mathrm{pl}, \mathrm{a}, \mathrm{Rd}}=\mathrm{A}_{\mathrm{a}} \cdot \mathrm{f}_{\mathrm{yk}} / \gamma_{\mathrm{a}}=113 \cdot 35,5 / 1,1=3647 \mathrm{kN}
\end{aligned}
$$


Druckzonenhöhe im Beton:

$$
x_{c}=\frac{N_{p l, a, R d}}{0,8 \cdot b_{m} \cdot 0,85 \cdot f_{c d}}=\frac{3647}{0,8 \cdot 225 \cdot 0,85 \cdot 3,0 / 1,5}=11,9 \mathrm{~cm}<28 \mathrm{~cm}=h_{c} \checkmark
$$

Momententragfähigkeit des Verbundquerschnitts:

$$
\begin{aligned}
\mathrm{M}_{\mathrm{pl}, \mathrm{Rd}} & =\mathrm{N}_{\mathrm{pl}, \mathrm{a}, \mathrm{Rd}} \cdot\left(\mathrm{h}_{\mathrm{a}} / 2+\mathrm{h}_{\mathrm{c}}-0,4 \cdot \mathrm{x}_{\mathrm{c}}\right) \\
& =3647 \cdot(29 / 2+28-0,4 \cdot 11,9) / 100=1376 \mathrm{kN} / \mathrm{m}
\end{aligned}
$$

\section{(c) Querkrafttragfähigkeit des Querschnitts}

Zur Querkraftaufnahme wird nur der Steg des Stahlträgers entsprechend EN 1993-1-1 [1.12], 6.2.6 angerechnet.

$$
\begin{aligned}
A_{V} & =A_{a}-2 \cdot t_{a} \cdot b_{a}+t_{a} \cdot\left(2 \cdot r_{a}+s_{a}\right) \\
& =113-2 \cdot 1,4 \cdot 30+1,4 \cdot(2 \cdot 2,7+0,85)=37,75 \mathrm{~cm}^{2} \\
V_{p l, R d} & =A_{V} \cdot \tau_{p l, a, R d}=37,75 \cdot \frac{35,5}{\sqrt{3} \cdot 1,1}=703,4 \mathrm{kN}
\end{aligned}
$$

\section{(d) Nachweis der Tragfähigkeit im GZT}

$$
\begin{gathered}
\frac{M_{S d}}{M_{p l, R d}}=\frac{1353}{1376}=0,98<1,0 \\
\frac{V_{S d}}{V_{p l, R d}}=\frac{601,7}{703,7}=0,86<1,0
\end{gathered}
$$

Eine Interaktion der Momenten- und Querkrafttragfähigkeit ist nicht notwendig, da das maximal Moment an einer anderen Stelle auftritt als die maximal Querkraft.

\section{A 3 Verformungsberechnung}

\section{A 3.1 Allgemeines}

Die Berechnung der kurzzeitigen Verformungen erfolgt mit dem in Abschnitt 7.2 beschriebenen quasi-elastischen Berechnungsansatz für das effektive Trägheitsmoment des Verbundträgers. Die Zunahme der Verformung infolge Kriechen und Schwinden wird rein elastisch berechnet, siehe Abschnitt 8.5. Als Eingangswerte für die Berechnung sind die elastischen Querschnittswerte des Verbundquerschnitts erforderlich. Die Querschnittswerte des Betonquerschnitts werden dabei mit den elastischen Werten der mittragenden Breite des Scheiben- bzw. Biegezustands bestimmt, siehe Abschnitt 4.2.

\section{A 3.2 Zeitpunkt $\mathrm{t}=0$}

\section{(a) Elastische Querschnittswerte des Betons}

Mittragende Breite des Betongurts, siehe Abbildung 4-6:

$$
\begin{aligned}
& b / L=3,5 / 9=0,39 \\
& \Rightarrow \quad b_{m, S} / b=0,52 \rightarrow b_{m, S}=0,52 \cdot b=0,52 \cdot 3,5=1,81 m \\
& \Rightarrow \quad b_{m, B} / b=0,91 \quad \rightarrow \quad b_{m, B}=0,91 \cdot b=0,91 \cdot 3,5=3,19 m \\
& A_{c}=h_{c} \cdot 2 \cdot b_{m, S}=28 \cdot 2 \cdot 181=10.136 \mathrm{~cm}^{2}
\end{aligned}
$$




$$
I_{c}=h_{c}^{3} \cdot 2 \cdot b_{m, B} / 12=28^{3} \cdot 2 \cdot 319 / 12=1.167 .000 \mathrm{~cm}^{4}
$$

Reduktionszahl der Betonquerschnittswerte zum Zeitpunkt $\mathrm{t}=0$ :

$$
\mathrm{n}_{0}=\mathrm{E}_{\mathrm{a}} / \mathrm{E}_{\mathrm{c}}=21.000 / 3.200=6,56
$$

Reduzierte Querschnittswerte des Betons zum Zeitpunkt $t=0$ :

$$
\begin{aligned}
& A_{c, 0}=A_{c} / n_{0}=10.136 / 6,56=1.545 \mathrm{~cm}^{2} \\
& I_{c, 0}=I_{c} / n_{0}=1.167 .000 / 6,56=177.896 \mathrm{~cm}^{4}
\end{aligned}
$$

\section{(b) Elastische Querschnittswerte des Stahlträgers}

$$
\begin{aligned}
& A_{a}=113 \mathrm{~cm}^{2} \\
& I_{a}=18.260 \mathrm{~cm}^{4} \\
& h_{a}=29 \mathrm{~cm}
\end{aligned}
$$

(c) Elastische Querschnittswerte des Verbundquerschnitts

$$
\begin{aligned}
& \mathrm{a}_{\mathrm{St}}=\left(\mathrm{h}_{\mathrm{a}}+\mathrm{h}_{\mathrm{c}}\right) / 2=(29+28) / 2=28,5 \mathrm{~cm} \\
& \mathrm{~A}_{\mathrm{i}, 0}=A_{\mathrm{a}}+\mathrm{A}_{\mathrm{c}, 0}=113+1.545=1.658 \mathrm{~cm}^{2} \\
& \mathrm{z}_{\mathrm{i}, 0}=\mathrm{A}_{\mathrm{a}} \cdot \mathrm{a}_{\mathrm{St}} / \mathrm{A}_{\mathrm{i}, 0}=113 \cdot 28,5 / 1.658=1,94 \mathrm{~cm} \\
& \mathrm{~S}_{\mathrm{i}, 0}=\mathrm{A}_{\mathrm{c}, 0} \cdot \mathrm{z}_{\mathrm{i}, 0}=1.545 \cdot 1,94=2.997 \mathrm{~cm}^{3} \\
& \mathrm{I}_{\mathrm{i}, 0}=\mathrm{I}_{\mathrm{a}}+\mathrm{I}_{\mathrm{c}, 0}+\mathrm{S}_{\mathrm{i}, 0} \cdot \mathrm{a}_{\mathrm{St}}=18.200+177.896+2.997 \cdot 28,5=281.511 \mathrm{~cm}^{4}
\end{aligned}
$$

\section{(d) Effektives Trägheitsmoment des Verbundträgers}

Das effektive Trägheitsmoment $\mathrm{I}_{\mathrm{i} \text {,eff }}$ des Verbundträgers berechnet sich nach Gleichung (7-1) in Abschnitt 7.2.2. Hierfür muss zusätzlich zu den elastischen Querschnittswerten das Rissmoment $M_{\text {Riss }}$ des Verbundquerschnitts, der Abstand $z_{c u, 0}$ des unteren Betonrands zur Schwerlinie des Verbundquerschnitts und die Belastung im Grenzzustand der Gebrauchstauglichkeit berechnet werden.

$$
\begin{aligned}
& \mathrm{z}_{\mathrm{cu}, 0}=\mathrm{h}_{\mathrm{c}} / 2-\mathrm{z}_{\mathrm{i}, 0}=28 / 2-1,94=12,06 \mathrm{~cm} \\
& \mathrm{M}_{\text {Riss }}=\frac{f_{\mathrm{ct}} \cdot \mathrm{I}_{\mathrm{i}, 0}}{\mathrm{z}_{\mathrm{cu}, 0}} \cdot \mathrm{n}_{0}=\frac{0,29 \cdot 281.511}{12,06} \cdot 6,56=44.407 \mathrm{kNcm}=444 \mathrm{kNm} \\
& \mathrm{q}_{\mathrm{GzG}}=\mathrm{g}_{\mathrm{k}}+\mathrm{p}_{\mathrm{k}}=(56,3+8,7)+30,6=95,6 \mathrm{kN} / \mathrm{m} \\
& M=\mathrm{q}_{\mathrm{GZG}} \cdot \mathrm{L}^{2} / 8=95,6 \cdot 9^{2} / 8=968 \mathrm{kNm}
\end{aligned}
$$

Zur Berechnung des effektiven Trägheitsmoments $\mathrm{I}_{\mathrm{i}, \mathrm{eff}}$ muss der Abminderungsfaktor $\alpha_{c}$ berechnet werden:

$$
\alpha_{c}=\alpha_{\text {Mat }} \cdot \alpha_{Q S} \cdot \alpha_{M}=0,849 \cdot 0,373 \cdot 1,836=0,581
$$

mit

$$
\begin{aligned}
\alpha_{\text {Mat }} & =\left(\frac{f_{c t}}{f_{c t, 0}}\right)^{-0,154} \cdot\left(\frac{f_{c}}{f_{c, 0}}\right)^{0,146} \cdot\left(\frac{f_{a y}}{f_{a y, 0}}\right)^{0,152} \\
& =\left(\frac{0,29}{0,1}\right)^{-0,154} \cdot\left(\frac{3,0}{3,0}\right)^{0,146} \cdot\left(\frac{35,5}{35,5}\right)^{0,152}=0,849
\end{aligned}
$$




$$
\begin{aligned}
\alpha_{Q S} & =\left(\frac{I_{c, 0}}{I_{i, 0}}\right)^{0,06} \cdot\left(\frac{S_{i, 0} \cdot a_{S t}}{I_{i, 0}}\right)^{0,565} \cdot\left(\frac{z_{i, 0}}{a_{S t}}\right)^{0,15} \cdot\left(\frac{b}{L}\right)^{-0,125} \\
& =\left(\frac{177.896}{281.511}\right)^{0,06} \cdot\left(\frac{2.997 \cdot 28,5}{281.511}\right)^{0,565} \cdot\left(\frac{194}{28,5}\right)^{0,15} \cdot\left(\frac{3,5}{9}\right)^{-0,125}=0,373 \\
\alpha_{M} & =1,357 \cdot\left(1-\frac{M_{\text {Riss }}}{M}\right)^{-0,493}=1,357 \cdot\left(1-\frac{444}{968}\right)^{-0,493}=1,836
\end{aligned}
$$

Damit kann das effektive Trägheitsmoment $\mathrm{I}_{\mathrm{i}, \text { eff }}$ berechnet werden:

$$
\begin{aligned}
\mathrm{I}_{\mathrm{i}, \text { eff }} & =\mathrm{I}_{\mathrm{a}}+\alpha_{\mathrm{c}} \cdot\left(\mathrm{I}_{\mathrm{c}, 0}+\mathrm{S}_{\mathrm{i}, 0} \cdot \mathrm{a}_{\mathrm{St}}\right) \\
& =18.200+0,581 \cdot(177.896+2.997 \cdot 28,5)=171.183 \mathrm{~cm}^{4} \\
& =0,60 \cdot \mathrm{I}_{\mathrm{i}, 0}
\end{aligned}
$$

\section{(e) Mittendurchbiegung des Trägers zum Zeitpunkt $t=0$}

Die Mittendurchbiegung des Trägers kann mit Hilfe des effektiven Trägheitsmoments $\mathrm{I}_{\mathrm{i}, \mathrm{eff}}$ und den normalen Mitteln der technischen Biegelehre berechnet werden:

$$
\begin{aligned}
f_{0} & =\frac{5}{384} \cdot \frac{q_{G Z G} \cdot L^{4}}{E_{a} \cdot I_{i, e f f}}=\frac{5}{384} \cdot \frac{95,6 \cdot 9 \cdot 900^{3}}{21000 \cdot 171.183}=2,27 \mathrm{~cm}=22,7 \mathrm{~mm} \\
& =L / 396
\end{aligned}
$$

\section{A 3.3 Zeitpunkt $\mathbf{t}=\infty$ (nach Abschluss von Kriechen und Schwinden)}

Wie in Abschnitt 8.5 beschrieben werden die Verformungen infolge Kriechen und Schwinden elastisch berechnet. Hierfür müssen die elastischen Querschnittswerte zum Zeitpunkt $t=\infty$ berechnet werden:

\section{(a) Hilfsgrößen}

Die Kriechzahl ergibt sich für ein Belastungsalter von 28 Tage und eine trockene Umgebung gemäß DIN 1045-1 [1.8] zu $\varphi_{28 d}=2$,2, für ein Belastungsalter von einem Tag zu $\varphi_{1 d}=4,1$ und das Endschwindmaß für ein Belastungsalter von einem Tag zu $\varepsilon_{\mathrm{cS}}=-48 \cdot 10^{-5}$.

Des weiteren sind folgende Querschnittskenngrößen für die weitere Berechnung erforderlich:

$$
\begin{aligned}
& \alpha_{T}=\frac{A_{S t} \cdot I_{S t}}{A_{i, 0} \cdot\left(I_{i, 0}-I_{c, 0}\right)}=\frac{113 \cdot 18.200}{1.658 \cdot(281.511-177.896)}=0,0120 \\
& \alpha_{I}=\frac{I_{S t}}{I_{S t}+I_{c, 0}}=\frac{18.200}{18.200+177.896}=0,0928
\end{aligned}
$$

\section{(b) Elastische Querschnittswerte des Verbundquerschnitts für ständige Lasten}

Zunächst müssen die Kriechbeiwerte, Reduktionszahlen und Querschnittswerte des Betons berechnet werden:

$$
\begin{aligned}
\psi_{\mathrm{F}, \mathrm{B}} & =\frac{1}{1-0,5 \cdot \alpha_{\mathrm{T}} \cdot \varphi+0,08 \cdot\left(\alpha_{\mathrm{T}} \cdot \varphi\right)^{2}} \\
& =\frac{1}{1-0,5 \cdot 0,0120 \cdot 2,2+0,08 \cdot(0,0120 \cdot 2,2)^{2}}=1,013
\end{aligned}
$$




$$
\begin{aligned}
\psi_{\mathrm{I}, \mathrm{B}} & =\frac{1}{1-0,5 \cdot \alpha_{\mathrm{I}} \cdot \varphi+0,08 \cdot\left(\alpha_{\mathrm{I}} \cdot \varphi\right)^{2}} \\
& =\frac{1}{1-0,5 \cdot 0,0928 \cdot 2,2+0,08 \cdot(0,0928 \cdot 2,2)^{2}}=1,110 \\
\mathrm{n}_{\mathrm{F}, \mathrm{B}} & =\mathrm{n}_{0} \cdot\left(1+\varphi \cdot \psi_{\mathrm{F}, \mathrm{B}}\right)=6,56 \cdot(1+2,2 \cdot 1,013)=21,2 \\
\mathrm{n}_{\mathrm{I}, \mathrm{B}} & =\mathrm{n}_{0} \cdot\left(1+\varphi \cdot \psi_{\mathrm{I}, \mathrm{B}}\right)=6,56 \cdot(1+2,2 \cdot 1,110)=22,4 \\
\mathrm{~A}_{\mathrm{C}, \mathrm{B}} & =\mathrm{A}_{\mathrm{C}} / \mathrm{n}_{\mathrm{F}, \mathrm{B}}=10.136 / 21,2=478 \mathrm{~cm}^{2} \\
\mathrm{I}_{\mathrm{C}, \mathrm{B}} & =\mathrm{I}_{\mathrm{C}} / \mathrm{n}_{\mathrm{I}, \mathrm{B}}=1.167 .000 / 22,4=52.098 \mathrm{~cm}^{4}
\end{aligned}
$$

Damit ergeben sich die elastischen Verbundquerschnittswerte für ständige Lasten zu:

$$
\begin{aligned}
& A_{i, B}=A_{a}+A_{c, B}=113+478=591 \mathrm{~cm}^{2} \\
& z_{i, B}=A_{a} \cdot a_{S t} / A_{i, B}=113 \cdot 28,5 / 591=5,45 \mathrm{~cm} \\
& S_{i, B}=A_{c, B} \cdot z_{i, B}=478 \cdot 5,45=2.605 \mathrm{~cm}^{3} \\
& I_{i, B}=I_{a}+I_{c, B}+S_{i, B} \cdot a_{S t}=18.200+52.098+2.605 \cdot 28,5=144.541 \mathrm{~cm}^{4}
\end{aligned}
$$

\section{(c) Elastische Querschnittswerte des Verbundquerschnitts für Schwinden}

Die Berechnung entspricht dem für ständige Lasten beschriebenem Vorgehen:

$$
\begin{aligned}
& \psi_{\mathrm{F}, \mathrm{S}}=0,5+0,08 \cdot \alpha_{\mathrm{T}} \cdot \varphi=0,5+0,08 \cdot 0,0120 \cdot 4,1=0,504 \\
& \psi_{\mathrm{I}, \mathrm{S}}=0,5+0,08 \cdot \alpha_{\mathrm{I}} \cdot \varphi=0,5+0,08 \cdot 0,0928 \cdot 4,1=0,530 \\
& \mathrm{n}_{\mathrm{F}, \mathrm{S}}=\mathrm{n}_{0} \cdot\left(1+\varphi \cdot \psi_{\mathrm{F}, \mathrm{S}}\right)=6,56 \cdot(1+4,1 \cdot 0,504)=20,1 \\
& \mathrm{n}_{\mathrm{I}, \mathrm{S}}=\mathrm{n}_{0} \cdot\left(1+\varphi \cdot \psi_{\mathrm{I}, \mathrm{S}}\right)=6,56 \cdot(1+4,1 \cdot 0,530)=20,8 \\
& \mathrm{~A}_{\mathrm{c}, \mathrm{S}}=\mathrm{A}_{\mathrm{c}} / \mathrm{n}_{\mathrm{F}, \mathrm{S}}=10.136 / 20,1=504 \mathrm{~cm}^{2} \\
& \mathrm{I}_{\mathrm{c}, \mathrm{S}}=\mathrm{I}_{\mathrm{c}} / \mathrm{n}_{\mathrm{I}, \mathrm{S}}=1.167 .000 / 20,8=56.106 \mathrm{~cm}^{4}
\end{aligned}
$$

Damit ergeben sich die elastischen Verbundquerschnittswerte für Schwinden zu:

$$
\begin{aligned}
& A_{i, S}=A_{a}+A_{c, S}=113+504=617 \mathrm{~cm}^{2} \\
& z_{i, S}=A_{a} \cdot a_{S t} / A_{i, S}=113 \cdot 28,5 / 617=5,22 \mathrm{~cm} \\
& S_{i, S}=A_{c, S} \cdot z_{i, S}=617 \cdot 5,22=2.631 \mathrm{~cm}^{3} \\
& I_{i, S}=I_{a}+I_{c, S}+S_{i, S} \cdot a_{S t}=18.200+56.106+2.631 \cdot 28,5=149.290 \mathrm{~cm}^{4}
\end{aligned}
$$

\section{(d) Verformungen infolge Kriechen}

Für die Berechnung wird davon ausgegangen, dass $60 \%$ der Nutzlast ständig wirkt. Die ständige Last beträgt damit:

$$
q_{B}=g_{k}+0,6 \cdot p_{k}=(56,3+8,7)+0,6 \cdot 30,6=83,4 k N / m
$$

Die elastische Kriechverformung der ständigen Lasten beträgt damit:

$$
\begin{aligned}
f_{K} & =f_{0} \cdot \frac{q_{B}}{q_{G Z G}} \cdot\left(\frac{I_{i, e f f}}{I_{i, B}}-1\right) \\
& =22,7 \cdot \frac{83,4}{95,6} \cdot\left(\frac{171.183}{144.541}-1\right)=22,7 \cdot 0,87 \cdot 0,18=3,7 \mathrm{~mm}
\end{aligned}
$$




\section{(e) Verformungen infolge Schwinden}

Die Schwindnormalkraft und das Schwindmoment ergeben sich zu:

$$
\begin{aligned}
\mathrm{N}_{\mathrm{S}} & =\varepsilon_{\mathrm{cS}} \cdot \mathrm{A}_{\mathrm{c}} \cdot \mathrm{E}_{\mathrm{c}} \cdot \mathrm{n}_{0} / \mathrm{n}_{\mathrm{F}, \mathrm{S}} \\
& =48 \cdot 10^{-5} \cdot 11.995 \cdot 3200 \cdot 6,56 / 20,1=6.013 \mathrm{kN} \\
\mathrm{M}_{\mathrm{S}} & =-\mathrm{N}_{\mathrm{S}} \cdot \mathrm{z}_{\mathrm{i}, \mathrm{S}}=6.013 \cdot 5,22 / 100=314 \mathrm{kNm}
\end{aligned}
$$

Damit kann die elastische Schwindverformung mit Hilfe des Prinzips der virtuellen Kräfte berechnet werden:

$$
f_{S}=\frac{0,5 \cdot L \cdot M_{S} \cdot L / 4}{E_{a} \cdot I_{i, S}}=\frac{0,5 \cdot 900 \cdot(314 \cdot 100) \cdot 900 / 4}{21.000 \cdot 149.290}=1,01 \mathrm{~cm}=10,1 \mathrm{~mm}
$$

(f) Gesamtverformung zum Zeitpunkt $t=\infty$

$$
\begin{aligned}
f_{\text {ges }}(t & =\infty)=f_{0}+f_{K}+f_{S}=22,7+3,7+10,1=36,5 \mathrm{~mm} \\
& =L / 247
\end{aligned}
$$

\section{A 4 Weiterführende Hinweise}

\section{A 4.1 Teilweise Verdübelung}

Das vorgestellte Vorgehen gilt strenggenommen nur bei starrem Verbund zwischen Stahl und Beton. Bei teilweiser Verdübelung kann das Vorgehen angewendet werden, wenn der Nachweis geführt wird, dass die rechnerische Dübelbeanspruchung im Grenzzustand der Gebrauchstauglichkeit kleiner ist als die rechnerische Tragfähigkeit im Grenzzustand der Tragfähigkeit, vgl. DIN 18800-5 [1.5], Element (1027). Da bei Verbundträgern niedriger Bauhöhe und Slim-Floor Trägern der Verdübelungsgrad in der Regel vergleichsweise groß ist, ist diese Bedingung in vielen Fällen eingehalten.

In anderen Fällen kann eine Abschätzung der Verformungen durch Erhöhung der mit dem vorgeschlagenen Berechnungsansatz ermittelten Verformung zum Zeitpunkt $t=0$ mit dem elastischen Erhöhungsfaktor der teilweisen Verdübelung erfolgen. Wie in [2.4] dargestellt sind dabei die Langzeitverformungen infolge Kriechen und Schwinden nicht zu erhöhen, da diese durch eine nachgiebige Verdübelung kleiner werden.

\section{A 4.2 Eigenspannungen im Stahlträger}

Wie in Abschnitt 7.5.3 beschrieben können Eigenspannungen im Stahlträger zu einer Vergrößerung der Verformungen führen. Bei den durchgeführten Vergleichsrechnungen zeigte sich, dass bei Trägern mit Eigenspannungen kleiner $30 \%$ der Streckgrenze der Einfluss auf die Verformung vernachlässigbar ist. Bei höheren Eigenspannungen kann der Einfluss jedoch $10 \div 20 \%$ betragen. In diesen Fällen ist unter Umständen eine genauere Berechnung notwendig. 




\section{Lebenslauf}

Name:

geboren:

Familienstand:

1992

1992-1993

1993-1998

1998

1999

$1998-2000$

2000-2005
Andreas Rieg

20.03.1973, Schwäbisch Gmünd

ledig

Abitur am Scheffoldgymnasium, Schwäbisch Gmünd

Studium der Physik, Universität Stuttgart

Studium des Bauingenieurwesens, Universität Stuttgart

Abschluß Diplom-Ingenieur

Artur-Fischer-Preis der Universität Stuttgart

Tragwerksplaner bei weidleplan consulting $\mathrm{GmbH}$, Stuttgart

Wiss. Angestellter am Institut für Konstruktion und Entwurf, Universität Stuttgart 